



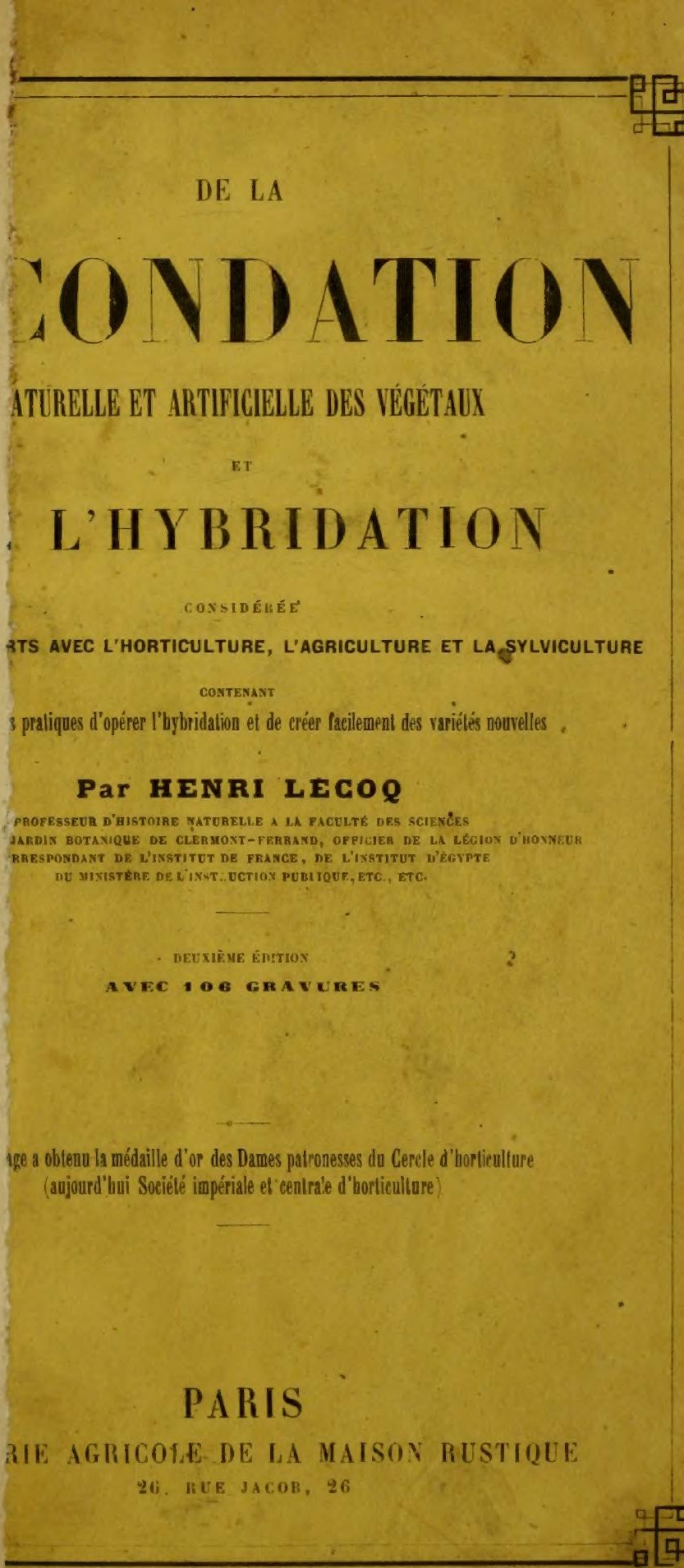



J)E I.A

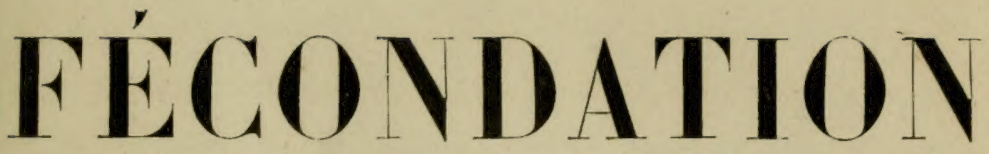
NATLRELLE ET ARTIFICLLLE DES VËGETALXX

E. $T$

\section{DE L'HYBRIDATION}

CกNSIDE์ E. E

DANS SES FAPPORTS AVEC L'HORTICULTURE, L'AGRICULTURE ET LA SYLVICULTURE

CONTENANT

Les moyens pratiques d'opérer l'byghridation et de créer facilement des variélés nourelles

\section{PAT HENRI LECOQ}

PROFESSEUR D'HISTOIRE NATURFLLE A LA FACEITÉ DES SCIEVCF

DIRFCTFUR DU IIRDIN BOTANIQUE DE GLERMOXT-FERRAND, OFFICIER DV LA LFGIOX D'HOVNELR. CORAFSPONDANT DE L'INSTITUT DE FRANCE, DE L'INSTITUT n'F́GYPTE

DU MINISIKAE DE L'INSTRUCTION PUBLIQUE, ETC.

MEบXIẼME ĖDITIOS

A VEC T G GRAVITES
- LiBRARY

NEW YORK

BOTANICAL,

OARDBN

Cet ouvrage a obtenu la médaille d'or des Dames patronnesses du Cerele d'bortienlture (anjourd'bui Société impériale el centrale d'borlicullure)

\section{PARIS}

LIBRAIRIE IGRICOIE DE LA MAISON RISTIOZE

26, RUE JACOR, 26

1862

Tuns droits réservés 


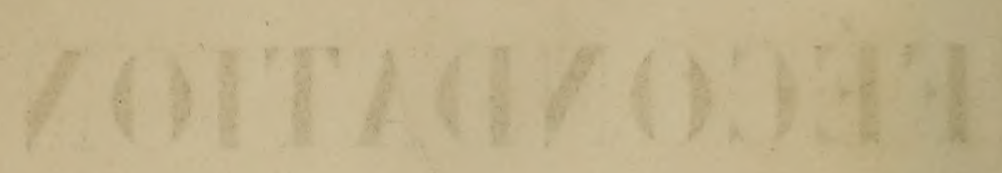

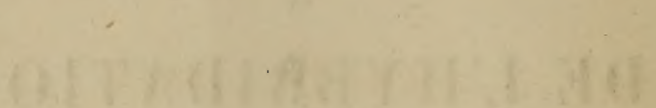




\section{INTRODUCTION}

Nous sommes arrivés à une épóque où la culture tend à sortir de la routine, dans laquelle l'ont si longtemps laissée l'ignorance et l'incapacité. Ceux qui se sont voués à la belle profession d'horticulteur ont senti la nécessité d'une instruction solide et variée, qui leur permit de baser leurs travaux sur une pratique éclairée et sur la connaissance exacte des grands phénomènes de la vic végétale. Aussi cette profession devient de plus en plus honorable, à mesure que des hommes plus instruits viennent y appliquer les lumières qu'ils ont reçues d'une bonne éducation.

C'est pour favoriser, autant que je puis le faire, cet élan général et ce progrès réel de la science des jardins, que je publie aujourd'hui mes observations sur la fécondation et l'hybridation des plantes. Un essai, je pourrais presque dire une première édition de cet ouvrage, parut en 1827, sous le titre de Recherches sur la reproduction 
des végétaux. Ce travail, présenté et soutenu comme thèse devant une école de Paris, dut subir nécessairement de nombreuses modifications; j’ai dû alors, dans celte thèse, upprimer la plupart des observations pratiques, et me contenter d'exposer les géneralités relatives ì la fécondation.

Depuis lors je n'ai cessé de recueillir des matériaux, j'ai cherché à réunir tous les faits que j'ai pu observer sur cette importante fonction des plantes. La forme, la position, la structure, les rapports des étamines et des pistils, relativement à l'acte si curieux et si varié de la re production, ont été l'objet de mes études; et je ne crois pas ètre entré dans une serre ou un jardin, ni avoir fait une promenade à la campagne, sans avoir ajouté quelques notions à celles que j'avais déjà acquises.

Un travail comme celui que je publie aujourd'hui devrait être le résultat d'une longue pratique et le résumé des tentatives de tous ceux qui se sont occupés de cette partie de l'horticulture. Malheureusement il n'a pu en être ainsi. L'hybridation, comme phénomène physiolo. gique, a bien été le sujet de quelques consciencieux el savants mémoires, mais pratiquement peu de personnes l'ont exercée, et encore parmi celles-ci plusieurs ont fait mystère des moyens qu'elles employaient, et surtout des procédés qui leur avaient le mieux réussi.

Je dois dire cependant que bien des améliorations se sont opérées depuis que j'ai publié, en 1845 , la première édition de ce trailé d'hybridation. J'ai lieu de croire que 
ce livre n'a pas été inutile à l'horticulture, et qu'il a pu justifier la haute récompense que le cercle d'horticulture de la Seine (aujourd'hui réuni à la Société impériale et centrale) lui a décerné, la médaille d'or des dames patronesses, comme étaint l'ouvrage le plus utile à l'horticulture.

Cette seconde édition, que je publie après seize ans d'observations nouvelles, pourra mieux encore guider tous ceux qui aiment les fleurs dans cette voie toujours nouvelle des créations.

La fécondation artificielle exige du temps et de la palience; il est donc presque impossible que le même individu puisse obtenir des résultats positifs sur un grand nombre d'espèces. D'un autre.côté, peu de personnes s'occupent de cette partie assez délicate de l'horticulture, sans y avoir un intérêt direct. Les amateurs qui, disposant de leur temps, devraient être les sectateurs les plus zélés de cette pratique, sont arrêtés dans les essais qu'ils voudraient faire par la crainte de mal opérer, quelquefois aussi parce qu'ils regardent cette opération comme plus difficile qu'elle ne l'est réellement.

I'ai essayé de diminuer les difficultés qui accompagnent l'opération si curieuse de l'hybridation, en décrivant les phénomènes physiologiques que présentent les organes sexuels, et en mettant ainsi chacun à même d'opérer ì peu près sûrement tous les croisements possibles. En ayant fait moi-même un grand nombre, et ayant d'ailleurs tenté tous les modes praticables, j'ai cru me rendre utile aux horticulteurs en publiant mes obser- 
vations, bien que je reconnaisse toute leur insuffisance. C'est ainsi que j'aurais désiré pouvoir mentionner un plus grand nombre de genres, mais il eût fallu les connaitre autrement que par des descriptions, et même de bonnes figures; il eût fallu les voir pour examiner leurs organes, savoir l'époque de l'épanouissement des fleurs, celle de l'anthèse, étudier enfin sur le vivant la physiologie de la fécondation. Je n'ai pu le faire pour une foule de plantes nouvelles, et j'ai voulu, autant qu'il était en moi, présenter un travail pratique, incomplet sans doute, mais contenant le moins d'erreurs possibles, tout en reconnaissant aussi la difficulté d'arriver à cet état d'infaillibilité auquel la science n'a pas le droit de prétendre.

Il sera, du reste, assez facile de suppléer à l'absence des détails relatifs à des plantes dont je n'ai pu parler, en se reportant aux genres qui en sont voisins.

Souvent le mode de fécondation est le même dans toute une famille, et la description d'une seule plante suffirail pour faire connaître ce phénomène dans le groupe tout entier.

Malgré mon regret très-fondé de n'avoir pu me procurer plus de matériaux, j'ai dù abandonner une portion de ceux que j'avais recueillis pendant plus de quarante années. J'ai laissé de côté toutes les notes qui n'avaient qu'un intérêt purement scientifique, et je me suis borné à celles qui concernaient les plantes le plus généralement cultivées. Je dois dire cependant que j’ai trouvé peu de documents dans la plupart des recucils qui sont consacrés 
ì l'horticulture. Ies notes, les mémoires relatifs ì l'hybridation y sont rares et clair-semés. D'um autru coite, jai en la satisfaction de trouver répétées dans quulques-mude ces mémoires la plupart des notions que javais indiquées dans ma première édition. C'étaient quelqurfois des observations isolées qui venaient confirmer les miennes, quelquefois des réminiscenees des faits yur javais publiés depuis longtemps.

Si j'avais voulu délayer ces notes anu lieu de les abréger, étendre mes descriptions au lieu de les restreindre, j’anrais pu publier deux gros volumes sur le sujat que j’ai essayé de traiter. J'ai préféré tout résumer dans le petil ouvrage que j'offre aujourd'hui à ceux qui s'occupent dr la culture des plantes. Ie me suis efforcé de le rendre aussi clair que possible, afin que les hommes pratiques ne soient pas effrayés par un étalage inutile de science of d'érudition. L'hybridation tonche aux plus hautes questions de physiologie végétale, et j’aurais pu peut-ètre, si f'inais cherché à les approfondir, ajouter quelques faits el quelques idées thioriques à ceux qui ont été émis sur er point, par le petit nombre de sarants qui s'en sont occupés. J'espere que les horliculteurs, dont le tempss est précieux, me sauront gré de ma réserve. J'ai cru que les mombreux amateurs pour lesquels le jardin est le délassement de tous les instints, de qui ne sont pas toujours initiés aux mysteres el au langage de la hotanique, appriciemient aussi mon désir de leur itre utile par ma concisint. 
Quelque restreint que soit un parterre, quelque exigu que puisse ètre le coin de terre dont un amateur peul disposer, que d'expériences utiles el d'essais curieux ì tenter, et que de jouissances à obtenir, quand, par une fécondation artificielle, il aura dolé son jardin, ses amis, son pays même d'une création nouvelle, qui devra le jour ì ses soins, ì sm intelligence! Que de plaisirs surtout pour celui qui, s'occupant de plantes de collection, rerra naitre presque à son gré, et chaque année, des nuances nouvelles, des coloris imprérus; qui verra les corolles grandir, ou les pétales se multiplier à l'infini! La satisfaction ne serit-1-elle pas aussi grande pour celui qui, se vouant à la cullure des arbres fruitiers, en perfectionnera les races, whliendra de nouveaux types, et sera le créateur de fruits ineommus qui, sans atteindre toujours la perfection des inciens, pourront cependant les dépasser aussi, et seront d'ailleurs, dans tous les cas, une conquête pour l'homme, 'fui souvent préfere le changement à la perfection?

L'hybridation ouvre une ère nouvelle au jardin potager; celui qui ne peut culliver qui ${ }^{2}$ Chou et un Xaret ne peut-il pas espérer de les croiser et de gagner de nouveaux légumes, comme celui qui ne possède qu'une bàche peut 'réer des espèces remarquables de Helons ou d'Ananas?

Si la science des jardins peut tirer un si grand parti de I'hybridation, l'agriculture n'a-t-elle pas aussi le droit de revendiquer sa part de ces végétaux, si utilement modifiés par l'homme? Quoique nous possédions plus de quatre cents vilriétés de froment, il est possible que nous n'ayoms 
pas encore le meilleur, et d'ailleurs ne peut-on pas ainsi obtenir des modifications nouvelles, des plantes dont les qualités soient mienx appropriées à tel sol, à tel climat, i telle convenance de celui qui les cultive; et hors des céréales, n’ y a-t-il jas mille perfectionnements à cherchru dans les espices fourragères, les plantes textiles, Jes olé:gineuses et tomtes celles enfin qui servent à nos besoins, it notre nomriture, ou qui alimentent notre industrie?

Liart forestier pent aussi tirer parti de ce levier puissant, el changer les arbres comme des végétaux herbacés. Une lécondation artificielle ne peut-elle modifier les Chènes, les Ormes, les Sapins, les Frènes, elc.?

Tous ceux qui aiment la culture, tous ceux qui tromvent du charme dans la contemplation des auvres du Créilteur, ne peuvent-ils pas se rendre uliles, de se procurer de vives jonissances, par des essais d'hybridalion? Comme noms l'avons vu, chacun peut agir dans sa sphère, dans son eoin, se taire s'il ne réussil pas, ce qui est rare, et s'enorgurillir, ì juste litre, si un gain remarquable ust venu couromner ses efforts.

Combien d'horlicultems marehands, surtout en Anghelerre el en Belgique; ont dhi leur réputation at leur forlune ì des hybridations operées avee arlesese, intelligence, et souvent aussi avec mystère!

Lavanlage que les ilrangers onl en sur nous lemial uniquement à l'emploi frópuent et raisonné qu'ils ont su faire de la fécondation artificielle. Dans ces contrées oì des hommes du plus grand mérite se sont voués an rom- 
meree des vigélaux, of oì un puissint intépè les joussait à la production de variétés nouvelles et lueratives, il n'est pas fommant quils aient obtenu des résultats i différents far leur valeur de ceux que le hasaril prísentait accidentellement à l'incapacité de quolques-ums de leurs collègues. Pourquoi les Français ne réussiraientils pas aussi bien? Le climat les favorise, l'instruction ne peut leur namquer, leur amour-prop̣re est un sûr garant de leur succès.

Les hỵbrides sont une preuve de la bonlé et de lir puissance de Dien, qui permet à l'homme de morlifier ses nurres, en se servant de l’intelligence divine qu’il lui a prêtíe pendant sa vie. Il est impossible de suivre les mulations suceessives d'un régétal, sommis aux influences variées de la culture el de l'hy̧bridation, sams itre pénétré de reconmaissance jour celui qui semblo céder ì l'homme une partie de ses droits, et qui l'autorise it soulever un faible coin du voile impénétrable qui rache tous les secrels de la création. Comment se fait-il qu'ì notre époyue, à Londres, l'ume des capitales du monle civilisé, des horticulteurs se soient élevés are: violence, dans une société créée pour étudier spécialement les mutations ou les changements que peurent subir les organes des végétaux, contre toute lentative deslinée à modifier les auves do Créateur? Est-ce présomplion, ignorance on intolírance religieuse, ou plulôl n'est-ce pas absence complète du sens commun che\% les membres de la sociéle morphologique qui, dans cello 


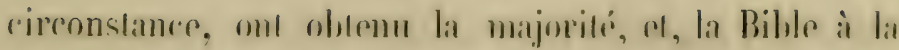

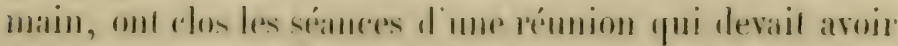
la plus hemrense influener sur lat science des champer el des jardins?

Si ces rélés sectaleurs de la céalion primilive chaient forcés de se conleuler, pour lígumes, de la Carotte des champes el des tiges durcies de la Chicorée saurage, el sils élatent condammés à so rafraîchir avec les fruits du Poirier ef du Pommier tols qüils sont sortis dles mains du Createur, of qüils existent encore dans nos bois, its arlmeftraient sans doute quelque restriction ì lemr vole ridicule, at recomuailnaient à l'auteur de la nalure le pumoir de faire le lien, en emploỵant toutefois des intelligenees supérieures à celle qu’ils mul montrée daus res pilovables discussions.

L'hỵhridation est done un levier d'ume puissance infinie, dont le Créateur a permis à l'homme de disposer pour son plaisir on son arantage. Il a donné à celui qui pourra s'en servir avec habileté le moxen de changer li forme du règne végétal, de développer sous diverses influences les organes des plantes, et de les transformer it von geré, pour les arlapter à ses besoins. Les hỵbrides, ou du moins les varialions que nous pourons oblenir, arrireraient pent-ilte un jour sur la terre, car penr la nature le lempes ne es lion, alle ne se hàle pas; mais l'hommes a

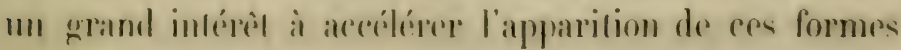
nowvelles, car le temps le pousse of l'entraine avere ume ineroyahle rapidile. Examinons en pen do mols les movens 
qu'il doit employer ponr arriver, le plus promptement possible dans cette direction, au but qu'il se propose d'atteindre.

Le premier point à obtenir pour faire varier les plantes est d'ébranler leur stabilité, ef de faire perdre à un végétal son habitude. Supposons un instant qu'une plante quelconque soit unique, on se pourra pratiquer l'hỵl)ridation, si tous les individis sont absolument semblables. Il faudra donc tâcher d'obtenir un changement quelconque dans cette plante, en semant les graines sous diverses conditions de climat, de température, de terrain, d'humidité, etc. Après plusieurs semis, il arrivera proiablement que quelques individus auront varié plus ou moins, quelquefois très-légèrement. Pour peu qu'ur mutation quelconque se soil opérée, il faudra recueillir la graine sur le pied qui présentera ce changement. Lal stabilité ou l'habitude étant un peu ébranlée, ces graines donneront sans doute des changements nouveaux. Ciest encore sur ces plantes que les semences seront choisies, et ainsi de suite. Il est rare qu'après plusieurs généralions on n'ait pas obtenu quelques modifications aux caractères naturels. Ils dépendent alors de phénomènes morphologiques, c'est-à-ilire que ce sont des changements de forme naturels sans hybridation.

Une fois parvenu à ce point, il faut eroiser, hybrider les variétés nouvelles, et d'autres leur succéderont. N'est-ce pas l'histoire si moderne des Dahlia, des Rhododendrum, des Azalea, des Achimeres et d'une foule d'aulres gemres? 
$\mathrm{Si}$, dis le principe, on a plusieurs mpèces voisines capables de s'hybloider, il sera inutite de chercher ou l'attendre des chamgements morphologiques; le croisement les donuera beaucoup plus vile; $\mathrm{d}$, quand une fois des races nouvelles auront applaru, il n'y a aucune raison pinur qui en suivant ces mème's procédés, on ne les multiplie pass indéfniment; pourvu, toutefois, que nons leme continuions nos soins, car si nous les abantonnons, elles dégéniment très-promptement, et retournent aux types saurages dont nous arons pu momentanément ébranler la stabilité, sans ponvoir leur communiquer une habitude nouvelle.

Les hỵbrides se produisent souvent dins la nature; ils somt gínéralement plus vignureux gue leurs ascendants. Ils dument des graines ordinairement fertiles et ils se ronserveraient, sans ancun doute, s'ils n'itailent pas abandommés it eux-mèmes, ot si l'habitude des lypes primitifs me tendait tonjours a reprendre ses droits.

La greffe vient alors au secours de l'homme qui veul. conserver le résultat do ses patientes recherches. Cetle admirable oprération est encore une hỵbridation; c'est le mélange de deux séves et de deux existences, el quoiqun jusyu'à frrésent on n'ait considéré pour ainsi dire le sujet que comme le support de la grefle, je ne doute pas qui il n'ait une grande influcnee sur lo bourgeon quion lui confie.

Cie serail sortir complétement de mon travail, que dexaminer ces conrieuses relations de denx ibres con- 
dammés ì vivre en enmmun, d'étudier leurs exigenees particulières el les modifications diverses qu'ils peuven mutuellement s'imprimer : je me contente de les rappeler ì l'attention des horticulteurs, et d'engager ceux qui venlent créer et multiplier les esprees et les variétés ì semer, hybrider et greffer, et à recommencer successivement les mèmes opérations. IIs seront eux-mêmes étonnés des résultats qui viendront progressivement s'offrir à leurs yeux comme récompense digne des efforts qu'ils auront tentés, et de l'intelligence avec laquelle ils auront essayé d'imiter la nature, en s'initiant à l'un de ses mystères les plus profonds.

Je répète ici ce que je disais au commencement de cette introduction; je publie un livre très-incomplet, je laisse de nombreuses lacunes à combler, un nombre infini d'expériences à faire. J'ai tàché de réunir en un seul faiscean les connaissances acquises sur ce point; chacun pourra concourir à l'augmenter, et je m'estimerais très-heureux si mes conseils et mon exemple pouvaient répandre des procédés qui permettent à l'homme de former lui-même, dans certaines limites, des êtres nouveạux dont le Créateur, dans sa bonté infinie, n'a pas même gardé le monopole.

Je n'ai plus qu'un mot à dire sur cetle seconde édition. I'ai dù y introduire quelgues changements, mais en petil nombre. En revanche, les atditions ont éte nombrenses; c'est un livre presque entièrement neuf par le nombre des faits nouveaux que j'y ai rapportés.

Ma première édition, accueillie avec heaucoup de hien- 
veillance, a reçu dans tous les journaux d'horticulture laceueil le plus llatleur; mais elle a élé vivement critiquic dans un rapport fait à la Société impériale el centrale par Poiteau et Lniseleur de Longchamps, ce qui n a pas empèché la Société de lui décerner une mention honorable. Iai dù répondre à des eritiques qui, dans ma pensée, pouvaient arrèter les horticulteurs dans la voie qui leur élait ouverte depuis longtemp̧s. Mais je n'ai pas cru devoir consigner ici cette réponse, car le temps et les résultats pratiques de l'hybridation ont fait justice des ubjections qui m’étaient alors adressées. 



\section{ORIORE DES CHAPITRES}

Cinarine 1. - Féconmatos saturelle. Des differents modes de fécondafion que l'on observe dans les plantes... . I

11. - Ve L espèce et de ses vahiations. . . . . . 16

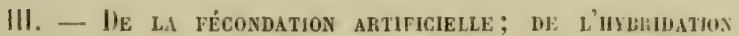
ET IES MOYENS DE L'OPÉnER . . . . . . . 23

lleurs hermaphrodites. . . . . . . . 2i

Fécondation directe et indirecte, inégalité de développement des organes. . . . . . . . . 2li

Fleurs monoïques. . . . . . . . . . . 28

kleurs dioïques. . . . . . . . . . . . 50

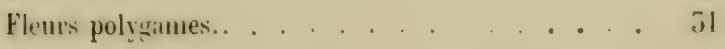

Choix des sujets.............. . . 51

De la couleur des variétés. . . . . . . . . ii.

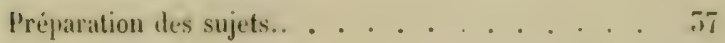

Opérations relatives a la fécondation artificielle.. . . Il

Castration on enlevement des élamines. . . . . 位

I'ullen. - Cunservalion. . . . . . . . . . . iti 
De lapplication du pollen ou de la fécondation frowprement dite. . . . . . . . . . . . . 1!

Action du pollen. - Sa struclure. . . . . . . jz

Des nectaires et les insectes. . . . . . . . bil

Quelques considérations générales sur les livbrites. . lit

IV. - Dicotrlédones thalamilohes, ou plantes a pitiles distincts insérẻs sur le réceptacle. . . . . . . S"

Y. - Jicotylébones calyciflones, ou plantes à prilites libres ou plus ou moins soudés, insérés sur le calice..................... 100

V. - Dicotylédones conolliflores, ou plantes à pétales soudés en une corolle hypogyne.. . . . . . 261

VII. - Dicotilédones monochlamydées, oll il périgone simple. . . . . . . . . . . . 249

VIII. - P'lantes monocotrlédones. ......... Jjét

(Voir, à la fin du volume, la table alphabétique des genres et des familles.) 
. 


\section{.}




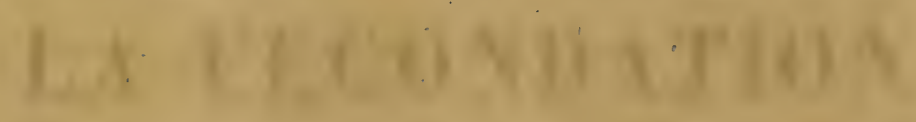

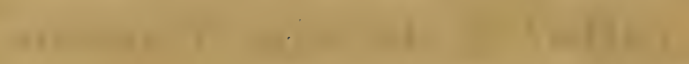

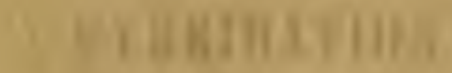

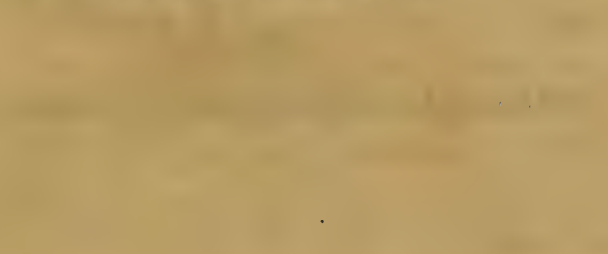

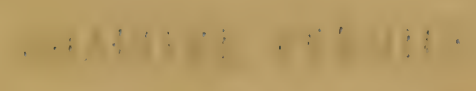

$$
+\quad \cdot, \quad, \quad .
$$

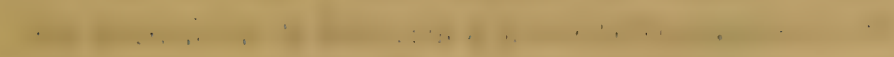
$\ldots \quad \cdots, \ldots, \ldots$ . $, \quad, \quad+$ 

l)

\section{LA FECONDATION}

NATURLLLE ET ARTIFICILLLA DES VEGETAUX

L. TH:

\section{L'HYBRIDATION}

C ONSI DẺ II É:

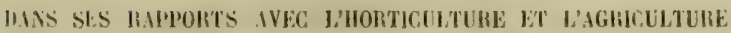

\section{CHAPITRE PREMIER}

FECONDATION NATURELLE.

WES DHFÉRENTS MODES DE FÉCOXDATIOX QGE JUN OLSERE DANS LES PLANIES.

Les fonctions de la fleur sunt d'opérer la fécondation, el de pourvoir de cette manière à la conservation de l'espèce.

Chacum des organes qui la composent est appelé à concourir à cet acte. Ie pistil, ou organe femelle, offre à sa base l'ovaire yui contient les ovules destinés à former les graines après la fícondation, et à sa partie supérieure le stigmate qui doit recevoir le pollen. Les élamines présentent l'anthère qui contient le pollen, dont les fonctions sont de vivifier les ovules en pénétranl dans l'oviare par le sligmate. Le style sourent placé entre l'oraire et le stigmate, les filets qui soutiennent presque toujours les antheres, semblent destinés les uns et les autres à pla- 
cer les organes quils supportent à la hauteur la plus convenable pour que le contact du pollen avec le stigmate puisse avoir lieu.

Le périgone simple ou double entoure les organes sexuels, el sert à les abriter, à les garantir des vents et de la pluie; peut-être aussi, comme le pensait Bernardin de Saint-Pierre, à réfléchir de diverses manières la lumière et la chaleur du soleil;

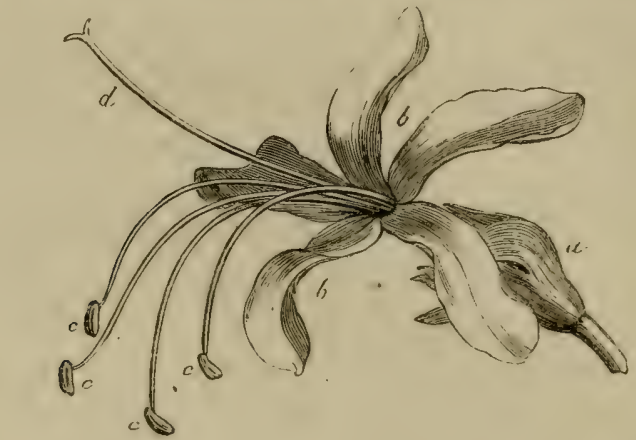

Cirav. 1. - Fleur de clerodendron. - $a$, calice. $-b b$, corolle. ccec, étamines. - $l$, pistil.

il sert aussi à la fécondation, en recevant le pollen el le transinettant quelquefois au stigmate.

Ainsi tout ce brillant appareil, tout ce luxe de cilices, de corolles admirablement nuancées, concourent an même but, à cet alte important qui assure la conservation de l'espèce en

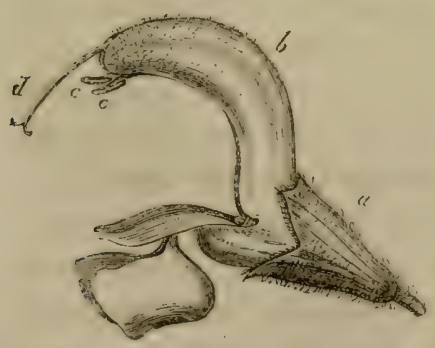

lirut: 2. - Hleur de sauge. - u, calico. l, corolle. - ce, étamines - $d$, pistil. multipliant à l'infini les individus qui la composent.

Pour que la fécondation ait lieu, il est nécessaire que le pollen se trouve en contact avec le stigmate (grav. 1 et 2 ).

L'enlèvement d'une partic du stigmate n'empêche pas toujours la fécondation d'atvoir lieu; mais, si on l'enlève mtièrement, les ovules restent inféconds. Plusients fois j’i (n- 
levé le stigmate de la Couronne impériale, du Lis, de la Bellede-muit, elc., avant la déhiscence de's anthères, et les graines sont restées stériles, tandis qu'en conpant la moitié des styles phumeux des Graminées et particulièrement du Mais, la fécondation avait également lieu, parce que les spongioles pistillaires, an lieu d'ètre disposées en tete comme dams le Lis, lit Belle-de-nuil, ete., sont siluées latéralement sur le style.

Lat nature a employé, pour assurer ce contact, soit entre urganes de lit même fleur, soit entre étamines et pistils do fleur's différentes, des moyens qu'on ne pent se lasser d'adunirer. Quoique la majeure partie aient été décrits, je les rappellerai sommairement pour en faire voir la diversité.

Lorsque les étamines et les carpelles sont réunis dans lia mème fleur, les étamines placées autour de ces derniers ont leurs anthères situées de manière qu'elles touchent ou aroisinent le stigmate. Si ce dernier se troure placé au-dessus, ses livisions, sonvent réfléchies sur les étamines et les fleurs penchies, permettent an pollen de s'arrèter sur l'organe femelle. La sligmate en outre sécrète souvent me humeur visqueuse qui retient les grains du pollen. Ceux-ci ne tardent pas à éclater: lia liqueur et les gramule's qu'ils renferment pénétrent dans liavire et vont porter la vie aux orules.

Le mode de dihiscence des inthères contribue aussi à assurer le contact du pollen aree le stigmate. Blles s'ourrent presque tnujours du còti du pissil, et cette déhiscence a souvent lieu subitement; les antheres sourrent par des pores placés à leur sommet dians les Solanum, a leur base dans les Pyroles, par de's valuules dans les Lumier's, les Berberis, l'Épimede des Alpes; elles se fendent longitudinalement dans le Lis, lat Tulipe; transversalement dans la Latemule, ete. Outre ces difleirents modes de déhiscence, elles affectent encore des mourements très-sensibles qui tendent toujours it diriger le pollen vers le stigmbile. Hams le Lis snperbe ef qundques autres espèces du mime genre, les anthères, dis qu' elles commentent it s'ourir, deviement mobiles sur leurs filets, of s'approchent sensi- 
blement du stigmate l'une après l'autre, puis s'en éloignent presque aussitòt qu'elles ont répandu leur pollen sur cet organe.

Dans la Tulipe, les anthères, fixées latéralement sur leurs filets, deviennent horizontales, et tournent visib!ement sur leur pivot à l'époque où elles répandent le pollen. Desfontaines a fait la même observation sur l'Amarillis formosissima et sur plusieurs Puncratium.

Les étamines des Rues, du Butome ombellé, riennent successivement une à une, deux à deux, trois à trois, appliquer leurs anthères contre le stigmate, et s'en éloignent cusuite pour reprendre la place qu'elles occupaient primitivement.

Dans les Dictames, les Capucines, les filets se courbent pour rapprocher les anthères du stigmate à l'époque de leur déhiscence. Dans la Belladone, les étamines d'abord courbées se redressent ensuite, et répandent leur pollen quand elles sont à lat hauteur du pistil.

Dans les Kalmiu, les dix étamines out leurs anthères placées dans des pelites fossettes de la corolle, et l'on voit le filet se courber en are pour retirer l'anthère, à l'époque où elle doil s'ouvrir.

Dans la plupart des Renonculacées, les étamines sont serrées: contre le pistil, et s'en écartent successivement après leur déhiscence, comme si elles étrient derenues inutiles.

Dans les Asarum, les filets sont courbés el se redressent deux i deux pour amener les deux anthères ver's charjue stigmate, dont le nombre est précisément la moitié de celui des étamines.

Dans quelques plantes, telles que les Ciste's, l'Epine-rinetle, on détermine à volonté les mouvenents des étanines, en less irritant avec la pointe d'une épingle.

Dans l'Ortie diö̈n̨u, le Murrer, ete., les lilets sont plie's en arc, et maintenus dans cette position par les parois du calice. Lorsque le soleil parait le matin, le calice s'ourre, et l'on voil toutes les étamines se redresser tout à coup, et lancer au loin leur pollen. 
Les organes femelles ne présentent pas à l'époque de la lécondation des mourements aussi marqués que les étamines; cerpendant les styles et les stigmates de quelques plantes paraissent aussi doués d'une certaine irritibilité, comme Desfontaines la fait observer pour les Passiflores, les Épilobes, les Nigelles, les Mimules, ie Lis superbe, etc.

On observe souvent d'assez grandes disproportions entre lit longueur du style et les filets des étamines; et, quoique généralement les fleurs dont le style dépasse les itamines soient penchées, tandis qu'elles sont droites quand l'inverse a lieu, un trouve pourtant un grand nombre d'exceptions à cette règle.

Mais, dans ces exceptions, il arrive que la fécondation sopère, soit avant l'épanouissement, soit lorsque la corolle se détache pour tomber. Dans le premier cas, les anthères s'ourrent avant la fleur, et le contact a eu lieu quand celle-ci s'épanouit : rest ce que l'on remarque daus les Verbascum, les Campamul's et la majeure partie des Synathérées. Dans ces deux dernières familles, les stigmates, en s'allonıeant dans le tube staminilëre, enlèvent le pollen qui doit féconder leurs ovules; dans le second cas, la fécondation n'a lien que lorsque la corolle vient à tomber, et c'est presque toujours dans les fleurs à pétales soudés que nous en trouvons des exemples. Le jollen tombe dans le fond de la lleur, et se rassemble dans les poils dont la gorge est garnie, en sorte que le stigmate se trouve nécessairement en contact aver le tulie de la corolle quand celle-ci se détache, et sourent même elle est retenue par cet organe, autour durguel elle se dessèche et finit par toniher en l'entrainant dans sal chute. On peut olserver ce mode de fécondition sur un yrand nombre de Véroniques.

Plusieurs plantes, dont les anthères sont extrorses, profitent. anssi du secours du périgone. Ainsi dans les Iris, les Aristo. loches, etc., les anthicres sourrent en dehors, et le pollen est recueilli sur les parois du périgone, sourent garnies de puils on l'aspérités; quand le périgone commence à se flétrir, si le pollen en réchant niest pas déja tombé sur le stignate, il s'y 
trouve appliqué par les enveloppes florales, qui, dans ces exemples, se dessèchent et se roulent plus ou moins sur elles. mêmes avant de tomber.

II s'en laut de beaucoup pourtant que la fécondation soit aussi certaine dans les fleurs à anthères extrorses que dans les autres; et nous en avons des preuves dans les Aristoloches, dont mm grande partie des fruits avorte ordinairement.

Dans un assez grand nombre de plantes, les périgones sont garnis de poils plus ou moins nombreux qui concourent à la fécondation en recevant le pollen des étamines, et le transmettaut au pistil placé sur un des bords, et ordinairement du mêne côté que les étamines, de manière à en pouvoir recevoir directement le pollen. C'est ce qui a lieu dans presque toutes les fleurs non symétriques des Labiées, des Persommées, etc.

Dans les diverses espèces de végétaux que nous venons de citer, le pollen est à l'état pulvérulent, et rien alors ne s'oppose ì la fécondation; mais il en est d'autres où il est plus ou moins épais, quelquefois même solide, et dans ce cas il est plus difficile de voir son contact avec le stigmate.

Dans les Asclépiadées, le pollen est en petites masses contenues dans les anthères; mais, celles-ci étant ordinairement appliquées sur'le stigmate lui-même, rien ne peut s'opposer à la fécondation.

llans le Laurier-rose, le pollen est composé d'une multitude. le petits grains agglutinés qui ne sortent pas toujours des anthères. Mais, si l'on examine les étannines avant l'épanonissement de la fleur, on voit que chaque anthère est en communication avec le stigmate par un petit prolongement placé entre les deux loges, et, si l'on veut séparer avec force ces deux organes, il reste sur le stigmate cinq petites protubérances dues au déchirement de ces appendices.

Il parait que dans ces plantes les grains de pollen se vident et fécondent le stigmate sans sortir de l'anthère; en sorte qu'il y a un véritahle accouplement. Dans plusieurs lleurs pourtant on voit distinetement des grains de pollen qui sortent, par une 
fente longitudinale de l'anthère, et se trouvent placés immédiatement sur le stigmate, comme daus les Orchidées.

Dans cette dernière famille, le pollen offre plusicurs modifications : tantòt il forme des masses composées de grains solides, réunis entre eux par une matière élastique, cemme dans les Orchis, les Ophris; tantôt ces masses sont tout ì fait granıleuses, comme alans les genres Epipuctis, Loroglossum, ete., ou hirn elles sont formées par une substance solide et rompacte, comme dans les genres Malaxis et Corallorliza.

Dans l'une on l'autre de ces plantes, il est nécessaire cependant, pour que la léeondation ait licu, que le pollen se trour 'n contact avec le stigmate; mais il faut remarquer que dans cetle famille les étamines sont épigynes, et qu'elles ne sont jamais appliquées contre lo stigmate, comme dans les Asclépiadées. Sil'on suit attentivement. les phases de la floraison de ces plantes, on voit qu'à une époque quelconque qui n'a rien dr. lixe, les masses polliniques sortent des anthères, et plusieurs d'entre elles tombent sur les stigmates visqueux qui ramollissent le corps élastique auquel adhère le pollen, et retiennent celui-ci sur leur surface. Souvent mème, comme on peut l'olss'rver sur l'Orchis bifolia, les masses polliniques sortent enlièrement, et s'accrochent, soit au stigmate, soit aux diverses parties de la fleur, par un petit disque visquenx qui se trouve i leur hase. Tantit e'est avant la floraison, ou lorsqu'elle commence, que les masses polliniques se détachent; tantôt elles restent dans l'anthère jusqu'à ce que le périgone, en se desséchant, les chasse vers le stigmate.

Il s'en fiut de beaucoup pourlant que toutes les fleurs des Orchis sorent fécondées; car, si l’on examine attentivement les pieds qui ont flenri, on voit ordinairement avorter une partie des fruits; si l'on recueille les graines de cenx qui paraissent sains et lécondés, el qu'on les sème dans les circonstances les plus farorables a leur germination, il n'y en a jamais qu'un très-petit nombre qui lève: ce qui tendrait à prouver que les fleurs sur les stigmates desquels le pollen s'est ar- 
rêté sont les seules qui puissent donner des graines fertiles.

On voit d'après cela que l'antra seminalis n'est pour rien dans la fécondation de ces plantes, et qu'il ne peut remplacer le contact du pollen.

D'autres faits tendent encore à prouver que le contact immédiat du pollen est nécessaire pour obtenir des graines fécondes. Tous les botanistes savent que les plantes aquatiques viennent épanouir leurs fleurs au-dessus de l'cau, et que la fécondation a lieu dans l'air: c'est dı moins ce que nous remarquons dans celles dont le pollen est pulvérulent, comme les Huttonia, Myriophyllum, Potamogeton, Nymphxa, Vallisneria, etc. Quand des plantes aquatiques fleurissent sous l'eau, et qu'elles sont munies d'enveloppes florales, ces dernières sécrètent de l'air, et la fécondation est accompagnée des mèmes circonstances que dans l'atmosphère, comme on peut l'observer dans le Ranunculus aquatilis, I'Alisma natans, Illecebrun verticillutum, le Pilularia, etc. Il existe pourtant des plantes aquatiques dans lesquelles les enveloppes florales manquent; il en est d'autres dont les fleurs mâles sont séparées des fleurs femelles, et qui pourtant restent constamment plongées sous l'eau; mais, si l'on examine ces plantes, on verra que dans la plupart le pollen est liquide, ou au moins capable d'être dissous et entrainé par l'eau. Or il est tout aussi facile de concevoir le transport d'un pollen liquide ou dissous par l'eau, que d'admettre la dissémination d'uu pollen pulvérulent par l'air, comme cela a lieu dans les plantes dioïques. On sait du reste que beaucoup d'animaux marins sont aussi fécondés à distance et dans l'eau.

Dans celles de ces dernières plantes qui fleurissent dans l'air, le pollen est quelquefois plus fin que dans celles où les sexes sont réunis; il est aussi plus abondant, et les fleurs femelles sont ordinairement rassemblées en cônes ou en épis munis de bractées. Ces circonstances farorisent beaucoup le contact du pollen; il arrive pourtant assez souvent, comme nous le verrons par la suite, que ce contact n'a pas lieu, et que les "riaines sont stériles, et c'est, je pense, ce qui arrive aussi anse' 
fréquemment pour les plantes aquatiques dont les sexes sont séparés.

Examinons maintenant quelques rapports qui existent entre - l'inflorescence et la fécondation, soit dans les plantes monoïques, soit dans celles dont les sexes sont réunis dans la mème lleur.

On dit communément que dans les plantes monö̈ques les lleurs màles sont presque toujours placées au-dessus des fleurs femelles, et le lait est généralement rrai; mais, si l'on considère la manière dont la fécondation s'opiere, on trouvera de grandes différences, dont la cause sera dans le mode d'inflorescence of dans l'époque de l'épanouissement.

Les lleurs mâles peuvent être placées à l'extrémité d'un rarmieau, et les fleurs fernelles au-dessous, comme dans l'Aune, le Toisetier, cte., et, dans ce cas, ces fleurs doivent être fécondées: par les lleurs males, sous lesquelles elles se trourent; mais li mène chose n'a pas lieu dans toutes les plantes. Dans les l'ins, Ies rạmeaux sont disposés avec assez de régularité, et tous, ainsi que clacune de leurs divisions, présentent à leur jartic supérieure une ou plusicurs fleurs femelles placies un peu andessous du sommet. Les écailles qui les entourent forment un petit còne propre à recevoir le pollen et ce petit cône est dirigé par en liant. Les fleurs mâles que portent les rameaux sont tonjours latírales et situées au-dessous des fleurs femelles de la mème branche, en sorte que les lleurs mâles du ramean supérieur correspond'nt aux fleurs femelles du rameau rui se trouve an-dessous, les fleurs màles de celui-ci i la branche quil recouvre, et ainsi de suite.

Faisant ahstraction des causes qui dispersent ou dévient le pollen, celui d'une branche tomberait par son propre poids sur Ir pistil de la fleur femelle qui se trouse sur la branche qui lui est inférieure, et ainsi de suite. Chaque branche prise isolémen! féconderait et serait fécondée; mais elle aurait besoin du concours des autres branches. Or, comme on peut, avec quelque raison, regarder chaque rameau d'un arbre comme un indi- 
vidu parfait, mais seulement adhérent à l'ensenble qui constılur l'arhre entier, ces plantes pourraient être considérées comme dioïques.

Ce mode de fécondation, bien remarqusble dans un granı nombre de Conifères, se présente d'une manière plus frappante, et far une autre cause, sur quelques plantes monoïques, et notamment sur les diverses variétés de Courges et de Concombres. Quoique ces plantes portent sur le même pied des lleurs miles et des fleurs femelles, il arrive sonvent que ces lleurs ne s'épanouissent pas en mème temps, et la fécondation a lieu rioïquement, quoique les plantes soient monoïques. I'ai raltivé, il y a quelques années, une variété de Cucurbita pepo, dont je n'avais qu'un seul pied, et dont je n'ai pu obtenir d. fruits à cause de cette alternance des fleurs mâles et des fleurs femelles.

Dans la Pimprenelle (Poterium Sanguisorba), les fleurs, rilrement hermaphrodites et presque toujours unisexuées, sont disposées en capitule et terminent chaque rameau. Presque toutes les lleurs dı capitule supérieur sont mâles. Les étamines sont pendantes; les fleurs du capitule inférieur sont en partie mâles, en partic femelles : ces dernières sont toujours placées au sommet, et les mâles à la base du capitule. Il serail naturel de supposer que les fleurs femelles de chaque capitule sont fécondées par les mâles de ce même capitule; mais on remarque bientôt la difficulté de ce mode de fécondation, en observant la situation inféricure des fleurs mâles et leurs étamines pendantes; et l'on voit qu'elle est impossible, si l'on fait atlention que les fleurs femelles d'un capitule sont flétries quand les mâles s'épanouissent. Il faut admettre ici la même chose que dans les Pins: les fleurs femelles des capitules inférieurs sont fécondées par les étamines des capitules qui leur sont supérieurs, et ainsi de suite (grav. 11, page 52).

Il y a pourtant queliques circonstances où, les fleurs mâles étant au-dessous desfleurs femelles, la fécondation a licu conme à l'ordinaire; c'est quand les fleurs sont disposées en épis moi- 
lié màles, moitié fernelles, el que les épis sont penchés commur dans le Carex gracilis et plusieurs autres.

C.e que nousvenons d'observer diuns plusiens plantes monoïgues, nous le retrouvons aussi dans yuelques plantes hermaphrodites, et notamment dans les Graminées.

Les fleurs de ces plantes sont disposces en épi on en panicule. n re sont toujours les fleurs inlérieures qui s’épanouiscent les premières.

Si nous prenons le Phleum ou le Seigle pour expmple, nous verrons les étamines de la premiere fleur qui sourrira rester pendantes, de manière it rendre la lëcondation très-difficile: mais les rtamines de la fleur qui se troure immédiatement andesists sont également pendantes, et leurs anthires se trouvent justement placées dans la fleur inférieure, ou du moins d'une maniere tris-propre a faroriser le contact de leur pollen avee le stigmate de la première fleur. A mesure que la floraison s'opèr, les lleurs sont snccessivement fécondées par celles qui sont placées immédiatement all-dessus.

lans les Graminies à panicule, les épillets offrent la mème disposition que les épis; mais, en outre, ils sont placés entre rux ì peu pris comme les rameaux des l'uis, en sorte que lit fírondation est plus certaine encore que dans les fleurs tout it fait hermaphrodites ${ }^{2}$.

Fuliu, on retrouve encore fréquemment la féeondalion indireete quand les fleurs sont disposées en ombelles, en corymbrs 011 en capitules.

Dans les diffirents rxemples que je viens de riter, le pollen

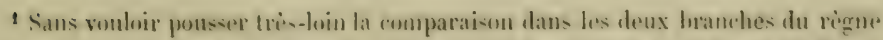

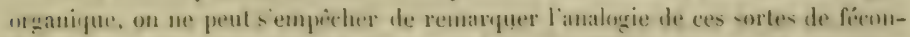

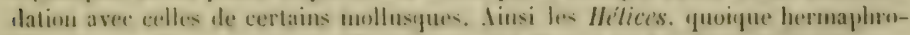

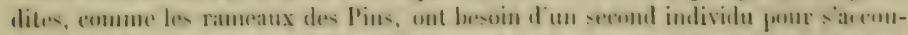

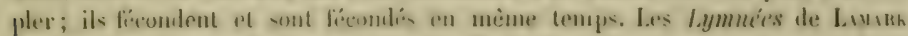

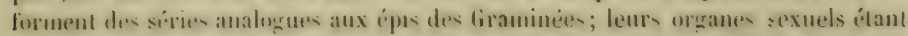

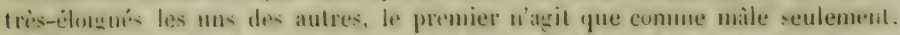

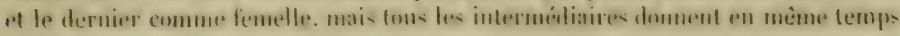
'pu'ils recoivent. 
des fleurs inférieures est entièrement perdu, et les fleurs supérieures des épis, des capitules supérieurs et du rameau terminal, doivent nécessairement rester stériles: c'est ce qui a lieu quelquefois, mais rarement, parce que deux causes s'y opposent.

La première, c'est que ces plantes, telles que les Pins, les Grrminées, les Joncs, la Pimprenelle, cte., vivent en sociétí, et peuvent, par conséquent, très-souvent ètre fécondées dioïquement.

La seconde, c'est que les vents, les insectes et un grand nombre de causes secondaires et accidentelles peuvent changer. la direction du pollen, le dévier de sa route, le transporter is de grandes distances; et qu'ensuite sa quantité est si considérable, que le hasard pent hien aussi aroir quelque part dans res fécondations.

Mais, parmi les morens dont se sert la nature pour accomplir toutes ces fécondations indircctes, le plus fréguent et le plus sùr est, sans contredit, le transport du pollen par les insectes. C.e sont les véritables méliateurs dans les mariages des fleurs.

Leur rôle est souvent indispensable dans l'acte de la fécondation. Une foule de plantes exotiques restent stériles dans nos serres, parce que nous n'avons pas importé arec elles les insectes étrangers qui butinaient sur leurs fleurs. Si, par une ruse innocente, nous promenons douccment un pinceau sur ces fleurs; si, pénétrant jusqu’au fond du calice, nous offrons aux fiancés abrités sous les tissus des corolles la coupe du nectar qui doit sceller leur union, nous avons plus tard la presue y y'un être virant, appartenant à un autre règne, manquait à la consécration de l'hṛménée.

Que deviendraient ces plantes diöques dont le pollen, visqueux comme celui des Saules, ne peut ètre emporté par le vent, si des légions dinsectes qui éclosent en même tempss que ces fleurs printanières ne leur prêtaient le concours de leurs ailes et leur turbulente vivacité? 
Que lon me permette de citer ici, an sujet des insectes, un paragraphe d'un ourrage récenment pullié'.

"Un papillon aux ailes blenes voltigeait au milieu des hranches et cherchait i prendre son essor au-dessus du taillis. Je le suivais des yeux. Peul-être, me disais-je, innocent messager, il porte aree lui les soupirs d'annur d'une fleur isolée. Sans doute jarais deviné, car bientôt, descendant sur le bord d'un ruissean aux rives lleuries, je vis ses ailes de saphir s'étaler sur la corolle rose et étoilée d'un Lychnis. On sait que dans celte plante les sexes, séparés, vivent souvent isolés à de grandes distances. C'est ainsi que l'insecte que nous voyons hourdonner dans les champs pent ètre l'ambassadeur d'une noble fleur exilće, et peut, à notre insu, transporter les plus. importants et les plus doux secrets de la nature.

"Ce rôle mystérieux accompli pendant tout l'éclat du jour par ces petits ètres bruyants, à locomotion si rapide, se renouvelle plus lard prur ces fleurs limides qui n'osent braver les rayons du soleit, qui attendent la nuit pour eacher leurs amours. Aussi d'autres acteurs se montrent dès que le crépuscule arrive. Arant l'apparition des planètes brillantes qui précèdent sur la route du firmanent les scintillantes ètoiles, les Sphinx au corps innelé ef aux ailes rapides sortent de leur retraite et rolent en hourdonnant. Leur trompe, roulée en spirale, s'étend dins toute sa longurur. C'est une pompe aspirante qui descend jusqu'au fond des fleurs et qui fonctionne pendant que le Papillon, soutenu par l'invisible et mipide mourement de ses ailes, reste un instant immobile devant la fleur qu'il il choisie. Quelle abondance de vie! quelle chaleur développér par cel incessant mourement! Singulicre existence, un mois daus l'wul, trois mois en chenille, sept mois en chrysalide, un mois a peine de vie aérienne, de viritable vie; pendant ce peu de jour's, une ou deux heures rhaque soir da itation fébrile,

1 La Vie des Fileurs, par 11 . Lecoq, 1 vol. in-18, 5 fr. 50 . (70 tableau, les lleurs s'épanouissent, les amours voltigent près d'elles.) 
d'existence poussée à l'excès; puis un repos absolu jusqu'au retour du crépuscule. Voilà la vie du Sphinx, du plus beau et du plus vif de tous les Lépidoptères. Pendant cette existence animée, il courtise mille lleurs différentes; il enlève sur son trompe quelques grains de poussière fécondante, et dans sa turbulente in!quiétude il distribue sans discernement ces étincelles de vie qui ne sont pas toujours accueillies.

" $\Lambda$ la nuit close, quand les étoiles brillent de tous leurs leux, ct surtout si la lune s'élève argentée aı-dessus des arbres de lat forêt, des Phalènes aux larges ailes volent doncement sur les fieurs nocturnes, cachant aux yeux des hommes les charrriantes peintures dont elles sont ornées. Puis les Yoctuelles, plus variées que les Papillons du jour, aux nuances délicates et fondues, aux ailes d'or ou d'argent, voltigent à leur tour sous la feuillée, descendent butiner sur les Bruyères fleuries, cherchent le 'niel des corolles, puis s'endorment de fatigue sur des lits parfumés d'amour. Que se passe-t-il dans ces royages, dans ces ébats des Papillons des nuits? Nous ne pourons les suivre. N'estil pas à craindre, malgré les jeux brillants de ces élégantes Noctuelles, qu'elles ne favorisent d'inutiles ou de coupables liaisons?... Mais un nuage passe sur la lune et eache sa humière; il est nuit close, laissons les fleurs et les Papillons débattre entre eux leurs mystères d'amour.

"La nature offre aus insectes, pour prix de leurs messages, le nectar parfumé servi dans les vases les plus somptueux au milieu de ces féeriques palais.

"Si les parfums et le nectar attirent leur's brillantes légrions sur les fleurs du printemps, d'autres émanations appellent d'autres tribus et d'imnombrables essaims. Certaines espèce's resteraient infécondes sans leur concours. Je citerai seulement les Raflesia, ces curieuses productions des climats chauds de l'Asie. Ces lleurs gigantesques et solitaires, dont les sexes séparés sont toujours situés trés-loin les uns des antres, ne peuvent compter sur le zéphyr, qui, dans les campagnes, transporte l'encens des llenrs et leur viviliante poussiere. le calme de 
l'atmosphère dans ces sombres forèts et la nature visqueuse du pollen des linflesiu ne permett nt pas aux vents de déposer sur de larges stigmates les émanations fécondantes quïls sout impuissants at soulever. Les insectes seuls sont chargés de ce soin : trompés par l'odeur eadavéreuse de ces lleurs, ils descendent par my̧riades dans leur calice charnu, et se chargent d'un pollen visqueux analogue a celni des Siaules el des Urchidées. Ils s envolent, appelés par une erreur nouvelle; ils vont accomplir an loin des destinées qu ils ignorent et lavoriser de létides amours dont la nature les a rendus les innocents complices, et dont la brise a refusé d'ètre ì la fois l'interprète et la messagère.

"Sunvent les insectes se plaisent à troubler les ménages les plus heurenx, les liaisons les mieux assorties. Ils portent, le jour ou la nuit, la poussière fécondante d'une fleur sur une antre, et, avant que le pincean intelligent de l'horticulteur ait remplacé les hasards de leur course ragabonde, c'est aux insectes que nous avons été redevables des panachures et des variations d'un grand nombre de fleurs de nos jardins.

"Ainsi l'insecte aux élytres étincelantes, le Papillon aux ailes de nacre et de rubis, rivalisent de beanté avec de firaiches corolles qui leur ofirent la coupe ciselée du plus délicieux nectar, en échimge de leur médiation. Mais, sous le ciel brùlant des réfions tropicales, des Oiseaux rapides comme le Sphinx, irisés comme l'opale et simulant le leu des pierreries, disputent aus insectes le roile que seuls ils remplissent dans nos contrées. Le's Oiseaux-mouches et les Colibris sont les conlidents discrets des anuours des fleurs, ct celles-la sont aussi les dépositaires du berceau et de l'hyménée de ces légers habitants des airs. Lat l'rovidence a voulu que les plus gracienses créations de la terre: musent des liens communs: Elle a rendu solidaires te honheur ul la beauté. "

(IIexn Lecoe, lal Vie des fileurs.) 


\section{CHAPITRE 11}

DE L'ESPECE ET DE SES VARIATIONS.

Avant d'entrer daus les détails pratiques de l'hybridation, que l'on nous permette de préciser quelques termes que les jardiniers emploient à tort comme synonymes, et sur lesquels les naturalistes eux-mêmes ne sont pas toujour's d'accord.

II sagit d'abord de reconnaître ce que l'on entend par 'spèce, car c'est là l'unité en botanique. C'est l'horizon audessus el au-dessous duquel se développent toutes les formes désignées par les botanistes, sous des noms particuliers de système ou de classification. Ainsi le Blé ou Froment est une espèce, mais le Blé barbu, le Blé de printemps, le Blé de miracle, le Blé de Tangarock, ete., ne sont pas des espèces distinctes dı Blé. Ce sont des différences désignées sous les noms de races, de variétés, ctc. Ce sont des varations inférieures à l'espèce. Ce sont les formes diverses du Triticum sutivum.

Le Rrisin muscat, le Chasselas rose, le Iraisin de Malaga, el cette multitude de Raisins divers cultivés en France et dans le midi de l'Europe, ne jroviennent pas d'espèces différentes de Vignes, mais ce sont des variétés de la Vigne ordinaire, Vitis vinifera; il en est de même des formes si variées de nos Pommes, de nos Poires, de nos Choux, de nos Pommes de lerre, etc.

Mais, si nous comparons le Groseillier rouge, ic Groseillier noir ou Cassis, le Groseillier épineux ou Groseillier à maquereau, 
ainsi que les Groseilliers doré, sanguin, etc., nous aurons des espèces distinctes.

Ces Groseilliers, semés, pourront nous donner des variétés; mais le Groseillier rouge ne produra jamais du Cassis, le Groseillier épineux ne dommera pas naissance an Groseillier sanguin, réciproquement.

L'espece est done composée de tous les individus qui se re'ssemblent plus entre éux quils ne ressemblent ì d'uutres, et qui se reproduisent indéfiniment, mais avec des variations limitées, par les yraines qu'ils produisent.

Telle est la manière dont nous devons considérer l'espèce, sans nous occuper ici de sa permanence, de sa transformation 011 de sa fixité absolue.

Les especes qui se ressemblent, étant réunies, forment des genres, degré de réunion plus élevé que les espèces. Les genres Groseillier, Rosier, Iraisier, renferment toutes les espèces qui peuvent se ranger sous ces titres.

l.es genres analogues, étant réunis, constituent les familles. Le Blé, le Seigle, l’Orge, l'Irraie, la Brise, appartiennent à la famille des Graminées; le Persil, le Cerfeuil, la Carotte, le Panais, à celle des Ombellifères; la Renoncule, l'Anémone, la Cilématite, à celle des Renonculacées; ete., etc.

L'horticulteur s'attache à l'espèce, et cherche à la modifier par différentes méthodes pour obtenir des plantes nouvelles, et tout changement de forme dans les individus d'une mème espèce s'appelle variation.

(quand celte variation se montre sur quélques individus seulement, soit dans la forme, soit dans la conleur, on la nomme varieté ou sous-variéte. Ces accidents ne se reproduisent pas, ou ne se reproduisent qu'accidentellement par les semis.

II ais si, à force de soins et de choix faits successivement sur les porte-graines, on arrive a fixer une variéte, de telle sorte yu elle se reproduit indefiniment par les semis, on a une ruce permanente. 
Une race permanente est presque une espèce que nous avons créée.

Nous devons supposer que la nature crée encore des espèces, et si nous ne voulons pas donner ce titre aux formes permanentes obtenues par l'homme, nous pourons assurer au moins qu'il a créé des races.

\section{De la variation.}

On donne le nom de variations, terme vague et général, à toutes les modifications que l'on observe dans une espèce. Nous pensons que l'on devrait le réserver aux modifications obtenues par les semis, sans employer les fécondations croiscés ou les hybridations. C'est avec cette restriction que nous allons en parler.

Lors de la publication de la première édition de cet ouvrage, les critiques portèrent principalement sur ce que j’attribuais à l'hyybridation de simples variations produites par le hasard, et pour l'obtention desquelles l'hy̧bridation était inutile.

Je conviens que plus des trois quarts des plantes que les jardiniers mettent en rente sous le nom d'ingbrides ne sont que des variations, et qu'ils ont abusé et abusent continuellement de ce mot; mais nous demandons que l'on ne nous accuse pas de confondre ces deux termes, et, à plus forte raison, ces deux choses.

Réservons l'hybridation pour le chapitre suivant; nous nous occuperons seulement ici de la variation.

Examinons done les plantes livrées aux seules forces de lit nature; ne laissons pas intervenir l'hybridation avec ses moyens de création artificiels. " Laissez faire la nature, disaient les patriarches de l'horticulture, elle fait bien ce qu'elle fait. " C'est un réritable abus de confiance. La nature travaille pour elle, pour ce magnifique plan d'ensemble qui lui a été tracé par l'Auteur de l'univers, ct dont les scènes majestueuses, se déroulant devant nous, commandent notre silence et notre respec- 
tueuse admiration. Yais l'homme doit s'aider de ses lumières et de sa propre intelligence, et deux grands moyens sont en sil puissance pour obtenir des especes ou des variétés nouvelles. Il doit diriger la variation ou pratiquer l'hybridation.

C'est toujours par les semis que l'on obtient la variation. Les boutures, les marcoltes, les greffes, la division des pieds, an un mot, toutes les multiplications par gemmes ou bourgeons, reproduisent identiquement le pied mère. Il n'en est pas de mêne des graines, La plupart des plantes ont une tendance à se modifier par ce mode de reproduction, et nous pensons que les modilicitions que doivent présenter les nombreux individus issus d'une même plante possèdent diuns la graine le germe de leurs modifications. Nous ne eroyons pas, par exemple, qu'une graine de Belle-de-nuit à fleur blanche puisse produire une plante it fleur rouge ou à flem jaune, par suite de la nature chimique ou phrssique du sol dans lequel elle sera cultivée, par suite du plus ou moins de séchieresse ou d'humidité, ete. Si la variation s'établit, ce qui peut arriver, elle sera indépendante des causes que nous venons de citer.

La variation limitée est la régle générate dans les semis. Toutes les plantes issues de graines sont comme sollicitées par deux forces contraires : cette tendance à la variété, qui n’est qu'une conséquence du mourement 't du progrès, auxquels obéissent tous les ètres de la création; et la stabilité ou l'habitude acquise, qui les retient et compense en partie la première de ces forces.

Mais, si l'homme intervient et qu'il protége efficacement la première de ces forces, la tendance au changenent, et qu'il combatte ouvertement la seconde, la stabilité et la force de l'habitude, nous verrons bientòt les générations de ces plantes se ranger de son côté el virre sous sa protection.

supposous done quiune plante queleonque répande ses: graines, et que tous les individus qui en proviennent soient placés sous nos reux. Tous ces individus feront partie d'une espèce type. Mais chacun de ceux obtenus par ce semis séloi- 
gnera un peu du type par une modification légère de l'un de ces organes. Que doit faire alors l'horticulteur qui veut obtenir des nouveautés? C'est d'étudier soigneusement les changements de caractère qui se présenteront, de poursuivre pendant plusieurs générations le développement de ces caractères qu'il veut fixer, de ne recueillir ses graines que sur les individus qui offriront ce caractère développé au plus haut degré, et de s'éloigner toujours du type par cette méthode que l'on nomme la voie de sélection.

Reste à choisir l'organe dont il veut poursuivre le développement ou l'atrophie.

Peu lui importe, par exemple, qu'un Chou produise des variations dans sa tleur, dans ses siliques ou dans ses graines; il s'attachera surtout à celles de ses variations qui peuvent avoir lieu dans ses feuilles ou dans ses racines.

Il ne s'occupera ni des feuilles ni des racines dans une Rose ou dans une Pensée, mais il suivra attentivement les variations qui surviendront dans les lleurs, dans la forme, le nombre et le coloris des pétales.

Les Carottes, les Betteraves, les Pommes de terre, ne l'intéresseront que par leurs racines ou par leurs tubercules; les Poires, les Cerises, les Pêches, que par leur développement c't la saveur de leur péricarpe charnu; les Amandiers, les Pois, les llaricols, par leurs graines; les Asperges, par leurs bourgeons; les Artichauts, par leur réceptacle, etc.

Puisque les plantes peuvent varier, au moyen des semis, par la plupart de leurs organes, il y aura un grand nombre de variations, qui n'auront aucun intérêt pour le cultivateur. S’il fallait, par exemple, créer aujourd'hui la Carotte des jardins, il faudrait chercher parmi les individus sauvages ceux qui présentent les racines les plus développées, et semer leurs graines. Dès la première génération on rencontrerait dans la multitude des différences notables dans divers organes. Il faudrait alor's choisir comme porte-graines quelques-unes des plantes dont les racines auraient acquis le plus de volume et contiendraient le 
plus de principe sucré. On chercherait les mêmes caractères dans les générations suivantes, et ainsi de suite jusqu'à ce qu'on ait obtenu des Carottes mangeables. C' est ainsi que II. Vilmorin a transformé en quelques ammées la Carotte des champs en racine comestible. Des semis successifs de la Carotte cultivée ont sans doute donné les variétés distinguées sous les noms de Carotte blanche des Vosges, Carotte à collet rert, Carotte de Ilollande, Carotte courte, etc; et si maintenaint on s'attachait à liaire de nouveaux semis de ce légume, non au hasard, mais par voie de sélection, en recueillant les graines sur des plantes dont les racines seraient les plus grosses ou les plus blanches, tes plus rouges ou les plus sucrées, on parviendrait encore à améliorer ce légume, sans savoir où serait le terme de ces modifications successives. C'est par ce mème moyen de séleclion que L. Vilmorin, employant un procédé des plus ingénieux, parvint à obtenir par semis successifs des variations de Betterave, contenant plus de sucre que celles que l'on cultivait lıabituellement.

Dans ces deux cas et dans tous ceux du même genre, on essaye autant que possible de s'éloigner du centre de l'espèce pour suivre les modifications d'un seul organe et pour déterminer la naissance d'individus nouveaux jouissant de caractères différents de ceux du type.

Ces individus constituent done ce qu'on nomme des variatlions. L'hybridation leur est complétement étrangère.

Les plantes sont d'autant plus disposées à produire des variations qui elles sont elles-mêmes de création plus récente. I insi une variation qui vient de se montrer pour la première fois avec la tendance que l'on cherche à dérelopper doit être préférée pour porte-graine à tous les autres types plus anciens. La culture tend done à modifier continuellement les espèces, ì changer quelques-uns de leurs caractères; mais toutes ce's formes nouselles ont hesoin d'acquérir la stabilité, qui ne peut leur être dévolue qu'en les maintenant pendant un certain temps sous l'empire des mèmes conditions qui les ont fait naître 
Les anciennes variétés de fleurs et de fruits, cultivées depuis des siècles dans nos jardins, ont, pour ainsi dire, acquis l'habitude et la stabilité des véritables espèces. Leur origine et leurs types sont perdus. Nous avons fait le Blé et la Rose à cent feuilles; leur culture s'est propagée à travers les siècles, ils ont acquis la stabilité, en perdant leur acte de naissance.

Il n'en est pas de même de nos Dahlias, de nos CEillets, de nos Tulipes. Des semis les plus soignés, des graines les plus précieuses, il sort encore une infinité de plantes qui ne rappellent plus les rangs multiples des fleurons, les vives panachures, ni les brillants coloris de la couromne de leurs ancêtres. Une partie de ces plantes de semis semblent retourner à l'état primitif, et si les soins les abandonnent, ce sont les plus rustiques qui donnent la plus grande abondance de graines, qui acquièrent le plus de vigueur et qui, bientôt, détruisent les. autres cn s'y substituant. Ils reprennent ainsi leurs anciemnes habitudes un instant contrariées par le capric et l'intelligenee de l'homme. 


\section{CHAPITRE III}

\section{DE LA FECONDATYON ARTIFICIELLE; DE L'HYBRIDATION ET DES MOYENS DE LOPERER.}

I'our arriver plus vite à des variations que l'on attendait anIrefois du hasard, du temps ou de circonstances incommues, on a imaginé (et la nature nous en avait donné l'exemple) de malier des espéces roisines, presque toujours du meme geure, of d'élever leurs familles. Bon nombre de ces mariages ont réussi, et leur produit a reçu le nom général d'hybride. L'acte même de l'accomplissement de ces mariages se nomme hybridution.

Ce sont les actes et les cérémonies usités dans ces mariages ınixtes, les procédés délicats à employer pour vainere parfois la répugnance des contractants dans ces sortes d'union, rue nous arons réunis dans ce petit volume, car dans l'hybridation réside en partie l'avenir de l'horticulture appliquée à nos parterres, ì nos potagers, à nos vergers et mème à nos forêts.

On doit distinguer deux degrés dans les hỵbrides : le premier est le résultat du croisement de deux espèces bien distinctes romme les hybrides des Mirabilis, Jalapa et $\mathrm{H}$. Iongiflora, du Cierens speciosissimus et du C. frandiflora, du Digitalis purpurea et du I). luteu, ete., etc.; le second degré est le produit du croisement les variétés entre elles, et iri le succès est hien plus certain; telles sont les variations qui résultent des mariages des Auricules entre elles, des Tulipes entre elles ou du moins de la Tulipe ordinaire, des Mirabilis Jalapa entre eux, ete., etc.

On a proposé de réserver le nom d'hybrides au produit 
croisé de deux espèces, et de donner celui de métis aux plantes nées du rapprochement de deux variétés.

Ces dénominations seraient utiles si on pouvait toujours les appliquer; mais dans la pratique de l'horticulture il arrive qu' un hybride est fécondé à son tour par ses propres parents, qu'un hybride féconde un métis ; puis il devient tout à fait impossible de se reconnaitre dans tous ces croisements de différents degrés. Nous en avons des exemples dans nos innombrables variétés de Fuchsia, de Pelargonium, de Rosiers, de Gladiolus, etc., etc.

La fécondation est artificielle toutes les fois qu' clle est opérée par l'homme. Nous venons de passer en revue, dans un des chapitres précédents, quelques-uns des moyens employés par la nature pour l'assurer; examinons maintenant quels sont cens que l'homme peut imiter, dans quelles circonstances il doit agir, quelles précautions il doit prendre, et quels résultats il peut espérer.

Pour les plantes de serre et pour plusieurs de celles que nous cultivons dans nos jardins, la fécondation artificielle est nécessaire pour en obtenir des graines, et c'est alors avec leur propre pollen, ou mieux avec le pollen d'individus distincts mais de même espèce, que cette fécondation s’opère; mais le plus souvent on l'emploie pour croiser les races et obtenir des plantes intermédiaires, en fécondant une espèce ou une variété par une autre. C'est ce que l'on nomme hybridisation ou plus simplement hybridation, et ce que l'on pratique souvent, mais trop rarement encore en horticulture.

Du reste, que l'on opère sur une même espèce avec son propre pollen, ou que l'on essaye de croiser deux plantes différentes, les procédés sont les mêmes; mais, dans ce premier cas, il y a bien moins de précautions à prendre que dans le second.

Nous ne séparerons donc pas, dans les généralités qui vont suivre, ces deux sortes de fécondations artificielles, et nous décrirons successivement:

L'état de la fleur, relativement à la situation des organes 
sexuels; nous examinerons les fleurs hermaphrodite, monö̈que, dioüque, polyyame, la fécondation directe et indirecte, et l'inégulité de développement dans les organes.

- Nous étudierons ensuite le choix et la préparation des sujets que l'on veut hybrider, et nous terminerons par les opérations pratiques nécessaires pour effectuer l'hybridation on fécondation artificielle.

\section{Fleurs hermaphrodites.}

Quand les deux sexes sont réunis daus une même fleur, olle rst hermuphrodite, et la fécondation artificielle est moins facile à opérer que sur les plantes dont les sexes sont séparés. Il faut alors retrancher les étamines ou au moins les anthères d'un certain nombre de fleurs, et ensuite poser le pollen au pincean (grav. 5 et 4).

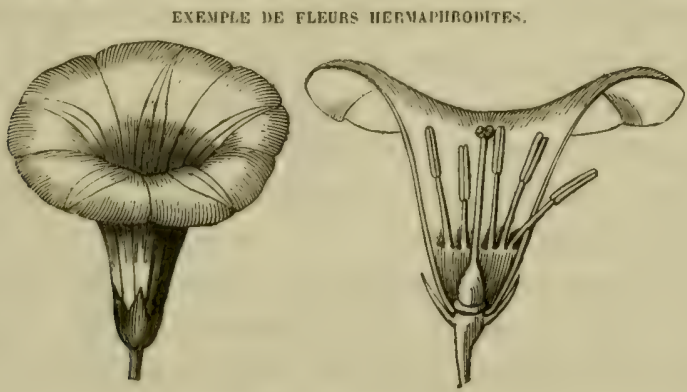

Grav. 5. - Fleur du Liseron.

Il est remarquable que dans la plupart des fleurs hermaphrodites des enveloppes plus ou moins nombreuses et plus ou moins développées entourent les organes sexuels, arrêtent en quelque sorte le pollen qui tendrait à s'échapper, et rendent ainsi la fécondation nalurelle plus certaine, en pré- Grav. 4. - Frur des firaminées (Festucu). servant les organes de la plupart des causes perturbatrices qui viendraient gèner leurs fonctions. II est done essentiel que les 
anthères en soient totalement enlevées avant leur déhiscence, avant que le pollen ait pu même toucher les enveloppes florales, qui souvent, en se flétrissant, viennent encore l'apporter sur le stigmate. Il s'en faut cependant, comme nous le verrons plus loin, que les enveloppes de la fleur soient un obstacle à l'accès d'un pollen étranger.

\section{Fécondation direete et indirecte.}

INÉGALITÉ DU DÉVELOPPEMENT DES ORGANES.

La fécondation est directe daus les fleurs hermaphrodites, quand elle a lieu dans chaque fleur au moyen du pollen contenu dans ses propres étamineś; c'est ce qui n’arrive pas toujours, lors même que ces organes sont entourés, comme nous venons de le dire, par des enveloppes protectrices, et alors nous n'avons rion à ajouter aux observations que nous venons de faire sur les fleur: hermaphrodites; mais elle est souvent indirecte, quand, la fleur étant hermaphrodite, son pistil n'est pas fécondé par ses propres étamines. Celte espèce d'anomalie peut avoir deux causes : la première, la position des organes; la seconde, leur inégalité de développement et d'aptitude.

Dans le premier cas se trouvent, comme nous l'avons déjà vu en parlant de la fécondution naturelle, le Blé, un grand nombre de Graminées, d'Ombellifères, de Synanthérées, etc.; dans le second, les Pelargonium, où les anthères n'ont plus de pollen, quand le pistil est propre à le recevoir, et une infinité d'autres plantes que nous citerons, à mesure que nous étudicrons dans les différents genres les caractères de leurs organes. La réunion d'un grand nombre de fleurs dans les épis, dans les ombelles, dans les calathides, est souvent une cause de fécondation indirecte (grav. 5 et 6 ). II nous suffira ici de faire remarquer l'avantage que présentent, pour l'hybridation, les lleurs hermaphrodites à fécondation indirecte; elles rentren! alors dans la catégorie des fleurs unisexuées. On peut, au besoin, se dispenser d'enlever les étamines; mais, comme dans: 
DIRECTE ET INDIRECTL.

ces cireonstances il y a presque toujours un grand nombre de

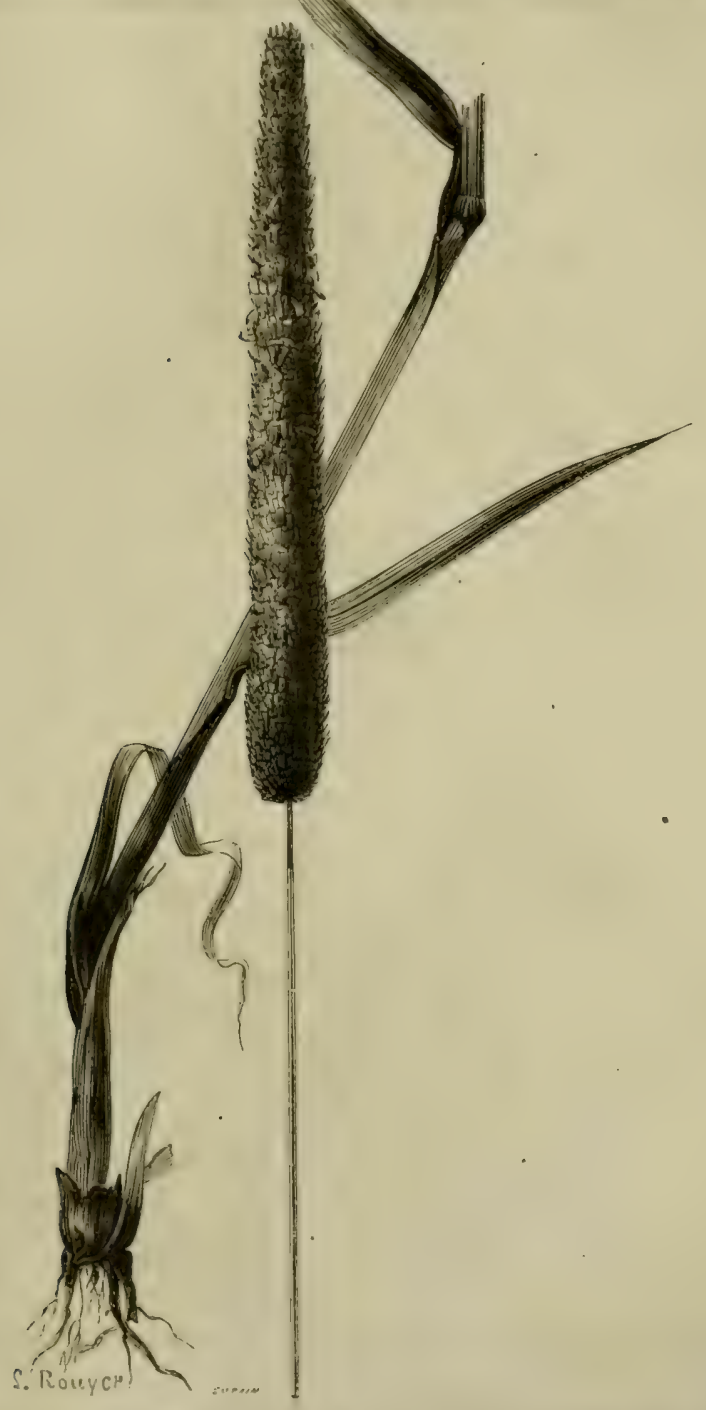

Grav, 3. - Exemple de fécondation indirecte dans l'épi dela Héole des prés.

lleurs qui sépanouissent it la lois, en suivant un ordre très- 
serré de développement successif, il vaut beaucoup mieux enlever les anthères à mesure qu'elles paraissent.

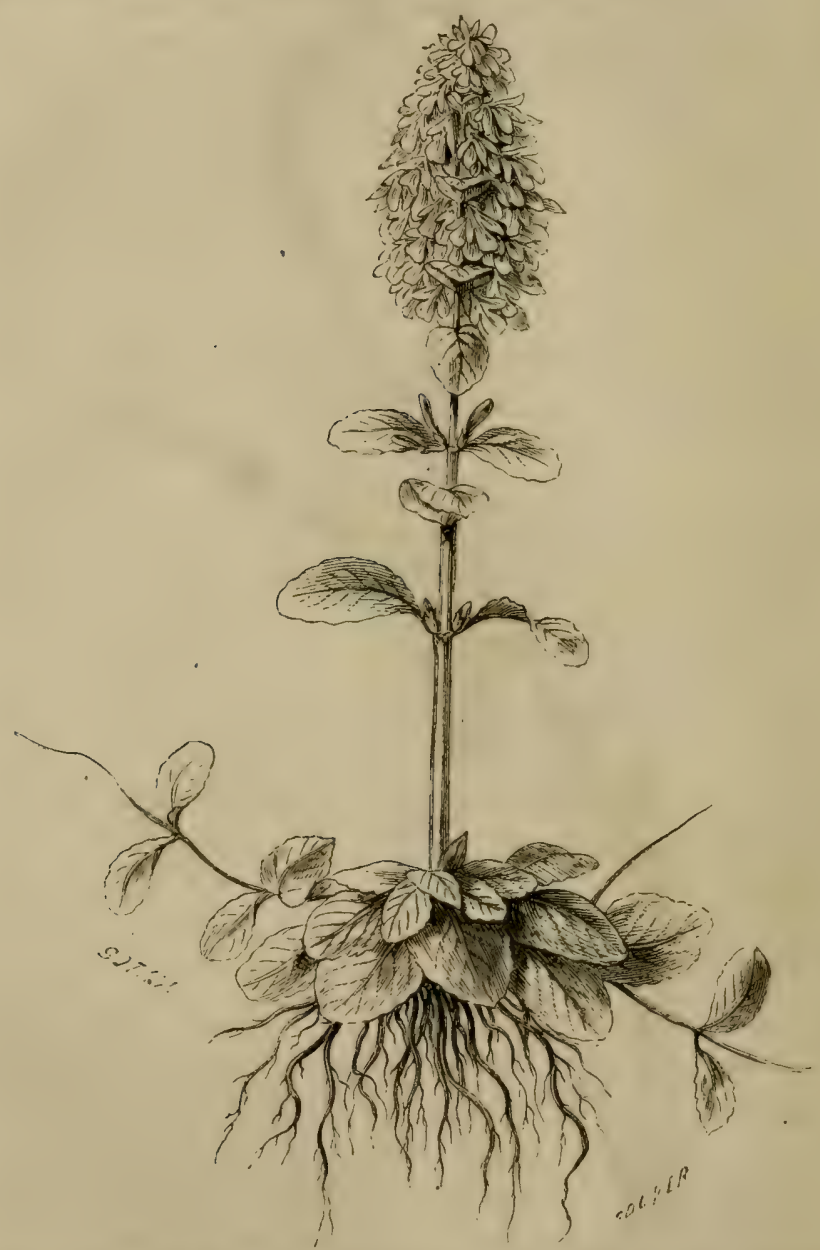

Lirav. 6. - Exemple de fécondation indirecte slans l'épi de fleurs de la Bugle rampantr.

\section{Fleurs monoiques.}

On nomme ainsi les fleurs unisexuées qui sont portées sur 
la même plante, exemple: le Melon, le Yoisetier, le Noyer, dont certaines fleurs sont mâles et d'autres femelles. Ces dernières seules donnent des fruits. On conȩoit qu'il est facile sur ces plantes de retrancher les lleurs mâles encore en bouton et à mesure qu'elles paraissent, de manière à rendre la fécondation impossible; quelquefois même, ces fleurs ne paraissant pas exactement à la même époque, l'imprégnation ne peut avoir lieu sans le secours de l'art (grav. 7 et 8 ).

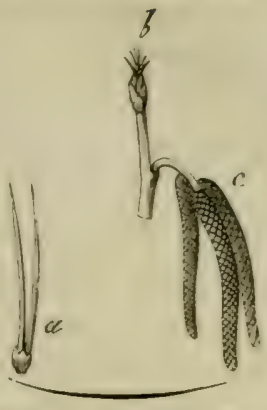

Grav. 7. - Fleur du Noisetier. $-a$, pistil isolé. $b$, lleurs lemelles. - $c$ fleurs mâles.

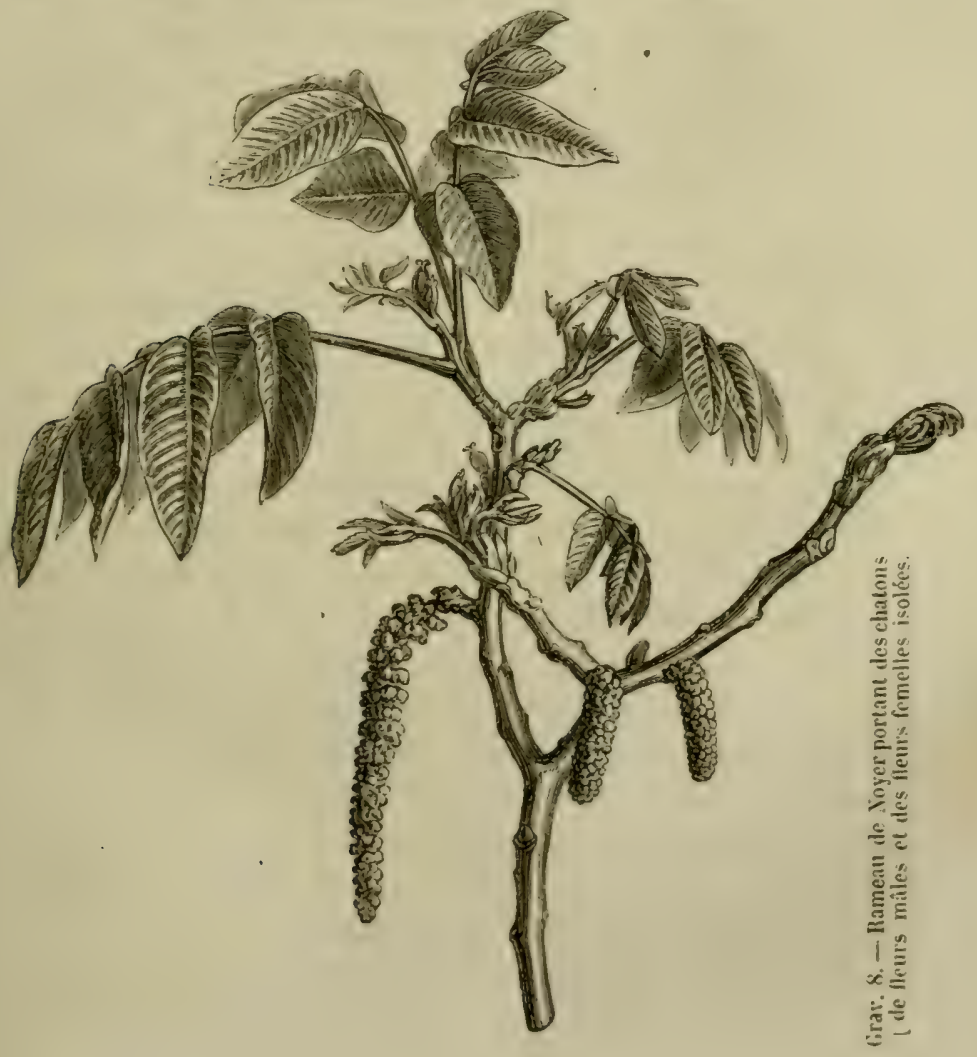


On peut done profiter de cette disposition pour faire des fécondations artificielles plus facilement que sur les fleurs her’. maphrodites.

\section{Fleurs dioïgnes.}

La diocie a lieu quand les fleurs unisexuées sont portées sur des pieds différents, comme dans le Chanvre, le Dattier. II peut arriver alors que la fécondation naturelle ne s'effectue pas; mais il faut pour cela absence complète de l'un des'sexes, car,

EXEMPLE DE DIECHE.

Fleur mâle et fleur femelie provenant d'individus séparés.

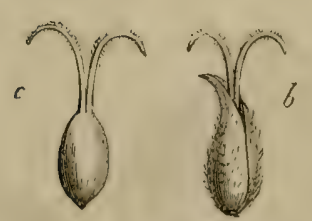

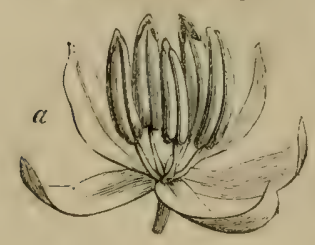

si les deux existent à une certaine distance, qui pent même être assez éloignée, la fructification s'opère le plus ordinairement. T'out le monde sait que les Dattiers femelles restent stériles, si l'on ne va au loin chercher des branches fleuries des Dattiers mâles que l'on vient secouer sur leurs grappes épanouics, précaution qui devient tout à fait inutile si l'on plante quelques Dattiers mâles au milicu des autres (grav. 9).

La fécondation artificielle paraît plus facile à exécuter sur ces plantes que sur 6rav. 9. - Chanve communu les autres, puisque les fleurs femelles fenelle.- $c$, la même, privée se trouvent isolées; mais elle offre cele son envaloppe pour montrer le pistil. pendant d'assez grands obstacles qui sont dus à la difficulté d'isoler complétement les sujets. La nature a suppléé à l'éloignement des sexes en multipliant à l'infini les fleurs mâles, en plaçant dans chaque anthère une grande quantité de pollen, si fin et si léger, que le moindre vent l'emporte et que souvent l'air est rempli de poussière fécondante; c'est au point que quelquefois on a cru à l'existence de pluies de soufre, à l'époque où les l'ins, quoique monoïques, répandaient dans l'air leur pollen jaune et abondant.

D'un autre côté, si la nature a pris parfois des précautions 
pour retenir le pollen des lleurs hermaphrodites autour des pistils, au moven de corolles et de calices enveloppants, elle a agi d'une manière opposée pour les fleur's unisexućes : elles sont souvent privées de toute espèce de téguments; le pollen peut s'en échapper facilement, voyager dians les airs, et nul obstacle ne s'oppose à ce qu'il vienne se placer sur les fleurs femelles, également dépourrues d'enveloppes et exposées à tous les conrants de latmosphière, qui sert de véhicule au pollen. L'attention de l'horticulteur doit done se porter ici, autant que possible, sur l'isolement des sujets l'emelles.

\section{Fleurs polygames.}

On nomme polygumes les plantes qui portent en mème temps des fleurs hermaphrodites, des fleur's mâles et des lleurs femelles, comme l'Érable, la Pimprenelle, les Gleditzic et quelques autres végétaux (gr. 10 et 11). On conçoit tout de suite EXEMIE DH: FLEI Ji- POIVGAMES tout le parti que l'horticulteur peut tirer de cetétat de choses, en ne laissant sur le pied mère que des fleurs femelles; et retranchanit toutes les autres. On opère alor's comme sur les

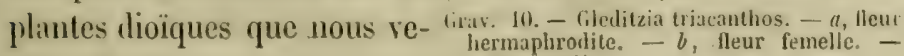
nons de citer.
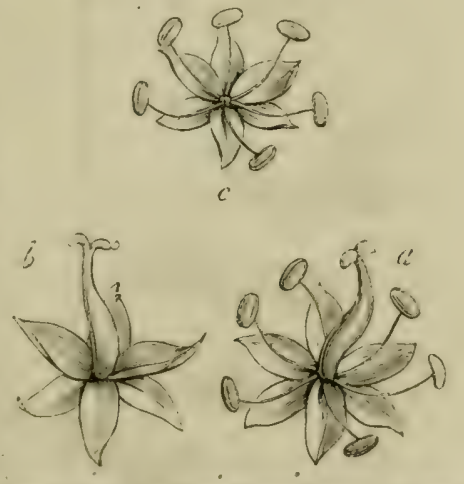
c, fleur mâle.

\section{Choix des sujers.}

Il est de la plus haute importamee, quand on vent tenter des croisements entre des espèces on des variétés d'un mème grenre, de choisir aree soin le porte-graine of la plante fécondante. En général, le produit tient des deux; mais jai remirrqué, dans un très-gramd nombre de croiscments que j’ai opérés arec tons les soins possibles, que les hỵbrides 
tiemment plus des porte-graines ou de la mère que du père. EXEMPLE D'L'NE I'LANTE ROLYGAME.

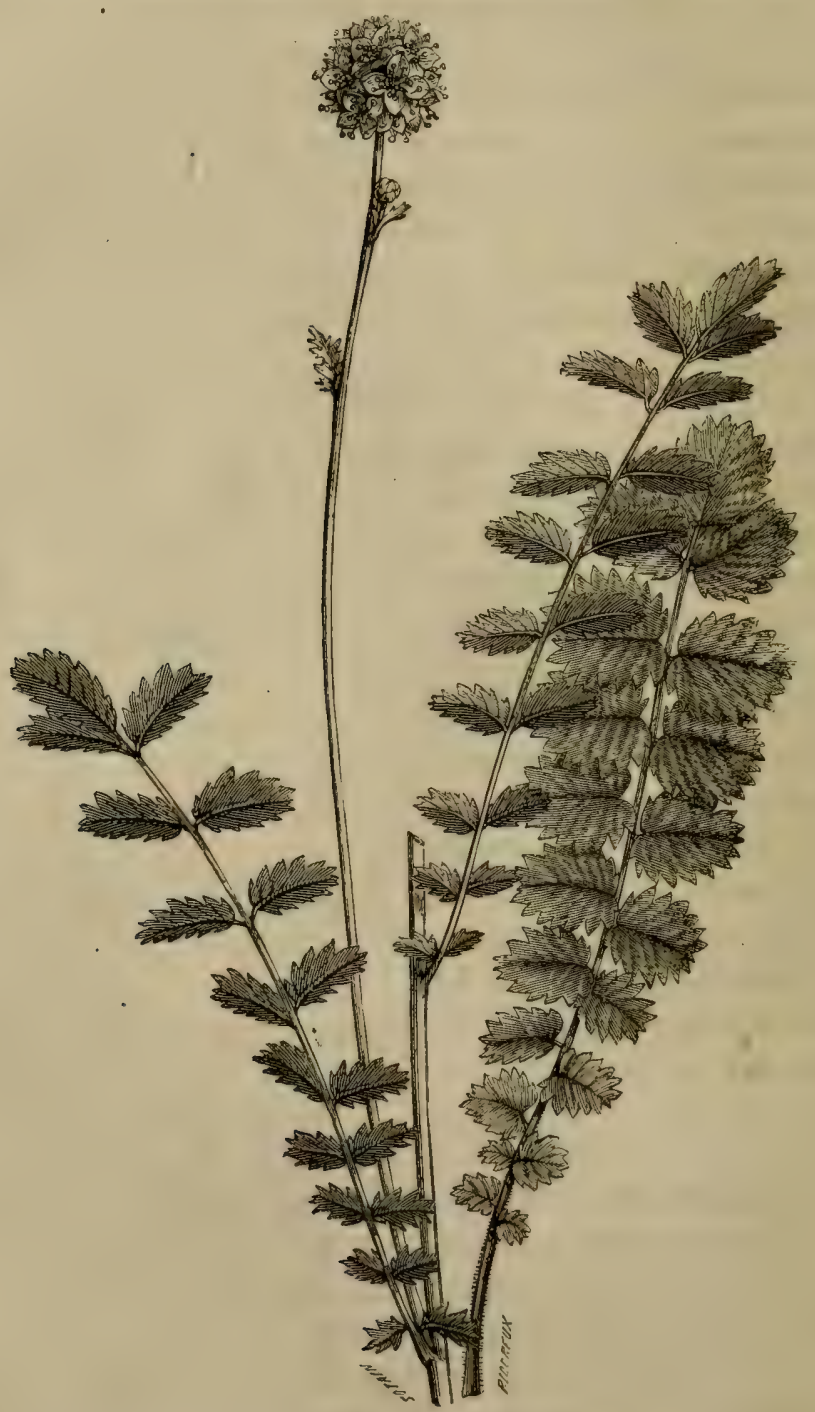

Grav. 11. - Pimprenelle sanguisorlse. 
Ainsi donc, que l'on opère sur des fruits, sur des légumes ou sur des plantes d'agrément, le premier soin devra se porter sur la mère. On ne peut cependant toujours choisir librement, car il peut arriver que la variété préférée dome difficilement des graines, et l'on peut ètre forcé d'en prendre une autre. bans tous les cas, le choix ne doit pas être fait légèrement; on ne loit rien laisser au hasard. Le choix du père exige le's mêmes attentions, et quand on a su réunir des plantes dont les qualités et les défauts peuvent s'unir, se combattre ou se modilier, on a fait déjà une bonne partic du travail. Si, par exemple, on veut améliorer un fruit précoce, c'est de le féconder par une autre variété meilleure, et qui s'éloigne le moins possible de l'époque de maturité du premier. Si l'on cherche it obtenir un fruit qui se conserve longtemps, mais qui soil plus volumineux que celui que l'on possède, il faudra croiser ce fruit tardif avec une variété plus belle, plus volumineuse, mais dont l'époque de maturité soit un peu retardée iussi:

Les qualités sucrées, farineuses, aromatiques, etc., que l'on recomnait dans les légumes, pourront également être augmenlíes ou modifiées par des croisements raisonnés, aussi bien que leur volume ou leur précocité.

Les caractères de beauté et d'élégance qui font rechercher certaines variétés de fleurs de collection éprouveront aussi de notables améliorations par des hybridations bien entendues; mais dans tous les essais il faut se garder d'employer des types inféricurs, c'est-à-dire des plantes qui ont déjà été dépassées de beaucoup par des gains nouveaux. On peut parvenir à obtenir aussi beau que ces nouveaux gains, mais on perd du temps pour y arriver, tandis que si on les prend pour point de départ, on a la chance de les perfectionner encore. Ainsi il ne fiut pas craindre de se procurer de belles variétés, bien qu'elles soient en petit nombre. Il laut prendre toutes les précautions possibles pour opérer convenablement et ètre sûs de ses graines. Ce n'est jamais la quantité qu'il faut chercher, mais la 
qualité, et lorsqu'on s'est donné la peine de faire des hybrides, de les cultiver et de les amener à fleurir, il est pénible de n'avoir rien de beau et de ne pouvoir faire un choix de quelques variétés nouvelles et méritantes.

Il est encore un point sur lequel nous devons appeler l'attention des horticulteurs, c'est sur la stabilité des espèces et l'ilvantage que l'on trouve à hybrider les variétés ou espèces nouvelles, plutôt que les anciennes.

Les plantes, comme tous les êtres vivants, les espèces et les variétés, comme les individus, sont soumises à l'habitude. Une variété que l'on vient d’obtenir donnera des graines qui produiront plus facilement des variétés nouvelles que les graines des anciens types. Cette variété n’aura pas encore acquis cette stabilité que donne l'habitude. Ainsi nous avons dans nos jardins des plantes dont nous avons conservé les variétés à force de soins, en les maintenant toujours dans des circonstances favorables à leur stabilité, et nous leur avons enfin donné l'habitude. Tout ce qui est de création nouvelle ne l'a pas encore acquise et varie avec la plus grande facilité. Pendant longtemps on a cultivé le Dahlia sans changement de couleur; dès qu'une nuance différente s'est montrée, elle en a bientôt fourni de nouvelles qui se sont multipliées à l'infini. Si, au lieu de semer les graines de la première variété obtenue, on avait persisté à semer celles du type, un long espace de temps se serait sans doute écoulé encore avant l'apparition de nouvelles couleurs. Aussi, dès que nous avons obtenu dans nos jardins quelques différences dans les formes ou les couleurs des individus d'une mème espèce, la voyons-nous ensuite se modifier de mille manières, soit en variant simplement par semis, ou par hybridation naturelle ou artificielle. Le plus difficile était d'ébranler la stabilité de la première race, de rompre son habitude, el, une fois l'impulsion donnée, la variation commence, et il n'en est aucune dont nous soyons sûr de connaître les limites. Avec le levier puissant de l'hyloridation, le pouvoir de l'horticulteur est illimité. Nous recommandons, d'après des expériences posi- 
tives, d'opérer toujours sur les plantes les plus belles et surtout les plus nouvelles, et de ne jamais juger un gain à sa première lleur ni à son premier fruit. Bien des plantes méritantes ont certainement été perdues par trop de précipitation dans le jugement que l'on a porté sur elles.

Les fleurs doubles méritent aussi une attention particulière; on pent les obtenir accidentellement dans un semis, mais on a la presque certitude de les acquérir si l'une des deux plantes que l'on a croisées a elle-même sa corolle doublée. Il n'est pas nécessaire que les deux plantes soient pleines, il suffit que l'une des deux le soit, et il arrive souvent, dans ces sortes de lleurs, que les pistils restent intacts malgré la multiplication des pétales, comme dans la Rose à cent feuilles et la plupart des Pivoines, ou que l'on parvient aussi à trouver au centre de la fleur quelques anthères échappées à la transformation, et contenant assez de pollen pour opérer une fécondation.

Deux plantes à fleurs demi-doubles donnent souvent des varićtés très-pleines, mais il est rare que deux espèces à fleurs simples en produisent de doubles immédiatement.

J'ai fait sur l'hybloridation des variétés très-doubles une observation assez curieuse. Dans quelques circonstances deux variétés doubles hybridées m'ont donné des individus à fleur's tellement pleines, que les boutons crevaient et ne pouvaient s'ouvrir, comme dans quelques Pivoines et un grand nombre d'Eillets. Mais il m'est arrivé aussi de n'avoir que des fleurs simples ou de les avoir en majorité. On remarque un fait semblable dans les animaux. Deux serins luppés, réunis, produisent souvent des serins à tête pelée, comme si les deux huppes se neutralisaient.

Un est done plus sûr d'obtenir des fleurs doubles si l'un des deux parents seulement présente ce caractère.

\section{De Ia couleur des variétés.}

Nous venons de voir que les variétés nouvelles participaient des qualités de leurs parents à des degrés différents, mais qu'en 
général, d'après mes observations particulières, la mère semblait communiquer ses propres caractères d'une manière prépondérante. Il en est de même pour les couleurs. Elles tiennent souvent le milieu entre celles des deux variétés qui viennent d'en produire une troisième; il y a, il est vrai, une foule d'exceptions à cette règle, et ceux qui ont fait des croisements un peu en grand et sur des plantes dont les couleurs changent litcilement ont vu combien il y avait de variations dans le coloris. Au milieu de ces perpétuelles variations, j’ai cru cependant reconnaitre quelques règles dont l'expérience confirmera plus tard la vérité ou affaiblira la constance des résultats.

On sait depuis longtemps que le blen, le ronge et le janne, qui sont les trois couleurs primitives, doment du brun par leur mélange sur la palette; elles en donnent également quand elles apparaissent toutes trois dans la même corolle. Un grand nombre de croisements faits sur des Primevères et des Auricules m'ont démontré qu'une Primevère jaune, fécondée par une Primevère violette, c'est-à-dire bleue et rouge, produisait souvent des variétés brunes à couleurs fausses; tanäis que le rouge fécondé par le jaune ou par le violet donnait des teintes plus pures, parce que les trois couleurs y étaient plus rarement mélangées dans la même corolle. J'ai fait la mêrne observation sur les Auricules, dont les fleurs jaunes, croisées par les violettes; ont aussi donné un grand nombre de fleurs brunes, à couleur de bois ou d'acajou sale.

Il est donc essentiel de combiner, autant que possible, les couleur's une à une et non deux à deux ou même une à deux, quand on veut avoir des couleur's pures, telles que des violets, des orangés, des rouges, des jaunes ou des bleus; mais, si l'on recherehe ces teintes brunes qui ont quelquefois. tant d'éclat quand elles appartiennent à des tons rabattus, c'est-à-dire qui semblent contenir du noir, il faut le mélange des trois couleurs ou l'action de l'une d'elles sur une plante dont la corolle est déjà brune.

Le plus ordinairement les couleurs se fondent et se mélangent 
par l'hyloridation, comme si on les réunissait sur une palette, et il en résulte une teinte moyenne et fondue; mais, dans quelques espèces, au lieu de se fondre, elles s'isolent et paraissent en panachures dans la corolle, comme dans la Belle-de-nuit, les Tulipes; en stries, comme dans la Peine-Marguerite, les Mufliers; en pointillé, comme dans certains Dahlias; en bordure, comme dans quelques Primevères et Awricules, etc.

Il est une autre couleur qu'il faut autant que possible chercher à introduire dans l'hybridation, c'est le blanc pur, quand on peut se le procurer. Les types à fleurs blanches sont souvent. les meilleurs porte-graines, et ils reçoivent facilement toutes les couleurs, qu'ils affaiblissent en leur conservant cependant toute leur pureté. Ce n'est que lorsque le type sauvage est naturellement blane qu'il faut éviter de le prendre pour porte. graines.

Quand on oblient, par des semis, des variétés à couleurs remarquables, avec de mauvaises formes, il faut les conserver et en semer les graines; on a l'espoir de voir rẹnaitre les mêmes teintes avec des fleurs mieux faites.

L'étude des couleurs et de leur mélange par les croisements des lleurs pourrait donner lieu à des considérations beaucoup) plus étendues; mais nous nous bornerons à l'exposition de ces détails, qu'il était nécessaire de joindre au sujet que nous traitons. Nous renvoyons, pour des considérations nouvelles sur les couleurs, à notre grand travail sur la géographie botanique. de l'Europe (9 vol. in-8).

\section{Préparation des sujets.}

II ne suffit pas d'avoir fait un bon choix des plantes que l'on veut croiser, il faut encore savoir les préparer de manière à laciliter la maturation de leurs fruits, et ì éviter le contact du pollen des antres fleurs avec celles que l'on vent hybrider.

Quand les plantes ont les fleurs rumies en ombelles, en corymbes, en srappes, en panicules ou en houquets, on enfin 
chaque fois que, sur un même pied, un grand nombre de fleurs se trouvent épanouies ou sur le point de s'ouvrir, la première opération est de se rendre maitre de son sujet, en élaguant tout ce qui n'est pas utile, mais en réservant les fleurs de la base dans les grappes et les épis, les fleurs extérieures des ombelles et des capitules, et les prenant, autant que possible, sur l'inflorescence terminale de la plante si l'on veut obtenir des individus très-développés, et sur les inflorescences latérales si l'on recherche des fleurs doubles. Ainsi presque toutes les branches à fleurs seront coupées, à moins qu'il ne doive s'écouler un certain temps entre leur floraison et celle des rameaux sur lesquels on veut opérer.

On choisit donc seulement une, deux ou trois grappes, on bouquets de fleurs. Ce choix étant fait, on retra nche à chaque groupe de fleurs une bonne partie des boutons, n'en laissant que deux ou trois, quelquefois moins, sur chaque branche; c'est ainsi que l'on prépare son sujet, et l'on trouve à cela deux avantages.

Le premier est de disposer la plante à donner de meilleures fleurs, à nouer plus facilement ses fruits et ì former des graines plus nourries.

Le second est de ne pas être assailli par le pollen de toutes ces fleurs que l'on a retranchées, et de n'opérer que sur un petit nombre de boutons que l'on surveille plus attentivement. II ne faut jamais perdre de vue que ce n'est pas le nombre des graines qu'il faut rechercher, mais leur bonne qualité, et l'assurance qu'elles proviennent d'une fécondation croisée. Un seul fruit lécondé, avec toutes les précautions nécessaires, peut donner plus de résultats que cent autres dont on ne sera pas sûr, et pour lesquels on perdra le temps et le terrain nécessaires à l'éducation des jeunes plantes qui en proviennent.

Quand on opère sur des arbres, il est souvent impossible de leur enlever la plupart des branches, il vaut mieux alors en choisir une arec un ou deux houquets de fleurs, ce qui peut avoir lieu de deux manières: soit en enveloppant la branche d'une 
gaze gommée, soit en la plaçant sous une cloche. Ce dernier moyen est préférable au premier, car la pluie peut détruire la gaze gommée, il est quelquefois difficile de l'appliquer convenablement, et quand elle est fortement serrée autour d'une Iranche, l'air renfermé daus l'intérieur se dilate parfois à tel point, par l'action des rayons solaires, qu'il peut faire déchirer la gaze, tout en s'échauffant trop fort pour les fleurs qui s'y trouvent plongées; il vaut donc mieux employer une cloche. 1 cet effet; on fabrique une petite table en clouant une planche sur un piquet, on pratique une fente sur un des côtés de la planche, et, après avoir placé cette petite table à la hanteur de la branche dont on veut féconder les fleurs, on fait entrer la branche par cette fente et on l'assujettit. On couvre ensuite la petite planche de mousse que l'on humecte légèrement, on perce cette planche de plusieurs trous assez larges, qui sont recouverts par la mousse et permettent cependant à l'air de circuler, puis on recouvre le tout d'une cloche qui isole tout ì fait la petite branche, el en fait en quelque sorte un arbre à part quoique tenant à un autre. On peut ombrager la cloche pendant la plus grande chaleur du jour, etl'on doit opérer le matin, et, autant que possible, à l'abri du vent. Dès que les fruits sont. noués, on peut enlever la cloche, en ayant soin de la lever un peu les jours précédents, de manière à sevier la branche, comme on le ferait pour une bouture. Par ce moyen très-simple on est maitre de la plante, on modifie la chaleur, on humecte ou on dessèche son atmosphère selon les besoins, c'est-à-dire selon les espèces de fruits et selon les variations atmosphériques; il faut ensuite enlever une bonne partie des fruits non fécondés artificiellement, car on sait que la maturation est une cause d'épuisement. Non-seulement quand on voudra pratiquer la fécondation artificielle sur une plante on coupera la majeure partie. de ses fleurs, de ses fruits, mais, si on le peut, on les retranchera déjà l'année précédente, pour les arbres surtout. Quand un arbre n’a pas fleuri l'année précédente, et lors mème qu'ayant lleuri il na pas domé de fruits ou qu’ils ont éte retranchés, la 
floraison suivante est préférable, et les fruits qui en proviennent ont plus de volume et de vigueur. C'est peut-être parce que ces plantes sont moins fatiguées puisqu'elles ne donnent pas de fruits, que les variétés à fleurs doubles s'épanouissent presqur toujours les premières, comme on le remarque dans les Hépatiques et plusieurs autres plantes.

S'il n'est pas difficile de détruire une partie des fleurs surabondantes que donment certains végétaux, il arrive très-souvent que l'on ne peut au contraire obtenir la floraison de quelques autres; mais les horticulteurs savent que l'on y parvient souvent en coupant quelques racines, en plaçant les végétaux dans un sol plus maigre, en laissant, le plus longtemps possible, les plantes sans eau, pour leur en donner ensuite à satiété; enfin, en greffant ou bouturant celles qui ne veulent pas se mettre à fruit; mais ce serait sortir de notre sujet que d'entrer dans ces détails, et nous nous contenterons de prendre nos plantes fleuries.

Un moyen que l'on ne doit pas négliger non plus pour assurer la fécondation naturelle ou artificielle, c'est de supprimer les bourgeons à bois, s'il s'en trouve dans le voisinage des fleurs, comme cela se présente souvent pour certaines plantes qui lleurissent de bonne heure, et notamment pour les Camellias.

L'isolement des plantes entières est tout aussi nécessaire que celui des branches; quand ces plantes sont petites, il y a presque toujours avantage à les placer sous cloche, et souvent même ce moyen très-simple fait fructifier naturellement des espèces qui ne donnent pas toujours des graines. Les espèces printanières, exposées aux pluies, à la neige, aux dernières gelées, traversent les mauvais jours sous une cloche, et, pour peu que le pinceau de l'horticulteur vienne suppléer aux insectes et aux courants d'air qui manquent sous cet abri, la fécondation est certaine. C'est ainsi qu'elle s'opère, quelle que soit la saison, sur les Crocus, les Iris de Perse, etc.

Pour les grandes plantes, les légumes, les céréales, il faut aussi les isoler dans le coin d'un jardin, et les éloigner, autant 
que possible, de tous les autres individus dont le pollen pourrait opérer une fécondation qui muirait à celle que l'on veut obtenir.

\section{Opérations relatives à Ja fécondation artificielle.}

Elles consistent dans l'enlivement des étamines, la conservation du pollen et son application sur le stigmate. Une petite pinee, une aiguille, un canif, de petits ciseaux et quelques pinreaux, semblables ì reux dont on se sert pour l'aquarelle ef la

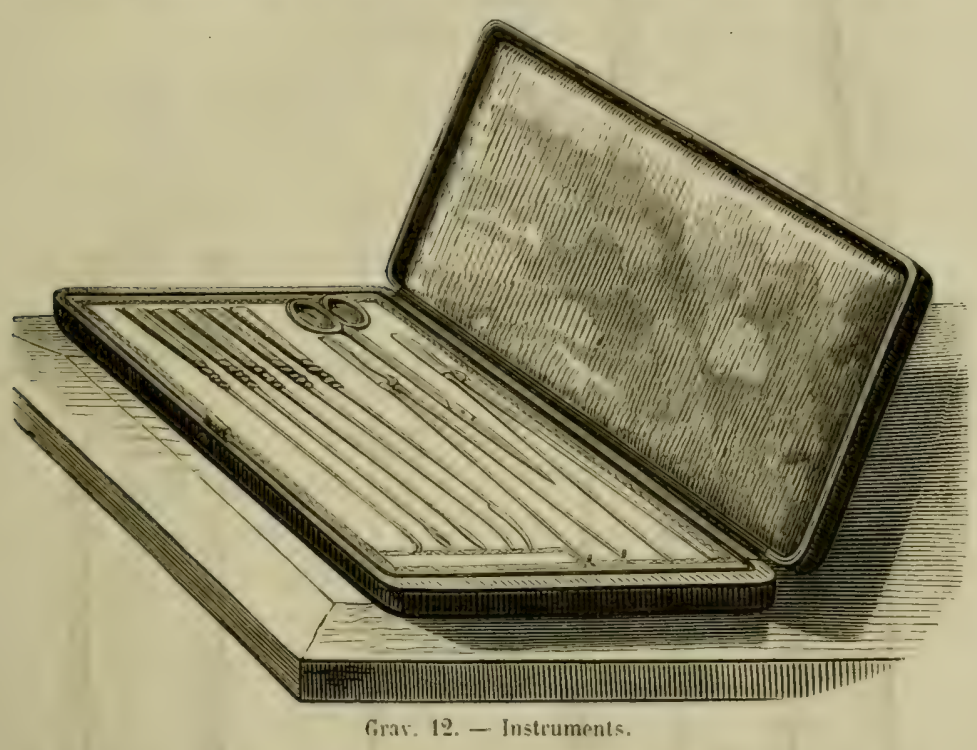

miniature, composent la trousse de lhorticulteur; c'est un néressaire aussi utile que la bêche et la serpette, et qu'un jardinier habile doit toujours avoir sous la main. Un peu d'adresse, heancoup de soins, sont les conditions indispensahles au succès, et chacun peut ensuite créer à son gré des plantes nouvelles, sans craindre que la nature se hasse des demandes réitérées qu'on lui adresse.

Nous reproduisons ici les figures d'instruments qui ont éti domnées déjà dans la Revue horticole du 16 septembre 1858 , 
et nous accompagnons ces figures de la note qu'y a jointe M. V. R.

“ Les instruments nombreux dont nous domnons ici le dessin sont destinés à favoriser les travaux de ce genre (fécondation naturelle et artificielle des végétaux). Ces instruments, d'abord fabriqués par M. Bernard, ont été considérablement. perfectionnés par M. Groulon, dont nos lecteurs connaissent
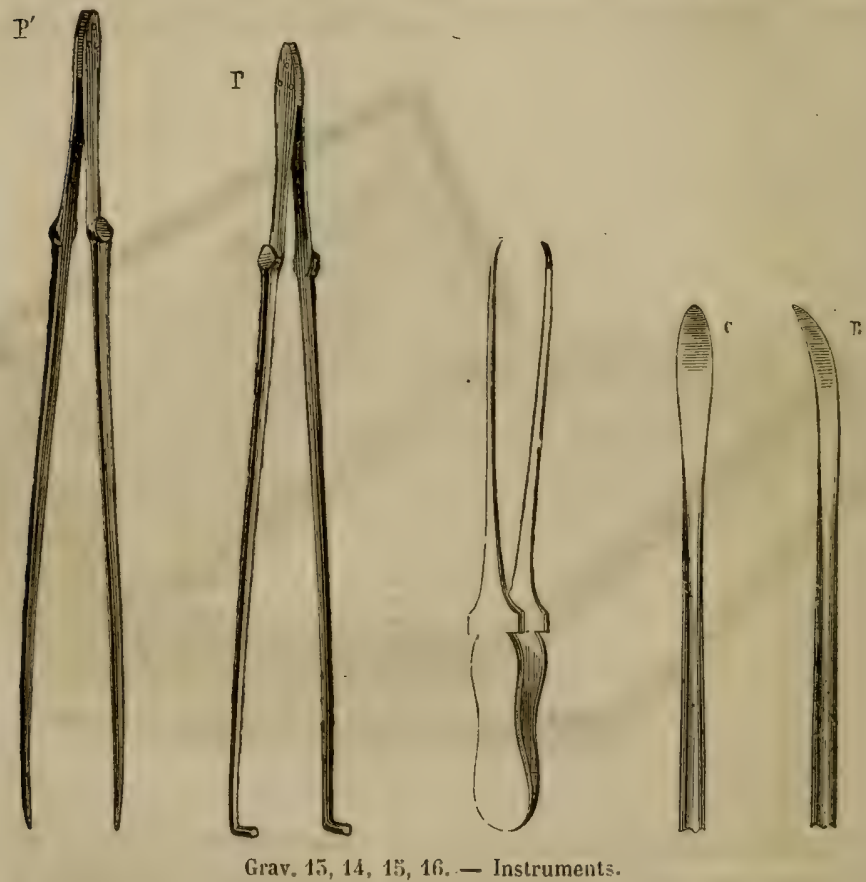

les bons produits. Nous avons placé à côté des outils les plus délicats un détail de grandeur naturelle, afin que ceux de nos lecteurs qui voudraient en faire fabriquer chez eux puissent donner à leur coutelier un modèle suffisant. Les figures nous paraissent suffire pour faire connaitre l'emploi des instruments qu'elles représentent, et rendre toute description superflue. ") (Grav. 12, 15, 14, 15, 16, 17, 18, 19, 20, 21.) 
INSTRUMENTS.
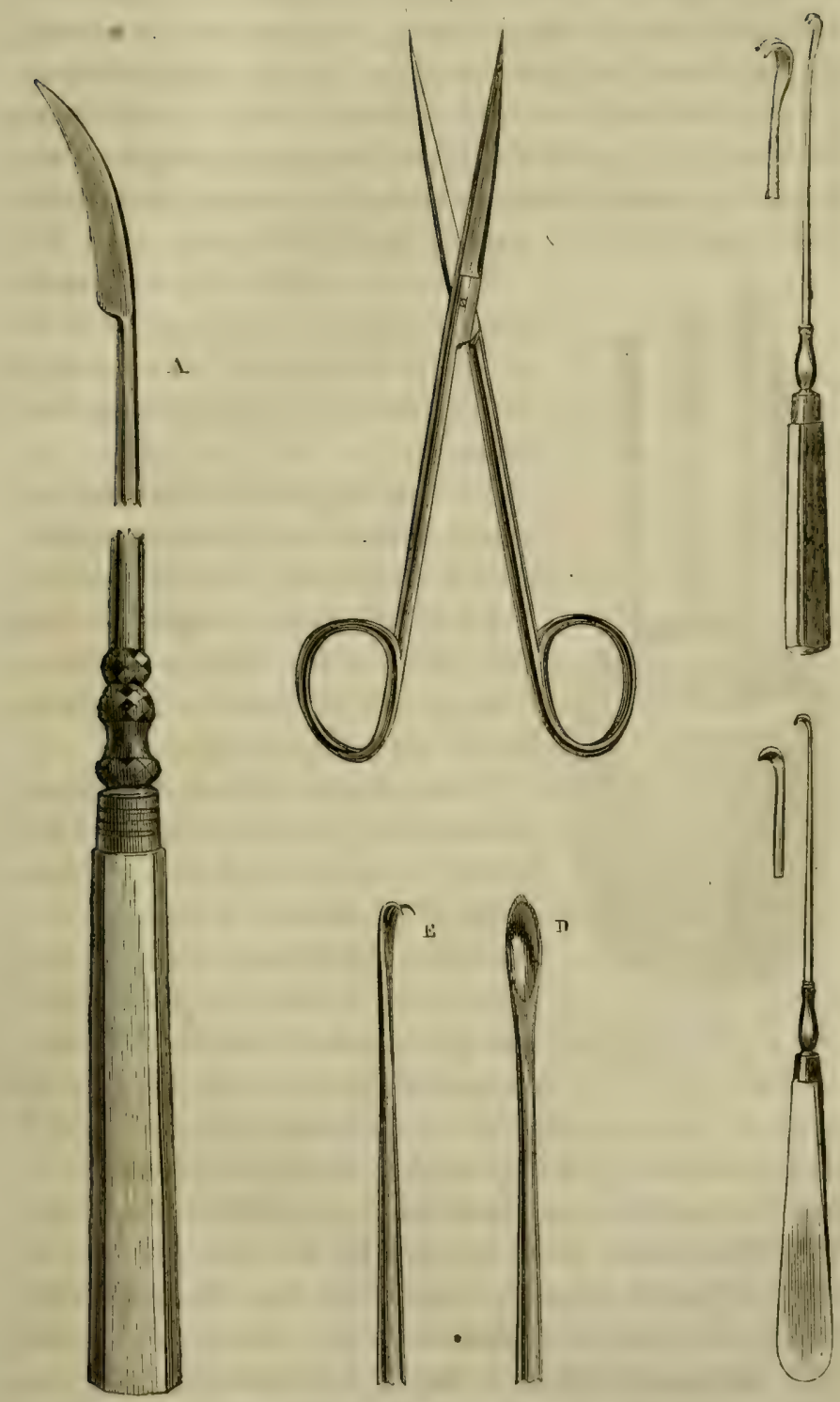

lirav, 17, 18, 19, 20, 21, - Instruments. 
Mon nécessaire pour hybrider a toujours été composé de deux gros étuis en bois ordinaire, et un seul suffit au besoin. On y renferme les pinces, les ciseaux, le canif et les pinceaux.

Le second étui porte, dans sa partie la moins profonde, un bouchon de liége ordinaire, lequel est percé de plusieurs coups de canif. Ces petites fentes permettent d'y insérer plusieurs pinceaux, par l'extrémité opposée au poil de blaireau (grav. 22).

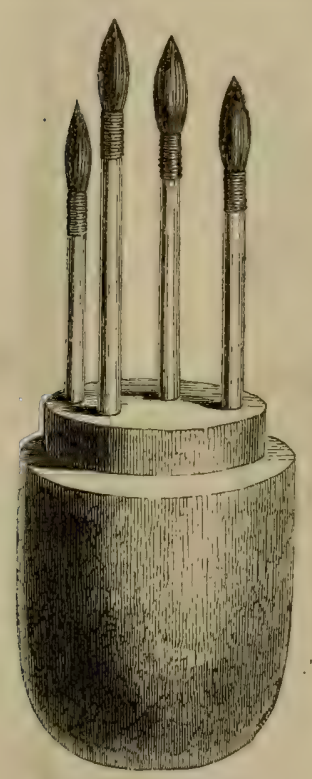

firav. 29. - Pinceaux placés dans un étui et destinés ì recueillir et à transporter le pollen.

Il n'est pas difficile de comprendre que ces pinceaux sont destinés à recueillir du pollen, que l'on emporte et que l'on conserve quelquefois très-longtemps.

Un coup de pinceau donné sur une variété nouvelle peut donner naissance, par l'intermédiaire d'une autre variété, à des hybrides remarquables, et plus d'un larcin a été commis au moyen d'une parcelle de poussière fécondante, fournie par une plante généreuse.

J'étais un jour chez un amateur qui possédait une très-belle collection d'Auricules, et qui m'en faisait les honneurs avec beaucoup d'aménité. " Je vous offre, me dit-il, des œilletons de la plupart de ces plantes. " Je le remerciai et lui demandai seulement l'autori. sation de charger mes pinceaux de lem pollen. Il n'y comprenait rien, et me laissait faire avec un sourire d'incrédulité, qu'il ne pouvait dissimuler. Je lui domnai rendez-vous dans deux ans, pour visiter la collection d'Auricules que jallais former à ses dépens. Un nouveau sourire, plus significatif que le premier, accueillit ma nouvelle proposition. Rentré chez moi, mes pinceaux furent essuyés sur un pied d'Auricule jaune, ì fleurs larges et bien faites, et, deux ans après, je montrais à cet amateur étonné des coloris qu'il 
ne connaissait pas, des fleurs plus larges quine pièce de cing francs, et je lui remettais, en échange cette fois d'un sourire de gratitude, mes pinceaux, qui venaient de caresser mes plus belles variétés.

Examinons dabord la castrution ou l'enlevement des étatmines, la conservation du pollen et son application; nous étudicrons ensuite son action et celle de la liquenr nectarifère.

\section{Castration ou enlèvement des étamines.}

Quand lit fleur s'épanouit et que l'on aperçoit ses organes dans son intérieur, rien de plus lacile que de les enlever avee la petite pince; quelquefois mème on peut les òter avec les doigts, comme dans certains Lis; mais on peut et l'on doit mème, dans la plupart des fleur's, derancer l'époque de l'épanouissement pour enlever les étamines, surtout quand celles-ci se fendent de bonne heure et répandent leur pollen sur les stigmates. On ouvre alors mécaniquement la corolle ou le calice, et on extrait adroitenent les étamines ou au moins les anthères, à l'aide d'une petite pince. Cette opération doit être laite avec quelque adresse; il faut ériter de les serrer trop fort, ce qui les écraserait et pourrait leur faire répandre le pollen; il laut éviter de toucher le pistil en les retirant, et, au lieu de les jeter par terre au pied de la plante, où elles mûrissent exposées au soleil et d'où le rent pent transporter le pollen sur les sligmates, il laut les recueillir dans une petite boite ou un cornet de papier. On les jette plus loin, ou bien on les conserve, si l'on a besoin du pollen pour opérer d'autres fécondations.

La castration ne présente, comme on voit, de difficultés que dans les cas où il faut ouvrir la fleur arant son épanouissement, ou bien lorsque, celle-ci étant épanouie, les anthères sont cachées au fond d'une corolle en entonnoir ou dans un tube allongé, comme cela a lieu daus un grand nombre de plantes monopétales. Diuns ces différentes circonstances, on peut quelquefois dérouler la corolle et enlever les anthires; mais le plus 
souvent il faut la fendre elle-même, soit avec la pointe du canif', soit avec celle de l'aiguille, au besoin même enlever un morceau du tube, assez grand pour qu' on puisse, dans tous les cas, aller chercher les anthères qui y sont enfermées. Il est rare que cette opération nuise à la plante; la fleur s'épanouit presque toujours comme à l'ordinaire, et l'on attend cet épanouissement naturel pour y poser le pollen étranger.

Les observations de Gærtner comme les miennes n'assignent a la corolle qu'un rôle insignifiant dans la fécondation. On peut li couper, la mutiler et l'enlever entièrement, sans que cela nuise à l'imprégnation du stigmate.

II y a des plantes dont les anthères s'ouvrent entièrement et restent pendant plusieurs jours couvertes d'un pollen adhérent qui acquiert sa maturité, puis se détache et va féconder le stigmate. Il vaut mieux ne pas attendre qu'elles soient ouvertes pour les enlever; mais, si l'on doit agir sous cette condition délavorable, il faut couper les filets avec les ciseaux pour nc pas donner de secousses, et enlever adroitement et très-légèrement les anthères avec les pinces.

Enfin, si les étamines sont très-nombreuses, et qu'il ne soit pas indispensable de les enlever, ce qui arrive souvent pour des plantes de serre où le pistil peut rester couché sur un lit d'étamines sans ètre imprégné, comme dans la plupart des Cactus, on isole le pistil dans un petit tube de verre pendant que l'on coupe toutes les étamines, ou l'on se contente de le placer dans un tube de papier, ouvert des leux côtés, et l'on féconde au pinceau, sans toucher à la fleur, dont le pollen, un peu filandreux ou glutineux, reste ainsi forcément éloigné du stigmate.

\section{Follew: - Coinservation.}

II arrive assez souvent que l'on ne peut se procurer le polleu l'ume plante à l'époque précise où l'on en aurait besoin pour en féconder une autre; dans diverses circonstances, on peuty 
remédier, en avançant l'une et retardant l'autre des deux plantes par les moyens connus; mais il arrive aussi que la chose est impossible. On peut alors conserver le pollen, en recueillant les authères quand elles sont mûres et au moment où elles vont s'ourrir. On place ces anthères dans de petits verres de montre, que l'on colle deux à deux avec un peu de gomme arabique légèrement posée sur les bords; on a soin de les laisser auparavant quelques heures ouverts avant de les coller, pour que le pollen puisse se dessécher et perdre la majeure partie de son humidité. Il ne doit être séché qu'à l'air libre, sinns chaleur artificielle, et, une fois enfermé, il faut entourer les verres de montre avec une petite feuille d'étain, semblable à celle dont on se sert pour première enveloppe du chocolat.

Cé procédé, indiqué déjà par M. Ragomnot Godefroy dans la Revule horticole, a été essayé par un de nos honorables collìgues, M. Giraud, secrétaire de l'ex-Société d'horticulture de l'Auvergne. On doit à cet habile expérimentateur une tentative très-remarquable sur la conservation du pollen. Voicr comment il s'exprime à cet égard: “ Il est aujourd'hui bien prouvé que le pollen des fleurs peut être gardé pendant un temps plus ou moins long; mais quelle peut en être la durée? C'est là ce que des expériences répétées pourront nous apprendre plus tard. Nous pouvons cependant aujourd'hui assurer que nous avons conservé, depuis 1842 jusqu'en 1845 , du pollen de Lis blane, qui nous a servi à féconder des fleurs de Lis auxquelles nous avions entièrement enlevé toutes les anthères, et que nous avons obtenu des fruits et des graines dans les meilleures conditions."

M. Ilaquin, de Liége, a fécondẻ avec succès des Lis avec du pollen extrait depuis quarant-huit jours; des Azalées avec du pollen de quarante-deux jours, et des Camellias qui ont fructifié avec du pollen de soixante-cinq jours. M. Haquin coupe les étamines dès l'épanouissement des tleurs, les place dans du papier bien collé, dépose ce paquet pendant vingt-quatre heures dans un endroit sec et chaud. Au bout de ce temps, la poitssière fé- 
condante est tout à fait développée; alors il ôte le pollen du papier, le place dans une feuille de plomb laminé, mince comme du papier, et renferme le tout dans un papier étiqueté, et dans un endroit froid sans être humide.

M. Ilay Brown, horticulteur anglais, a obtenu un hybride de l'Épiphyllum Jenkinsoni, fécondé par le polten du Cereus grandiflorus, qu'il avait conservé cinqà six semaines enveloppé dans un morceau de papier ${ }^{1}$.

M. Perottet a rapporté qu’à la Guadeloupe M. Barrau conservait pendant trois mois le pollen des fleurs mâles d'un Dattier dont la floraison avançait du même laps de temps sur celle d'un Daftier femelle.

Au reste, la connaissance de ce fait que le pollen se conserve longtemps date de loin, car Linné a conservé six semaines le pollen du Jatropha urens et s'en est servi avec succès pour fëconder des fleurs femelles.

M. Brown pense que si certaines plantes nefructifient pas, c'est que dans nos serres le pollen ne peut acquérir assez de maturité; tel est, par exemple, celui du Ciereus grandiflorus, dont la fleur reste ouverte une seule nuit. Il croit aussi qu'en conservant le pollen quelque temps il devient apte à donner des variétés plus distinctes qu'à l'élat frais, chose qui n'est pas impossible, puisque déjà l'expérience a démontré que les graines de certaines fleurs, telles que les Giroflées, donnent plus d'individus à fleurs doubles quand elles sont vieilles que lorsqu' elles sont nouvelles.

Quand on opère tout de suite et sur quelques plantes seulement, on trinsporte le pollen an bout du pinceau; mais, quand on pratique en grand, soit comme simple amateur, soit comme horticulteur marchand, pour livrer au commerce des graines remplies d'espérances ou pour obtenir des gains nouveaux de ses semis, on est obligé de récueillir le pollen dans de petites boites, et, sous ce rapport, je ne connais rien de plus utile

1 Horliculteur universel, rédigé par Lemaire, t. 11, p. 185. 
'qu' ume petite boite d'étain ou de bois, en forme de tabatière allongée, et séparée en plusicurs compartiments. On recueille dans chacune des cases le pollen d'une variété de couleur, et I'on a ainsi séparées les poussières fécondantes qui représentent des teintes différentes et dont on peut approximativement garder le souvenir en collant au couvercle au-dessus de chaque compartiment un pain à cacheter d'une couleur analogue à celle de la fleur qui a fourni le pollen. Si l'on veut plus de précision, rien n'empêche de mettre une étiquette: on évite, par ce moyen, de féconder des variétés james entre elles, de mettre du pollen représentant du ronge sur un stigmate où l'on voudrait appliquer du blanc; chaique case doit avoir son pinceau. Un raisome, on calcule, rien n'est livré au hasard, et, bien que ce dernier ait obtenu souvent de grands succès, je crois qu'il les doit surtout à ce que pendant longtemps il a conservé le monopole de la création des variétés.

\section{Pe l'applieation du pollen ou de la fécondation proprement dite.}

Rien de plus simple, assurément, que de poser du pollen sur un stignate; il suffit de prendre ce pollen au bout d'un pinceau et de toucher très-légèrement le stigmate avec ce pinceau. Un voit bientôt cet organe couvert de poussière lécondante, qui peut y rester très-longtemps adhérente. Il faut ì la rigueur très-peu de pollen pour opérer la fécondation; mais, quand il y a plusieurs styles ou que le stigmate offre seulement plusieurs lobes, il faut que ses différentes parties soient touchées, ciar chaque stigmate représente un carpelle ou pistil particulier, et leur soudure dans un grand nombre de lleur's n'entraine pas leur réunion complète. Si un seul stigmate reģoit le pollen, la loge à laquelle il correspond est la senle qui soit féconde.

La quantité de pollen appliqué sur un stigmate a done une assez grande importance sur la fécondation. Si l'on enploie le pollen de la mème espèce, il 'en fiut moins; mais, si l'on se sert d'un pollen étranger, il en liut davantage, parce que beancoup 
de grains ne se trouvent pas dans les circonstances favorables pour développer leur tube pollinique. De là nécessité de l'appliquer à plusieurs reprises sur le même stigmate et à des heures différentes de la journée.

Quand le stigmate est saillant, toute espèce de pinceau peut y poser le pollen; mais, quand il est inclus, il est nécessaire d'en avoir de très-petits qui puissent pénétrer dans les tubes des corolles et atteindre cet organe.

Il arrive aussi que l'organe femelle est tellement enfernęe ou phacé si bas dans la corolle, que l'on ne peut l'atteindre et que toute fécondation deviendrait impossible, si l'on n’avait recours ì de véritables opérations. Il ne faut pas craindre, dans ce cas, de l'endre la corolle, ce qui souvent a déjà été nécessaire pour l'extraction des étamines. On profite de cette même ouverture pour y placer le pollen. J'ai plusieurs fois pratiqué de semblables incisions sur des Jacinthes, sans nuire en rien à la production des graines; il faut seulement avoir grand soin de ne pas blesser le pistil avec l'instrument.

D'autres fois, les pistils de quelques Synanthérées, des Chry. santhèmes, par exemple, sont placés si profondément dans des lubes allongés, qu'ils sont inaccessibles au pollen. On doit les lendre longitudinalement avec la pointe d'une aiguille, ou, alı hesoin, commencer par raccourcir toutes les corolles avec les ciseaux, jusqu’à ce qu'on puisse arriver au stigmate. Une fois lit fécondation assurée, il est toujours utile de couper avec des ciseaux, et non d'arracher li majeure partie des pétales des lleurs doubles et des Synanthérées : on évite la pourriture, qui, à moins l'une année extraordinaire et d'ume sécheresse prolongée, ne manque jamais d'altérer les graines.

Une fois qu'un stigmate est imprégné par un pollen étranger, le propre pollen de la fleur elle-même n'a plus d'action sur lui.

Dans lout ce que nous voulons faire, il faut, autant que possible, imiter la nature, et, à plus forte raison, quand il s'agit d'un de ses principaux mysteres. Il faut donc altendre, pour 
poser le pollen sur le stigmate, l'heure ordinaire de l'épanouissement des fleurs. Les unes ne s'ourrent que le matin, d'autres à midi et en plein soleil, quelques-unes le soir ou la nuit, et il II est pas douteux que ce ne soit à ces époques diverses, si rariées pour chaque espèce, que la fécondation s'opère. Comme plusieurs de ces plantes n'ont leurs fleurs épanouies que pendant un temps très-court, il faut savoir saisir l'instant et en profiter; pour d'autres, on peut attendre, car les lleurs restent longtemps épanouies, et cola tient en général au mode de fécondition. Les fleurs doubles, qui n'ont pas d'étamines, restent longlemps ouvertes, cornme si les pistils attendaient, pour se llétrir, l'arrivée du pollen. Dans celles où la fécondation s'opère lentement, parce que les étamines se défleurissent successivement pendant plusieurs jours, les enveloppes florales attendent pour tomber que cette grande opération soit terminée. Entin, si les ctamines et les pistils ne sont pas aptes en mème temps, la corolle persiste jusqu'à ce que les deux sortes d'organes aient alecompli leurs fonctions.

Les fleurs dioüjues ou monoïques sont aussi sourent plus durables que celles qui sont hermaphrodites. En général, e’est le matin que la fécondation artificielle doit être tentée de préférence, puisque toutes les fleurs sont alors complétement épanonies entre sept et neul' heures; quelques-unes, comme celles des Graminées, répandent leur pollen aux premiers rayons du soleil, et c'est aussi l'instant qu' il fiut saisir. Le matin est, dinns la plupart des fleurs, l'épocque où le stigmate c'st le plus apte à retenir le polleri. La nuit lui a permis de sécréter un peu de cette liqueur visquense dont toutes ses papilles sont imbibées, "t le soleil n'a pu l'enlever encore. Enfin, il y a des fleurs dont la durée est si courte, que c'est au lever du soleil seulement 'fu'on les trouve dans un développement parfait; bientòt après, les pétales se détachent et tombent inmédiatement.

C'est done ordinairement le matin, quelquefois dans la journée, mais presque toujours avant midi, qu'il fiut poser le pollen au pinceau sur les stigmates, excepté cependant pour les lleurs 
nocturnes, où il faut attendre la nuit; encor's plusieurs de ces plantes, qui ne s'épanouissent qu’à l'obscurité, peuvent-elles aussi être fécondées le malin, au lever du soleil, un peu avant yu'elles se flétrissent. Quand les lleurs durent plusieurs jours, il est utile de recomnencer à poser le pollen tous les matins ei plusieurs jours de suite, afin d'être sûr d'arriver au point convenable, c'est-à-dire au moment où le stigmate est apte à le recevoir. Si l'on attend, pour féconder une fleur, l'époque de l'épitnouissement ou plutôt la puberté du stigmate, il faut avoir soin d'opérer le r'etranchement des étamines bien avant cette époque, comme nous le verrons en nous occupant des genres el des familles.

Disons seulement d'une manière générale que l'on peut reconnaître, avec M. Fermond, quatre époques distinctes dans l'accomplissement du phénomène de fécondation, relativement a la durée de l'épanouissement :

$1^{\circ}$ Fécondation dans le bouton avant l'épanouissenıent;

$2^{\circ}$ Fécondation au moment de l'épanouissement;

$7^{\circ}$ Fécondation pendant la durée de l'épanouissement;

$4^{\circ}$ Fécondation au moment où les enveloppes se ferment.

C'est qu'en effet, si la corolle est inutile à la fécondation dans une foule de plantes, il en est d'autres où, par ses mouvements de torsion, d'involution ou de chute, elle favorise le contact du pollen avec le stigmate. Nous avions fait depuis longtemps cette remarque; elle a été confirmée, il y a quelques années, par M. Fermond, particulièrement sur les lridées, dont les anthères sont extrorses, sur les Malvacées, les Campanulacées, certaines Papilionacées, etc.

\section{Action du pollen. - Sa strueture.}

Le pollen ou poussière fécondante des plantes a été éludié far de nombreux observateurs. Dès l'année 1711, Geoflioy avait consigné ses observations dans les Mémoires del'Académie des sciences. Il a décrit et figuré la forme des grains de pol- 
len dans un grand nombre de plantes, formes très-variées, comme on le sait, mais n'ayant peut-c̀tre aucune influence sur relles des granules qui s'y trouvent enfermés.

Linné avat aussi fait des observations sur le pollen et sur son action fécondante. "Chaque globule de poussière séminale, dit-il, renferme dans ses enveloppes une substance élastique, qui, quoique très-déliée et presque invisible, se répand cependant au moyen de l'eau chaude et souvent avec force; lorsque les plantes sont en fleur, la poussière séminale s'échappe des anthères et est dispersie en dehors, comme les semenees sortent du fruit et se répandent çà et là à l'époque de la maturité. En mềme trmps que la poussière fécondante sort des anthères, le prstil présente son stigmate; cet organe est alors dans l'état le plus parfait, et parait couvert d'une humeur particulière au moins pendant une partie du jour; il est entouré des étamines, ou, si les fleurs sont naturellement penchées vers la terre, ces mêmes itamines sont couchérs, de manière que la poussière séminale peut être portée aisément sur le stigmate; elle y est retenue, non-seulement par l'humeur qui enduit cette partie, mais encore par le fluide qu'elle contenait et qu'elle répand. Ce qui sort des globules de la poussière séminale, mêlé avec les lluiḍes du stigmate, est porté jusqu'aux rudiments des semences. ") (Limné, traduit par Broussonet, Journal de plingsique, 1711, p. 451.)

Linné, quoique très-bon observateur, se laissait entrainer Ipuelquefois par son imagination, et des études ultérieuresn'ont. pas confirmé, à beaucoup près, ni ses principes généraux sur la position des fleurs mâles au-dessus des fleurs femelles, ni les détails que nous venons de lire sur l'imprégnation.

Les grains de pollen offrent des formes très-différentes dans: Ins diverses familles du règne régétal el souvent mème dauss les genres. Ces grains, qui nous paraissent semblables à une poussière très-fine, ne sont que les grossières enveloppes d'un liquide fécondateur que l'on nomme forilla.

Nous reproduisons ici les figures coloriées de quelques es- 
pèces de pollen, telles qu'elles ont été dessinées par Guillemin dans les llémoires de la Société d'histoire naturelle de Paris, en 1825 (voyez planches 1 et 2 ).

II. A. Brongniart considère ces grains comme formés de deux enveloppes. La membrane externe est la plus solide, et elle parait percée de distance en distance par de petits orifices, au travers desquels la membrane interne fait des espèces de bosses ou hernies saillantes, et, lorsque ces grains sont en contact avec les stigmates, les hernies s'allongent et se transforment en tubes allongés, qui s'ourrent à leur extrémité et par lequel la forilla s'échappe. Ce tube ou boyau fécondateur, observé d'abord par M. Amici sur le pollen du Pourpier, a èté retrouvé depuis par M. Brongniart et plusieurs autres observaleurs. Il n'est donc plus douteux maintenant que la fécondalion ne s'opère dans les plantes par l'introduction de ces boyaux férondateurs entre les cellules du stigmate, comme l'indiquent la dernière figure de la planche 2 et les gravures $2 \overline{5}$ et 24 .

La matière visqueuse qui couvre ce dernier organe et dont la liqueur miellée ou nectarifère n'est peut-être que l'excédant, parait destinée à faire ẹ́clater les grains polliniques. L'eau produit le même résultat, mais le tube du pollen semble plutòt s'y dissoudre que s'y allonger, comme dans la liqueur visqueuse du stigmate. Ce qu'il ! a de certain, c'est que l'eau tombant sur le pollen en trop grande quantité s'oppose à la fécondation, ce que l'on remarque souvent quand des pluies surriennent pendant la floraison de la Vigne et des Céréales et font couler on arorter leurs fruits.

Ces accidents n'arrivent pas avec la liqueur visqueuse de charque stigmate, qui paraît appropriée au pollen qui doit y adhérer. On peut mème remplacer cette liqueur par celle des nectaires ou même par l'eau miellée; le miel, employé dans celte circonstance, n'étant en quelque sorte qu'une restitution inexacte, sans doute, aux plantes qui l'ont fourni aux aheilles.

Quand les grains de pollen sont posés sur le stigmate, ils restent souvent longtemps, quelquelois plusienrs jours, sans 
laisser échapper les tubes qui doivent répandre la fovilla. Pour assurer les fécondations artificielles, j’ai quelquefois humecté, is plusieurs reprises, le stigmate avec la liqueur des nectares, et je suis parvenu ainsi à faire fructifier des plantes qui hien rarement domaient des graines dans nos serres.

Malgré ce que nous venons de dire sur le pollen, il n'est pas probahle que dans toutes les plantes les tubes fécondateurs

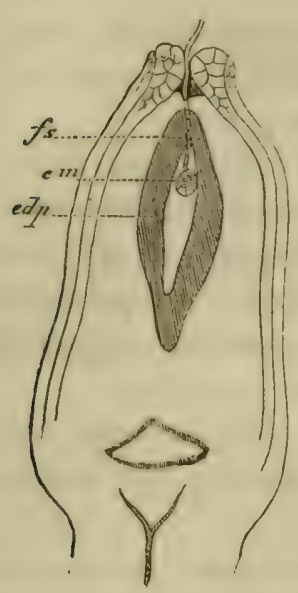

Lav. 25.-Coupe longitudinale d'un ovule ile Polygonum dans lequel on voit la naissance de l'embryon, $e, m$, et le fil suspenseur $f ; s$, qui le soutient; $e, d, p$, indiquent le commencement de la formation de l'endosperme de la graine.

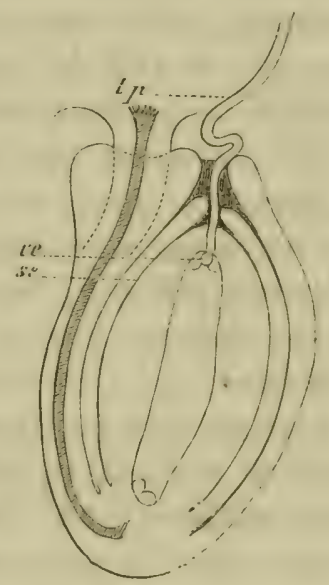

Grav. 21. - Coupe longitudinale d'un ovule de la pensée dans lequel pénètre le tube pollinique, $l, p$, il entic daus le micropyle et se prolonge jusqu'au nucelle, oì il alteint le sac embryonnaire, $s$, $e$; près du microprle se trouvent les vénicules embryonnaires, $v, c$, cellules très-rléli- cates qui naissent à l'époque le l'imprégnation.

soimt produits par ccs globules; tout porte à croire qu'il suffit, pour plusicurs d'entre elles, que le pollen puisse se rompre el répandre ses granules sur les papilles du stigmate.

L'adhérence du pollen an stigmate est sourent. un signe de fécondation. Ce n'est pas toujours le liquide visqueux du stigmate qui en relient les gramules, mais souvent ce sont les tubes polliniques qui ont prenctré dans les lissus et qui font adhérer les grains. C'est pour cela que le pollen même d'une plante 
semble s'y attacher plus facilement et plus promptement qu'un pollen étranger.

M. Brongniart a déterminé la grandeur ou plutòt l'extrême petitesse des granules contenus dans la liqueur qui sort de chaque grain de pollen, granules qui sont conduits par les boyaux fécondateurs dans les tissus du stigmate, et qui, selon toute apparence, arrivent jusqu'à l'orule. Il a reconnu que les uns étaient globuleux, arec un diamètre de 1/456 à 1/700 de millimètre, et les autres ellipsoïdes ou cylindroïdes avec des diamètres de $1 / 46$ de largeur sur 1/550 de longueur, ou de $1 / 456$ de largeur sur 1/700 de longueur.

Ces dimensions sont déterminées pour chaque genre, et II. Brongniart pense, avec raison, que ces granules sont adaptés aux méats intercellulaires de ces genres, et que de cette proportion peut dépendre la possibilité ou la non-possibilité de la formation des hybrides parmi des plantes congénères ou analogues:

Les recherches plus récentes de M. Mohl attribuent aux gramules de la fovilla des dimensions beaucoup plus petites que celles indiquées par İ. Brongniart.

Pour que l'on puisse prendre une idée de l'extrême variété qui règne dans la forme et dans l'organisation des grains de pollen, nous allons donner un aperçu de la classification proposée par M. Hugo Mohl.

Ce n'est pas que la forme si différente de ces grains soit un obstacle direct à la fécondation entre espèces dont le pollen ne présente pas la même configuration, puisque le grain lui-même n'est que l'enveloppe grossière du liquide ou des organismes fécondateurs. Le grain de pollen llétri reste sur le stigmate et le tube fécondateur seul pénètre entre les tissus ou à travers les tissus jusqu'à l'ovule.

Mais la différence extérieure du grain entraine des dissemblances physiologiques que nous ne pouvons apprécier, el nous pouvons être certains d'avance que des plantes à pollen dissemblable ne peuvent pas se croiser. 
On remarque une grande analogie entre les pollens d'espèces du même genre et souvent encore entre genres différents de la même famille.

Le volume des grains de pollen offre aussi de très-grandes variations, et il en est de mème de celui des granules ou molécules organiques contenus dans le liquide appelé fovilla et que le tube pollinique conchit jusqu’à l'ovule, avec la liqueur dans laquelle ils nagent.

Le professeur. Mohl partage d'ahord le pollen en trois grandes séries :

1. Pollexs a vise selle membrine. - Cefte spiri ne comlient que le pollen des Asclépiadées.

2. Pollens a neux membranes.

* 5. Pollens a trois membranes.

\section{POLIENS A DEUX MEMBRANES}

Cotte seconde division est partagée en quatre groupes, d'après l'organisation plus ou moins compliquée de la membrane extéricure :

A. Membrane externe sans plis ni pores;

I. Membrane externe présentant des plis longitudinanx.

(i. Membrane externe présentant des pores.

I). Membrame extèrne présentant des plis Iongitudinanix ef des pores.

Premiire division. I. Membrane externe granuleuse : Strelit:in reginx, Calla pulustris, Crocus vermus, Ramunculus arvensis, heancoup d'Euphorbiacées. II. Membrane exterme papilleuse, Camma indica, Bauhinia furcatu. III. Membrane externe rellu. leuse : Aleurites triloba, Phlox undulata, Tribulus terrestris.

A la suite viennent les formes dérivées qui résultent de lil rémion de plusieurs grains souvent soudés quatre à quatre: Periploca yraca, Juncus Jacquini, Iu:ula vermalis. 
B. Deuxième division. I. Un seul pli longitudinal : la plupart des Monocotylédons. Il. Deux plis longitudinaux: Watsonia plantaginea, Tamus communis. III. Trois plis longitudinaux: Cynomorium, Nelumbo, Quercus robur. IV. Quatre plis longiIndinaux, Sideritis scurdioides, Solanum tuberosum. V. Six plis longitudinaux : une partie des Labiées et des Passiflorées. VI. Plus de six plis longitudinaux: heaucoup de Rubiacées, Sesamum orientale.

Formes dérivées. M. Mohl considère comme des dérivés le pollen du Pin, du Sapin, du Nymphæa Lotus, le pollen prismatique de la Capucine et les formes qui tendent au tetraèdre, au cube el au dodécaèdre, comme dans le Corydalis capmoides, le Fumaria spicata, le Rivina brasiliensis, etc.

C. Troisième division. J. Un seul pore : Graminées, Cypéracées. II. Deux pores: Colchicum, Broussonetia. III. Trois pores : Dombeyacées, Onagrariées, Protéacées, quelques Amenlacées et Passiflorées. IV. Quatre pores: Myriophyllum verticillatum, Campamula rotundifolia, Impatiens noli tangere. V. Plus de quatre pores: Alnus glutinosa, Campanulu speculum, heancoup de Nyctaginées, de Convolvulacées, de Chénopodées, de Caryophyllées, Gucurbita pepo, Malvacées, Polygonées.

Formes dérivées : assemblage tetraédrique du Jussiæn ererta; réunion des grains de pollen des Mimosa.

D. Quatrième division. I. Trois pores et trois enfoncements: beancoup de Dipsacées et de Géraniacées. II. Trois plis et trois pores : la plupart des Synanthérées, Syringa vulguris, Ligustrum vulgare. III. Plus de trois plis, dont chacun contient mn pore: la plupart des Boraginées, les Polygalées. IV. Six ì neuf plis, domt trois contiement un pore : Lythrarices, Melastomacées, Combretacées. V. Trois à quatre plis, avec six à huit papilles : Hyræa odlorata. VI. Trois plis et trois papilles, nom situées sur les plis : Carolinea campestris.

Formes dérivées : assemblage tétraédrique des grains : Éricinées, Epacridées, Vacciniées. Forme cubique et dodécaédrique: Gendichandia suffruticosu, Malpighiacées. Formes polyé- 
driques : la plupart des Lactucées, Trayopoyon pratense, Crepis rubra, etc., etc.

\section{POLLEN a TROIS MEMBRANeS.}

Cette division peu nombreuse contient le pollen de plusieurs plantes de la famille des Conifères.

II. Mohl termine ces observations, dont nous n'avons domń fir un extrait très-abrégé, par les considérations suivantes :

"Il résulte de ce (jue l'on vient de voir que la structure plus simple ou plus composée des grains de pollen n'est pas in rapport direct arec la place plus ou moins élevée que les plantes oreupent dans la série des familles, mais la nềme forme de pollen se trouve dans des familles différentes, et sonrent très-éloignées par tout le reste de leur organisation.

" A la vérité, on peut dire, en général, que chez les Monointylédones dominent les formes à un seul pli longitudinal, ou ì un seul pore, et que celles à trois plis ou à trois pores se trouvent presque exclusirement chez les Dicotylédones; e’est presique la seule règle que nous trouvions dans le partage des tormes entre les différentes fimilles, et encore est-elle sujette i des exreptions. Les mềnes formes de pollen se rencontrent frìguemment dans des familles très-éloignées, ce que Guillemin et .I. Brongniart ont établi d'une manière incontestable. An rontraire, ces deux auteurs voulaient, et leur opinion it cel igard a été soutenue par d’autres observateurs, que les plantes de. la même famille eussent une même forme de pollen, règle dont M. Brongniart excepte toutefois les Cucurbitacées. Commue ire rapport, ontre la grande rraisemblance qu' on lui trouse d'ahord, est encore appuyé sur des exemples frappants, tels uque ceux des Graminées, des Cypéracées, des Thymélées, des Protéacées, des Onagrariérs, des Dipsacéres, des Ericinées, des Eiparridées, des Polygalées, des Grossulariées, des Chénopodées, des Silinées, des Myrtarées, des Mélastonarces, des Lumrinées, f'est it ma gramde surprise que jiat trouvé dans le cours de mes 
recherches que la forme du pollen varie extrêmement, nonseulement dans les genres d'une famille, mais aussi dans les espèces d'un même genre, et que même, dans plusieurs plantes, la mème anthère contient des grains de pollen de formes assez diverses. Un petit nombre d'exceptions ne peuvent pas renverser une loi générale; mais iei les exceptions se multiplient tellement, que l'assertion de la conformité du pollen dans la même famille ne peut être considérée comme vraie, prise dans cette extension. ")

M. Mohl produit un tableau des formes du pollen dans deux cent onze familles, en groupant les espèces de chaque famille d'après la forme de leur pollen, et il ajoute :

"Quoique ces observations soient contraires à la loi en question, toutefois, d'un autre côlé, il faut reconnaitre que les différentes formes de pollen ne se trouvent pas répandues an hasard dans le règne végétal, mais que, sinon les familles, sonvent un certain nombre des genres d'une famille, ou du moins des espèces d'un genre, présentent des pollens semblables on analogues. D'où l'on peut déduire, comme règle générale, que les plantes très-voisines forment des groupes, qui ont des formes de pollen semblables ou rapprochées. Toutefois il ne fant pas oublier ici que les groupes formés d’après les formes du pollen ne coïncident pas du tout d'une manière régulière aves les genres et les familles admis dans la botanique systématique. » (Änn. des sc. nat., $2^{2}$ e série, t. III.)

\section{Des nectaires et des insectes.}

(On trouve, dans le plus grand nombre des fleurs, des glandes d'un tissu tout particulier, diversement logées dans les autres organes, et auxquelles on donne le nom de nectaires, parce que leurs fonctions sont de sécréter une liqueur sucrée dont le rôle nous est encore à peu près inconnu. Beaucoup de botanistes ont négligé les neetaires, d'autres ont donné re nom aux appen- 
dices particuliers qui les portent, ou bien l'ont appliqué à tout ce qui dans la fleur ne pouvait se rapporter aux organes sexuels ni aux pièces du périgone, se débarrassant ainsi, par un mot jeté dans une description, de tout ce qui pouvait nuire à cet ancien arrèt porté par les botanistes, que les fleurs étaient toujours formées du calice, de la corolle, des étanines et du pistil, plus quelquefois des nectaires.

Les glandes nectarifères existent dans presque toutes les plantes, el si très-sourent on ne les a pas apergues, c'est qu'elles n'étaient pas entourées d'un appareil particulier qui les faisait remiarquer, conme, par exemple, dans les Ifellébores, où clles. nceupent le fond d'un cornet; dans la Capucine, où elles sont situées an fond de l'éperon du calice; daus la Courome impériale, où elles tapissent d'admirables fossettes, véritables sources de liquide nectarifère.

Plus souvent, le nectaire s'étend, sous la forme d'une couche standuleuse plus ou moins épaisse, sirr le torus ou sur le réceplacle des fleur's, et là distille sa liqueur, quelquefois si abondante, 'qu'elle s'écoule d'elle-même de la fleur et tombe sur le sol, comme dans le Strelitzia reginx. Lin somme, il y a peu de plantes qui, à l'époque de l'anthèse, manquent de cette sécrétion de l'humeur miellée, soit sur les papilles de leur stigmate, soit sur des organes particuliers.

La fréquence des nectaires dans les végétaux a fait penser à quelques botanistes que l'humeur miellée avait un rôle à remplir daus la fécondation, et l'on doit au célèbre Vaucher, de Genève, une foule de remargues du plus grand intérêt sur les lunctions du nectaire on plutôt de la liqueur qu'il sécrète. J'ai puisé souvent, dans l'ouvrage ${ }^{1}$ de cet excellent observaleur, des documents du plus haut intérét pour le travail que je rédige. Son livre est une mine inépuisable de recherches dirigées dans: une voie yui, malheureusement pour la science, est peu suivie par ses adeptes.

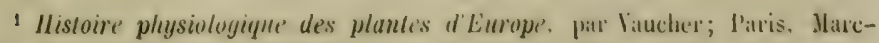
Aurel, 1841 . 
L'humeur miellée est-elle destinée, comme le croit Vaucher, it recevoir le pollen et à renvoyer l'aura seminalis sur les stigmates? J'avoue que je crois peu à une fécondation qui n'est pas opérée par le contact immédiat du pollen sur le stigmate, et, comme la plupart des stigmates sont glanduleux el qu'ils sćcrètent une sorte de liqueur, ne serait-il pas plus rationnel de considérer l'humeur miellée comme destinée à être absorbée parr l'organe femelle, à l'époque de l'imprégnation?

Il y a toujours un fait certain, c'est que le pollen, mis en conlict avec la liqueur des nectaires, ne tarde pas à éclater et ì :e trouver dans les conditions nécessaires à lia fécondation. Aussi, charque fois que j’ai opérésur des fleurs à nectaires, j’ai préalitblement imprégné le stigmate de la liqueur niellée, et j’en ai obtenu d'excellents résultats.

Le procédé pratique est très-simple; il consiste à prendre avec un pinceau très-propre un peu d'humeur miellée dans la fleur même sur laquelle on opère, ou sur une fleur semblable s'il laut la détruire pour en obtenir, et à lit poser très-délicatement sur le stigmate, en ayant soin de ne pas noyer cet organe. Avec un autre pinceau, on recueille le pollen et on l'applique avec les mèmes précautions. On sait que l'humidité est nécessaire ì l'explosion du pollen, et je me suis bien trouvé, quand les flantes sécrétaient peu de matière sucrée, de mouiller le pinceau avec de l'eau, de le passer sur les glandes nectarifères pour culever le peu de matière sécrétce et l'appliquer sur le stigmate.

J'ai même employé avee succès de l'eau sucrée avec du miel ordinaire, et même de l'eau pure sur des genres entièrement dépourvus de glandes, comme les Pelargonium.

On sait, llu reste, qu'unc pluie douce favorise souvent la lécondation, et que de grandes pluies font couler les fleurs, c'estì-dire produisent un effet contraire. L'imbibition préalable du stigmate est donc une opération qui doit être faite avec tous les ménagements possibles; mais ellc est souvent nécessaire, car, avec des organes parfaitement secs, l'imprégnation n'a pas lieu. 
Du reste, dans la fécondation que l'on opère sur des lleur's à nectaires, rien n’empêeche de fiaire tomber avec le pinceau du pollen surbla liqueur miellíe, qui peut-être agit aussi comme le soupçonne Vaucher.

Koelreuter croyait que le fluide sécrété par le stigmate était luvileux et il recommandait de lubréfier cel organe avec une groutte d'lumile fraiche de noix ou d'amandes douces. Le docleur Aldrige, de Dublin, ayant observé que les acides prodursaient la déhiscence des grains de pollen, a essayé de poser sur les stigmite's de quelques plantes du papier de tournesol, yui a rougri. Il en al conclu que la fécondation s'opérait par l'action de l'acide du stigmate qui faisait éclater les grains et produisait les lubes polliniques. Nous n’avons essayé ni l'huile recommandée par Kicetreuter, ni les acides faibles du docteur Aldrige.

La présence de la liqueur micllée, et şa sécrétion plus abondante a l'époque de l'anthèse, ne rentreraient-elles pas dans ces moyens si variés et si mystérieux que l'Auteur de la nature a mis en æurre pour assurer ses immuables volontés? Ce micl n'altirerait-il pas ces tribus ragabondes d'insectes bourdonnints dont le eorps, convert de mille pinceaux, disperse le pollen sur toutes le's parties de la fleur, et concourent ainsi, en butinant pour leur propre comple, au grand curre de la reproduction? Long('mups les liorticulteurs ont laissé faire les insectes, sans comprendre celte grande legen quilis recevaient tous les jour's, et li récolte d'une cellule de miel a peut-être produit les premières variélés de Jicinthes el de Tulipes qui sont venues décoperer nos parterres.

Le transport du pollen par les insectes est un fait parfaitenent avéré. Il y a des plantes gui, sans eux, seraient constamment inlécontes, et si plusicur's espèces de nos serres chaudes ou tempérées restent stériles, c'est souvent parce que nous n'avons pas importé arec elles les insectes qui vivaient sur leurs fleurs; uni pénétraient dans leurs corolles, et qui, secouant leurs ailes sans cesse agritées, échangeaient avec elles des bienlaits dont la nature seule connaissait la valeur et arait dicté la réciprocité. 
Nous aurions pu nous étendre beaucoup plus sur ces généralités, mais nous n'avons dit que ce qui nous semblait indispensable, réservant pour chaque genre ou pour chaque famille les détails dont nous aurions pu faire ici le résumé. Il est impossible de faire un travail comme celui-ci sans répétitions; peut-être quelques-ines d'entre elles sont-elles nécessaires. Nous arons voulu, avant tout, faire un ouvrage de pratique, non un livre de science, ni une œuvre de littérature.

\section{gLELQUES CONSIOÉRATIONS GÉXÉRALES SUR LES MYBRIULS.}

Un a singulièrement abusé, conne nous l'avons déjà dit, du mot d'hybride, en l'appliquant sans discernement ì de simples vitriations ou à de véritables espèces. Personne, sous ce rapport, :'a porté l'abus aussi loin que Linné, qui admettait les hybrides entre genres différents, et surtout entre genres limiéens, car, depuis cette époque, les hybrides sont venus bien souvent attaquer la légitimité de plusieurs genres modernes.

Nous ne savons pas où s'arrète la possibilité des croisements, mais nous devons supposer qu'ils ne peuvent avoir lieu qu'entre des plantes qui ont entre elles beaucoup d'alfinité, entre espèces du même genre, entre genres de même fanille, tout au plus, et rarement encore.

La crédulité de certaines personnes est parfois poussée bien loin. Un jeune homme vint un jour me confier qu'après avoir bien étudié mon livre sur l'hybridation, il avait fait depuis plusicurs années des tentatives multipliées pour croiser les Primevères de la Chine avec son plus beau Fuchsia (ce dernier agissant comme père), et me demandait sérieusement s'il ı' eût pas mieux valu employer la Fuchsia comme porte-graine.

Si la nature aidée par l'homme se refuse obstinément à ces unions ridicules, elle accomplit seule de très-nombreux mariages adultérins dans les espèces saurages.

Le nombre des hylorides qui se forment naturellement dans 
les campagnes et dans les jardins est considérable. C"est à peine si dans les écoles de butanigue, où les plantes sont rapprochées, on peut conserver les t!pes des Ticotiuna, des Digjitalis, des Dianthus.

Dans les chanjs, les Verbascum se croisent tellenent, qu' il devient difficile de déterminer les hybrides et de recommaitre leurs parents.

Les Cirsium se sont hybridés si souvent, que lioch, dims la seconde élition de son Sinopsis, en décrit 56 appartenant senlement au centre de.l'Europe.

Et que savons-nous si la Rosomanie, la Rubomanie, la llicraciomanic et autres manies dont quelques botanistes sont gratvement atteints, nont pas pour causes, outre les varialions naturelles, de fréquentes hybridations?

Lne des grandes questions relatives aux hybrides est de sitvoir s'ils sont ferliles. J'ai été vivenent attaqué, lors de la première édition de cel ouvage, pour avoir allimé qu'il y avait plus d'injbrides fertiles que d'hybrides stériles, el depuis cette époque jai été pleinement conlirmé dans cette opinion.

Les expériences si nombreuses de M. Taudin sur les hybrides lui ont démontré, comme à moi, contre l'opinon généralenent reçe, que la plupart des hybrides sont fertiles, et que, saul l'arortement des gỉains polliniques, ee qui a lieu quelquelois, lous pouraient le devenir dans certaines conditions dàge et de culture.

.lais la grande question, comme le reconnait aussi M. Naudin, est de savoir " si les hybrides fertiles par leur propere polIn conservent indéfiniment, par voie de grénération, leurs caractires mixtes ou leurs anomalies, te manière à lane sonche d'espèces. „) (Naudin.)

M. Saudin ne le pense pas. Il en domne pour raison que lit nature, qui a liat les especes parce quiclle en arait besoin, et qui les a organisces pour des fonctions détcrninées, n'a que faire des hybrides, qui ne répondent pas à son plan; atusi les fiail-elle généralenent disparaitre an un pelit nombre de gé. 
nérations, et quelquefois dès la première, en leur refusant la faculté de se reproduire. »

Nous ne savons ni pourquoi ni comment la nature a fail les espèces, et nous doutons qu'elle ait grand besoin de toutes celles qu'elle a faites; aussi avons-nous bien plus de confiance dans les expériences patientes et ingénieuses de ce savant naturaliste que dans des idées sur les besoins de la nature. Toutefois nous ne croyons pas la question résolue par la négalive, et nous pensons, au contraire, qu'en choisissant parmi les hybrides fertiles les individus, quelquefois en petit nombre, bien semblables aux types dejà hybrides, qu'en agissant pendant longtemps, par voie de sélection, sur ces hybrides, on parvient à fixer certains types et à les conserver iudéfiniment. Par ce dernier mot nous ne voulons pas dire que le nouveau type fixé ne variera jumais; il y aura bien quelques écarts; nous voulons dire qu'il restera, comme les autres espèces, sensiblement fixe, tant qu'il se trouvera entouré des mêmes conditions. On ne pent nier ni la fertilité ni l'indépendance de certains hybrides de Mirabilis et d'Eyilops.

Nous reconnaissons dans tous ces hybrides fertiles une grande tendance à l'atarisme, c'est-à-dire une propension à retourner Liuntòt à l'un des types, tantôt à l'autre, plus souvent à la mèrc qu'au père. Mais l'atavisme, comme l'indique son nom, remonte au delà des derniers parents, il rappelle souventle grandpère, la grand'mère, les bisaïeuls, et l'on est tout étonné, au bout de quelques générations d'hybrides, de voir reparaitre des formes que l'on croyait perdues. Cette tendace à l'atavisme, ubservée par tous ceux qui se sont occupés de croisements, lait que souvent les plus belles variétés de Pélargonium, d'Azaléa, de Rhododendron et d'une foule d'autres plantes ne produisent par les semis que des formes très-ordinaires et souvent moins helles que la plante qui a fourni la graine. II faut soutenir ces belles variétés par des hyybridations nouvelles el successives. Tous ceux qui ont pratiqué longtemps savent par expérience et par cette seule voie que certaines variétés, qui ne sont pas tou- 
jours les plus belles, sont les meilleurs porte-graines, celles qui doment les plus heaux gains. (Qu'on le demande i II. Souchet, pour ses Glaieuls.)

"II est à remarquer, dit M. E. Regel, que, parmi les milliers de plantes qu'on obtient chaque année dans le jardin de Zurich au moyen de fécondations artificielles, il y en a toujours un trèsgrand nombre qui ressemblent aux formes ordinaires on qui s'en écartent fort peu, tandis que, dans les mèmes semis, on n'en trouve qu' un fort petit nombre qui s'écartent notablenent de ce que l'on comnait. La raison en est simplement que la fécondation entre des espèces voisines et des lyybrides est proporliomellement facile el donne en abondance de bomes graines, landis que celle qu'on opere artificiellement sur des hybrides arec une plante qui n'est pas l'un des parents est toujours difficile et domne peu ou pas de bounes graines. Mais le petit nombre de plantes qu'on obtient daus ce dernier cas constitue des nouveautés remarquahles. De là il résulte qu'on obtient les meilleurs résultats en prenant des hybrides pour porte-graines. La nature conduit du reste elle-même à cette méthode; car le plus souvent les hybrides ne produisent pas de bon pollen, et la conséquence en est 'fu'on peut rarement les employer comme pères. L'état délectueux du pollen est souvent un moyen cerlain pour distinguer un hybride d'une variété. II faut cependant rappeler qu'il existe quelques hybrides qui produisent toujours de bon pollen, tels que le Matthiola maderensi-incana, ceux des Petunia, 'quelques-uns de ceux de Begonia, etc. " (Soc. imp. et centrale d'hortic., t. I, p. 252.)

Si l'hy̧bridation a pour résultat de produire sousent des plantes d'une vigueur extrème, l'inverse a également lieu. Ainsi, dans nos nombreuses hybridations de Mirubilis, nous arons: tous les ans quelyues sujets qui doment des boutons cn abondance, mais des houtons qui se dessèchent et tombent sans s épanouir. Il est rai que ce phénomène ne se présente jamais sur les individus ubtenus diredement par la lécondation du pistil du .M. jalupu par le pollen du .M. Iongjfloru, mais seulement 
à la seconde génération provenant des graines de ces hybrides.

Charles Morren cite un fait sernblable au sujet des Hymenocallis disticha et rotata. Leurs hybrides, quand le temps de lit floraison était arrivé, produisaient des lıampes stériles où les boutons étaient décolorés et mourants.

Il est encore une observation du plus grand intérêt relative à la vigueur des sujets ou à l'hybridation. Plus on développe le sujet, plus on le nourrit, plus en l'arrose, en un mot, plus on développe ses organes foliacés et ses bourgeons, moins on a de. chance de réussir dans J'hybridation. Ce fait est la conséquence d'une loi que l'on désigne sous le nom de loi du balancement des oryanes.

Les plantes ont deux moyens de se reproduire: par gemmes ou bourgeons, et par semences. Or la vigueur d'un individu le pousse toujours à produire de nouveaux bourgeons, et ceux-ci sont formés aux dépens des organes de la reproduction ou des graines. Si au contraire une plante souffre par une cause quelconque, elle tend à fleurir et à fructifier. Elle s'empresse de le laire comme si elle craignait que sa race fût perdue. On sait du reste que, pour mettre à fleurs ou à fruits certains végétaux rebelles, il faut leur faire le plus de mal que l'on peut: les faire souffrir de la soif longtemps, courber leurs branches, les couper ì moitié, les mutiler à coups de bâton, etc., pour diminuer leur vigueur. Cet excès de développement des organes de la végétation sur ceux de la reproduction est la cause qui rend souvent les hybrides infertiles, et par la mème raison on peut ètre assuré qu'un individu souffrant sera plus apte à fruclifier et à recevoir' le pollen d'une autre espèce que s'il était en pleine végétation.

Le développement complet et normal du péricarpe par suite du contact d'un pollen étranger est une chose quel'on voit souvent sans que pour cela les graines soient fertiles. Quelquefois même les graines manquent totalement.

La maturation du fruit d'une plante hyloridée est ordinairement plus longue que celle du fruit fécondé par le propre pollen de la plante. L'ovaire semble hésiter avant de s'accroitre, 
et quand il a commencé il mûrit un peu moins vite. Dans tous les cas, le fruit et lit graine conservent exactement la forme habituelle à la plante mère, et rien n'indique, avant la nouvelle génération qui doit en sortir, les caracteres de l'espèce qui a fourni le pollen.

Telle est du moins l'opinion généralement admise, mais qui laisse encore des doutes dans l'esprit de plusieur's horticulteurs; c'est de savoir si l'hybridation réagit sur les péricarpes et sur les graines des fleurs hybridées.

L'exemple que nous citons plus loin du Pommier d'Abheville semblerait répondre affirmativement à cette question. Liopinion, généralement recue, que de mauvais Melons placés près de variélés méritantes en altèrent la saveur immédiatement, al]puierait encore cette manière de voir' nous devons dire qu'elle est partagée aussi par un pomologiste distingué, M. I. de Liron d'dirolles, qui, nous l'espérons, parviendra à domner par expérience une solution à cette grave question.

Il est certain toutefois que dans les expériences assez nombreuses que jai tentées, mais à la vérité sur des fruits à péricarpes secs, je n'ai jamais pu reconnaitre, ni sur les péricarpes, ni sur les graines, aucun signe qui pût m'indiquer que l'hỵbridation avait en lieu. Parmi les quantités de graines de Mirobilis que jai obtenues, aucune ne différait des graines frovenant de lleurs non hybridées de la mème plante. Il était de Ioute impossililite de les distinguer, et pourtant les graines des Mirabilis varient de forme et de grosseur avec la plus grande facilité.

Dès que ces graines-levaient, il etait facile de constater les différences, ct les plantes hybrides domaient elles-mènes des graines qui tenaient souvent le milieu entre celles des deux espèces; mais, jusqu’à cette génération, l'embryon paraissail avoir seul subi l'action du pollen modificateur.

En dehors d'expériences dircetes il est un fait qui, dans celte question, lerait pencher pour la négative; c’est yue le mene arbre fruitier donne constamment des fruits semblit- 
hles. Nous ne trouvons pas sur le même Pommier plusieurs variétés de pommes, sur le même Cerisier des cerises distinctes par la forme et le coloris, sur un pied de Prunier des prunes intermédiaires entre la reine-Claude et les mirabelles, etc.; et cependant le vent et les insectes ne peuvent ètre restés étrangers à la fécondation de toutes ces fleurs.

Le développement des fruits des plantes liybridées se fait souvent d'une manière très-inégale. Ainsi l'on voit les péricarpes se développer comme.à l'ordinaire sans contenir de graines. Dans d'autres conditions, on trouve des péricarpes et des graines, mais celles-ci sont sans embryon. Dans de meilleures circonstances quelques graines sont fertiles. Enfin, dans le plus petit nombre des cas, toutes les graines sont honnes. La quantité de pollen appliquée, laquelle doit toujours être considérable, peut faire varier ces résultats. C'est à tel point même, que dans les espèces fécondées naturellement par leur propre pollen, si les graines sont très-nombreuses, comme dans les Pavots, les Tabacs, les Digitales, on rencontre toujours un certain nombre de graines qui ont échappé à l'imprégnation.

Il résulte d'observations très-précises qu'il y a même des plantes stériles par elles-mèmes dans certaines circonstances, qui se laissent féconder par un pollen étranger. William IIerbert rapporte que les Zephiranthes carinatu et $Z$. tubispatha ne donnent pas de graines en Angleterre; mais, si le dernier est fécondé par le pollen du premier, il fructifie et produit des graines fertiles. Le mème fait s'est reproduit sur des Amaryllis cultivés par M. IIerbert. On l'a reconnu également sur quelques Passiflores et sur plusieurs autres plantes.

Pendant que j’écrivais ces lignes, j’ai reçu de M. Charles Darwin un Mémoire tiré des Proceedlings de la Société linnéenne de Londres, et qui m'a fait d'autant plus de plaisir qu'il a trait au sujet dont je m'occupe et qu'il rient confirmer des observations que j’avais faites depuis longtemps sans en tirer les ingínienses conséquences que H. Darwin' a pm y voir. Il s̀agit du 
dimorphisme des organes sexuels de plusieurs plantes, mais particulièrement du genre Primula.

On savait, et ceux qui hybrident le savent mieux que les autres, que dans les P'rimevères de nos prairies, comme dans les Auricules et les Primevères de Chine, on distingue deux formes très-différentes par la longueur du style et par la position des étamines; mais on n'en savait pas davantage.

Dans l'une de ces formes, le stigmate est inclus et les étamines se montrent à l'issue du tube de la corolle; dans l'autre, ce sont les étamines qui sont enférmées et le stigmate qui fait saillie, porté par un long style. Ceux qui cultivent les Auricules appellent clouées celles qui présentent ce dernier caractère, ils donnent le nom de paillettes aux ctamines saillantes, et désignent sous le nom d'ail la réunion des étanines au sommet du tube quand le stigmate est inclus.

Après avoir recomnu que dans la plupart des Primevères, el peut-être dans toutes, il y arait un nombre à peu près égal d'individus cloués et d'individus aillés, M. Darwin en a recherché la cause. Il a fait précéder cette recherche des olsserrations suivantes:

Les Primevères longuement stylées ont une pistil beancoup plus long, aree un stigmate globuleux et beaucoup plus rugueux, situé bien au-dessus des anthères. Les étamines sont courtes; les grains de pollen moins volumineux et de lorme oblongue. La moitié supérieure du tube de la corolle est plus renflée, le nombre des graines produites est relativement plus faible.

Les Primevères brièvement stylées ont un pistil court dont la longueur est moitié de celle du tube de la corolle, arec un stigmate lisse aplati, placé au-dessous des anthères; les étimines sont allongées; les grains de pollen sphériques et plus volumineux; le tube de la corolle conserve son mème diamètre jusqu’à son extrémité supérieure; le nomlire des graines produites est relativement plus grand.

" Jai examine, dit M. Darwin, un grand nombre de flents, 
et, quoique la forme du stigmate et la longueur du pistil soient variables, surtout dans la forme à court style, je n'ai jamais vu aucume transition graduelle entre ces deux formes. Il n'y a jamais le plus léger doute relativernent à la forme sous laquelle on doit classer l'individu, jamais je n'ai rencontré les deux formes sur la même plante. »

Après ce court résumé des longues observations de M. Darwin, on se demande avec lui si ce dimorphisme nindiquerait pas une tendance à la dicecie, et si ces plantes à long style ne tendraient pas à devenir femelles ou à en jouer le rôle, tandis que les individus à étamines saillantes rempliraient les fonctions de mâles? M. Darwin est arrivé à reconnaitre cette tendance, mais avec cette différence que ce sont les plantes à court style qui seraient les femelles. Ce sont les plus fertiles dans la propartion de 41 à 54 .

“Quoi qu'il en soit, dit M. Darwin, la possibilité du passage lent et graduel d'une plante à l'état dioïque mérite d'autant plus d'être mentionnée que le fait pourrait facilement échapper à l'observation. ”)

En poursuivant ses expériences sur le plus ou le moins de fertilité des Primevères, M. Darwin eut l'idée de les isoler au moyen l'une gaze et de mettre ainsi les ombelles fleuries à l'abri des insectes turbulents qui pourraient venir contrarier ses essais.

Il obtint ce résultat curieux que des plantes à court style, munies ensemble de 27 ombelles de fleurs, ne produisirent que 50 graines, et 18 plantes à long style, pourvues de 74 ombelles, n'en donnèrent pas une; d'autres plantes abritées dans la serre furent également stériles. Ici, comme dans la plupart des plantes diö̈ques, lintervention des insectes est donc indispensable.

Mais il faut remarquer que dans le transport du pollen par les insectes la fécondation est souvent indirecte, c'est-ì-dire qu'ils peuvent prendre sur ume fleur le pollen dont ils saupoudrent le stigmate d'une autre fleur, ct c'est ce qui arrive dans les Primevères. 
Lia plus curicuse peut-être des expériences de II. Darwin est d'aroir fécondé artificiellement, d'un côté, les plantes à court style par leur propre pollen, celles à long style aussi par leur propre pollen, et, d'un autre còté, celles à court style par le pollen de celles à long style, et réciproquement: ce qu'il appelle fécondation homomorphe dans le premier cas, fécondation hétéromorphe dans le second. Toutefois, dans les fécondations homomorphes il a pris soin encore de prendre le pollen sur une fleur différente de celle qui était destinée à le recevoir.

Or les fécondations hétéromorphes ou entre plantes dissemMables ont toujours été plus fertiles que les autres, et cela dans la proportion de 6.4 à 40 pour le Primula sinensis, et de 54 à 5.) pour le P. veris. Nous reproduisons ici la ligure au mosen de laquelle U. Darwin montre les rapports qui existent dans la fécondation réciproque des plantes à long stỵle et à court style (grav. 25).

\section{Union hétéromorphe. \\ Complète fertilité.}

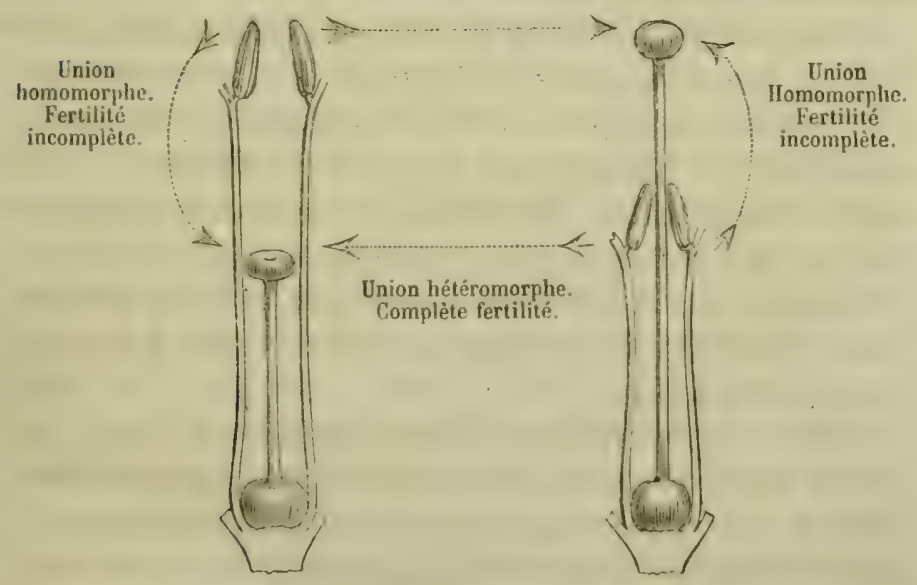

Grav. 23. - Forme i long style.

Forme à court style.

" Ia signification et le hut de l'existener, dans les Primula, de deux formes en nombre à peu pres éral, avee leur pollen ap- 
proprié à une union réciproque, est suffisamment claire : le but est de favoriser le croisement entre individus distincts. Parmi les végétaux il y a de nombreuses combinaisons qui tendent à cette fin, et on ne peut comprendre la cause finale ou la structure d'un grand nombre de fleurs si l'on ne tient compte de ce fait. 》

Quoique généralement le pollen d'une plante soit plus approprié au stigmate de cette plante que celui d'une espèce très-voisine, nous ne savons pas si nous n'obtiendrions pas des variétés nouvelles, ou des plantes plus vigoureuses, ou des individus plus fertiles, en fécondant artificiellement le stigmate par le pollen de la mème espèce, mais provenant d'un individı différent. La nature accomplit souvent elle-même ces sortes de mariages par une foule de moyen indirects dans lesquels les insectes, comme nous l'avons reconnu, jouent un rôle très-actil'.

M. Darwin croit tellement à la nécessité de ces croisements, qu'il est persuadé que le pollen d'une Primevère, de l'une des leux sections à court ou à long style, doit être préféré par le stigmate de la forme opposée.

“Les deux formes, dit M. Darwin, quoique présentant chacune les deux sexes, sont en fait diöques ou unisexuelles. Quelque avantage qu'il puisse y avoir à la séparation des sexes, séparation vers laquelle nous trouvous une tendance si friquente dans la nature, cet avantage est ici si exactement réalisé, qu'une des deux formes est fécondée par l'autre el réciproquement; et cela parce que la poussière fécondante de chaque forme a moins d'action que celle de l'autre forme sur son propre stigmate.

"Que l'état dimorphe des Primula, continue M. Darwin, ait ou non quelque rapport avec d'autres points d'histoire naturolle, il a de l'importance en ce qu'il montre comment la nature s'efforce, si je puis m'exprimer ainsi, à favoriser l'union sexuelle d'individus distincts de la même espèce. Les ressources de la nature sont sans bornes; et nous ne savons pas pourquoi les espèces de Primula ont acquis ce nouveau et curieux secours, pour empêcher de continuelles fécondations 
de la plante par elle-même, au moyen de la séparation des individus, et deux groupes d'hermaphrodites possédant une puissance sexuelle différente, au lieu de la méthode plus fréquente de la séparation des sexes, ou bien de l'aptitude à des périodes distinctes des organes mâles et femelles, ou, enfin, de tout autre artifice. "

M. Darwin cite ensuite un grand nombre de cas de dimorphisme plus ou moins complet, et plus ou moins apparent.

Il est évident pour moi que le dimorphisme n'est autre chose qu'une de ces modifications employées par la nature pour obtenir des fécondations indirectes. La séparation directe des sexes ou diøcie est le cas le plus exceptionnel. Dans la monœcie, les sexes, comme nous l'avons vu, sont disposés de telle manière, que la fécondation est presque toujours indirecte. La fleur femelle d'un rameau, contrairement à l'opinion de Limné, que l'on a reproduite sans y regarder, ne reçoit guère le pollen que de la fleur mâle d'un autre rameau: or, les végétaux pouvant être considérés comme des agrégations d'individus, la fécondation monoïque, dans les conditions que je viens d'indiquer, devient presque dioïque.

J'ai publié dès l'année 1827, dans une thèse soutenue à l'École de pharmacie de Paris, de nombreuses observations de fécondations indirectes, sujet dont on s'occupait peu ì cette époque. La question des hybrides, étudiée avec soin, est venue confirmer mes idées sur le croisement des types à des degrés différents. J'ai un partout la fécondation indirecte plus fréquente que la fécondation directe, même dans les fleurs hermaphrodites, et j'arais comparé mème ces fécondations indirectes à celles de certains mollusques, tels que les Helix dans une circonstance, les Lymnées dans une autre.

La nature semble avoir antipathie pour les fécondations directes des plantes, comme pour les alliances consanguines des animanx. Seulement, l'inconvénient de ces alliances direetes entre parents parait d'autant plus sérienx que les êtres sont plarís plus hant dims la sírie. Faibles dans les plantes et daus 
les animaux-inférieurs, les conséquences fàcheuses de ces unions deviennent plus graves chez les oiseaux et les mammifères, si terribles dans l'espèce humaine, qu'une grande partic des dégradations qui touchent même à l'intelligence proviennent de mariages entre parents.

Parmi les plantes hermaphrodites se présentent des causes nombreuses d'unions indirectes. Nous pouvons citer : la situalion relative des organes, souvent contraire au contact du pollen avec le stigmate ou le dimorphisme, la disproportion des dimensions entre les filets et les styles, la viscosité du pollen ou son adhérence, l'ouverture cxtrorse des anthères, les différences entre les époques d'aptitude des organes mîles et des organes femelles, l'avortement plus ou moins complet d'un des organes essentiels, ce qui nous conduit à la séparation complète des sexes.

Dans le mois d'avril dernier (1862), j'eus la bonne fortune de communiquer ces observations sur les fécondations croisées cutre individus de même espèce à la Société centrale d'horticulture de Paris. Mes savants collègues voulurent bien écouter avec attention et bienveillance l'exposition de faits qui touchaient si directement à l'horticulture, et je reçus sur ce sujel quelques communications d'un grand intérêt.

II. Rivière me dit avoir inutilement essayé de féconder un Oncidium Cavendishiamum par son propre pollen; les lleurs restèrent stériles. Mais, en prenant le pollen sur un pied différent, il obtint immédiatement des fruits fertiles. Il fit alors la contre-partie de l'expérience, e'est-ì-dire qu'il prit le pollen du pied devenu fertile pour le poser sur le stigmate de ceiui qui venait d'agir diö̈quement, et il obtint les mêmes résultats. La fécondation directe était done sans résultat et la fécondation réciproque parfaitement assurée.

M. le docteur Pigheaux a bien voulu aussi m'adresser une note que je transcris presque littéralement.

"Les arbres fruitiers, dit mon savant collegue, aiment ì vivre en famille; ils sont d'autant plus féconds qu'un espace 
limiti ne contient que des fruits de même espèce. Il est même profitable de les rapprocher d'autant plus les uns des autres, que leur floraison est simultanée el quiils fructifient dans la même saison. Cette observation porterait à ç̣oire que la poussière pollinique de plusieurs individus concourt, soil par l'intermidiaire des insectes, soit par les vents, à fëconder des individus moins aptes a imprigner leurs propres lleurs.

" Dans un grand jardin, un arbre unique de son espèce est en gieneral peu fécond. L'. Imandier, qui le premier nous montre ses fleurs au printemps, est de ce nombre. J'en ai eu deux dans: mon jarlin; il n'y en avait jamais qu'un seul qui fructifiail, celui qui était sous le vent du sud-ouest, qui souflle presque constamment sous le climat de Paris. Depuis la mort de l'arbre fécondateur, l'autre est resté presque stérile.

"Les Cerisier's de la reine-Ilortense sont peu cultivés, i cause de leur délicatesse et de leur peu de fécondité habituelle. Eh bien, si l'on en constitue un petit groupe dans unc encoignure de jardin, on roit leur fertilité égaler celle de toutes les antres espèces; mais les plus féconds, comme mon Amandier, sont toujours sous le vent.

"Quand on cultive des arbres à haute tige et des arbres a hasse lige, soit en rangée, soit en quinconce et suflisamment espacés et alternant entre eux, les plus fertiles d'abord sont ceux qui reçoivent par leur position infërieure l'inlluence pollinique du plis élevé, et en tout temps les plus féconds sont toujours ceux qui sont situés sous le rent du sud-ouest, ce qui lait prisumer que les vents ont au moins d'autant d'inHuence que les insectes pour féconder les lleurs.

" En Syrie, les Dattiers mâles agissent par leur pollen à de grandes distances quand ils sout dans certaines conditions hien connues des Arabes, qui s'en rapportent tous a l'inlluence du vent du sud-ourst. Ils ne fécondent artiliciellement, à l'aide de fleurs males prises sur les Dattiers saurage's, que ceux qui sont it contre-vent du sud-ouest, el encore ont-ils reconmu leur moindre fertilité et leur assignent-ils une valeur hien moindre. 
"Je pourrais multiplier à linfini ces exemples; mais, cumme ils ont tous la mème signification et la mème portée, ceux que jai citis suftisent pour conlirmer de tous points rotre manière de roir. Tos obserrations ont d'autant plus de valeur que la nature seule sest chargée d'en tournir les éléments. "

Tous pourons done, en enrisageant la question au point de vue pratique le lhỵbridation, remarquer dans les diffẻrents modes de fécondation des degres dirers que nous allons citer, dans l'ordre de leur éloignement de la fécondation directe et hermaphurodite.

P'́revier degré. - La fleur est lécondèe par son propre joullen, c'est-à-dire par les étamines de celte mème fleur où existe le stigmate.

SEcord negrê. - La fleur est fécondée par le pollen d'une autre fleur, appartenant à la mème grappe, au mème épi, ou enfin à la même inflorescence.

Trorsième degné. — La fleur est fécondée comme ci-dessur. mais par le pollen done fleur appartenant à une autre inflorescence ou à un autre rameau florifère du meme inclividu.

()catrièye degné. - La tleur est lécondie par le pollen de la mème espèce, mais pris sur un individu différent.

Cirotiène detié. - La fleur femelle est fécondée par une fleur màle, appartenant au mème rameau ou à la mème inflorescence.

Sixième degrit. - La fleur femelle est fécondée par une fleur màle, appartenant à un rameau différent ou à une inllorescence différente, mais sur le même pied.

Septièue degrié. - La fleur femelle est fécondée par le polleu d'une fleur màle, située sur un pied différent.

HLntéme degré. - La fleur hermaphrodite ou unisexuée est lëcondée par le pollen d'une autre variété.

Xecrièye tegré. - La fleur hermaphrodite ou unisexuée, al tëcondée pai le pollen diune espèce diférente.

IhaĖue uegré. - La fleur hermaphlırudite un unisexuce 
hybridée est fécondéc par le pollen d'une autre fleur, également hybride.

On comprend tous les intermédiaires qui peuvent exister entre ces derniers degri's, et toutes les exceptions que les insectes peuvent apporter partout, en troublant les unions les plus régulières.

Les détails que jai réunis dans ce livre se rapportent pres. yue entièrement aux derniers de ces degrés de fécondations, c'est-it-dire aux hybrides de variétés et d'espèces, et aux hybrides d'hybrides.

Les essais de fécondation entre individus distinets de la nème espiece riont été pratiqués, à ma connaissance, que sur les Primula, par II. Darwin, et sur les Mirabilis, par moi-mème, 't pendant plusieurs années. J'ai acquis la certitude que la génération y̧ui résultait de ces croisements était extrêmement vigoureuse, plus robuste que les parents, et cette sorte de vitalité oul d'énergie atteint son maximum dans les hybrides d'espèces.

Certaines plantes, qui n'acceptent pas leur propre pollen, deviendraient peut-ètre fertiles, si, au lieu de recueillir lo pollen sur le porte-graine, on se la procurait sur un individu distinct de la même espèce. T'oute une série de nombreuses expériences reste ì faire sur ce sujet, et le travail de M. Marwin ouve une ire nouvelle à tous ceux qui voudront entrer daus celte voie si curieuse et si pleine d'avenir et de progrès.

Sans vouloir donner aux insectes plus de pouvoir qu'ils n'en exercent réellement dans les grandes harmonies de la nature, nous devons rappeler encore une fois l'importance de leur ròle dius les fécondationis indirectes. Il est évident qu'ils lécondent souvent des fleurs par le pollen de la même espèce, mais par de's pollens pris sur des individus différents, et qu'ils croisent ainsi des générations qui, sans cet intermédiaire, auraient lieu entre proches parents.

L'importance bien constatie d'un rucher à proximité d'un verger ou d'un jardin vient appuyer cette opinion du ròle actil les insectes pour favoriser les fécondations indirectes. 
On ne sera pas étonné de cette importance, si l'on se rappelle les curieuses conclusions qu'llubert de Genève a tirées de ses nombreuses observations. D'après lui, les abeilles d'une ruche voyagent dans une circonférence dont le rayon moyen est de quatre kilomètres. En donnant pour population moyenne à chaque ruche douze mille habitants, en attribuant à chaque abeille quatre voyages par jour, on obtiendra quarante-lıuit mille chances multipliées par le nombre de fleurs que chaque insecte pourra visiter par voyage. Nettons dix fleurs seulement, et nous aurons quatre cent quatre-vingt mille visites qui s'augmenteront dans une énorme proportion si un autre rucher est situé tout près du domaine qu'il devia exploiter. Si nous ajoutons aux abeilles de notre ruche ces innombrables hyménoptères sauvages que nous voyons partout hutiner sur les corolles, si nous supputons les myriades de mouches, de papillons et d'imperceptibles insectes qui trouvent aussi leurs aliments ou leurs plaisirs au sein des fleur's, nous serons forcés de reconnaitre que chacune d'elles a bien des chances pour recevoir la joussière fécondante d'une autre fleur.

Nous terminons en citant en leur faveur une observation faite dans les serres du Luxembourg par MI. Rivière, dont personne ne conteste le talent et l'habileté, et rapportée par M. Duchartre à la Société de botanique (séance du 27 décembre 1861).

Un Agave, l'A. potatorum, Zuce., ayant développé ses fleurs, II. Rivière fit sur elles divers essais de fécondation artificielle. "Dans ce but, il transporta sur le stigmate de ces fieurs le pollen que les anthères ouvertes offraient en abondance, et, afin d'avoir plus de chances de succès, il se servit de tous les moyens

- habituellement employés dans ce but. Il prit done le pollen avec un pincean fin, avec des barbes de plunc, avec les doigts, et le déposa sur les stigmates des fleurs épanouies. D'un antre côté, pensant que l'heure à laquelle l'opération était faite pouvait exercer quelque influence sur le résultat, il agit sur ces stigmates à des moments très-divers de la journée, toujour's sans 
exception, la fécondation artificielle échoua, et pas un seul oraire ne sulit un commencement apprécialie d'aecroissement. Cependiant une circonstance inprérue et tout ì fait accidentelle amena le resultat désiré, an moment où l'on désespérait de l'obtenir.

"Lidgave fleuri se trouvait dans le tambour ou le vestibule vitré qui sert d'enltre à me serre: un essaim de mouches s̈introduisit dans cet endroil, et peu de jours après II. Rivière recommut, avec unc vire surprise, que les fleurs de sa plante étaient fécondées, que lés oviares commenģaient à frendre un développenent rapide. Ainsi solfrait à ses yeux me nouvelle preuse de l'utilité, déja plusiems lois constatée et cependant rncore anjoud hui contestée par certaines personnes, qu'ont les insectes pour la lécondation des diverses plantes. "

Te se pouraait-il pas que l'insuceés de M. Riviere tint à ce yuiil a recueilli le pollen yu’il a pose sur le stigmate dans la lleur mène oi ce sligmate so trumait placé, opérant ainsi une léeondation directe, larfuelte sonvent ne réussit pas"? Les insectes, au contraire, recueillaient indistinctement la poussière fécondante sur toules les lleurs, appartenant aussi a d'autres branche's, et croisident les unions aulant que cela peut avoir lieu sur le même individu.

D'un antre còti, et en supposant que cet Igave que je ne commais pas ait eu de la linueur miellér, Ir. Riviere a-t-il posé sur lestigmale un peu de cette liqueur lubréliante qui détermine l'apparition des tubes polliniques dque les insectes posent souvent aree le pollen dans les flenr's yutils ront courtiser? J'ai manque de's fécondations de Strelitsiu pour aroir négligé de poser an pincean sur le stignate un g en de celle liqueur nectarifère.

Ca sont de simples indicitions que je dome icianx botanistes et anx horlicultems pum we rien megher dins l'application d'une patique (l'hybridationi is langelle j'attache toujours une grande importance. 


\section{CHAPITRE IV}

\section{DYGOTYLÉDONES THALAMIFLORES}

PLANTES- $\Lambda$ PÉTALES DISTLNCTS INSÉRÉS SUR LE hÉCEPTAGLE.

\section{FAMILLE DES RENONGULACÉES.}

\section{Genre Clématite. - Clematis.}

Les organes reproducteurs des Clématites sont nombreux et presque à découvert, car les sépales de ces plantes sont quelquefois caduques, et n'opposent par conséquent aucun obstacle à la fécondation indirecle. Les étamines sont en assez grand nombre et entourent les pistils, qui sont eux-mèmes assez multipliés. Les étamines extérieures répandent les premières leur pollen, qui peut atteindre non-seulement les pistils de la mème fleur, mais encore celles du mème bouquet et celles des branches inférieures; les étamines plus rapprochées des pistils s'ourrent ensuite, puis successivement celles qui les fouchent, en sorte que si les pistils ne sont pas aptes à recevoir dès les premières émissions du pollen, ils le deviennent nécessairement pendant la longue anthèse nécessitée par la maturité progressive de toutes les anthères. Ajoutez à cela que les pistils sont souvent plumeux, qu'ils semblent avoir des mouvements qui les éloignent du centre en les rapprochant des étannines, et vous 
remarquerez que lontes les conditions d'une léeondation trèssûre sont admirablement remplies.

Une léemulation artilicielle dervint done assez diflicile dans

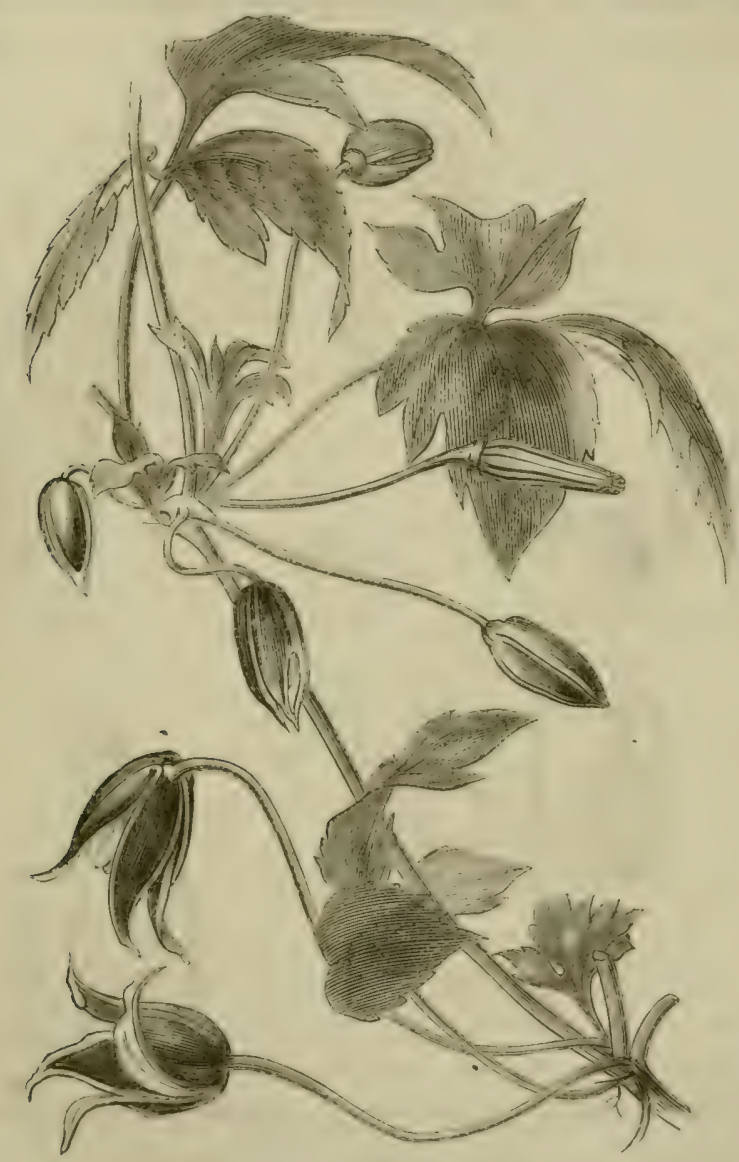

Grav, wi, - Clematite barbelée .

les Clematites, et expendant que de hedles varietes pourraien

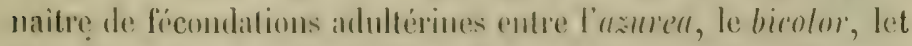
peregrina et mème le vilullat de pense ropendant pu'il serait 
facile d'y parvenir, en employant quelques précautions. D’abord, lisolement du sujet, indispensable toutes les fois que les plantes hermaphrodites peuvent ètre fécondées dioïquement, et mème l'isolemint des fleurs, quand, par la même raison, le pollen d'une autre fleur située sur la même plante peut venir déranger

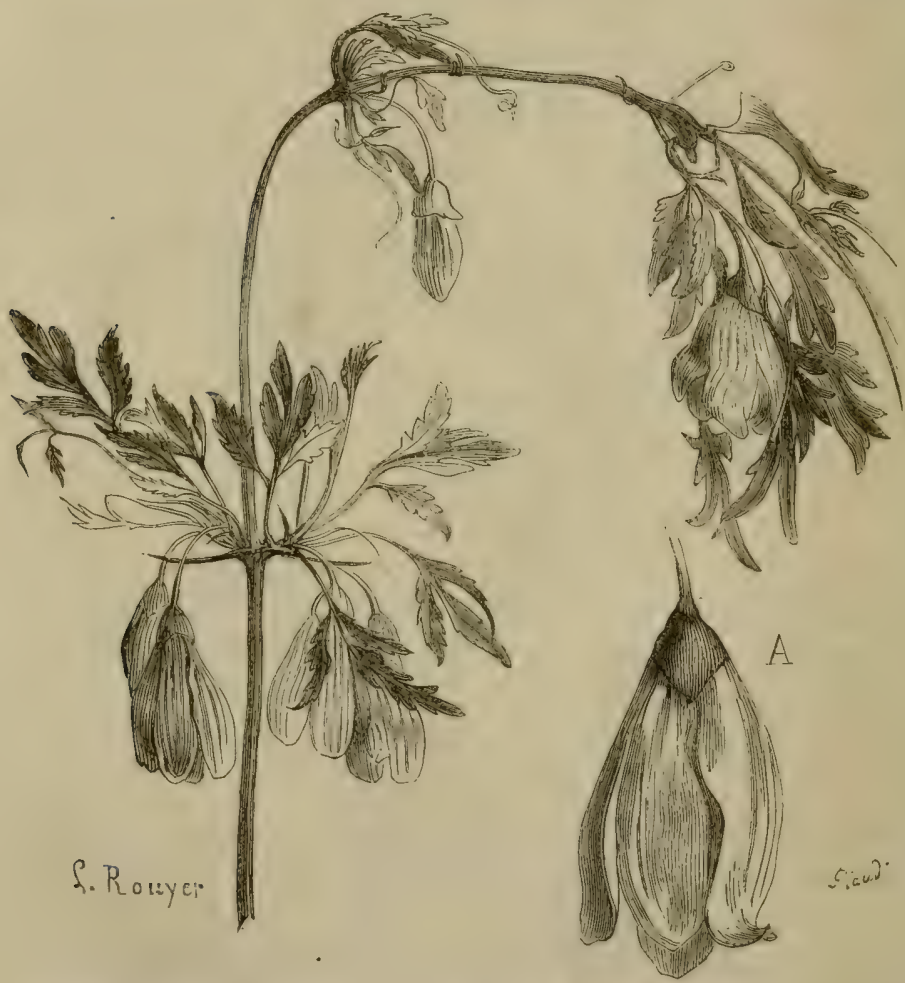

Grav. 27. - Llameau de la tilématite ì calice, moitié grandeur naturelle, et fleur de grandeur naturclle.

ct rendre inutiles les précautions que vous employez. Si donc vous n'avez rien à craindre des fleurs voisines, épiez le moment de l'épanouissement, que vous pouvez au besoin accélérer de quelques heures mécaniquement, et cnlevez avec les pinces tous les organes miles. C'est seulement le lendemain, ou nème plu- 
sieurs jours apres, que vous pourrez applinuer le pollen étrangrer, car dans res plantes les pistils ne sont pas apte's le jour de l'épanouissement; mais ils le deriemnent bientot et successivement, suivant, comme les étamines, un ordre de développenent progressif que la nalure a employé, dans son admirable préroyanee, pour assurer la conservation des especes.

Quelyues Clématites étrangères monoigues ou diö̈nues se prèteraient plus facilenent à la fécondation artificielle (grar. 26 et 27).

Nous devons sans doute rapporter déjà à des croisements une partie de ces nouvelles variétés de Clématites qui viennent maintenant orner nos jardins de leurs larges fleurs blanches ou azurées.

\section{Genre Pigamon. - Thalictrum.}

Les organes sexuels sont moins nombreux dans les Thalictrum que dans lés Clématites; mais leur mode d'inflorescence rapproche ces deux genres. Un trì-grand nombre de fleurs s'épanouissent a la fois dins la plupart des espreces; elles sont disposées en panicule, et les anthères vacillantes entourent, au moindre vent, la plante d'un véritahle nuage de pollen. La fécondation est donc à la fois directe et indirecte, et at lieu d'autant plus facilement, que les sépales se détachent presque toujours dés l'épanonissennent, et laissent les pistils partiitement nus. Il serait done difficile de féconder les Thalictrum; on ne pourrait le faire qu'en employant les moyens yui ont été indiqués pour les Clématites. Il y a cependant de's Thulitrum dont les fleurs ne sont pas paniculées et d'autres qui sont

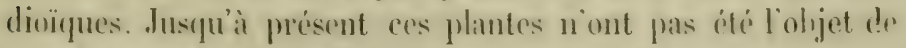
grands soins de la part des horticultenrs; olles mériteraient ore

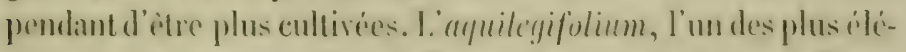

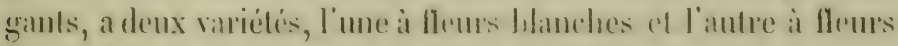

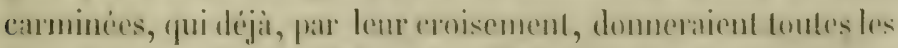
teintes intermadiaires. Tontes les dillicultés que les botanistes 
éprouvent à classer les Thulictrum et à en différencier les nombreuses espèces nous font supposer qu'il pourrait y avoir parmi eux des hybrides naturels.

Cenre Anémone. - Anemone.

Le genre si remarquable et si beau des Anémones a déjà donné une multitude d'hybrides, qui peurent prendre place arec les Roses, les Jacinthes, les Tulipes, etc., parmi les plus admirables productions du règne végétal. Les pistils et les étamines sont nombreux, comme daus la plupart des Renoneulacées, mais leur déreloppement est successil. Tantôt, comme dans l'Anémone des jurdiniers, les extérieures s'ouvrent les premières; tantôt ce sont les intéricures, comme dans l'arborea, ou bien les intermédiaires, comme dans celle de Haller, en sorte que le premier soin doit être de les retrancher toutes soigneu-. sement dès que les pétales s'entr'ouvrent.

Ia f'́condation artificielle peut s'opérer facilement sur l'A némone des lleuristes, sur les doubles surtont, qui sont privées d'étamines, pourvu qu'elles conservent quelques pistils, ce qui a toujours lieu dans les semi-doubles. C'est sur celles-ci, quand elles conservent des étannines, et au besoin sur des simples bien colorées avec un anneau de couleur différente au fond de la fleur, quil faut recueillir le pollen, pour le porter au pinceau sur les pistils des porte-graines.

Les simples donnent aussi de très-beaux résultats par les fécondations croisées; leurs pistils forment une tête assez longue au milieu de la fleur, et ne deviennent pas tous aptes à la mème époque. En sorte que si la plante avait déjà domné du pollen avant la castration, l'hybridation pourrait encore avoir lieu en supprimant ou ne recueillant pas les graines inférieures, qui auraient pu être fécondées arec leur propre pollen.

les Anemone patonina, coronaria, stellata et palmata, si communes dans les champs du midi de la France, ont produit 
naturellement une si grande (unanlité $d^{\prime} h_{1 y}$ ] rides intermédiaires, que l'on ne peut presque plus distinguer les types. Ce sont toutes des plantes extrèmenrent belles, sur lesquelles on pourrait tenter avec succès des fécondations croisées, et qui augmenteraient

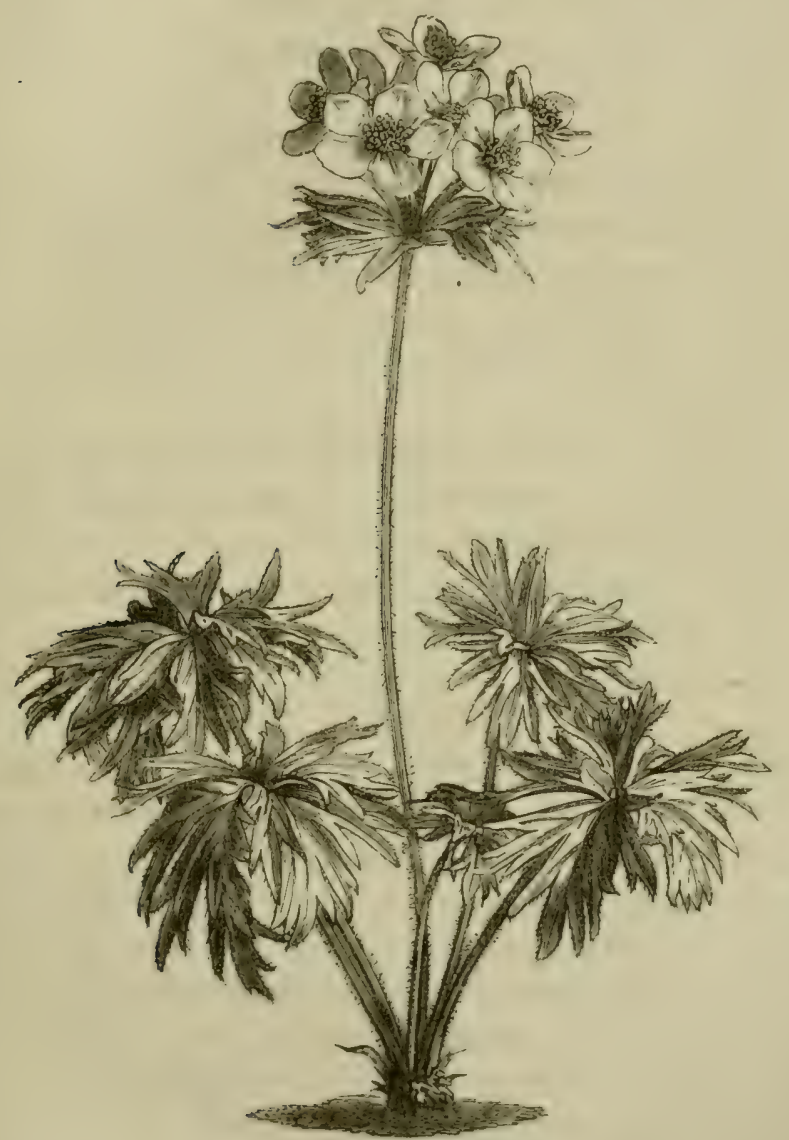

Grav. 2R. - Anemone narcissillora.

certamement nos richesses en lielles Anémones. Cie sont des essais que je recommande aux horticulteurs du Mlidi, notamment à ceux qui habitent le littoral, entre Gènes et Toulon. 
Les mêmes procédés d'hybridation pourraient s'appliquer aux différentes espèces de la section des Pulsatilles; mais ces plantes se ressemblent tellement, que je ne verrais aucun avantage à ces croisements, à moins que l'on ne puisse obtenir des hybrides entre les Pulsatilles à fleurs brunes ou bleues, et cette belle Anémone des Alpes, dont les variétés à fleurs blanches ou soufrées décorent les pelouses de toutes les régions montagneuses.

Les fécondations artificielles n'ont pas été tentées non plus sur la belle Anémone bleue des Apennins, ni sur les Sylvies, telles que les nemorosa, syluestris, trifoliatı, ramunculoides, plantes dont on possède déjà de très-jolies variétés qu'il serait très-possible d'accroître. Quoique toutes ces espèces soient un peu différentes, il est probable qu'elles se féconderaient réciproquement.

L'Auémone à fleur de narcisse serait certainement une de celles qu'il faudrait chercher à varier; mais elle forme un trpe séparé qu'il serait difficile de croiser. C'est done du temps qu'il faut attendre quelque variété naturelle qui sersirait ensuite à créer des variétés intermédiaires (grar. 28).

\section{Genre Hépatique. - Hepatica.}

On trouve peu de dificultés pour la lécondation des Hépatiques, et M. Rodegaze, de Saint-Trond, a obtenu de grands succès dans cette opération. II a fourni à II. Van Iloulte, de Gand, de nouveaux types que cet habile horticultenr a lui-même trisheureusement croisés. La bleve, la blanche et la rouge s'hybrident réciproquement ef donnent d'admirables résultats. II y a aussi, je crois, deux espèces étrangères, moins lielles que la nôtre, mais que l'on pourrait peut-être introduire dans les croisements, avec d'aulant plus de facilité qu'elles sont fitites sur le même type que l'H. triloba. Il faut beaucoup de précautions pour cnlever les étanines yui śourrent de l'extéricur à l'intérieur, pendant sept à huit jours de suile, ct lemr pollen est fiacilement retem par les petits stignates sessiles et tinement 
glimduleux. Les fleurs étint tres-nombreuses, le porte-graine a becoin dètre préparé par la suppression de bon nombre de Joutons à fleurs; il peut être fícondé an pot, re gui est plus facile; mais il y a plus de chances de succes en pleine terre et sous cloche. Les grain's mûrissent vite; quand on s'aperçoit qu'elles approchent de la maturité, on remet la cloche, et pardessous un morceau de papier blane ou de carton, sur lequel toutes les semences riennent se déposer.

Les varietes doubles sont difficiles à obtenir, parce que ces plantes, comme les Inémones, perdent leurs pistils en donhant an moins la bleue ef la rose. Il faut donc choisir des varivtes semi-doublers, on chereher dans les tleurs doubles si l'on un. trouse pas un pistil ou quelque étamine échappée à la transformation.

\section{Genre A flonis. - Adomis.}

Ces plantes font un des plus beaux ornements des champs; mais elles ne sont pas assez répandues dans nos jardins, où l'on ne rencontre presque jamais ces beaux Adonis à fleurs jaunes, qui śépanouissent au premier printemps dans les Pyrénées ef In midi de la France. Les . ddonis à fleurs rouges, malgré quelques varietés à fleurs citrines on jaunattres, forment un groupe très-distinct et très-net. Lés fécondations croisées ne seraient pas plus difficiles sur ces plantes que sur les Anémones; mais le retranchement des étamines exige beancoup de soin, car elles s'ourrent successivement; leurs filets s'allongent après l'ṕpanouissement, et finissent par dominer le pistil, puis ce dernier s'allonge à son tour, c'est-à-dire que l'axe qui porte les carjelles so développe un peu plus tard, en sorte qu'au milieu de ers mouvements il est difficile que tous les earpelles ne soirnt pas completement et naturellement fécondés. Il faut done retranther toutes les etamines avant d'appliquer le pollen au pinceau.

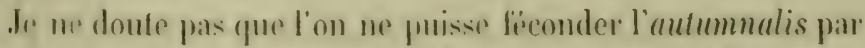
l'astiralis ef rériproupuement, el je présume, en cas d'impossi- 
bilité, que l'on pourrait au moins féconder l'une par l'autre les variétés de couleur de ces jolies plantes. C'est une culture négligée qui peut amener de tris-beaux résultats.

Il ne faudrait pas songer à féconder une section par l'autre :

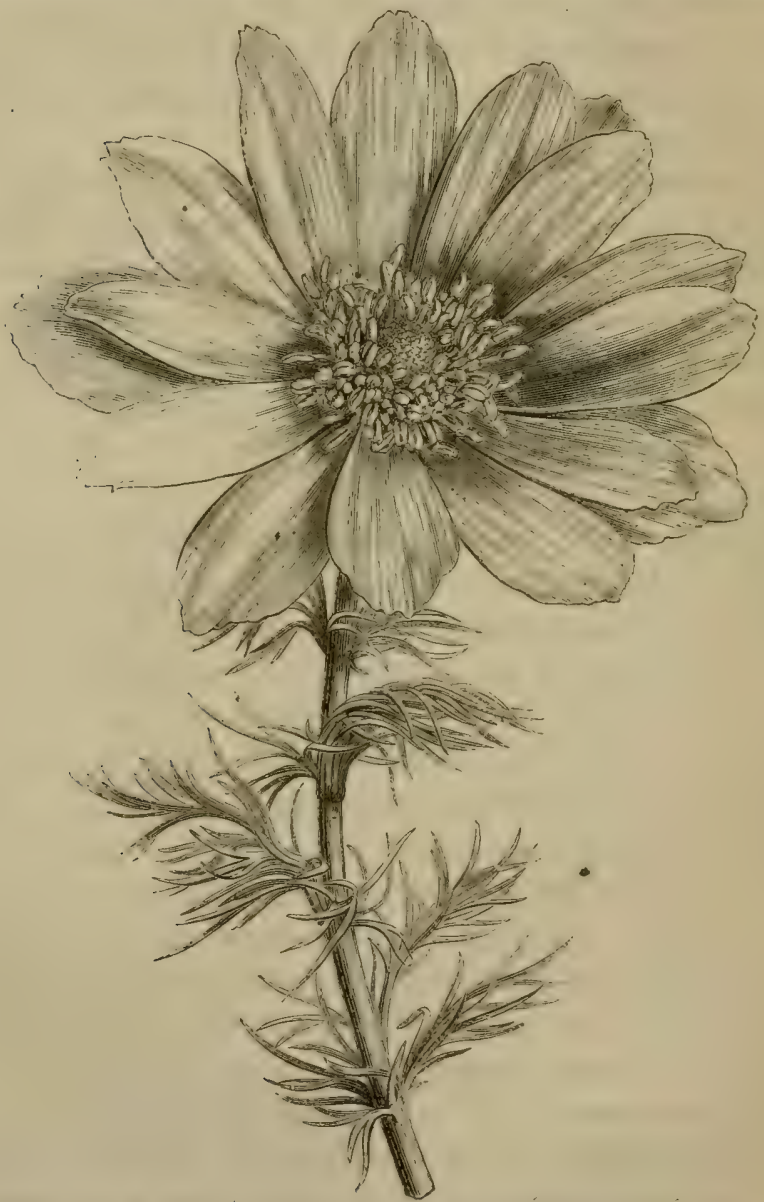

Grav. 29. - Fleur d'Adonis printanière de grandeur naturelle.

les différences sont trop grandes; si cependant un tel résultat pouvait être obtenu, il dédonmagerait certainement d'un grand nombre de tentatives infructueuses (grav. 29). 


\section{Genre Renonenle. - Ramunculus.}

Ces plantes ont, comme les précédentes, lon nombre d'étamines et de pistils, entourés de pétales concares, qui forment un réritable bassin, dans lequel la fécondation naturelle s'opère arec la plus grande facilité. Comme dans toutes les fleurs qui contiennent beaucoup d'élannines et de pistils, la fécondation est lente et s'opère successivement. Ainsi les étamines placées sur plusicurs rangs s'ourrent les unes après les autres de dehor's on dedans. Les carpelles, disposés en tète ou en épi court, retardent générilement sur les étamines, et deviennent aptes à l'impréchation, en commençant par les plus inféricurs. Il résulte de celte disposition que somvent ce sont les dernières citamines qui fécondent, et que les plus extérieures sont inutiles. Il est rai de dire aussi que le pollen reste assez longtemps adhérent à l'anthère et peut se conserver pendant plusieurs jours. La fécondation artificielle est donc presque assurée dans ces plantes, car, lors même quon aurait négligé d'enlever assez tôt les organes males, il n'y aurait jamais que les premièrs carpelles qui auraient reçı le pollen, et l'on pourrait encore imprégner les autres au pinceau. C'est done pendant plusicurs jours de suite qüil faut opérer, jusquà ce que les nombreux carpelles du centre de la fleur se soient tous épanouis.

C'est surtout pour obtenir de nouvelles rariétés de la Renoncule d'Asie que l'on emploie les fécondations croisées, en choisissant très-soigneusement ses porte-graines parmi celles des plantes les plus doubles qui ont conservé des carpelles. Le pollen est pris sur les semi-doubles de belle forme et de belle conleur.

Huant aux autres especes de ce genre, je ne crois pas que l'on ait essayé leur hỵluridation, et je ne pense pas non plus quien ras de suecis on oblieme rien de hien remarquable. 


\section{Genre Trolle. - Trollius.}

Les diverses espèces de plantes exercent sur nous une sorte d'influence que l'on ne peut définir, et pour mon compte ces magnifiques Trollius qui courrent les prairies alpines de leurs fleurs soufrées et globuleuses ont toujours produit sur mon esprit une impression des plus vives. Cela tient sans doute à un souvenir d'enfance que l'on m'excusera de rapporter. A une époque où j'ignorais encore le nom des fleurs, j'avais aperçu dans un jardin de ma ville natale, la ville d'Aresnes, dans le département-du Nord, une touffe de Trollins enropxus qui fleurissait admirablement. Le jardin de mes parents n’élant sćparé que par un mur, je passais des heures entières sur ce mur pour admirer ces fleurs et pour attendre leur ćpanouissement complet, qui n’arrivait jamais. Depuis lors je n’ai pu roir sans émotion cette belle plante, à l'état saurage, disputant la place dans les prairies des montagnes au Narcisse des poëtes, aux Pédiculaires, aux Gentianes et à ces nombreuses tribus de plantes alpines qui constituent les grands parterres de la nature.

Bien que le T. europæus soit, sans contredit, le plus beau, il en est un autre, le $T$. asiaticus, dont les fleurs sont moins graindes, mais orangées. Je n’ai jamais tenté d'hybrider ces deux plantes, soit entre elles, soit avec les autres espèces du nord de l'Asie; mais il parait que des hybrides se sont formés tout naturellement dans quelques jardins du Loiret, car voici ce qu'écrit M. Bailly dans le numéro du $1^{\text {er }}$ juillet 1861 de la Piexue horticole :

" Nous ignorons si aucun horticulteur s'est livré à des essais de fécondation artificielle entre ces deux espèces roisines pour en obtenir des produits hybrides qui ajoutassent à nos richesses horticoles; mais, que ces tentatives aient eu lieu ou non, la nature s'est clıargée d'opérer ce croisement dans le jardin d'uì de nos amis, M. Bariller, hahitant de la ville de Gien (Loiret), lequel cultivait colte à còte les denx espèces de Trollius. Des 
graines recueillies par lui sur le Trollins usiaticus et confiées à III autre amateur très-éclairé de la nème ville, II. Abicot, produisirent non pas l'espèce d'Asie type, mais tout un groupe de plantes plus ou moins semblibles à chacune des deux espèces, sans ètre comptétement identiques à l'une d'elles. Ce sont autant de plandes hybrides qui présentent des caractères propres aux Trolles d'Europe et de Sibérie, mais qui en diffèrent assez pour former des plantes nouvelles, qui peurent tourner au bénéfice de l'horticulture, et dont une des plus belles est celle que nous indiquons aujourd'hui, en la recommandant fortement à l'attention des horticulteurs, qui en obtiendront facilement du plant ou de la graine en s’adressant à II. Abicot, à Gien.

“Des semis faits avec ces graines, outre l'intérèt horticole gui s'y rattache, pourront servir à éclaircir une question encore olsscure de plyssiologie végétale, à savoir, le degré de fécondité des hyjlorides végétaux. On sait en effet que, dans le règne animal, les produits de deux espèces roisines sont entièrement privés de la faculté de se reproduire ou sont donés d'une fécondité qui ne tarde pas à s’éteindre dans des générations successives; précaution admirable de la nature, qui ne veut pas laisser altérer' son œurre en l'abandonnant aux hasards de nos caprices. Eh bien, cette loi du règne animal, la retrouve-t-on également puissante dans le règne végétal" Voit-on ici la nature se départir de ses droits et permettre à l'homme de créer en quelque sorte, par le croisement, des espèces intermédiaires, qui se reproduiraient fidèlement par une suite indéfinie de générations, comme on le voit dans les espéces naturelles?

"L'opinion des lootanistes se trouve encore partagée sur ce point: tandis que quelques-uns, en petit nombre, il est rrai, croient à la persistance des hy̧brides végétaux par voie de semis, les autres pensent que l'existence de ces mèmes hybrides se suspend après un nombre variable de générations, de telle sorte que la race nouvelle périrait bientòt par labsence el l'infertilité des germes ou par le retour des produits à l'un ou l'autre des types dont ils procèdent. Nous le répétons, des semis successifs 
faits avec les graines du Trollius hybride que nous amnonçons aujourd'hui pourront devenir le sujet d'observations précieuses pour la solution d'une question importante, et qui ne peut ètre résolue autrement que par des expériences nombreuses et poursuivies avec persévérance.

« M. Mbicot, de qui nous tenons cette plante, est déjà entré dans cette voie. Il nous écrit : "Il y a au moins cinq ans que “ j’ai obtenu mon premier gain; j’ai semé depuis des graines " récoltées sur ce premier gain, et j'ai ru avec plaisir qu'il se re" produisait, je pourrais dire très-exactement, peut-être mème " plus beau. » Plus loin il ajoute: "J'ai au moins quatre génć" rations successives de cette variété. J'ai semé quelques graines " en 1859; j’ai dans un terrine cinq à six pieds qui ont réussi, “ un seul a fleuri en 1861 et parait identique à mon hybride; " les autres ne fleuriront que l'année prochaine. J'ai semé, en " 1860 , dans deux terrines; le plant est levé en ce moment, je le " soignerai. " Comme on peut en juger par ce passage, ce premier essai domnerail quelque espérance que celte variété nouvelle puisse être fixée et se reproduire de semis; toutelois nous sommes d'avis qu'on ne saurait, des aujourd'hui, juger définitivement la question, et que, pour être concluante, cette épreuve doit être prolongée encore pendant une assez longue série d'années. )

"Nous dirons quelques mots, en terminant, sur les principaux caractères de la variété qui fait l'objet de cet article. Comme la plupart des hybrides végétaux, elle offre une vigueur el des dimensions supérieures à celles des parents. Les tiges tubuleuses, striées el teintes parr places de violet foncé, forment de fortes touffes hautes de $0^{\mathrm{m}}, 50$ ì $0^{\mathrm{m}}, 70$. Le feuillage palmé, à cinc lobes incisés-dentés, se rapproche plus de celui du Trollius e’tropæus en ce qu'il est moins découpé et à dents moins aiguës que dans l'espèce d'Asie. La fleur terminale, d'un beau jaune orangé clair, est un charmant intermédiaire cntre les deux parents. C'est un énorme bouton d'or dont le calice globuleux, it demi ouvert, laisse voir au centre un groupe de nombreuses éta- 
mines orangées, entourées de pétales itroits, d'une belle teinte orangé foncé, connue ceux du Trolle d'Asie, mais moins longs que daus cetle derniere espece. C'est, en somme, une tris-belle Renonenle, fort ornenentale, el, conme beaucoup de plante: de ce dernier grme, précieuse en ce qui clle domne ses fleurs à une époque oì la décoration de nos jardins ne repose que sur un petit nombre d'espèces.

"La culture est facile, la plante est vivace el se multipliera de graines et d'éclats. Elle préfëre une terre légère, lumide, arec un pen d'ombre. Dans de telles conditions elle fournit une grande quantité de fleurs, qui paraissent en arril-mai. "

Lémon a aussi indiqué une variété dı $T$. érołæus obtenue di semis, et dont les fleurs étaient, dit-il, plus grandes que celles du Trollius ordinaire. Nous ignorons si célait un liybride ou une simple variété qui se serait perdue depuis longtemps.

C'est le Trollius e'uropaxus qu'il faut prendre pour portegraine, écarter de bomme hemre ses pélales, et enlever les étamines très-nombreuses, dont les extérieures s'ouvrent les premières, comme dans la plupart des Renonculacées.

\section{Genre Irellebone. - Helleborus.}

Les nombreuses étamines des Ilellébores emploient un temps tris-long pour s'ouvrir, en sorte que la floraison est très-prolongée. Ce sont les extérieures qui répandent les premières leur pollen, puis, successivement, celles qui sont le plus rapprochées des pistils. Ces derniers sont serrés dans le niyjer, et au contraire écartés dans le viridis. Rien de plus facile que d'opérer des fécondations sur ces plantes, puisque l'on peut tonjours, longtemps d'avance, en enlever toutes les étanines; mais je ne sache pas que, jusqu'a présent, on ait tenté de les croiser. Le niger, le seul qui soit réritablement ornemental, n’offre que deux variétés, une rosée plus srande et une blanche plus petite et plus florifire. Il ny aurait aucum avantage a obtenir des internédiaires. Si done on tantait quelque hịjoridation, 
il faudrait opérer entre le niyjer et l'orientalis, ou le viridis, l'odoris, l'utrorubens, le dumetorum ou le purpurascens; encore tous ces derniers sont-ils d'une autre section, celle des Ilellébores à tiges multiflores. Ils se féconderaient certainement entre cux, car ils se ressemblent beaucoup, et, à l'exception de l'orientalis, ne sont peut-être que des variétés les uns des àutres.

L'époque de la floraison de ces plantes rend la cloche nécessaire au succès de l'opération. On obtiendrait peut-être de fort belles variétés avec un peu de persévérance.

\section{Genxe Isopyre. - Isopyrum.}

J'ignore si l'on pourrait hybrider ces jolies plantes, dont je u'ai cultivé qưune seuble, le thalictroides. Elles ont peu d’élamines et devraient se traiter comme les Hellébores el sous cloche. Elles sont d'une délicatesse extrême. Le thalictroides donne tous les ans des graines dans mon jardin; peut-être obtiendrait-on des hybrides avec le fumarioides, assez commun dans nos cultures, et avec le grandiflora ou l'adoxcides, si l'on pouvait se procurer vivantes ces deux dernières espèces.

\section{Genre Vigelle. - Nigella.}

Dans ce genre et dans le Garillella, qui en est très-voisin, les étamines sont nombreuses, comme dans la plupart des Renonculacées, et disposées tantôt sur un rang, comme dans l'urientalis, tantôt sur plusieurs. Alor's les anthères se fendent successivement de dehors en dedans. Chaque anthère est munie de denx petites valves qui s'ouvrent, courertes de pollen et le conservent assez longtemps. Aussitôt que le pollen est répandu, les étamines se déjeltent en dehors. Les pistils ont des stigmates quelquefois terminaux, mais d'autres fois latéraux, el qui toujours se développent très-tard. Ils s'allongent alors et se 
recourbent, de manière à atteindre les étamines, dont les anthères extrorses se trouvent précisément dans la position la plus favorable pour abandonner leur pollen. Cette inégalité de développement dans ces denx organes rend les croisements très-faciles; mais, malgré l'élégance de plusieurs espèces de ce beau genre, je ne crois pas que l'on se soit encore occupé d'hybridation.

\section{Genre Aneolie. - Iquilegia.}

Ce grenre, composé de magnifiques espices, nous offre en outre un grand nombre de variétés plus ou moins remarquables par leurs belles coulcurs et $\mathrm{f}$ ar la forme de leurs fleurs. Celles-ci, en effet, douhlent, par la multiplication et l'emboite. ment des cornets, par leur renversement, par le nombre de leurs sépales, el se distinguent cncore par des dégénérescences particulières. Toujours ou presque toujours elles portent graine, en sorte que, l'ancolic pouvint facilement s'hybrider, cette plante est appelée à joner un grand rôle dans les jardins, par ses nombreuses et élégantes variétés toutes printanières.

Les étamines sont serrées contre les pistils et semblent disposées par rangées, quoique, en réalité, elles soient placées par faisceaux allongés et situés sur des rayous partant du centre de la fleur, caractère que cependant on ne trouve pas dinns le myrenaica.

A l'opposé de la règle générale, ce sont les étamines intérieures et les plus rapprochées du pistil qui s'ourrent les premières, hien qu'à cette époque les stigmates soient 'n retird conme dauns les Nigelles. Il y a souvent une grande quantité de pollen répandu, et les styles ne sunt pas encore allongés; il est done ficile d'enlever les étamines à un petit nombre de fleurs et de porter sur les pistils, à l'éporque de leur développenent, le pollen d'autres especes ou variétés. On peut ourrir lextrémité des comels et laroriser la fécondation en posant sur les stigmates un preu de la liqueur nectarilere que les plimtes y sí- 
crètent. Linc capsule contient toujours beaucoup de graines, et l'on est sûr du succès. Je recommande aux amateurs ces plantes remaryuables. Ils choisiront de préférence, pour porte-graines, la variété blanche à fleur double, et éviteront la bleue, celleci tendant assez à reprendre cette couleur. Il serait curieux aussi d'essayer le croisement de l'A. canadensis, qui for'me un type séparé, avec les autres espèces qui ont les plus grands iapports entre elles. L'Ancolie deviendra une des plus belles plantes de collection dans les mains qui sauront en tirer parti.

M. Lemaire cite un $\boldsymbol{A}$. blanda né dans le jardin de M. Werschaffelt et qui proviendrait de l'A. vulyaris fécondé, trèsuraisemblablement, et, par accident, par l'A. leptoceras ou vice versia.

\section{Genre Dauphinelle. - Delphinium.}

Ces plantes ont encore des étamines nombreuses, dout les antheres s'ouvrent par des valves comme celles des Ancolies. Ces étamines se redressent une à une contre les pistils, el presque toutes ont fini de répandre leur pollen quand les stigmates sont aptes à le recevoir. Il devrait résulter de cette inégalité de développement des organes de nombreux hybrides, si des pétales en capuchon ne mettaient obstacle à l'arrivée des poussières étrangères; malgré cela, il est probable que la féconda. tion s'opère monoïquement, et cela explique peut-être le peu de différences qui existe entre les espèces de chaque section de ce genre nombreux.

Il serait done facile de pratiquer des fécondations artificielles sur les Delphinium entre les espèces ou variétés de même section. C'est ainsi que les Ajacis ou Pieds-d'alouette des jardins suraient facilement croisés, non-seulement entre eux, ce qui serait, du reste, inutile, ces croisements ayant licu tout naturellement par fécondation indirecte, mais encore avec le consolidu, qui donne lui-même de lien belles variétés dans nos parterres, et pent-ètre aussiavec le flavum, la seule espèce à fleur jaune, et gu'il serait curieux de cultiver pour essiyer les croisements. Déjà on 
a obtenu aussi de très-helles plantes probablement hrbridées dans la section des Delphinastrum, ceux qui sont ì longrs épis de fleurs blenes, et il y en a bien d'autres à espérer. Le I). Burlovii, l'ušreum, qui en est voisin, le Burlovii hybridum el quelques espèces roisines douneront déji de très-lecaux hybrides, et le genre tout entier promet de grandes richesses à l'horticulture.

Les sujets à féconder derront ètre préparés en retranchant la majeure partie de leurs lleurs.

Depuis que j'ai écrit cet article, en 18 '.i, les grands Delphinium à fleurs hleues, issus du formosum ef de plusieurs autres, unt èté croisés si fréquemment et si heureusement, qü ils sont, pour ainsi dire, tombés dans le domaine public de l'horticulture. On nien recommait plus les types ni les espèces. Ce sont d'admirables plantes.

Nous ignorons si des hỵbridations ont été tentées avec le pollen du Delphinium curdinale, assez difficile à cultiver, et nos beaux Delphinium bleus, si rustiques et si floriferes.

\section{Genre Sconit. - Aconilum.}

Ce genre est un des plus curieux de la famille des Renonculacćes. Les organes sexuels sont enfermés dans un pétale qui a la forme d'un casque et qui les isole assez complétement, surtout dans la section des napels.

Les étanines et les pistils se déreloppent généralement à des époques différentes, en sorte que la fécondation doit ètre indirecte; d'un autre côté, les stigmates sont quelquefois avortés, surtout dins les napels, en sorle que ce's causes rémie's, et surtout l'isolement produit par le casque, rendent les féconditions naturelles assez rares dans les Aconits, excepté cependint dans la section de's Lycoctomum, nù ils sont plus fréquents.

Comme les Aconits sont de fort helles plante's dont l'horticulture s'est emparée aree raism, il importe d'essayer d'en obtenir des graines hỵ hidées. Le meilleur moyen a employer consiste a bien préparer le sujet con s'y prenant longlemps d'avance; on enleve plus de la moitic des jeunes boutons d'une 
branche, dès que ces boutons paraissent, et une quinzaine de jours plus tard on ne laisse que trois à quatre boutons sur cette mène tige. On donne ainsi un plein développement à ces

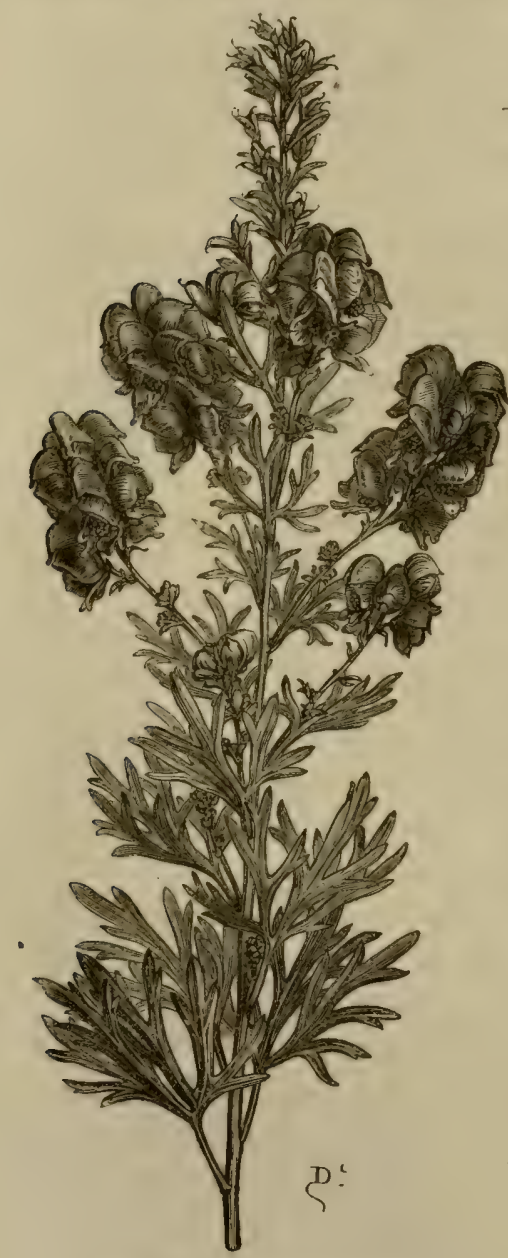

Grav. 50. - Aconitun cminens. fleurs, et l'on parvient à leur faire compléter leurs stigmates, qui, malgré ces précautions, avortent quelquefois encore dans la section des napels. A l'époque de la floraison, il faut attendre que les fleurs soient très-avancées, mais préalablement on peut enlever les étamines avec les pinces. Les plus intérieures se développent les, premières, mais bien avant que les stigmates soient propres à l'imprégnation. Si le casque gêne pour enlever les étamines ou pour poser le pollen sur le pistil, on peut le couper, mais il suffit ordinairement d'écarter les pé-

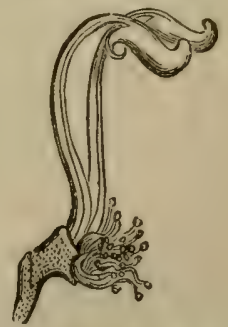

Grav. 31. - Fétales cucullés et organes de la fécondation d'un Aconit.

Lales avec les doigts, pour être à portée de saisir toutes les étanines, dont celles qui ont répandu le pollen se déjettent 
immédiatement. Les graines, une fois nouées, mûrissent assę facilement et exigent heaucoup' de précautions pour les semis, de l'ombre, de la fraicheur et un abri (grav. 50 et 54 ).

\section{Genre Pivoine. - Pronia.}

Voici le genre le plus éclatant de toute la famille des Renonculacées, et l'un de ceux sur lesquels la fécondation artificielle a donni les plus beaux résultats. Les nombreuses étamines des Pivoines sont très-délicates et très-mobiles; elles répandent par le sommet et ensuite par les côtés de l'anthère un pollen abondant. Les plus intérieures s'ourrent les premières et sourent aussi avant le développement des stigmates. Ces derniers sont latéraux, velus et ordinairement colorés en rose. La fécondation s'opère seule très-facilement dans les Piroines quand elles comservent quelques-unes de leurs nombreuses étamines; mais celles-ci ont une si grande tendance à se changer en pétales, que l'on voit sourent des Piroines entièrement pleines et privées d'organes mâles. Dans ce cas, les pistils qui persistent peurent être fécondés artificiellement en posant doucement le pollen sur les stigmates roses. Comme il arrive souvent que les trois à quatre stigmates de la Pivoine ne sont pas aptes à la même époque, il faut renouveler l'opération deux à trois fois à deux ou trois jours d'intervalle. Dans les Pivoines très-doubles, le pistil lui-mème est souvent stérile par manque de nourriture; on peut remédier à cet inconvénient en arrachant, dès le commencement de la tloraison, mais avec précaution, une bonne moitié des pétales, ou en les coupant près de l'onglet.

Toutes les belles variétés de Pivoines arborescentes s'obtiennent ainsi par des fécondations croisées sur des individus dont les étamines ont été enlevées avant l'anthèse. La mème chose a lieu pour les herbacies, qui forment maintenant de magnifiques plantes de collection.

On est quelquefois embarrassé pour trourer le pollen sur les étamines des Pivoines, mais il e'st toujours facile de le recueillir sur le fond de la fleur, sur tes pétales, qui en sont quelquefois 
couverts. Des arrosements très-fréquents sont nécessaires après la fécondation pour que les capsules ne soient pas entièrement vides. Les Pivoines sont longtemps à se développer, mais ce sont des planies tellement belles et si chères, que les horticulteurs marchands ne doivent pas craindre de s'en occuper et d'y consacrer quelques expériences.

L'introduction en France des Pivoines en arbre ne date que dı commencernent de ce siècle. Trois variétés parurent en mème temps : la Pivoine Noutan, la Pivoine à odeur de rose, la Pivoine papavéracie. Sont-ce trois espèces distinctes ou trois variétés? Celte dernière supposition est plus probable. Il est certain qu'elles peuvent s'hybrider entre elles, car il y a plus de cinquante ans que Noisette avait obtenu de belles viriétés, en fécondant entre elles ces trois Pivoines.

Depuis lors, on a continué les croisements avec les hyjbrides. On lcs a modifiés au moyen de types nouveaux, arrivés de la Chine, et l'on est parvenu ì ces créations infinies et variées qui ne permettent plus de recounaitre les formes types dont elles proviennent.

Beaucoup de ces Pivoines sont tellement doubles, que les organes de la reproduction sont atrophiés ou mal conformés. J'en ai même obtenu qui tendaient à être prolifères, comme certains Boutons d'or. En général, il reste des étamines, et les Cétoines aux élytres d'émeraude qui viennent butiner et dormir sur ces lits de Pivoines suffisent quelquefois pour échanger les pollens et pour vivifier les ovules.

Il vaut mieux, avec le pinceau, couvrir de pollen tous les stigmates bien conformés. Quelques graines, sourent une seule, nouent donc leur follicule. Ce fruit s'ouvre en été, offrant la belle nuance de car'min qui colore son intérieur et montrant au soleil ses belles graines à découvert. Il faut les laisser mùrir complétement jusqu'à ce qu'elles se détachent, les semer ou les stratifier immédiatement. Elles lèvent un ou deux ans après et fleurissent de six à dix ans.

Dernièrement encore, M. Werschaffelt à mis dims le com- 
merce, sans le nom d'.llexandre II, une magnifique variété ohtenue du croisement direct du $P$. paparerarea area le $P$. rosea. Ses grandes fleurs, pleines et odorantes, aux larges pétales éclatants, sont colorées en pourpre, marginées de blanc carné et de franges. Le Triomphe-de-Grand de Tan-Gert présente aussi d'inormes et d'admirables fleurs.

Tous ne connaissons rien de plus beau que ces massifs de Pivomes arborescentes renues de semis et constiluant de's buissons d'une extrême vigueur.

Toute une race d'autres Piromes hybridées est sortie des Piroines herbacées désignées sous les noms de $P$. erdulis on fruyrans, $P$. sinensis et $P$. albiflor $a$, Pallas. Celte trinite, comme la précédente, a été croisée d'abord par Lémon, qui en a obtenu des plantes charmantes, offrant comme les parents tous les coloris, depuis le blanc pur, le rose le plus tindre, jusgu'au carmin et au jaune sulfureux. L'odeur mème du $P$. frayprons est restér dans un grand nombre de rariétés et s'exhate des globes rolorés que présentent ces Pivoines dans ces moments de finicheur et d'íclat où elles ront notis montrer toutes leurs riches parures. M. Lémon fils, M. Jaques, M. Guérin-IIodeste, ont continué l'œurre de Lémon père, qui date de 1828.

P'usieurs de ces Pivoines ont les filets des étamines élargis en pétales et en languettes, présentant ainsi l'aspect d'énormes Anémones à fleurs doubles, tandis que d'autres sont pleines comme les Roses à cent feuilles.

Malgré mes tentatires, je nai jamais pu faire fructifier le P. anemonxflora; peut-être y aurail-il toute une race nouvelle i créer avec le Péoniu udonidifoliu, si l'on parrenail à la léconder avec une autre espèce herbacée.

\section{FAMILE DIES MIGNOLIACEES.}

Genre Magnolier. - Magnoliu.

On trouve dims les organes sexuels des Matymoliu une disposilion tris-singuliere, at qui semble sopposer it la fécondit- 
tion : e'est la position des pistils au-dessus des étamines et le redressement desfleurs, en sorte qu'il est à peu près impossible que le pollen puisse atteindre les organes femelles. Les anthères s'ouvrent en dehors dans le grandiflora, qui est le type d'une section de ce beau genre, et en dedans dans le yulan, qui forme le type d'une seconde division; la position des organes rend les hybridations très-faciles dans les Maynolia; mais le succès est bien incertain. D'abord, les Magnoliers de la première section, et notamment le grandiflora, ont leurs stigmates avortés dans toute l'Europe septentrionale; ce n'est qu'à partir du $45^{\circ}$ degré que l'on rencontre de bons stigmates, et par conséquent que l'on peut espérer d'obtenir des graines, ce qui arrive souvent et tout naturellement dans l'Europe australe. A Ifyères, le Magnolier fructifie quelquefois.

L'yulan, le pumila, le discolor, ne donnent jamais de graines; ainsi, avant de chercher à les hybrider, il faudrait tâcher de les féconder avec leur propre pollen, ou bien se servir du pollen des Marmolia de cette section pour féconder ceux de la section précédente. Il est douteux que de semblables croisements réussissent. Ainsi ce que l'on doit chercher pour le moment dans ce genre, c'est de faire fructifier les espèces en aidnnt la nature, et d'obtenir des graines pour semis. On considère cependant le Magnolia Soulangiana comme un hybride.

\section{Genre Tralipier. - Liriodendron.}

Le Tulipier est la seule espèce du genre, par conséquent l'hybridation ne peut être tentée; mais il est possible que l'on puisse obtenir des graines fertiles en fécondant la plante avec son propre pollen. Le secours de l'homme parait d'autant plus nécessaire dans cette opération que les étamines du tulipier s'ouvrent en dehors par deux rainures longitudinales; le pollen se répand sur les pétales et non sur les stigmates. Les plantes exoliques, n'ayant pas dans nos climats les insectes qui les fréquentent dans leur pays natal, restent souvent infertiles. Il paraitrait mêne qu'en Amérique la fécondation du Tulipier est souvent 


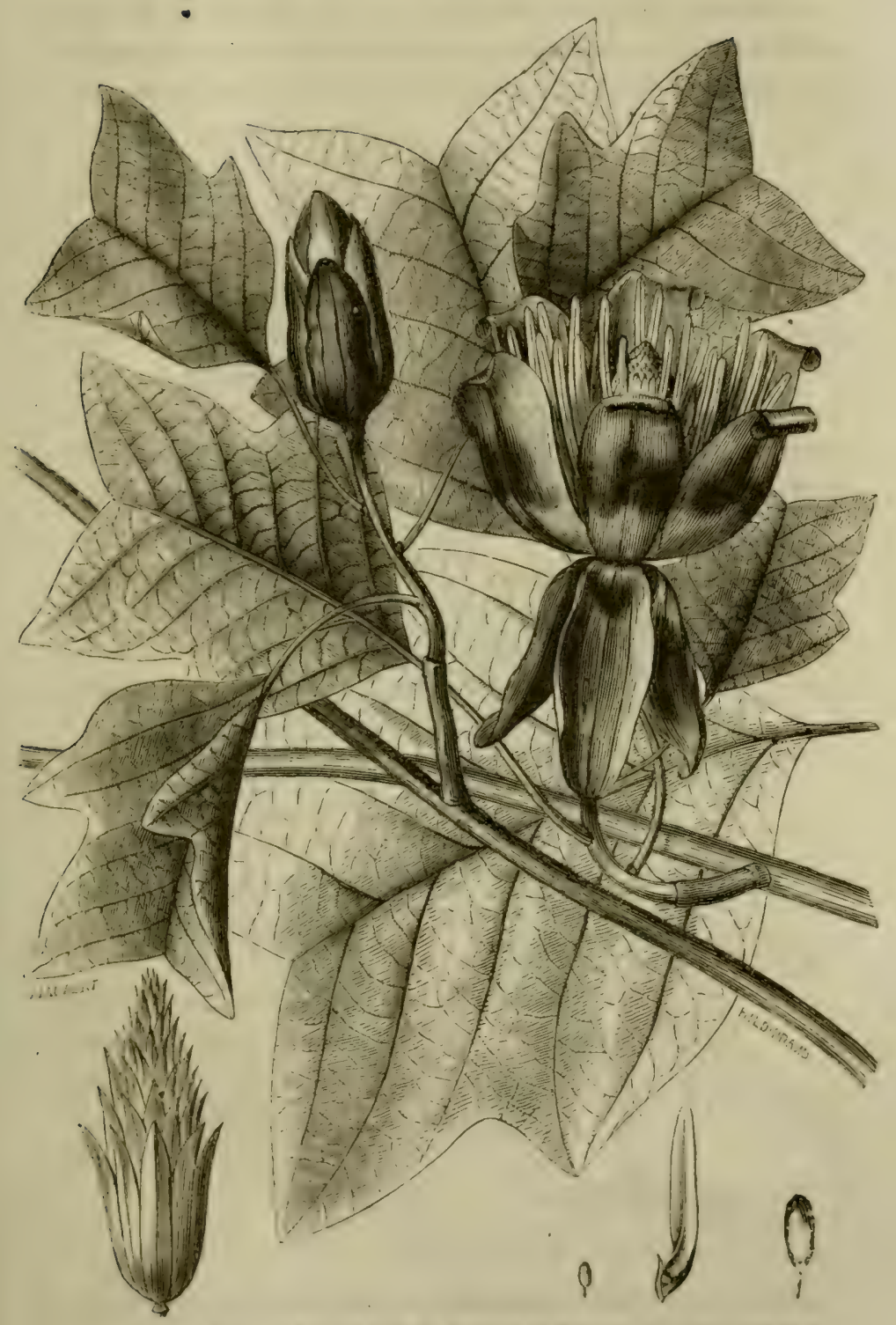

firav. $: 2 .-$ Raneau, 1lıu et fruil du Tulipier de Virginie, aux deux tiers de la grandeur naturelle. 
nulle ou imparfaite, car les graines que l'on reçoit ne sont pas toujours bonnes. On a obtenu du Tulipier ordinaire une variété qui donne l'espoir d'en acquérir d'autres avec le temps (gr. 52).

\section{FAMILLE DES BERBÉRIDÉES.}

\section{Genre Epine-vinette. - Berberis.}

La fécondation des Berberis s'opère avec une telle précision, qu'il serait difficile de la remplacer par une imprégnation artificielle. Les deux loges de chaque étamine se fendent à l'époque de l'anthèse, et deux petits fragments, attachés par le haut, souvrent comme deux véritables portes, se relèvent, et, par un mouvement tantôt spontané, tantôt excité par des insectes, viennent s'appliquer immédiatement sur le stigmate; qui est à l"in-

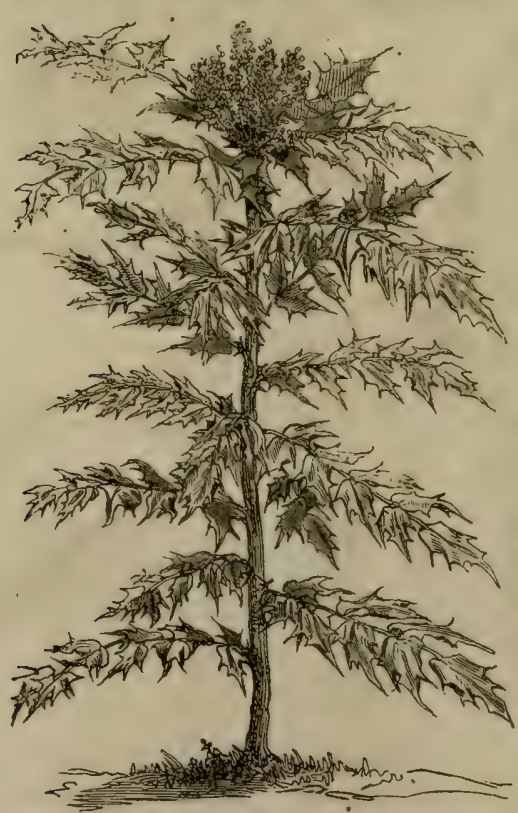
stant recouvert de pollen; aussi chaque fleur est fertile. Il faudrait donc, pour hybrider entre elles les différentes espèces de Berberis, enlever de très-bonne heure des étamines, avant que les anthères soient ouvertes. On connaît de belles variétés d'Épine-vinette, qui sont cultivées comme plantes d'ornement, et l'on doit espérer encore des modifications nouvelles qui se rangeront peut-être un jour au rang des arbres fruitiers.

firav. $\tilde{5}$. - Port de la plante du Mahonia realii.

Genre Mahonie. - Mahonia.

Les organes sexuels des Muhonia présentent it pen pris la 
même disposition que ceux des Berberis, et ce que nous avons dit de ces derniers peut leur être appliqué. On pourrait essayer

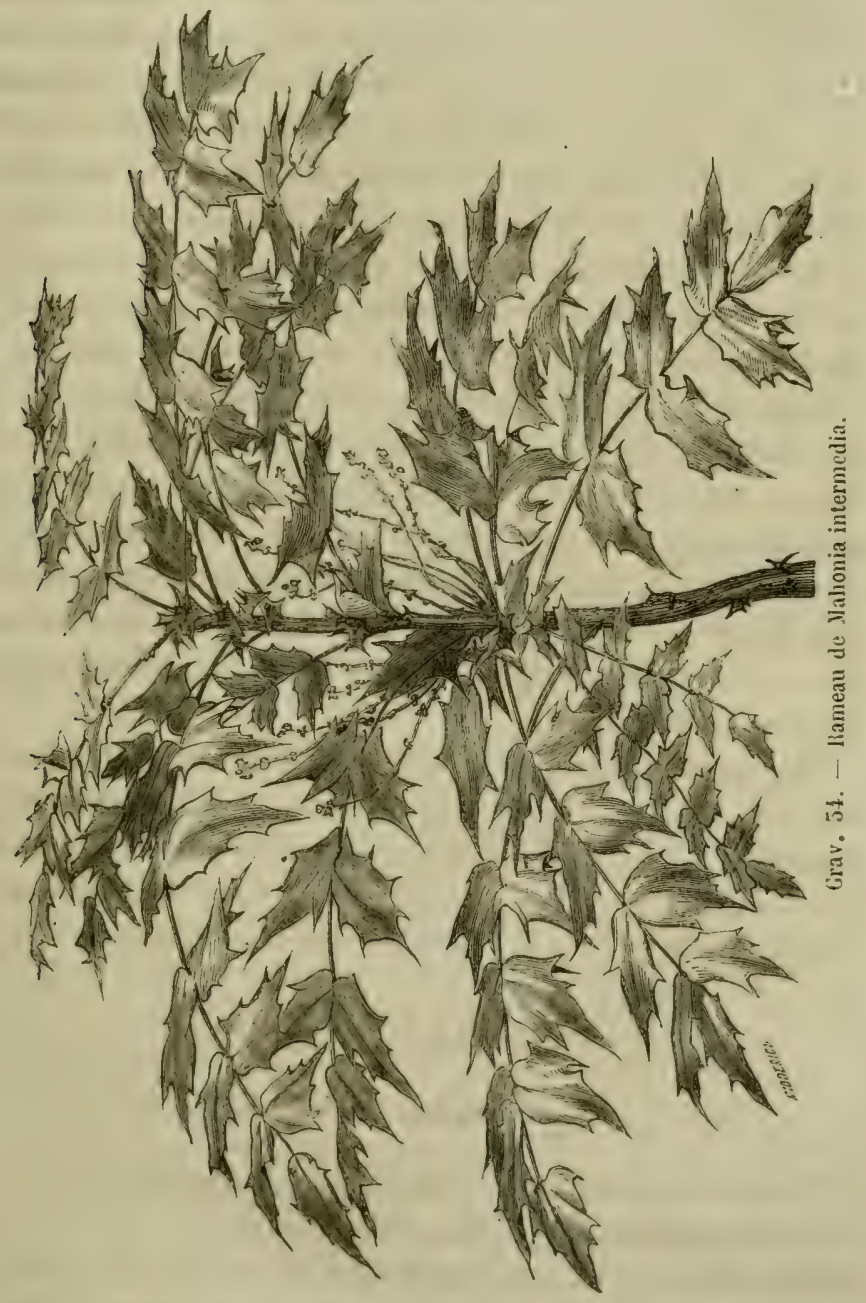

I'hybrider les belles et nombreuses espèces qui le composent et qui ont entre elles de grands rapports (grav. 55, 54). 


\section{Genre Épimède. - Epimedium.}

Ce genre contient des espèces très-jolies et très-délicates, toutes remarquables par la structure et l'élégance de leurs fleurs, 'munies de quatre nectaires tubuleux. Les étamines, également au nombre de quatre, sont extrorses, et le pollen toucherait difficilement le stigmate sans l'admirable arrangement d'une petitc valvule qui se détache de chaque loge de l'anthère, et se roule de bas en haut de telle sorte que, garnic elle-mème d'un pollen qui alors a acquis toute sa maturité, clle vient s'appliquer sur le stigmate et opère la fécondation. Les huit plaques polliniques forment une espèce de petit chapiteau au-dessus du pistil. On voit alors que, pour les hybridations, il faudrait enlever les étamines avant l'évolution de ces valvules, et l'attendre au contraire pour les sujets destinés à fournir le pollen. Les charmantes espèces à fleurs blanches et violettes, actuellement connues, ont déjà donné d'élégants hybriles : les Epimedium atroroseum, lilacinum, rubrum, sulphureum, versicolor, sont des hybrides obtenus par Donkelaar.

Un journal anglais, le Gardener's Chronicle, contient aussi une note sur un hybride d'Epimedium, né en 1855 de la fécondation de l'E. colchicum par le pollen d'un E. macronthum. La couleur jaune pâle des fleurs est presque intermédiaire entre le jaune et le blanc des fleurs des parents.

\section{FAMILLE DES NYMPHÉACÉES.}

\section{Genre Nelombo. - Nelumbium.}

Parviendra-t-on jamais à faire fructifier sous nos climats ces végétaux éclatants de majesté et de magnificence qui déjà sont cultivés et fleurissent dans le midi de la France? Les fécondations artificielles seraient faciles sur des ovules presque nus et placés chacun dans une cavité. Les étamines, quoique nombreuses, scraient facilement retranchées, et d'ailleurs ce serait sans doute 
inutile, car ce ne sera certainement qu'en imprégnant les ovules an pinceau que l'on pourra espérer d'obtenir un jour des graines fertiles; pourquoi alors les deux variétés rouge et blanche du Nelumbium speciosum ne donneraient-elles pas des intermédiaires? pourquoi n' essayerait-on pas leurs croisements arec le N. luteum? Le luxe des serres n'est-il pas arrivé déjà à cultiver en pleine eau ce que les lacs de l'Asie et de l'Amérique présentent de plus admirable?

\section{Genre Nénuphar. - Nymphæa.}

L'hybridation n’a pas abdiqué sa puissance en face des brillantes Ninmphéacées, et nous ne savons pas encore où elle arrèt'ra ses conquètes. Ces belles plantes sont munies d'étamines nombreuses dont les anthères s'ourrent successivement et dont il est indispensable de priver entièrement le porte-graine. Le pollen doit ètre appliqué sur les côtés du stigmate, qui a la forme d'un écussọn, et non à sa surface supéricure, qui n'est pas apte à le recevoir.

En 1851, M. Ortgies obtint des graines fertiles en retranchant les étanines des fleurs du $N$. rubra, qui fleurissait dans l'aquarium de Van Houtte et en saupoudrant les stigmates vierges de cette espèce avec le pollen du N. Ortgiesuna. Les graines semées aussitôt après la récolte ne tardèrent pas à lever. M. Planchon nous apprend que ces plantes fleurirent en 1852. "Elles étaient intermédiaires entre le père et la mère par leur couleur rose, tenant du premier par le mode, le temps et la durée de leur épanouissement. Ajoutez à ces qualités une vigueur insolite de croissance, la faculté de fleurir presque à l'air libre, une prolificité telle, que, jusqu'en décembre, le même pied étalait parfois jusqu à sept fleurs en un jour. » (Flore deṣ serres et des jardins; t. VIII, p. 69.)

Des observations pleines d'intérêt ont été faites à ce sujet par II. Ortgies et par I. Planchon. Le N. hybride nommé Ortgiesu. no-rubra est stérile, et cela malgré des organes reproducteurs 
parfaitement conformés, malgré un pollen qui émet fixilement ses tubes remplis de fovilla. Ce pollen, stérile sur sa propre fleur, peut en fertiliser d'autres. On observe ici ce fait singulier, que l'on a déjà remarqué dans les Pașifllores et dans quelques autres plantes.

M. Planchon propose avec réserve une explication de cette singulière anomalie. Après avoir constaté dans la fleur des Nymphéacées une augmentation de température lors de la fécondation naturelle et nocturne de plusieurs plantes de cette famille, accroissement qui fait défaut dans celle de l'hybride, il suppose que c'est à l'absence de ce dégagement de chaleur que la plante peut devoir'sa stérilité.

Nous croirions plutôt qu'il faut attribuer cotte infertilité à cet ordre de faits que nous avons déjà signalés dans nos généralités, c'est-à-dire à la tendance qu'ont les fleurs hermaphrodites ì être fécondées de préférence par d'autres fleurs, et, mieux encore, par d'autres individus de la même espèce.

M. Planchon termine en exposant les résultats obtenus jusqu'à ce jour (1852) dans le croisement des Nymphéacées. Déjà les hybrides sont nombreux, non-seulement entre Nymphæa de la même section, mais entre espèces de sections différentes. D'autres essais sont restés infructueux, sans qu'il faille en conclure à l'impossibilitẻ du croisement.

Nous ne pouvons trop recommander de nouveaux essais pour hybrider ces admirables plantes. Tout le monde n'a pas à sa disposition l'aquarium d'une serre chaude; e'est donc un devoir, pour ceux qui sont dans cette disposition exceptionnelle, de courir les chances d'expériences aussi belles et aussi intéressantes.

Voici, d'après l'article de la Flore des serres et des jardins, le mode opéraitoire à suivre pour la fécondation du Nympllax.

"La première fois qu'uue fleur s'ouvre, les anthères restent constamment fermées, le pollen n'est pas tout à fait mûr; la coupe stignatique est remplie d'un liquide clair. C'est alors que l'on enleve soigneusement avec un canif les étamines de la fleur destinće à devenir porte-graine. Quand cette fle ur s'ourre 
pour la seconde fois, le liquide du stigmate a disparu; ce stigmate est tout prèt à recevoir le pollen de l'espéce qui sert de fuale, pollen que l'on prend bien mùr et que l'on fait tomber en abondance sur la coupe stignatique. Aprés cela, si la troisieme fois que la flemr s'ourre les processus stigmaliques iou parastigmates, se montrent fortement courbés en dedans, c'est un signe que l'imprégnation s'est faite. D'autres symptònes, du reste, annoncent promptement la réussite ou linsuccès del'opération. Si l'ovaire et la partie supérieure du pédoneule jaunissent, peine perdue; si ces parties restent vertes, si l'ovaire grossit, espoir de récolte.")

Ln IS:T, . .J. Bonché, inspecteur du jardin botanique de berlin, a obtenu aussi un joli Nymphxe à fleurs d'un rose tendre en croisant le $N$. rubra avec le pollen du $N$. lotus.

On doit encore a M. Bouché de magnifiques hybrides de ce bean genre. I'rolitant, en 1857 , de la vaste étendue d'un hassin alimenté par l'eau chaude d'une fabrique près de Berlin, il y cultivait de nombreux. Iymphxa; il en a décrit alors seize hylrides, sept provenaient de la fécondation des fleurs du Nymphaxe rubra aver le pollen du $N$. lotus, et pour les autres il a opéré sur ces premiers hỵbides en appliquant sur leur stigmate le pollen du $N$. lotus.

Ionlielaar a fécondé le Tictoria reyia avec du pollen pris sur différentes espèces de Nymphax, et réciproquement. Il en a obtenu des graines qui ont parfaitement levé, mais dont le résullat n'a rien produit de tres-heureux. (Belgique horticule, t. IIII, 1). 280.)

\section{FAMILLE DES PAPATERACELS.}

\section{Genre Pavot. - P'(tpaver.}

Il est difficile de renentres ungene de plantes dont la féceondation nilurelle soil plus assurie que dans le Parol. On trouve souvent dans chapue fleur plusieurs centaines d'éta- 
mines qui inondent un large stigmate à écusson de leur poussière fécondante. La floraison presque éphémère de ces plantes est en rapport avec l'ouverture pour ainsi dire instantanée des anthères, en sorte que, si l'on voulait hybrider des Pavots, il faudrait enlever les étamines au moment même où le calice s'entr'ouvre pour l'épanouissement des pétales. Dans les fleurs doubles, l'hybridation parait plus facile, mais il faut remarquer que, malgré le nombre très-considérable de pétales qui se développent, il reste presque toujours quelques étamines. Je ne crois pas, du reste, que le croisement puisse avoir lieu entre toutes les espèces de Pavots, mais seulement entre les variétés nombreuses des Pavots des jardins, entre celles également belles et multipliées des Coquelicots doubles, et enfin entre ces belles espèces de lavots écarlates, telles que le bracteatum, I'orientale, etc. On pourrait sans doute obtenir encore dans ces diverses plantes des variétés nouvelles, et toutes produisent beaucoup d'effet.

L'hybride entre ces deux éclatantes espèces est connu et cultivé depuis plusieurs années. Il a été obtenu à Anduze par le docteur Miergue. Il a sufti d'enlever toutes les étamines du $P$. orientale, au moment de l'ouverture du calice. Les stigmates qui ne sont pas aptes dans le bouton le deviennent immédiatement après l'épanouissement; leurs papilles, qui étaient blanches ct à peine saillantes, se redressent et ressemblent alors à des bandes de velours violet posées sur l'ovaire, et sur lesquelles le pollen doit alors être appliqué.

- Le spectabile, qui, malgré son nom, n’est pas le plus besu, pourrait peut-ètre se croiser avec les coquelicots et donner ainsi des nouvelles plantes très-remarquables.

Broussonet dit avoir vu dans le jardin d'Édimbourg un liybride du Pavot d'Orient et du Pavot somnifère. Cet hybride était connu de Linné.

Genre Sanguinaire. - Sanguinarta.

Les fleurs du Sanguinaria n'ont qu'une courte durée; la 
lëcondation sopere très-rite, comme dans les l'avols, an moyen de nombrensé étamines qui fécondent très-fiacilentent un stigmate à deux lobes épaissis.

L'hybridation serait facile en supprimant de bonne heure les étanines: mais je ne sais si on pourrail l'opérer avec le S. Bartonis, que je ne commais pas, ou s’il existe d'autres espèces ou variétés voisines.

\section{Genre Escholtie. - Escholzia.}

Dinls ce genre, comne dans les autres de la mème fanille, les étamines, souvent au nombre de douze, an moins dans le ralifornica, répandent très-promptement leur pollen sur des sligmates lililormes ou épaissis, mais toujours très-rapprochés des antheres. Un pent maintenant tenter des croisements entre les enèces, puisquelles grainent très-facilement sous notre climat, en prenant les mèmes précantions que pour les Parots.

\section{WAMILE DES WUMARIEES.}

1) cultive dans nos jardins un certain nombre de Fumuriées appartenant surtout aux genres Corydalis et Dielytru. Leur lécondation naturelle est assure par la position des six anthères, souvent portées sur deux filets unis, appliques sur le sligmate mème. Cet appareil est ensuite enfermé dans la corolle, en sorte que les fécondations croisées ne doivent janais awoir lieu naturellement dans ces plantes. Je ne erois pas non plus qu'il y ait grand motif de les tenter artiticiellenent: mais: purtant elles seraient possibles en enlevant de bome heure les antheres sur quelques fleurs que l'on anait ménagées en préparant le sujet.

Depuis l'introduction de ce noureau el admirable Ilelytro, qui a fait si grande sensiation lors de son entrée dans le monde horticole et depuis lapplarilion du ll. cucullaria, il ! aurait certainement des essis de rroisement i faire sur ees plantes ornementales. 


\section{FAMILLE DES CRUĊFÈRES.}

\section{Genre Giroflée. - Malhiola.}

Dans ce genre se trouvent le Mathiola annua, désignć sous le nom de quarantain, qui donne dans nos jardins de si belles variétés, et l'incana, qui en produit de plus belles encore, dout la vie se prolonge quelquefois plusieurs années, et les variétés désignées sous le nom de cheiri, qui appartiennent au glabra. Cies belles plantes sont connues sous la dénomination générale de Giroflées.

Le pistil est surmonté d'un stigmate épaissi, contre lequel viennent s'appliquer les six anthères des étamines. La fécondation directe est done entièrement assurée; les anthères ne s'murent, du reste, qu'après l'épanouissement de la fleur, en sorte qu'il est facile de les enlever et d'imprégner ensuite le stigmate au pinceau.

Ce n'est que sur les lleurs simples que l'on peut opérer l'hybridation, car les doubles sont dépourvues de pistils, ce qui rend la reproduction impossible. Il est vrai que les pieds à fleur's simples produisent des graines qui domnent souvent des individus à fleurs doubles; mais il n'y a rien de certain ni de constant. J'engage toutefois les amateurs de ce beau genre à tenter les croisements pour obtenir encore de nouvelles varićtés. Il arrive, il est vrai, mais rarement, dans les $M$. aînuu, yræcu, incana, que l'on trouve gà el là quelques pistils, el par suite quelques graines sur des individus à fleurs doubles, et ces graines reproduisent assez exactement la mère.

Le docteur Messer de Cabo a publié, en 1828, un Mémoire dans lequel il assure que l'on peut, à volonté, obtenir des fleurs doubles des Giroflées, en recueillant les graines sur des fleurs simples dont toutes les anthères sont retranchées avant l'anthèse. Il dit que, sur cent plantes de quarantains, il en obtenait toujours soixante à soixante-dix à fleurs pleines, tandis 
qu'en ne supprimant pas les anthères du porte-graine il n'obtenait pas plus de 20 à 50 pour 100 de plantes à fleurs doubles. Il ijoute que, si les étamines sont encore trop jeunes lor'squion leur fait subir la castration, l'ovaire arorte. Wuand clles ne: lat subissent que lorsfu'elles sont mieux formées, mais tonjours avant qu’elles aient répandu leur pollen, l’ovaire se développe et devient fruit; mais, au lieu de contenir de quarante à cinquante graines, il n'en renferme que cinq ou six, toujours plus courtes et autrement configurées. (Annales de Fromont, avril.1855.)

Si ce fait très-curienx est exact, le résultat ne tiendrait-il pas a unc lécondation croisée, opérée par les insectes entre plantes de mème espice, mais entre individus distincts, conme nous l'avons fait pressentir en rappelant les ingénieus essais de II. Ch. Darwin sur les Primula?

Il ast done probable que les fleurs privées dautheres sont lécondees par le pollen d'autres variétés, et il est possible yue I'hyloridation seule produise ce singulier résultat. Ce sont de curieuses expériences à répéter.

\section{Genre Lunaire. - Lumarill.}

On cultive dans le's jardins le biennis et le redivien, qui paraissent trop distinctes pour qu'on puisse les croiser; mais, comme le biennis oflie des variétés à fleur's blanches, on pourrait obtenir tous les intermédiaires entre le pourpre et le blanc. Toutefois il faut convenir que ces plantr's ne valent pas la peine quion leur sacrifie un temps qui serail mieux employé it opeirer sur une multitude dautres rigétaux. Cette observation sinplique à presque tous les gemres de cetle limille, tels qur les Arabis, Turrilis, Sysimbrium, Ciardamine, Lrysimum, ete., yui n'appartienment pas aux plantes d'ormement proprement dites, et dont les procédés d'hyblidation sont, du reste, absolument les mèmes que ceux de toutes le's plantes de ce groupe.

II. Reuter décrit, dans son supplément an calalogue des plantes de Genève, une nouvelle espèce d'Arabis, qu il nomme 
116 FÉGONDATION NATURELLE ET ARTIFICIELLE.

hybrida, parce quelle tient le milieu entre les A. stricta et les A. vernalis. Ces trois espèces croissent ensemble dans les rocailles du pied de la montagne de Saleve, et la lorme intermé-

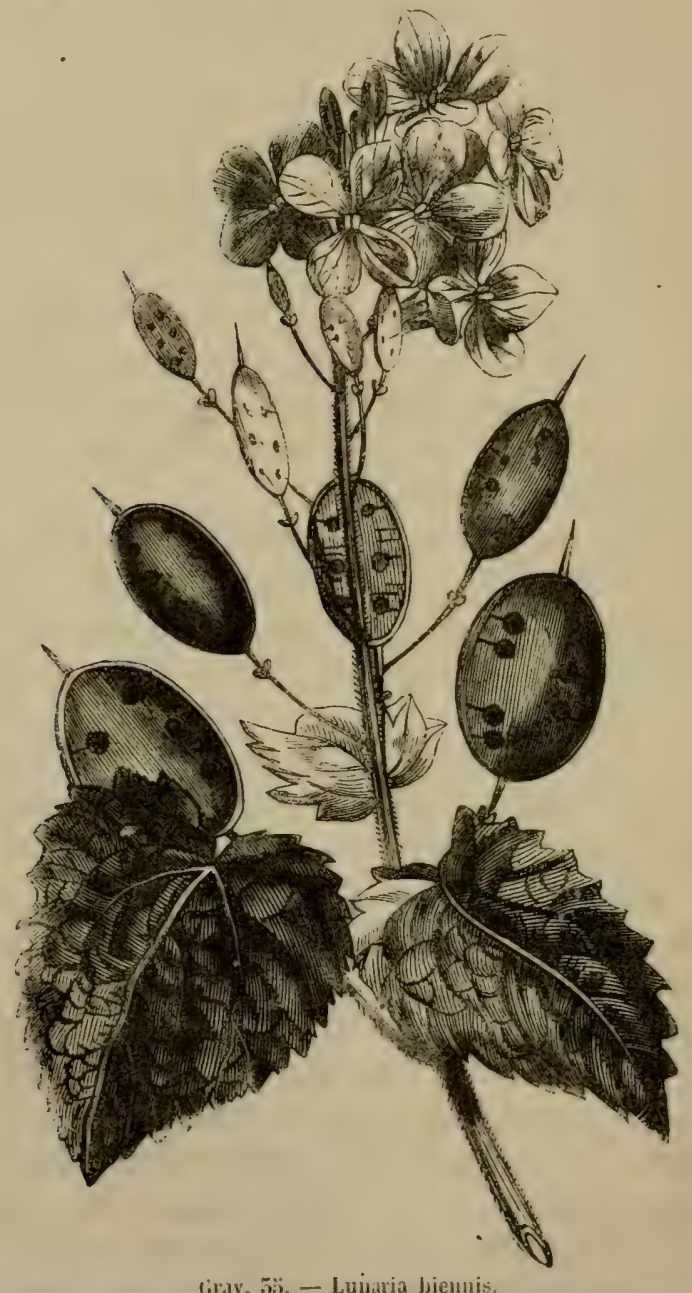

diaire y est assez abondante pour faire supposer quelle est du nombre des hybrides qui se reproduisent de graines (grav. 55 ). 


\section{Genre Ibéride. - Iberis.}

II y a peu de croisements a faire dans ce genre, quoique ses espèces soient fort nombreuses; mais elles ne sont pas toutes ornenientales. Le semperforens, seul de sa section, ne peul certainement pis shybrider avec les lheris herbacés. Il serail facile de croiser l'umbellatu, qui est le plus cultivé, aver Io ciliuta et le temuifolia, ou mène avec lo nana et le spathulutu, on du moins ces plantes ont de si grands rapports, que l'on pourrait presque les considérer comme des rariétés.

II en serail sans doute do mème des croisements que l'on voudrait essaver entre l'umura, l'odorata et le pimnafa, puisqu" l'intermedia est déjà peut-itre un de ces hỵbrides.

Enfin, on trouve aussi les plus grands rapports entre le sempervirms, le suxulilis, le conferta et quelques autres.

L'inflorescence de's Iheris est ordonnée de telle manière, que presque toutes les ombelles s'épanouissent en mème temps, an sorte qu'elles sont littéralement couvertes de fleurs, et produisent beancoup d'effet dans les parterres. Il faudrait done, pour assurer le succés des fécondations artificiçles, isoler le sujet et le préparer par le retranchement de presque toutes les ombelles et de la majeure partie des lleurs de celles que l'on conserverat.

Genre Julienne. - Hesperis.

Line espèce de ce genre, le matronalis, a doublé dans nos jardins, et produit ces belles variétés blanches ou lilas que l'on désigne sous le nom de Girardes. Leurs fleurs, commr relles des Mathiola, perdent leurs pistils en doublant, en sorle quion les multiplie de boutures ou en séparant les vieux pieds, rarement par les graines. Celles-ci sohtiement facilement sur les pieds simples, far dans ces plantes la fécondation est diantant plus certine, qu'elle s'opire avant l'épanonissement, on du moins les étanines sont deja ouvertes quand la corolle s"itale. II est vai que les deux stignates qui sont appuyés l'un 
contre l'autre semblent un peu moins hâtifs que les étamines; mais il faudrait toujours, pour une liybridation, enlever celles-ci avant le déroulement complet des pétales; peut-être le matronalis pourrait-il ètre hybridé par le runcinata ou l'heterophiılla.

\section{Genre Chou. - Brassica.}

Le Chou est un des légumes les plus importants pour la nourriture de l'homme et des animaux. Il est remarquable par la grande quantité de variétés ou d'hybrides que le hasard a fait obtenir et que la culture a conservés.

Je suis convaincu que l'on est loin encore de connaitre toutes les variétés possibles de cet excellent légume, et je pense que l'on peut arriver sûrement, par les procédés d'hybridation, à en créer un grand nombre de nouvelles. Avant d'émettre queiques idées sur ce sujet, il convient d’ètre fixé sur les principaux types de ce genre, et le résumé que nous allons donner, en suivant la elassification adoptée par de Candolle, suffira pour remplir ce but.

1. B. Olẹracea. Cette espèce est la plus importante; on la croit originaire des rochers maritimes de l'Europe. Elle a produit cinq grandes races, dont nous allons eiter les principales variétés.

A. Choux romms. 0 . capitata; à grosse tète allongée, ronde ou aplatie, verte ou rouge, tels que les Choux d'Yoreli, petit et fros. - Caur de bouf. - Pain de sucre. - Rouge, petit et yros. - Vert de Vangirard. - De Hollande à pied court. Pomme de Saint-Denis. - D'Allema!me ou Quintal. - I)e Hollande tardif. - Vert glucé d Amérique. - Trapu de Brunswick; etc.

J. Choux ne Mas.s. 0 . bullala; frisés à lète ronde ou ohlongue à jets gemmifères. Là se trouvent les diverses variétés de Choux de Bruxelles. - Les milans hâtifs. - Pied court. - Doré ù tête longue. - Le pancalier de Touraine.

C. Crour verts xox rommis. O. acepluala; à grosses coites, 
vert, blond, frangé, et les jolies variétés vertes, roses, lilas, carminées, a feuilles laciniées, désignées sous le nom russe de lictpousta, et qui sont de véritables plantes d'ornement.

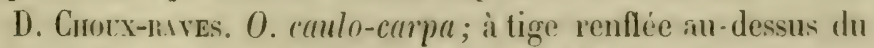
collet; tels sont : - Le blane de Siam. - Le violet. - Le nain hâtif.

E. Carour-fleuss. O. botritis; à corymbes charnus ef renflés, dans lequel on distingue: - Le tendre. - Le demi-dur. Celui d'Angleterre. - De Hollande et ues brocous. - Violet. - Pommé vert. - Blane d'Angleterre.

2. B. Campestris. Indigène des chanps de l'Europe, a produit trois variétés principales, le colzu, le pabularia ou chou à laucher, et le napo-brassica, différent du navet.

En croisant le Chou et le Navet, Sageret a. ohtenu un hylbrile entièrement semblable au colza, et il en a conclu qur. cette dernière plante était une espèce nouvelle, créée par hybridation et stabilisée par une longue habitude.

3. в. Mapa. Type de la navette à graines oléagineuses el de lit rave à racine comestible, dont on conmait aussi beaucoup de sous-variétés.

1. B. Napus. Qui a prorluit une variélé oléilère, désignce sous le nom de navette d'hiver, et un grand nombre de variétés Lubéreuses, connues sous le nom de navets, et que l'on partage en trois types:

Texones. Des Vertus. - Des Sablons. - Rose du Palulinat. - Gros-long d'Alsace. - Blanc-plat hatif. - Ronyje mlat hâtif. - De clair-fontaine. - Tumeps. - Dem-tennass. Janne de IIollande. - Janne d'Écosse. - Janme de Malte. Noir d'Alsuce. - Gris de Morigny. - Sacs. Freneuse. - Mn We'mux. - Saulien. - Berlin. - Jaune long des Etuls-Unis.

5. B. Praceox. N'est cultivé que comme plante oléilìre.

II est bien probable que plusieurs des variélés rangées sous ces ciny types sont de riritables hybrides entre les esprees, et que l'on pourrait en obtenir d'autres encore; mais il y aurail certaimement avantage i croiser an moins les races de la pre- 
mière espèce, ou les variétés de chaque race entre elles. On trouve souvent dans les jardins des hybrides entre le Chou de Vilan et le cabu, et entre ce dernier et le Chou rouge, et ces liybrides sont quelquefois préférables aux types.

Le colza, croisé par d'autres variétés du $\boldsymbol{B}$. campestris, ou par le $B$. præcox ou les erucustrum, donnerait peut-être unc race encore plus séminifère que celle qui est cultivée pour l'huile que l'on en extrait.

Toute la série des $B$. ropa et napus, en $y$ ajoutant même les Choux-ranes et les rutabayas, pourraient sans doute former encore des variétés nouvelles, plus grosses, plus tendres, plus précoces ou plus tardives, plus duuces, on d'une plus longue conservation; enfin, je crois qu'il reste dans ce genre un grand nombre de découvertes à faire, et, du moment où l'on admet le mérite d'une variété nouvelle de Rose ou de Dahlia, on ne peut cortester celui d'un légume nouveau qui vient augmenter nos richesses,

Il serait possible aussi qu'en croisant les diverses espèces de la section des erucustrum, on obtienne des plantes oléifères, mains productives peut-être que le colza, mais susceptibles de croitre dans les saljles ou des terrains médiocres, où le colza ne pourrait végéter.

Enfin, le pe-tsaïe ou Chou chinois, qui fleurit et fructifie si facilement dans nos jardins, sans donner un légume bien remarquable, ne pourrait-il pas être fécondé par nos races curopéennes, ou les féconder à son tour, et produire ainsi des lígumes tout à fait nouveaux et précieux peut-être par leur végétation hâtive? M. Pépin cite des pe-tsaïs, semés le $\mathrm{I}^{\mathrm{er}}$ avril, et dont les graines étaient récoltées le 16 juillet.

L'hybridation est facile à faire dans le genre qui nous occupe. Il suffit de laisser au sujet une seule branche de fleurs, et, comme celles-ci s'épanouissent lentement et successivement de bas en haul, on enlève soigneusement et de boune heure toutes les étamines de plusieurs fleurs, ef l'on abat le reste de. la branche au-dessus de la dernière, que l'on a artificiellement 
fícondée. Si deux ou-trois siliques seulenent réussissent, on it assez de graines pour comnatre, dis l'annce suivante, les résultats de ses essais.

Les mèmes remarques sappliquent aux Simupis et aux Riophamus. Haus les limilles tris-naturelles, comme celle des Cruciferes, ou les genres sont à peine distincts les uns des antres, l'hybridation a quelquefois lieu entre cux. Ainsi Sagerel, qui a porti dans l'étude des hỵbrides la science et le talent d'observation qur l'on remarque dans tous ses écrits, a obtenu un lyghride entre le Chou et le Radis noir: ce dernier était le portewraine. L'hỵhride avait des siliques doubles, c'est-à-dire placées l'une au-dessus de l'autre ef tres-distinctes par la forme; l'me resemblait i celle du Chou et l'autre à celle du Radis.

\section{Genre Radis. - Raphunus.}

Le R. suliuus ou Radis a produit, comme on le sait, de nomhreuses variétés que l'on pourrait multiplier encore. On les. partige en trois sections. Les roxns, qui renferment le rose ordinaire, le blane hitifde Hollande, le violet haitif, le saumonné, le jaume ou romx. Les Loxas, que l'on désigne aussi sous le nom de raves, ou l'on trouve le blane, le rose, le rouge et le petil hâtif. Enfin, les sontis, qui contiennent le violet de Chine, le rose d'hiver, le blane ordinaire et le gros blane i'Angshoury. Avec un si grand nombre de modifications, il serait très-facile d'en obtenir d'autres.

\section{FAMILLE DES CAPPARIDEES.}

\section{Genre Caprier. - Capparis.}

Les Cilpriors ne sont pas assez cultivés comme plantes dornement ot forment cependant an des plus beanx genres qui existent. Leurs fleurs ont pent de durée; leurs étmmines sont tris-nombreuses et le pistil est mique. Malgré la rourte existence des fleurs, les chtamines ne śnurent pas en même temjs 
que la corolle, et l'on peut facilement les retrancher avant l'anthèse, en sorte que les croisements seraient faciles et pourraient peut-être s'obtenir sur le spinosa avec l'odoratissima, le pulcherrima, le inpestris, l'xgyptia, etc.

Genre Cléome. - Cleome.

Il est extrêmement facile d'opérer l'hybridation sur les Cleome, puisque les pistils sont très-distincts des étamines et que l'on peut très-aisément retrancher ces dernières avant l'anthèse; mais j̈ignore si les croisements, qui auraient lieu nécessairement entre espèces assez distinctes, pourraient réussir.

\section{FAMILLE DES PASSIFLOREEES.}

\section{Genre Passifore. - Passiflora.}

Vaste et magnifique genre qui renferme près de deux cents espèces d'un éclat et d'une originalité remarquables. La fleur offre cinq étamines dont les anthères sont pivotantes et renversées. Les styles, au nombre de trois, se terminent chacun par un stigmate épais et glanduleux. Les Passiflores peuvent s'lybbrider, car M. Delaire, directeur du jardin de botanique d'Orléans, qui s'est depuis longtemps occupé d'hybridation, et qui est, je crois, un des premiers qui aient fait des expériences positives dans les serres du Museum de Paris, oì il était alors jardinier, a remarqué que plusieurs Passiffores, qui ne pouvaient être fécondées avec leur propre pollen, fructifiaient en les croisant avec d'autres espèces.

Ces plantes, déjà très-belles sans qu'elles soient croisées, ont donc offert déjà des hybrides remarquables. Une des plus belles à fleurs d'un rouge vif est le Passiflora amabilis de M. Schachter, obtenu à Loos-lès-Lille, du $P$. princeps (mère) et du $P$. alata (père), fait d'autant plus curieux que le $P$. princeps ne fructific pas ordinairement avec son propre pollen, ou du moins sans fécondation artificielle. Les P. Lemicheziana, Londoni, Colvillii, 
princeps, racemosa, sont encore de jolis hybrides de ce genre.

II. Belot, de Ioulins, a obtenu aussi, en 1847, une remarquable plante en fécondant le $P$. alatı par le $P$. carrulea.

Au sujet du $P$. Lemicheriana, M. Lemichez m’écrivait en 1845 rque ret hybride prosenail du $P$. alata, féconde par

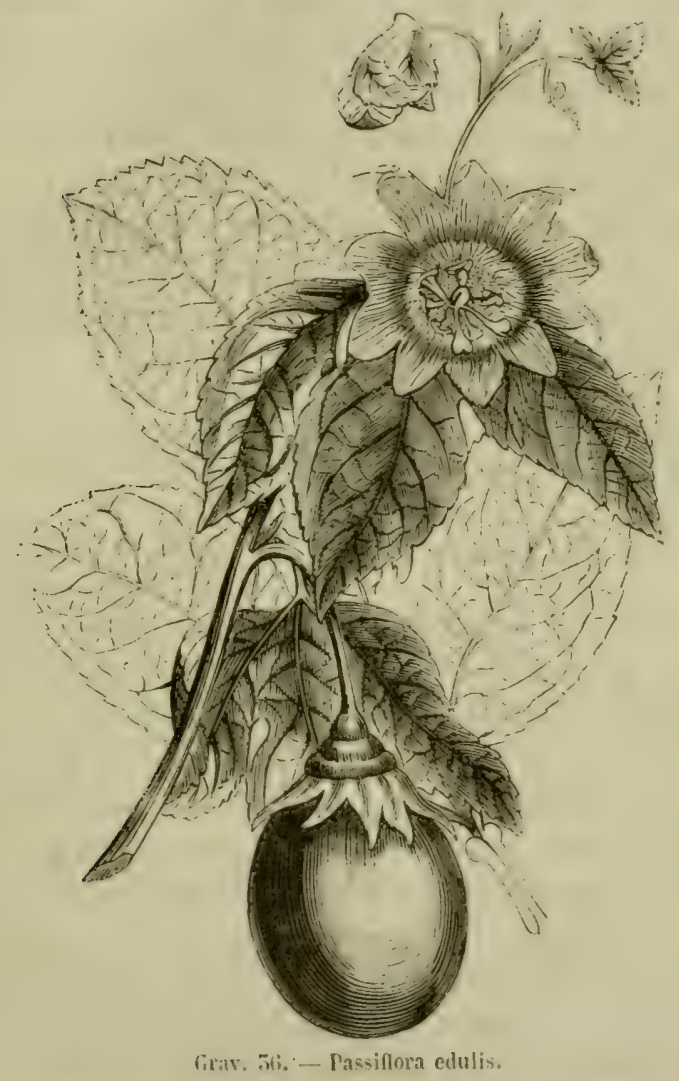

le pollen du P. liermesina, lepuel faisait toujours fructificr le $P$. alata, tandis que l'inverse n'avait pas lieu.

Si une fois on s'oceupait de ce tenre, comme on l'a fail pour Ires Pelaryonium, on finirail saus doute aussi par perdre la trace des espiees, et on doterait l'horticulture de plantes admirables. 
Il est vrai qu'avant de chercher à faire de nouvelles plantes il vaudrait mieux peut-être se procurer d'abord celles qui existent dans la nature, déjà si riche en formes variées et singulières. La fécondation artificielle sera toujours utile pour les Passiflores comme pour les autres plantes de serre, pour en obtenir des graines, qui souvent, sans cette opération, manquent totalement. Ainsi, soit qu'on opere daus cette intention, soit que l'on cherche à croiser les espèces, il faudra poser le pollen sur les stigmates et humecter ceux-ci au pinceau avec l'humeur miellée que l'on cherchera au fond de la corolle. Si c'est un croisement que l'on opère, on retranchera les anthères avant qu'elles soient ouvertes; mais les Passiflores laissent tout le temps dr: faire cette ablation, car ce n'est guère qu'ì la défloraison que la fécondation sopère (grav. 56).

\section{FAMILLE DES VIOLARIÉES.}

Genre Violette. - Viola.

Ce genre offre de très-grandes différences dans la forme du stigmate, qui tantôt est pointu et courbé en forme de bec, comme dans la Violette odorante, tantòt bilobé, comme dans le biflora, ou creusé en entonnoir, comme dans les Pensées. Les 'étamines, au nombre de cinq, presque adhérentes entre elles, sont collées contre le pistil, et leurs anthères sont terminées par deux petites lamelles brunes qui forment une sorte de couronne autour du stigmate. Ce dernier est apte avant les étamines, en sorte que l'on pourrait pratiquer la fécondation artificielle sans enlever ces organes; c'est peut-être ainsi naturellement que se sont formés plusieurs hybrides naturels. M. Godron a signalé un Viola hirto-alba. II. Timbal-Lagrave en a décrit plusieurs dans les Némoires de l'Académie impériale de Tonlouse. C'est peut-être aussi à des croisements que sont dues les nombreuses variétés de l'altuicu.

On a supposé du moins que c'était le $V$. ultcricu qui avait. 
communiqué à notre $V$. tricolor la tendance à produire des lleurs d'un grand dianètre et de formes arrondies. Il est également positif que les plus belles Pensées couronnées en Anglt:terre sont provenues par l'hybridation naturelle et artilicielle de l'alluica (La Pensée, par le baron Biedenfeld, Duchartie, lietue, t. I, p. 95. Si ces croisements ne sont pas possibles entre les especes de cette division, ils ont eu lieu an moins entre les variétés obtenues par la culture, et il est peu d'espèces qui aient produit autant de variations que la Pensée ordinare. C"est du reste wae de ces plantes que l'on pourrail appeler changeantes, car on troure, sur le mème pied, des fleurs entièrement différentes; aussi lesplus belles variétés obtenues sontelles dues au hasard. On ne prend plus la peine de féconder un plante qui dome d'elle-mème de si admirables changements, et qui n'attend mène pas que l'on preme la peine de semer ses: graines pour les produire.

M. Fermond explique la lécondation de la l'ensée par l’inLermédiaire de la corolle. Les anthères sourrent assez longtemps avant que la lleur s'épanouisse et le pollen arrive au conlact de la gorge dr la corolle, dont trois pétales se trouvent, a ret endroit, munis de poils collecteurs destinés à retenir ce poll'n; mais, pour sépanouir, la corolle s'accroit, le tube s'al.longe, et, en s'allongeant, il glisse le long.du style et va porter sur le stigmate le pollen qui s'y était attaché.

\section{FAMILLE DES POLYGMLES.}

\section{Genre Polygala. - Polygulu.}

La fécondation des Polygalas n'a pas toujours lieu liacilement dans nos serres, on tres-probablement on obtiendrait plus souvent des graines fertiles en imprégnant au pinceatu.

Lés élamines, an nombre de huit, entourent un stignate i deux lères, dont lointérieur seulement parait être le véritable stigmate. Je ne crois pas que l'on ait tenté d'hybrider les Polygalis, mais on le pourrait certinement, en enlevant de bome 
heure les anthères et appliquant sur le stigmate le pollen d'une espèce voisine, car ces plantes forment plusieurs sections assez nombreuses, et l'on ne pourrait espérer de croisements entre des espèces de section "différente. Le P. Dalmaisiunu, récenment obtenu, paraît être un hybride du grandiflora, fécondé par le cordifolia.

\section{FAMILLE DES RESÉDACÉES.}

\section{Genre Itéséda. - Reseda.}

Les plantes de ce genre ne présentent rien de gracieux ni d'élégant, et la seule espèce cultivée, l'odorata, ne l'est que pour le parfum qu'elle répand. Il n'y a done rien à tenter pour des fécondations artificielles qui scraient faciles, car dans les résédas, comme dans les Pelaryonium, le stigmate n'acquiert son aptitude qu'après l'anthèse des étamines, de sorte que la fécondation naturelle n'a lieu qu'indirectement par les fleurs supérieures de l'épi ou par les épis voisins, comme dans la plupart des céréales.

\section{FAMILLE DES CISTINEES.}

Genre Ciste. - Cistus.

Je ne sache pas que l'on se soit occupé sérieuseinent en France des Cistes sous le rapport horticole; ils forment cependant un bien beau genre, qui semble avoir un grand avenir, mais dont les fleur's ont l'inconvénient de ne pas durer longtemps. Les étamines sont nombreuses, les anthères répandent le pollen par des fentes qui partent du sommet, et la fleur entière en est quelquefois couverte; les mouvements que l'on observe souvent dans ces organes facilitent la dispersion du pollen, qui est recueilli par un stigmate en forme de tête tellement situé, qu'il est impossible que la fécondation ne soit pas parfaitement assurée.

Les fleurs des Cistes s'épanouissent de très-bonne heure, et 
c'est au lever du soleil, ou mienx un peu anparavant, quil fitudrait enlever les étanines. On peut, pour faciliter l'opération, écarter les pétales, ou mêne les couper et les supprimer arec les étamines. Le stigmate est apte à receroir inmédiatement, ou du moins peu d'heures après. l'époque de l'épanouissement.

L'hyjloridation peut done se faire lacilement, et déjà elle a eu lieu dans la nature, car il est impossible que toutes les formes de Cistes que l'on observe antour du bassin de la Méditerranée, et seulement en France, soient des espèces dislinctes.

Ce genre est divisé en deux sections assez naturelles, et ce n'est qu'entre les espèces de même section que les croisements peuvent s'opérer. Ainsi on peut considérer comme des hybrides naturels : le florenlinus, intermédiaire entre le monspeliensis et le salvifolius; le ledum, qui parait provenir du laurifolius et du monspe. liensis. Ce dernier semble en-

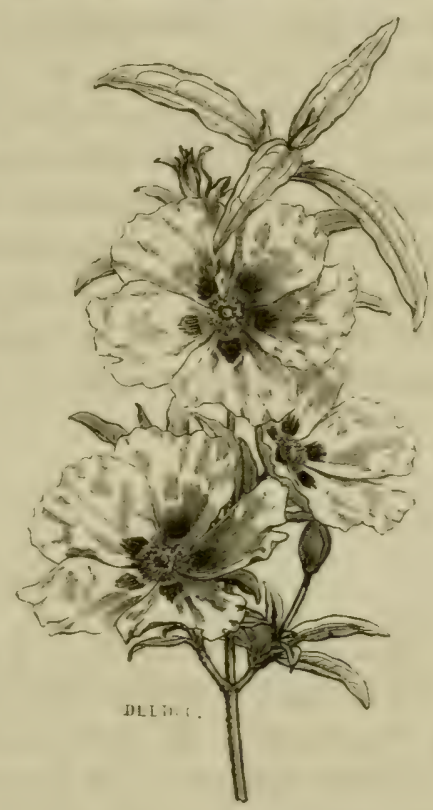

Grav. $\overline{1} .-$ Cistus ladaniferus. core aroir doriné naissance au longifolins en fécondant le jumnlifolius. Les croisements opérés par les espèces à fleurs simples sur les variétés doubles déjà obtenues par la culture donneraient, sans aucun doute, de bien belles nouveaules, comme on en obtient maintenant dans les Roses, par le mème procédé. Le Cistus vanjinutus de Ténírife est une des plus helles espèces que l'on pourrait employer dans les croisenents. Le C. ladaniferus est aussi un des plus remarquables (grav. 57). 


\section{Genre Iélianthème.-Helianthemum.}

Ce que nous venons de dire des Cistes s'applique aussi it ce genre, qui présente, dans les organes sexuels, de très-grandes ressemblances, mais dont les sections, assez distinctes du reste, ont pour principal caractère différentiel la longueur du style, qui tantôt élève le stigmate au-dessus des étamines, et d'autres fois le tient en dessous, ou bien enfin le place dans une région mojenne. Dans tous les cas, il peut recevoir directement le pollen. La fécondation est sûre comme dans les Cistes; elle a lieu de très-bonne heure, et les croisements doivent être aussi laciles entre espèces voisines seulement, car il y a dans ce genre des berbes et des arbustes, des plantes vivaces ot d'autres qui sont annuelles, des plantes dressées et des espèces rampantes dont les analogies sont trop éloignées pour qu'on puisse, dans ces circonstances, regarder l'hybridation comme possible.

\section{FINILLE DLS CARYOPIIYLLLESS.}

\section{Genre Eillet. - Dianithus.}

Danis ce genre nombreux, quatre espèces, qui sont maintenant le type de nombreuses variétés, se disputent nos parterres: le $D$. chinensis, aux fleurs éclatantes; le barbutus, chargé de bouquets multicolores; le plumarius, type de nos charmantes mignardises; et enfin le caryophyllus, qui a produit cette prolusion de variétés désignées par les noms d'OEillets flamand: et de funtaisie. Il faudrait encore ajouter à ces plantes le superbus ì pétales frangés, le cæsius, le glacialis, le viryineus et l'ulpinus, dont les gazons s'étendent sur les rochers qu'ils cachent sous leurs fleurs parfumées.

Toutes ces plantes ont des étamines dont les anthères sont portées sur des filets généralement assez longs pour qu’elles sortent du tube, quoique cependant elles y restent quelquefois enlermées. Il y en a presque toujours cinq grandes et cinq plus 
petites, qui ne s'ourrent pas à la fois. Les styles sont allongés au nombre de deux ou trois et garnis sur la majeure partie de leur longueur de stigmates papillaires. Les pétales sont velus ou au moins veloutés à la base, et regoivent le pollen. Ce n'est quelquefois qu'à la défloraison que le pistil se trouve fécondé par son contact arec les étamines elles-mèmes ou avec les pétales couverts de pollen.

La fécondation artificielle est donc très-facile dans les (Eitlets, car les styles sont saillants et les étamines ne le sont pas toujours, et précèdent assez souvent le développement des stigmates. Ces derniers avortent quelquefois.

On rencontre assez, souvent des hybrides dans la nature. I'lusieurs sont cités dans les ouvrages de botanique. lls appartiennent surtout à la section des plumarius, et nous-méme avons signalé le D. Monspessulano-Segnieri, assez répandu sur les pelouses des montagnes de l'Aurergne. Les caractères de ces hybrides rarient selon qu ils ont pour père le D. Monspessullamus ou le D. Seguieri.

Le chinensis et le barbatus ont une si grande tendance à rarier, que l'on ne tente guère de croiser leurs fleurs. On pourrait cependant féconder les variétés doubles par les simples nuancées d'autres couleurs. Le barbatus, désigné aussi sous les noms de bouquet tout fait ou bouquet parfait, présente le singulier phénomène de fleurs diversement colorées sur le même pied et dans le même fascicule. On en voit de rouges et de blanches qui se touchent. Les plantes qui offrent ce caractère sont déjà des hybrides qui viennent appuyer une observation faite depuis longtemps par Sageret et que mes remarques ont également confirmée : c'est qu'au lien d'obtenir toujours un hybride qui tient le milieu entre le père et la mère, on est souvent étonné de trouver des sujets dont tel ou tel organe appartient complétement à l'un des ascendants, sans avoir èté modifié par l'autre.C'est sans doute à cette raison qu'il faut attribuer les fleurs de couleur différente sur un même pied, comme cela se présente dans l'Willet qui nous occupe. 
L'apparition du D. Heddewigii, avec ses grandes et magnifiques tleurs et sa facilité de croisement, a fait naitre dans cette section des races nouvelles et des plus éclatantes. L'arenir cependant nous réserve bien d'autres surprises, et tend à mêler dans nos jardins les diverses espèces de Dianthus, comme il a confondu les types des Roses, des Fuchsia, etc.

Les mignardises se fécondent très-bien; les doubles conservent assez souvent quelques étamines, et d'ailleurs on peut prendre le pollen sur les semi-doubles et même sur les simples. Peut-être même y a-t-il déjà des hybrides entre le plumuriụs et le caryophyllus.

Quant à ce dernier, c'est par l'hybridation que l'on a acquis ses nombreuses variétés; mais ces croisements, comme la plupart de ceux obtenus avant notre époque, sont entièrement dus aux insectes. Ce n'est que dans ces derniers temps que l'on a su pratiquer l'opération si simple de l'hybridation. Elle est extrêmement facile sur les CEillets : il suffit d'attendre le développement des stigmates et d'y porter le pollen au pinceau. II est même inutile d'enlever les étamines de la fleur fécondée, quand elle en conserve. Si on ne trouve pas le pollen sur de belles fleurs doubles, il faut le chercher, comme dans le plumarius, sur les semi-doubles, et, au besoin même, sur les simples; ınélanger les flamands et les fantaisies, dont les graines pourront alor's domner les deux races; mais féconder entre eux les beaux flamands, si l'on tient à conserver la race pure; choisir avec soin ses porte-graines, et, une fois le choix fait, sur un seul pied, de deux à trois fleurs ayant les pistils bien conformés, supprimer toutes les autres.

Les Eillets ne donnent pas tous de la graine, surtout les flamands, qui manquent quelquefois de pistils et souvent d'étamines, à cause de leur transformation en pétales. Presque toutes les variétés dites fantaisies en produisent, et l'on conçoit alors toute la facilité qu'elles présentent à la fécondation artificielle el le nombre infini des modifications qu'elles peurent subir, si les couleurs sont habilement croisées. Les flamands, 
moins féconds, offrent cependant d'excellents porte-graines que l'on peut hybrider entre eux ou quelquefois léconder avec d'autres qui conservent queliues étamines. Voici, d'après le baron de Ponsort, les types que l'on doit s’attacher à cultiver pour lit récolte des graines : Pocnpres. Philippe de Belmas. - Léonidas ocelle. - Mannoss. De Coussemalier. - Icehus. - Bailde. Puis en déclinant, néanmoins dans leur ordre de mérite, Quatre-feux. Mutten. - Loridan. - Martin. - Schiller. Bizanie feu. Goethe.-Crayoisi cerise. Arius.-Violet. Olinde. - Rose. Orphée. - Bizanes noses. Saur Isabelle. - De Coussemaker. - Reine de Chypre.

Nous trourons dans la Flore des serres et des jardins, t. II, 1. 29, une note extraite du Floricultural cabinet, où nous lisons ce qui suit sur l'hybridation des (Eillets :

"On choisit les fleurs parlaitement doubles, et pour que ces lleurs puissent produire des graines, il est nécessaire de laisser tous les boutons arriver à fleur. Lorsque les fleur's sont enlièrement ouvertes et que les pistils prennent une apparence glacée, on prend une autre flcur à moitié épanouie et on l'oavre de manière à décourrir les anthères. On enlève une de celles-ci; si elle n’est pas déjà ouverte, on l'ourre et on la promène le long des stigmates jusqu'à ce que l'on voie quelques grains de pollen s'y attacher. Si cette opération est bien faite, la fleur se lerne en deux ou trois heures. Dans le cas contraire, il faut recommencer. Deux ou trois jours après cette fécondation artificielle, on enleve tous les autres boutons et l'on porte la plante dans un lieu bien exposé au soleil, on l'arrose abondamment el l'on protége la capsule contre la pluie au moyen d'un morceau carré de planche mince que l'on fixe au sommet du tuteur. Un cueille ce fruit lorsqu'il est mùr ot on le conserve tout entier dans une fiote bien bouchée. ")

\section{Genre Silène: - Silene.}

de ne cile ce genre que pour faire voir avec quelle facilité on peut croiser les espèces. Il y a évidemment dans les Silènes 
des plantes qui sont intermédiaires et peut-être lyybrides; mais ce qui rendrait les croisements très-faciles, c’est l'inégalité de? développement des organes sexuels; non-seulement plusieurs sont dioiques, mais ordinairement les dix étamines se développent en deux séries : les cinq premières avant les trois styles, et les cinq autres en même temps qu'eux, et quelquefois plus tard, en sorte que la fécondation d'une fleur a généralement lieu par les étamines d'une autre, ce qui rendrait très-faciles les essais d'hybridation. Ce genre nombreux n'offre, du reste, qu'un petit nombre d'espèces cultivées dans les jardins.

\section{Genre Lychnide. - Lychnis.}

Très-beau genre qui fournit à nos jardins un grand nombre d'espèces; clles ont dix étamines et cinq styles. En général, les anthères ont jeté leur pollen arant que les stigmates soient aptes il le recevoir; en sorle que la fécondation, qui est réellement dioïque dans certaines espèces, comme dans le sylvestris et le dioica, est presque toujours monoïque, quoique les fleur's soient hermaphrodites. Il serait done facile de former des hybrides entre les plantes de ce genre qui ont le plus de rapports : il suffirait d'attendre le déreloppement des stigmates pour les imprégner au pinceau. Les variétés du Lychnis chalcedonica ou croix de Jérusalem pourraient s'augmenter encore de cette manière. Les variétés doubles des Lychnis dioica, sylvestris et flos-cuculi pourraient se croiser ou du moins être fécondées par les simples, et réciproquemeut, quand elles ont des styles et des stigmates bien conformés. Il y aussi des hybrides à espérer entre le fulgens, le Bunjeana et les belles espèces à grandes fleurs récemment introduites. La fécondation artificielle pourrait au moins leur faire donner des graines, mais les Lychnis, comme la plupart des Caryophyllées, se croisent si facilement, que l'hybridation de ces belles espèces serait une chose trèsprobable.

Déjà, en 1854, M. Pépin a indiqué dans les Annules de Flore et de Pomone un Lychnis hybride provenant du Luclenis ou 
Agrostema flos-Jovis et du $L$. ou A. coronaria. "Cette variété, dit M. P'́pin, est d'un bel effet par ses grandes fleurs. Elles ne forment pas le corymbe comme dans la première espèce, mais elles sont moins dichotomes que dans la seconde; enfin, le calice est moins renflé et ses angles moins saillants que dans celle-ci, et, an contraire, plus saillants et plus allongés que dans celle-là.

\section{Genre Lin. - Linum.}

Ces plantes produisent des fleurs très-éclatantes, mais éphémères, qui s'ourrent de grand matin, et dont les pétales tomhent de bonne heure. Elles ont cinq étamines et cinq stigmates, quelquefois réduits au nombre de trois. Les anthères s'ourrent en dedans, en dehors ou sur le côté, et les stigmales papillaires, aptes à recevoir immédiatement le pollen, sont disposés de telle manière, que la fécondation est à peu près certaine.

Les Lins ont cependant une grande tendance à s'hybrider, comme Kølreuter l'avait reconnu depuis longtemps. Les nomhreuses espèces à fleurs bleues, voisines du perenne, n'en sont peut-être que des variétés. Ne pourrait-on pas, avec ces espices, hybrider le lin commun et produire de nouvelles variétés textiles? Les Lins à fleurs blanches et roses pourraient aussi se croiser; ils forment une section particulière du genre comme ceux à fleurs jaunes en forment une autre. Viennent ensuite ces beaux Lins à grandes fleurs rouges qui deviendront peut-être la source de nouvelles variélés.

Il faut, du reste, se lever matin pour tenter les croisements sur ces plantes. Il est nécessaire d'enlever les anthères de trèsbonne heure, car, peu de temps après l'épanouissement, la lécondation est opérée, et les pétales tombent.

\section{FIMILLE DES MALVACÉES.}

Genre Mauve. - Malva.

Un assez grand nombre de Haures sont cultivées dans les serres et dans les jardins, bien que ces plantes ne soient pas, à 
beaucoup près, aussi éclatantes que plusieurs autres de la même famille. Leurs anthères, très-nombreuses, sont situées au sommet de filets qui ne sont libres quà leur partie supérieure et qui sont soudés à leur base. Les stigmates forment une espèce de houppe au-dessus des anthères, et les styles passent au milieu du tube formé par la soudure des filets (grav. 38.) Quelque-

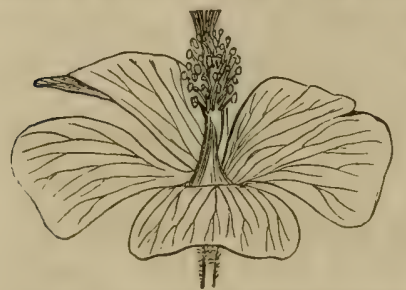

Grav. 58. - Étamines monadelphes d'une Mauve. fois ces stigmates sont aptes en même temps que les anthères, d'autres fois ils ne se développent qu'après; mais, comme le pollen des Mauves et des autres Malvacées est garni de papilles, il en reste assez de grains adhérents à toutes les parties de la fleur pour assurer la fécondation. Il serait, dans tous les cas, fucile de la pratiquer artificiellement, en enlevant de bonne heure les étamines et imprégnant au pinceau. Je ne vois pas quelles variétés ornementales pourraient être créées avec les Mauves, et je pense que si l'on voulait prendre la peine d'hybrider des plantes de cette famille, ce serait dans les Althæa, et surtout dans les Hibiscus, qu'il faudrait chercher ses sujets.

\section{Genre Guimauve. - Althra.}

La principale espèce cultivée de ce beau genre est l'Athæa rosea ou Passe-rose, admirable plante qui commence à lutter dans nos jardins avec le Dahlia, qu'elle surpasse en éclat, qu'elle atteindra en variétés de couleur, mais moins facile à conserver comme plante vivace. Les Passe-roses, quoique trèsdoubles, donnent presque toujours de la graine, car elles conservent une partie de leurs nombreuses étamines, soudées par leurs. filets comme dans les autres Malvacées. Le pistil reste ordinairement intact, et développe ses stigmates assez longtemps après que les élamines ont répandu leur pollen, en sorte que la fí- condation artificielle peut s'opérer au pinceau, après avoir en 
toute facilité pour retrancher les étamines. On peut même négliger cette précaution, mais alors il faut, avant la défloraison, entourer le stigmate d'un petit tube en carte ou en papier pour empècher son contact avec la corolle, qui se tord et se rapproche du stigmate, lui apportant ainsi une partie des grains polliniques qui lui sont restés adhérents. On peut féconder les simples par les doubles, ces derniers par des semi-doubles; hybrider le rosea par le ficifolia, le pallida par le sinensis, et réciproquement : on obtiendrait ainsi des variétés naines ou élevées, de couleurs extrềnement variées. On en a déjà de bordées de coulcur différente; on aurait des striées, des veinées. Ce sont des plantes dont le croisement, très-facile comme celui des Malvacées, mérite toute l'attention des horticulteurs. Après l'hybridation de cing a six fleurs sur un pied, il faut couper l'extrémité de l'épi; les graines sont meilleures, et l'on n’a plus à craindre le pollen des fleurs supéricures; si l'on ne veul pas se priver d'un reste de floraison, on ôte seulement les boutons qui sont placés immédiatement au-dessus des fleurs fécondées, et dont le pollen pourrait altérer les oprirations. Une senle fleur, bien fécondée, donne une grande quantité de graines.

Ce que je viens de dire de l'Althax roseu peut s'appliquer à toutes les especes de ce genre qui sont cultivées dans nos serres of nos jardins. Le gemre Malope et la plupart des Malvacées doivent être traitées de la mêmé manière.

Genre Lavatère. - Lavatern.

L'organisilion des Lautìres est la mème que celle des Haures et des Althxa; nous ne les mentiomnons que pour engager les horticulteurs i tenter quelque hybridation sur ces helles espèces, renvoyant, pour tout re qui est relatif ì cette opération, au genre précédent.

\section{Genre Hubeus. - Ilibiscus.}

On cultive dans nos serres un grand nombre d'espèces de re 
156 FÉCONDATION NATURELLE ET ARTIFICIELLE.

beau genre, qui, chez nous, fructifie assez rarement, malgré un '

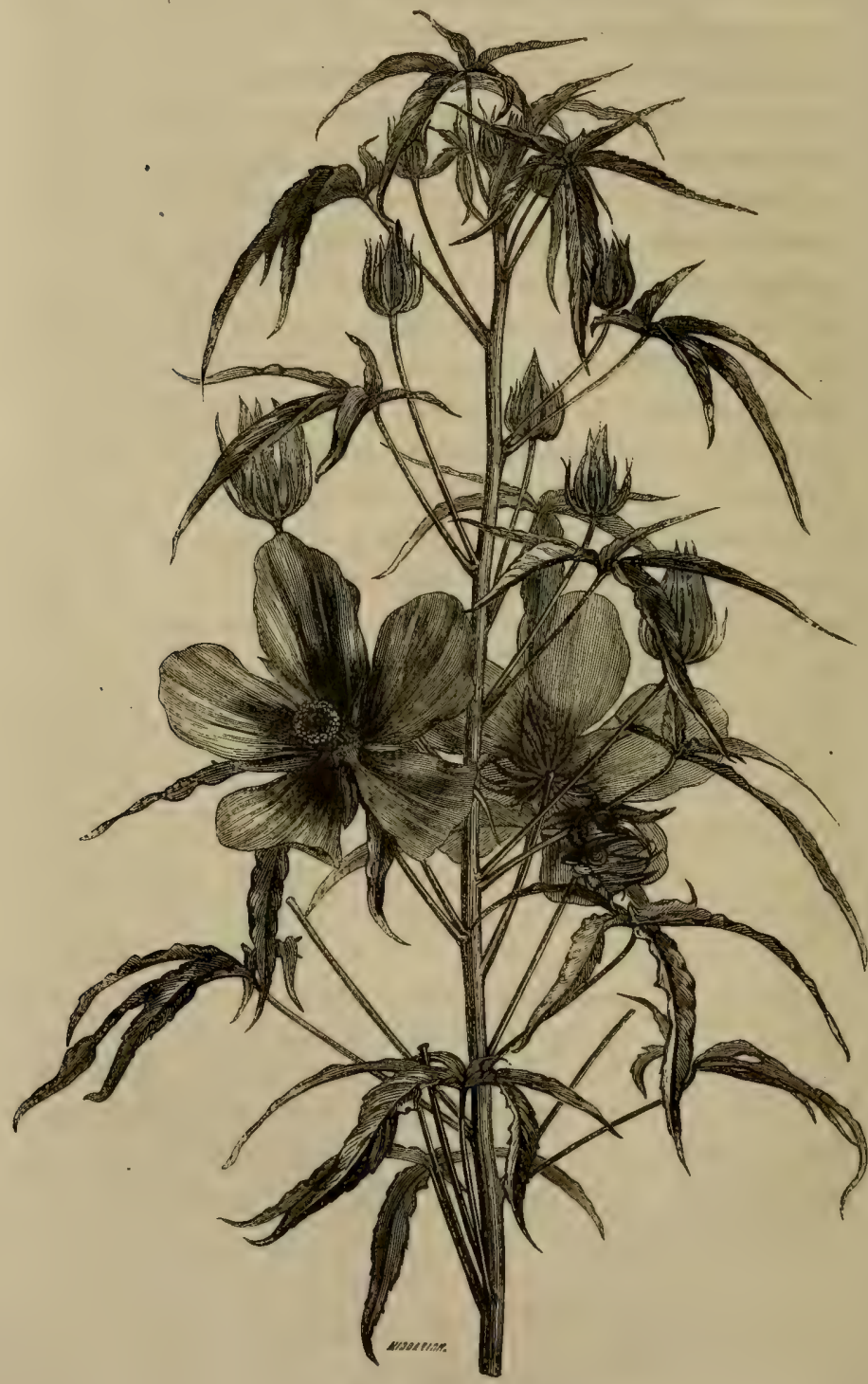

Grav. 39. - Hibiscus speciosus. 
bon nombre d'étamines et cing stigmates bien conformés, qui généralement s'épanouissent à l'époque où les anthères répandent leur pollen. La fécondation artificielle, avec le pollen de la mème fleur, faciliterait sans doute la fructification de plusieurs espèces, et l'hỵbridation pourrait aussi avoir lieu entre les espèces trìs-roisines sculement et dérivant du mème type, car il y a dans ce genre nombreux une douzaine de sections particuculières formant chacune des groupes assez nettement séparés.

L'opération serait, dans tous les cas, très-facile; les fleurs sout peu nombreuses, les étamines peuvent ètre enlevées sans difficulté, et les stigmates peuvent être préservés par des tubes. C'est un genre brillant qui récompensera dignement l'horticulteur qui le prendra sous sa protection (grav. 59).

\section{Genre Sida. - Sida et Abutilon.}

Les Sida et les Abutilon présentent un mode de fécondation analogue à celui des autres Malvacées, et doivent être traités de la même manière. On s'est peu occupé d'hybrider ces plantes, mais la fécondation artificielle rendrait sans doute productifs presque tous ceux qui restent stériles dans nos serres.

II. Lambotte, de Namur, a exposé en 1855 à la Société d'horticulture de Gand des hybrides qu'il dit avoir obtenu en fécondant l'Abutilon striatum par le pollen du Sida albida. Quelle que soit l'origine de ces plantes, elles ont sans doute du mérite, puisque la Société d'horticulture a cru devoir donner un prix à ces nouvelles variétés.

\section{FAMILLE DES TILIACEESS}

\section{Genre Tilleul. - Tilia.}

Ces beaux arbres constituent un genre assez nombreux dont. less espèces penvent être groupées sous deux sections. La pre- 
mière contient nos Tilleuls ordinaires, parvifolia, grandifolia, rubra, intermedia; il n'y aurait peut-être aucun avantage à chercher à les hybrider. La seconde renferme les belles espèces nommées argentea, petiolaris, glabra, mubescens, laxiflora, qui pourraient entre elles produire des arbres forestiers nouveaux. Ce serait, du reste, chose assez difficile que d'isoler les fleurs de ces Tilleuls; il faudrait, comme pour les arbres fruitiers, faire porter une branche sur un support et la recouvrir d'une cloche, ou l'envelopper dans une gaze imperméable. Les fleurs de Tilleul ont peu de durée; les étamines sont nombreuses et répandent iumédiaternent leur pollen; il faudrait les enlever de bonne heure et appliquer le pollen tout de suite, au pinceau, sur le stigmate qui termine le style unique.

\section{FÄNILLE DES HIPPOCASTANÉES.}

\section{Genres Marronmier et Pavia. - Esculus el Pavia.}

Les Asculus et les Pavia forment deux types séparés que l'on ne peut pas espérer de croiser, mais on pourrait obtenir des hybrides dans chacune de ces deux sections. Leurs fleurs disposées en grappes ne fleurisșent pas en même temps. Celles de la base s'ouvrent les premières, et parmi celles-là seulement se trouvent quelques fleurs dont le pistil est bien conformé et dont les pédicelles, destinés par cette raison à supporter des fruits, sont plus fermes que ceux des fleurs mâles ou inf rtiles. Il faut donc choisir celles de ces fleurs qui sont munies d'un style et d'un stigmate, et y poser le pollen des espèces voisines en supprimant ensuite toute la partie supéricure de la grappe. Il est bien entendu qu'il faut auparavant enlever, dans la fleur à féconder, les étamines, qui y sont souvent au nombre de sept.

Si le Marronnier à lleurs doubles a son pistil bien conformé, on obtiendrait probablement de nouvelles variétés à fleurs doubles, en le fécondant avec le type à flẹurs simples. 


\section{FIVILLE DES IIYPÉRICINÉES.}

\section{Genre Millepertuis. - Hypericum.}

Les Millepertuis ont de nombreuses étamines réunies par les lilets en plusieurs faisceaux qui entourent trois à cinq styles distincts, surmontés par des stigmates papillaires.

Les fleur's souvrent le matin dassez bonne heure, et les styles semblent doués de mouvements qui tendent à les rapprocher des étamines. Ce serait done de bonne heure, et presque dans le bouton, qu'il faudrait faire l'ablation des organes mâles, si l'on voulait tenter quelques croisements sur le genre si nombreux des Hypericum.

\section{FAMILLE DES SARMENTACEES.}

\section{Genre Vigne. - Vitis.}

On ne comprend pas qu'ume plante aussi importante que la Vigne n’ait pas donné lieu plus souvent à des croisements rationnels opérés avec soin sur ses nombreuses variétés. Il en serait résulté, sans aucun doute, des races nouvelles, qui bientôt se seraient répandues par la culture, et auraient présenté chacune des qualités ou des avantages particuliers. Ces variétés nouvelles doivent rarement se produire naturellement, car le stigmate, garni d'ume lumeur gluante, ne peut manquer de recevoir le pollen de quatre étamines serrées au-dessus de lui par quatre pétales capuchonnés et soudés par la pointe. Quand ces pétales tombent, les filets s'étendent, les anthères s'ouvrent et l'imprégnation a lieu. Cette structure indique tout de suite la maniere d'opérer pour pratiquer des fécondations artificielles. C'est de couper, dis le commencement de la floraison, avant que le pollen se répande, les authères et les pétales, tout à la fois, ee qui est tres-ficile, avee de petits ciseanx. Cette opé- 
ration faite pendant quelques jours sur un certain nombre de fleurs, on coupe le reste de la grappe, ainsi que celles qui naîtraient encore sur le cep, à moins qu'on ne les opère de la même manière. Il est essentiel d'isoler les Vignes sur lesquelles on reut opérer, car le pollen de ces plantes est fin et léger, et le vent peut le transmettre dioïquement à de grandes distances.

Les diverses variètés de raisins noirs, blancs, roses ou rouges, s'hybrideraient facilement, et de nouveaux chasselas, ou des variétés plus précoces, plus sucrées ou à grains plus volumincux, ne tarderaient pas à paraitre comme admirable résultat des $\mathrm{cx}-$ périences qui seraient tentées.

Depuis que nous avons écrit ces lignes, de nombreuses tentatives d'hybbridation ont été faites sur la Vigne, et plusieurs d'entre elles ont été couronnées de succès. Plusıeurs de ces essais ont été faits aux États-Unis.

Les détails des expériences faites par M. Fisk Allen sont contenus dans une lettre adressée par lui à la Société d'horticulture de l'État de Massachusetts, et publiée dans l'avant-dernier numéro du journal de New-York, the Working Former. Le journaliste fait remarquer que M. Allen est une excellente autorité pour tout ce qui se rapporte à la culture de la Vigne.

L'an dernier, M. Allen a commencé à obtenir les fruits de plusieurs Vignes hybrides, parmi lesquelles quelques-unes lui ont donné des raisins de très-bon goût. Plusieurs de ces gains ont figuré à différentes Expositions des États-Unis. Dès 1848, ¡l avait planté un pied d'Isabelle dans une serre, dans le but de le féconder et avec l'espoir d'en obtenir une variété qui mûrît de bonne heure et qui fût, dès lors, plus avantageuse que les variétés déjà cultivées en grand dans le pays. Afin d'éloigner, dit-il, toute incertitude relativement à l'origine des graines, il a procédé de la manière suivante : le pied de Vigne sur lequel il voulait agir se trouvait seul dans une serre dont la plus grande partie était occupée par des Pêchers. Dans le but d'enpêcher que les aheilles ou des causes extérieures quelconques ne produisissent une fécondation hybride et ne vinssent ainsi 
contrarier l'expérience, II. Allen força sa Vigne au mois de janvier, de manière à la faire fleurir avant que les Vignes en plein air fussent entrées en végétation. Lorsque les jeunes grappes approchèrent du moment de l'épanouissement, il choisit les mieux fournies, et il supprima les autres, du moins celles jui se trouvaient dans leur voisinage. Il éclaircit encore les grappes conservées, n' laissant environ que le quart des boutons, choisis parmi les plus vigoureux et les nieux placés. Lor'sque les fleurs commencèrent à s'épanouir, il les surveilla avec la plus graude attention, et il enleva les anthères dès qu'il put les saisir avec de petits ciseaux pointus. Cela fait, il appliqua sur le stigmate, avec un pinceau très-doux, le pollen de quelques variétés européennes. Ce pollen avait été pris sur des lignes forcées dans une serre, et il arait été mélangé dans une boite; il appartenait aux variétés Chasselas, Prince noir, IIambourg noir. Lorsque la fécondation avait lieu, l'ovaire grossissait très-promptement; dans le cas contraire, il restait dans le même état qu' aupararant. "J'étais ainsi assuré, dit M. Allen, que toutes les graines obtenues produiraient des Vignes hybrides. A la maturité des fruits, les graines furent soigneusement recueillies et ensuite semées dans une terre où l'on était certain quil n'y avait pas déjà d'autres graines de Vignes semées accidentellement. Les jeunes plants obtenus de ces graines ont été soignés constamment par M. Allen lui-mème. Il les a lui-même dépotés et rempotés, et ensuite plantés en pleine torre. Celles de ces Vignes qui ont deja fructifie ont donné toutes du raisin noir, une seule exceptée; leur fruit s'est montré d'une précocité remarquahle, beau et très-sucré. Ces - jeunes plantes ont été exposées à l'action de l'hirer dès qu'elles ont eu pris quelque force. Le's plus délicates ont péri, et il en est resti une vingtaine qui sont dès lors assez rustiques pour résister anx hivers de New-York, aree un. simple paillis léger sur leurs racines. Cette nouvelle ligne a cté nommée Hybride d'Allen. On ne sela pas encore issez certain de son rieirite avant deux ou trois ans pour pouroir l'introduire avec toute 
assurance dans la culture en pleine terre. ") (Soc, imp. et cent. d'hortic., t. I, p. 252.)

De magnifiques produits d'hybridations ont été décrits et figurés dans la Flore des serres et des jardins, éditée par Van Houtte.

Un de ces produits a reçu le nom de Raisin doré de Stocliwood. On le donne comme un produit métis né du raisin noir de Hambourg, fécondé par le pollen du chasselas de Hollande. C'est M. Busby, jardinier à Stockwood, qui a obtenu cette magnifique variété à grains énormes et dorés.

Une autre superbe variété de raisin noir qui a paru aussi dans le même recueil, le muscat hamburghs, est aussi sans doute un hybride.

Comme il faut souvent attendre longtemps la mise à fiuit d'une vigne de semis, on peet la greffer sur un pied vigoureux et abréger ainsi de beaucoup les amées d'altente.

\section{FAMILLE DLS GERANIACEES.}

\section{Genre Pelargonium. - Pelargonium.}

Les Pelaryonium, comme les Camellia, ont été croisés de Loules les manières, et à tel point, qu'il est presque impossible maintenant de reconnaitre les véritables types qui ont donné naissance à de si nombreuses variétés. Ce genre a été entièrement changé par la culture, et il n'en est aucun dans le règue végétal qui présente des couleurs plus éclatantes et une plus belle inflorescence. Toutes les espèces de Pelaryonium ne s'hybrident pas entre elles; et l'essentiel, en croisant ceux qui admettent les fécondations artificielles, c'est de bien choisir ses couleurs, afin d'obtenir quelque chose de nouveau en éclat, en grandeur, en coloris:

Dans la plupart des Pelaryonium, l'hybridation est très-facile par le développement inégal des anthères et des stigmates. Ces derniers sont plus tardifs, et les anthères de chaque fleur 
ont ordinairement fini de répandre leur pollen quand les stigmates s'écartent et acquièrent leur aptitude. La fécondation

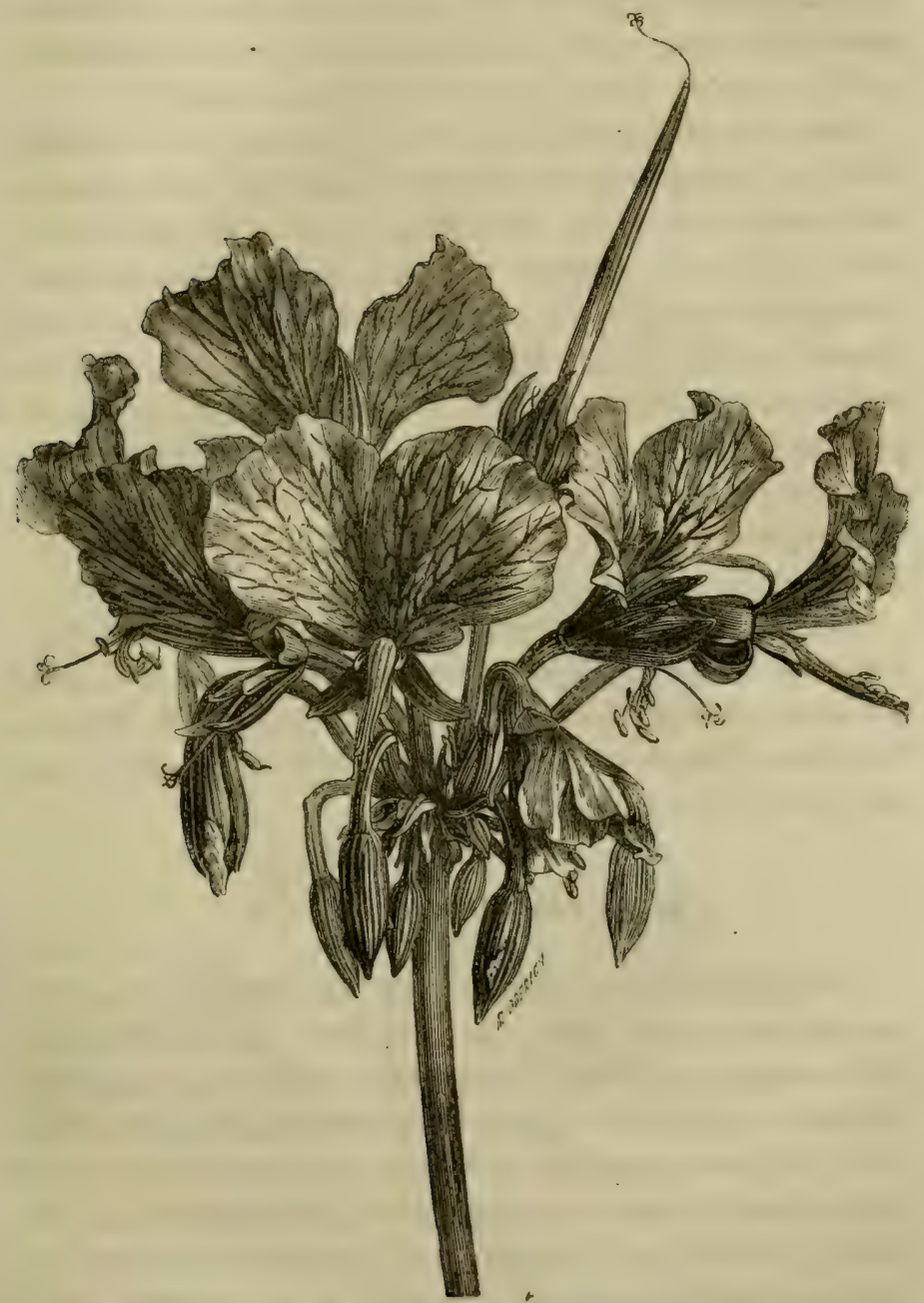

Grav. 40. - Pelargonium Endlicherianum.

naturelle chant indirecte, l'hỵbridation est facile, puisqu'il sulfit de recueillir le pollen au pinceau et de l'appliquer sur les stig- 
mates. Je me suis bien trouvé de mouiller très-légèrement ces or'ganes, avant l'application du pollen, avec un peu d'eau miellée, qui facilite l'explosion des grains polliniques. La fécondation des Geranium, opérée avec réciprocité sur une douzaine de variétés choisies, peut donner d'admirables résultats.

Cette fécondation doit se faire en serre bien claire et bien aérée, et toujours dans la matinée. On ne doit l'opérer que sur des variétés nourelles et très-belles. Si on ne possède pas ces variétés, il faut les acquérir; et tous les ans des gains nouveaus viennent surpasser les anciens (grav, 40). Ce genre a été tellement travaillé, et par des hommes si habiles, que si on ne prend pas leurs derniers et plus beaux succès pour point de départ, on restera toujours en arrière. Alors on perd son temps, et il vaut mieux l'employer à hybrider des geures moins perfectionnés, et dont on a déjà à sa disposition les plus lielles variétés.

La section des Zonule, cette éclatante parure des massifs, a beaucoup encore à gagner, et pourtant combien de variétés méritantes obtenues même celte année encore par MM. Babouil-. lard, Dufoy, Delpaute, Lemoine, Van Houlte, Rendlater, et par les horticulteurs anglais!

\section{Genre Capueine. - Tropæolum.}

Les corolles les plus bizarres et les plus éclatantes ornent les espèces de ce beau genre, dont les fleurs ont huit étamines, trois ovaires, un style et un stigmate trifide. Je ne sais si les différentes espèces lı Capucines pourraient s'hybrider entre elles; elles se ressemblent assez pour qu'on puisse l'espérer, et l'on obtiendrait ainsi des plantes bien curieuses et bien élégantes. Les variétés de couleur de la grande Capucine, que l'on cultive partout, se croisent d'elles-mêmes dans les jardins, où il n'est pas rare de voir des fleurs panachées de jaune et d'orangé, d'orangé et de brun, provenant évidemment des diverses planles plus ou moins colorées que nous possédons maintenant. On 
FAMILLE DES GÉRANIACÉES.

peut obtenir le croisement entre variétés de la manière la plus assurée, et peut-être entre espèces, en enlevant les huit arthè-

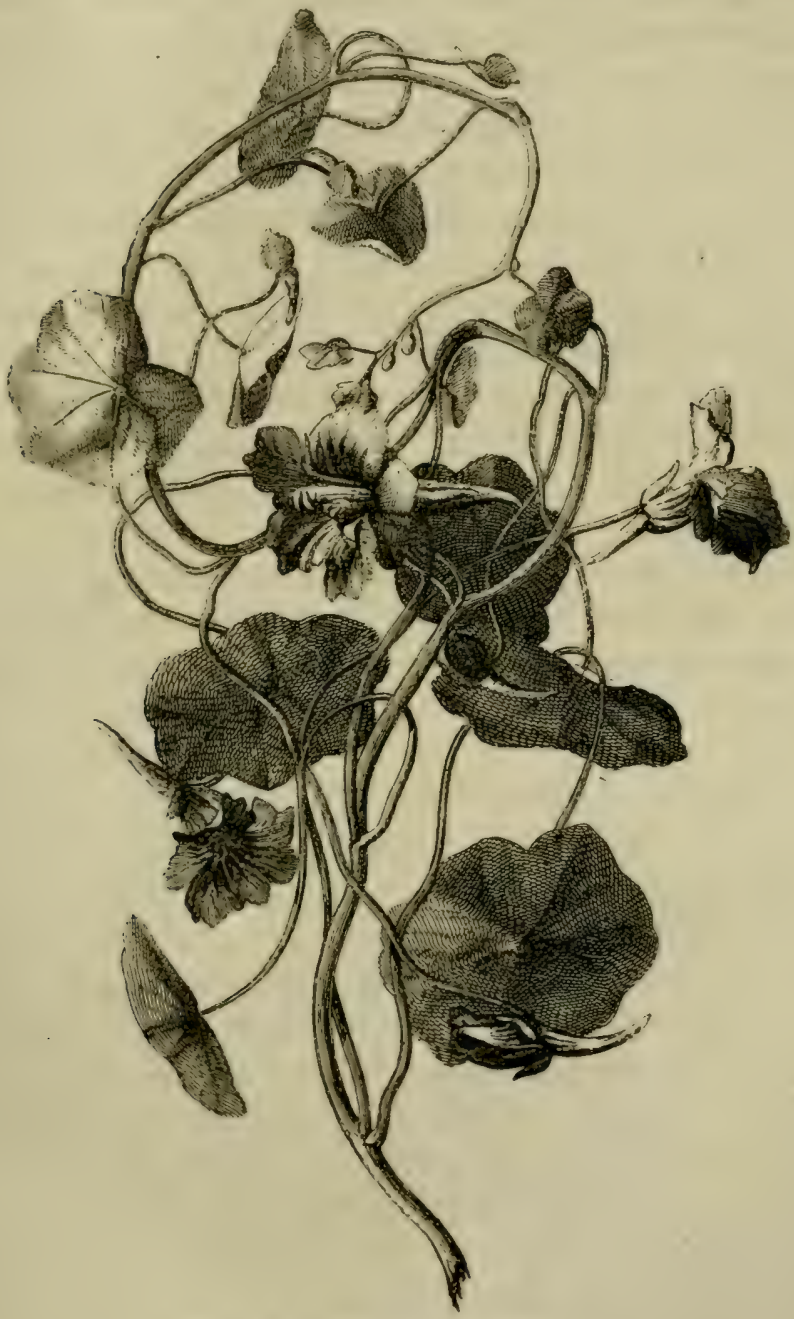

Grav. 41. - Troprolum Lobhianum.

res le jour de l'épranouissement et posant, ce jour mème ou le lendemain, sur les trois stigmates, la poussière fécondante; on 
brise immédiatement l'extrémité de l'éperon, on enlève au pinceau un peu de la liqueur sucrée qu'il reuferme, et on en touche les stigmates; aucune graine ne manque.

Ces belles plantes peuvent donc s'hybrider assez facilement, mais on ne connait pas bien encore les espèces qui se prèteraient le mieux au croisement. Depuis longtemps déjà on a signalé plusieurs hybrides entre le Tropæolum Moritzianum et le T. majus, entre ce dernier et le T. Lobbianum, entre celui-ci et le T. Stockeanum.

Les T. Lobbianum, Stoch. et T. Smithii, D. C. ont domé aussi un grand nombre d'hybrides.

M. Ortgiès indique comme les plus méritantes de ces plantes croisées : Louise Koller, Lilli Smith, massiliense, triomphe du Prado, Zanderi ypandiflorum, Zipseri, Zipseri majus, et Chaixianum.

Nous ne commaissons encore ancun croisement parmi les Capucines de serre, si multipliées et si belles, dont les fleur. bleues, ronges ou tricolores se présentent toujuurs avec gràce et avec originalité (grav. 41).

\section{Genre Oxalide. - Oxalis.}

Un cultive maintenant un assez grand nombre d'Oxalis, parmi lesquels il existe de véritables espèces el des variétés. Dans toutes, il y a dix étamines, dont cinq grandes et cinq plus petites, ainsi que cinq styles qui portent leurs stigmates, tantôt entre les denx systèmes d'étamines, tantòt en dessus, et quelquefois en dessous. Les anthères s'ouvient de bonne heure, et la corolle, en se roulant sur elle-même, opère leur rapprochement avec les stigmates. La fécondation artificielle ne serait donc possible qu'aulant qu'on opérerait le premier jour de la floraison, qui, du reste, a lieu très-facilement quand on expose la plante au soleil (grav. 42).

\section{Genre Balsamine. - Balsamina.}

Les Balsamines ont cinq anthères portées sur de très-courts 
filets, et qui enveloppent exactement un ovaire ovoïde surmonté de cinq stigmates. La fécondation est done assurée dans ces plantes, et pour la pratiquer artificiellement il fiut beaucoup

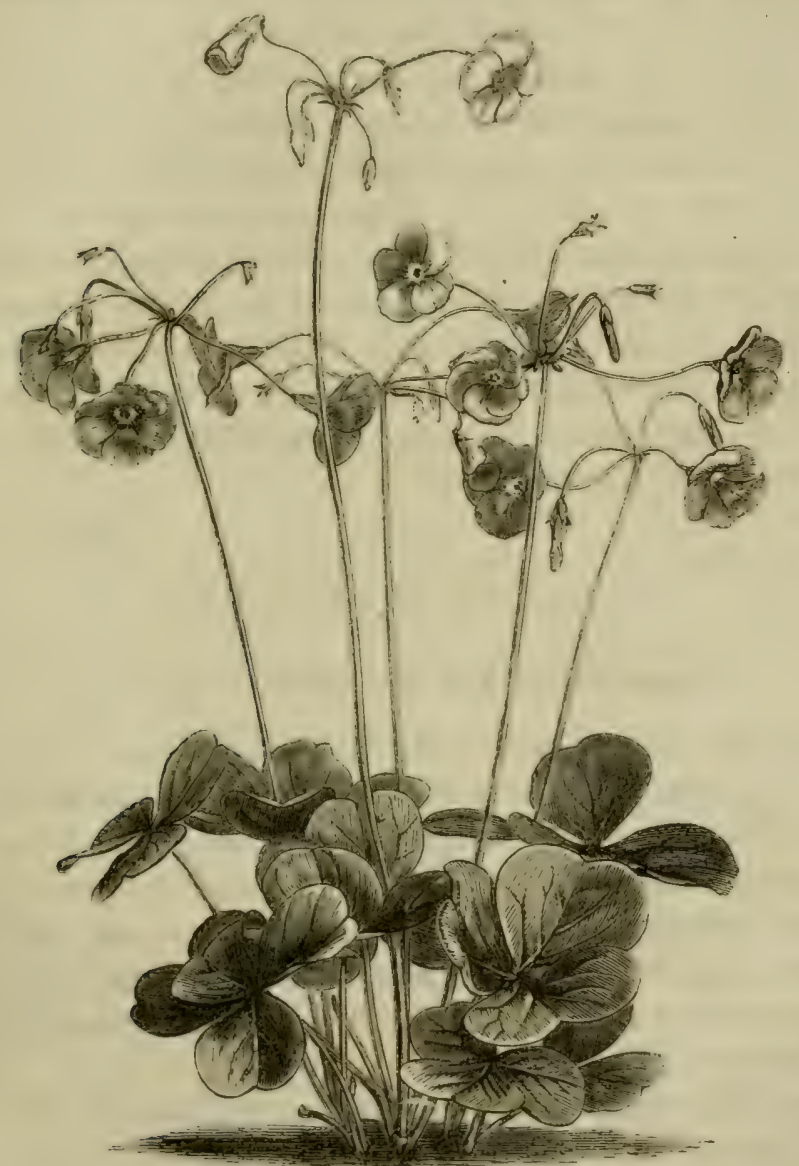

,...?.?.?

frav. 42. - Oxalis Bowici.

d'adresse pour enlerer les étamines dans le bouton, un peu avant l'épanouissement, et ne pas endommager l'ovaire. C'est une peine que l'on ne prend pas habituellement, car les Balsa- 
mines non croisées donnent elles-miêmes d'assez nombreuses variétés, quoique les graines proviennent d'un seul pied dont la couleur, cependant, est toujours dominante. Quelques fleurs, et surtout les dernières des rameaux, manquent d'une tache jaune nectarifère et restent infertiles. $0 \mathrm{n}$ a introduit, dans ces derniers temps, plusieurs espèces nouvelles de Balsamines. Elles sont remarquables par leur belle végétation, mais ne méritent pas les éloges dont quelques journaux d'horticulture se sont plu à les glorifier. Si ces plantes pouvaient acquérir quelque importance ornementale, ce ne serait qu'autant qu'on parviendrait à les hybrider avec la Balsamine des jardins et ses nonbreuses variétés. Cos croisements donneraient certainement, s'ils pouvaient réussir, des plantes nouvelles d'un grand intérêt et d'une grande richesse. Peut-être parviendrait-on à les obtenir avec le ylanduliferi.

\section{FAMILLE DES HESṔERIDEEES.}

\section{Gienres Oranger et Citronnier. - Cilrus.}

La culture et les semis ont déjà produit tant de variétés daus: ce groupe si remarquable du règne végétal, que si l'on ne connaissait l'inépuisable fécondité de la nature, on croirait que l'on ne peut plus rien obtenir de nouveau. 'lelle n'est pas ma conviction. Je crois possible et très-facile de inultiplier et d'améliorer les races d'Oranger's, car je crois que dans le pays où ils fructifient arec une si grande facilité, on ne s'est pas encore occupé de les hybrider.

Dans l'état actuel de nos connaissances, et d'après M. Risso, qui a publié d'importants travaux sur ce genre, on peut ranger sous cinq types les espèces cultivées :

$1^{\circ}$ Le Cédrat ( $C$. medica), à pétioles nus, fruits oblongs, à écorce ridée, épaisse, chair acidule, 5 variétés;

$2^{\circ}$ Bergamotte (C. limettri), à fruits globuleux, couronnés en bouclier, 7 variétés ; 
$5^{\circ}$ Citron (C. limonum), à pétioles peu ailés, à fruits oblongs, écorce mince, suc très-acide, 25 variétés;

$4^{\circ}$ Oranger (C. aurantium), fruit d'un beau jaune, orangé, globuleux ou un peu déprimé, à suc doux, 19 variétés;

$5^{\circ}$ Bigarade (C. vulgaris), à pétioles ailés, fruits tuberculés, ì pulpe amère, 11 variétés.

II est certain que les variétés réunies dans chacune de ces espèces peurent se féconder réciproquement, et peut-être aussi pourrait-on hybrider les espèces elles-mêmes, puisqu' elles ont dẹjà de grandes alfinités. L'isolement d'un rameau est nécessaire, et, pour opérer plus facilement, on peut enlever toutes les lleurs et tous les boutons, sauf celui que l'on veut féconder. C'est dans le bouton même qu'il faut soustraire les anthères. On le presse légèrement entre les doigts, et les pétales se séparent; s'ils résistent ou si les fleurs qui s'ouvrent ainsi ont déji répandu leur pollen, il ne faut pas craindre d'inciser le bouton avec un canif, en prenant toutes les précautions nécessaires pour ne pas endommager le stigmate. On peut même couper entièrement un morceau de la corolle, de manière à introduire une pince pour enlever les anthères. On attend alors l'époque de la tloraison, et, dis le premier jour, on pose le pollen sur le stigmate élargi qui termine le style. Les anthères, au nombre de vingt ì trente, réunies par les filets en plusieurs faisceaux, donment abondamment du pollen au moment de l'épanouissement des fleurs.

On connaît un hỵbride de Citronnier et d'Oranger dont les linits sont sourent orange d'un còté, citron de l'autre.

\section{FAMILLE DES CAMELIEES.}

\section{Genre Camélia. - Camelia.}

šil est un genre sur lequel la fécondation artificielle a été pratiquée avec succis, c'est certainement celui qui nous occupe rol er moment. Les milliers de variétés obfenues sont dues, 
pour la plupart, à ce nouveau perfectionnement de l'horticulture. Il est à regretter que plusieurs jardiniers français ne soient pas encore suffisamment initiés aux mystères de cette opération, qui pent apporter autant de jouissance anx amateurs que de réputation aux horticulteurs marchands.

Dans le Camélia, les étamines sont nombreuses, réunies par paquets, soudées par les filets. Les anthères contiennent beaucoup de pollen, et s'ourrent ordinairement en dehors. L'ovaire est simple et surmonté par deux à cinq stỵles souvent soudés ì leur base, jusqu'à la moitié et même aux deux tiers de leur longueur. Les stigmates qui les terminent sont simples et papillaires.

Rien de plus simple que la fécondation du Camélia : il suffit d'attendre le développement de l'organe femelle, qui a lieu deux à trois jours après l'épanouissement, et d'y poser légèrement le pollen au pinceau. Si l'air de la serre n' est pas chaud et humide, il fandra élever un peu la température, et poser doucement, sur le stigmate couvert de pollen, une petite goutte d'eà miellée avec un pinceau. Pourvu que le pistil soit hien conformé, l'ovaire nouera, et le Camélia donnera des graines, en qui, cependant, lui arrive assez rarement quand on l'abandonni a lui-même. Il est inutile d'enlever les étamines du sujet que l'on féconde; il faut seulement éviter de les toucher avec le pinceau qui porte le pollen d'unc autre espèce; mais, si elles sont trop rapprochées des stigmates, il faut les supprimer. Tous les Camélias ne peuvent pas domner des graines; il en est qui sont trop doubles et dans lesquels le pistil même a disparu; mais on obtiendra de nouvelles races, en fécondant tous les semi-doubles avec le pollen d'autres variétés également semidoubles. Il y a bien moins de chances d'avoir des fleurs pleines, en hybridant les simples par les semi-doubles, quoique cependant cela arrive quelquefois. Fn employant les procédés faciles que j’indique, j’ai toujours réussi dans la fécondation des Camnlias.

Reste done à choisir les plantes que l'on vent allier. Less pro- 
duits ne participent pas toujours des qualités de leurs parents; mais cela arrive souvent, et l'on aura toujours avantage à choisir les fleurs les mieux laites, les couleurs les plus pures, les arbustes les plus prolifères, ceux dont la floraison s'effectue le plus: facilsment et cenx dont la culture présente le moins de difficulté.

Les suivants paraissent, jusqu'à présent, les meilleur's portegraines: C. carnea, Donclileari, Weimarii, waratah, marmorata, king, press-eclips, lady Henriette, nobilissima, picturata, rariegata, striata, punctuta, Cliviana, Camphelli, conspicurt de Loddliges; ce sont du moins ceux que M. Haquin, de Liége, emploie pour ses croisements. Le C. althexfora est ordinairement muni d'étamines el de pistil ; le reticulatı a produit aussi des fruits très-volumincux.

11. Coquet a fécondé le $C$. expansa par le vulra variegatu, cl a obtenu de très-belles variétés, dont une porte son nom.

Les premiers voyageurs qui virent au Japon des Camélias rapportèrent que dans cette merveilleuse contrée on royait des Roserers grands comme des Chènes of dont le feuillage restait vert et luisant comme celui des Lauriers. Bien entendu que l'on l'en crut rien jusqu'à l'époque où le jésuite Kamel, missionnaire au Japon, en rapporta deux pieds en 4759 ; ils étaient rouges et simples. Ces Camélias, placés en serre chaude, périrent ì foree de soins. Un petit nombre de variétés furent introduites dirertement du Japon et bientôt les hybridations commencèrent, hors de France, et les rariétés de semis se multiplièrent à l'infini.

Nous ignorons de quelles variétés sortent les nouveaux Camélias de notre époque. Rien de mieux fait ni de plus heau que les: C. Pozzi, vera Leopold, bicolcre de la reine. inmtesse de Derby, tricolor angela cocchi, etc., etc.

\section{FAMILLE DES RUTACEES.}

\section{Genre Diosma. - Diosma.}

Un trouve dans les serres de nombreuses espèces de Diosmu, qui ressemblient it de petites liruyères et qui fleurissent pendanl 
l'hiver. Leurs fleurs, petites, souvent rapprochées en tète au sommet des rameaux, présentent cinq étamines et un pistil bien conformé. Le pollen reste longtemps adhérent aux anthères sans toucher le stigmate, en sorte que les Diosmas sont souvent stériles. Fécondés artificiellement, ils donnent assez facilement des graines. On peut aussi essayer de les croiser, et il est même probable que plusieurs de ceux que nous cultivons ne sont autre chose que des hybrides.

\section{Genre Boronic. - Boroniı.}

Il faut espérer que ces jolis arbrisseaux se multiplieront dans nos serres, comme les Pimelea, et que l’on parviendra aussi à les hybrider, ou au moins à féconder artificiellement leurs fleurs. Leurs étamines, ạu nombre de huit ou de quatre, ré-

- pandent un pollen assez abondant qui reste souvent éloigné des quatre stigmates, et qu'il serait facile d'en rapprocher aı pinceau.

\section{Genre Correa. - Correa.}

Depuis quelque temps les Corren ont attiré l'attention des horticulteurs, et de nombreux hybrides ont apparu, laissant loin derrière eux les anciennes espèces qui leur ont servi de type.

L'hybridation n'est pas difficile dans ce genre, où les huit étamines peuvent être enlevées très-facilement, dès l'ouverture de la corolle, et où le pollen peut être posé au pinceau sur un stigmate quadrifide. C'est donc, comme dans le Boronia, wne fécondation simple à opérer, et aussi facile que celle des $F_{11-}$ chsia, que les Correa semblent remplacer en hiver. 


\section{CHAPITRE V}

\section{DICOTYLEDONES CALICIFLORES}

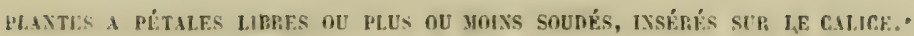

\section{FAMILE DES RIIAMNEES.}

\section{Gienre Fusain. - Enonymus.}

Cos arbressont cultivés pour la beauté de leurs fruits, ot leurs expeces, assez voisines, pourraient peut-être s'hybrider. Léjà on a obtenu des variétés dans la couleur du fruit de l'enropæus. Les Fusains ont quatre étamines, et le stigmate n'est pas apte quand celles-ci répandent leur pollen, en sorte que la fécondation est probablement indirecte. I.es croisements seraient done tris-faciles à opérer; on pourrait mème séggliger l'ablation des anthères. Les lutifolius, verrucosus, et peut-êtr. même les espèces américaines, telles que l'obovalus atl'americamus, pourraient sans doute s'hybrider avec l'europarus et produire des plantes i fruits éclatants de couleur.

\section{Genre Moux. - Ilex.}

Les Houx, presque tous diö̈ques, pourraient probablement s'hybrider. Le Houx ordinaire lemelle graine facilement et les autres especes, qui se ressemblent assez, pourraient, quand elles 
fleurissent dans nos climals, donner le pollen pour le croisement. Un Houx femelle, cultivé seul dans un jardin, éloigné d'individus mâles, et par conséquent infertile, serait précieux pour de telles hybridations, qui donneraient sans donte des produits très-remarquables.

Les croisements seraient d'aulant plus faciles dans les Houx que le pollen visqueux ne peut être transporté que par les insectes. Ces derniers sont largement récompensés de leurs messages par te miel que les fleurs des deux sexes sécrètent en abondance.

\section{Genre Jujubier. - Zizyphus.}

Les fleurs du Jujubier ordinaire sont polygames, et l'on pourrait alors très-facilement essayer l'hybridation avee d'autres espèces, et notamment avec celles dont les fruits comestibles surpessent en qualité nos jujubes ordinaires. Il faudrait alors choisir les fleurs femelles et retrancher toutes les autres, opération d'autant plus nécessaire que, dans l'état ordinaire du Jujubier, - il ne conserve que très-peu de fruits sur chaque grappe. La difficulté est de se procurer le pollen des autres espèces qui sont peu cultivies dans les jardins; mais on pourrait l'obtenir par correspondance, car le pollen des plantes unisexuées peut se conserver longtemps et parvenir très-loin sans altération.

\section{Genre Phylique. - Phylica.}

Nos serres contiennent ẹ abondance ces jolis petits arbustes qui attendent l'hiver pour fleurir et qui ressemblent à des Bruyères. Leurs fleurs sont petites et munies d'étamines presque dépourvues de filets, mais très-rapprochées de deux ou trois sligmates, qu'elles fécondent quelques jours après l'épanouissement Elles restent ouvertes ou plutôt entr'ouvertes assez longtemps. Je ne sais si on obtiendrait mieux que re que l'on a, en fécondant ces plantes artificiellement et en rroisant les espèces. Il est probable que l'hy̧bridation aurait lieu, puisque ees plan. tes sont voisines, peut-être même déjà hybridées en partir ou 
susceptibles de l'être par celles qui appartiennent à la même section, car le genre présente deux tỵpes assez distincts.

\section{PAMULE DES WEGUINEUSES.}

\section{Genre Choryzème. - Choryzemu.}

Ihans ce gente, conmm dans ceux qui en sont voisins, tels que le Podolobium, l'Eutaxiu, le Brachysema, le Daviesia, el qui appartiemnent tous à l'océanie, la fécondation s'opère naturellement par la position des dix étamines qui entourent le stigmate. Ces organes sont enfermés ensemble dans le pétalr inférieur plié sur lui-mème et qui a recu le nom de carène. Cette carine s'ourre presque toujours d'elle-mème à l'époque. de la fécondation, mais alors l'anthèse a eu lien. Si done on voulait essayer de croiser les espices connues de Choryzemu, qui lleurissent facilement dans nos serres, il faudrait ourrir la "arène et enlever les anthères; il suffirait ensuite de poser le pollen au pinceau et d'humecter, au bosnin, légìrement t: stigmate aree un pen d'eau miellée.

\section{Genre Hovéc. - Hovell.}

Daus ce genre, comme dans le Platylobium, les anthères s'ourrent de bonne heure, mais ì cette époque la carène érarte ses deux pétales et la férondation s'opère à l'air libre. On pent done, sans difficultés, féconder ces plantes pour les faire grener dans les serres ou tenter do les hybrider par des croisement:.

\section{Genre Cytise. - Cytisus.}

Dans res jolis arbrisstaux, lat fécondation s'opère daus l lintirieur do la carène et avant l'épanonissement complet de la fleur, an sorte que si l'on voulait rsayer des crnisements, il faudsait rnlever les antheres dans la earène lorsque le houton rommener it s'ourrir. Plusients espères sunt tellement wisines, que l'hy- 
bridation aurait certainement lieu; tels sont les $C$. canescens, albicans, capitatus, elongatus, supinus, hirsutus, etc. Le C. laburmum s'hybriderait aussi avec ses diverses variétés et peutêtre avec quelques espèces voisines; mais il est douteux qu'il puisse en résulter un arbre plus élégant que celui qui décore au printemps nos jardins et les parties inférieures des montagnes alpines.

Le $C$. Adami est considéré comme un hybride des $C$. laburnum et C. purpureus; il arrive même quelquefois que l'hybridation se révèle dans quelques bourgeons par la réapparition de branches isolées appartenant complétement à l'un ou à l'autre des parents.

\section{Genre Luzerne. - Medicago.}

Excepté l'arborea et quelques autres, ces plantes sont ex'lues des jarlins d'ornement, et reléguées dans les champs, oì deux espèces, le lupulina et le sativa, mais cette dernière surtout, constituent un fourrage très-abondant et très-estimé. C'est peut-ètre la plante fourragère la plus utile. Ce ne serait lone que sous le point de vue agricole que l'on pourrait essayer l'hybridation des Luzernes, et encore est-il permis de se demander si l'on obtiendrait mieux que ce que l'on a. On arriverait peut-être à des variétés plus robustes, plus capables de résister au froid dans les pays du Nord, ce qui serait un grand avantage; mais le croisement du sativa ne pourrait guère avoir lieu qu'avec le falcata, et ce mélange s'opère quelquefois natırellement par le pollen du sutiva sur le fulcata; il en résulte des plantes infertiles, en sorte que, cette expérience étant faite naturellement, ce serait l’inverse qu'il faudrait essayer, c'est-à-dire de poser le pollen du falcata sur le sativa, et d'avoir, par conséquent, ce dernier pour porte-graine.

Le lupulina pourrait sans doute s'hybrider avec toute la section des Luzernes à fruits épineux et contournés, mais je ne vois pas quel avaritage agricole on pourrait retirer de tels hybrides. II n’y a pas nécessité de crier de nouvelles plantes, tant qu'ou 
n'aura pas essayé les avantages ou reconnu les inconvénients de celles qui existent depuis la création.

La fécondation artificielle est diffieile dans la Luzerne; les fleurs sont très-petites, le's anthères à peiue yisibles, el, comme dans la plupart des Légumineuses, l'anthèse a lieu un peu avant l'épanouissement; mais il arrive quelquefois qu'à cette éporque le pollen n’a pas encore touché le stigmate, en sorte quion peut espérer de réussir.

\section{Gemre Trène - Trifolium.}

Cie que nous venons de dire des Lusernes peut sapplinger parlaitement aux Trifles; leurs fleurs petites rendent l'operation du croisement tris-difficile, et je ne sais réellement pas ce que l'anriculture aurait i y gagner. Les T' pratense, repens, hybridum et incarnatum, peuvent remplir toutes les indications, occuper la plupart des terrains et constituent d'excellents lourrages.

\section{Genre Clitorie. - L'litoriu.}

On trouve daus ce genre de très-belles espèces, dont plusieur: sont assez voisines pour quon puisse espérer de les croiser. Les lleurs sont grandes, renversies, souvent solitaires, et il est extrèmement facile d'en enlever les étamines et de léconder ensuite le stigmate an pincean. On peut toujours employer ce moyen sur les plantes qui ne doment pas de graines delle'smemes dans nos serres et qui fructifient sourent, quand m opere artificiollement le contact du pollen et du stigmate.

\section{Genre Itohinier. - Robinil.}

Conme lit plupart des légumineuses, les Robinia ont leus: étamines enfermées dans la carene, qui cependant s'ouve légèrement à l'époque de la lécondation. Le pseudo-ucaciu donne abondamment des graines, et c'est sur celui-lit que l'on pourrait essayer d'hybrider les autres en y portant leur pollen 
au pinceau. La fécondation artificielle, pratiquée avec le pollen même de la plante, ferait peut-être nouer quelques fleurs de l'Acacia rose, qui est généralement infertile. On l'a vu cependant fructifier à IIyères, chez II. Farnous, et malheureusement un coup de vent a brisé l'arbre qui portait les graines. Il y at lant de choses à tenter avec l'hybridation, tant d'avenir dans cette opération bien conduite, que l'on ne sait pas où s'arrêteront ses résultats.

\section{Cenre Sainfoin. - Onobrychis.}

Le Sainfoin ordinaire ou Onobrychis sutiva pourrait trèsprobablement être hybridé par le saxatilis, le procumbens, le petræa, l'alba, le conferta, le supina, etc. Mais je ne pense pas qu'il puisse résulter de ces croisements une plante plus utile à l'agriculture que l'espèce ordinaire. Si cependant on voulait tenter l'hybridation, on devrail, comme dans les autres Légumineuses, agir de bonme heure, car la fécondation s'opère dans la carène, au montunt mème de l'épanouissement.

\section{Genre Fève. - faba.}

L'hybridation peut aroir lieu entre les diver'ses variétés de Fèves que l'on cultive comme plantes maraichères ou agricoles. Elle peut s'opérer comme dans les autres genres de Légumineuses, par l'application directe du pollen sur le stigmate, un enlevant de très-bonne heure les anthères. On en connaît déjà de très-bonnes variétés. Les plus cultivées sont les Fève's de maruis, - de Windsor, - julienne, - i longue cosse, - mazagran, - toujours verte, - violette, - naine hattive, - i lleur pourpré ; et toutes pourraient sans doute s'hybrider.

\section{Genre Pois. - pisum.}

Dans ce genre encore, la fécondation s'opère dans l'intérieur de la carène, et cependaut cetle plante a donné un grand nombre de variélés, que l'on pourrait sans doute multiplier encore 
(in employant les procédés opératoires que nous avons dẹjà indiqués plusieurs fois pour les plantes de cette famille. Quand la carène est gènante, on peut, avee des ciseaux, en retrancher ume portion sans nuire au résultat que l'on agttend. Ce n'est guère, du reste, qu'entre variétés que les croisements peuvent se faire, car les autres espèces, peu nombreuses, ne servent ni ì l'alimentation ni à l'ornement.

Voici, d'après II. Courtois-Gérard, la liste des meilleures variétés, puisque ce sont celles que cultivent les maraîchers de Paris, et que nous copions textuellement dans son excellent Traité de la culture marcấchère :

A èeosser. - Le plus hitif, - Michunx de Pueil, - Michanxordinaire, - Michaux à ceil noir, - d'Aurergne, — nain hatif, - domine, - nuin de Hollande, - très-nain de Bretuyne, - gros nain sucré, - nain vert gros, - nain vert petit, - nain impérial, - de Clamart ou carré fin, - de Harly, - sans pareil, - carré blane, - curré vert, - yros vert, - rilé ou knight.

Pois sans parehemin ou mange-tout. - Nain hitif de $\mathrm{Hol}$ lande, - nuin ì la moelle d'Espunne, - à flenr rouge, — crochu à laryes cosses, - très-nuin ou éventail, — ture ou comronué, - ture äflewr ronge, - géant sams purchemin.

\section{Gienre Gesse. - Lathyrus.}

Dauns ce grenre très-nombreux, peu d'espèces sont cultivées dans les jardins: la principale est le $L$. odoratus, Gesse odorante un Pois de senteur, dont on connait maintenant six à sept variétés distinctes, et dans laquelle les fécondations artificielles en leront probablement décourrir encore de nouvelles. Les étamines, enfermées dans la carène, répandent leur pollen avant l'épanouissement; mais ce n'est ordinairement que pendant la floraison accomplie que la fécondation s'opère. Il est donc assez. facile d'imprégner le stigmate d'un pollen étranger. Ce serait la varieté blanche qu'il faudrait choisir pour porte-graine, afin 
d'obtenir des panachures ou des fleurs bleues et blanches qui n'existent pas encore. Quant aux autres espèces de Gesse, il est frobable qu'elles ne s'hybrideraient pas entre elles, et on n'atlrait d'ailleur's aucun intérêt ì l'essayer. Il y a cependani le L. grandiflorus, que l'on devrait tenter de croiser avec notre l'ois de senteur; il est vrai que e'est une plante vivace qui ne supporterait peut-être pas nos hivers; mais, si l'hybridation avec le Pois de senteur n'avait pas lieu, elle réussirait sans doute avec le latifolius, qui a déjà donné une belle variéti blanche.

\section{Genre Orobe. - Urobus.}

Plusieurs espèces de ce gemre sont cultivées dans les jardins, où elles ont jroduit déjà un assez grand nombre de variétés. Cie sont principalement le vermus et le varius qui ont donné celles que nous connaissons, telles que l'asureus, albus, roseus, speciosus, venosus. On cultive encore l'alpinus, le luteus, le purpureus, etc.

Presque toutes ces plantes sont susceptibles de s'hybrider, et, quoique le pollen sorte des étamines avant l'entier épanouissement, la fécondation ne s'achève que plus tard, et l'on peut, en s'y prenant dès l'ouverture de la corolle, praticjuer assez fitcilement la fécondation artificielle. Ces plantes constituent un beau genre très-printanier, qui conmence à ètre très-recherché dans les jardins, et qui vaut bien la peine que l'on s'en occupe.

\section{Genre Kennedie. - Kemedia.}

Cie genre ne renferme que des plantes de la Nouvelle-Hollande, dans lesquelles la fécondation s'accomplit de bonne heure, comme dans la plupatt des légumineuses. Un en cultive déjà un certain nombre d'espèces très-élégantes que l'on pourra sans doute hybrider, et qui méritent bien l'attention que les horticulteurs voudront leur accorder. Le K. Maryattæe est une des plus belles espèces. 
Genre Haricot. - Phaseolus.

Comme dans toutes les plantes cultivées depuis longtemps, il est difficile de retrouver les especes dans les Haricots annuels que nous connaissons dans nos jardins. On y reconnait facilement plusieurs races; on y distingue des types particuliers; mais il n'y a, pour ainsidire, pas de limites ou chaque espèce vienne siarrèter. Il y a donc eu sourent hybridation dans ces plantes, comme on peut s'en convaincre par les formes et les couleurs variées de leurs graines.

In's especes viyaces sont peu répandues dans les collections, ef je n'ai jamais été à mène d'observer leur's fleurs, et, comme l'ilileurs elles domnent difficilement des graines daus nos climats, il y a peu de croisements à espérer entre elles.

II n'en est pas de même des espèces potagères; on en connail, il est wrai, un si grand nombre, et qui répondent si bien à tous. les usages auxquels on les destine, qu’il est pent-ètre inutile de chercher à en augmenter les modifications; mais il est toujou's curieux pour l'amateur ou le physiologiste d'opérer sur des plantes aussi susceptibles d'liybridation que le sont les Ilaricots, ut sur lesquels on peut si promptement s'assurer, par la couleur des graines, du succès de l'opération. Arant l'épanouissenent complet de la fleur, les anthères des llaricots commencent ì répandre leur pollen, et aussitôt la carène se contourne, enIrainant avec elle les filets soudés, qui s'allongent en même temps comme s'ils voulaient atteindre le stigmate emporté par le style et l'ovaire, qui prement aussi de l'accroissement. Toutelois le pistil s'allonge moins que les étanimes, et la fécondation ne tarde pas d'avoir lieu. Il suffit donc, pour l'opérer artiticiellement, d'scarter un peu la carene, et de poser, au pinceau, te pollen sur le stigmate. Ce dernier le retient facilement, et conme le pollen des llaricots n est pas très-pulvérulent, mais souvent un peu piteux, on peut se dispenser d'ôter les étamines, soccupant seulement de féconter le pistil dès que l'état de la lleur permet d'y parvenil. 
Il est à peu près certain que tous les Ilaricots ne se féconderaient pas réciproquement; il faudrait donc choisir au moins les sections telles que les multiflores, les volubiles, les nains, les espèces à graines globuleuses, celles à légumes et scrnences aplatis, etc.

II. Fermond a publié des détails très-intéressants sur le mode re fécondation des Phaseolus. La carène de leurs fleurs est en lorme d'hélice el renferme les organes de la reproduction. Cette carène est ouverte au sommet et dome passage aux stigmates papillaires situés au sommet du style. Le stigmate est lonc extérieur pendant que les étamines sont enfermées, et ce seul fait suffirait pour explicquer les nombreuses variatious des Phuseolus. Conme tous les Ilaricots annuels conmus ne forment probablement qu'une seule espèce, l'hybridation de toutes les variétés doit se produire avec la plus grande facilité, surtout si le pinceau vient aider encore l'action du vent et la bruyante inimation des insectes.

La fécondation directe et naturelle est plus difficile et s'opère par l'écartement des ailes et de l'étendard de la corolle, mouvements qui, après avoir fait sortir le style, le font rentreir et le mettent en contact avec les anthères.

II. Naudin n'admet pas la fécondation croisée dans les llaricots, ni daus la plupart des Légumineuses. Il se fonde sur ce que les anthères sont ouvertes longtemps avant l'épanouissement et sur ce que la carène enveloppe si bien les organes de lat génération, que le vent et les insectes, médiateurs ordiniares des mariages clandestins, sont impuissants devant ces obstacles. Malgré notre confiance dans les observations de M. Naudin, nous ne pouvons nous refuser à voir dans le Haricot d'Espagne, hicolore par sa lleur et par sa graine, un hybride fertile ou plutôt un métis des deux variétés à fleurs blanches et à fleurs écarlates.

Ce n'est pas, comme il le dit, « une simple et légère altération individuelle sans fixité. ) La plupart des Haricots, si variés dans leur forme et dans leurs couleurs, se reproduisent presque exactement. 
Nous ne pouvons admettre non plus, comme M. Naudin, que l'on puisse inroquer, comme causes de rariations ou de modifications dans les plantes cultivées, la nature minéralogique du terrain, le plus ou moins de chaleur', de sécheresse, d'humidité ou de lumiere. Nous pensons au contraire que, sanf le plus ou moins de développement, les modifications de l'individu préexistent dans la graine qui doit le produire.

\section{Genre Lupin. - Lupinus.}

Lhorticulture a pris depuis longtemps possession de ce genre, remarquable par l'abondance de ses fleurs, son bean port et son élégant feuillage.

Il y a dans les Lupins, comme dans les llaricots, des plintes viraces el des espèces anmuelles. Il n'est pas probable qu'elles puissent se féconder réciproquement, mais il est certain qu'elles peuvent se croiser entre elles. Le stigmate est en forme de tète to ordinairement entouré d'une petite couronue de poils, les anthères s'ouvrent de bonne heure, et, chose trìs-remarquable, il y en a cinq qui sont pointues et cinq qui sont arrondies, contenant toutes le mème pollen, mais s'épanouissant successivement, les pointues d'ahord, les rondes ensuite, de sorte que si le stigmate échappe aux premières, il est atteint par les secondes, et la fícondation a double chance pour' s'effectuer. On est loin d'avoir obtenu toutes les variétés que peuvent fournir les Lupins. II faut maintenant chercher a agrandir leurs lleurs, i allonger leurs épis et it obtenir de nouveltes patnichures.

Il fiut enlever les antheres de très-bome heure, et n'opérer que sur deux ou trois fleurs sur le mème épi, dont on coupe tout de suite les boutons supéricurs qui pourraient donner du pollen, et dont on abat ensuite la pointe pour arrèter définitivement la floraison (grav, 45).

\section{Genre Errythrine, - Erylhrinu.}

Dans ces plantes, la carine s'ourre au sommet et laisse aper- 
164 FÉCONDATION NATURELLE ET ARTIFICIELLE.

cevoir les organes, ce qui permet d'opérer la fécondation au

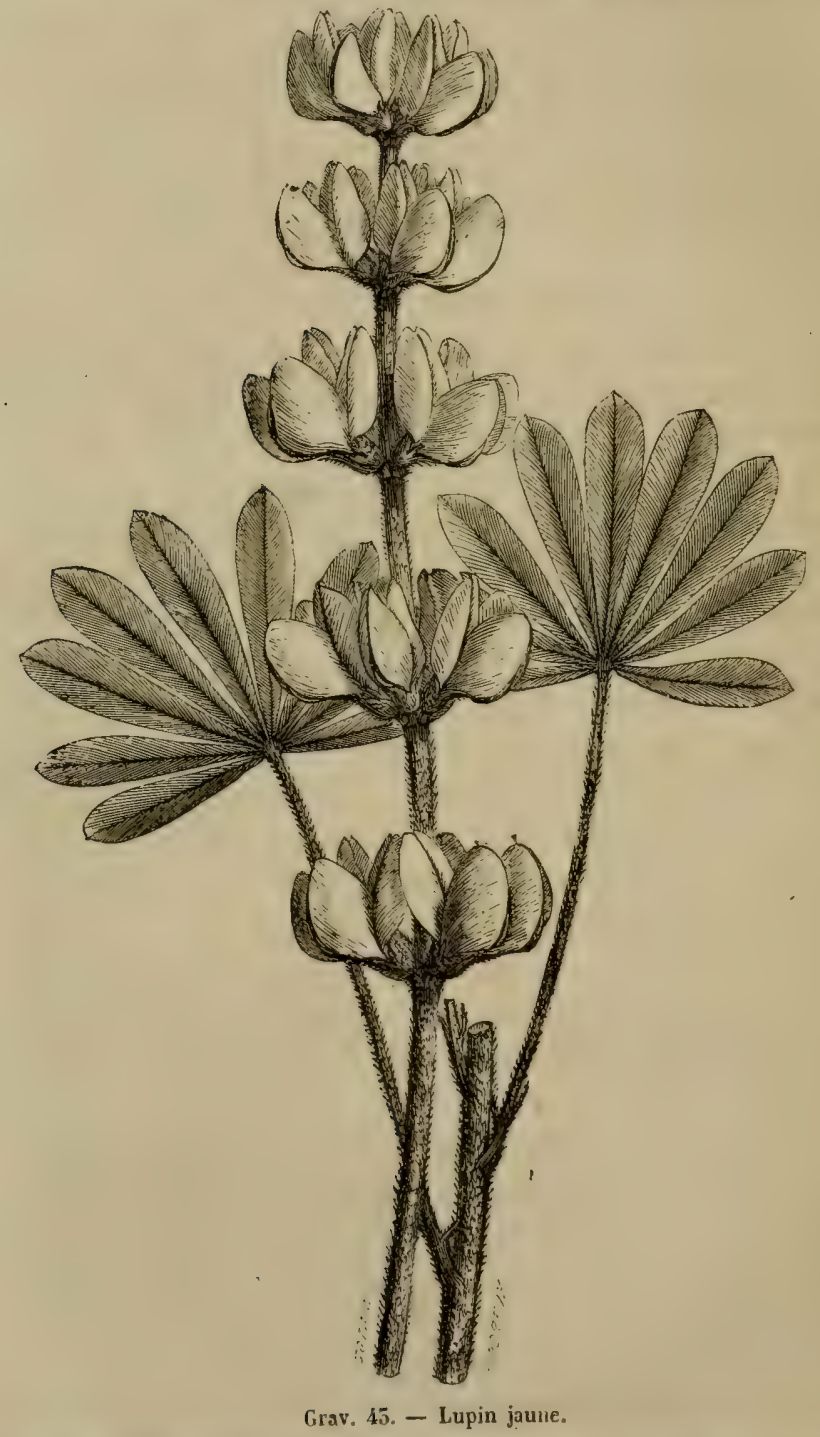

pinceau sans le moindre obstacle. Les $E$. cristagalli, laurifo- 
lia, speciosa, resupinata, peuvent s'hybrider, et l'on peut aussi proliter de la fécondation artificielle pour faire fructifier, dans nos serres et dans nos jardins, ces diverses espèces d'Erythrina qui donnent mème quelquefois des graines naturellement. $0 \mathrm{n}$ est certain de réussir en prenant le pollen sur un autre individu, on le posant au pinceau et appliquant ensuite, sur le stigmate, un peu de la liqueur sucrée pue la fleur sécrète au fond de sa corolle.

On comprend que l'on ait cherehé ì civiliser et ì soumettre à nos caprices un genre aussi remarquable que les Erythrines. II. Duchartre nous donne (Jourmal de la Soc. imp. et cent. d'horticulture, 1. VII, p. S1), le point de départ des variéties nouvelles, qui ne peurent manquer de nous arriver. " M. Bellanger, ancien jardinier à Charonne, a obtenu, dit-il, son point de départ en férondant I'E. cristu galli, L., par l'herbacea, H. L. Le résultat de cette fécondation croisée a été la production de trois hỵlrides, assez peu remarquables par eux-mêmes, mais qui oni pu servir ensuite à la formation de métis beaucoup phus beaux. En outre, M. Bellanger a fécondé ses lyybrides et métis par l'une ou l'autre des deux espèces trpes et réciproquement. Ainsi la plante qu'il a nommé Marie Bellanger, qui semhe ètre le terme le plus brillant de la série, a été obtenue par lui en 185 ă, à la suite d'une fécondation de l'E. crista galli par une de ses rariétés métissées à łaquelle il n'avait pas donní de noin. Elle a été choisie parni quatre-ringt-onze formes plus ou moins variées, ventues des graines qu'avait dommées cette fécondation. "

On connaissait cependant, selon M. Otto, depuis douze it quinze ans, sous le nom de $L$. Bidwillii, une plante élégante rt curieuse gui a en pour mère l'E. herbacen et pour père l'E. crisla galli.

Des espères assez nombreuses de ce beau genre ne peuvent manyuer d'attirer l'attention des horticulteur's et de dommer lien a de nouveaux hỵbrides. 
Genre Casse. - Cassia.

A l'exception du falcata et du marylandica, les Casses fructifient trop rarement dans nos clinals pour qu'on puisse pen. ser à croiser leurs espèces; et si je mentionme ici ce genre si

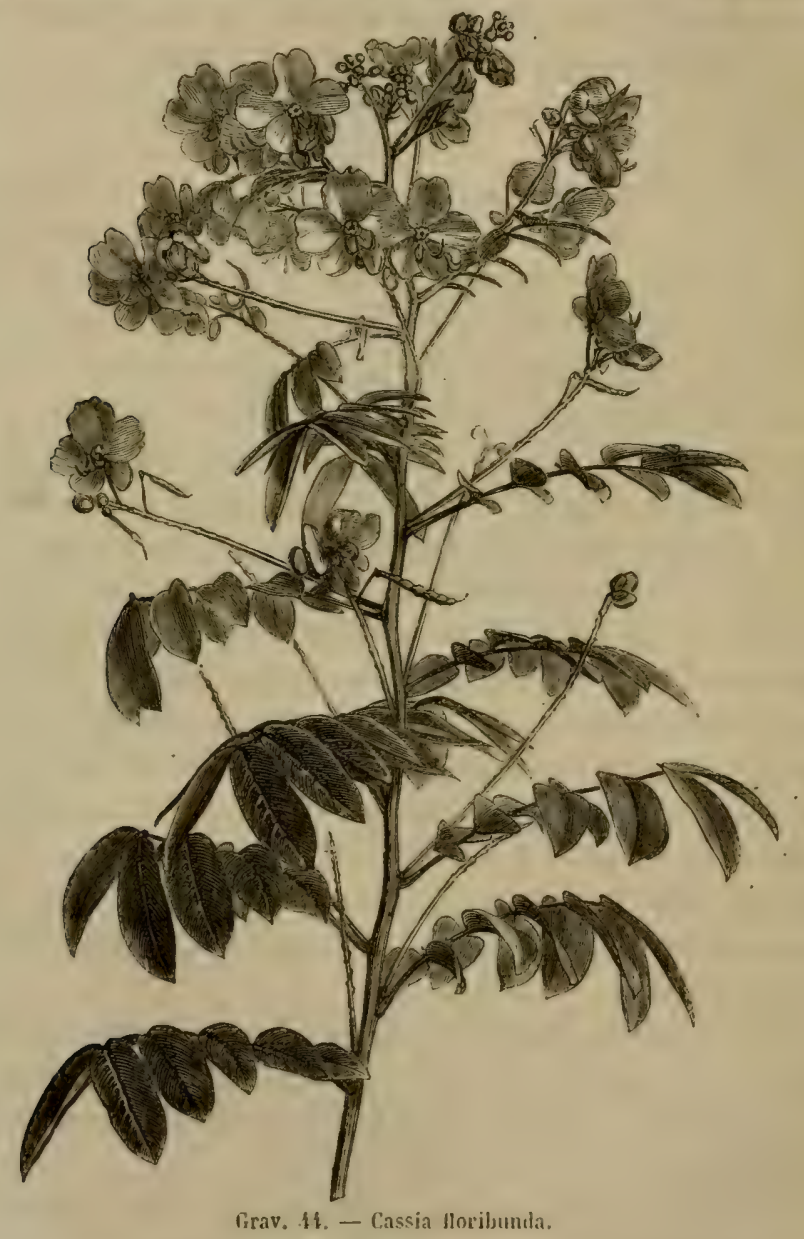

nombreux en belles plantes, e'rst pour rappeler que l'on pourrait probablement obtenir des graines de plusicurs d'entre elles en les fécondant au pinceau (grav. 44). 
Genre Mimose. - Nimosu el Acacir.

Peu de Mimosées fructifient dans nos serres, dont elles sont rependant un des plus beaux ornements. On ne peut done esprirer de faire des hybrides dans nos climats; mais on peut souvent obtenir, par des fécondations artificielles, des graines qui, sans cette opération, ne se seraient pas montrées.

Les étamines des. Mimosa 'sont nombreuses et forment, dans chargue flenr, de petits pinceaux qui produisent abondamment le pollen. Plusieurs fleurs, et ordinairement la plus grandr. partie, sont mâles, quelques-unes hermaphrodites, et le plus: petit nombre femelles. Ce sont surtout ces dernières, plus encore que les hermaphrodites, qu'il faut féconder: elles réussissent plus ordinairement, et l'on peut, par ce moyen, fair porter graine à des Mimosa jusiqualors infertiles. Les Acuriu, les Ilesmanthus, doivent ètre traités de la même manière, puisyu'ils présentent la même organisation.

\section{FAMILE DES ROSACEES.}

Genre Amandier. - Amygdtulus.

Les étamines assez nombreuses de l'Amandier sourrent successivement aussitôt que la corolle est épanouie, et répandent leur pollen sur le seul pistil de la fleur. Il faudrait done, si on voulait hygbrider une variété de cet arbre par une autre, enlever de bonne heure les étanines sur la branche que l'ou aurait isolée, puis couper l'extrémité de cette branche, une fois que l'on aurait préparí deux ou trois fleurs. On connait pru de variétés de cet inlore; la seule hybridation que l'on pourrail Ienter serait peut-être celle the l'Amandier ordinaire, it wros Iruits doux, avee l'Amandier de Tours.

L'Amandier et te Pécher ne sont pas incompatibles; Sageren an a déji obtemu des hybrides. Il serail done possible de les eréer encore. 


\section{Genre Pècher. - Persicn.}

Valgré le grand nombre de variétés que nous avous obtenues du Pêcher, il en reste encore beaucoup à découvrir, et on les devra maintenant aux fécondations artificielles raisonnées. Après avoir isolé un rameau, ou suivra attentivement l'époque de l'épanouissement, et aussitôt avec les brucelles on enlèver:i les étamines pourprées, avant qu'elles aient répandu leur pollen. Alor's on fécondera le stigmate au pinceau avec le pollen de la variété que l'on aura choisie. Les chevréuse hâtive et bononurier, les maydeleines rouge, blanche et grosse violette, la belle de Vitry et la belle de. Paris, la belle Beausse, la grosse mignonne et la violette, lit vinense de Fromentin, sont autant de variétés que l'on peut essayer de croiser, certain. d'obtenir de bons fruits, s'ils ne dégénèrent pas de leurs parents.

Les pavies, dont la chair est adhérente au noyau, sont généralcment moins bounes que les autres pèches, mais elles se conservent plus longtemps, mûrissent plus lard, et possèdent ainsi quelques arantages dont on pourrait tirer bon parti, si l'on parvenait à obtenir dans cette série d'aussi bons fruits que dans les pêches proprement dites.

Non-seulement on pourra essayer de féconder entre elles les variétés du Pêcher, mais il est possible que l'on puisse obtenir des hybrides avec l'Abricotier et le Prunier. Déjà ces plantes admetlent la greffe réciproque, et les tentatives d'hybridation ne seraient probablement pas sans succès.

Jusqu’à présent, la majeure partie des nouvelles variétés de fruits (qui ne valent pas toujours les anciennes) ont été obtenues par hasard; il est temps que l'on arrive à prévoir aı moins le résultat de ses essais.

Dès 1819 Andrew Knight a présenté à la Société d'horticullure de Londres des pêches provenant d'un Amandier à fruils doux fécondé par le Pècher ordinaire, et ces fruits tenaicnt 
heaucoup plus du père que de la mère. La plus grosse de ces pèches avait sept pouces anglais de circonférence. Le fruit etait doux, fondant et très-juteux; mais il avait peu de saveur. Il est rrai quil avait souffert pendant le transport. Ic noyau élait gros, relativenent aux dimensions de la pèche; it était à peu près rond, assez rahoteux, et asait une petite pointe à l'un de ses houts. On remarquait tout autour, à sa surface, une assez grande quantité de cette farine qui on aperçoit sur les amandes fraiches. Le noyau se séparait aisément de la chair; trìs-pen de filaments lui restaient adhérents. IAnnales de chimie et de physique, t. XIII, p. 551.)

On considere généralement les Brugnons comme des hybrides de Pècher et de Prunier, et il se pent qu il en soit ainsi. Des horticulteurs distingués ne voient dans ce fruit qu'une simple variation du Pècher. Ces derniers citent, à l'appui de leur opinion, l'apparition, en plusieurs circonstances, d'une branche de Brugnon sur un Pècher ordinaire. Ce fait est parfaitement constâté, et ne peut laisser aucun doute; mais, d'un autre côté, le Brugnon se reproduit de noyau, en sorte qu'il est bien difficile de tracer ici une ligne de démarcation entre: l'hybridation et la simple variation.

\section{Genre Abricotier. - Armoriaca.}

La fécondation de l'Abricotier s'opire comme celle du l'icher. Par conséquent, les moyens ì employer pour le féconder artificiellement sont absolument les mèmes, ot le résultal doil ètre aussi certain.

Les essais devront être tentés entre les variélés suivantes: A. pêche, A. blane, A. royal, albergier de Tours, anyoumois, A. de Portuyal. I.e croisement aree des variètés précoces, telles que le précoce d'Espérin, produirait peut-être des race's hâtives, convenables aux pays du Nord.

Les diverses variéte's d'Alricots ancmus, nouvellement annoncées, sembleraient aussi très-propres ì domer des hyybrides; 
mais les fruits, plus que les plantes d'agrément encore, ont besoin d'être connus avant d'être admis.

\section{Genre Prunier. - Prunus.}

C'est encore la même fécondation que celle du Pècher, et nous renvoyons non-seulement ì ce genre, mais aux généralités dans lesquelles nous parlons de la fécondation des arbres.

Il y a deux races dont il faut chercher à augmenter les va. riétés dans les prunes, celles de table et celles pour pruneanx. Dans la première se trouvent d'abord toutes les reines-claudes, et surtout la verte; et celle de Bavay, plus grosse, plus tar-

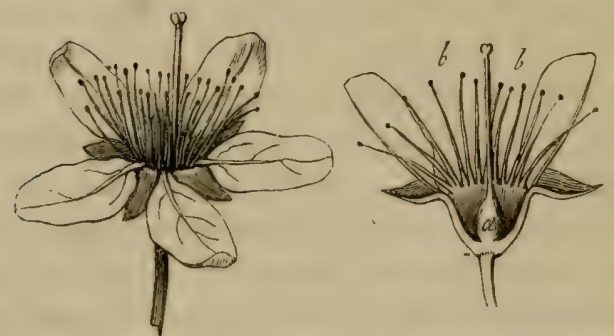

Grav. 45. - Fleur de prunier. - $a$; ovaire. $-b b$, étamines attachées au calice.

dive et récemment découverte par le major Espérin; la P. Washington, celles de Monfort, les mirabelles, la royale de.Tours; et, dans la seconde, la prume d'Ayen, la virginale ì yros fruits, la Suinte-Catherime jumme, la prune altesse, la youtte d'or, etc. En opérant entre ces diverses variétés, on aura certainement chance d'obtenir de honnes et nouvelles races (grav. 45).

Genre Cerisier. - Cerasus.

Ce que nous venons de dire du Prunier s'applique au Cerisier, qui n'est, proprement dit, qu'un démembrement dı même. genre.

II reste, je crois, beaucoup à laire dims celle division des 
arbres fruitiers, les cerises aigres, les guignes el les bigarreaux: forment des types que l'on peut encore améliorer, soit en croisant les especes de elarque type, soit en hybridant les types eux-mèmes entre eux. Sous l'un ou l'autre rapport, nous conseillons d'essay er les croisements entre les varietés suivantes : la royule hutine, la royale tardive, la belle de Chitenay, li belle undigeoise, la belle de Choisy, les Montmorency, le roi de Prusse, la cerise de Spa, la monstruense de Bavay, la tardive du Mans, le gros gobet, les bigarreaux gros couret, d'Espérin, à fruit rose ou d'Espungue, la cerise admiruble de Soissonis.

\section{Genre Spirée. - Spiræa.}

Malgré le grand nombre de Spirées qui sont maintenant cultivies dans les jardins, on ne s'est pas encore suffisamment occupé de ce beau genre, qui renferme des espèces trìs-élégantes et très-rustiques. Il y a certainement déji quelques hybrides dans celles que nous avons, mais on pourrait en augmenter le nombre par des croisements. Ces plantes sont cependant difficiles à hylhrider. I.es fleurs sont si nombreuses, quiil faut élaguer en quantité avant d'opérer, et ensuite le grand nombre des étamines à retrancher, et la prọmptitude avec laquelle les anthères déposent le pollen, sont encore des olstacles. C'est le inatin qu'il faut enlever les anthères, et vers midi qu'il faut poser le pollen en humectant les stigmates avee le nectar des fleurs qui en sont pourvues.

Il n'y a pas bien longtemps que l'on connait des hybrides de Spirées, ces charmants arbrisseaux qui menacent d'envahir nos hosquets et nos jardins. Une des plus jolies especees, le S. cullosu, se fait remarguer par sa tendance à l'hyluridation. Ainsi il y a plus de trois années que M. Ch. Koch a fait connaitre, sous le nom de Sp. Samssoucimun, in hyluride qui avait éti obtenu par hasard à Sans-Sonci, près de Potsdium, de ce Sprirea féeondé aver te pollen du Spr. Douglusii. Plus récem- 
ment, un hybride semblable a été obtenu en Angleterre par M. Noble, de Bagshot, dans le comté de Surrey, et il a reçu de M. W. Hooker, dans le Botanical-Maga:ine (pl. 5169), la dénomination de $S_{p}$. Nobleana. - Un autre hybride entre les deux mêmes espèces a reçu le nom de $S_{p}$. pachystachys, cesta-dire Spirée à grappes serrées, qui lui convient fort bien. Celui-ci est peut-être le plus heau de tous. Il se rapproche beaucoup du Sp. Sanssouciana, mais ses fleurs forment une grappe ombellée, plusieurs fois composée, en pyramide raccourcie, et non unc panicule serrée comme chez celui-ci. Au bout de ses hranches se trouvent cing à huit rameaux presque entièrement sans feuilles, et dont chacun se termine par ume inflorescence, de sorte que toutes ses fleurs réunies forment une masse totale, large souvent de seize à dix-huit centimètres. Quant à ses feuilles, elles ne paraissent pas différer de celles dı Sp. Sanssouciana. Il existe encore dautres hỵbrides dı S. Fortunei, Planc., qui ont été obtenus avec les Sp. expansu, Wall., et latifolia, Borkh. (carpinifolia, Willd.), et qui ont aussi beaucoup d'intérêt. Liun de ces hybrides obtenus avec la dernière espèce est dù à M. Billiard, de Fontenay-aux-Roses, et a reçu de là le nom de $S_{p}$. Billiardi; il est intermédiaire ì ses deux parents; mais, pour le port, il lient surtout du Sp. latifolia. - Sous le nom de Sp.eximia, II. Booth a livré au commerce une plante qui est aussi connue sous le nom de $S p$. californica et qui est très-roisine du $S p$. Billiardi, si mème elle n'est identique avec celui-ci. D'autres formes rappellent, les unes le Sp. Fortunei, les autres le Sp. latifolia. Parmi ces dernières se trouve le $S_{p}$. Bethlehemensis rubra des horticulteurs. Le Sp. Bethlehemensis n'est, d'après M. Ch. Koch, qu'une simple forme du Sp. latifolia, dans laquelle les feuilles ont les dents plus aiguës; on ne sait pas trop bien d'où vient le nom de cette plante, à moins que ce ne soit de Bethléliem, l'une des villes de ce nom qui se trouvent dans l'Amírique du Nord. Ce Sp. Bethlehemensis rubra a tout l'aspect d'un Sp. latifolia, avec ses feuilles rétrécies a coin a lemr 
base; en mème temps il a les fleur's colorées comme dans le Sp. Fortuncei, et le dessous de ses feuilles est grisâtre comme dans cette derniere espece. La forme qui se rapproche le plus du Sp. Forlunei ou callosa, Hort., non Thunb., auquel elle a emprunté lis couleur des fleur's et la l'orme de l'inflorescence, est

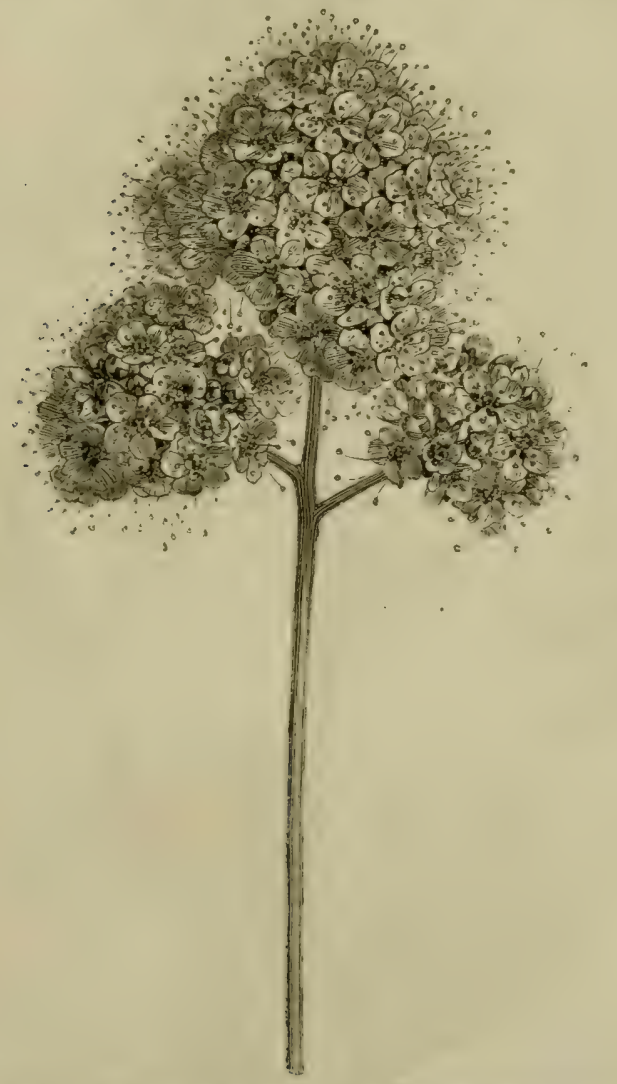

dirav. 16. - Lanicule de fleurs du Spirza Fortunci paniculata de srandeur naturelle.

celle qui parait ètre venue accidentellement dans la pépiniere royale de Berlin, et à laquelle M. Ch. Koch a domé le nom de Sp. calluso-latifolia. C'est également à la mème catégorie qu'appartient un autre hybride nommé Sp. Régeliana. 
174 FÉCONOATION NATURELLE ET ARTIFICIELLL.

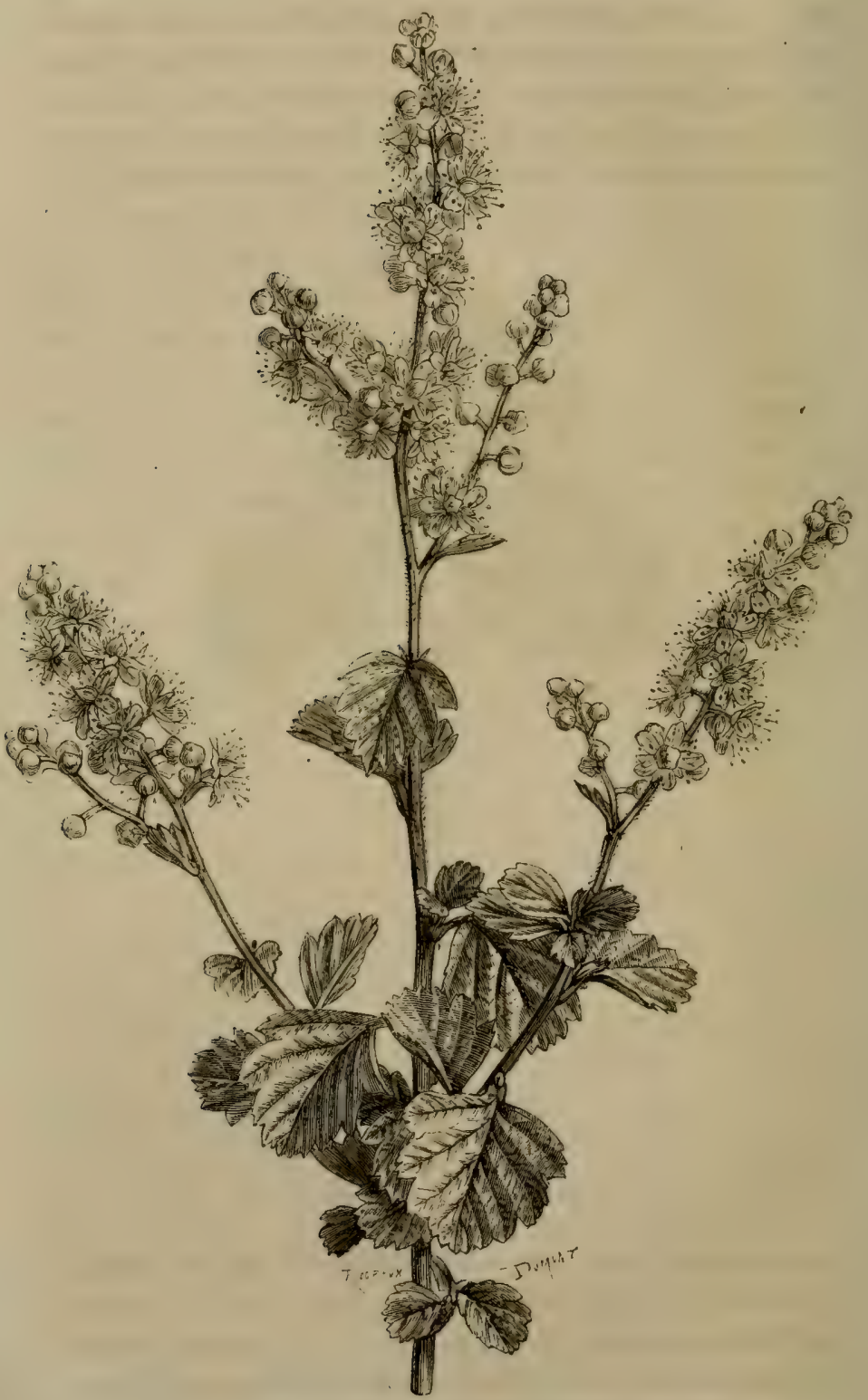

Grav. 47. - Spiræa Boursierii au dixième de grandeur naturelle. 
Une troisième scirie de formes est provenue de l'hybridalion du Sp. Fortunei (cullosu, Ilort.) arec le Sp. expansa, Wall. On (1) cultive surtoul deux, qu'on noume Spiræa expansa rubra et Sp. expansa hyfrida. La première des deux est très-roisine du Sp. eximia; son inflorescence en panicule dense ot non en grappe ombellè composie fitit penser à M. Ch. Koch que e'est plutòt un hybride de l'une de ces deux espèees avec le Sp. latifolia. II a la mène opinion sur l'origine du Sp. expansa hybrida. En terminant, II. Ch. Koch rappelle que lat Revue horticole $(1859,1 \%$. 457) a publié sous deux noms nouveaux deux sprirés conmues depuis longlemps; l'une, nommée dans le journal francais Spiræu speciosu, n'est, dit-il, que le Spr. hypericifolia, L., tandis que l'antre, appelée Sp. flagelliformis, n'est pas autre chose que le Sp. conescens, Don. (cumeifolia, Wall.). Jouinal de la Soc. imp. et centr. d'hortic., t. VII, p. 284. (Grav. 46 et 47. )

\section{Genre Benoite. - Geum.}

Les belles lleurs des Geum leur ont domé entrée dinns nos jardins, où depuis quelques années elles occupent me place distinguée. Leurs étamines nombrenses s'ouvrent de home heure, mais successivement, et les pistils, plus nombreux encore, ne se développent que les uns après les autres et de lit circonférence au centre, ce qui rend la fécondation croisée trèsl'ucile, car, en supposant que l'on enlève les anthères un peu trop' lard, les premiers pistils seulement, e'est-it-dire ceux de la rilronférence, sont fécondés, ch les antres sont encore aptes, a mesme qu ils se développent, à receroir le pollen. Aussi, dins ces plantes, comne dins celles à pistils nombreux, il est bon de poser, à plusieurs reprises et à des époques différentes et disLimles de douze henes environ, le pollen de la plante qui doit servir ì hybrider. Nous citerons, comme croisements à essayer, les espèces suivantes : G. coccineum, grandiflorum, album, splendens, sanguineum, et les G. montanum et triflorum, donl on a fait le genre Sieversia. 
Genre Ronee. - Rubus.

lie genre fournit, a la fois, des arbres fruitiers et des plantes d'ornement. Les fleurs, dans l'un et l'autre cas, 'sont confol'mées à peu près comme celles des Geum. Les étamines égalenent nombreuses entourent des pistils multiples, disposés en séries étagées et dont les plus inférieurs sont aptes les preniers. Il suffit donc d'enlerer les étanines le plus tôt possible et de féronder ensuite atu pincean. Si l'on craignait que les premiers pistils eussent déjà reçu le pollen, on se contenterait, pour les graines, de recueillir celles de la partie supérieure des fruits.

Les Framboisiers, quoique ayant déji fourni de très-belles variétés, peuvent, selon toute apparence, domer encore d'exectlents lybrides. On peut les tenter entre toutes les variétés con!nues, mais principalement entre celui des Alpes on des qualre saisons et le bifêre, les Framboisiers de Hollande, à fruits allongés ou arrondis, à fruits jannes, noirs, couleur de chair, entre les Framboisiers communs et celui du Chili, ou avec une ispèce nouvellement découverte en Angleterre, et désignée sous Ic nom de yonell's fastolff raspberry.

Les Rubus odoratus, qui forment un groupe séparé, s'hỵbrideraient entre eux, mais, quelle que soit la couleur de leurs fleurs, c'est par le leuillage principalement yue ces plantes se lont remarquer, et l'hy̧bridation ne le changerait pas, à moins: qu'on ne puisse les croiser avec les Ronces, ce qui n'est pas pro.bable.

Certaines variétís du Rubus fruticosus et du R. Cæasius donnent des fruits que l'on peut manger, quoique saurages, et si ces plantes étaient cultivées avec soin, on arriverait sans doute à les transformer, soit par hybridation, soit autrement, en véritables arbrisseaux à fruits, qui seraient même préférables au Mûrier noir.

C'est surtout comme plantes d'ornement que l'on cultive les Ronces; plusieurs variétés à fleurs doubles blanches, roses ou carminées, sont réellement très-belles, et comme elles ont 
„énéralement les pistils bien conformés, on en obtiendrait facilement des fruits, soit en les croisant avee le cæsins, le spectubilis, les Ronces laciniées, de. Ces plantes valent la prine que l'on s'en occupe.

\section{Genre Fraisier. - Fraguriu.}

C'est depuis quelques années seulement que l'on s'est occupé sérieusement du semis et de l'hybridation des Fraisier's, et il liut convenir que si on u'a pas encore dépassé, pour la saveur et le parfum, la Fraise que bieu fait natre dans nos hois et nos montagnes, on a du moins acguis d'admirables variétés de forme, de volume, de coloris et mème de saveur.

Comme dans la plupart des liosacies, les étamines sont nombreuses dans les Fraisiers, et elles forment une couronne autour des pistils; les mes sont situées devant les pétales, et d'autres, qui sourrent toujours les prenieres, occupent les intervilles. I.es pistils sont nombreux, situés sur un réceptacle qui, plus tard, devient charnu et succulent, qui s’allonge après la fécondation, et éloigne ainsi les pistils supérieurs des étamines.

La fécondation artificielle est, comme on le roit, très-facile à lirire, d'autant plus que quelques Fraisiers sont diörques. Les insectes sont les médiateurs le julus généralement employés daus leur fécondation. Le pinceau peut les remplacer, ce que faisait déjà le P. Duchesne quand il s'oceupait de la monographie des Fraisiers. La fécondation peut s'opérer entre Fraisiers de la même section, et même entre types différents, car les liraisiers ont été divisés en six classes qui finiront par se confondre à cause des croisements.

Dans la première se trourcut les Fraisiers communs, tels que ceux des bois, de Montrenil, des Alpes ou des quatre suisons, de yaillon et le fraisier-buisson, dont plusieurs ont des variétés à fruits blancs.

Dans la seconde, les étoilés ou ceanuelins, viennent se ranger l'hétérophylle, la cineuse de Champagne, le bargemont et le parvifolia. 
Dans les caproniers se placent les caprons royul ef commun, ainsi que le mâle, qui ne sert qu'à féconder le commun, dont les fleurs sont femelles. Ces fraises ont une chair très-parfumée, et il serait à désirer qu'on pût les croiser avec celles de la secfion des écarlates ou des ananas.

Dans les cearlates, on rencontre le Fraisier de Virginic, le rose berry, l'écarlate oblongue, la grimstone, le duc de Kenl, l'écarlate américaine.

Les amanas, qui, pendant longlemps, ont été ceux qui donunient les plus gros fruits, contiennent de belles variétés, telles que les Fraisier's de Bath, ananas ordinaire, ananas rouge, myall, elton, Dowton et la reine des fraises ou fraisier Queen.

Entiu, les eliiliens, qui produisent maintenant de si beaux fruits. Parmi ce genre on distingue le chili couleur de chair, la silpirbe Wilmot, le chili ordinaire, le fraisier de Paris.

Quelques nouveaux Fraisiers obtenus en Angleterre el en France méritent aussi de servir de porte-graines pour des lécondations croisées; tels sont le British Queen, le prince Alberl, Delport pine, le black prince la fraise maryuerite, la victovia, elc.

Il faut, dans les croisements des Fraisier's, beaucoup de soins pour isoler les sujets.

\section{Genre Potentille. - Polentilla.}

La structure des fleurs de ce genre est la mème que celle dı liraisier, et les fécondations artificielles sont tout aussi faciles. On cultive un assez grand nombre de Potentilles, parmi lesquelles on distingue de très-belles espècès ou variétés, telles que los $P$. atropurpurea, marginata, aurantiaca, bicolor, con. fertiflora, sulphurea, Traversii, oliveriana, Demayana, crocea, picla, el une foule d'autres qui, par leur croisement, pourraient donncr des variétés plus belles encore.

On peut toutefois essayer les croisements entre les Potentilles de couleurs diverses, telles que les jaumes, qui forment une série à part, et les rouges et blanches, qui en forment une 
autre; et entre les Potentilles à feuilles palmées el celles à feuilles pinnatiséquées, qui sembleraient aussi s'exclure dans les croisements.

(Guant a la couleur des fleurs, je ne veux parler que des espèces sauvages, car, si l'atropurpurea ou l'atrosanyuineu, dont les fleurs sont naturellement rouges, ont produit des rariétés écarlates ou orangées, il est clair que l'on pourra les hybrider avec d'autres variétés blanches ou rouges.

Les Potentilles forment un beau genre, appelé peut-ètre à joucr un grand ròle dans les jardins, et qui mérite d'être étudié et travaillé avec patience.

II. Lemoine s'est occupé avec 1 remarquable succès des l'otentilles. "Les espèces sont si nombreuses, écrivait l'habile horticulteur de Nancy à la Société impériale et centrale d'horticulture (t. VII, p. ji 8 , et si roisines les unes des autres, yu'il est tonjours difticile de se prononcer catégoriquement pour l'une queleonque d'entre elles, du moins quant aux variétés cullivées aujourd'hui en Europe. Les Potentilla nepalensis, Hook., atrosanyuinea, lood., et recta, Lin., ont dù jouer un grand rôle dans la production des variétés à flur's simples qu'on possède anjourd'hui. Jinsi la première est représentée par plusieurs variétés, telles que le $P$. Bainesiana, etc.; le $P$. atrosanguinea l'est par le $P$. spectalilis, etc., ct le $P$. recta a, de son còté, une nombreuse descendance.

“Ver's 1852, d'un semis fait au hasard, j'ai obtenu une vilriété à fleurs jaunes semi-doubles. Les graines avaient été recueillies sur des plantes de ma collection, parmi lesquelles il n'existait pus une seule fleur junne, mais bien desfleurs de divers tons orangés et minium. A cette époque, M. Van Iloutte mettait dans le commerce et décrivait ${ }^{1}$ sa sous-rariété dı P. atrosanynineu Menziesii, à fleurs semi-doubles, d'un jaune orangé, lavées de rermillon au hord externe des pétales.

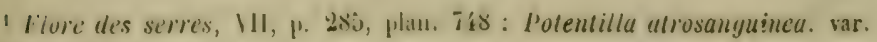
Ilensiesii flore pleno. 
"Je me suis servi du pollen de ces deux variétés pour opérer des fécondations sur les plantes vigoureuses, à fleurs sinples, que je possédais. J'olbtins ainsi, en 1857, deux variétés que je nomme aurantiaca plena el ramunculiflora plena. Le jaune dominait chez ces deux variétés, quoique les porte-graines fussent d'un rouge minium plus ou moins mêlé d'orangé.

“ En même temps, en 1856-1857, Miellez, de Lille, annonça successivement deux variétés qu'il nomma Mlauvieri flore pleno et Wazemmi. Ces deux variétés venaient d'un M. Mauvier, de Wazemmes (Lille). En en faisant l'acquisition, je reconnus que c'étaient des plantes chétives, délicates, dont les fleurs à peu près semi-doubles avaient les pélales intérieurs de moitié plus petits que les extérieurs: ces fleurs étaient colorées en rouge-minium très-foncé.

،. C'est en portant le pollen de ma Potentille jaune semidouble, de celle de M. Van Iloutte, des deux de Miellez sur les miemes de toute nuance et sur de belles finurs simples trouvées dans mes semis, que j’ai obtenu les variétés que j’ai eu l'homucur de présenter à l'Exposition de la Société impériale el centrale d'horticulture. Une remarque importante que je crois devoir faire au sujet de la filiation de ces plantes, c'est que, pour les obtenir, je n’ai jamais semé de Potentille jaune, ni simple, nii semi-double, et que cependant, parmi les plantes venues de mes semis, il y a cu toujours moitié environ de jaunes pour les plantes à fleurs simples, et deux tiers de la même couleur pour celles à fleurs semi-doubles.

"Une Potentille hybride existait déjà dans les jardins de la Grande-Bretagne, et elle a été plus récemment importée en France : c'est le Potentilla Russelliana, Lindl., qui tient des $P$. nepalensis et atrosanguinea par ses feuilles comme par ses fleurs. Le $P$. atrosanguinea peut bien être pour quelque chose dans toute cette séric de nuances qu'on possède maintenant.

“Mes Potentilles semi-doubles conservent l'aspect et les caractères des variétés du $P$. nepalensis; leurs feuilles inférieures sont quinque-foliolées, profondément crénelées et pileuses; 
leurs pétales sont obcordés; leurs fleurs sont nombreuses et disposées en une sorte de panicule. Quclques-unes ont la tige dressée, hérissée, et sembleraient tenir du P. hirtu, Lin. Quelques-unes encore ont les feuilles cotonneuses en dessous.

"Jai oublic de rappeler que M. Ambroise Verschaffelt a public, dinns l'Illustration horticole $(\mathbf{1 8 5 7} 7, \mathrm{pl} .145)$, sous Ie nom de Potentilla Mulleri (hṛbr.), une Potentille à lleur jaune, semi-double, semblable à celle que javais moi-même ohtenue en 18.52 ; mais l'auteur de l'article concernant celte plante dit qu'il n'en connait pas les parents. ")

Avant les magnifiques hybrides obtenus dans ce genre par M. Lemoine, de Nancy, on avait déjia eu des gains remarqualles. La Flore des serres et des jardins avait figuré le P. Smoutli obtenu par II. Smoutti, pharmacien à Nalines, of dont les belles fleurs jaunes veinées d'écarlate rappelaient une parenté un peu douteuse à laquelle prétendaient à la fois los P. Russeliana, atrosanguinea, nepalensis et bicolor.

Les P. Mac-Nabiana, Russeliuna, Menziesii, étaient anssi considérés comme des liybrides.

\section{Genre Rose. - Rosi .}

Je ne crois pas quilit y ait un genre de plantes plus cultivé of plus digne de l'être que celui qui nous occupe en ce moment. II n’en est pas sur lequel les horticulteurs aient fondé plus d'espérances et sur lequel ils aient obtenu plus de succès. Les véritables types ont disparu, et une multitude infmie de variélés sont venues les remplacer, offrant chacune leur avantage, leur beauté particulière et l'attrait de leur nonveauté. Le hasard a, pendant longtemps, présidé seul à ces admirables créations, et l'insecte qui hourdonnait de fleur en fleur a été, pendant plusieurs siècles, l'agent inconnu de ces mystérieuses transformations. De nos jours encore, il produit de charmantes variétés, ct si l'on ne m’a pas trompé sur l'origine de notre plus belle fleur, la Rose de la reine, le hasard aurait été eneore plus heurenx que de contume. 
On ne doit plus maintenant compler sur l'inconstance d'une chance plus ou moins favorable ni sur le vol d'un insecte pour créer de nouvelles Roses; c'est le pinceau qui doit les produire, c'est le goût, aidé de l'expérience et de l'intelligence, qui doit le diriger. C'est en effet par ce moyen que des horticulteurs, que l’on a crus longtemps privilégiés, ont obtenu des succès qui étonnaient même leurs confrères. C'est par ce moyen que les jardiniers anglais et les belges nous ont si longtemps derancés dans la plupart des genres. A notre tour maintenant, à nous surtout les Roses; e'est l'horticulteur français qui doit les eréer, et nous qui devons les répandre dans l'univers.

"Le Rosier, dit M. Rouillard (Journal de la Société imprériale et centrale dhorticulture, t. VII, p. F 80 ), est une des gloires les plus complètes de la lloriculture française, qui, depuis les Descemet, Dupont, Cartier, Écoffé, Desprez, IIardi, Vibert, Laffay, jusqu'aux Verdier, Portemer, Margotin, F.Fontaine, Lévêque dit René, Ducher, Guillot, Lacharme et antres semeurs de notre époque, a produit toutes ou a peú près toutes les variétés méritantes de Rioses, non remontantes ou remontantes. Ces variétés, olstenues en France, ont été propagées par les semeurs français et répandues, non-seulement. sur le continent européen, mais encore dins toutes les partics de l'univers civilisé, où elles peurent rroitre avec ou sans soin. ")

Quand on examine les fleurs iles Rosiers, on en trouve ale simples, de doubles et de semi-doubles.

Les étamines, nombreuses dans les simples et disposées sur plusieurs rangs, sont aussi abondantes dans les semi-doubles; les anthères s'ourrent de bonue heure, peu de temps apris l'épanouissement. Les styles, tantôt soudés, tantòt seulement serrés les uns contre les autres, forment une colonne on paquet au centre de la lleur, et se terminent chacun par un stigmate papillaire ou capité, qui correspond à un ovaire ou à ume seule graine. Dans les fleurs doubles, l'organe femelle reste souvent intact, d'autres fois il se transforme en III lon- 
ton, en une branche ou en un ramean; le Rosier devient prolifère, et ne peut plus produire de graines. Quand les styles et stigmates persistent, il y a quelquefois des étamines qui restent, qunique la fleur soit très-pleine, ou bien on trour seulement quelques anthires dont les filets sont changés en pétales, ef lo plus sourent dans les fleurs hien doubles res anthẹres ou étamines accidentelles sont dépourvues de pollen. II faut done, pour opérer la fécondation artificielle dans les lioses, choisir d'abord celles qui ont le pistil bien conformé, rnlever leurs étamines si elles en ont, et les òter de bonne licure, poser le pollen an pinceau, plusicurs jours de suite, si cola est possible, supprimer une partie des boutons el arroser Ins Rosiers pendant tout le temps de la fructification, pour pen qu'il y ait sécheresse. Il faut laisser mûrir complétement los fruits el ell semer immédiatement les graines.

On distingue, dans les Rosiers, un assez grind nombro de races, dont lia pureté commence déjà à s’altérer par des mélanges et des croisements.

On est certain de féconder entre elles les espèces de chaque série connue, les thés, les bengales, les bourbons, les cent fenilles, etc.; mais on est parvenu aussi à mélanger les trpes et is former de véritables hybrides avec les cent feuilles, arec les: bourbons, etc. Nous ne reproduirons pas ici les catalogues, ni les elassifications plus ou moins bien faites des diverses especes: et variétés de Rosiers, nous recommandons seulement aux horticulteurs qui s'occupent sérieusement de ces plantes de rhercher à multiplier encore les Rosiers remontants, cette conquêtre remarquable de l'horticulture moderne, à hỵbrider encore les rent fenilles, les mousseuses, les thés et les hengales, à essayrer If croisement des banks entre eux ou avec les multiflores, et i chrreher surtout les coloris jaunes, sonfrés, orangés on hlanes purs, qui sont encore les plus rares.

Il est essentiel de choisir les Rosiers qui peuvent donner des sraines, car il en est qui n'en donnent jamais. On peut considé. per enmme porte-graines presque tous les Bengales of une 
bonne partie des Provins; parmi les Thes : lady Granville, Caroline, belle Allemande, the janne, Lyonnais; dans les Mousseuses: l'éclatante single crimson et Luxemboury; dans les Églantiers: le double janme et celni d Harrison; dans les Bourbons: Gloire de Rosumine, Augnstine Lelienr, Delisle, Duboury, bouquet de Flore; parmi les'roses de Chine : camellin blanc, Henri V, madame Bureun, belle Elvire, Thérèse Stravius; et dans les Iybrides: Céline, princesse Augusta, duc de Devonshire, nec plus ultra, Hemi Barbet, général Allard, et tant d'autres acquisitions nouvelles dont la nomenclature seute remplirait notre volume. D'après M. Laffay, l'hybride du bengale Athalin est un des meilleurs porte-graine; il l'a lécondé avec une Rose mousseuse, et cet horticulteur habile, i qui l'on doit déjà la belle Rose de la reine, ne regarde pas les Roses comme arrivées à leur apogée pour les variations. Quant à mui, je partage tout à fait son sentiment, et je suis persundé que, pour les Roses; comme pour les Duhlius et toutes les antres plantes de collection, les variétés ne cesseront de se produire que si l'on cesse de s'en occuper. La fécondité de la nature est infinie, et les hybrides sont des créations nonvelles dont nous accélérons l'apparition sur la terre.

\section{Genre Aube-Ëpine. - Cratæguts.}

La fleur des Gratryus n’a plus la mème structure que ceile des Rosacées que nous venons de passer en revue. Les étamines, il est vrai, sont nombreuses et placées comme dans les autres genres, en double couronne sur le haut du calice; mais les styles, ordinairement libres, sont au nombre de deux à cinq seulement. Peu d'heures après l'épanouissement des fleurs, les anthères sont aptes à répandre leur pollen; en sorte que, pour des croisements, il faudrait les enlever de bonne heure et aver soin.

On a déjà de belles variétés de Cratægus, dont les unes ornent, par lat multitude de leurs fleurs, quelquefois doubles, el d'autres, par l'abondance de leur's petits fruits colorés. 
Les épines à fleurs doubles, blanches ou roses, conservent généralement leurs pistils, en sorte qu'on peut, en les fécondant artificiellement, soit avec leur pollen, dont elles ont souvent encore une certaine quantité, soit avec un pollen étranger, recueillir des graines parfaitement conformées.

D'un autre còté, on a obtenu, dans les Crutæonus, des variétés à frnits plus ou moins gros, à fruits jaunes, a fruits violets, variés, dont le nombre peut rertainement s'augmenter par hybridation, et qui produisent un effet très-pittoresque daus les jardins paysagers.

Il est probable aussi que ces jolies Rosacées pourraient s'lı- brider entre espices, et que les variétís de l'oxyacantha on Iubépine, dont nous venons de parler, pourraient se croiser avec le pyracantha ou buisson ardent, le crus galli, lasarolus, l'aronia, le florentina, et peut-être même avee quelques-unes: des espreces de la Chine, de l'Inérique ou de l'Ifrique.

\section{Genre Vèlier. - Mespilus.}

Les Néfliers ont un assez grand nombre d'étamines, destinées à féconder deux à cinq stigmates qui correspondent, par un mème nombre de styles, à des ovules qui se changent en osselets. Il y a peu de croisements à faire dans ce genre, qui ne conlient guère que le .I. Smithii et le germanica, car le juponica ne semble pis appartenir au même ț̣pe. Le yermanica a donné, par la culture, une variété à gros fruits et une autre à fruits sans osseles, une à fruits allongés et une précoce. L'hyyloridition pourrait sans doute en domne "encore de nourelles, surtout en se servant pour porte-graine au Néflier à gros fruits.

\section{Genre Poirier. - Pyrus.}

Plusieurs espèces composent ce genre, qui a été l'objet de fant d'essitis et qui a produit peut-être le meilleur fruit qui rxiste. Les espèces sauvages sont : l'achras, le pyrastru, le polu!ylleriana, le salvifolia, l'amygdaliformis, le salicifolia, le 
nivalis, le nussia, l'xeleagnefolia, etc. On cultive ces espèces dans les jardins botaniques, mais les deux premières paraissent avoir donné toutes nos variétés de poire de table, et le salvifoliu a fourni une partie des poires à cidre.

le hasard, les croisements faits par les insectes, ont produil cette multitude de variétés de Poiriers; dans lesquels il est inpossible de se reconnaitre aujourd'hui.

Les étamines sont souvent disposées dans la fleur par petit: faisceaux de trois ou de quatre. Il y en a en tout une vingtaine.

Le pollen sort des deux poches par une fente longitudinale; mais, avant qu'il se détache, les parois des deux lobes s'étalent. et forment comme deux petits disques chargés de pollen jaune it grains ovoïdes d'une extrême finesse. Duhamel avait déji rrmarqué que ces anthères s'ouvrent au lever du soleil par une secousse, et qu'il en jaillit à linstant un petit tourbillon de poussière qui s'attache à toutes les portions de la fleur.

I.es stigmates, au nombre de cinq, ont la forme d'une petite trompe frangée sur ses bords.:

La fécondation s'opère directement et facilement; les filets sont recourbés avant l'anthèse et se relèvent à mesure que les anthères répandent leur pollen. Il faut done s'y prendre de très-bonne heure pour enlever les étamines, et, quoique souvent l'aptitude des stigmates ne soit pas en rapport avec l'ouverture des premières anthères, il est toujours prudent de châtrer dans lc bouton.

La fécondation artificielle est donc facile, elle exige seulement l'isolement d'un rameau et l'enlèvement matinal des anthères.

Il n'y aurait aucun avantage à chercher à hybrider entre elles les races sauvages, mais on obtiendrait sans doute de nouveaux types sil'on parvenait à croiser les espèces cultivées avec les espèces sauvages. Dans les variétés si nombreuses des poires cultivées, on en distingue qui surpassent toutes les autres en qualité. En première ligne se présentent les beurrés et notam- 
ment le beurré gris et celui d'Arembery, qui mûrissent en atttomne; les bons-chrétiens, dont la maturilé commence dès lo mois d'aout, pour se prolonger jusqu'au milieu de l'hiver; les crassames nu bergamoles, qui se conservent aussi; les colmars, les. Saint-(ro'rmain, qui se gardent si longtemps et ne mûrissent que dans les fruitiers, et une foule d'autres variétés qui sont rneore très-honnes; mais pour essayer des croisements, il faut Ies tenter entre les meilleurs fruits et ticher de ne pas grossir inutilement des catalogues déjà trop volumineux. Il faut cependant choisir les variétés qui présentent, an plus haut degré, les qualités que l'on cherche: c'est ainsi que, si l'on vise à la précocité, ce ne sera pas sur les colnars qu'il faudra opérer, tandis que, si l'on veut, au contraire, lirher d'avoir des espèces encore plus tardives que celles que nous connaissons, ces demires derront avoir la préférence pour les hỵbridations.

Si les reherehes sont dirigées sur les poires à cidre, les variétés ne sont plus les mèmes; ce sera surtout le saugé ou salvifolius qu'il faudra choisir pour porte-graine, et tenter les croisements avec le rayuenet, le sabot, les moque-friands, le yros vert, le curisi, le trochet de fer, le grosmenil, ete.

Il y aurait aussi à s'occuper des Poiriers sous le rapport ornemental, car tous tiennent un rang distingué par leurs belles fleurs, que déji on est parvenu ì doubler. Les croisements amèneraient sans doute de nouveaux résultats, surfout en prenant pour porte-graine la rarieté it fleurs doubles si, comme je le suppose, son pistil est bien conformé.

Les croisements que l'on tentera sur les arbres fruitiers, comme sur les autres plantes, derront, autant que possible, avoir lieu entre variftés nourellement obtenues, entre des plantes yui, comme nous l'avons dit au second chipitre de ret ouvage, et comme Viun Mons l'a prouvé depuis longlemps par experience, n'ont pas eneore acenuis la stabilité de l'habitude. 


\section{Genre Pommier. - Malus.}

Les Pommiers ont la même structure florale que les Poiriers, dont ils ne diffèrent que par la forme des fruits et par les styles, qui sont plus ou moins soudés à leur base. Ce que nous avons dit des organes du Poirier et du mode de fécondation artificielle s'applique donc également aux Pommiers.

Quelques espèces, en tête desquelles il faut placer le spectabilis, sont cultivées comme arbres d'ornement, tels sont encore les $M$. coronaria, sempervirens, microcarpa, baccuta, que l'on pourrait peut-ètre hybrider, mais dont les intermédiaires seraient sans doute moins beaux que le spectabilis. Ces deux derniers pourraient aussi produire des fruits nouveaux en les hybridant avec diverses variétés du Pommier ordinaire.

Sageret a inutilement tenté d'hybrider des Poiriers par des Pommiers et réciproquement, mais il a hybridé entre elles les diverses races des variétés de Pommier et en a obtenu des variútés nouvelles plus vigoureuses que leurs ancêtres.

Quant aux races cultivées, elles dérivent toutes de deux types, le $M$. acerba, qui a fourni les pommes à cidre et le $M$. communis, qui a donné celles de table ou pommes à couteau.

Les Pornmicrs à cidre, cultivés seulement en Normandie, présentent de très-nombreuses variétés, parmi lesquelles on distingue les doux, les amers et les acides, que l'on mélange dans la fabrication du cidre. Je ne pense pas qu'il y ait d'hybridation à tenter parmi ces pommes, car, indépendamment de leur saveur variée, on a des arbres très-productifs et donnant à des époques différentes; il vaut donc mieux employer son temps à croiser les races deś pommes à couteau. Nous recommandons aux soins des horticulteurs les croisements entre les calvilles et les reinettes, et toutes les variétés à courte queue, appelées courtpendus, les apis, le pigeonnet, les rambours, les fenouillets de toutes couleurs. Nous recommandons surtout, comme portegraine, la belle jomme Adam, dont la chair est si tendre et le 
volune si considérable. Il y a beaucoup à faire encore dans les rariétés de Ponmier. On a des pommes énormes et l'on arrivera i de plus grosses encore; entin, plusieurs variétés se conservent très-longtenps, et ce sont surtout les pommes d'hiver dont il laut chercher à multiplier les races.

Mustel a observé que des fleurs de Pommiers dont les pétales et les étamines araient été mangés par un limaçon avaient produit des fruits en plus grand nombre à proportion, et plus heaux que les fleurs dont les pétales el les étamines étaient tombés d'eux-mêmes. Mustel a essayé sur quelques fleurs de conper les mimes parties avec des ciseaux, et toujours avec le mème succès. Hais il ne dit point qu'il ait pris des précautions pour que les étamines n'eussent pas répandu leur poussière arant d'ètre retranchées ou pour que la poussière des étamines des bouquets voisins n'y füt point portée. (Mém. de l'Académie (les sciences, 1771, p. 46.)

Nous reproduisons ici la description curieuse du Pommier lemelle de Saint-Valery, insérée dans le t. III, p. 164, de la Société linnéenne de Paris. Elle est due à M. Tillette de Clermont.

“ II. Alix, propriétaire à Saint-Valery-sur-Somme, possède un Pommier dont il ignore l'origine et qu'il croit âgé de trente à quarante ans. Cet arbre en tout semblable au Pommier com. mun par les feuilles et la disposition des fleurs, en diffère d'une manière très-remarquable par la stucture de ces dernières et par ses firuits.

"Un pédoncule tomenteux soutient une fleur composée d'un calice à dix folioles soudées par la base, disposées sur deux rangées alternes, les intérieures un peu plus courtes. La corolle cl les étamines manquent; les styles, au nombre de quator\%, légèrement velus ì la base, sont surmontés d'un sigmate oblique, très-vigoureux.

«Les premières anmées que l'arbre a fleuri, son propriétairr, ctomné, disons mieux, très-mécontent de ne lui roir porter aucun fruit, allait l'abattre, quand un médecin, instruit de 
cette bizarrerie de la nature, conseilla la fécondation artificielle, qui réussit parfaitement. Depuis ce temps, chaque année, à l'époque de la floraison, c'est à qui des dames et demoiselles de Saint-Valery ira faire sa pomme (c'est l'expression consacrée). On applique sur chaque fleur une fleur hermaphrodite, cueillic par un temps sec sur un Pommier quelconque, en l'y abandonnant jusquà ce que, la fécondation achevée, elle tombe naturellement, puis on attache un ruban de couleur au bouquet fécondé pour que, quand l'automne sera venu, chacune reconnaisse le fruit que sa main a créé.

“ Ces fruits diffèrent entre eux par la grosseur, la saveur et la couleur, mais ils se rapportent aux especes hermaphrodites qui les ont fécondés. Ils sont très-remarquables par un étranglement situé vers les deux tiers de leur longueur, ainsi que par quatorze loges disposées sur deux plans parallèles, dont cinq, placées comme celles des pommes ordinaires, occupent le milieu du fruit; les neuf autres, plus petites, la partic roisine du sommet. liarement toutes ees loges contiennent des graines. Dans le très-petit nombre de fruits que nous avons ouverts, elles variaient de trois à neuf.

"Plusieurs botanistes, entre autres Wildenow et M. Poirel, parlent de Pommiers uni-sexuels très-différents de celui dont nous nous occupons. Dans les leurs, il y a avortement des pétales et des étamines; dans le nôtre, il y a bien aussi avortement des mêmes organes, mais il y a augmentation en nombre loujours constant des autres parties de la fructification. Chez eux, le calice est simple, à cinf folioles; ici, il est évidemment double, l'intérieur semblable à l'extérieur, cornme lui persistant et ne pouvant être assimilé à une corolle. Dans les preuier's, on trouve cinq styles et stigmates; dans le nòtre, quatorze. Les pommes ordinaires n'ont que cinq loges; celles-ci en renferment quatorze sur deux rangs et représentent deux pommes soudées bout à bout, dont la coupe longitudinale a la figure d'une feuille panduriforme ou en violon.

“M. Alix a porté des greffes de cet arbre sur la moitié d'un 
Ponmier voisin qui reste constanment stérile, quoique les fleurs soient parfaitement semblables à celles du premier, et que les mêmes moyens de fécondation soient employés. Dés graines semées ont très-bien levé; le plant, trop jeune, n'a pas encore fleuri. »)

\section{Genre Alisier. - Aria.}

Lee grenre approche encore beaucoup du Poirier, et présente la même disposition dans les étamines et les pistils; les styles sont ilı nombre de deux ou trois dans les véritables Aria, et de deux à cinq dans les Torminariu. Ces plantes, assez différentes, pourraient cependant s'hydrider, car il y a de grands rapports de structure, mais on les cultive peu, et ce n'est que par leurs liruits ou leur fenillage yu'elles deciennent ornementales ot sont admises dans les grands jardins.

\section{Genre Sorbier. - Sorbus.}

Lincore la mème conformation que les Poiriers, et encore deux à ciny styles. Les Sorbiers se distinguent par leur's fruits, dont une espèce, celui du Cormier, est comestible. Il y aurait peut-ètre possibilité d'hyybrider les Sorbier's; on pourrait tenter le croisenent entre l'Aucuparia, qui est le plus beau, celui l'Amérique, le Cormier, le pleureur, nouvellement obtenu, l'asplemfoliu, également très-noureau, l'argenté, le quercifolia, celui de Laponie, etc. C'est un genre où il reste encore i liare, car les Sorbiers sont de trìs-beaux arbres qui ornent par l'élégance de leur fenillage et de leurs fruits.

\section{Genre Coignassier. - Cydoniu.}

Les Civignissiers ont aussi de nombreuses étamines yu il fiut enlever de bonne heure si l'on vent tenter des fécondations: artilicielles, et cing styles munis de stigmates papillaires et glanduleux. Une espèce domne des fruits comestibles, et déjà on en connait plusicurs variétés, à fruit long, à fruit rond, le 
coing de Portuyul et celui de la Chine, qui est une espèce dilférente. Il serait à désirer que l'on tentàt des croisements entre ces plantes, dont les fruits, très-parfumés, finiraient peutêtre par s'adoucir et devenir mangeables sans préparation.

Le Coignassier du Japon, dont l'espèce primitive, à fleurs rouges éclatantes, a déjà donné une variété à fleur blanche, une à fleur jaune et une à fleur rosée, désignée sous le nom de Rosalba, produirait certainement d'autres nuances par l'hybridation. Sa précocité le rend très-recommandable, mais il ne fructifie pas toujours facilement. En résumé, les Coignassiers semblent former quelques types qui appellent l'attention des horticulteurs. Ils deviendront peut-être un jour la souche de nouveaux fruits, ou celle d'une série d'arloustes de collection, à fleurs variées et éclatantes.

\section{FAMILLE DES CALYCANTIIÉES.}

Genre Calycanthe. - Calycanthus.

Il est si rare d'avoir des fruits de Calycunthus, qu'il ne liaul guère penser à hybrider ces plantes, dont les stigmates sont presque toujours avortés et qui peut-être sont dioïques dans leur pays natal. Cependant on voit quelquefois des fruits provenant de fleurs bien conformées, et il est probable que, si on donnait quelque attention à la structure des fleurs, on en trouverait quelques-unes femelles ou hermaphrodites qui, fécondées au pinceau, noueraient très-certainement. Les G. floridhis, fertilis, bullatus, edulis, heterophyllus, pourraient sans doute produire ensemble, et le C. pracox donnerait aussi des modifications nouvelles arec ses propres variétés albus et grandiflorus. Il fructifie à Hyères, et M. Henon assure qu'il mûrit également ses graines sous le climat de Paris, pourvu que l'arhre ait atteint douze à quinze ans. Nous en avons nous-mème obtenu des graines en Auvergne. 


\section{RANILLE DES MELLASTONACEES.}

\section{Genre Mélastome. - Melasloma.}

Cìs plimtes ne peurent être cultivées en Europe que daus lil serre chaude, oi elles ne fructifient pas. Il en existe un tresgrand nombre d'espèces, mais peu d'entre elles ont été introduites en France. Leur fécondation est remarquable en ce que les antheres sont enfermées dans le tube au moyen d'un filet qui est plié cn deux, et qui se déploie en lés anenant successivement sur le stigmate, qui recoit leur pollen d'autant plus facilement, que chaque anthère dirige sa pointe sur lui et se trouve percée de pores pour domner issue il la poussière lécondante. Il n'y aurait donc aucume dilficulté à hybrider ces plantes, mais, auparavant, il serait nécessaire de pouroir les firire fructifier, 'n les fécondant avec leur propre pollen, c'esti-dire avec le pollen de la même espèce.

\section{RAIILLE DES MIR'TACEES.}

Genre Grenadier. - Plmic

De nombreuses étamines sont attachées au calice et répandent leur pollen peu de tempss après l'épanouissenent. Le stignate est garni de papilles et a la forme d'un petit bouclier; il est trislacilement imprégné.

L.e Grenadier est cultivé partout et généralement it lleurs doubles, en sorte qu'il perd ses étamines et mème son pistil, ou du moins presque toujours; on rencontre quelquefois des fleurs semi-dumbles, que l'on pourrait féconder, soit aree celui à fleur jaune, soit avec le Grenadier à fleur blanche de la Chine on arec le nain des Antilles. Comme on ne sest jamais occupé du croisement de ces arbrisseaux, ce serait une étude intéressante pour les horticulteurs méridionaux, et ‘ui 
leur domnerait certainement de beaux résultats, sous le rapport des fruits et des lleurs.

\section{Genre Seringat. - Pliladelphus.}

Les Seringats sont des arbrisseaux qui se ressemblent par le port ct par leurs fleurs blanches odorantes ou inodores. II y aurail peu d'intérêt à chercher à les hybrider, car il y a trop peu de différence entre eux. Le croisement serait probablement facile, car les quatre à cinq styles sont très-apparents, ct les nombreuses étamines qui les entourent ne sont aptes que peu de temps après l'épanouissement. Ce genre renferme de très-belles espèces.

\section{Gemre Mélaleuque. - Melalenca.}

On ne cultive qu'un petit nombre d'espèces de ce genre, et assez souvent elles donnent des graines dans nos serres. La fécondalion artificiclle serait facile, car les étanines nombreuses sont rémies en cinq faisceaux par la base des filets, et ces laisceaux sont recourbés dins l'intérieur du tube, en sorte qu’il suffirait de les couper à leur hase et de les enlever. L'opération devrait se fiare de bonne heure et arant l'épanouissement, car les anthères s'ourrent avant le redressement des étamines. Le style est terminé par un stigmate en tête.

\section{Genre Metrosideros. - Metrosileros.}

Le's étamines très-nombreuses et très-saillantes des Metrosideros rendent la fécondation arlificielle assez difficile; il faut, comme dans les Melaleuca, les enlever de honne heure, mais elles ne se tiemnent pas et sont séparées. Le style et le sligmate sont isolés au milieu d'elles. Il est probable que plusiemrs Metrosideros s'hybrideraient et domneraient de helles viriélés; les Callistemon et les Leptospermum derraient itre traités comme les Metrosideros. 
Genre Nyrte. - Myilus.

Les Myrles, quonque assoz nombreux en esperes, sont peu répandus dans les serres. On ne cultive guère que le Mỵte commun, qui abonde dans le Midi de la France, où je l'ai rencontré sous dés formes très-différentes. Il offre, en elfet, plusieurs variétés ì fenilles petites, moyemnes, élargies, et les M. romain, de Belgique, de I'ortugal, de Tarente, ne sont que des modifications de l'ordinaire. Toutes ces variétés se croiseraient par des fécondations artificielles, en enlevant de bonnc heure les nombreuses antheres de la fleur à féconder, et en appliquant le pollen sur le stigmate lobé.

\section{FAMILLE IUES CUCURBITACEES.}

\section{Genre Calebasse. - Lagenaria.}

Lappareil de la fructification se présente dans la famille des Cuculbitacées avec des formes particulières, et, dans le genre qui nous occupe, les fleurs sont monoïques et les étamines sont réunies en trois masses incigales, deux plus grosses et une plus petite. les stigmates des fleurs fenelles sont renllés, souvent partagés en deux lobes et couverts de poils glanduleux, destinés a relenir facilement le pollen. Riin de plus simple que de prendre ce pollen au pinceau sur les fleurs màles et de le porter sur le stignate des flems femelles, qui sont bien moins nombroses que les mâles. On peut ainsi multiplier it l'inlini

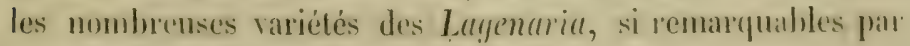
les singulieres formes de leur's fruits.

Genre Melon, Concombre. - Cucumis.

Les espees atsez nombrenses du gemre Cucumis ont ité introduites dans nos jardins depuis tris-longlemps, et elles ont 
domé béaucoup de variétés alimentaires. Les principales sont les Concombres et les Melons.

Dans ce genre, les fleurs sont monoïques et rarement hermaphrodites; les ciny étamines sont réunies en trois paquets inégaus, et la fleur femelle offre trois stigmates épais et bifides, couverts de papilles glanduleuses. La fécondation artificielle s'opère d'autant plus facilement que les sexes sont séparés, et, par conséquent, la castration devient inutile; mais, si l'on veut faire des essais avec soin, il est nécessaire de bien isoler le porte-graine et de lui enlever toutes les fleurs mâles avant leur épanouissement. On les reconnait au bouton, qui ne porte pas en dessous de petites boules formées par l'ovaire infère. On prend au pinceau le pollen sur le pied que l'on a choisi et on le porte sur le stigmate. Quand cette opération est faite, on prend au fond de la fleur femelle, et avec un autre pinceau, un peu de liqueur miellée que l'on pose très-légèrement sur le stigmate fécondé.

Le Cucumis sutivus ou Concombre ordinaire a produit, surtout en Angleterre, où cette plante est trìs-recherchée, de nombreuses variétés alimentaires. On peut les augmenter encore par les fécondations croisées, en suivant les procédés que nous venons d'indiquer.

Le $C$. melo ou le IIclon en a donné plus encore peut-être, et maintenant on les a rangées sous trois types qui appartiennent eependant ì la même espèce. Voici classíes, sous ces trois divisions, les principales variétés, dont nous avons extrail la liste de l'excellent Manuel de culture maratichère de M. CourtoisGérard.

Brodés. - Marciicher. - De Lanjeais. - Sucrin de Tuar's. - A chair blanche. - Ananas ì chair verte. - De Honfleur.

- De Coulommiers.

Cantaloups. - Orange. - Noir des Carme's. - De vingthuit jours. - Fin hitif d'Angleterre. - Cantaloup noir d'Amerigue. - Prescote fond blanc. - Cientatouy boule de 
Siram. - Gros noir de Hollande. - Gros de Portugal. - A chair blanche. - A chair verte.

A ceoree lisse. - De Malle ì chair blanche. - Rounge. -

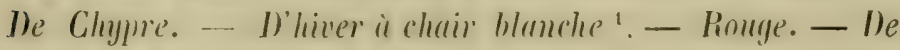
Perse ou d'Odessu (grav. 48):

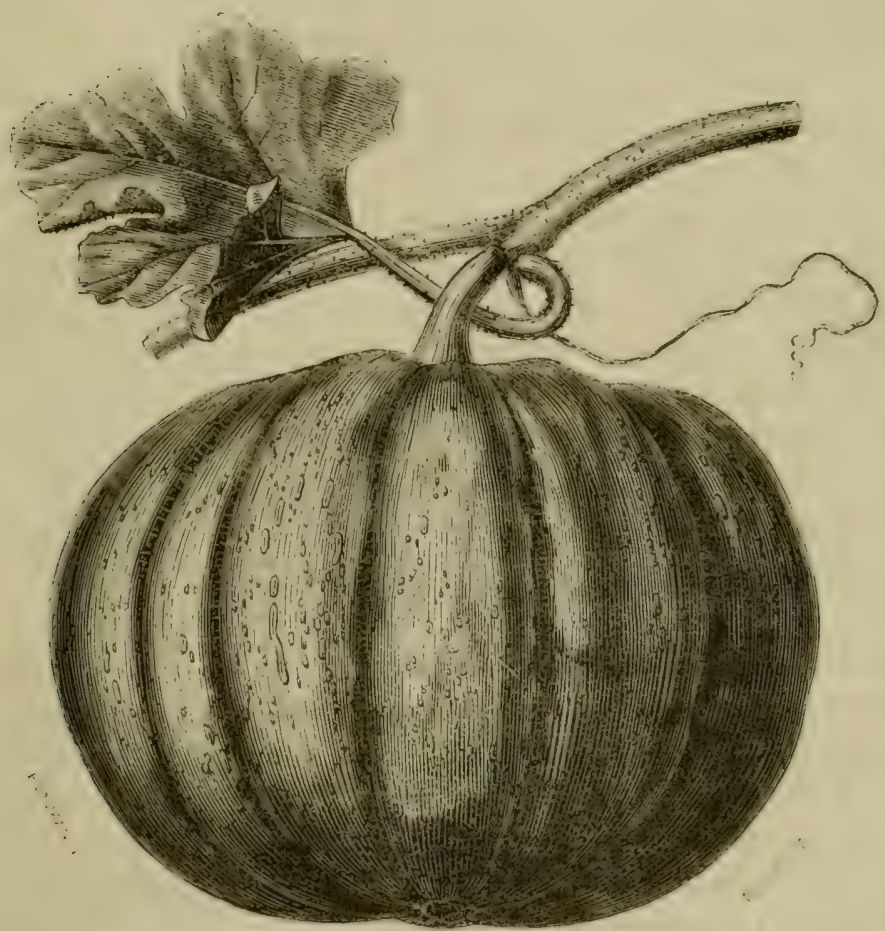

Cirav. 1S. - Je!on cantaloup sucré, an tiers de sa granteurnaturelle.

Cos trois types peuvent être croisés entre eux, et, à plus forte raison, on peut ohtenir l'hỵbridité entre les variétés qui forment chacun d'eus. On opiere en tout comme nous venous de le dire pour les Concombres.

II y a sourent avautage, sunon à croiser, du moins à prati-

1 Ces melons d'hiver, dépooés daus un lien sece ee ennecrrent sans altération jusqu'en janvier et quelquefois plus longtemps. 
quer la fécondation artificielle avec leur propre pollen sur ces différentes variétés de Melon, en prenant autant que possible le pollen sur un autre individu de la même espice. Cela est utile, surtout pour les Melons de printemps, dont les fleurs femelles coulent facilement, c'est-à-dire tombent sans nouer. On prévient souvent cet accident par la fícondation au pinceau. D'autres fois, certaines variétés ne donnent que des fleurs femelles au commencement de la floraison, et l'on est obligé de prendre le pollen d'une autre variété pour suppléer aux fleurs mâles qui manquent.

La variété que l'on désigne sous le nom de Melon hybride de Lanvian provient d'un Melon de Valparaiso, accidentellenent hybridé en France, et il s'est constamment reproduit avec fidélité. C'est, selon II. Bossin, une des meilleures variétés.

La même opération peut se pratiquer sur le $C$. citrullus, dans lequel se trouvent la Pastèque et le Melon d'eru, plantes qui ne réussissent bien que dans le Midi. Il faut remarquer toutefois que le Melon d'eau présente quelquefois des fleurs hermaphrodites an milieu des fleurs unisexuées.

On trouve encore dans ce gente la Coloquinte ou $C$. prophe. tarum, le C. flexnosus ou serpent à très-long fruit, qui donne même des variétés que l'on peut aussi hybrider entre elles; mais les espèces proprement dites ne se croisent pas et restent pures de tout mélange, à l'exception du flexuosus, qui se croise peut-être avec les Melons.

Le fécondation artificielle est d'une utilité pratique journalière dans toute la famille des Cucurbitacées.

\section{Genre Citrouille, Potiron, Courge. - Cucurbita.}

Ce que nous venons de dire sur la fécondation des Cucumis, Melons et Concombres, s'applique tout naturellement a celle des Cucurbita. Dans ceux-ci, les cine étamines sont soudíes ì la fois par les filets et les anthères, et les trois stigmates des fleurs femelles sont épais et partayés chacun en deux lobes. Ce genre 
est aussi très-nombreux; les espèces sauvages sont peu connues. On ignore mène somrent quels sent lestypes des variótés cultivées. Quoique lenrs modilications soient trè-multipliées, il reste encore heaucoup à faire sur ces plantes, qui s'hybrident très-facilement, mais entre varictés de mèmr espèce.

Sagreret, qui s'est necupé d'une manière spéciale de l'hybridation des Cucurbitacies, indique les groupes suivants comme ne se mêlant pas ensemble et n'arant ancune influence fécondante les uns sur les antres.

Pepo potiron, comprenant le fiotiron ef tontes ses variétés:

Pepo cirrullus, où se trouvent le Giranmont et ses variétés, commues sous les noms de Citronille, Courye ì la moelle, Pustisson, Bonmet d'électemr, Colonnimelle, Colorpuinte oranye, Coloquinte poire, etc.

Pepo mosehatus, Potiron musqué, ou Potiromon;

Pepo malabarieus, ou Courye retyée et mouchetée.

Sageret ajoute à ces quatre types le Lagenaria, dont nous avous parlé, et le Melon d'én, que nous avons placé dans le genre précédent. Les belles variétés de Giraumont, le Potiron d'Espoume, les diverses variétés de Citromilles, les Comryes d'Italie, la C. sucrière du Brésil, sont les races sur lesquclles il faudrait essayer les croisements, qui n’ont, pour ainsi dire, aucune limite entre les variétés des planles cultivées.

II. Naudin n'admet pas non plus les hybrides dans ces plantes, malgré l'opinion contraire si répandue. Il a cultivé ensemble leurs diverses espéces; il a aidé de son pmecaul'íchange dis poussières fécondantes; il a livré ses planches de Courges it l'incessante activité des abeilles, "l n'a pu obtenir une seule graine lerrtile.

"Conchons-en qüici, dit M. Naudin, malgré f'apparence, malgré surtont leur étonmant polymorphisme, les espèees sont tres-distimetes, fres-nettement arreteers d absolument incapables de laisser salterer par hybridation leur sai caracliom. Con-

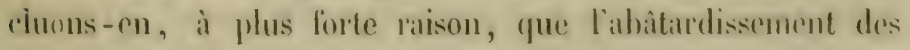


races de Melons par les Courges n'a aucune probabilité, et qu'il n'y a là qu'une de ces erreurs populaires qui tombent dès qu'on les soumet au contrôle d'une observation sérieuse. "

Il est bien entendu qu'il s'agit ici d'espèces distinctes de. Courges, et non de variétés.

\section{FAMILLE DES GENOTHÉRÉES.}

\section{Genre Fuchsia. - Fuchsiu.}

Les Fuchsia ont tous huit étamines et un style comronné par quatre stigmates libres ou plus souvent soudés. Dans un certain nombre d'espèces, comme les thymifolia, parvifolia, microphylla, serpyllifolia, les étamines restent dans l'intérieur dı tube, et il faudrait les aller chercher avec de petites pinces; on fend le tube lui-même pour les extraire, mais, comme ces espèces sont peu cultivées, il est à peu près inutile de tenter sur elles des fécondations artificielles.

Il n'en est pas de même des autres espèces, toutes très-belles el qui ont fourni dejà une très-grande quantité de variétés nouvelles. Dans toutes ces plantes, les étamines sont saillantes et pendantes; elles ne s'ouvrent quelquefois que deux à trois jours après l'épanouissement, et leur pollen, qui n'est pas très-pulrérulent, ne peut même pas toujours atteindre le stigmate. Rien n'est donc plus facile que de retrancher les anthères el d'hybrider les Fuchsia.

Les fleurs tiennent peu au pédoncule, ou plutôt la corolle adhère peu à l'ovaire, en sorte qu'il faut les toucher avec précaution, et ne pas attendre trop longtemps pour féconder le stigmate.

On trouve dans le Jardin et la Ferme un article sur l'hybridation des Fuchsia qui indique les moyens employés par quelques jardiniers anglais pour pratiquer le croisement. II consiste. 
i recueillir le pollen des variétís diverses dans les vingt-quatre cases d'une petite boite, muniez chacune d'un morceau d'étoffe de la couleur de la plante qui fournit le pollen, et à attacher la livrée du père au porte-graine adopté. Ce moyen peut être bon pour la généalogie des variétés futures, mais il est presque inutile, puisque l'on ne peut même plus savoir quelles sont les véritables espèces qui ont procréé toutes les formes différentes que nous cultivons maintenant.

II. II. S. a publié dans un journal anglais the Florist, de mai 1859) quelques conseils surl'hybridation du Fuchsio: " La plupart des Fuchsia qui existent aujourd'hui dans les jardins, dit-il, mûrissent très-bien leurs graines; mais, à moins que celleś-éi n'aient été produites par hybridation, c'est à peu prìs perdre son temps que de les semer, car les plantes qu'elles donnent sont rarement égales en mérite aux porte-graines, et souvent mème elles leur sont inférieures. Or la fécondation artificielle est très-facile à opérer sur les Fuchsia, leur pistil étant très-saillant et leurs anthères contenant beaucoup de pollen.

"Lorsque l'on a fait choix des plantes que l'on veut hybrider, il ne faut pas perdre de vue que celle qui portera la graine communiquera son port à l'hybride, et que peu importe le port du pied qui fournira le pollen, pourvu que ses fleurs aient du mérite. Après avoir choisi les fleur's que l'on veut féconder, on en supprime les étamines aussitôt après l'épanouissement, et, lorsqu'elles sont parfaitement ouvertes, on applique sur leur pistil le pollen des fleurs dont on veut communiquer les qualités.

"Quand les fruits sont parfaitement ınùrs, on sépare les graines de la pulpe en lavant le tout dans de l'eau limpide; les bonnes graines vont au fond.

"On sime ensuite ces graines ainsi nettoyées, au printemps, en pots remplis d'une terre légère et tenus en orangerie. Il leur linut peu de chaleur pour germer. Il ne fint ni les pincer, ni les tailler, jusqu’à ce qu'ils fleurissent. "

Le premier Fuehsio fut déeouvert en Amérique, à la fin du 
dix-septième siècle, par le père Plunier, qui le dédia à Fuchs, botaniste allemand. L'importation en Europe ne date que de 1788; depuis celte époque, des espèces nouvelles ont successivement paru, et l'hybridation s'est emparée de ces plantes comme d'une véritable proie. L'apparition des variétés à pétales violets, à pétales blanes, à calices carnés, les Vémus victrix, Queen Victoria, blonde Ariane, Lady Franlilin, Espérance, Vénus de Médicis, Roi des blancs, Virgo Maria, etc., etc., a donné le signal d'une production inouie et de variations qui ne s'arrêtent plus. Au lieu des petites fleurs des Fuchsia primitifs, on a maintenant des boutons globuleux ou allongés qui entraînent les branches vers le sol. Les pétales sont veinés, doublés, multipliés, et, quoique les Fuchsia doubles aient jerdu une partie de leur grâce el de leur perfection naturelles, ils n'en sont pas moins très à la mode et très-estimés.

Quand les belles espèces de Fuchsia fulgens, corymbifera, cordata, ont fait leur apparition, les horticulteurs intelligents ont compris tout de suite limportance des croisements qu'ils allaient opérer avec les anciennes espèces. Si toutes les tentatives n'ont pas réussi, il faut pourtant admettre que c'est à partir de cette époque, déjà ancienne, que ce genre est entré dans cette voie admirable de créations nouvelles, qui sont loin de s'arrêter.

Toutes ces formes, dont nous ne pouvons retrouver les ancêtres ni les types, sont fertiles et nous montrent la puissance de l'homme sur ces belles productions de la nature.

Les Fuchsia atteignent en ce moment leur plus beau développement. On a des corolles blanches, violettes, lilacées, carnées, veinées, des pétales panachés, comme dans le $F$. lord Clyde, Youell. On a des fleurs énormes dans le $F$. Béranger, etc., etc.

\section{Genre Onagraire. - OEnother .}

Les OE. biennis, speciosa, grandiflora, fruticosa, ylauen, 
noctifloru, taraxacifoliu et plusieurs antres, ont èté adınis dans nos jardins, et sont, en eflet, de tres-belles plantes. Je ne crois pas fue l'on ait cucore tenté de croiser ces espèces, dont quelques-unes ont cependant entre elles des rapports assez marqués pour que l’on puisse en espérer quelques suceès. Tontefois l'hỵridation dans ces plantes offre une difficullé, c'est l'enle-

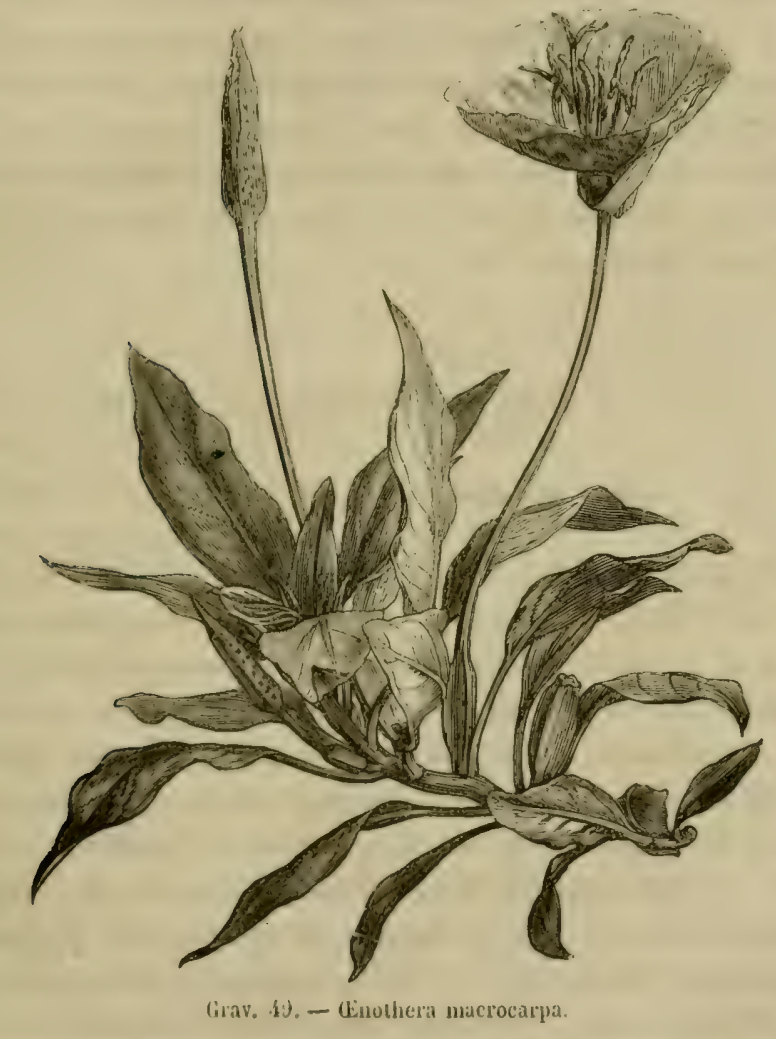

vement des élamines arant l'ouverture des anthères. Il faut, pour cela, ourrir le bouton l'avant-veille de son épanouissement, en le fendant longitudinalement aver la pointe d'un canif', sans s̈inquiéter si l'on coupe plusieurs pétales roulés les uns sur les antres. La seule précantion il premble est de ne pas murir me 
des huit étamines qui sont appliquées sur le pistil, et déjà prêtes à répandre leur pollen, dont les grains sont visqueux et liés par une foule de petits filaments. On les enlève avec la pince et l'on pose immédiatement, sur le stigmate quadrifide et très-grand, le pollen que l'on vient de recueillir dans le bouton d'un autre CEnothère. On peut attendre, pour placer le pollen, que la fleur à féconder commence à s'ouvrir. Lors même qu'en opérant on aurait blessé un des stigmates, les trois autres rempliraient leurs fonctions, et comme une seule des quatre loges de la cipsule d'un Trnothère contient une grande quantité de graines, on est. toujours sûr d'en aroir assez si l'on parvient à féconder seulc. ment une ou deux fleurs. On enlève alors toutes celles qui sont supéricures (grav. 49).

\section{FAMILLE DES FICOÏDES.}

\section{Genre Fieö̈de. - Mesembryanthemum.}

Les espèces sont tellement multipliées dans les Ficoïles, que l'on doit y supposer, comme dans la plupart des genres nombreux de la pointe australe de.l'A frique, des lybbrides et des croisements presque continuels. Ce qui autorise encore à faire cette supposition, e'est que, dans la plupart de ces plantes comme dans les Pelargonium, les stigmates ne sont aptes qu'après la floraison, ou du moins à l'anthèse des dernières étamines. Ce sont donc plutôt des plantes monoïques ou diöques qu'hermaphrodites. Les ítamines sont nombreuses, disposées sur plusieurs rangs et les carpelles ordinairement au nombre de cinq. Il n'y a aucun doute que l'on réussirait à croiser les $1 /$ esembryanthemum, en choisissant des espèces assez voisines el en posant le pollen pendant que la fleur est dans son plein épanouissement. 


\section{FAMIILLE DES PORTULACÉES.}

\section{Genre Pourpier. - Porlulaca.}

Ce genre renferme des espèces très-différentes, dont une, le Pourpier cultivé, est considérée comme plante alimentaire et a déjà domé plusieurs variétés. C'est un légume qui a trop peu d'importance pour qu'on s'en oceupe sérieusement, mais il n'en est pas de même des espèces d'ornement. Le grandiflora, le Thellussonii, le Gillesii, le carminea, sont de fort belles plantes qu’il serait avantageux d'hybrider et de multiplier. Déjà le frandiflor' a donné une variété d'un l'ouge orangé qui pourra produire des individus à teintes de plus en plus jaunes, et l'on aurait déja fait un grand pas, si l’on obtenait des variétés entièrement jannes. La fécondation s'opère naturellement d'une manière très-sûre dans les Pourpiers, car les étamines sont nombreuses, et les stigmates, an nombre de huit ì neuf, sont étalés de manière à recevoir très-facilement le pollen. Il faudrait donc, pour hybrider les Pourpiers, enlever de grand matin, et avant l'épanouissement, toutes les anthères, puis attendre que le soleil, frappant sur la plante, l'oblige d'ouvir sa corolle. Alors on pose le pollen au pinceau, et la lleur se relerme quand le soleil l'abandonne.

Toutes ces variétés, dont je préroyais l'arrivée en 18 \%ั, se sont présentées, et bien d'autres encore, en sorle que l'on a des lleur's rouges, orangées, james, citrines, blanches, pimachées, striées, etc.

\section{FAMILIE DLS CACTEES.}

\section{Genre Mamillaire. - Mamillaria.}

Cie genre ne renferme que des plantes chammes dont les espèces, très-varićes, ont été placées dans plusieurs sections. Les 
étamines sout nombreuses el placées sur plusieurs rangs. Elles s'ouvrent presque toutes en même temps et répandent en abondance leur pollen sur un stigmate bien conformé à ciny à sept rayons. La fécondation commence peu de temps après l'épanouissement, et souvent elle ne s'effectue pas, à cause de la

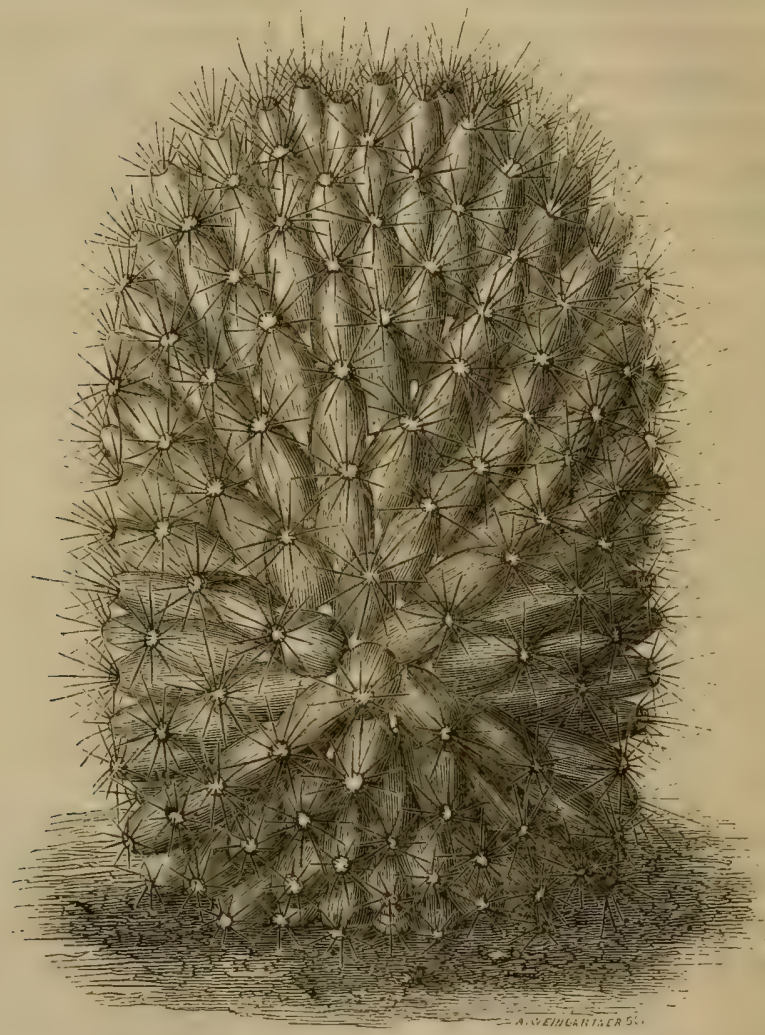

Grav. 50. - Namillaria simplex de grandeur naturelle.

consistance du pollen, qui n'est pas très-pulvérulent et qui reste attaçhé aux anthères, sans tomber sur le stigmate. Aussi la fécondation artificielle est très-facile dans ce genre, et quelquefois même il n'est pas nécessaire d'enlever les étamines; il sulfit de poser au pinceau le polleu étranger sur le stignate. Il est plus 
sir cependant, ou de suppriner les anthères, ou de platere autour du stignate un petit tulie pour l'isoler, comme il est convenable de le laire pour les Gereus.

Les Mamillaria sont tellement nombreux, qu'il n'est pass douteux qu'il existe parmi eux un bon nombre d'hỵlyides, et rien ne serait plus facile que d'en augmenter les variétés. Les plantes appartenant aux diverses sections peuvent saus doute se féconder réciproquement, car les Cactées forment une vaste famille qui s'hybride tous les jours. Les 1 . pusilla, simplex et quelyues autres, doment facilement des graines tgrav. 50 ).

\section{Genre Mélocacte. - Melocactus.}

La floraison des Melocuctus est sensiblument la mème que celle des Mamillaria; les étamines, très-nombreuses, sont égallement disposées sur plusieurs raugs et le style est terminé par ciny stigntites. On peut done opérer la fécondation artificielle dins ces plantes comme dans les Mamillaria, soit en laissint les ćanines, soit en les enlerant ou en isolant le pistil. On en connait une dizainc d'espéces on de variétés qui pourront probablement s'hybriler et qui, en général, domnent facilement des granes. Le communis parait etre $u$ bon porte-graine que l'on pourait croiser avec le violaceus, le myramidalis, ete. P'eutère même pourrait-on hybrider ces plantes arec les Gerérus et les Mamillaria.

\section{Genre Ĺchinocacte. - Echinocaclus.}

La mème structure florale se retrouve dans les Echinocactus ut les Melocuctus; par conséquent, ce que nous arons dit des autres gemes s'applique également à celui-ci; mais, comme plusieurs espèces ne sourrent que pendant une muit on pendant un jour, el que leurs flemrs se ferment alors tout i lait, il faut choisir le moment convenable pour opérer la fécondation. Il y a déja très-certainement, dans ces plantes, un très-grand nombre d'hybrides, ce qui tient à l'état particulier de leur pollen, qui, 
quoique très-abondant, reste adhérent aux anthères et a besoin naturellement du concours des insectes pour se déposer sur le stigmate. On devra tenter aussi le croisement avec les Cereus et les Melocatus; l' $E$. Ottonis donne très-facilement des graines (grav. 51; 52, 55, 54, 55).

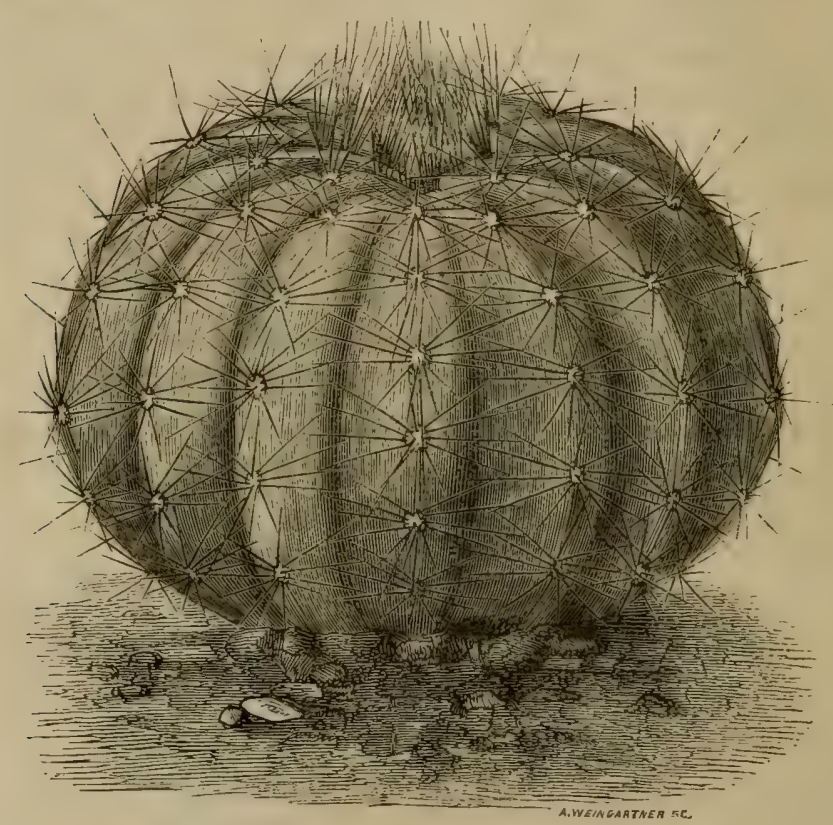

Grav. 31. - Echinocactus 0ttonis, aux deux tiers de la grandeur naturelle.

Genre Cierge. - Cierelts.

Il est peu de plantes qui contiennent un si grand nombre d'étamines que les Cereus, et presque toutes les anthères s'oulvrent en mème temps pour répandre leur pollen. Le stigmate, placé à la même hauteur quue ces organes dans la plupart des espèces, est sourent couché sur un lit de pollen; mais ce dernier est un peu pitteux, et la fécondation manque le plus ordinairement, tandis que, si on l'opère au pinceau, on est presque sûr de réussir. II faut gutler avec attention l'heure de l'é- 
panouissement dans quelques-unes de ces belles plantes, et entourer immédiatement le pistil d'un tube en pinpier ou en carton qui l'isole des ctamines, qu'il serait difficile de retrancher à cause de leur nombre et de la quantité de pollen dont elles sont chargées. On peut alors poser au pincean un pollen étranger, pris non-seulement sur les expèces dn genre, mais encore sur

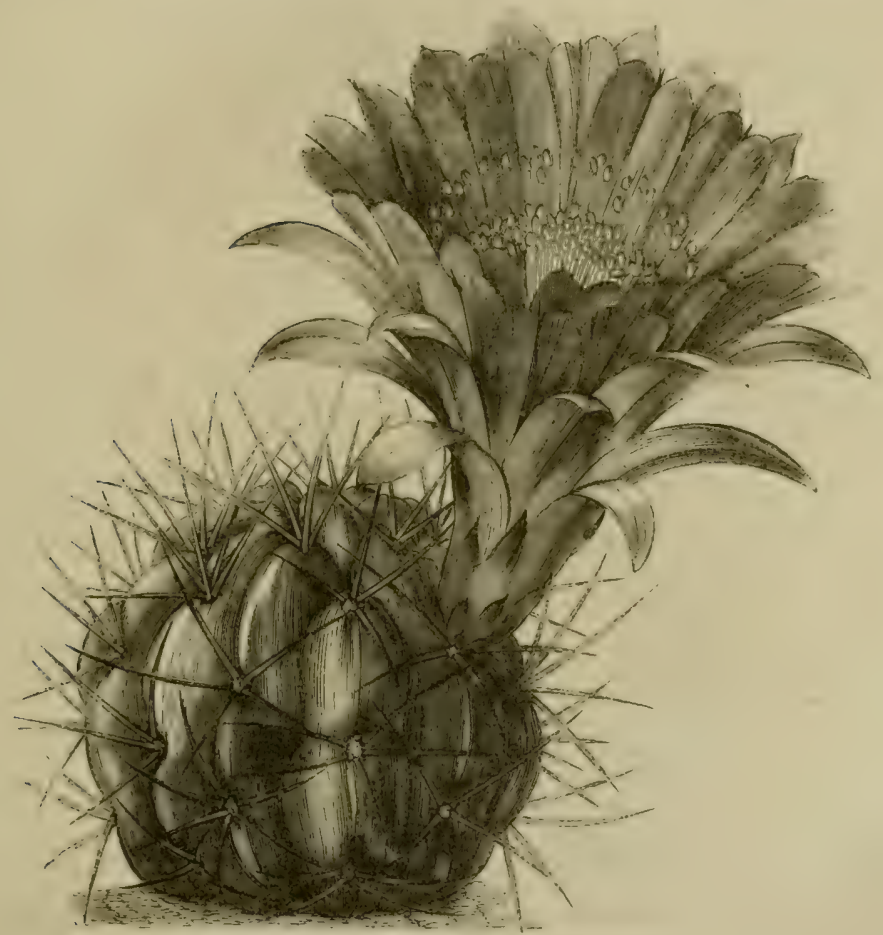

Grav. 5\%, - Echinopsis ou Echinocuctus.

celles des genres voisins, car il est peu de familles où les plantes se eroisent aussi facilement que dins celle des Cactées. Il m’est arrivé, sur cinquante fleurs de Cerens speciosissimus, d'obtenir dix fruits senlement, ct celit paree yue je navais fécondé que dix fleurs soit aree lem propre pollen, soit aree celui de quelyues Eichinocuelus ou d'antres Cerens. Le speciosus 
et le speciosissimus sont deux très-bons porte-graines qui entploient quinze à dix-huit mois, lc dernier surtout, pour amener leurs fruits à parfaite maturité. Il est toujours très-utile dans ces plantes, comme dans beaucoup d'autres, de poser légèrement au pinceau un peu de leur liqueur miellée sur le stigmate.

On a amnoneć il y a environ seize ans, chez Mil. Davies 't $\mathrm{C}^{\mathrm{ie}}$, de Londres, un Cereus hybride du grandiflorus et du speciosissimus; cet liybride est à fleur rouge pàle, de couleur

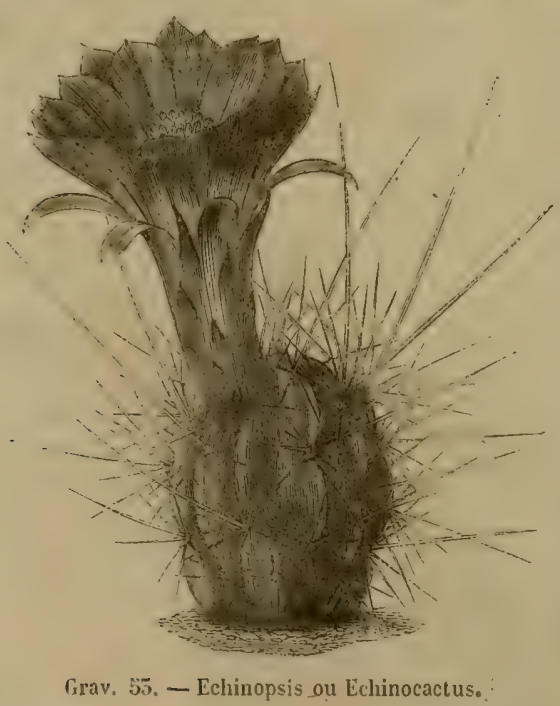

pourpe all centre; les lleurs mesurent vingt-cinq centimètres de diamètre, s'ouvrent ver's sept heures du soir et restent épa nouies la journée du lendemain.

Le mème fait s'est reproduit il y a quelques années seuleınent. On a donné à l'hybride le nom de Gautus Maynardi. C'esi encore le produil du Ciereus speciosissimus aux couleurs si brillantes, fécondé par le pollen abondant de la fleur parlimée du Cereus grandiforus. M. Et. Kenny, jardinier de M. Minynard, et auquel on doit ce remarquilble liybride, lait ob- 
server que cette plante a conservé à peu près la tige et les formes florales de la mère: tandis que son père en a modifié le volume et le coloris, qui varie du rouge vif au plus brillaut cramoisi. Ses fleurs restent ouvertes pendant deux ou trois jours, comme celles du C. speciosissimus.

En 185̃2, II. Jacques a déjà signalé sous le nom de Cereus

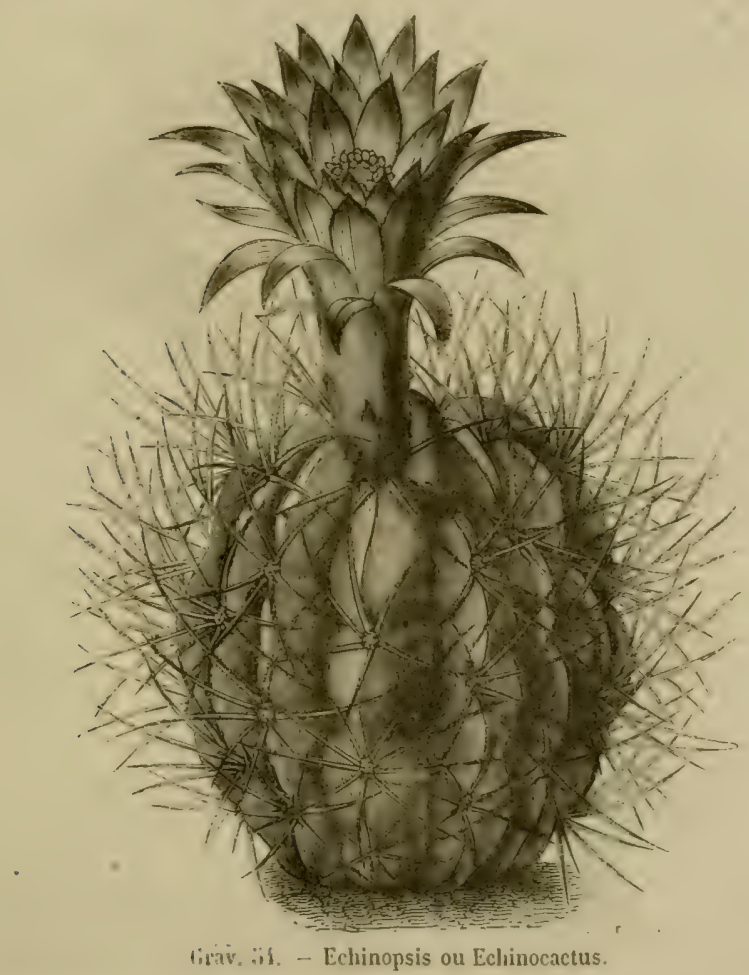

Guillardeti un hybride remaryuable mais accidentel du Gerens: speciosissimus.

En 18 א. I. Cirisart du sianlget a fécondé le Cactus Acliermanni, qui lui-mème est un hỵhide, par le Caches ou Coreus flaygelliformis, et il a obtenu de graines ferliles dix plantes d'itbord eylindriques, mais qui, en croissant, se sont divisées en lit- 
212 FÉGONDATION NATURELLE ET ARTIFICIELLE.

nières plus ou moins épaisses, plus ou moins crénelées ou unies.

M. Grisard décrit sommairement la floraison et le port de ces plantes, toutes différentes, et dont la plupart sont admirables.

“ La diversité de ces fleur's, dit-il, provenues d'une même fécondation, prouve l'inexplicable puissance de la nature el

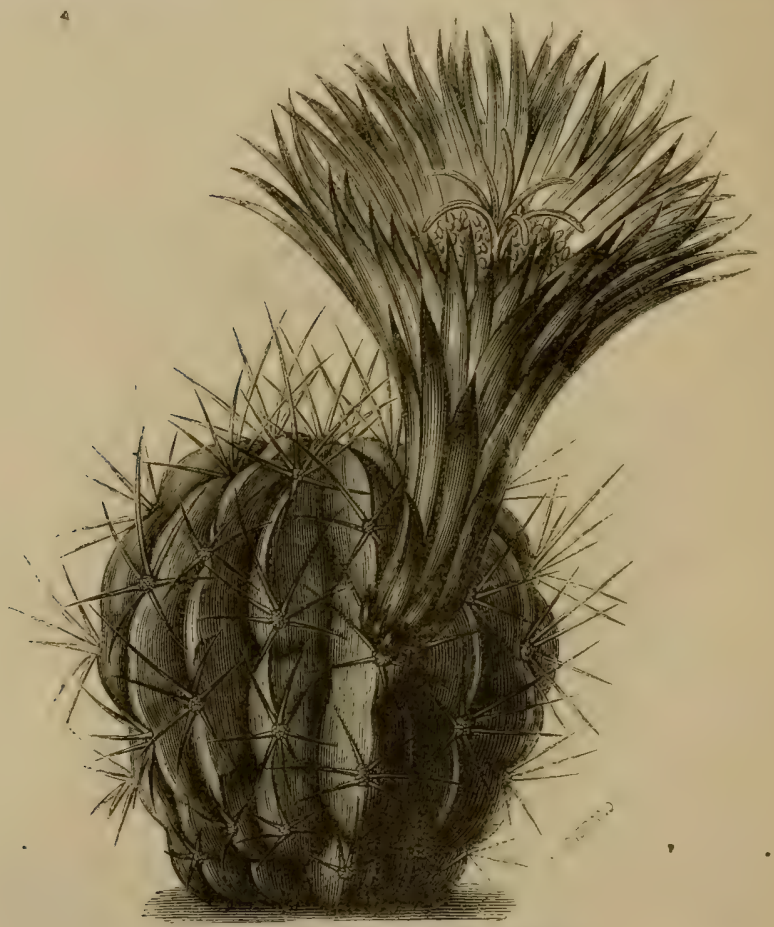

Grav. 5̋̀. - Echinopsis ou Echinocactus.

doit éveiller chez les amatem's le désir d'employer ce moyen. " (Journal de la Soc. imp. et centrale d'horticulture, vol. V, p. 594.)

On annonce aussi, comme gagné par M. R. Herrington, un noureau Cereus à grandes fleurs doubles, d'une belle couleu' rouge, avec une tache couleur pourpre au centre de chaque 
pétale. On le considere comme liybride du longissimus avec le iruncatus.

Que n'obtiendrait-on pas arec cette facilité de croisement des Cereus, si l'on se servait pour l'un des parents de ce magnifique C. Mac Donaldia, indigìne de Itonduras et dont les immenses fleurs nocturnes dépassent en grandeur et en beauté celle du C. grandiflorus!

\section{Genre Raquette. - Opuntia.}

Les Opuntia ou Cactiers raquettes ont encore la même organisation florale que les autres Cactées, mais leurs nombreuses étamines paraissent irritables et répandent plus facilement leur pollen sur des stignates également nombreux; aussi ces plantes domnent-elles plus sourent des fruits que les autres Caclées. Elles exigent aussi un peu plus de soins pour les croisements; il faut s'y prendre plus tôt pour enlever les étamines ou isoler le pistil. Tous les Opmtia ne fleurissent pas dans les serres, et, malgré la bizarrerie de leurs raquettes, ils sont moins recherchés que les Cereus et Echinocactus.

\section{FAMILLE DES GROSSULARIÉES.}

\section{Genre Groseillier. - Ribes.}

Ce genre très-nombreux forme, à lui seul, une petite famille dans laguelle se trouvent des espèces assez distinctes par leur organisation.

Les ụnes sont cultivées pour la récolte de leurs fruits, et les autres pour leurs fleurs, qui, dès le printemp̨s, décorent nos parterres.

Les Groseilliers ont quatre à cinq étamines et un seul style terminé par deux stigmates entiers ou divisés chaciu en deux pauties. La fécondation soprère presque aussitòt que l'épanouissement commence, et elle manque rarement; aussi, pour croi- 


\section{FÉCONDATION NATURELLE ET ARTIFICIELLF.}

ser les espèces ou les variétés, faut-il s'empresser de retrancher les étamines en arrachant les anthères avec une brucelle dès que la fleur' s'entr'ourre. Il ne faut laisser que deux ou trois fleurs sur chaque grappe et couper les autres avec des ciseaux. Le pollen s'applique facilement au pinceau.

Le Groseillier commun ou à maquereau, Ribes wa crispu, a produit maintenant un si grand nombre de bonnes variétés, que l'on ne sait récllement où s'arrêtera cette création. L'hybridation entre toutes ces rariétés s'opère facilement et permet encore d'obtenir du nouveau.

J'ai croisé souvent les plus belles variétés des Groseilliers épineux. J'ai choisi d'abord les plus beaux types anglais et je les ai indistinclement mélangés. Les graines des fruits hybridés, semées en terrines, aussitôt après la maturité du fruit, lèvent très-bien au printemps suivant. Jai toujours obtenu des fruits magnifiques, de toutes les couleurs, de formes variées et souvent délicieux.

Le R. triflorum, le speciosum, le cynosbati, qui appartiennent au mème groupe, pourraient sans doute se croiser entre cux et augmenter ainsi nos arbrisseaux d'ornement.

La section des Ribesia ou des réritahles Groseilliers, dans laquelle se trouvent le G. noir, le rouge et le blanc, ainsi que les beaux Groseilliers sanguins, est la plus nombreuse du groupe. Je présume que l'on obtiendrait des fruits nouveaux en hybridant le Cassis ordinaire par le sangumeum, et, si les fruits n’étaient pas préférables à ceux du cassis, il est probable que les fleurs compenseraient déjà la peine que l'on aurait prise. II y aurait aussi une multitude de croisements à tenter entre ces beaux sangmineum, atrosanguineum, glutinosum, malvaceum, qui ne sont que des variétés, et les nouvelles espèces introduites, comme plantes ornementales, dans nos jardins (grav. 56).

II. Satter, en croisant avec des variétés communes de Groseilliers les fleurs des Ribes pulmutum et aureum, est parvenu à leur faire porter des fruits très-mangeables, sans finire perilre 
a ces arbustes aucune des qualités qui les recommandent comme végétaux diornenent. Jnmmal de ayriculture de Bixio, $2^{\mathrm{e}}$ série, t. III, pl. 156.)

Les especes fruitieres, telles yue le: G. romege, le blenc, lo

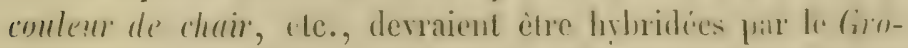

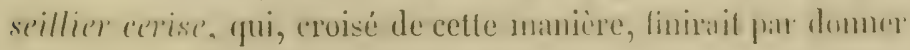

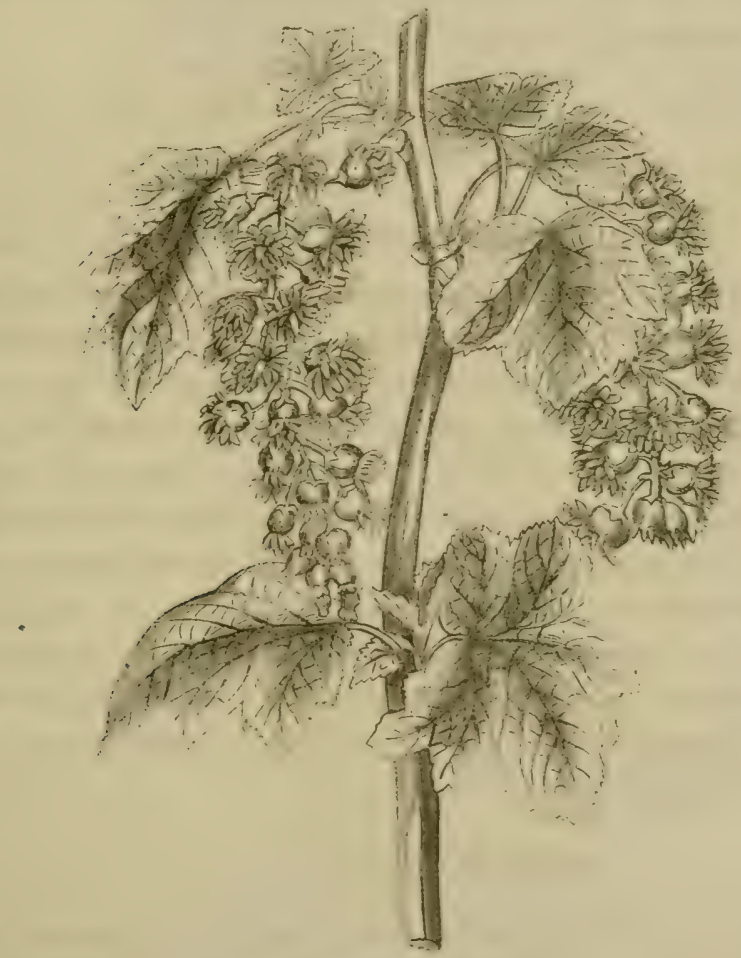

Grav. ili. - Groseillior sanguin.

ansi des varietes hlanches of roses phus rolumineuses encore que celles dẹjà obtenues.

Entin, les li. anrermm al flurum, yui forment encore ume

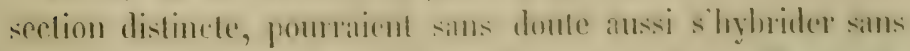

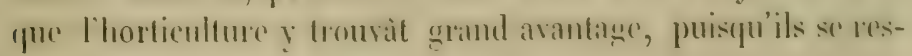
semblent ieja beaucoup. 
Parmi les espèces sauvages, il en est deux, l'alpinum, qui est dioïque, et surtout le petræum, qui, s'ils pouvaient ètre hybridés par le Groseillier rouge ordinaire, donneraient peut-être des variétés de fruits moins acides, et qu'il serait très-intéressant de rechercher.

En résumé, le genre Ribes offre à l'horticulture un vaste champ de recherches et d'essais, quoique déjà il ait été l'objet de nombreuses ohservations.

\section{FAMILLE DES CRASSULACÉES}

\section{Genre Crassule. - Crassula.}

Les Crassules forment un genre nombreux, dont les espèces viennent se ranger sous plusieurs sections assez naturelles, parmi lesquelles on obtiendrait certainement des lybrides, sinon entre les sections elles-mêmes, au moins entre les espèces qui sont placíes dans chacune d'elles. Peut-être même leurs nombreuses espèces proviennent-elles déjà d'hybridations naturelles. Comme dans toutes les plantes de serre, la fécondation artificielle est facile; mais, jusqu'à présent, on s'est peu occupé des Crassules, et surtout de leurs graines, car ces plantes reprennent si facilement de boutures, que c'est le moyen dont on se sert ordinairement pour les multiplier. Elles ont cing étamines et cinq carpelles.

\section{Genre Rochea. - Rochea.}

Les Rochea ont, comme les Crassules, cing étamines et cinq carpelles. On peut, au besoin, se dispenser d'enlever les organes mâles pour poser sur les stigmates glanduleux un pollen étranger; car ce pollen un peu glutineux reste longtemps fixé sur les anthìres, et les fleurs sont très-souvent infécondes.

Il serait donc possible de croiser les Rochea, et ce sont d'assez. belles plintes pour chercher à obtenir des variétés nouvelles. Les falcata, coccinea, odoratissima, versicolor et quelques ra- 
riétés que l'on a déjà obtenues permettraient d'opérer de nouveaux croisements et d'augmenter le nombre de ces beaux végélaux.

\section{Genre Sedum. -- Orpin.}

Les Sedum ont dix étamines et cinq carpelles. Ces derniers sont munis de stigmates qui ne sont aptes que quelque temps après les anthères; en sorte quil est bien probable que la fécondation est indirecte, et comme les fleurs se succèdent longtemps et que leur disposition est presque toujours étagée, la fécondation est à peu près certaine. Cette disposilion rend le croisement très-facile à tenter, et il serait à désirer que l'on pût obtenir des hybrides avec les Sedum Sieboldii, telephium et ses variétés, populifolium, album, acre, refexum, dasyphyllum, pulchellum, cristatum, etc.

L'hybridation ne devrait toutefois être tentée qu'entre espèces à fleurs jaunes d'une part, ou d'un côté, entre espèces à fleurs blanches, rouges ou bleues. Le caractère des feuilles plates ou cylindriques est aussi à. examiner pour ne réunir que les espèces dont les feuilles présentent la même forme.

\section{Genre Joubarbe. - Sempervivum.}

On trouve dans les Joubarbes plusieurs sections distinctes, telles que les arborescentes, les vivaces et les annuelles. Les étamines sont toujours nombreuses et en nombre double des pétales. Elles sont placées sur deux rangs; celles qui sont opposées aux sépales s'ouvrent les premières, mais après l'épanouissement de la fleur seulement; puis viennent ensuite celles de l'autre rang opposées aux pétales, qui répandent aussi leur pollen et assureraient ainsi la fécondation des pistils, si leurs stigmales, comme dans les Sedum, n'étaient pas en retard sur les étamines. On a done tout le temps nécessaire pour enlever celles-ci et essayer des fécondations croisées qui, jusqu ici, n' ont pas été tentées (grav. 58). 
218 FÉCONDATION NATURELLE ET ARTIFICIELLE.

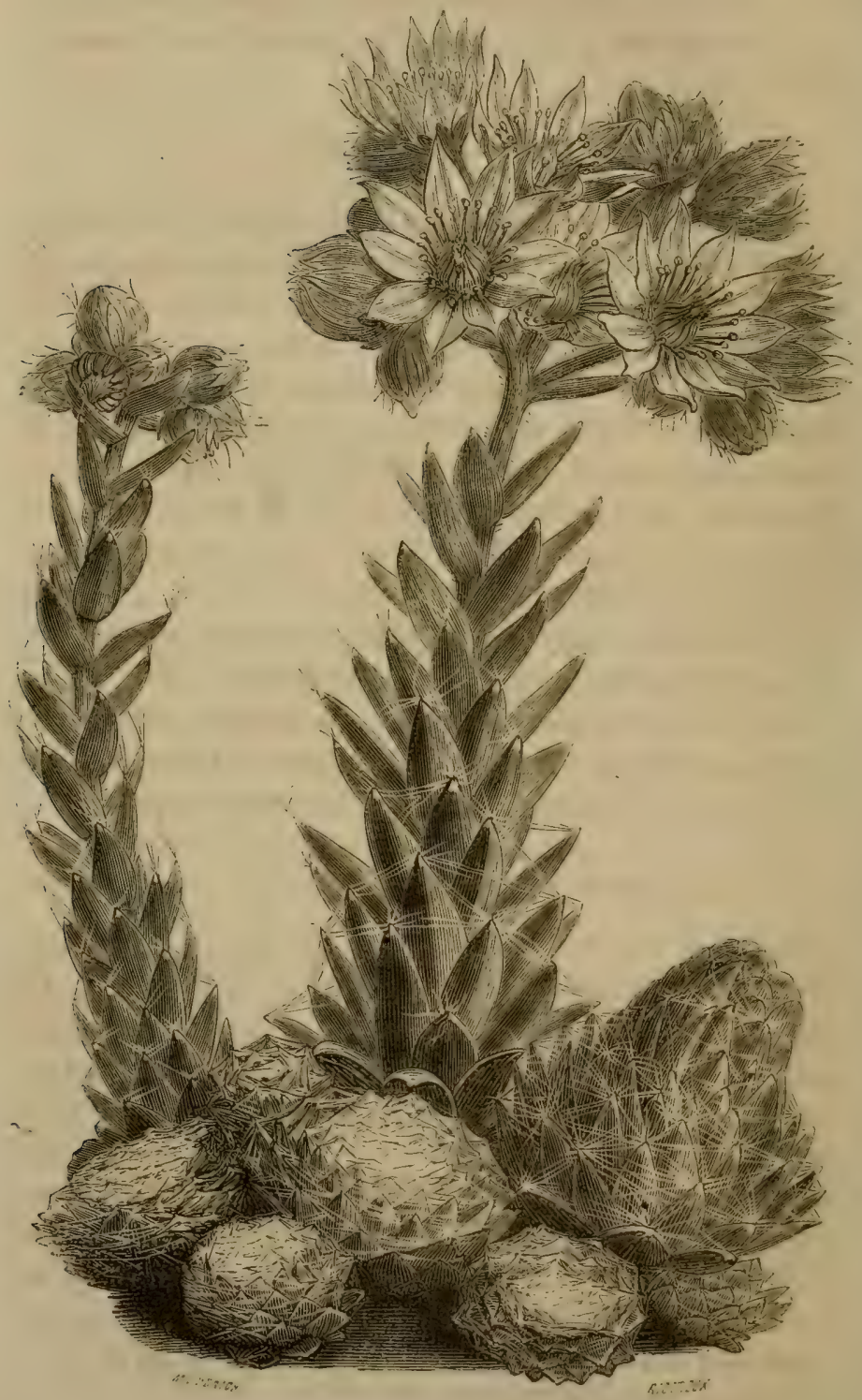

lirav. 57. - Joubarbe tomenteuse, grandeur naturelle. 
Lu's Joubarbes shybrident tout naturellement, et je rapporlerai ici la note que II. Lamolte ni adressait cetle année (1862):

"Arant la publication de mes Notes sur quelques plantes monvelles du platern central, ancun hyglode narait été indi'qué daus le genre Sempervium. Dans ces notes, après aroir décrit une forme inédite de Sempervirum, sous le nom de S. Pomelii, jai énis l'opinion que cette plante derait être un hybride des $S$. arachooideum et arvernense, opinion qui n’ètait fondée que sur la stérilité et sur les caractères distinctilis qui paraissaient être la fusion des caractères principaux des deux espèces précédentes. Depuis lor's, cn étudiant le S. Pomelii dans son lieu natal, $j$ ai aequis la certitude qu’il est un produit anomal dû à la fécondation du S. arachnoideum par le S. arvernense. La première de ces espèces est donc la mère; il ne peut y aroir aucun doute à cet égard, les touffes de rosettes du S. Pomelii étant toujours placées très-près ou autour de celles du $S$. arachnoideum.

" Jai trouvé dans la vallée de Saint-Yectaire, à Champeix, une seconde forme adultérine, produite par les mèmes e:pèces. Mais il est très-probable que pour celle-ci les ròles ont été changés et que le $S$. arvermense a élé porte-graine; car c'est près de lui et à une assez grande distance du S. arcleluoideum que croit ce deuxiène hybride, que javais d'abort nommí S. villosum, mais qui doit, daprès la nomenclature généralement adoptée, prendre le nom de $S$. arvernensi-arachnoidenm, "t la premiere celui de $S$. arachoideo-areremense.

"Outre ces deux hybrides, qui appartienment à l'Auvergne, jai constaté l'hybrulite de trois formes que jai regues des Alpes du Iauphiné, dont l'une a été décrite el publiée comme sepere ligitime. II. Lorret a également fait connatre (Bulletin dela Soc. bolunique, 1. T, p. 147) deux h! Jorides dus i l'union des S. arachnoideum et S. Boutignianum et croissunt dans les Pyrénées. - Le fait le plus singulier et le plus dignes de re.

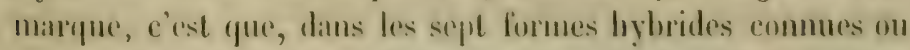
que je ne tarderai pas a fare conmaitre, le $S$. arachnoiderum 
est toujours un des parents remplissant tantôt le rôle de fécondateur, tantôt recevant l'influence du pollen d'autres espèces; c'est le cas le plus fréquent. Tous ces hybrides ont la pointe de leurs feuilles terminée par une houppe de longs poils soyeux, caractère dû à l'influence du S. urachnoideum; tous ont des graines infécondes. Le pollen est bien conformé; certaines loges polliniques sont complétement remplies, d'autres sont presque vides dans la même fleur. Le stigmate est moins développé, moins papilleux que dans les espèces normales.

“ Voici la nomenclature des sept hybrides que j’ai étudiés; elle est provisoire pour plusieurs, qui devront prendre les noms combinés des père et mère, lorsque ceux-ci seront connus. Je les range ici d'après leur plus grande affinité avec le $S$. arachnoideum:

“ S. pseudo-arachnoideum, Lamt., inéd.;

"S. piliferum, Jord.;

"S. Jorlani, Lamt., inéd.;

"S. arachnoideo-Boutignianum, Lorr.;

"S. Boutigniano-arachnoideum, Lorr. (S. rubellum, Lamt.);

"S. arachnoideo-arvernense, Lamt. (S. Pomelii, Lamt.);

"S. arvernensi-arachnoideum, Lamt., inér. (S. villosum, Lamt. olim).

“ Le S. pseudo-arachnoideum est l'hybride qui a les plus grandes fleurs, les plus belles et les plus colorées du genre; le père m'est inconnu, mais il pourrait bien avoir un peu de séve du $S$. montamum dans ses vaisseaux. Les $S$. piliferum et Jorlani ont probablement les mêmes parents, dont les fonctions ont été interverties. M. Lorret a reconnu, dans les localités où croissent les deux hybrides qu'il a décrits, que le père de l'un était la mère de l'autre, et réciproquement; il doit en être de même des S. Pomelii et villosum. »)

Outre ces hybrides indigènes, on a cité un hybride obtenu par Doukeclaar et provenant du croisement du S. speciosum avec le $S$. tabulæforme. 


\section{FAMILLE DES SIXIFRAGÉES.}

\section{Genre Hydrangée. - Hydrangea.}

Nous ne mentionnerons ici l'Hortensia et les autres especes de ce genre que pour appeler l'attention des horticulteurs sur la stérilité de ces plantes. Jusqu'à présent, ni l'Hortensia, ni le niv'rilis, ni le quercifolia, ni le rosea ou japonica, récemment introduit, ne présentent toutes leurs fleurs bien conformées. Si les étamines existent, le pistil avorte, ou du moins les stigmates manquent ou sont incomplets. Il faudrait done rechereher si l'on peut décourrir quelques fleurs femelles bien conformées, et les féconder au pinceau avee les étamines qui seraient alors en bon état. Une bonne préparation du sujet pourrait, dans cette circonstance, comme dans beaucoup d'autres, amener sa fructification.

Si l'Hortensia.est ordinairement stérile chez nous, il peut fructifier dans des climats plus chauds; c'est ce qui est arrivé aux îles Borromées. II. René Rovelli, ayant retranché une par. tie des fleurs de l'Hortensia, a obtenu de celles qui restaient et qui avaient acquis plus de vigueur des ovaires qui grossissaient visiblement. Il a opéré artificiellement la fécondation de ces fleur's arec celles de l'Hydrangea japonica, et alternativement, mais il a observé que les capsules de l'Hortensiu étaient beaucoup plus grosses et plus rondes que celles de l'Hydrangea. Un seul corymbe porte plus de cent fruits. "Jai remarqué, dit II. Rovelli, que, sur les corymbes qui ont été déchargés d'une partie de leurs fleurs, celles qui furent conservées formèrent en peu de temps, à la base du pédoncule, une espèce de collet qui tomba plus tard. Je conclus donc de ce qui précède que c'est ordinairement à un trop grand atsicmblage de flen's sur un mème corvmbe quil faut attribuer lat sterilité de l'Hortensiu. ")

Genre Saxifrage. - Saxifragu.

Ces plintes forment un des gemres les plus nombreux du ré- 
gne végétal et présentent toutes une organisation florale tresanalogue : dix étamines et deux styles terminés chacun par un sligmate. Les deux styles sont généralement appliqués l'un con-

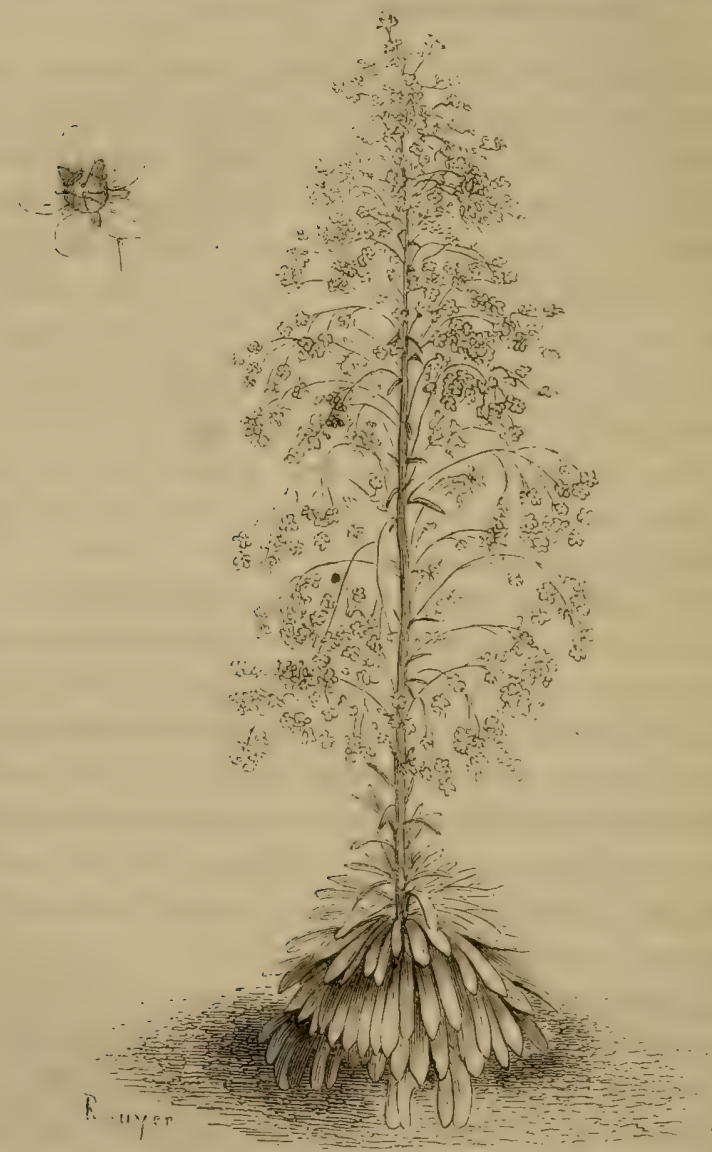

lirav. :38; - Saxifrage pyramidale au quart de la grandeur naturelle. Fleur isolée de grandeur naturelle.

tre l'autre; puis ils écartent les stigmates pour les rapprocher des étamines à l'époque où ils sont aptes, mais il est rare alor's que les étamines le soient; en sorte que la fécondation est indirecte, et les fleur's, rendues monoïques et presque diö̈ques de 
cette maniere, admettent facilement un pollen etranger. C"est peut-être a celte cause quil fint altribuer le gramil nombre d'especes de Siavifrages qui conrent les rochers ef lés éboulements des hautes monlagnes. la fécondation artificielle ne présente aucune difficulté, mais ju ne sache pas que, jusqu'a présent, elle ait été pratiquée, ni sur le gramulata it flems double's, ni sur les crussifolia, corduta, ligulatu, crocen et autres helles especes que l'on cultive dans les jardins. Ce sont, en général, de trés-belles plantes printanières qui mériteraicul d'altirer l'attention des horticulteurs (grav. วิ8).

\section{FAMILLE IIES OMBELIIFERTES.}

\section{Genre Astrance. - Astrantia.}

Dunique les (Imbelliferes senlerment peu de plantes douneneme, on un peut reluser ce titre aux Astremtich gui sont tresClequantes, qui offrent déja des variétés dans li nature, et quï, par consinguent, en offriratent sans doute par la culture. Elles: domment lacilement des graines. Lenrs cing étamines sont plicés sur les filets avant l'anthise, et les deux styles et stimmales sont aptes à peu près à la mème éporque. Il y a sourent des lleurs unisexuces dans les Astrances, en sorte que lit fécondation artilicielle serait licile, en choisissant les fleurs fémelles pour les imprégner. Il faudrait, si l'on roulait essayer les croisements sur le major, choisir de préférence l'ombelle centrale, dont les fleurs sont toujours mienx conformées, et. si l'on opérait sur. l'Astruntia minor, choisir les lleurs fémelles qui se trouvent dans les ombelles latérales et supprimer tout le reste.

Genre sche. - Ipium.

Le Persil et to Céleri offrent chacum plusieurs variétes, dont le nombre pourait sans doule ètre augmenté par des croisements, et ceur-ci sunt faciles à opérer entre les variétés 


\section{FÉCONDATION NATURELLE ET ARTIFICIELLE.}

de chacune de ces espèces. Comme dans ces plantes les stigmates ne sont aptes qu'après l'ouverture des anthères, il faut, après avoir préparé le sujet par un très-grand nombre de retranchements, ne conserver qu'une partie de l'ombelle principale, ne prendre, dans cette ombelle, que les rayons de la circonférence, et dans les ombellules du dehors enlever encore les lleurs centrales, qui donnent des graines moins vigoureuses. On retranche soigneusement les étamines dès l'épanouissement et avant l'anthèse, et l'on attend, un jour ou deux, que les stigmates soient développés pour y poser le pollen étranger.

Les Persils commun, frisé, de Naples, nain, très-frisé et i grosses racines, pourraient être hybridés, et parmi les Céleris, le plein blanc et le creux, le tardif, le Céleri rave, sont dejjà des modifications sur lesquelles des fécondations croisées ne manqueraient pas d'en produire d'autres.

\section{Genre Carotte: - Daucis:}

La section du genre Dutcus, qui renferme les Carottes cultivées, contient encore plusieurs espèces que la culture pourrait peut-être rendre potagères, et avec lesquelles on derrait tenter des croisements sur l'espèce ordinaire; d'un autre còté, cette dernière offre déjà de si nombreuses variétés, que bien certaincment leur croisement en fournirait encore d'autres, et ce genre a élé peu travaillé par les horticulteurs. Indépendanment de ses qualités comme plante potagère, la Carotte a une grande importance comme espèce agricole, à cause de sa racine essentiellement alimentaire. Elle mérite donc l'attention des cultivateurs. Le mode de croisement est le même que pour le Persil et le Cerfeuil, dont nous venons de parler.

La Carotte blanche et celle à collet vert sont cultivées très en grand pour la nourriture du bétail, et parmi les variétés culinaires on distingue principalement les suivantes:- Rouge hâtive de Hollande. - Rouge longue. - Rouge prile de Flandie. - Janne courte. -- Janne longue. - Violette d'Espagne. Quelques-unes des variètés que l'on obtient en croisant lia 
linolte sanvige avec celles qui sont cultivées, montent en grame dès la première année, si on les sème trop tard, remarque déja laite par Vihnorin, daus ses Essuis sur l'amélior tetion de la carotle sentange non croisée. On obvie à cet inconvénient, en semant seulement à la fin de mai.

de ne meétendrai pas davintage sur la fécondation des Onbellifères, qui s'oprire à pen près toujour's de la mène manière. Les tilets allongés se dédoublent pour élever leurs anthères à l'époque oi eltes rejandent le pollen. Les stigmates sont aptes quelquefois an moment de l'anthèse des étamines, et le plus ordinimenent, ils ne le deviennent quapris, en sorte que diuns ces plantes la fécondation est indirecte, et ce sont les fleurs du second ou du troisieme rang de l'ombelle qui fécondent le premier rang. Les étamines de ce premier rang sont donc inutiles, et ce sont aussi les lleurs qui ont les pistils le mieux conlormés et celles qu'il faut féconder pour avoir de

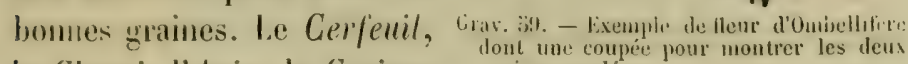
le Chervi, l'Anis, la Corian-
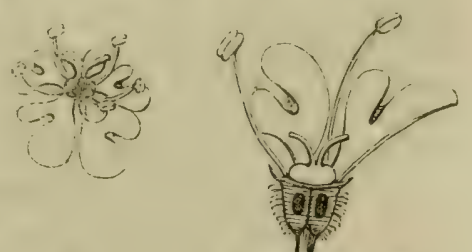
ovaires soudés.

dre, le Pennis, et plusiems antres gemes ont encore de

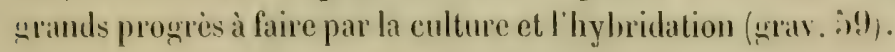

\section{FAMLLE IUS ARULACEES.}

Genre Aralie. - Iralia.

(1) cullive inaintenant un grand nombre d'Aralia dont le Japon a l'oumi les principales espèces. Un ou deux seulement lleurissent dans nos jardins, et l'on parviendrait peut-ètre à les farre fructitier par fécondation artificielle. Si, comme on doit le supposer, les antres especes viennent aussi a fleurir dans nos serres, on pourra tenter les croisements en retranchant de hom heure les ciny chanimes et portant le pollen étranger sur 
les cing styles et stigmates des fleurs. Il faudra, comme dans les Ombellifères, enlever un grand nombre de boutons.

\section{FAMILLE DES CAPRIFOLIACERS.}

\section{Genre Viorne. - Viburnum.}

Ces arbrisseaux pourraient sans doute s'hylurider en essayant entre les espèces qui se ressemblent le plus, comme par exemple entre le timus, le rugosum el le rigidum, entre le lantana et le lantanoüdes, etc.

Il serait à désirer que l'on pût trouver, dans les Boules-deneige cultivées, quelques fleurs fertiles ou seulement munies d'un pistil, mais elles semblent toutes complétement stériles. Ne pourrait-on pas essayer de bonue hemre de retrancher la majeure partie des boutons sur quelques corymbes? Pour croiser les Viburnum, il faut d'ailleurs leur cnlever la majeure partie de leurs lleur's et laisser dans l'ombelle celles qui ont les: pistils le mieux conformés, car un grand nombre avorte toujours par l'imperfection de cet organe. Les extérieures sunt quelquefois mâles ou privées de stigmates; le choix des fleur's étant fait, enlever de suite les cing étamines et poser ensuite le pollen au pinceau sur les trois stigmates.

L’introduction assez récente du $V$. macrocephalum et de quelques autres permeltra peut-être d'obtenir des arbrisseaux encore plus beaux que la Boule-de-neige ordinaire, malgré toute sa fraîcheur et toute son élégance (grav. 60 ).

\section{Gemie Chèrefenille. - Lonicera.}

Les nombreuses espèces de Chèvrefeuille n'ont pas pu, selon toute apparence, s'hybrider naturellement, car il est peu de plantes dont la fécondation soit plus sûre. Elle s'opère dans l'intérieur de la corolle avant l'épanouissement. ILes cinq étiinines ont leurs filets courbés, et les anllières répandent leur pollen sur le stigmate, qui porte un style généralement assez 
PAMLLE DES CAIRIFOLIACEES.

lony ; quelyudois cependant lat ficontation se termine à l'air

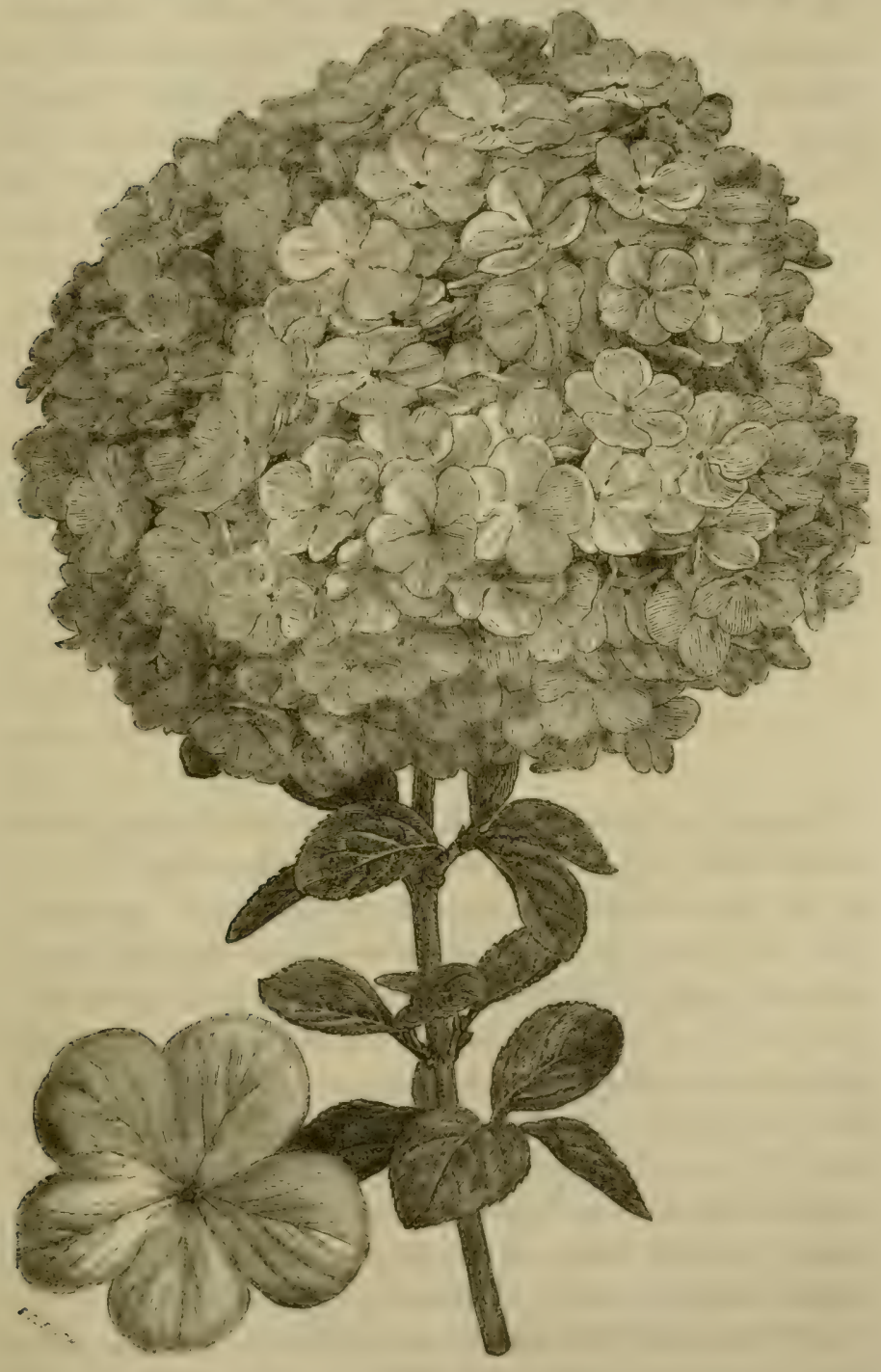

(irav. 60. - Vierue à grosse tc̀te.

libre. Si l'on roulait essayer les croisements, il fitudrait done 
retrancher les anthères avant l'épanouissenent, et imprégner' ensuite le stigmate d'huneur miellée et de pollen. On cultive maintenant de chrmantes espèces de Chèvreféuille et de Chamécerisier, qui lorment une section particulière dans ce genre. Ces plantes, de section différente, ne se croiseraient probablement pas entre elles, mais tout porte à croire que les espèces de chaque division s'hybrideraient. Les Lonicer'ı etruscu et Caprifolium, qui fructifient si bien dans nos jardins, pourraient ètre croisés avec le sempervirens, le llexuosa, le Ledebourii, le japonica, le pubescens, etc.

Les Chamxcerasus, moins nombreux, offrent aussi de belles espèces à hybrider, tels que ceux de Tartarie it grandes flents roses, et sa variété blanche obtenue par M. Irovost, avec le xylosteon, l'alpinum, etc.

ce sont des plantes qui, en général, ne sont pas assez cultivées.

\section{FAMILLE DES DIPSACEES.}

\section{Genre Scabieuse. - Scabiosa.}

Dans ce genre très-nombreux, les fleurs sont réunies en cilpitules serrés et présentent, comme dans les Synanthérées, tantòt des fleurs hermaphrodites, tantôt des fleurs unisexuées. Elles ont quatre étamines et un seul pistil terminé par un stigmate simple, élevé hors des fleurons dans les fleurs fertiles, inclus dans celles qui sont stériles. Les étamines, au contraire, sont saillantes par le dédoublement spontané des filets, quand elles contiennent du pollen, et incluses quand elles sont avortées. Il a presque toujours, dans le même capitule, les trois sortes de fleurs, et quelquefois même tout à fait à la circonlérence, des fleurs stériles et privées d'organes sexuels, comme dan's les Boules-de-neige, les Bluets, etc.

La floraison des Scabieuses commence presque toujour's par li partie moyenne du capitule, rarement parla circonférence, jamais par le centre. La floraison continue ensuile par les verticilles su- 
périeurs et inlérieurs it ceux qui occupent la partie moyenne.

La léeondation artificielle pent sopérer facilement sur les lleur's femelles, en ayant soin de retrancher entièrement les fleurs mîles, sur le capitule conservé pour l'opération. II laut, autant que possible, choisir les lleurons femelles les plus extérieurs et non ceux du centre qui sont toujours moins robustes.

Le Scabiosa atropurpurea, qui offre un grand nombre de rariétés, en produirait sans doute de nouvelles en croisant celles que l'on a déjà obtenues; d'un autre còté, ce genre est si nombreux et présente des espèces si voisines, que très-probablement on parviendrait à les hybrider en opérant avec quelque soin. Si les hybrides des plantes annuelles n'ont pas un bien grand intérèt pour l'horticulteur commerçant, elles peurent procurer

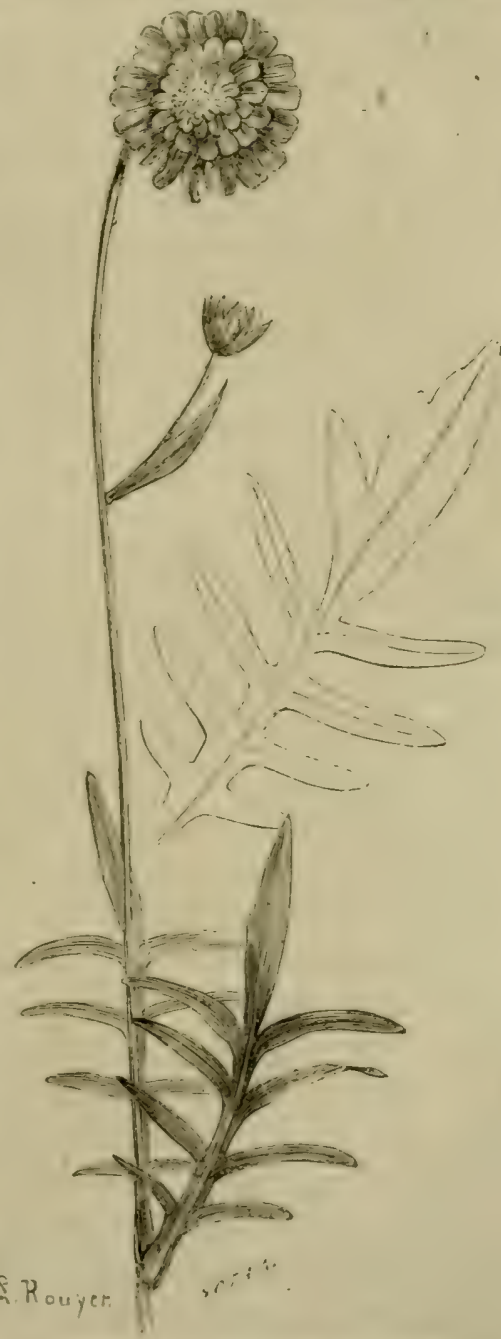

firav, 61. - Sealieuse des clamps. a l'amateur le snjel de tres-interessuntes olservations et des

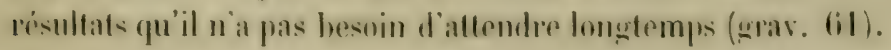




\section{FUILLE DES COUPOSLES OU SYNINTIETEELS.}

\section{fremre Aster. Reine marguerite. - Asler.}

Ces fleurs ont, comme la plupart des Radićes, une couronne de fleurons entièrement femelles, et un disque composé de fleurons hermaphrodites: de telle sorte que, non-seulement les cing itamines des fleurons du centre fécondent leur propre pistil, mais encore le pistil des lleurs femelles gui les entourent. On peut done espérer de faire facilement des hybrides dans les Asters, qui composent d'ailleurs un gemre tellement nombreux, que l'on peut supposer des espèces formées par croisements naturels.

Tous les essais que nous avons faits diuns ce genre ont été tentés sur une seule espèee, la Reine marguerite des jardins, qui offre maintenant de si belles et de si nombreuses variétés.

La structure des fleurs de la Reine margucrite simple est la mème que celle des autres Lsters; une courenne de fleur's femelles, un disque couvert de fleurons hermaphrodites. Par la culture, la fleur a changé; le nombre des ravons de la courronne a augmenté, et l'on est parrenu à obtenir des fleurs toutes formées de fleurons plats ou ligulés, de couleurs trèsvariées et présentant seulement l'organe fenelle, qui est presque toujours bien conformé dans ces fleurons. II reste ordinairement dans le centre quelques fleurons en entounoir, jaunes et pourvus d'étamines; parlois cependant ils mancuent entièrement, mais les petites fleurs du centre ne sont pas entièrement en languettes et conservent encore des élamines fertiles.

D'autres variétés de Reine marguerite ont quelques lleurons en languettes yui forment une couromie à la circonférence, et tont l'intérieur cu fleurons tubulés trìs-grants et très-heaux. Enfin, il ch est grii ont tous lis fleuruns en entomoir, comme la variété dite Anémone ronye de Russie, et qui est cependant très-prolifèr?. Dans ees dernières variétés, prestgur tous lis 


\section{FIMIITE DES COMIOSERS OI STYAXTIIEREES. 2JI}

fleurons fulsulés sont munis de pistils bien conformés, mais sourent les étamines sont arortées, et les plantes donnent beaucoup moins de graines que les simples et les semi-doubles, dont la lécondation est assmée par les ctamines qui entourent le pistil.

On troure aussi plusieurs de ces fleurons développés outre mesure, et formant par leur réunion, d'admirables fleurs dépourvues de stigmates, parfois, mais rarement, de toute espece d'orgaues. Dans ces organisations diverses, qui empèchent ynelquefois totalement la plante de grener, on voit que l'on peut toujours arriver artificiellen.cnt à féconder les pistils. Il suffit de prendre le pollen sur de belles plantes et de le porter : $u r$ les stignates de la couromme qui manque d'étamines. Si lon peut le faire, il est préférable de prendre du pollen dans les fleurons en entomoir qui sont placés vers le centre de la fleur. Il serait très-difficile de féconder les stigmates des fleurons jaunes des semi-dloubles, car en sortant du tube stamifère ils sont dẹjit imprégnés, et, d'ailleurs, les graines, provenant des lleurons du centre, sont loin d'ètre aussi bonnes que celles qui proviennent de fleurs femelles de la circonférence.

Vilmorin, dans le Bon jardinier, conseille de recueillir les graines de Reine marguerite, sur les petites fleurs latérales, et il assure, avee raison, que l'on a plus de chance d'avoir enstlite des plantes à fleurs doubles. Si l'on fait attention à la struclure de ces fleurs, on remarque hientòt que ces petites fleurs sout généralement doubles, mème sur les pieds qui n'ont que des fleurs semi-doubles, et les fleurons sont presque tous femelles. Il laut donr qu'ils soient fécondés par les fleurs voisines, el e'est en effet ce qui a lieu le plus somvent. La fleur terminale de la plante, et qui s'épanouit la première, est la moins pleine, elle conserve presque tonjours quelques-uns de ces lleurons jaumes qui ne sont jamais stériles. Ciest cependant la fleur la plus large ef lit plus vigoureuse. Or, l'ohservation de Vilmorin devait laire supposer que les graines provenant de fleurons mumis de corolle domnaient plutòt des plantes à fleurs 
doubles que celles qui avaient été produites par les petits fleurons jaunes. Cette supposition vient d'ètre pleinement confirınée par l'expérience ingénieuse d'un de mes amis, horticul-

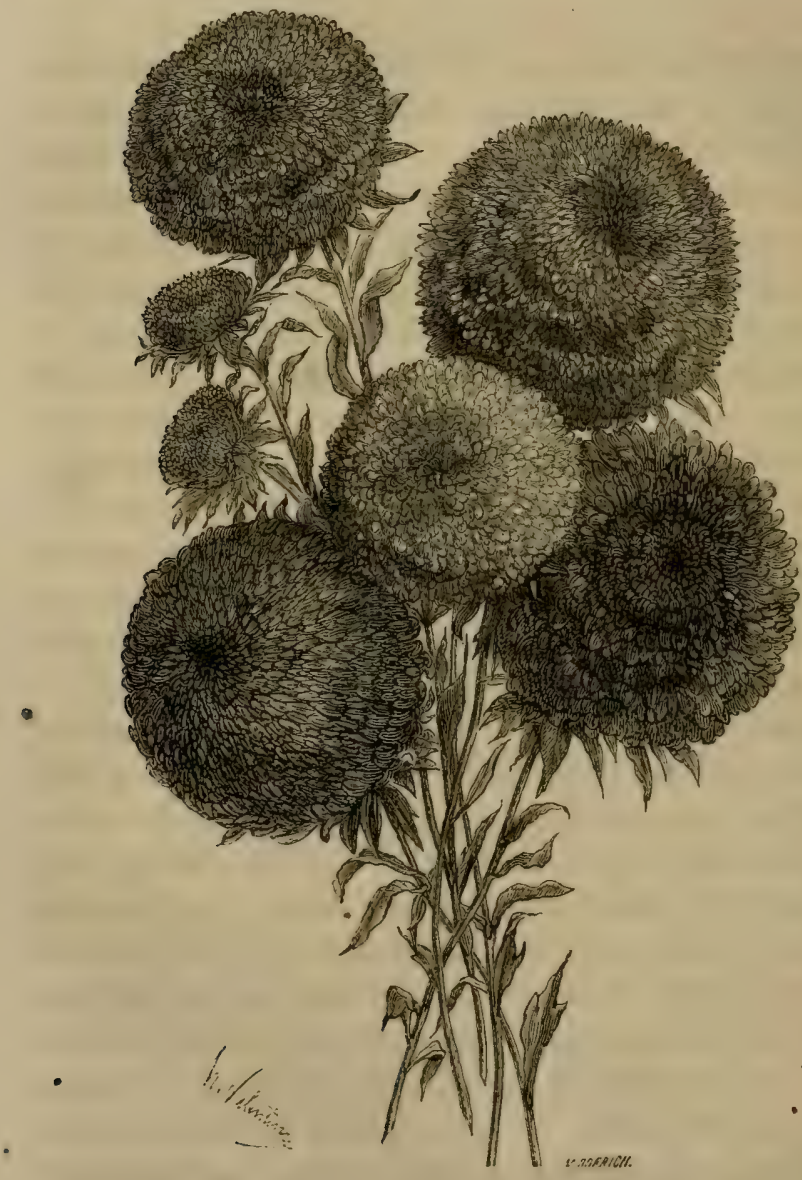

- Lìn, 62. - Reine marguerite pyıamidale it fleurons ligulés.

leur des plus distingués, le docteur Maisonneuve, I'Ambert. Ilabitué à manier le scalpel, il n’a pas craint d'opérer les lieines marguerites et de leur enlever, des le commencement 
de l'épanouissement, tous les fleurons jaunes du centre. Dès lors il a été sùr de n'obtenir des graines que des fleurons de lit circontérence, et il est parvenu, de celte manìre, à créer des plantes yui donnent presque toutes des fleurs doubles, i conserver et ì améliorer les belles rariétés pyramidales à pétales plats, rui sont encore extrêmement rares. La Reme marguerite doit arriver ainsi à un haut degré de perfection. J'ai opéré sur elles lés fécondations artificielles arec lout le succès possible, et jai même tondu des fleurs tubulées de manière à mettre leur pistil an niveau du tuhe, pour y appliquer plus facilement le pollen (grav. 62).

\section{Genre Marguerite. - Bellis.}

Ciette jolie plante, dont on connait maintenant un grand nombre de variétés, serait sans doute susceptible d'en produire plusieurs autres, et c'est une des espèces qui mérite le plus les soins que l'on voudrait lui donner.

Notre Piatquerette peut donner des graines par la férondation intificielle. Toutes les variétés sont munies de pistils qui, ì lat verrité, manquent souvent de stigmates et rendent les fleurons infertiles, mais il y a toujours, dans un capitule, assez de llenrons bien organisés pour donner des graines. Dans les espèces dont les lleurons tubulés se fendent, c'est-i-dire dans toutes less varietés intres que lia rouge ordinaire, les pistils peuvent itre atteints au pinceau, en écartant simplement les fleurons. bans la ronge, il faut les couper aux deux tiers ou les fendre avec une aiguille. Le pollen ne se trouve pas sur toutes les lleurs; il est plus abondant daus la variété toute hlanche, puis dinns la rose on panachée. On ne pent l'obtenir qu'à la fin de la floraison du capitule, de sorte qu'il faut lemplover sur d'autres rappitules moins avances, où les stigmates soient en bou ital.

La Mere-gigngne, en retranchant ses fleurs latérales, devienIrait peut-ètre un bon porte-graine. On trouse aussi, in létit

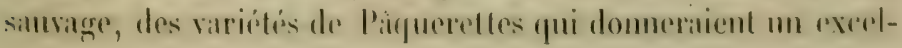


lent pollen, et peut-être parviendrait-on à croiser le Bellis pevennis de nos jardins avec l'ammu, si frais et si florifère, qui tapisse si élégamment les pelouses des bords de la Méditerranée.

\section{Genre Dahlia. - Dahliu.}

Le Dahlia est, sans contredit, la plante ornementale yni a le plus occupé les horficulteurs; il dispute le pas à la Rose et au Camellia, mais l'emporte sur tous deux par l'inconcevible variété de ses couleurs. Le hasard a pendant longtemps été le seul guide pour le croisement des Dalulias, ou plutôt les mouches et les insectes peurent, comme pour les Tulipes, revendiquer l'homneur des premières hı̣ bridations. On est arrivé à un tel point de perfection pour cette fleur, qu'il est impossible d'agir de la même manière, mais on est sûr an moins de créer presque toujours de belles plantes par le procédé que je vais indiquer, et que jai moi-même mis en pratique avec succis.

La fleur du Dahlia double, le seul dont nous ayons ì nous occuper, est composée d'une nombreuse série de rangées concentriques de pétales en languettes ou en cornets plus ou moins fermés. Tous les rangs extéricurs sont neutres, c’ést-idire qu'ils ue contiennent ni étamines ni pistils; mais à mesure que les rangs approchent du centre, on commence à trouver, dans l'intérieur de quelques fleurs seulement, des stigmates à deux branches un peu écartées. Le rang tout entier est loin d'ètre muni de stigmates; le plus intérieur en contient quelquefois un plus grand nombre; enfin, ce n'est qu'en approchant successivement du centre que l'on peut espérer d'en rencontrer quelques-uns. $\Lambda$ mesure que ces organes se mulliplient, la grandeur des cornets qui les renferment diminue: en sorte que la vigueur de l'organe femelle est presque torjours en raison inverse de celle de la corolle qui le contient.

En dedans des cornets, la plupart des Dallias finissent par 'laisser sortir quelques fleurons jaunes, mumis d'étamines et de pistils aree des stigmates bien conformés, et qui donnent tris- 
facilenent des graines fertiles. It y a rependant des variétés qui manquent tont à fiit de ces fleurons, et ce sont généralement les plus helles; elles ne montrent jamais leur cenur. Les cornets qui contiemnent des stigmates manqueut souvent dans les quimze à ringt rangées extérieures, et jai ru des fleurs qui n'en offraient en tout que trois ou quatre. Le choix des plantes dins lesquelles on peut rencontrer un certain nombre de stigmates est indispensible pour les porte-graines.

Lne fois le pied mère choisi, on lui coupe une partie de ses lnanches, an moment où il va commencer à fleurir, et l'on réserve seulement huit it dis lleurs qui doisent produire les graines.

A mesure que la floraison s'opère, on cherche dans les cornets ceux qui ont des stigmates, ou, si l'on reut ériter cette peine, on plonge le pincean, couvert de pollen, dans tous les cornets des rangées intérieures, en recommençant plusieurs fois de suite, afin de ne pas manquer la meilleure époque pour l'imprégnation. Quand les fleurons jaunes du centre paraissent, on les enlève un à un asec la pince avant le développement de leurs étamines. Il est bien entendu que sur les plantes qui doivent produire te pollen, on les laisse, au contraire, se développer librement, mais it arrive aussi quelquefois, quoique rarement, que l'on trouve des étamines dans des cornets voisins du centre, et leur pollen doit être préféré.

Quand la fleur fécondée commence à se flétrir, on coupe avec deseiseaux tous lescornets, en commençant par les plus extéricurs et finissant, quelques jours phos tard, par ceux qui sont les plus voisins du centre, at que l'on doit particulierement ménager. si l'on négligeait cette précantion, les phuies feraient une masse compacte et boneuse de ces cornets flétris, et les graines seraient exposées à pourrir, au moins dans nos elimats. Si on les arrache, an licu do les comper, on s'expose à enlever en même tempss les jounes ovaires qui ne sont pals encore assez allherents. Ces opúrations terminées, il faut attendre patiemment la maturité des graines, qui doit arriver en septembre, 
car les pieds mères et ceux qui doivent fournir le pollen ont besoin d'être plantés de bonne heure pour fleurir en juillet ou au commencement d'août. Les graines restent assez longtemps ì mûrir, et si la gelée arrivait avant leur maturité, il faudrait couper les branches et les suspendre dans im appartement sec et pas trop chaud, où elles finissent de mûrir. On obtient, par ce procédé, fort peu de graines, mais toutes lèvent sans jamais manquer el presque toutes donnent de belles plantes it lleurs pleines, parmi lesquelles on peut retirer plusieurs bonnes variétés nouvelles: Les fleurs du centre domment aussi quelquefois des lleurs doubles, mais bien plus rarement que les autres.

Les Dahlias blanes, jaunes, et les variétés pointées et hordées sont celles qui doivent servir de préférence de picds mères, en les fécondant avec toutes les autres nuances.

Les plantes obtenues des semis, et qui présentent des nuances nouvelles, ne doivent pas être rejelées, quoique de mauraises formes; leur croisement avec des plantes de couleurs voisines, mais bien faites, peut donner de tris-bons résultats, et leur semis, sans fécondation artificielle, peut aussi amener, dans li mème coulcur, des fleur's mieux formées on des plantes d'ume meilleure tenue.

Malgré tout ce que l'on a fait sur les Dahlias, il y a encore heancoup à espérer; et, si les unicolores ont tous été obtenus, re que je ne pense pas, les pointés, les rayés, les bordés sont encore dans l'enfance, et les maculés ou à taches fondues, comme cerlaines Balsamines, sont encore ì naitre.

\section{Genre Kinnia. - Zimvifl.}

Les Zimia out, comme la plupart des Radiées, une couromne de lleurons en languette, portant des graines, sans étamines, ef des lleurons en entomoir qui sont hermaphrodites. Ce sont ees derniers qu'il faut enlever avec des pinces à mrsure qu'ils sont sur le point de s'ipanouir. On fécomile alors les. 
stignates des fleurons en languette, et l'on obtient des graine's bien conformées. Jïnnore si les différentes espoeces de Zinniu preuvent se féconder entre elles, mais les variete- déja nomhrenses shishrident avec la plus grande facilite, et lon prent obtenir toutes les teintes comprises entre le muge, le violet. le jaune pàle et le blane.

L'apparition toute récente des Zimnia a llents doubles vient ourrir un viste champ productif i tous ceux qui voudront soccuper de leur hybridation. In ne posside encore qu'un trispretil nombre de nuances de ces helles lleurs. Le croissment arec les Zimnia simples, diversement colorées, amèner: bientot des coloris nouveaux. Si, comme je le suppose, les flenrons des Zinnia doubles ont conservé leur pistil bien conlimé, la livrée future des Zinniu arrivera peut-itre i luller arế la varieté des bahlia et de nos antres plantes de collection.

\section{Genre coreopsis. - Cioreopsis.}

L.hylnidation est dillicile dans ce genre, parce que les demi. lleurons, yui forment la couronne sur lesquels s'opère ordinairement la fécondation artificielle dans les Composées sont neutres dans toutes les espèces.

Ce ne est done que sur les lheurons du prenier rang extériem yu'il faut agir, et il n'est pas aisé d'enlever les étamines arrant quielles naient répandu leur pollen; aussi, comme jusqui present on n'a pas, à ma connaissince, cherché à croiser les: Correopsis, les variétés nbtcunes l'ont été par hasard, et ne sont pent-itre pas le résultat de croisements même alceidenteds.

\section{Genre Chrysanthème. - Cihrysanthemum.}

Après le Buhlia, le Chrysinthème est la plante la plus ormementale de la lamille de's Composées. Elle a acquis maintenamt un degré de perfection renarqualhe, et tend à multiplier encore ses admirables variéties. Il finul, du reste, que celte plante 
puisse varier avec une grande facilité, car jusqu'à présent les graines ont été généralement recueillies dans le Midi, où l'on ne s'est pas occupé de féconder artificiellennent les lleur's. Actuellement on obtient des graines dans tout le centre de la France, et probablement mêne qu'elles pourront mûrir à Paris, puisquielles réussissent à lierne, où le colonel May de Buren, l'honorable président de la Société d'horticulture suisse, en a obtenu et m'a indiqué le moyen de les faire mûrir dans les appartements, moyen qui consiste à couper les fleurs à l'éporque où elles sont fanées et à les suspendre par bouquets dans un lieu sec et aéré.

Ia fécondation artificielle s'opère avec facilité sur ces régélaux; leur structure s'y prêle parfaitement bien. On troure, en effet, dans les Chrysanthèmes doubles ou semi-doubles une grande quantité de lleurons en languettes qui presque tóus sont munis de bons stigmates, et qui restent pendant longtemps aptes à recevoir le pollen dont ils sont presque toujours privés. Iu centre se développent ordinairement des fleurons hermaphrodites qui donnent un pollen orangé assez abondant of un peu pâteux, mais que l'on recueille facilement au pinceau pour le porter sur les stigmates des fleurons femelles.

Quand les fleurs sont tres-doubles, comme dans Jupiter, roi les roses, et il n'y a pas toujours de ces fleurons, mais presque tous cependant en sont poursus et il est rare qu un Chrysanthème ne donne pas au moins quelques fleurs sur lesquelle's on puisse récolter la poussière fécondante.

II est quelquefois très-difficile d'arriver aux stigmales des lleurs femelles, car presque toujours les fleurons sont tubuleux i leur base, et souvent dans toute leur longueur; rarement le stigmate est à nu, comme dans Achmet-Bey, Hadame Hardy, etc. II faut alors fendre les fleurons avec des ciseaux pour ne pas endommager le stigmate, ou bien arec la pointe d'une aiguille, que l'on pique à la base et que l'on relève lentenent pour séparer les fleurons en deux parlies. Quand ces derniers sont très-longs, conme dans tubulosum, ornitum, moulin rose; 
magicum, ele., il vaut mienx tondre la fleur en laissant le tube des fleurons assez court pour yu on puisse atteindre le stignate arec un pincean. Celle mulilation ne nuil en rien i la fécondation; il est mème nécessiare de la liare sur toutes les fleurs que l'on a opérées lorsqu'elles commencent à se fimer, alin d'éviter ia pourriture qui se développe très-souvent sur ces plantes enfermées daus nus serres. On enlève aussi, à la pince, tous les lleurons jaunes du centre, frour naroir que de bonnes graines. Le's Chrysanthimes ayant une tendanee a domer des fleurs blanches on très-pàles, qui sont, à la vérité, les plus belles, ce sont surtout les plus foncés qu'il faut choisir pour portegraines.

Si, nalgré la précaution que l'on prend de dimimer un peu les arrosements et daugmenter la chaleur, les fleurs avaient encore quedgue tendance à se pourrir, il faudrail les couper de suite tris-bias sur la plante, on abattre celle-ci tout entiere el lit suspendre an plafond (grav. (35.)
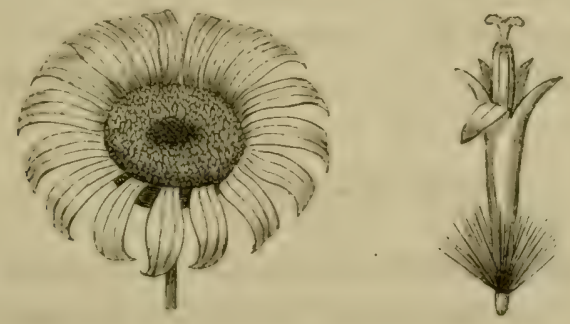

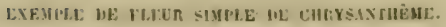

Lirav. $65^{-}$- lleuron du centre détaché et grossi.

L'hunidite, et par suite la pourriture peurent farie perdie les graines que l'on atlend de's Chrysanthemes hybrides. Te ne puis lrop recommander de n'en pas réunir dams la mêne serre une trop srande quantite, de ne laisser aux portegraines que quatre à cing fleurs, de les mettre en pots longtemps avant la floraison, et de les arroser avee du purin mélangé d'eau, ou une bome macération de guano avec un peu de sulfate de fer ou couperose verte. 
Depuis que l'ou a obtenu les variétés à petites fleurs, on a multiplić à l'infini les couleurs et les formes de ces jolies plantes. Les variétés pompons, croisées avec celles à grandes: lleurs, ont donné les plantes à capitules moyens qui sont maintenant si belles et si variées.

\section{Genue Cinéraire. - Cineraria.}

Les lleurons en langnettes qui lorment la jolic conronne des Cinéraires sont lemelles et bien conformés, en sorte que ciest sur leur stigmate qu il faut appliquer le pollen des variétés qur l'on veut croiser. leejà il existe un grand nombre d'hybrides: dans ce genre, et on en obtient tous les jours de nouveaux. Il reste encore à chercher les cinéraires à lleurs ravées, striées ou pointées. On devra surtont s'attacher à celles qui ont un bon port, des corymbes droits, bien fomnis, et ne pas négliger non plus la grandeur des fleurs, hien que souvent wlle soit en raison inverse du nombre. Les C. cruenta, anritu, éhinatu paraissent être les types d'où sont sorties les nomlneuses variétés de Cinciraires maintenant cultivées dans les: jardius.

\section{fienre souci. - Colendula.}

Les lleurous extérieurs sont femelles el munis de stigmates lintiles, ceux du centre sont quelquefois hermaphrodites el plus souvent mètés, de telle sorte que, si l'on voulait essayer d'hybrider les Soucis, rien ne serait plus aisé que d'atteudre le développenent des stigmates et d'y poser le pollen d'une autre espèce ou d'une variété. Il existe sans doute dans la nature des croisements de ce genre, qui se sont opérés naturellement par cette conformation des fleurs.

Genre Centanuée. - Centaurea.

Les capitules des C'entaurées sont composés de fleurous neu- 
tres à l'extéricur et de fleurons hermaphrodites au centre. On ne peut done penser à obtenir des graines des fleurons dé la couronne, et, si l'on voulait hybrider ceux du centre, il fiudrait de bonne heure fendre, avec la pointe d'une aiguille, le tube anthérifère, en dégager le style et le stigmate, et, un peu plus tard, le féconder avec le pollen d'une autre espèce ou variété; mais, quoique les Centaurées contiennent de très-belles espèces, je ne pense pas que jusqu’à présent on ait essayé de les croiser et d'en obtenir des variétés. Celles que l'on connaît daus le Bluet sont entièrement l'effet du hasard et non le résultat d'une hybridation calculée.

\section{Genre Chicoréc. - Cichorium.}

On commaît déjà plusieurs variétés de la Chicorée sauvage, et surtout de l'endive cultivée, autre espèce du même genre. On rencontre encore, sur les côtes africaines de la Néditerranée, le C. divaricalum et probablement le pumilum. Si toutes ces espèces ne peuvent s'hybrider, on peut du moins espérer de réussir entre les variétés de l'endive et la Chicorée sauvage. La grande culture et les jardins maraichers peuvent encore espérer des plintes nouvelles dans ce genre, malgré les difficultés de l'hybridation. En effet, tous les fleurons de chaque capitule sont hermaphrodites, tous fleurissent en même temps, et la lécondation s'opère le même jour que l'épanouissement. Il faut donc de grand matin guetter l'épanouissement de ces fleurs, enlever entièrement une partie des fleurons du centre, détacher le tube anthérifère après l'avoir fendı longitudinalement, et poser le pollen sur le stigmate qui se déreloppe aussi en même temps que les fleurs.

Les C. de Meanx. - Fine d'Italie ou d'eté. - Les Scaroles, ordinaire, - blonde, - ì fleur blanche, et la $C_{\text {. }}$ panachée sont des types qui produiront encore des hybrides. 
Genre Salsifix. - Tragupogon.

On connait peu de variétés de ce légume, mais il est probable que l'on pourrait en obtenir de nouvelles en essayant de croiser les espèces sauvages, qui se ressemblent toutes, avec celle qui est cultivée. Il y aurait ici la même difficulté que dans les autres Chicoracées, car tous les fleurons sont hermaphrodites, et il faudrait enlever les tubes staminifères avant l'anthèse. Cettc opération est facilitée par le mode d'épanouissement de ces plantes. La floraison s'exécute de six à dix heures du matin, el à midi elle est ordinairement terminée. Cette floraison n'a lieu que pour un seul rang de fleurons, qui est le plus extérieur; le lendemain, le second rang s'ouvre et se féconde; le troisième jour, le troisième rang, et ainsi de suite. Si donc on attend le prumier jour, il faudra couper entièrement les fleurons, dont ou ne peut pas très-facilement empêcher la fécondation naturelle, mais on coupera aussi les étamines du second rang, qui ne doivent s'ouvrir que le lendemain. La fleur se refermera comme à son ordinaire, et le lendemain matia, quand elle s'ouvrira avec ses stigmates bien développés, on pourra alors opérer la fécondation artificielle. On agira de mème sur le troisième rang, et ainsi de suite; mais on fera bien, après avoir opéré sur deux rangées de fleurons, de couper les autres pour éviter toute cause d'erreur.

On sait que Linné a obtenu un hybride entre le Tragopogon mratense et le $T$. porrifolium, lequel tenait à la fois des deux espèces. Voici, du reste, les propres expressions du grand naturaliste :

"Le Trayopogon hybridum fixa mon attention, l'automue dernière, dans une partie de mon jardin où j’avais planté le T. pr' $l$ tense et le T. porrifolium; mais l'hiver détruisil toutes ces sc. mences. L'année dernière, lorsque le T. pratense était en fleur, j'enlevai de bonne heure, dans la matinée, toute la poussière séminale des fleurs en les frottant; à huit heures du matin je fis tomber sur ces mêmes fleurs de la poussière prolitique du $T$. por- 
rifolium, et je marruai avec un fil les fleurs sur lesquelles j’avais fait cet essai. Je cueillis les graines aussitòt qu'elles furent mùres; et je les senai dès cette antomue; elles levèrent très-bien, et elles ont produit cette année, 1759 , des fleurs pourpres, jaunes à la base, qui ont donné des semences; je doute qu'on puisse laire aucune expérience qui démontre plus clairement que celle-ci la génération des régétaux. "

" J'ai viu, dans le jardin botanique d'Édimbourg, une plante ligbride produite par le Parot d'Orient et le Parot somnifère. On arait eu soin de conper toutes les étamines des fleurs du Parot sommifère avant qu'elles fussent épanouies, et on plaçait les étilmines du Pavot d'Orient sur les pistils lorsqu'ils étaient bien développés. Cette expérience étriit répétée tous les ans avee un ígal succès. ») (Journal de Physique, t. XxxII, p. 459.)

\section{Genre Laitue. - Lactuca.}

Quoique lia culture ait déjà produit un trìs-grand nombre de variétés de Laitue, on est en droit d'en espérer encore. Le Lactuca sutiva, qui les a produites, ainsi que les quercifolia, Scariola, peut-ètre même le virosı et le saligna, seraient probablement susceptibles de se croiser, car toutes ces plantes ont entre elles de grands rapports. Le Lactuca peremis, que l'on mange dans plusieurs contrées et que l'on peut forcer et blanchir comme la Chicorée, ne pourrait-il pas être croisé avantigeusement avec quelques variétés de nos Laitues potagères?

lin prenant pour porte-graine ces énormes salades récemment introduites dans nos jardins, et les fécondant par les autres variétés cultivées ou par les espèces samvages, il y aurait certainement espoir d'aroir encore des plantes nourelles. C'est le soir que la plupint de ces plantes s'épanonissent, et c'est à celte époque quilil faudrait enlever adroitement le tube anthérifére et attendre le lendemain matin pour poser le pollen sur les stigmates. Souvent les capitules n'ont que cinq fleurons, et il est 


\section{FÉCONDATION NATURELLE ET ARTIFICILLLE.}

uécessaire d'opérer sur tous, et d'ébrancher considérablement la tige, pour pouvoir opérer en même temps sur tous les capi-

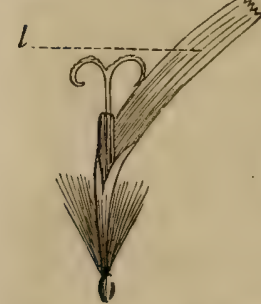

Griv. 64.-Fleuron ligulé d'une laitue, $-l$, ligrule ou pétale de la corole, soudée. tules qui s'ouvrent à la fois (grav. 64). Voici, d'après M. Courtois-Gérard, la liste des laitues cultivées par les maraichers de Paris, et dans laquelle on peut choisir des porte-graines pour des hybridations :

A. Pommées. Pommées de printemps. Grêpe ou petite noire. - Golte ou George. - Pommées d'été. Palatine ou rouge. Grosse brune paresseuse ou grise. De.Versailles. - Blonde d'été. - De

Berlin. - Trapue. - Royale. - Batavia blonde. - Brune ou Laitue chou. - De Malte. - Turque. - Grosse américaine. - Impériale. - De Gènes. - Cocasse. - Méterelle. - Ronsse à graines junnes. - Ronge chartrense. - Sanynine ou flayellée. - Pommées d'hiver. - Passion flayellée. - Morine. - De Groslay. - Coquille. - Laitues à couper. - A couper ou petite laitue. - Chicorée. - Épinard ou ì. F. de chêne.

B. Iomaines. Verte. - Hâtive. - Grise maraichère. - Alphange. - Blonde de Crunoy. - Panachée ou sanguine. Romaine rouge d'hiver.

Un arlicle de M. Pepin, dans la Revue horticole, indique une salade nouvelle, la Laitue incomparable, comme la meilleure romaine que l'on connaisse. Elle a été introduite par M. Bossin, et vient de la maison Cormack, de Londres.

On a aussi amnoncé, depuis lors, plusieurs variétés nouvelles de Laitue. Elles diffèrent peu de celles que nous venons de citer.

\section{Genre Artichaut. - Cynara.}

Les nombreux fleurons, rassemblés sur le large réceptacle de l'Artichaut, sont tous en entonnoir et munis d'étanines et de pistils généralement bien conformés. Les anthères doivent ètre 
retranchíes de bonne heure ou peu avant l'ipanouissement, et les styles se développent ensuite en toute liberté, ne devenant aptes qu'après. On doit aussi retrancher une bonme partie des fleurons du centre pour donner plus de développement aux graines de la circonférence.

Deux espèces de ce genre sont cultivées dans les potagers : l'une, le C. cardunculus, a produit les Cardons, dont on distingue plusieurs varictés, telles que celui de Tours, d'Espayne, le plein inerme, celui à côtes rouges et le nouveau Cardon de Chambéry, dépourvu de piquants et atteignant jusqu'ì deux mètres de hauteur. Ces variétés s'hybrideraient et en produiraient d'autres.

Ia seconde espèce est l'Artichaut proprement dit ou C. scolymus, dans laquelle on trouve le vert de Provence, le gros vert de'Laon, le violet, le camus de Bretagne. Les plantes qui proviennent de graines sont plus rustiques que celles que l'on mulliplie par ocilletons. On peuty trouver de bonnes variétés nouvelles, mais il y a toujours un certain nombre de pieds quu se sont rapprochés du typesauvage, qui grandissent comme des Cardons ou qui deviennent épineuses.

\section{FAMILLE DES CAMPANULACEES.}

\section{Genre Campanule. - Campanuir.}

Le beau genre des Campamules offre un mode de fécondalion lien remarquable. Les cing anthères s'ourrent longtemps avant l'anthèse et déposent leur pollen sur le sţ̧le, muni, ì sa partie superieure, d'un certain nombre de lignes de poils collecteur's. Plus tard, à l'époque où la corolle s'ouvre, on voit paraitre ses cing stigmates qui se roulent en dehors of recueillent ce pollen.

Si lon voulait hybrider ces plantes, il faudrait done lendre Ic houton asant son épanouissement, et en extraire les anthires avant qu'elles n'aient pu déposer leur pollen sur les 
poils collecteurs, attendre ensuite la floraison, et poser à la fois sur le stigmate et sur les lignes de poils la poussière fécondante avec laquelle on voudrait opérer le croisement.

On a déjà obtenu dans les Campanules plusieurs variétés à fleurs doubles, et si ces plantes conservent les styles intacts, c'est sur elles qu'il faudrait tenter 'd'opérer.

On introduit tous les jours de nouvelles espèces, et, parni les anciennes même, les grandis, media, pỵramidalis sont des plantes d'un admirable effet. Si on s'occupait sćrieusement de collectionner et d'hybrider ces beaux végétaux, on arriverait certainement à de nouvelles variétés plus belles encore que les types.

\section{Genre Lobélie, - Lobelia.}

Les Lobélies ont cinq étamines dont les anthères serrées les unes contre les autres, sont presque soudées. Ce tube est traversé par un style simple, ordinairement muni d'une partie couronnée de poils collecteurs pour rassembler le pollen, et un stigmate simple d'abord, mais qui, le plus souvent, se partage en deux lobes qui se retournent et s'imprégnent de pollen sur la couronne de poils, si déjà ils n’ont pas recueilli ce pollen auparavant.

Les anthères s'ouvrent de bonne heure dans les Lobélies. Si l'on voulait pratiquer des fécondations artificielles, il faudrait ourrir les fleurs avant l'épanouissement, et en culever adroitement les anthères, puis attendre la floraison pour poser, sur le stigmate el sur l'anneau qui l'entoure au-dessous, le pollen arec lequel on voudrait hybrider la plante. Ce genre contient de très-belles espèces à fleurs rouges, bleues et violettes. Il n'y aurait peut-ĉtre aucun avantage à croiser leurs coulcurs, mais, fécondées entre plantes de même couleur, on pourrait arriver à des variétés plus brillantes encore que leurs types.

Dès l'année 1771, Knelreuter avait hybridé les Lohélies, cl avait obtenu des résultats très-intéressants dans ses lybrides do L. cardinalis ot de L. syphilitica. Il avait été moins hen- 
reux en voulant croiser les L. wrens, Cliffortiana, Erimus arec le cardinalis.

Genre Syphocampilos, - Syphocampilos.

Les quatre étamines, sondées par les filets et les anthères, forment un tube que traverse le style. Celui-ci śélève encore au-dessus du tube anthérifere et s'épanouit en un stigmate bilahié. Le pollen sort abondamment des anthères quand on fend le tube, mais ordinairement il y reste emprisonné, en sorte que, pour féconder ces plantes, soit, avec le pollen de l'espèce, soit avec un pollen étranger, il faut aller le chercher dans le tube anthérifère, humecter légèrement le stigmate avec un peu de la liqueur miellée qui se trouve abondamment au fond de la corolle, et poser ensuite ce pollen, dont les grains crèvent humectés par cette liqueur. Le stigmate, d'abord très-court, recueille en s'allongeant, et au moyen des poils collecteurs dont il est muni au sommet, le pollen des anthères au moment où la fleur s'épanouit, mais ne

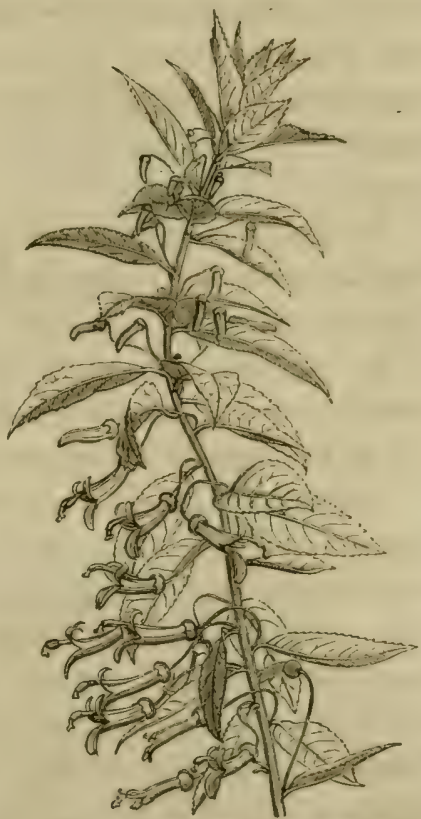

Grax. (ij. - Syp!weampilos betulifolius. devient apte, comme celui des Synanthérées, qu'un certain laps de temps après s'être couvert de pollen (grav. 65).

\section{FAMILLE DES GESNERIACEES.}

\section{Genre fesnerie. - Gesneria.}

On compte maintenant un assez grand nombre d'espèces ou 
de variétés de ce beau genre, qui semble exclusivement destiné à l'ornement des serres chaudes.

Ces plantes ont quatre étamines qui réunissent leurs anthères au sommet de la corolle, et forment un disque glanduleux dont la partie inférieure est couverte de pollen. Le style, qui s'allonge pendant l'épanouissement, amène le stigmate à une certaine distance des anthères, et les dépasse même dans quelques espèces. Cependant la fécondation ne s'opère pas toujours. On peut la pratiquer artificiellement en enlevant le disque anthérifère avant l'anthèse, c'est-à-dire au commencement de l'ouverture de la corolle, et en posant sur le stigmate le pollen d'une espèce voisine et un peu de la liqueur miellée sécrétée par la corolle.

De magnifiques hybrides ont été obtenus ainsi aux dépens des Gesneria, des Tydæa et de quelques autres genres démembrés des Gesneria.

Le stigmate bilabié de presque toules ces Gesnériacées semble indiquer par l'écartement de ses lèvres le moment précis où la fécondation doit être opérée.

Le Gesneria Douckelaarii a été obtenu, par l'habile jardinier dont elle porte le nom, par la fécondation artificielle du Gesneria discolor par le pollen d'un Gloxinia rubra, hybridité peutêtre douteuse. C'est une plante magnifique, portant une belle panicule de fleurs d'un beau rose en dehors et jaunâtre en dedans. Il faut encore considérer comme hybrides obtenus par Douckelaar le Gesneria Miellezii à fleurs lilas et le G. gloxinixflor à grandes fleurs carminées.

Nous trourons encore dans la Flore des serres et des jardins, sous le nom de Dircæa Gesneria purpurea Planchon, une trèsbelle plante que II. Van Iloutte, qui l'a obtenue, prétend issue du Gesneria Douglasii verticillata, fécondé par le Dircæa Cooperi, croisement qui a fourni des hybrides de diverses nuances. Le même horticulteur cite encore un hybride du Dircæa lobulata Lem., fécondé par le Gesneria Leopoldi Schwd., produits divers qui prouvent que le démembrement 
de plusieurs genres des Gesnérincées ne serait pas accepté aussi bien par la nature que par les botanistes.

\section{Genre Gloxinie. - Gloxinia.}

On peut enlever aussi les quatre étamines des Gloxinia dont les anthères se réunissent quelquefois en un disque comme celles des Gesneria. La fécondation s'opère de mème, et l'on peut pratiquer l'hybridation comme sur le genre que nous re. nons de citer. If Gaximia speciosa a déji domni de supertes

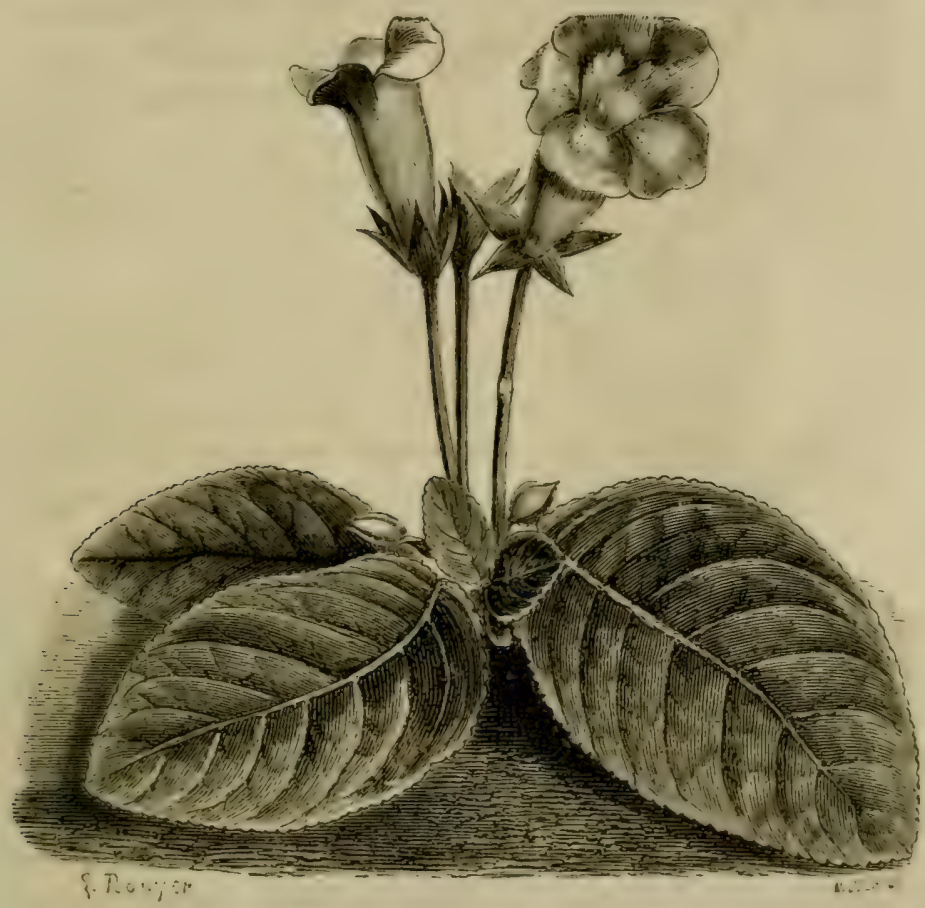

Givav. 66. - Exemple de Gloxinia.

variétés, qui peuvent elles-mèmes en produire de nouvelles, et parmi lesquelles nous citerons l'insignis, le bicolor, le Curtoni, l. mayniflora maxima, rubra rosalbu, etc. J'ignore si l'on pourrait hybrider ces plantes avee d'autres espèces, telles que te cerina et le hirsuta (grav. 66). 
M. Van Iloutte annonce, dans l'Horticulteur universel, qu'il a hybridé mystériensement les plus beaux Gloxinia, des maxima, des rubra, et qu'il n'a obtenu que des bleus ordinaires, c'est-à-dire le type de l'espèce. Il n'y a rien là cependant qui

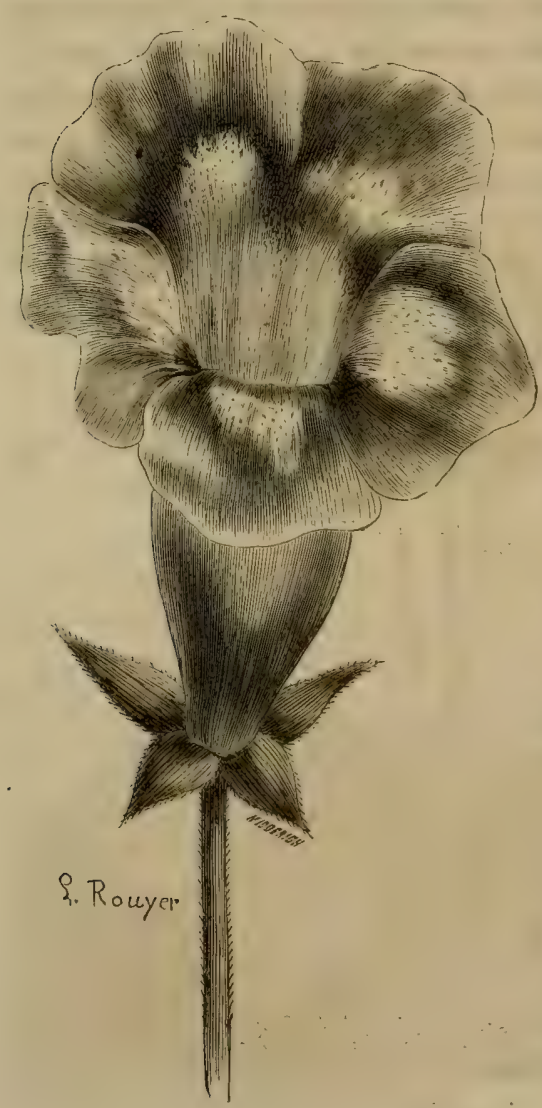

Grav. 67. - Fleur de Gloxinia.- Mno Pinard, le graniteuri, naturelle. doive décourager ceux qui voudraient s'occuper de ce beau genre. 0 n ne réussit pas toujours, lors même qu'on est hàbile, et d'autrefois on est dédommagé, témoin les belles Calcéolaires de II. Van Iloutte, qui ne peut, pour ce genre, demander de plus beaux succès.

Nous écrivions ces lignes en 1845, et depuis lors M. Van Houtle s'est amplement dédommagé de ses premiers insuccès.

En 1845, M. Fyfe, jardinier à Cothesay, a obtenu pour la première fois un magnifique Gloxinia à liampe droite ou plutôt à fleur droite et non inclinée, qui est devenu le type de tous nos Gloxinia Iressés (grav. 67). Un fait plus remarquable encore s'est produit: c'est le développement de la cinquième étamine et la forme parfaitement régulière de la corolle.

On suppose que cet hybride remarquable jrovient du G. spe- 
ciosa, V. maxima et du G. caulescens, mais on n'est pas certain de cette origine. On lui a donné le nom horticole de $F_{r}$. Fyfana.

Le G. Teichleri, si adruirablement panache de rose et de bleu, est encore un hyhiride du G. speciosa et du G. caulescens, deux espèces dont les mariages ont été si sourent heurenx.

Après tous ces gains brillants viennent les plantes vraiment extrandinaires récemment obtenues par II. Yan Houtte et figuries daus le tome $\mathrm{X}$ de la Flore des serres et des jurdins. On ne peut rien voir de plus admirable que ces charmants coloris adliptes aux formes les phus gracienses. Quelle descendance sortira done maintenant de ces merveilles? Comment concevoir que l'on puisse obtenir plus beau? Nous pouvons affirmer que dans des mains aussi hahiles que relles de II. Tan Iloutte l'hrbridition des Gloxinia n'est pas au bout de ses prodiges.

\section{Genre Achimènes. - Achimenes.}

On peut appliquer à ces plantes ce que nous venons de dire des Gloxinia et ce que nous avons dit plus haut des Gesneria. lenr structure forale est la même, et l'hybridation vieudra sans doute modifier et multiplier les formes d'un des genres les plus éclatants du règne végétal. Les A. grandifloru, longiflora, rosea, hirsuta, pedtunculatu, picta, alba ont déja fourni de nombreux hybrides.

I.es genres disers que les botanistes ont établis aux dépens des Achimenes né les empèchent pas de s'hybrider entre elles. Les Plectopoma, les Tydea (grav. 68), les Achimanes, les Trevirunia et même les Gloxinia, quoique très-différents, peuvent s'allier dans une foule de circonstances, lorsque l'on s'occupe aver quelque persérérance de ces unions illégitimes.

II. Regel cite des hrbrides remarguables des Treviraniu. II cite anssi un hybride beaucoup plus compliqué, quili dosisne sous le nom de Plectopoma fimlriatum Hanst., var. Edouard Ollo. Ciest un hrbiride entre le P. fimbriatum Hianst., ou 
252 FÉCOYDATION YATURELL ET ARTIFICIELLE.

Achimenes yloxinixflora Lem., ou Gloxiniu fimbricita, qui a fonmi le pollen, et l'Achimenes (ou Trevirania) cocrinea, déjà hybride lui-mème du Trevirania grandiflora et du Iniastema gracilis.

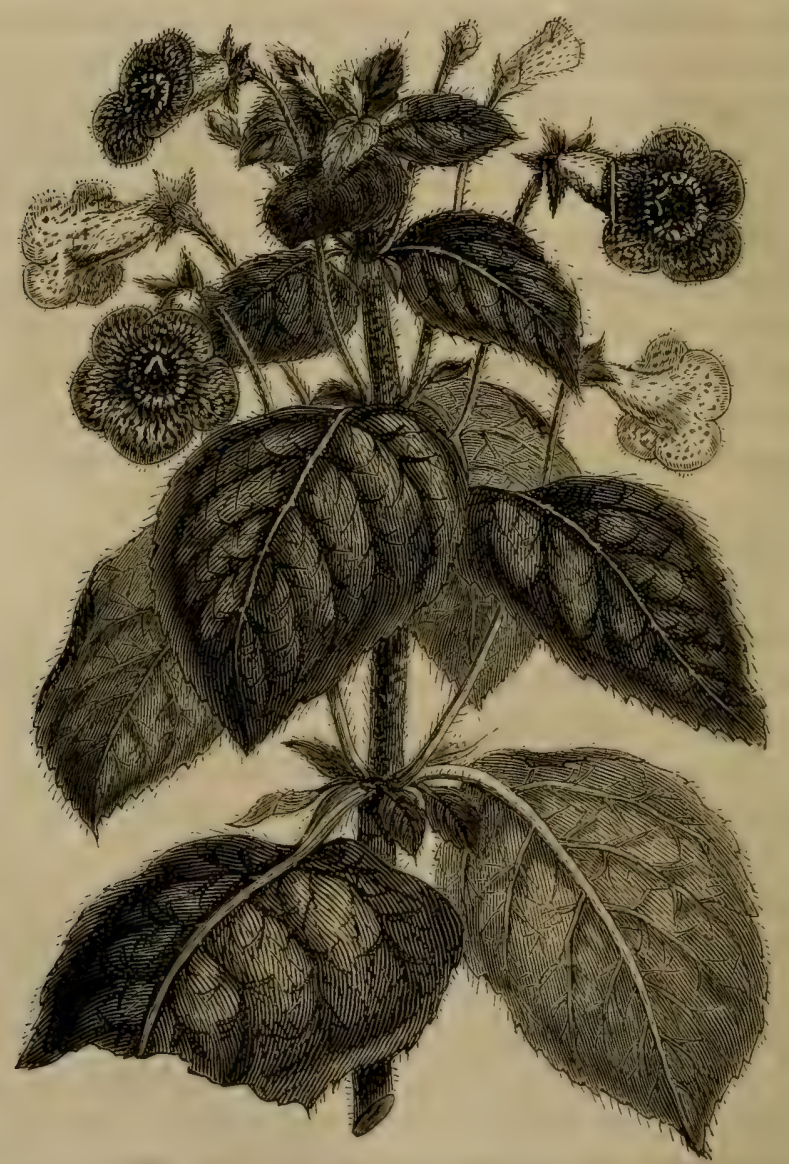

Grav. 68. - Tydæa amabilis, Un tier's de grandeur naturelle.

L'Achimenes Escheriana, ohtemu à Zurich par M. Regel, ost encore un joli hybride de l'A. rosea, fécondé par l'A. longiflora. Mais, dans ce genre, il faut avoir soin de recueillir le pollen dans le houton un peu avant l'épanouissement, et de 
chatrer les fleurs de très-bonne heure, en déroulant on en fendant la corolle.

M. Van Houtte a ohtenu, il y a quolques années, de magnifiques hybrides de Tydru. C'est it II. Riazh, un de ses chefs de culture, que sont dues ces plantes"si belles el si remarquables.

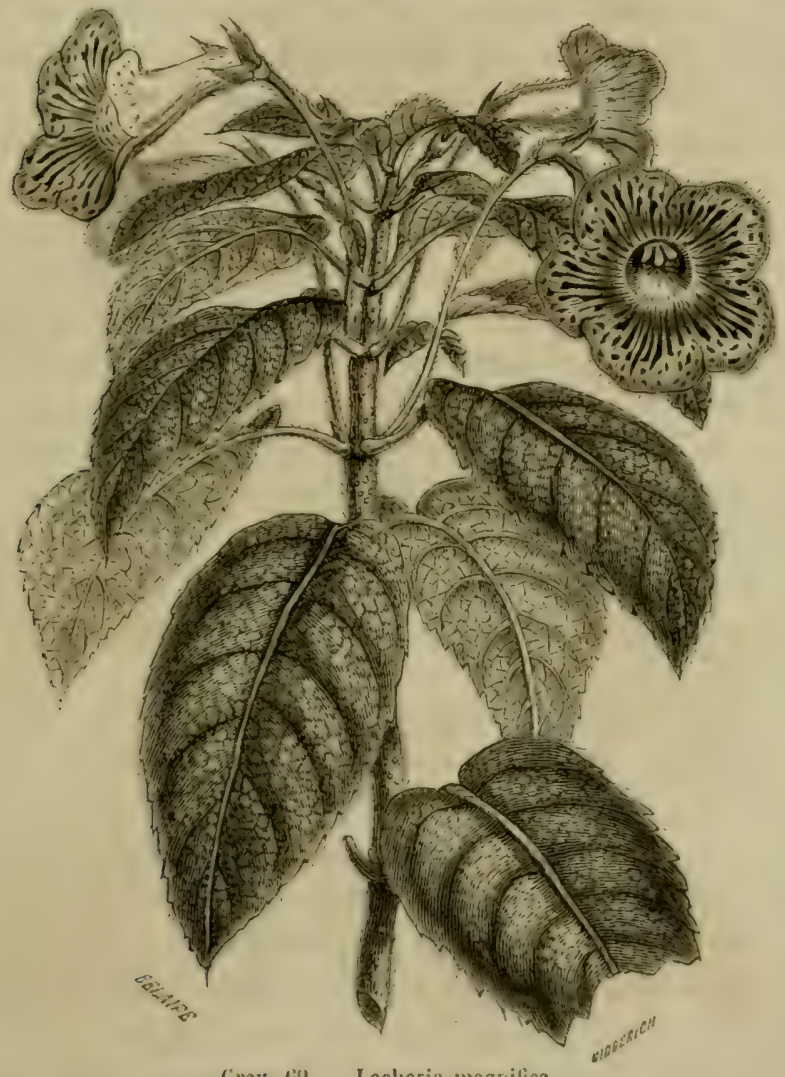

(irav. 69. - Locheria magrilica.

Les T. Warscencievii et T. pictu ont été lécondés liun par. l'aulre, "t récipropuement. C'est par centaines que ces hybrides ont fleuri, avec d'inormes proportions at des fleurs abonAimles. Le T. gigante'u, l'un de ces hybrides, prolonge tresslongtemps sa floraison. 
Le Gesneria zebrina, fécondé par le Scheeria mexicana, a encore donné à N. Rocal un magnifique hybride. Il a été publié par M. Planchon dans la Flore des serres et des jardins, sous le nom de Mandirola Nægelia Razlii.

"Sur des centaines de graines semées, dit N. Roul, deux seulement se sont trouvées fertiles. Pas une des fleurs de l'hybridation n'a les étamines fertiles. Elles n'ont pas de traces de pollen. ) Celte plante est aussi très-florifère.

Le Mandirola multiflora, fécondé par le Gesneria zebrina, a donné naissance au Mandirola Nægelia picturata Planchon, au feuillage marbré et aux grandes fleurs mouchetées. Les antthères, au lieu d'être rapprochées et cohérentes, comme daus la plupart des Gesnériacées, se présentent entièrement libres.

Un autre liybride plus beau encore, à fleurs plus grandes (que le T. gigantea, a paru dans les serres de Van Iloulte sous le nom de T. Ortyiesii. Il serait né du T. Warscewiezii, fécondé par le polten du Locheria magnifica (grav. 69).

Un autre encore, de toute heauté, est le $T$. Eechantei, obtenu aussi par M. Rowl au jardin Van líoutte, et produisant pendant près de six mois ses magnifiques pyramides de fleurs.

\section{FAMILLE DES ERICACEES.}

Genre Arbousier: - Arbutus.

Ce genre offre un pistil simple, a sligmate glutineux, devant lequel vieunent successivement se placer dix anthères qui s'ourrent chacune par deux pores, et répandent un pollen gras et pulvérulent. Il serait, par conséquent, facile d'enlever les anthères au moment où la fleur s'épanouit, et de féconder le stig̨nate immédiatement avec un pollen étranger. L'Arbutus Unedo, qui fructifie très-facilement dans nos serres, pourrait aussi servir de plante mère, sur laquelle on essayerait l'impréguation au mojen de joussière fécondante recueillic sur les mucronata, furinosa, xalupensis, chinensis, Cumminyii, Milleri, procera, etc. 
Genre Andromède. - Andromeda.

La lë́condation des Andromèdes est la mème que celle des Arbutus, mais toutes les especes cultives ne donnent pas des graines fertiles. On pourrait en obtenir de plusicurs d'entre elles en les lëcondant au pinceau, peut-ètre mème avoir des hybrides, en choisissant pour mères celles de ces plantes qui fructifient facilement. Déjà le caliculatu a domé des variétés, il scrait ì désirer que l'on pùt en obtenir avec le floribunda, le lucida rubra, le tetragona, le vaccinoïles, ete.

\section{Cenre Bruyère. - Erica.}

Le's huit étamines des Bruyères forment me espirce de couromne autom du style, et le stignate quadrifide s'élève au. dessus d'elles. Les anthères commencent à s'ouvrir dans le bouton, mais, malgré cela, le pollen reste encore quelque temps adhérent à l'anthère; et; comme le stigmate est issez élevé au-dessus d'elles, il est rare, dans nos serres surtout, que la fécondation s'accomplisse immédiatement. On pourrait done hybrider ces plantes sans enlever les anthères, el lor's même que cette opération serait nécessaire, elle pourrait être faite avant l'épanonis

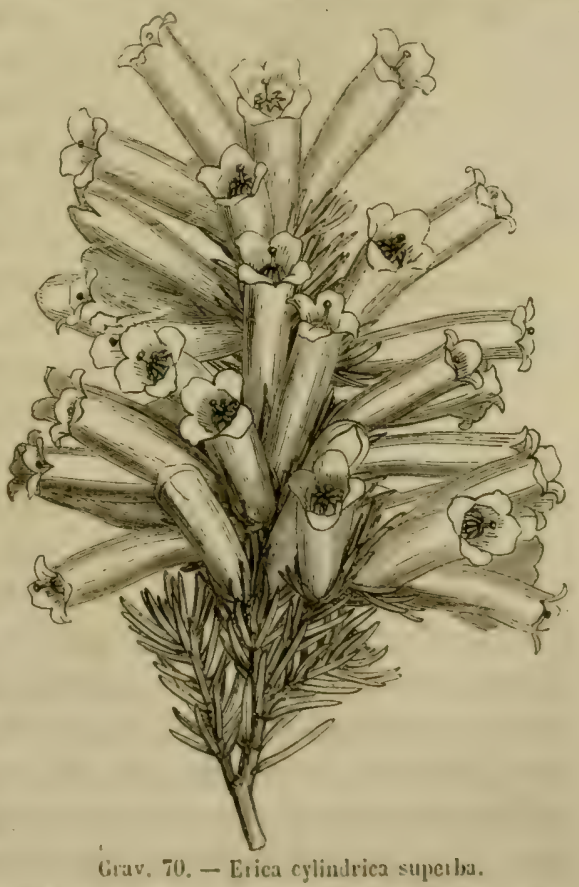
sement par une petile incision pratiquée sur le côte de la corolle. Le pullen sort par une ouverture ovale placée au sonmet 
de l'anthère. On trouve aussi des espèces de Bruyère dont les anthères sont saillantes hors du tube, mais, en général, elles sont incluses.

Ce genre, un des plus nombreux du règne végétal, semble avoir donné déjà une multitude d'hybrides, et plusieurs se sont fiuts naturellement dans les plaines du Cap, où abondent ces charmants végétaux. Peut-être nos belles Bruyères indigènes, qui se reproduisent avec tant de prodigalité, pourraient-elles servir de types ou pieds mères, pour des fécondations arti-

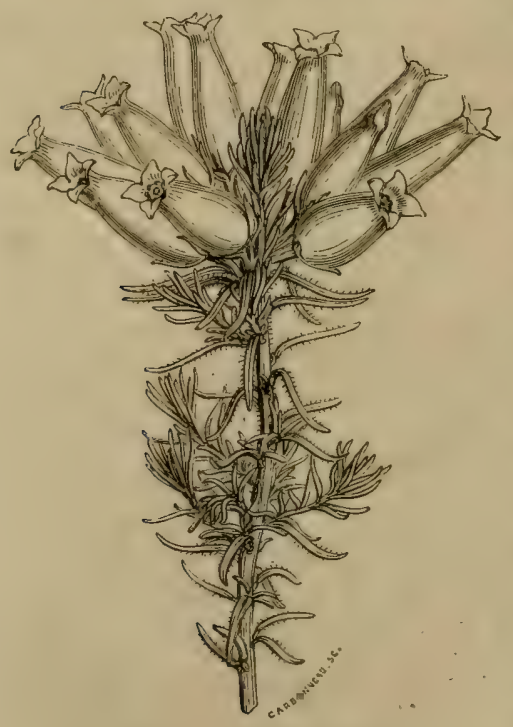

Grav. 71, - Erica ventricosit.

ficielles: l'arborea, le mediterranea, le tetralix, le vagans, le cinerea, toules si élégantes, recevraient peut-être un pollen étranger qui amènerait des variélés nouvelles, dans un genre déjà si nombreux.

Il y aurait sans doute quelques précautions à prendre dans les hybridalions de Bruyères, ce serait de ne croiser que des plantes analogues, ou au moins de même section. Ainsi la forme de la corolle serait un indice suffisant pour le rapprochementou l'́loignement des espèces, car elle est liée à certains appendice's des étamines. Quand les antlières sont nues, la corolle est en tube; quand elles sont terminées par de petites pointes, la corolle devient campanulée, et elle prend l'apparence d'un grelot, quand ces mêmes anthèrés sont frangées; malgré quuelques exceptions, ces données sont suffisantes pour guider dans le choix des espèces que l'on voulrail essayer de croiser.

On cite comme hybride un erica Hartnello-Hiemalis on 
E. Burnetti, charmante plante ì grelots roses que l'on attribue à l'E. Hartnelli, fécondé par l'E. Hiemalis de nos jardins (grav. 70, 71).

\section{Genre Azalée. - dzalea.}

Les Izalées constituent un des genres les plus nombreux en variétés cultivées; elles riennent se grouper en deux espèces: l'indisu et le pontica, auxquelles on peut joindre le mudiflor' et le viscosa.

L'indica a dix étamines, tandis que les espèces de pleine terre et d'Amérique n'en ont que cinq.

Dans les deux types, la fécondation artificielle peut s'opérer facilement. Le stigmate est porté sur un long style, et les étanines, libres et distinctes, peurent très-facilement être enlevées lors de l'épanouissement, et avant qu'elles n'aient répandu sur le stigmale un pollen glutineux qui semble attaché par des fils déliés, et qui sort par deux pores situés au sommet de l'anthère.

Le stigmate est également visqueux et relient parfaitement le pollen que l'orr y applique au pinceau. Il n'y a done rien d'étonnant qu' on ait obtenu de si nombreuses et de si belles variélés d'Azalées. Ce que nous venons de dire s'applique également aux Azalées de pleine terre et de serre froide. Nous recommandons surtout aux amateurs de s'occuper des fécondatıons artificielles, qui donnent de si beaux résultats; ils ne trouveront aucum gemre aussi facile que celui qui nous occupe en ce moment.

Le seul Azalea lateritia a fourni à N. Kenight vingt-deux variétés remarquables, qui ont été introduites en France. MI. IIaquin est parrenu à croiser les Azalées de l'Inde avec ceux de pleine terre.

Les Azalées ont trouvé a Gand des amateurs distingués, qui se sont spécialement occupés de ce beau genre. C'est au point uquil existe toute une série de ces plantes, série de pleine terre 
dans la terreau de feuilles, qui portent dans le commerce le nom d'Azalées de Gand, ou A. de Mortier.

"Feu Mortier, boulanger, à Gand, consacrait ses loisirs à croiser l'A. pontica de Turquie avec les A. nudiflora, viscosa, calendulacea de l'Amérique du Nord. Le pollen des yariétés hâtives, à corolle étroite, mais de coloris vif, servait à féconder les variétés tardives, et de là cette belle collection si indispensable à l'ornementation de nos jardins, et à laquelle viennent se joindre tous les jours de nouveaux gains, dus surtout à feu Van Castel, Wolf, Louis Verschaffelt et autres. "(Flore des serres et des jardins.)

On est obligé de convenir que les Azalées constituent un des genres les plus brillants que nous puissions cultiver, aussi éclatants que les Rhododendrons avec lesquels ils ont fait alliance, cussi variés que les Pelargonium et les Mirabilis.

Nous croyons devoir indiquer ici, d'après un journal anglais, un choix de variétés anciennes et nouvelles qui peut-être ne seront jamais surpassées, et qui témoignent hautement de la puissance de l'hyloridation.

Dans l'état actuel des choses (1860), les onze variétés que l'auteur anglais regarde comme les plus remarquables, sont les suivantes : Admiration, Griterion, Étoile de Gand, Extrani, Gem (Iveri), Gledstanesi, Sir H. Havelock (Frost), Iverniana, Juliana, Perfection (Frost.), Perryama, variegata. Certaines de ces variétés sont comparativement anciennes; mais leur mérite est tout à fait supérieur, et elles ne sont surpassées par aucune des variétés plus récentes. Après ces douze magnifiques plantes, les douze qui viennent immédiatement, par ordre de mérite, sont celles dont voici les noms : Beauty of Reiyate, Chelsoni, crispiflora, Distinction, Duc de Brabant, illustris nova, Lecuna, perfecta elegans, Roi Léopold, rosea elegans, rosea superba, Standard of Perfection (Epps.) Dans cette seconde série, comme dans la première, plusieurs variélés ne sont pas trèsmodernes, mais aucune parmi les nouvelles ne mérite d'être classée plus haut qu'elles. - Le mérite des vingt-quatre varié- 
tés dont on vient de voir les noms en fait essentiellement des plantes d'exposition; s'il s'agissait, au contraire, de faire un choix d'Azalées, en les envisageant seulement au point de sue décoratif, il faudrait admettre sur la liste un certain nombre d'autres variétís telles que arborea purpurea, Barclayanu, Eulalie ram Geert, Glory of Sunniny IIill, Lonis Napoléon, Miltoni Georgianu, petunix/lora, Sir Charles Napier, Stanleyana, Soncenir de l'Exposition, The Bride, qui toutes sont ou brillantes ou très-bien caractérisces.

Les Azalées ont accepté aussi l'union avec leurs voisins les Rhododendrons. Les Azalées que Smith a obtenues par ces croisements sont devenues les types d'une race nouvelle, remarquables par l'ampleur de leur corolle et leurs brillantes couleurs. Smith a obtenu du croisement d'un Rhododendrum ponlicum avec l'Azule' sinensis ces beaux Rhododendrons, à fleurs jaures, dont le premier portait s n nom, et qui ont donué depuis lors de si belles variétés.

I. Souchet a obtenu aussi un hybride de l'Azalea Danielsii lécondé par un Rhododendrum.

Cette belle Azalée courte de Hainaut, aux lleurs double's et carmines, ne serait-elle pas encore un hybride de l'A. indicu, daus lequel serait intervenu le pollen d'un Rhododendrum?

Cenre Iosage. - Rhododendrum.

Ce que nous venous de dire des Azalée's s’applique égallencm aux Rhododeudrum. Leurs dix étamines, ourertes at sommet pirr deux petits opercules, répandent aussi leur pollen attaché par des fils déliés, et peusent ètre rnlevées très-facilenent avant que cette émission se soit oprére. On trouve an fond de chaque lleur une belle goutle de liqueur miellec dont il faut ligirement impregner le sligmale. Les hybrides de ce genre se multiplient tous les jours et presque it linfini, et proviennent sutont des Rh. aboreum, muximum, c'uncasicum, ponti- 
cum. Ceux qui sortent de ces deux dernières espèces sont tous de pleine terre, les autres de serre froide. Leurs fleurs admirables offrent les plus riches coloris, et il serait difficile de faire un choix de porte-graines et de plantes fécondantes.

Parmi les innombrables hybrides de ce beau genre nous osons à peine citer quelques variétés, tant elles sont belles et nombreuses et tant leur origine est cachée dans la nuit des temps, comme le diraient les historiens.

Le Prince Camille de Rohan est un des plus beaux parmı les bouquets roses, carnés et maculés. On lui attribue pour origine un $R$. maximum fécondé par une variété de $R$. arboreum ou de $R$. caucasicum. Citons encore la fraiche image du R. étoile de Villers, pontico-catawbiense obtenu par MII. Lemichez, qui, des premiers en France, se sont occupés d'hybridatiun. Que voir aussi de plus frais, de plus beau que ce Rhododendrum neige et cerise provenant encore du $R$. arboreum et du $R$. catawbiense?

M. Smith, en fécondant un R. ponticum par le pollen d'un Azalea sinensis, a obtenu toute une lignée de plantes charmantes, parmi lesquelles nous devons citer les $R$. aureum, decorum, amœnum, carneum, elegantissimum, norbitonense, flavescens, et qui eux-mêmes ont produit des hybrides et dos variations nouvelles.

L'introduction dans nos jardins, vers 1846 ou 1847, du magnifique Rhododendrum javanicum aux larges fleurs dorées est devenue la souche de toute une belle série de nouveaux coloris.

L'apparition du R. Brookeanum, découvert par N. Low dans l'intérieur de Bornéo, avec ses énormes bouquets de fleurs orangées, deviendra sans doute, comme le $R$. javanicum, qu'il surpasse en beauté, le type de nouveaux hybrides.

Depuis que l'on a découvert les Rhododendron de l'Himalaya, depuis que l'on a vu les fleurs gigantesques du R. Nuttalii, on arrivera sans doute à croiser ces admirables végétaux et à faire du genre qui nous occupe ce qu’il est déjà, la plus belle 
fleur de nos jardins et le plus beau fleuron de la couronne de Flore.

Que ne ferait-on pas avec une collection de Phododendrons, d'Áalea, de Beyonia, de Calalium el de Fougères!

D'aprè M. Standish (The Florist, etc.; et Journal de la Société impériale et centrale d'horticulture, t. IT, p. 652), les espèces ou variétés desquelles sont provenus la plupart des hybrides de ce genre sont les R. arboreum, ponticum, murpureum, allum, cancasicum, companulatum, catawbiense, maximum.

Le Rhododendrum cutawbiense, fecondé par l'arborerum, i produit l'altaclarense; le catanbiense, fécondé par l'altaclarense, a produit le Blandyanum; le Blandyanum, par le Qneen Virtoria, a donné le Blandyanum superbum, le Manglesii, le. Robert Burns, le Menziesii, le Countess de Morella, le général Cabrera, etc.

Le Rhododendrum maximum, fécondé par l'arboreum, a donné naissance au Lindsayii; celui-ci, fécondé par le ponlicum allum, a produit un grand nombre d'hybrides à fleurs colorées de teintes purpurines fort délicates et presque blan. ches; à leur tour, ces derniers, fécondés par l'altaclarense, ont donné Paxtonii, mistress Beecher Stowe, Gem, Gulnare, Zuleilia; le Paxtortii, fécondé par le Lindsayji, a produit Climix et fimbriatum.

Du Rhododendrum ponticum purpureum, fécondé par l'altuclarense, est provenu le Queen Victoria, qui, à son tour, fécondé encore par l'altaclarense, a produit Vesurius; et celuici, arec le Blındyanum, a donné Brebnerii, Madame Titrens, etc.

Le Rhododendrum ponticum album, hyloridé par le caucasium, a domé naissance au Cunningham's White, qui, à son tour, aree le Claudyanum, a produit le limbatum, la plus belle de toutes les variélés bordées.

Les Rhododendrum cotaubiense hyluides, tels, par rexmple, que l'album elegans, hybrides par le pictum, ont 
produit Standislis Perfection, Minnie, mistress Standish, cte.

II. Standish donne les caractères des divers luybrides quil vient d'énumérer; nous ne reproduirons pas cette partie de son article. Il ajoute enlin quelques observations importantes.

“Les premiers hybrides, dit-il, qui aient été obtenus du - maximum et de l'arborenm, sont de tous les moins floriferes, mais ils résistent aux temps chauds mieux que tous les autres; ils ont les inflorescences très-compactes et les fleurs de nuances très-brillantes. A leur tour, ces hybrides du maximum, féconlés par d'autres hybrides, ont donné naissance aux métis les plus florifères; car, dit l'habile horticulteur anglais, j’ai toujours vu que les hybrides nés de deux hybrides sont beaucoup plus floriferes que ceux qu'on a obtenus entre deux especes. Pour produire des hybrides vigoureux, il est indispensable d'opérer sur des espèces et des hybrides qui aient entre eux beaucoup d'affinité, ou bien les plantes qu'ou obtiendra ne végéteront que faiblement. J'ai fécondé plusieurs de mes hybrides à fleurs blanches par le Falconerii, et, quoique j'aie obtenu beaucoup de bonnes graines, parmi lesquelles un grand nombre ont germé, tous les plants que j’ai eus ainsi ont péri, à l'exception de quelques-uns issus du cimnamomeum, fécondé par le Falconerii, qui paraissent devoir devenir des plantes vigoureuses. Il est curieux d'observer les plantes qui résultent de la fécondation de deux hybrides entre eux : elles retournent, les uns à l'un des deux parents, les autres à l'autre, tandis qu'il en est qui s'en écartent résolûment et qui prennent une manière d'être à elles propre. ")

Nous terminons ce long article sur les Fhododendrum en rappelant à ceux qui veulent s'occuper de l'hybridation de ce beau genre que leur pollen peut se conserver longtemps avec toutes ses propriétés, pourvu qu'on le préserve de l'humidité. " Ainsi, dit un journal belge, les amateurs de France, d'Allemagne et d'Angleterre peurent s'adresser à leurs amis de Pongique, à charge de réciprocité, et se faire envoyer du pollen 
des phus riches variétés de nos collections acturllement en lleurs; ils sont assuries que les croisements opérés avee ce pollen, quand même ils n'auraient lien que l'amée suivante, auront autant de chances de succes que si les lleurs sur lesquelles on a recueilli ee pollen s'étaient épanouic's côte i coite de celles de lat variéte choisie comme porte-graine."

\section{Gienre Kalmia. - Kalmiu.}

Les Kulmia ont dix étamines dont les anthères sont logées dans les petites fossettes de la corolle, et dont les filets, en s'allongeant, se recourbent jusqu'à ce que l'are se détende et enlive l'anthère qui projette son pollen sur un stigmate glutineux ì eing divisions très-petites. La fícondation n'est done opérée qu’à celte éporque, en sorte que, si on coupe les filets avee des ciseaux à l'époque de l'épanouissement et avant quils ne soient détendus, on peut enlever les anthères intactes et fíconder le stigmate par un pollen étranger.

On connait plusieur's espèces de Kolmia, mais elles n'ont donné eneore qu'un petit nombre de variétés, et sont loin, sous ce rapport, des Rhododendions et des Azulen.

\section{Genre Epaeris. - Epacris.}

Ces plantes ont le port des Bruyères et sont tout aussi éléfantes; elles ont cing étamines dont les filets portent les antheres it la hauteur d'un stigmate en tête et ghlutineux. L'anthèse a lieu un peu avant l'épanouissement, en sorte que la fécondation artificielle ne peut se pratiquer sur ces plantes que comme sur les Bruyères, avec lesquelles elles ont beaucoup d'imalogie.

Les Epacris altemuatu, elegans, lavigulu, refulyens, immessa, coccinea, compumulatu sont peut-être celles sur lesquelles il conviendrait de faire des essais, mais elles domnent difficilement des graines. 


\section{CHAPITRE VI}

\section{DYCOTYLEDONES COROLLIFLORES}

$\Lambda$ PÉTALES SOUDÉS EN UNE COROHLE UTPO

\section{FAMILLE DES JASMINÉES.}

\section{Genre Oliwier. - Olea.}

L'Olivier a deux étamines dont les anthères sont enfermérs daus le tube de la corolle et avoisinent un stigmate qui se présente comme une petite tête élargie, divisée en deux parties et couverte de petites papilles. Les anthères y répandent un pollen d'un beau jaune qui assure ordinairement la fructification.

Pour hybrider l'Olivier il faudrait done, après avoir isolé un rameau, détruire une partie des fleurs, et veiller la floraison pour enlever de suite les deux anthères; on appliquerait le même jour, au pinceau, le pollen étranger.

Il est fâcheux que l'Olivier ait une croissance aussi lente; il y aurait sans doute des essais d'hybridation à faire sur un arbre aussi important. En conservant pour pied mère l'Olivier ordinaire, ne pourrait-on pas essajer de le croiser avec celui de Madère, et avec cette variété de Criméc récemment annoncée comme pouvant résister aux froids de nos hivers du centre et du nord? Peut-être même un croisement avec le fragrans ou 
l'americana produirait-il, sinon des variétés utiles ì la culture, au moins des plantes ornementales par leurs fleurs ou par leur port.

\section{Genre Lilas. - Syringu.}

Le Lilas, comme les autres Jasminées, n'a que deux étamines qui sont enfermées dans le tube de la corolle, mais quì ne répandent leur pollen qu'au moment de la floraison, en sorte qu'en reillant l'épanouissement on peut les retrancher à la pince avant l'anthèse. Ce retranchement se fait d'autant plus facilement que les anthères sont placées près du sommet du tube, tandis que les deux stigmates portés sur un style court sont situés au fond du tube, où il faut aller les féconder avec un petit pinceau.

Ce genre est un de ceux où il ỵ a le plus de variétés à obtenir par les fécondations croisées. Déjà le Vurin est un hỵbride, et I'on a encore le Sungé, le Churles $X$ et l'ancienne espèce connue sous le nom de Lilas de Perse. On a introduit aussi le Lilas Vallet, le Lilas double de Noiselte, le Prince Nottyer et le Josilieu, véritable espèce très-distincte. Ce dernier et le Lilas ordinaire sont, je crois, ceux qui seraient préférables pour porte graines, ct qu'il faudrait féconder par tous les autres, quand la chose serait possible; car il arrive très-sourent, dans ces hybrides, le Saungé el même dans le Lilas de Perse, que les étamines arortent dans le tube. It est bien entendu qu'il faudrait préparer convenablement le Lilis avant de le féconder, et retrancher lit majeure partie de ses lieurs.

Parmi les nombrenses variétés obtenues dans ces derniers temps, nous n'en eiterons qu'une seule qui, par sa magnificence, devra ètre employée dans tous les croisements. C'est le Lilas de Lindley.

\section{Genre Frène. - Fraximus.}

Dans un genre aussi nombreux que le frêne, il est bien probable qu'il s'est formé naturellement des hybrides, et celte suppo. 
sition parait d'autant plus vraisemblable que les espèces sont polygames, et qu'alors, indépendamment des lleurs hermaphrodites, il y a aussi des fleurs mâles et d'autres qui sont femelles, souvent portées sur des individus distincts. On voit que la fécondation artificielle serait extrêmement facile, et que la seule difficulté consisterait dans l'isolement du porte-graine. Je ne crois pas que l'on ait jamais tenté d'hybrider les Frênes, qui, à l'exception de quelques variétés, doivent plutôt être considérés commie des arbres forestiers que comme des espèces d'ornement. II suffirait done, si l'hybridation est possible entre leurs espèces, de secouer des rameaux mâles sur des fleurs femelles. L'excelsior ou frêne ordinaire se croiserait peut-être avec ses propres variétés ou avec l'angustifolia, le parvifolia et le lentiscifolia. L'americana pourrait sans doute s'hybrider avec les autres espèces des mêmes contrées, comme le jubescens, le juylandifolia, le caroliana, ete.

L'importance des bois de construction ou des arbris forestiers devrait engager le gouvernement à faire tenter I'hybridation sur tous les arbres qui en sont susceptibles.

\section{Genre Jasuin. - Jasminum.}

Ces plantes offrent encore deux anthères placées i l'entréc du tube de la corolle, et que l'on peut enlever avec des pinces ì l'époque de l'épanouissement. Le stigmate à deux branches, caché dans le tube, est apte avant les étamines et peut receroir, au moyen d'un petit pinceau, le pollen étranger.

Il est douteux, toutefois, que les Jasmins à fleurs jaunes puissent être hybridés par ceux à fleurs blanches, qui ont d'ailleurs les feuilles opposées, tandis que les autres les ont alternes, et réciproquement; mais, cntre espèces de la même section, il est très-possible que l'hybridation puisse avoir lieu et qu'il en rísulte des variétés supérieures à leurs types. 
FAMILIE DES APOCVNEES.

Gesure Inclépiade. - Asclepias.

Ces phames offient un appareil de reproducton qui differe assez sensiblement de toutes les aulres especes. Toiri rom-

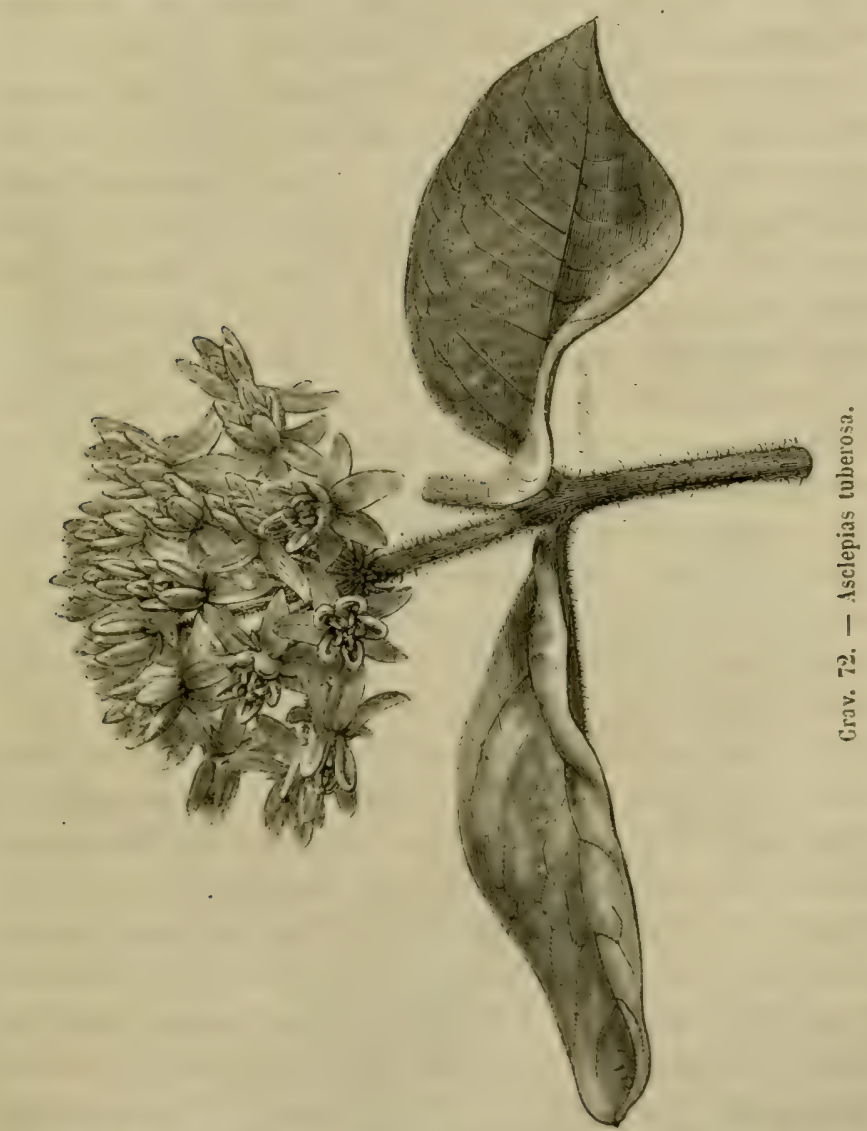

ment le décrit Vaucher, qui l'a observé ave beancoup de soin, rt dont nous reproduisons textuellement les expressions. "Je remarquerai 10 que les Asclépiodes que jai exami- 
nées ont leurs lobes anthérifères séparés, aplatis, formés d'une matière homogène, solide, et attachés par un pédicelle liliforme et articulé à un renflement discoïde; $2^{\circ}$ que toutes ces anthères sont logées dans le voisinage des stigmates plus ou moins papillaires et plus ou moins engagés dans le corps cylindrique el pentagone qui les recouvre, et auquel je donne le nom de couvercle; ordinairement ces stigmates sont presque libres, et dans le fruticosa ils sont d'abord couchés.

" Les cornets qui entourent le couvercle sont des poches nectarifères, car je les ai vus très-souvent remplis de l'humeur miellée qui, à la floraison, sort en si grande abondance, que non-seulement elle les remplit, mais qu'elle imprègne tout le couvercle où sont engagés les anthères, qu'elle entre par les ouvertures des lames cartilagineuses et se répand même en gouttelettes sur le terrain, comme on peut le voir dans l'Hoya; or, ll n'est pas difficile de comprendre qu'elle détrempe aussi la masse pollinique, dont elle transporte les molécules on les émanations sur les stigmates.

“ La manière dont s'opère ce phénomènc exclut toute forme hybride ou variété; aussi n'en remarque-t-on aucune dans les Asclépiades, dont les espèces sont d'ailleurs si rapprochées.

"Ce mode très-extraordinaire de fécondation a été étudié d'abord par Jaquin, Treviranus, Ehremberg, et ensuite Brongniart et Robert Brown; ces derniers ont constaté que les deux masses polliniques, jaunes et aplaties, de chaque anthère, étaient autant de sacs qui renfermaient les granules polliniques, réunis ordinairement en petits groupes anguleux; qu'à la fécondation, ces sacs se rompaient sur leur angle intérieur, et laissaient sortir les granules déjà pourvus de leurs queues ou boyaux, qui s'insinuaient à traver's les vides d'un tissu cellulaire allongé, arrivaient ainsi jusqu'au sommet papillaire et velouté des stigmates, pénétraient de là ì travers le tissu lìche des styles jusqu'à la cavité de l'ovaire, et transmettaient immédiatement aux ovules le' fluide prolifique, c'est-à-dire un fluide oléagineux, mèlé de molécules extrêmement pelites. Ces savants 
ne vont pas au delà et ne prononcent pas si l'ovule, non fécondé, contenait déjà l'embryon inerte, ou sı cet embryon ré-

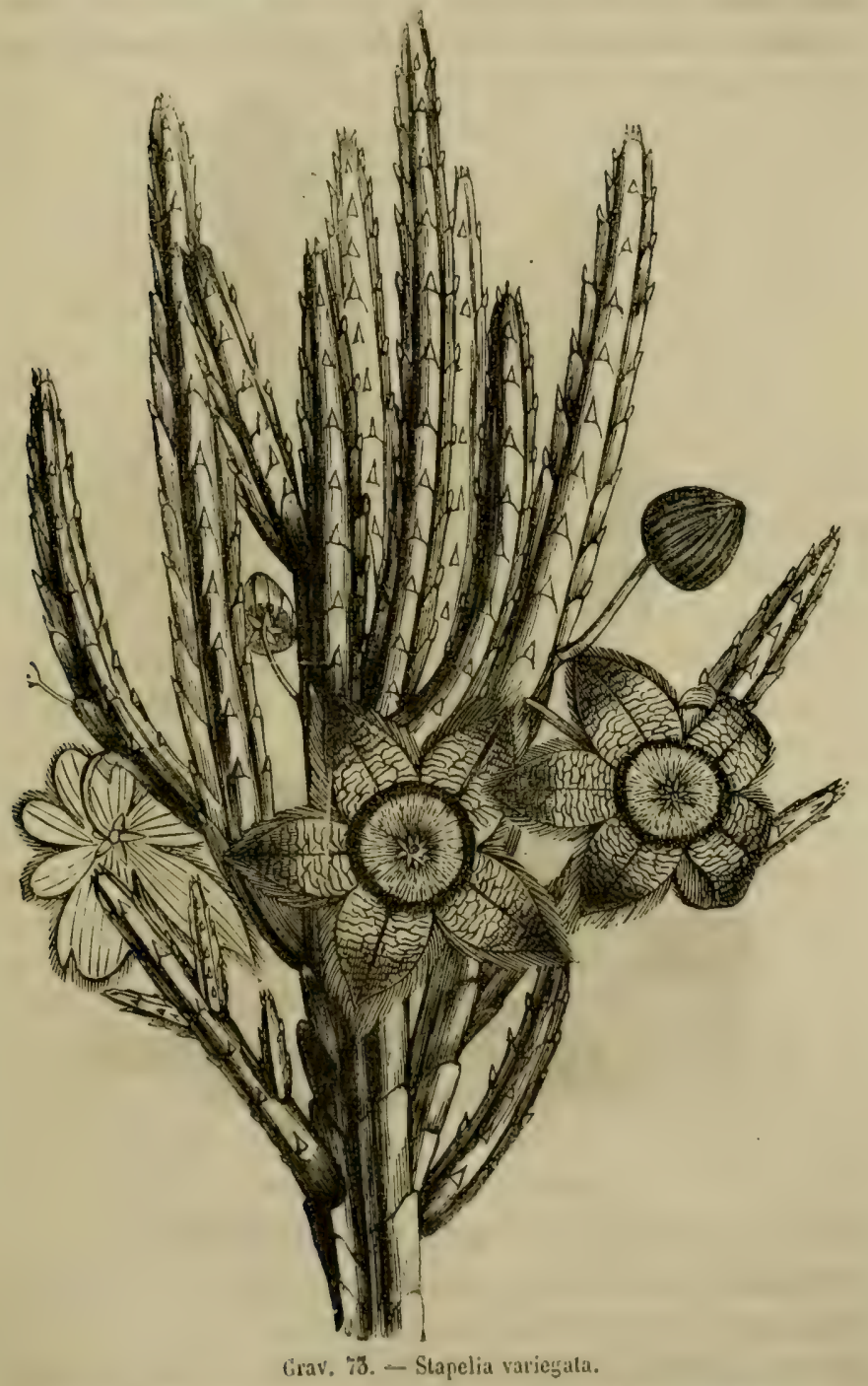

sulte du mélange des corpuscules du fluide fécondant avec ceux 
quii sont propres à l'orule : question qui, sans doute, ne sera janais résolue, parce que la suprème Sagesse ne l'a pas mise à notre portée. ") (Grav. 75.)

Nous ne pensons pas, comme le frofond ohservaleur que

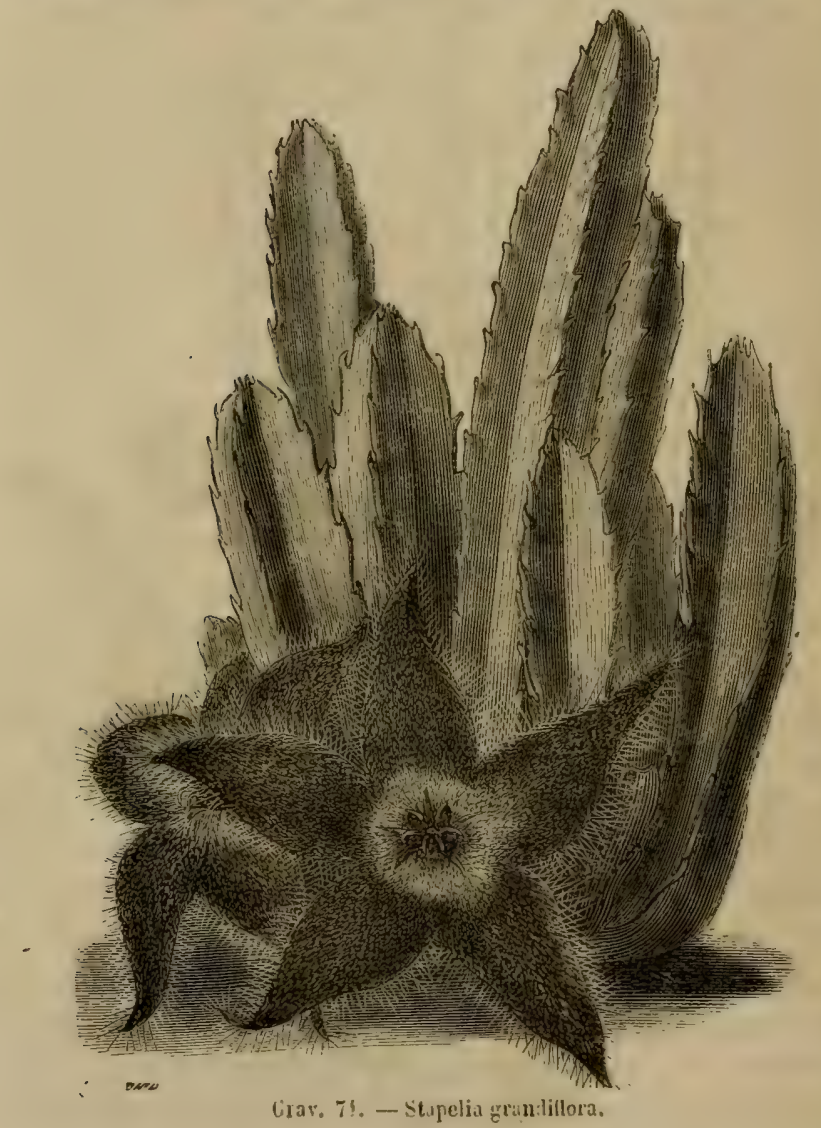

nous venons de citer, que l'hybridation soit impussible dans le: Asclépiades; nous croyons, an contraire, que les masses polliniques peuvent ètre détachées d'une espéce et portées dans lit liqueur miellée d'une autre, de manière à y ètre délavées et it féconder son stigmate. Les insectes ne pervent-ils pas opérer 
eux-menses celle mysterieuse fécondation? Ainsi, daus less Stıpelia, qui appartiennent à la même famille, l'odeur cadavéreuse des fleurs, qui est telle que les mouches y déposent coutinucllement leurs œufs, comme sur de la viande corrompue, n'a-t.elle pas pour but de faciliter la fécondation par le moyen des insectes, et si une mouche peut transporter le pollen d'une lleur sur une autre, pourquoi l'homme ne ferait-il pas plus sùrement ces hybrides, en enlevant les masses polliniques du Stapelia comme des Asclepias, et en les délayant au pinceau dans la liqueur nectarifère, dont il imprégnerait ensuite les stigmates (grav. 75, 7/).

\section{Genre Laurier-rose. - Nerium.}

Les cinq étamines des Laurier's-roses s'ourrent arant l'épanonissement de la lleur, ef entourent un anneau glutineux qui forme li base du stigmale, qui s'élève ensuite sous forme d'un petit cylindre. J'ai toujours trouvé les anthères ouvertes avant lés lleurs et le sligmate entonré de pollen, mais, comme ce pollen n'est pas pulvérulent, on peut encore enlever adroitement les anthères, poser sur le stignate un pollen étranger et appliquer cusuite l'humeur miellée que l'on troure dans le fond de la corolle. Les nouvelles variétés d'oléandres, obtenues par II. Mabire, les variations de couleurs que l'on connait, doivent engager les horticulteurs à s'occuper de ce beau greme, dont lit lécondation artificielle est difficile, mais promet de beaux résultits. Il arrive aussi quelquefois que, dans les nuurelles varietis de Nerium ì fleurs orangées, jaunâtres ou blanches, on rencontre quelques fleurs dont les étamines sont avortées 04 contiennent du pollen infertile. Il est facile, quand on le's trouse, de les léconder artiliciellement. Le nouvean Terium Jénne d'Are i lleurs odoranles, d'un blane pur, donnerait cerlainement des ganins nourcaux sil fructific facilement.

\section{Genre Pervenche. - l'inca.}

Les élannines, dans les Pervenches comme dans les Nerium, 
entourent un stigmate muni d'un anneau en forme de couronne et recouvert d'une étoile de poils. La fécondation est donc assurée avant mème que la fleur' ne s'ouvre, et l'hybridation me parait impossible. Malgré cette certitude d'imprégnation, malgré ce contact du pollen avec le stigmate, je n'ai jamais vu de fruit de la petite ni de la grande Perrenche, tandis que celle de Madagascar, ou le rosea, fructifie facilement daus nos serres.

Peut-être l'absence des fruits des Pervenches herbacées tient. elle à ce que le pollen glutineux manque du contact de lia liqueur miellée pour opérer la fécondation.

\section{FAUILLE DES BIGNONIACÉES.}

\section{Genre Bignone. - Bignonia.}

Ces plantes ont toutes quatre étamines didynames qui ouvrent leurs anthères à peu près vers l'époque de l'épanouissement de la fleur ou un peu après, et répandent une grande quantilé de pollen avant que le stigmate bilamellé, qui termine le stylc, soit entièrement développé. Ces conditions sont donc favorables à la fécondation artificielle que l'on pourrait tenter sur quelques especes, en ayant soin de rapprocher celles qui ont le plus de rapport entre elles, car on y observe des différences assez grandes pour motiver leur séparation en genres ristincts; ainsi, le catalpa et le longissima forment le genre Catalpa; le- radicans, le grandiflora, le capensis, etc., compensent le genre Tecoma; et le pandorea, l'unyui.s, le capreolata, etc., sont restés dans les Biynones.

\section{FAMILLE DES POLÉIONIACÉES.}

Genre Phlox. - Phlox.

Les cinq étamines des Phlox sont distribuées à des lauteurs différentes dans te tube de la corolle, de telle sorte que le 
stigmate, à deux ou trois divisions, se trouve toujour's placé à la hauteur d'une anthère et ne peut échapper à son pollen. L'anthère s'ourre en même temps que la fleur, circonstance qui rendrait toute fécondation croisée impossible, si on ne pouvait retrancher les cinq anthères dans le bouton. Il faut donc les enlever arec les pinces par une petite fente pratiquée à la corolle, et attendre, pour féconder le pistil, que ces fleurs opérées s'épanouissent comme les autres, que l'on a soin de retrancher immédiatement pour éviter les fécondations indirectes. On peut mêne, au besoin, attendre l'épanouissement pour enlever les anthères, car le stigmate ne devient apte qu'après les étamines; mais la difficulté de retrancher les anthères qui sont au fond du tule rend tonjours necessaire la petite incision, qui, du reste, ne nuit en rien aux fonctions de la fleur.

Les Phlox sont déja tellement perfectionnés que l'on est embarrrassé sur le choix des sujets à croiser; les diverses variétés du striala, du pyramidalis, du suffruticosa, le vincxplora versicolor, Van-Houttei, trimphans, picta, alba carulescens. et alba glomerala ont été la souche des admirables variétés que nous possédons maintenant et qui tendent, par leur heaute, a faire oublier leurs premiers parents. Ce beau genre est loin d'avoir atteint ses limites de perfection, et il y aura certainement toujours gloire et profit à s'en occuper.

\section{FAMILLE DES CONYOLTLLACÉES.}

Genre Liseron. - Convolvulus, Ipomea.

l.e genre très-nombreux des Liserons présente ses organes reproducteurs disposis d'une manière analogue. Cinq étamines, dont les anthères sourrent assez souvent à l'opposé du pistil, un syle terminé par deux ou trois stignates, quelquefois par un scul, et toujours papillaires. Ces stigmates deviemnent aptes le jonr mème de l'épanomissement, qui sonvent dure à peine le reste de la jonmée. La fécondation est presque tonjours retardée 
jusqu'au moment où la fleur, en se flétrissant, applique sa corolle couverte de pollen contre le stigmate. Avec de telles dispositions d'organes, on peut hybrider, ou du moins tenter l'hybridation, soit en retranchant, le matin, les étamines, ce qui est le moyen le plus sûr, soit en isolant le pistil fécondé et empèchant la corolle de se flétrir sur lui, au mojen d'un peu de coton ou d'un petit tube de papier. Les fécondations croisées ont déjà donné des variétés panachées, tricolores, rayées ou de couleurs uniformes très-différentes, dans l'Ipomea des jardins. Il serait à désirer que l'on puisse en obtenir de même entre les différentes espèces qui composent le beau genre Pharbitis, détaché des Liserons, et dans lequel on trouve le Learii, le longifolia, etc. La patate, Convolvulus patatas, à racine comestible, mériterait aussi toute l'attention des horticulteurs; les patates palmée et igname surtout ont déjà donné en serre des graines fertiles. Il n'est pas douteux que, si, en Italie, en Espagne, ou même dans le mıdi de la France, on s'occupait de leur culture et surtout de leur croisement, l'on obtint un grand nombre de variétés excellentes, comme cela a eu lieu pour les Pommes de terre.

\section{FAMILLE DES BORAGINÉES.}

\section{Genre IIéliotrope. - Heliotropium.}

Les cinq étamines des Héliotropes sont placées au-dessus d'un style conique, terminé par deux petits stigmates, en sorte que la fécondation est assurée dans ces plantes. L'anthèse a lieu en même temps que l'épanouissement, et, comme les anthères sont très-petites, il est difficile de les enlever. Il faudrait, du reste, faire cette opération le matin, de bonne heure, car la fécondation ne dure qu'un jour. Il y aurait peut-être de jolis hybrides à obtenir entre les Héliotropes, et surtout en prenant pour type l'Héliotrope du Pérou, dont les fleurs odorantes donnent facilement des graines dans notre climat. 
Les varialions ou les hybrides récemment obtenus dans ce genre ont dignement récompensé les efforts de ceux qui ont eu foi dans la rariabilité et dans l'hỵbridation des espèces.

\section{Genre Consoude. - Simphylum.}

Les Consoudes offrent quelques jolies espèces qui méritent de lixer l'attention des horticulteurs. Peut-être en obtiendrait-on des hybrides en enlevant de bonne heure les cing étamines et en posant au pinceau le pollen étranger. Les S. coccineum, orientale, echinatum, peregrinum sont ceux qu'il faudrait cherclier à croser:

\section{Genre Pulmonaire. - Pulmonaria,}

On cultive quelques Pulmonaires, dont les jolies fleurs s'épanouissent le matin et durent pendant quelques jours. Leurs cing anthères sourrent le premier jour de l'épanouissement, et peuvent être enlevées assez facilement du tube de la corolle. Comme les espèces sont assez roisines, l'hỵbridation doit avoir lieu, bien que je ne sache pas.qu'on l'ait jamais tentée. L'azurea, le sacharrata, le sibirica, le dahurica se croiseraient probablement, et non le virginica, qui se rapproche plus des Lithospermum que des Pulmonaria.

\section{FAMILLE DES SOLANEES.}

\section{Genre Morelle, Pomme de terre. - Solanum.}

Les nombreux Solamum forment un genre très-naturel, dont toutes les espices se ressemblent par les fleurs. Toutes ont cinq étamines à filets courts, à anthères pointues et appliquées les unes contre les autres, de manière a former un tube que traverse un style terminé par un stigmate en tète et glulineux. Les anthires répandent leur pollen par deux pores ou opercules, placés à leur sommet, et ne deviement aptes qu’après l'épanouissement, en sorte quion peut très-aisément les suppriner le 
jour de la floraison, et le lendemain ou surlendemain féconder le pistil.

J'ignore si l'on pourrait croiser les diverses espèces de Solanum que l'on cultive dans les serres. Quelques-unes sont fort belles; telle est, par exemple, le quitoense à grandes fleurs violettes. Mais cependant ce n'est pas dans ce genre qu'il faut chercher les plantes le plus ornementales. L'espèce qui donne à ce groupe sa haute importance, c'est le tuberosum ou la Pomme de terre, dont les variétés sont nombreuses, mais susceptibles de s'augmenter encore.

Il faut planter séparément les Pommies de terre que l'on veut hybrider, dans le coin d'une terre ou d'un jardin, supprimer une partie des ombelles de fleurs, en laisser deux ou trois à chaque ombelle et enlever les étamines à mesure que la floraison s'opère. On choisira ensuite le pollen sur d'autres variétés. Quelquefois on rencontre des anthères stériles, ou bien on ne trouve de pollen qu'à la partie supérieure de l'anthère.

Il n'est pas difficile, comme on le voit, d'avoir des variétés nombreuses de Pommes de terre, mais il faut en obtenir qui, sous certains points de vue, soient prélérables aux anciennes.

Ainsi on cherchera par l'hybridation des variétés plus précoces, d'autres plus productives en tubercules, plus riches en fécule, plus savoureuses pour la table, etc., en ayant soin de prendre toujours pour un des types une des variétés qui présentent au plus haut degré la qualité particulière que l'on cherche à obtenir.

Voici la liste de plusieurs variétés principalement destinées à la table ou remarquables par quelques qualités :

Kidney, jaune, longue, très-précoce. - Naine hative, jaune, ronde, très-précoce. - Truffe d'août, ronde, rouge pàle, très-précoce. - Fine hîtive, jaune, ronde, très-précocc. - Decroizilles, longue, rouge vif, se conservant bien. Châtaigne-Sainville, jaune, oblongue. - Vitelolte, rouge, longue, de conserve. - Ronge longue de Hollande, produil peu, très-bome. -- Cornichon janne, longue, très-bonne. - 
Petite clinoise, ronde, blanche, petite, très-honne, peu productive. - Des Cordillières, rouge, ronde, chair jaune, délicate, peu productive, très-honne. - Violette ronde, très-productive, bonne qualité. - Turdire dr Irlande, rouge, ronde, se conservant sans pousser jusqu' à la plantation. - Magdelaine, très-précoce. - Seyjonsac, végétation très-prompte. - Vierge, produits très-abondants et de bonne qualité.

On trouve encore dans ce genre deux espèces potagères, les S. melongena et $S$. persicum.

La première, connue sous les noms d'Aubergine, Melonyène, offre des variétés de couleur et de forme que l'on pourrait multiplier à l'infini par les croisements. L'ne des plus estimées est la longue blunche de la Chine, à pulpe fondante et peu filandreuse, que l'on pourrait choisir pour porte-graine.

La seconde est la Tomate, susceptible aussi de nombreuses modifications. On comnait déjà la yrosse rounge, la grosse janne, la petite jume et la petite rounge, celle en poire ou cerise, etc.

Ce que nous venons de dire des Solumum peut également s'appliquer aux Capsicum ou Poivres rouges, qui en sont tris-svoisins.

\section{Genre Cestre. - Cestrum.}

La corolle plus ou moins tuluulée des Cestrum renferme cinc étanines, au milieu desquelles se trouve un stigmate en tite aplatie. J'ignore si ces plautes peusent fructifier dans nos serres, où elles fleurissent ordinairement la nuit; mais, à cause de cette floraison nocturne, les essais de fécondation, soit avec leur propre pollen, soit avec celui d'une espice roisime, devront être tentís à l'heure où la corolle est totalement ¿́panouie. Le Cestrum rosenm a dejà produit des graines.

\section{Genre Stramoine. - Datura.}

Les grandes fleurs des Datur'a contiennent cing étamines, dont les anthères sont quelquefois soudées en un tulie, et dont 
le style, assez long, porte un stigmate à deux lèvres. Les uns ont les fleurs constamment droites, d'autres les ont penchées. Il y en a d'herbacés annuels et d'arborescents que l'on a séparés des autres pour en former le genre Brugmansia.

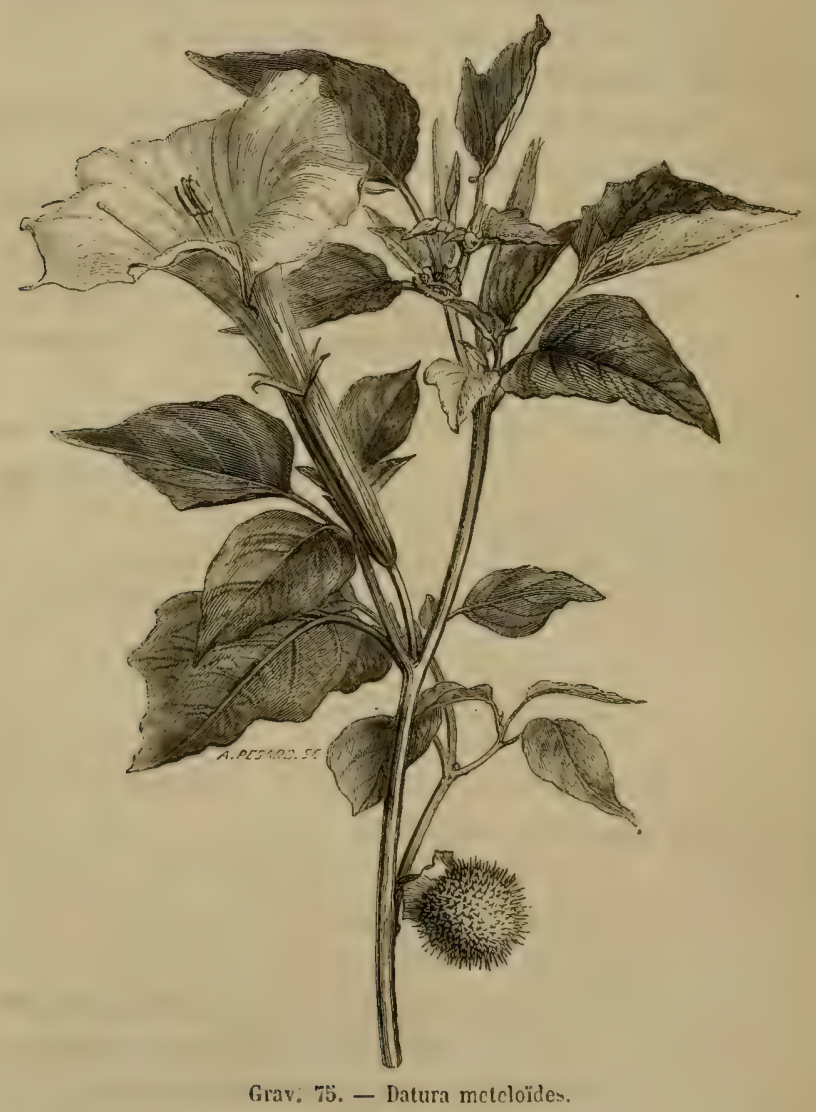

Les espèces herbacées donnent facilement des graines, et il I'y a aucun intérêt à les hybrider. Il en est certainement de même des arborescents, dont une espèce, le $D$. arboréa à grandes fleurs blanches, est trìs-commun dans nos jardins, où je ne l'ai jamais vu fructilier; peut-être réussirait-on en le fé- 
condint avec son propre pollen, ou an moins arec celui du suateolens, de l'arbuscula, du floribunda ou du Knigthii. Quant au bicolor, il a déjì donné deux variétés, le lutea et le sanguinea, qui se croiseraient entre elles sans grand résultat, mais que l'on peut espérer d'hybrider un jour avec les espèces que nous venons de citer plus haut.

II. Nandin, dans ces derniers temps, s'est occupé, avec tout le zìle et toute la sagacité qu'on lui connait, de l'hybridation des Datura. Il a obtenu facilement des hybrides, et nous renrojons à son mémoire les personnes qui voudraient renouveler ces croisements, plus importants au point de vue de la physiologie que sous le rapport horticole (grav. 75). J'ai tenté cette année ( $\$ 62$ ) de's croisements entre les magnifiques fleurs des Datura ceratocaulon et metelö̈les. Je ne puis connaitre encore le résultat de ces essais.

\section{Genre Tabae. - Nicotiana.}

Les fleurs des différentes espèces de Tabac ont cinq étamines placées dans le tube de la corolle, et un stigmate capité, un peu échancré sur ses bords.

Dis l'épanouissement de la fleur, on peut, avec les pinces, enlever trìs-facilement les anthires, et le stigmate est bientôt apte à être imprégné du pollen d'une autre espèce. Aussi l'hybridation du Tabac a-t-elle lieu très-souvent et même naturellement par les insectes. Il y aurait peut-être grand avantage pour l'agriculture à faire des essais d'hybridation sur ces plantes et surtout sur le Nicotiana tabacum. On arriverait peut-être, par des races croisées, à labriquer en Europe, et surtout en France, ces rariétés particulières de tabac étranger, qui sont évidemment supérieures aux nôtres, et qui ne doivent pas seulement au climat les qualités qui les distinguent.

On a annoncé, en 1852, un Tabac venant d'Alger, N. macroplyylla Link, qui pourrait peut-être entrer avec avantage dins les croisements que l'on tenterait sur les espèces de ce groupe. 
Ce genre est celui qui se prète le micux à tous les croisements, et c'est sur des Tabacs que Kolreuter a fait autrefois les. expériences les plus positives sur l'hybridation des végétaux.

\section{Genre Pétunie. - Petunia.}

Comme les Tabacs, les Petınia se croisent très-facilement et ont, comme eux, cinq étamines, dont les anthères occupent dans le tube des hauteurs différentes, et un sligmate renflé, épaissi, à deux lobes glutineux. Il faut enlever de bonne heure les anthères, car l'anthèse suit l'épanouissement. Déjà le nycta'giniflora à fleurs blanches et l'élégant à fleurs violettes se sont hybridés dans nos jardins, et le Petunia est devenu plante de collection. M. Van Houlte en a obtenu des variétés à fleurs énormes. Et, depuis lors, de nombreux essais ont été tentés avec succès dans l'hybridation de ces plantes.

Toutes nos variétés proviennent du croisement de deux espèces distinctes, mais voisines des $P$. myctaginiflora et $P$. violncea. M. Naudin a fait de nombreux essais sur le croisement de ces espèces.

On peut presque toujours appliquer aux nombreux hybrides de Petunia ce que disait avec esprit et poésie mon savant collègue Planchon, en parlant du Petunia meleagris : " On soupçonne, il est vrai, le $P$. violaceu, d'en être la mère; mais, par contre, la paternité n'est pas aisément établie chez des êtres androgynes, dont une corolle forme le lit nuptial; car mille petits butineurs ailés, friands de nectar et saupoudrés de pollen, se font à leur insu les instruments de tendres larcins, et, quand une fleur renchérit sur la beauté de sa mère, la médisance ne manque pas de l'appeler enfant de l'amour. "

\section{FAMILLE DES ANTHIRRINEEES.}

\section{Genre Mufnier. - Anthirrhimum.}

Quatre élamines, munies chacune d'une anthère à deux grosses loges, sont enfermées dans le tube de la corolle des 
Muffiers et des Linaires, et répandent leur pollen sur un stigmate bifide. Ces anthères ne s'ourrent qu'à l'époque de l'épanouissement, en sorte que l'on peut facilement les ôter à la pince et pratiquer la fécondation artificielle. Elle réussit parfaitement entre les variétés d'Antirrhinum majus, ou grundes gueules-de-liom, que l'on cultive maintenant dans tous les jardins, où elles produisent beaucoup d'effet.

\section{Genre Penstemon. - Penstemon.}

Les quatre anthieres du Penstemon, forınées chacune de deux grosses loges ovoïdes et pointues, s'ouvrent un peu après la fleur pour inonder de pollen un stigmate que le style amène à peu près à la hauteur des anthères. Le style, d'ahord appliqué sur la lèrre inférieure, et placé par conséquent aul-dessous des anthères, se relère ensuite dans la plupart des espèces et s'applique à la partie supéricure, tandis que les étamines défleuries vienuent, au contraire, prendre la place que le style occupait auparavant. Les anthères peurent s'enlever facilement arec les pinces, et l'hybridation s'opire au pinceau. On peut, au hesoin, humecter le stigmate avec un peu d'humeur miellée que chaque fleur sécrète à sa base.

Les Penstemons forment un genre nombreux en belles plantes, dont quelques-unes ne sont que des hybrides ou des variétés. Le gentiunoüdes, entre autres, a déjà beancoup varié, et sa variété cocrineus major est une des plus belles. Le commatus, le cobra, le laurifolius, le splendens, le venustus et une foule d'autres pourraient peut-être s'hybrider, et ce sont des plantes assez belles pour qu'on apporte quelques soins à leur croisement.

\section{Genre Digitale. - Digitalis.}

Les belles fleurs des Digitales renferment quatre étamines à anthères didymes et remplies d'une grande quantité de pollen; elles s'ourrent en mème temps que les fleurs ou peu après, et leur volume permet de les retrancher sans difficulté. C'est seu- 
lement deux jours, quelquefois trois, après l'épanouissement que le stigmate acquiert son développement et présente une ou deux lamelles aptes à recevoir le pollen. Au moyen de cette disposition, les Digitales sont des plantes qui offrent de trèshonnes conditions à l'hybridation. Aussi se croisent-elles naturellement, comme on le voit dans le purpurascens, hybride du purpurea et du parviflora, mais qui ne se reproduit pas de graines. Je ne sais si les Digitales indigènes ou à épis unilatéraux, et les exotiques à épis complets pourraient s'hybrider entre elles, mais, en supposant que cette fécondation croisée n'ait pas lieu, en admettant même qu'on n'obtienne, en croisant les indigènes, que des plantes moins belles que le purpurea, ce qui est probable, on pourrait au moins hybrider les diverses variétés de cette dernière, qui, jusqu’à présent, offre toutes les nuances du rouge pourpre foncé au blanc pur.

Dès l'année 1769, Kœlreuter avait hybridé les Digitales et en avait obtenu des hybrides dont plusieurs lui ont fourni des graines fertiles qui ont reproduit les hybrides.

Il a obtenu en premier lieu le Digitalis purpurascens que depuis on a rencontré çà et là à l'état sauvage. Il a eu des hybrides extrêmement fertiles par le croisement des $D$. purpurea et thapsi, des $D$. ferruginea et ambigua; les D. canariensis et purpurea lui ont aussi donné des hybrides.

M. Pepin, l'un des chefs si habiles du jardin de botanique de Paris, cite un hybride entre le purpurea et l'ambigua, et affirme que toutes les espèces de l'école perdent bientôt leurs caractères par l'hybridation.

\section{Genre Mimule. - Mimulus:}

Ce genre offre quatre étamines enfermées dans la corolle et un style terminé par un stigmate formé de petites lamelles élargies et papillaires extrêmement irritables; aussitôt qu'on les touche elles se rapprochent, se serrent et ne s'ouvrent plus. Cettesingulière irritabilité du stigmate permet d'opérer la fécon- 
dation artificielle sans enlever les étamines. On pose le pollen au pinceau sur le stigmate qui se ferme tout de suite et en conserve les granules. J ai souvent fécondé entre elles, et toujours avec succès, les diverses modifications de .I. yuttatus, et j’en ai obtenu de très-belles variétés. Je ne sais si on pourrait de même croiser les espèces entre elles, mais il me semble que déjà plusienrs paraissent intermédiaires ou hybrides. Le cardimulis serait aussi susceptible de produire des variétés nouvelles, surtout s'il pouvait recevoir le pollen des diverses variétés du guttatus, du luteus et du glutinosus.

On a de très-belles variétés hybrides de divers Mimulus, et les Diplucus, voisins des Nimulus, peuvent aussi être croisés, comme vient de le prouver M. Ingelrelst, et comme on peut s'en assurer à Nancy, dans les jardins de M. Y. Lemoine.

\section{Genre Calcéolaire. - Calceolaria.}

Ces jolies plantes n'ont que deux étamines munies de deux grosses anthères que l'on aperçoit tout de suite, en écartant un peu le sabot de la lèvre supérieure. Ces anthères répandent leur pollen aussitôt que la fleur s'épanouit; mais cette poussière reste longtemps adhérente sans toucher le stigmate. Ce dernier, très-pelit et très-simple, termine un style qui s'élève plus ou moins au-dessus des étamines, selon les variétés. Celles dont le style est le plus saillant sont celles qu'il faut préférer pour les fécondations croisées, parce que, au milieu de l'atmosphère tranquille d'une serre, le stigmate échappe au pollen de ses propres étamines, et peut être imprégné par un autre, sans qu'on prenne la peine de retrancher les anthères. An reste, cette opération est très-simple et doit se pratiquer avant l'épanouisscuent, yu’il faut allendre ensuite pour féconder le stigmate. On s'en dispense le plus souvent quand on veut faire en grand l'hybridation des Calcéolaires; mais un anateur, qui opérera seulement sur quelques pieds, sera beaucoup plus sûr du résultat en culevant à temps les étamines. 
Les résultats si remarquab!es obtenus à Gand par M. Van Iloutte, dans le croisement de ces plantes, doivent engager les amateurs à l'imiter et à créer chaque année des centaines de variétés toutes différentes, toutes plus belles les unes que les autres.

On fera bien de recueillir le pollen dans une boîte à compartiments, munis chacun de leur pinceau pour nuancer en espérance les plantes selon son goût ou ses prévisions.

Les organes de la reproduction sont très-petits dans les Calcéolaires, mais, comme la fécondation croisée n'a lieu qu'entre variétés de coloris, on peut se dispenser de retrancher les étamines et poser d'avance sur le stigmate un pollen préalablement recueilli, avant que celui des propres étamines de la plante se soit échappé.

C'est au père Feuillée que l'on doit, en 1714, la première Galcéolaire que l'on vit en Europe. Elle venait du Pérou. Fothergile en introduisit, en 1777, une seconde espèce des îles Falkland. Ces espèces restèrent pures, sans alliance ni bâtardise; mais, de 1825 à 1850, parurent les Calcéolaires du Chili, dont les espèces croisées sont devenues la souche de nos brillantes Calcéolaires.

On ne peut s'attendre à rien de remarquable, et surtout à rien de nouveau, si l'on sème des Calcéolaires qui n'ont pas été hybridées. Il est donc essentiel de réserver pour la graine quelques plantes de choix que l'on fécondera indistinctement les unes par les autres. Seulement il faut avoir la précaution de choisir sur chaque plante pour porte-graine, non pas les fleurs les plus larges et les plus brillantes, mais, au contraire, celles qui sont les moins belles. L'ampleur et la leauté de la corolle sont presque toujours en sens inverse du développement des organes de la reproduction. Les belles Calcénlaires sont comme certaines femmes qui ne déploient le luxe de leur toilette qu'aux dépens des objets de première nécessité dans le ménage. 


\section{FAMILLE DES RIIINANTACÉES.}

\section{Genre Véronique. - Veronica.}

Les deux étamines des Véroniques ne répandent leur pollen qu'après l'épanouissement, et peuvent être enlevées dès que la fleur s'ourre. Quand on a fécondé au pinceau les stigmates des lleurs ainsi débarrassées de leurs anthères, on coupe l'extrémité de l'épi. Les croisements ont déjà donné naissance à de très-helles plantes. On a donné le nom de $V$. Andersonii à une jolie léronique très-florifère, aux heaux épis bleus et blimes, que M. Anderson a obtenue près d'Édimbourg, en fécondant le V. sulicifolia ou Lindleyana des horticulteurs par le V. speciosa, charmant arbuste à leuilles laurinées. Cet hyloride, dit Lindley, est un des plus intéressints produits que l'art humain ait, en quelque sorte, créés.

II. Anderson posséderail encore, d'après N. Planchon Flore des serres et des jardins); un autre hybride obtenu avee le $V$. saxatilis et le $V$. finticulosa, et dont les fleurs sont bleues, veinées de pourpre.

\section{FAMILLE DES LABIÉES.}

\section{Genre Sauge. - Salvia.}

Le vaste genre des Sauges est un de ceux qui offrent le plus d'especes a l'horticulture et le moins de ressources à l'hybridation, car ces plantes sont très-distinctes el, par conséquent, peu susceptibles de se croiser. D'un autre côté, les Sauges ont peu de variétés, et, à l'exception du pratensis, qui varie du bleu au rose et au blane, et qui mériterait me place dans les jardins, peutètre aussi du Sulvia Grahummi, on ne voit pas les Sauges se modifier comme beaucoup d'autres plintes. Il y aurait done à tenter, dans ce genre, d'abord la férondation arti- 
286 FÉCONDATION NATURELLE ET ARTIFICIELLE.

ficielle avec le propre pollen des espèces qui ne fructifient pas

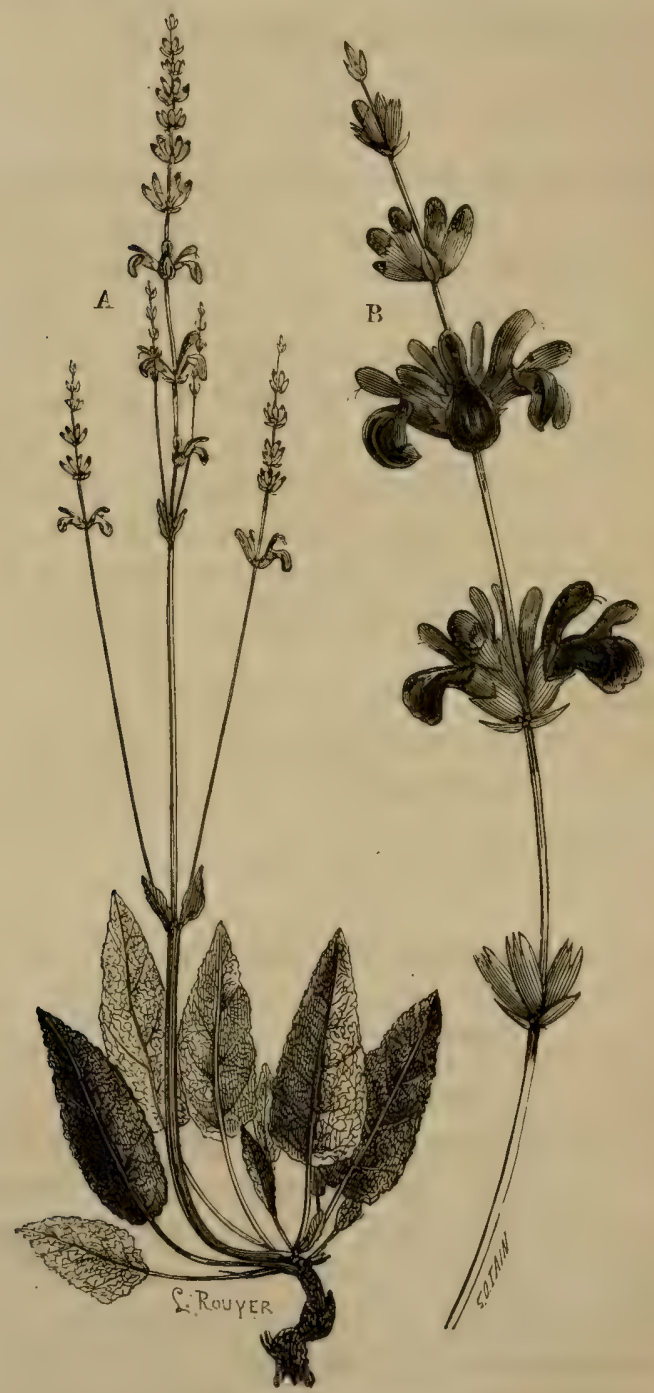

Grav, 76. - Sauge des prés.

dans nos serres; ensuite le croisement des espèces de méme 
section, et enfin l'hybridation des variétés que l'on obtiendrait des graines. Ces dernières tentatives réussiraient très-certainement arec quelques précautions.

Les deux anthères des Sauges s'ourrent avant l'épanouissement de la fleur, et le style, dont les deux hranches sont munies de stigmates papillaires, est plié pour ainsi dire sur le pollen. Quelquefois cependant il échappe à la fécondation. Il faut done dérouler la lèvre supérieure avant l'épanouissement pour enlever les deux anthieres, ou bien fendre par-dessous la lèvre inlérieure, et attendre ensuite, pour poser le pollen, que le slyle ait pris tout son développement après l'épanouissement des fleurs:

Les Salvia patens, Parsi ou dielytrö̈des donneraient de curieux ligbrides si on pouvait les croiser. Peut-être ne raudraient-ils pas le Splendens souchetti, la plus belle du genre (grav. 76).

\section{Genre Monarde. - Monarda.}

Les Monardes ont, comme les Sauges, deux étamines seulement, mais chaque anthère est munie de deux loges, tandis qu'il n'y en a qu'une seule dans les Sauges.

Comme dans ce dernier genre, les anthères s'ouvrent avant l'épanouissement de la' corolle, qu'il faut fendre ou dérouler pour les enlever; le style, assez long, est terminé par un stigmate à deux lobes que l'on ne peut féconder qu’après le développement de la corolle. Du reste, comme les différentes espèces de Monardes ont beaucoup de rapport entre elles, la fícondation croisée peut avoir lieu, et l'on connaît déja un certain nombre de variétés de l'hybride, qui est pent-être cllemême une espèce croisée.

\section{Genre Seutellaire. - Scutellaria.}

Ce genre contient de très-belles plantes, dont plusieurs espèces ont pénétré dans nos jardins. La fécondation s'opère dans l'intérieur de la corolle, au mogen de quatre étamines et d'un 
style terminé par deux stigmates, dont l'un supérieur, court et avorté, et un autre inférieur, plus développé, que l'on pourrait essayer de féconder artificiellement en enlevant préalajlement, par une ouverture pratiquée à la corolle, les quatre anthères qui devaient répandre le pollen.

Le S. japonica, le splendens et nos Scutellaires bleues indigènes sont des plantes extrèmement élégantes.

\section{Genre Dracocéphale. - Dracocephalum.}

Comme la plupart des Labiées, ces plantes ont quatre étamines, dont deux grandes et deux petites, el un style terminé par deux branches en alène couvertes par les papilles des stigrmates. La fécondation s'opère ainsi dans l'intérieur de la corolle, de telle sorte qu'il faudrait en ouvrir le tube avant la lluraison pour en retirer les anthères, et pouvoir ensuite tenter des croisements. Il est probable qu'ils réussiraient entre espèces de même section, et que les Dracocéphales, déjà très-nombreux, finiraient par donner des variétés. Ce sont des plantes qui méritent de fixer l'attention des horliculteurs.

\section{Genre Phlomis. - Phlomis.}

Ces plantes ont quatre étamines et un style terminé par deux branches faisant les fonctions de stigmates et dont la supérieure avorte presque toujours.

C'est encore au moment de l'épanouissement et quelquefois un peu avant que s'ourrent les anthères des Phlomis; ainsi, ce que nous avons dit de la fécondation des autres Labiécs s’applique également à ce genre, ainsi qu’à celui des Stachys, qui contient aussi quelques espèces cultivées comme plantcs d'ornement.

La beauté de cerlains Phlomis les a fait introduire dans les jardins, et rien n'est plus facile que de tenter entre eux des croisements d'espèces, les organes des Labićes s'y prètant parfaitement. M. Pepin a nnême signalé, en 1845 , des liybrides 
accidentels trinant le milien entre te $P$. Laciniala aux fleur: pourpes el le $P$. iberica aux flemrs janes. Les fleurs de ces hybrides rariaient de couleur et rappelaient ces deux especes ornementales.

\section{FAMILLE DES VERBENACEES.}

\section{Genre Lantane. - Lantana.}

On peut diviser les Lantana en deux sections, ceux qui sont épineux el ceux qui sont inermes. Il est probable que tous ceux d'une nême section se féconderaient entre eux.

Dins la première, les épineux, se trouvent les purpuré, mulabilis, variegata, striata, melissefolia, amethystina, hispida, etc., a fleurs rouges, roses ou lilas, les crocea, cumara, cremulutu, uculeatu, à fleurs jaunes ou orangées, et les nivea, alba et Lokartii, à fleurs blanches.

Dans la seconde section, on rencontre les mêmes couleurs, les recta, tencrifolia, odorata, hirta, Geroldiana, etc., à fleurs blanches; les Moril:iana, glitinosa et horrida, à fleurs jaune: orangées, et l'on compte quelquefois parmi celles à fleurs rouges, roses lilas, ou jaumes pàles, les salvixfolia, radulu, Sellowiana, lilacina, trifolia, involucrata, albopurpurea, ete.

Les espreces de ce genre ont quatre étamines, dont deux plus grandes, et qui restent enfermées dans l'intérieur du tube. Le style est surmonté d'un stigmate à deux lobes, qui reçoit directement le pollen des étamines. La fécondation s'opère peu de temps après l'épanouissement, et elle manque rarement dans les Lantana, car presque tous mûrissent leurs graines dans nos serres. Ces belles plantes ont dejà domé quelques variétés, et il n'est pas douteux qu'on puisse en obtenir de nouvelles par des fécondations artilicielles.

\section{Genre Verveine. - Verbena.}

Les Verbena melindra, incisa el létcroïdes, cette dernière surtout, ont produit de numbreux lịbrietes qui ornent main- 
tenant nos plus modestes jardins, et où elles ont acquis les droits les plus incontestables à nos soins et à notre admiration.

La fécondation artificielle n'est pas très-difficile dans les Verveines. Les quatre étamines ont leurs anthères à la haıteur du stigmate. Le pollen est répandu dès le commencement de l'épanouissement. La gorge de la corolle est garnie de poils qui en ferment l'entrée; mais les stigmates ne deviennent aptes qu'à l'époque de l'entier épanouissement de la fleur, en sorte que l'on peut espérer de pouvoir les féconder avec le pollen d'une autre variété. Il suffit d'aroir quelques piriceaux et de puiser le pollen dans les tubes où il est rassemblé et de porter le pinceau bien garui dans les corolles du porte-graines que l'on a choisi. Le pinceau amène presque toujours la fécondité de l'ovaire, souvent, il est rrai, avec le pollen de la même fleur. Si l'on veut être sûr de l'hybridation, il faut enlever la corolle dès qu' elle s'ouvre, et imprégner le stigmate de pollen le lendemain du jour où cet organe a été enlevé. Le stigmate étant alors saillant, on est presque sûr du succès, et l'on peut choisir à volonté les sujets que l'on veut unir, éludier leurs goûts et leurs inclinations, pressentir leurs tendances et deviner leur fulur coloris. On a cru remarquer que les rouges foncés, hybridés par les bleus intenses, ne produisaient que des coloris pâles et sans éclat; il faut donc choisir avec soin les individus que l'on destine à fournir les graines, et ne leur faire contracter de mariages qu'arec des sujets dignes sous tous les rapports de leur être unis.

Dans quelques variétés, on ne trouve que deux étamines, et, dans la plupart, les quatre qui existent sont placées sur deux rangs ou à des hauteurs différentes.

Le teucroïdes, qui a maintenant donné beaucoup de variétés et beaucoup d'hybrides avec ses congénères, a la fleur plus grande et se prète mieux que les autres à l'enlevement des étamines. On approche déjà du bleu, et c'est vers cette couleur et en mème temps vers la grandeur des fleurs quil faut diriger :es recherches. Toutefois, les Verveines suivantes, nouvelle- 
ment créér, peurent être ronsidérées comme des plantes dignes de devenir les ascendants d'une génération nouvelle; Greut-Eastern, Temensis, striatu perfectu, la belle des belles couronnée de rose sur fond blanc, elc., elc.

Nous pourions grosir indéliniment cette liste en citant dans ce beau grenre les dernicrs gains de MII. Dufoy, de Paris, Lemoine, de Kincy, Bouchanlat, de Lyon, Hoste, de Lyon, Nivert, Micllez, de Lille, Poulet, de Beaune, ete.

le Verbeu Monetii, si joli par ses stries et ses corolles bordécs, a donné anssi, par variation ou par hybridation, des varictés mowelles qui laissent leur tyge bien loin.

II. Laloy, horticulteur ì Louhans, a obtemu, en 1860, de nouvelles virictés de cette plante, dont le coloris dépasse encore tout ce que l'on commaissait. Nous ignorons si l'on pourra croiser cette espèce avee quelques-unes de nos belles varictés, mais an est en chenin d'obtenir, par hybridation des diverses variétés du Monetii, de charmantes et élégantes noureautés.

\section{FAMILLE DES PRIMULACEES.}

Genre Monrun. - Inagallis.

Les fleurs des Hourons s'épanouissent do bonne henre et durent tres-peu de temps. Aussi est-ce de grand matin qu il fant enlever les cinq étamines dont elles sont pourvues, et au moment où la corolle s'entrourre. Sur les huit it dis lieures, on pent poser au pinceau le pollen étranger sur un stignate en forme de petite tète et papillaire. Les helles especes et variétés, actuellement commes, permettent d'olstenir de grander virriations dauss ee grenré élégant. Les Anagullis Brewerii el ses rarietés, les Hemelli, Parlieri, rosalia, fleyens, ule., qui, presque tous, portent graine quand ils sont liecondie, donmeraient, par leur mélinge, d'admirables résultats, anxipuels fout hortienterur a le droit de prétendre. 
Genre Primevère. - Primula.

Ce genre est un des plus beaux du règne végétal, et l'un de ceux qui fournissent le p'us grand nombre d'espèces et de vatriétés à l'ornement des jardins.

Toutes ces plantes ont cinq étamines placées dans le tube de la corolle, et insérées tantôt à la base, tantôt au sommel de ce tube.

Le pistil a toujours un style terminé par un stigmate très-piapillaire, glutineux et en tête arrondie; mais tantôt ce stigmate est ćlevé, saillant, et dépasse de beaucoup les étamines; d'autres fois, il est caché dans le tube et recouvert par les cinq étamines que le jardinier nomme les paillettes dans l'Auricule.

Ces deux sortes de dispositions, dans des plantes qui malgré cela sont naturellement fertiles, rendent les opérations de fécondation artificielle également différentes. Si l'on choisit des fleurs moins estimées, à stigmates saillants, rien n'est plus facile que de les imprégner de pollen, même sans enlever les an. lic̀res; mais, si l'on préfëre celles qui ont le style inclus et les anthères saillantes, il faut enlever celles-ci de bonne heure, arant l'épanouissement, par une petite incision pratiquée dans le tube de la corolle, ou en développant ses pétales, mais le plus commode est de faire une petite fente à la fleur. Une fois les anthères enlevées, on peut attendre deux ou trois jours pour poser le pollen au pinceau.

On partage le grand nombre de Primevères connues en plusieurs sections qui, je crois, ne peuvent pas se féconder réciproquement, tandis que les espèces de chaque section, et, à plus forte raison, leurs variétés, se croisent très-bien entre clles.

Une de ces sections est formée des Primula acaulis, elatior et odorata ou officinalis, de Limné. Ces plantes passent un peu de l'une à l'autre dans la nature, et sont devenues les types des plus belles Primevères que nous cultivons dans nos jiridins. L'acuulis, dont les fleurs ont doublé, est lin souche des Primevires doubles it lleurs blanches, lilas, carminées, soufrées, ctc.; 
ef l'on trouve à l'état saurage une de ses variétés désignée sous If nom de umbellifera, ou variabilis de quelques auteurs, qui donne d'abord des fleurs solitaires at radicales comme le type, rt ensuite des ombelles comme l'elatior. Une grande partie de nos plus belles Primeveres ombellées appartiement a cette variété.

Le P. variabilis, comme l'a constaté If. de la Perrandière, est toujours un hybride du $P$. officinalis et du P. grandiflora. III. Codron et Grenier professent la même opinion, et d'ailleur: celte plante ue se présente que dans les localités où se trouvent les deux espèces types, tandis que, chose remarquable, elle exclut le $P$. elatior. Il arrive cependant que le $P$. variabilis est quelquefois fertile, et quil retourne au țpe probablement maternel du fremdiflora, mais à fleurs purpurescentes. J'ai ju constater ce fait aux environs de Grenoble, ot ce P. yrandifiorn i corolle rougeàtre est probablement une des souches des Primevères à grandes fleurs, de nos jardins.

M. Lehel, qui a observé le P. variabilis aux environs do Talognes, ne le regarde pas comme un liybride à cause de la rareté et de l'áloignement du P. officinalis. Il pense que ce pourrait être une autre espèce que le $P$. variabilis Goupil.

J'ai fait un grand nombre de fécondations croisées cntre ces diverses Primevères, et j’ai tonjours réussi et presque inujours obtenu la variété ombellifère, qui, d'abord, me donnait des fleurs radicales, et ensuite des ombelles. Ainsi, je sais par expérience que les Prinevères de cette section se férondent entre elles, et que les fécondations artificielles domnent, dans ces plantes, d’admirables résultats.

On doit préférer pour plantes mères celles dont les coulems snut neftes et franches, ear on ohtient toujours un asser grand nombre de pieds a couleurs fausses. Il faut choisir les plamtes qui ont un lont pédoneule et de grandes fleurs qui se présentent bien et ne sont pas trop penchés. La Primesere

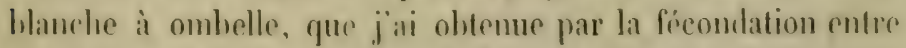
un elatior très-pâle of un acuulis hlane, est un excellent porte- 
graine qui produit une foule de variétés de couleurs tendren bien noins communes que celles à fleurs foncées.

J'ai essayé inutilement de fíconder la Primerère ordinaice par l'Auricule qui forme une suction différente dans ce genre.

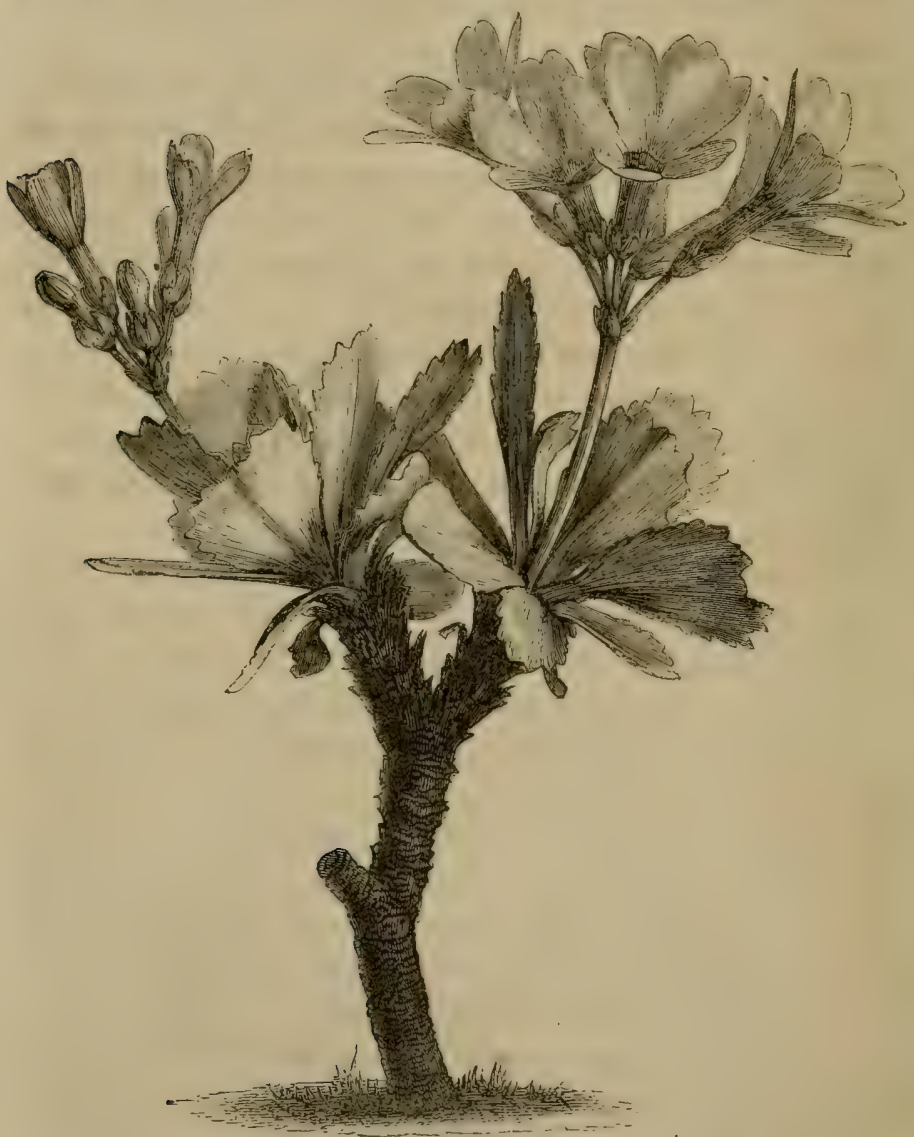

Grav. 77. - Primevère unarginée de grandeur naturelle.

Cette dernière espèce, l'Auricule ou Oreille d'ours, la plus belle, sans contredil, du genre Primulu, dérive du P. auriculi des Alpes, et probablement aussi des maryin!ta, viscosa, et peut-itre même des liybridalions du villosu, de l'hirsutu el in- 
tẹpifolia. Dans les jardins, res plantes ont donné de tris-belles variétés, et leur croisement en produit tous les jours de nouvelles. On y trouve, comme dans les autres Primevères, les deux sortes de fleurs a stigmates saillants ou inclus, et les une's 't les autres donnent des plantes qui ont les deux espèces de lleurs. On doit choisir pour porte-graines les fleurs larges et bien portées sur leurs pédoncules, et éviter les plantes à lleurs violettrs, car on a ohtenu maintenant toutes les nuances de violet, à moins que ces plantes ne soient bordées régulièrement. Les jaunes pure's, les rouges et les noires, sont d'excellents pieds mères, ainsi que toules les Auricules dont la gorge est blanche et nettement marquée. On ne doit pas laisser plus de quatre capsules par ombelle et les surveiller à la maturité.

On peut souvent attendre le commencement de l'épanouissement pour enlerer les anthires, quoique celles-ci soient déja ourertes, mais le pollen est resté adhérent sur les poches et liest pas encore tombé sur le stigmate enfermé dans le tube. Les choses se passent surtout ainsi quand les fleurs sont inclinées; mais, si elles ne le sont pas, rien n'empèche de les pencher vers le sol et d'arracher les anthères à la pince. On les relive ensuite ef l'on fait tomber dans le tube les antheres couvertes de. poussière du père que l'on a choisi. Les variétés s'infirident très-facilement, et un procédé qui ne serait pas sulfisamment exact pour tenter des croisements entre espèces suffit parfaitement entre variétés qui s'imprégnent avec le pollen d'une autre plus aisiment quavee le leur. Ce procédé pernet de prendre pour porte-graines, dans les P'rimevères romme dans les duricules, des rariétés à paillettes saillantes. Quvique ce caractire, si recherché des véritables anateurs, ne soit pas, selon moi, indispensable, et que son absence ne doive pas laire rejeter des lleurs, d'ailleurs bonnes de formes ou de coloris, il n'en est pas moins rai que les flenr's qui en sont douées sont généralement plus helles, et que, si on les prend pum porte-graines, elles doment plus de plantes à paillettes saillantes que celles dont le style ne est pas inclus. Si l'on obte- 


\section{6}

nait des couleurs remarquables avec de mauraises formes, il faudrait les conserver pour de nouveaux croisements.

Une petite section, composée des Primula farinosa, longifolia; cortusoüdes, donnerait peut-ètre des hybrides on des variétés, mais on n’a fait aucun essai pour les obtenir (grav. 77).

Enfin, dans une autre section du même genre se trouve le Primula sinensis, dont on connait des variétés blanches, carminées, frangées sur les bords, la belle panachée obtenue par M. Forest, celle à grandes fleurs de M. Fourquet, ainsi que des variétés doubles. Nul doute que la fécondation artificielle n'augmente encore les variétés de cette espèce. Elle offre aussi les deux sortes de lleurs, mais rarement les stigmates sont siillants, en sorte qu'une opération est nécessaire pour extraire les anthères avant l'épanouissement.

Plusieurs de ces Primevères appartenant aux différentes sectious ont des fleurs doubles. J'ai cherché souvent si ces fleurs contenaient par hasard quelques anthères au moyen desquelles on anrait pu hybrider des fleurs simples, mais quand j'en ai trouvé, elles étaient infertiles. Pent-être en découvrira-t-on, et l'on aurait alors une chance pour obtenir des variétés doubles en se servant de ce pollen.

Nous renvoyons pour plus de détails aux observations intéressantes de M. Darwin, dont nous avons analysé le mémoire dans le troisième chapitre de cet ouvrage.

\section{Genre Dodécathéon. - Dodecatheon.}

Ces élégantes espèces, au lieu d'avoir leurs cinq étamines cachées comme celles des Primevères, les ont, au contraire, saillantes et très-faciles à enlever. Le stigmate reste alors en saillie et isolé, on peut l'imprégner au pinceau. Les I). meadia, bicolor, elegans, gigantea, alba, etc., qui ont entre eux beaucoup de rapport, se croiseraient sans aucun doute et donneraient des graines fertiles. Il arrive souvent cependant que celles-ci sont en petit nombre, malgré la belle conformation ap- 
parente des rapsules. Les antherres doivent ètre entevées ansitit que le bouton s'épanouit, ef le pollen posé le lendenain ou le surlendemain.

\section{Genre Cyelame. - Cyclamen.}

Ce que nous venons de dire du Dodecutheon pent s'appliquer aux Ciyclamen, dont la fructilication est à peu près semblahlr a celle de ce premier genre. Il faut aussi enlever les authères de home heure. Le petit live si concis et si clair de M. Jongle sur les Cyclimens indique les espèces suivantes : C. coum, persicum, europaum, vermum, neupolitanum, hederxfolium, dont plusieurs ont donné des variétés jar le semis. Tous peuvent servir de porte-graine, mais je pense que l'hederafolium serait un des préférables en conservant le pollen des autres; car je l'ai toujours ru en fleur en automne, ce qui ne l'empèche pass de donner des graines mùres tous les ans dans mon jardin. On annonce aussi un Cyclamen africumm. 


\section{CHAPITRE VII}

DICOTYLEDONES MONOCHLAMYDEES

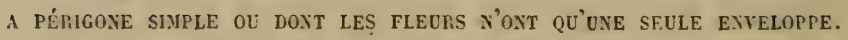

\section{FAMILLE DES PLUMBAGINEES.}

\section{Genre Dentelaire. - Plumbago.}

Les Plumbago ont cinq étamines et un style terminé par cinq stigmates. Les antheres s'ourrent de bonne heure, et si l'on voulait tenter l'hybridation, il faudrait les enlever avant l'épanouissement par une petite incision; mais, comme sonrent aussi les cinq branches du style ne s'écartent qu'après l'épanouissement complet, il faut attendre cet écartemcut avant de poser le pollen. Les $P$. rosea, capensis, auriculuta, zeylanica, sont ceux qu'il faudrait essayer d'hybrider ou au moins de féconder avec leur propre pollen.

\section{Genre Statice. - Statice.}

On peut croiser les espèces des Statice qui ont entre elles le plus de rapports, mais il ne faudrait pas espérer olstenir des hybrides entre toutes les belles espèces que l'on cultive maintenant dans les serres et dans les jardins. Après avoir diminué 
considérahlenent le nombre des fieurs, on conleve les cing antheres, à mesure que crolles qui restent sépannouissent, et l'on pose le pollen sur l'extrinité des cinq styles. Il y a beaucoup

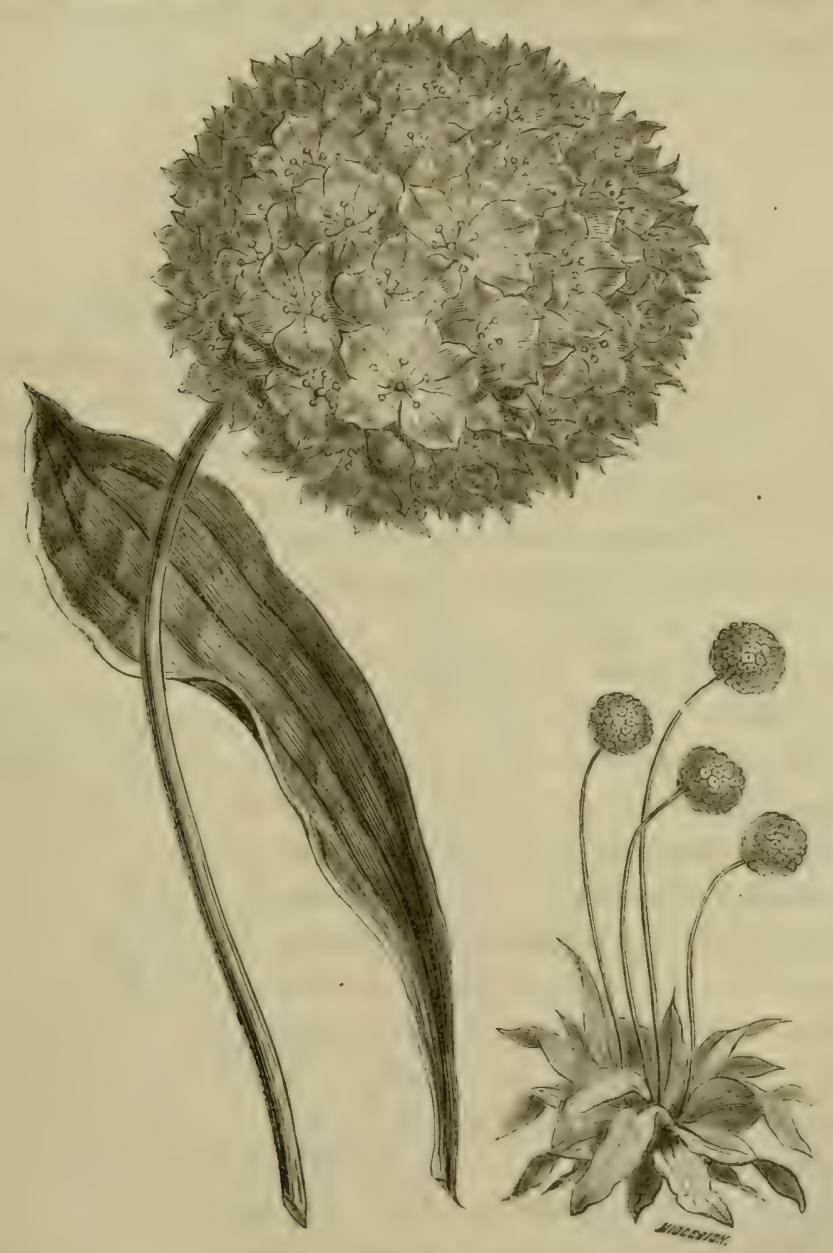

Grav, $78 .-$ Statice piscudo-armeria.

i) faire dans les statices, en (hoisissant aree discernement les épeces, et opérant aver la patience nécessaire jour la fécondation artificielle de toutes les petites fleurs. 
1I. Belot, horticulteur distingué, à Moulins, a mis dans le commerce, sous le nom de Statice intermedia, un très-bel hyJride produit de ses cultures. Le S. macrophylla, speciosa, Fortunei ct plusieurs autres donneraient sans doute aussi de beaux croisements (grav. 78).

\section{FANILLE DES NYCTAGINÉES.}

\section{Genre Belle-de-Nuit. - Mirabilis.}

C'est le soir seulement quıe s’épamouissent les brillantes corolles des Mirubilis; elles restent ouvertes toute la nuit et ne se ferment que sur les neuf heures du matin. Dès le soir on aperçoit les cinq étanines dont les anthères ne sont pas encore ouvertes, et le stigmate globuleux et papillaire porte sur un sigle assez long qui conserve, comme les filets, une partie de li courbure quil arait arant l'épanouissement. C'est le soir quil faut enlever les anthères, et c'est le matin de bonne heure qu'il faut poser le pollen sur le stigmate.

Le genre Mirabilis de Linné ne renferme, jusqu'à ce jour, qu'un petit nombre d'espèces, dont la plus commune, connue de tout le monde, est le $Y$. jalapa, cultivé dans tous les jardins pour la beauté de ses fleurs. On remarque, dans les mèmes lieux, le M. Longiflora L., dont les fleurs singulières répandent lous les soirs des émanations parfumées. La première de ces espèces est vivace et originaire du Pérou, selon les uns, des Indes-Orientales, suivant d'autres auteurs. La seconde, qui passe pour annuelle, est du Mexique. Une troisième espèce est le $M$. dichotoma L. du Mexique, rivace par ses racines; puis vient le 11 . hybrida Lepell. de la Nourelle-Grenade. Deux autres

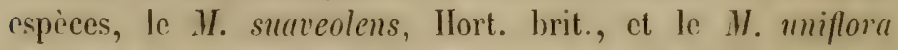
Schrank, sont indiquées la première comme du Mexique, lit seconde comme du Brésil. Eufin, jai reçı du jardin botanique de Bruxelles deux espèces désignées sous les noms de H. ambigna et 11 . planiflora, toutes deux à fleurs rouges, très- 
difficiles à distinguer entre elles, et différant à peine du .II. julapa, si ce n'est par leurs graines plus arroudies et plus fortement striées, caractire qui a peu d'iniportance dans le genre dont nous nous occupons.

C'est principalement sur les .11. julapa et les longiflora que nous arons tenté l'hyblridation, soit entre especes, soit entre variétés.

Le 1 . julapa, dont le type rouge est naturalisé et se reproduit de lui-même dans nos jardins, el surtout à la Guadeloupe, nous présente d'abord deux rariétés très-distinctes, la blanche et la jaune. Ces trois couleurs se sont depuis longtemps mélanwrees, et l'on obtint d'abord des variétés rouges et blanches, plus tard des panachures de rouge et de jaune, et enlin, plus lard encore, les Mirabilis blancs et jaunes qui restèrent longtomps ascez rares. Aujourd'hui, ces six variétés se reproduisent constamment de graines, ce sont des races lixées. On a mème obtenu accidentellement quelques pieds qui produisaient des lleurs où les trois couleurs prinitives des trois premières, variétés se trouvalient réunies sur le mème pied, mais cette variété tricolore que l’on fait très-facilement par lybbridation I'est pas constante.

Quant an . Mirabilis longiflora, éest une espece d'une grande constance que nous niarons jamais pu ébranler par la culture. Sé: lleur's sont restées constamment blanches, ses jemes pousse: Glutineuses, et le tube de sa corolle n’a pas rarié de longueur.

Hybrides entre variétés du Mirabilis jalapa.:

Nous avons vu plus haut que le nombre des variétés que nous: avions à notre disposition étaient de sept seulement, et que ces variétés diversés ont servi à nos essais. Nous ne prétendons pas que ce soient les seules commues, nous croyons mème que plusieurs autres coloris ont éte accidentellement obtenus; mais nous pensons qu'à notre époque ces sept variétés, tout au plus, se reproduisent sous notre climat, tindis que, sous un ciel plus 
chaud, il existe d'autres coloris qui ne sont également que des varialions du Mirabilis jalapa.

J'ai done tenté, en 1846, de nombreuses hybridations entre les six premières variétés que j’ai citées, pour obtenir des valriations dans la couleur du Mirabilis jalapa. On sait que dans cette espèce on n'obtient gnère de couleurs fondues de tons différents, comme dans les Auricules et les Primevères, mais plutôt, et presque toujours, des panachures plus ou moins complètes. Ainsi, les trois coulcurs, telles que le rouge, le blane et le jaune, restent séparćes dans une mème fleur et donnentales mélanges de deux de ces couleurs qui produiscnt un très-hel effet.

Rarement ces panachures sont régulières, et presque jamais elles ne se reproduisent identiques sur toutes les fleurs d'un même individu. Au contraire, on trouve sur le même pied toutes les panachures possibles, depuis une égale proportion des deux nuances employées, jusqu à une séparalion complète; puisque sourent on roit sur un mème rameau des flcurs unicolores et différentes, représentant chacune une des deux nuances qui panachent les autres fleurs. Il faut dire cependant que, dans ce cas, très-ordinaire pour la plante qui nous occupe, unc flcu à nuances uniformes qui naît sur un pied panaché participe souvent des deux couleurs fondues. C'est ainsi que le rouge et le jaune se fondent pour constituer des fleurs cuivrées ou d'un rouge briqueté, tandis que le blanc s'unit très-rarenent avec le rouge pour produire des fleurs couleur de chair ou d'un rose pâle.

Tous ces jeux de couleur, dans ce Mirabilis, me le firent considérer comme une espèce très-propre à quelques essais que je voulais entreprendre, d'autant plus que l'on sait trèsbien que cette plante reproduit exactement ses variétés par la graine. Je choisis done six pieds de Mirabilis jalapa, tous de couleurs différentes, trois unicolores et trois panachés, et jopérai avec soin une fécondalion croisée sur cent flcurs environ de chaque pied. Les sujets araient été bien préparés, he:ıcoup de loranches retranchées, bon nombre de fleurs supprimées, et 
après la fécondation toutes les fleurs ultérieures furent pincée's avant leur épanouissement.

Ce travail fut assez long, mais jobtins pris de six cents graines parfaitement mûres qui furent semées en 18.47

J'avais basé mon lı̣ hridation sur le désir que j’avais d'ohtenir des fleurs tricolores, et de roir si les couleurs, qui, réunie: - deux à deux, tendaient à rester distinctes au lieu de se fondre sur la mème fleur, continueraient à rester séparécs el moffriraient de triples panachures. Je fícondais donc une fleur unicolore par le pollen d'une plante possédant les deux autres munces, cherchant it réunir charque lois les trois couleurs, blanc, rouge el janne, en une seule, et jajoutais, par consiquent, la couleur qui mauquait quand j’hybridais des pieds déja panachés. Toutes mes plantes furent ainsi mises à même de me donner les trois couleurs réunies.

In résultat tout à filit inattendu vint me surprendre. Dins toutes ces hybridations, je n'obtins, sur six cents plantes, que denx ou trois pieds entièrement bines, que je suppose avoir échappé à la fécondation artificielle, un très-petit nombre de panachures blanches et rouges, un nombre plus considérable de panachures rouges el jaunes, et une quantité prodigieuse de rouges de toutes les nuances.

Il est évident, dans cette expérience, que le blanc a pour ains dire disparu, el que le rouge au contraire s'est étendu, tandis que lo jaune a joué un ròle mixte. Aucune plante ne m’a dome franchement des fleurs tricolores, mais plusieurs pieds m ont offert de temps en temps les trois couleurs entièrement séparées sur quelques-unes de leurs fleurs.

Exinnons mantenant, séparément, comment chaque couleur s'est comportée.

firsxi, - Dans foutes les hỵbridalions, lo blanc, comme nous venons de le dire, sist presque complétenent elfaci. Le pied mire, le couleur blanche, hybride par rouge et jaumr, ne m’a, pour ainsi dire, fommi que du rouge on un melange fondu de: jaune et rouge assez terne et comme cuirré. Je n'ai obtenu ni 
panachures ni rouge pâle, ou du moins très-rarement, et sur deux cents graines je n'ai eu que deux pieds blancs, que j'attribue très-positivement à des fleurs qui auront échappé à l'hybridation. Ainsi, chaque fois que le rouge et le jaune se sont trouvés en contact avec le blanc, non-seulement le blanc s'est comporté comme teinte neulre et sans influence, mais le jaune s'est combiné au rouge et a produit des nuinces cuivrées ou plus ou moins orangées. I y a eu cependant quelques plantes à fleurs d'un carmin très-pâle, dans lesquelles le blanc a eu une certaine action.

Rouge. - Cette couleur est certainement la nuance primitive du Mirabilis jalapa. Daus les variétés le plus ordinairement cultivées, on distingue surtout deux nuances de cette couleur : le rouge vif écarlate sur les bords de la corolle et le rouge carminé tirant un peu sur le violet.

Dans mes hybridations, un pied rouge écarlate d'une nuance Irès-vive a reçu le pollen de la variété blanche et jaune. Je n'ai obtenu de ces graines que des fleurs rouges; les unes ressemblant à la mère, et la plupart offrant une teinte de rouge contenant évidemment du jaune et tirant à l'orangé. Toutes les nuances saumonées se sont montrées dans ces différents rouges; quelques fleurs ont approché de l'aurore, des teintes cuivrées, mais le blane a disparu et l'attraction du rouge pour le jaune a été telle, que partout les deux coulcur's se sont associées. Après avoir attendu de mes nombreuses hybridations une foule de panachures, jai été surpris de voir surgir cette variété de nuances fondues qui provenaicnt toutes de mélange en proportions différentes de rouge et de jaune.

Malgré cela, l'orangé pur, si brillant dans la Capucine et dans la variété du Rosier églantier, ne s'est jamais montré, et cela tient évidemment à ce que le rouge du Mirabilis contient toujours un peu de bleu; et l'on sait que le mélange des trois couleurs primitives, surtout si les proportions sont inégales, donne des tons sales et brunâtres qui masquent toujours la vivacité des couleurs binaires. On voit pourtant dans la nature 
quelques exceptions. Ces nuances si suaves et si pures du chamois et de lat teinte saumonée sont formées de trois couleur: affiablies par du blanc qui agit en éloignant, en séparant chaque cellule diversement colorée, ct en empêchant le mélange intime yui n'agit plus sur l'œil de la mème manière.

Ainsi, dans la Belle-de-Nuit, la petite quantité de bleu qui dome aux fleurs rouges une teinte de violet s'oppose aux belles nuances d'orangé que domnerait le mélange du jaune et du rouge par les hy̧bridations.

Jusxe. - Nous venons de voir la grande attraction du jaune pour le rouge. Aussi, tous les mélanges dans lesquels le jaume a été fécondé par llane et rouge ont domné des fleurs cuivrée's ou fauve orangé, et enfin tris-différentes des belles panachures: que jaattendais. Le blanc a également disparu. Je n'ai pas obtenu de ces fécondations croisces beaucoup de plantes entierement jaunes, cependant plusieurs se sont montrées, quelquesunes d'un jaune assez pàle et d'autres d'un jaune plus foncé. Cette couleur n'a donc été remarquable que par sa fusion avec le rouge.

Paxachenes. - Peu satisfait des résultats que j’avais ohtenus, je repris, en 184i, mes hybridations; et cette fois je fécondai des pieds panachés et, par conséquent, bicolores par d'autres fleurs qui offraient aussi deux couleurs, dont l'une était difficrente de celles des fleurs que jhỵbridais. J'arais alors à ma disposition les panachures ordinaires qui sont des mélanges binaires de blanc et de rouge, de rouge et de jaune et de jaune et de blanc. Je n'avais pas vu encore de plantes franchement tricolores. Ces diverses panachures ont été hybridées soit entre

- elles, soit par des fleurs carminées, et jai pu reconnaitre c'ncore dans ces hỵlurides l'attraction du rouge pour le jaune. Diuns les plantes jaune et rouge, hybridées par rouge et blane, le rouge a pris le dessus et s’est souvent mêlé au jaune sans panachures. Jiantres fois les panachures sont restees, mais le rouge s'est montré sur du jaune affaibli par le blanc.

Dans les rouges et les blanches hyluridées par jaune et blane, 
le jaune s'est uni au rouge, qui, de carminé qu'il était, s'est orangé ou cuivré, et le blanc est resté intact.

Enfin, quand ces différentes plantes panachées ont été hybridées par la couleur rouge carminé, c'est-it-dire contenant un peu de bleu, ce bleu du carmin violacé s'est constamment uni au rouge, même dans les panachures sur fond jaune, et s'est soigneusement séparé de cette couleur complémentaire. Aussi ai-je obtenu de très-helles variétés dans les panachures jaunes, sur lesquelles le carmin violacé s'est montré par bandes ou macules, ou comme un pointillé plus ou moins fin.

Plusieurs pieds m'ont offert des fleurs tricolores, mais en petit nombre et très-remarquables. En sorte que, sous le rapport pratique, ce sont surtout les variétés panachées quiil convient d'hybrider entre elles ou avec des fleurs carminées.

En 18:8, jessayai ce que je nomme l'hybridation en mélange, c'est-à-dire qu'après avoir préparé quelques pieds, dont un tricolore, j’en hybridais les fleurs avec un mélange de pollen recueilli sur un grand nombre ou du moins sur plusieurs variétés. J'obtins ainsi en grande quantité des pieds à flcurs tricolores, provenant indistinctement ou de lis plante qui présentait déjà ces caractères, ou des autres qui avaient reçu l'imprégnation d'un pollen composé.

Je rappellerai ì ce sujet, que j’avais déjà pratiqué, sur diverses variétés de Primevères et d'Auricules, ces hybridations en mélanges, et que je suis presque convaincu, par les résultats que j’ai obtenus, qu'une graine fécondée peut avoir deux pères.

Mes essais d'hybridation entre variétés de Mirabilis jalapa furent continués aree persérérance jusqu'en 1862, et les fleur's obtenues celte anmée, ne m'ont pas semblé offrir de nuances ni de panachures que je n’eusse déjà remarquées.

Hylrides entre les Mirahilis jalupa et longiflora.

Nous avous déjà dit qu'il était facile de croiser ces deux 
plantes, et, des lsti, jarais antrepris des fécondations artilicielles qui mont comduit à des résultats assez curieux.

J'avais préparé un pied de Mirnbilis longifloru, destimé a itre fécondé par le jalapa, et un pied de julapu destiné à recevoir le pollen du précédent.

La premirre combinaison fut intertite, ot le Mrubilis Iongifloru, fécondé arec tous les soms possihles, ne me donna pas une seule graine.

Cependant les actes de la Société des curieux de la nature de lierlin de 177 ; rapportent l'expérience faite par J. Ch. E., qui at fécondé le Mirubilis longiflora par le pollen du. Mirabilis julap)a, et qui a obtenu une plante tout à fait internédiaire entre le père et la mère.

Mais si, a plusieurs reprises, je ne pus réussir en employant comme porte-graine le.11. longiflore, il n'en fut pas de mène e! prenant pour pied mère le $.1 \%$. jalapa. Je choisis d'abord un pied rouge. Les graines mûrirent comme à l'ordinaire, mais, craignant de n'avoir pas pris assez de précaution dans le croisement, je négligeai la récolte des graines, persuadé que les lleurs avaient été fécondées de nouveau et après moi, tous les soirs, par les Sphinx du Liseron, qui ctaient alors extrèmement commums.

Ce lut done par hasard et négligemment que je recueillis uguelques graines, qui furent mèlées à dautres provenant du .H. jalupu. Au printemps de $18 \% \overline{7}$, je remarquai aree surprise, au milieu des julapa, trois pieds qui avaient entierement l'apparence du .1. Congiflora. Certain de n'avoir semé que des .I. jalaja, je soupcomua bientôt ces plantes de provenir de graines hyluidées, et, en effet, elles présentaient des caratlères parfintement intermédiaires antre les derux espres. Les fleurs parurent et me conlirmèrent dans mon opinion lilles étaient anssi intermédiaires; leur couleur était blanche on d'un lilas violet, et souvent pamachées ou seulement partigés par ces deux couleurs. Lodeur était calle du .H. Ionyjfloru, et leur aspere géneral rappelail beauroup plus le pere que lat mere. 
Cependant la plante n’était pas visqueuse, le tube était raccourci et les trois pétales étaient, sauf quelques variations dans la couleur des fleurs, parfaitement identiques.

Vers le milieu de l'été, ces plantes fleurissaient en abondance, mais aucune fleur ne nouait, et mes trois pieds étaient stériles. Me promenant un jour avec un bâton à la main, je donnai, comme plaisanterie, une forte correction à une de mes plantes, sous prétexte de lui faire porter graines. Il restait à peine gquelques rameaux, et je fus très-étonné, peu de jours après, de remarquer que leurs fleurs donnaient des graines qui vinrent à maturité parfaite.

Les deux autres plantes, qui n'avaient pas été mutilées, m'ont aussi domé des semences, mais à la fin de l'automne seulement, quand les individus eurent perdu en partie leur vigueur.

Les racines de ces plantes étaient énormes; elles furent conservées, bouturées au printemps, et les boutures donnèrent une assez forte récolte de graines.

Voyant ce résultat, en 1847 , je m'empressai de préparer des sujets et de les féconder par le M. longiflora. Un pied rouge et surtout un jaune, furent destinés comme portegraines, et la récolte, assez abondante, fut soigneusement cultivée en 1848. J'avais un grand nombre d'hybrides, dont la plupart étaient blancs et lilas, comme ceux de l'annéc précédente; quelques-uns violets pâles montraient de temps en temps un peu de blanc. Un seul était jaune et très-différent des autres. Il n'avait pas le port du M. longiflora, mais ces fleurs avaient un long tube et un limbe très-rétréci. La fleur s'ourrait à peine. Le pied n'était pas vigoureux. Il ne m'a donné aucune graine, malgré des fécondations artificielles avec son propre pollen et avec celui des autres.

Quant aux graines de ces Mirabilis hybrides, elles reproduisent la plante, et il arrive aussi qu'elles donnent des sujcts très-roisins du M. jalapa, retournant ainsi à leur type maternel. 
J'ai reçu de II. Vilmorin des graines d'une variété violette du. Y. longiflora, qui m'ont doniné une plante très-diffẻrente par la couleur et la vigueur, des liybrides que jarais créés, mais qui est très-certainement un hỵbride comme ceux que j'ai cultivés. Sa lleur est plus petite, d'un violet plus foncé, it la plante est faible si on la compare aux M. longiflora et jalapa, et surtout sil'on se rappelle l'extrême vigueur des hỵhrides que nous avons obtenus. Cette plante violette donne très-peu de graines fertiles, et il est à remarquer que les hybrides, qui, comme elle, se reproduisent de graines, perdent peu à peu la vigueur du pied mère qui leur a donné naissance.

J'ai en ce moment (juillet 1862), dans mon jardin, un pied d'hy̧bride qui date de 1848, qui, depuis cette époque, a passé les hivers sans couverture, dont la racine a plus d'un mètre de longueur, et dont les branches forment un buisson d'environ trois mètres de diamètre.

Cet hybride, très-robuste et résistant en plein air depuis plus de dix ans, se courre chaque soir de plusieurs milliers de fleurs dont les ovaires avortent constamment pendant les mois de juillet et d'août, et souvent même pendant la première moitié de septembre. A parlir de cette époque, on voit quelques ovaires grossir, puis un plus grand nombre devenir fertiles; et enfin, au mois d'octobre, toutes les derniires fleurs doment de bonnes graines. On peut en récolter plus de mille sur un seul pied. Si l'on ne veut pas attendre l'automne, et si l'on vent avoir des graines plus tôt, il faut mutiler la plante, couper et briser ses rameaux ou les déchirer à coups de bìIon, ainsi que Braconnot le recommandait autrefois pour faire mettre ì fruit les arhres trop paresseux, c'est-à-dire trop vigroureux.

Les hylhrides se comportent done ahsolument comme cerlaines espèces bien caractérisées qui ne donnent presque jiamais de graines, paree que chez elles la reproduction gemmipare l'emporte sur lia génération par sexes. Il y a, daus tous 
les végétaux, comme dans les animaux inférieurs, lutte et halancement entre ces deux modes de multiplication de l'espèce. Si une tendance l'emporte sur l'autre, elle la diminue on l'anéantit; en sorte que, pour avoir des hybrides fertiles, il faut diminuer leur vigueur par divers moyens; de même que, pour hàter la fructification ou la maturation des semences, or courbe les branches des arbres, on leur enlève des anneaux d'écorce, on les meurtrit, ele., toutes pratiques qui ont pour but de rappeler à l'individu ou au groupe d'individus qui constitue un végétal qu'il y a pour lui possibilité de périr, et qu'il est temps, dans cet état de faiblesse, de réunir ses forces pour assurer la perpétuité de son espèce.

Je ne suis pas le seul qui ait obtenu un hybride entic les M. jalapa et longiflora.

II. Pépin a citć dans les Annales de Flore et de Pomone pour 1856 deux pieds d'hybride de $M$. jalapa et $M$. Iongiflor $a$. Ils étaient, dit-il, intermédiaires entre les deux espèces et avaient tous deux les fleurs d'un rouge violacé. La racine vivace était conservée dans le sol au moyen d'une couverture de feuilles et produisait chaque année un énorme buisson. II cite encore un pied de ce Mirabilis qui, au bout de dix ans, présentait une racine du poids de 41 lilos. Ces lybrides étaient fertiles.

Linné avait essayé, sans plus de succès que moi, de féconder le M. Tongiflora par le pollen du M. jalapa l'expérierice inverse qui m’a si bien réussi.

\section{Hybrides d'Iybrides.}

Lorsqu'en 1848 j’eus obtenu des hybrides très-nets et trèstranchés, parfaitcment intermédiaires par tous leurs caractècs entre les $M$. jalapa et longiflora, j'essayai de féconder ces lyybrides par lemrs antécédents et réciproquement. J'oblins difficilement quelques graines des hybrides fécondés par le M. julapu. Je ne pus en recueillir du M. Iongiflora croisé par 
les hybrides, ni récipropuement: mais les .1. jalapa hybrides par les luphrides me domerent des sranes nombreuses, el, par la suite, des plantes extremment curieuses et presque toutes fertiles. Ces expreriences ont dé continuces pendant plusieurs amécs, of l'an dernier, octubre 18 (sil, jai recueilli encore un cerlain nombre de graines qui me doment aujourdhui (juillet 1862 ) de curieux résultats.

I’ai toujours choisi pour pieds mères des $M$. jalapa, et surtout des plantes a lleurs jaunes ou panachies de rouge, mais jaii opéré aussi sur des tricolores et des plantes de toutes les couleurs.

Il me serait impossible de décrire les types qui sont nés de ces croisenents; ils sont en trop grand nombre, et tellement différents des. . jalapa el longiflora, qu on les prendrait facilement, du moins plusicurs d'entre eux, pour des espèces lout i firt distinctes. Ce quil y a de certain, c'est que les diflërences spécifiques étaient plus grandes que celles qui existent entre toutes les espéces de Mirabilis et le .H. jalapa.

Ouelques-unes de ces plantes ćlaient glabres partout, d'autres hérissées 't relues. Les tiges étaient couchées dans les unes et dressies dans les autres; les fleurs tantòt rares et éparses, tanlôt rassemblées el dressées en magnifiques bouquets. Le tube chait plus long que dans le jalapa, et l'odeur rappelait encore le M. longiflora.

Le limbe de plusiems fleurs mesurait $j 0$ à $j$.t. millimètres de diametre (une piece de 5 fr. en mesure 57 ), tandis que l'on (n) voyail de beaucoup plus petites que celles du .I. longiflora. L'hrure de l'épanouissement était très-différente, et, en général, elle retardait sur celle du .1\%. jalapu. Il y aviit mème des llaurs qui ne s'ourraient pas du tout, et les plantes qui présentaient ce caractere ne me domèrent pas de graines.

La forme des corolles offrait aussi beaucoup de variations. Un reneontrait de's limbes trés-profondément divisés et des lleurs étoilées; on en vogail d'autres parfiatement arrondis, sans échancrures, et simulant des Liserons. 
Quant aux couleurs, il m'est impossible aussi d'en rendre compte tant elles étaient variées. J'avais des fleurs à long tube, jaunes ou panachées de rouge et de jaune, et toutes stériles. J'avais des larges fleurs carnées, roses ou violettes, entièrement semblahles, pour l'aspect, à celles de la Pervenche de Madagascar. Certaines variétés était veinées à l'intérieur comme la fleur de la Jusquiame noire. D'autres, d'un blanc de neige, avaient le tube et la gorge violets. Les teintes de saumon, de jaune-soufre passant au rose, d'abricot, de fauve et d'orangé, se montraient fréquemment. Toutes les panachures imaginables : marbrures, macules, pointillé, bandelettes, stries, entin toutes les combinaisons possibles se présentaient, et certains pieds offraient des fleurs dans lesquelles il était facile de distinguer cinq à six nuances bien différentes.

Ces singulières modifications se sont encore manifestées sur les graines. Certains pieds n'en donnaient aucune; d'autres les produisaient en abondance, pas une fleur narortait, et quelquefois mème la plupart des fleurs avaient deux ovaires et donnaient deux graines mûres.

Leur couleur variait entre le noir, qui est la couleur de la grame du $M$. jalapa, et le brun moucheté que montre celle du M. longiflora. On remarquait tous les intermédiaires possibles entre ces deux nuances.

Les formes étaient plus curieuses encore. Les graines, rarement rondes, et plus courtes que celles du $M$. jalapa, étaient souvent plus longues et quelquefois même très-pointues, à côtes plus ou moins saillantes. Enfin la variété était telle, dans ces plantes obtenues par des fécondations faites au moyen de pollen en mélange, qu'il n'existait plus aucun moyen de séparer neltement les espèces et de reconnaître les types.

J'ai obtenu aussi cette année des hybrides entre les $M$. jalapa et dichotoma. Les fleurs sont restées jaunes, ou panachées de jaune et de blanc.

Les graines de ces nombreuses variétés ou espices, ou, pour ne rien hasarder, de ces curicuses modifications, n'ont pas tou- 
jours donné des plantes semblahles à celles dont elles provenaient. Elles ont produit des individus à couleur différente, et sont retournées la plupart au $M$. jalapa.

Ile nombreux essais restent encore à faire sur les Miralitis. On peut les considirer comme des plantes éminemment propres i mettre sur la voie de la valeur que l'on peut donner aux vitriations et aux liyglridations. Il serait à désirer que l'on puisse recueillir leurs différentes espèces, très-rares ou inconmues dans les jardins, et que les essais d'hy̧bridation et de croisement pussent avoir lieu sous différents climats.

\section{Considéxations générales sur Irfybridation des Mirabilis.}

On pent tirer de mes essais d'hybridation la conséquence que, dans les hybrides entre espèces, au moins pour les Mirabilis, le produit est exactement intermédiaire; nais on arrive aussi à cet autre résultat singulier, que les hybrides d'hybrides ne suivent plus cette loi et deviennent infiniment variés en s’éloignarit quelquefois beaucoup de leurs types. Ainsi, jai ohtenu des Miraliilis à fleurs étoilées et d'autres à fleur's de Liseron, des Mirabilis à fleurs étroites ou à fleurs fasciculées, qui s'éloigrnaient beaucoup de leurs ascendants. On peut aussi être certain d'un fail, c'est que tous les hy̧brides végétaux ne sont pas stériles, puisque nos plantes croisées donnent des graines en petite quantité, mais des graines fertiles, et qu'en croisant ces hybriles arec leurs propres parents, on obtient des sujets d'une grande fertilité.

D'un autre côté, nous voyons ces graines fertiles avoir une srande tendance au retour rers les anciens types, et nous voyons la force de l'halbitude, un moment suspendue par nos effints, se montrer de noureau, des que nous ne nous opposons plus ì son développement.

Il serait prématuré de tirer des conclusions générales d'un senl fail, quelque précis qu'il soil, el des études sur les Mirabilis ne peurent domuer le droil d'etablir des theories applicables 
à tout le règne organique. Nous pouvons cependant nous baser sur ces faits et sur ces expériences pour donner de la valeur à lopinion que j'ai émise depuis longtemps, que l'hybridation, quand clle est possible, est bien plus prompte que la variation pour modifier l'espèce.

Je suis de ceux qui croient à la filiation de l'espèce, ct, par conséquent, à la variation possible et même nécessaire de la succession des individus, et nous avons partout des preuves de cette variation.

Sans sortir de notre sujet, nous savons que lors même qu'une seule espèce de Mirabilis, le $M$. jalapa, était connue, cette plante a varié ses couleurs seulement, et nous a donné des variétés qui se sont maintenues et multipliées par la culture; mais ces variétés se sont montrées lentement, à plusieur's reprises, et si nous faisons abstraction de celles qui sont connues de tout le monde et que nous avons citées au commencement de cet article, les autres ne se sont montrées que de loin en loin et ne se sont pas conservées.

Si done jai pu, en quelques années, faire revivre ces anciennes variétés et en ajouter de nouvelles, au point que celte annéc j'ai pu en séparer quarante bien distinctes, c'est que j’ai eu à ina disposition un mojen plus actif que l'espoir des variations naturelles ou accidentelles, e'est que j’ai pu ébranler la stabilité des races et des variétés connues. L'hybridation m'a domé ce mojen, et une fois la plante dérangée de ses liabitudes, elle tend à les reprendre, il est rrai, mais elle donne alors de nombreuses variétés qu'elle n'aurait pu produire auparavant. Cela est si vrai que les graines de Mirubilis que je recueille maintenant en masse, et qui proviennent toutes d'individus autrefois hybridés, c’est-ì-dire ébranlés et ramenés par des croisements successifs, au M. julıpa, donnent seules et sams hybridation de nombreuses et nouvelles variétés, que je ne puis prévoir et dont je suis moi-même étonné.

Un phénomène semblable m'est arrivé pour les Primula: ilprès avoir créé le $P$. variabilis par la fécondation des $P$. acau- 
lis ct offecinulis, j'ai obtenu une race dont les variations ne s'arrètent pas, en choisissant toujours les grames sur de jeunes pieds et sur les variétés nouvelles.

Cactle facilite débranler les races et mème les espèces par l'h! bridation, de les rendre fertiles par de nouveaux croisements qui les rapprochent de leurs trpes, et de les rendre ainsi propres à fournir des variations el des modifications multipliées. ne serait-clle pass applicalile à de nombreuses espèces des deux grandes divisions du regne or contrihuer it faire entrer dans la domesticité des races qui s'y refusent, ou qui atlendent de la part de l'homme de nouveaux efforts ou des méthodes différentes de celles qui ont été employées?

Quand on considere ces fails, et que l'on pense à l'énergic de lit nature lorsque les espéces étaient encore jeunes, on se demande si des types, anjourdhui différents et stabilisés par une longue habitude, par un entourage prolongé des mêmes - ronditions el de's mêmes-milieux, ne proviement pas de sonches communes, dont tes virtialions et les hybridations les an. raient fait dériver.

Il est diflicile de ne pas admettre cette opinion pour les genres nombreux en esprices; il est presque impossible de ne pas croire à la filiation quand on roit ces mèmes formes se reproduire dans la série des àges, quand on observe ces créations parallèles si bien indiquées par 1. Geoffroy Saint-llilare, et quand on étudie géographiquenent l'aire d'extension des espéces dans tout le règne organique.

\section{FAMILLE DES MMARANTIIACEES.}

\section{Genre Celosie, - Celosia.}

Les flemrs nombreuses ef très-rapprochies des Calosin rendent la fécondation artificielle tris-diflicile; mais, comme ces plantes produsent heancoup de graines, que les sligmates sont 
aptes à recevoir le pollen de leurs cing étamines un peu avant que celui-ci ne se répande, on peut se dispenser d'enlever les anthères et poser partout le pollen étranger. Un certain nombre de graines sont ordinairement hybridées, du moins en opérant sur les diverses variétés du cristata.

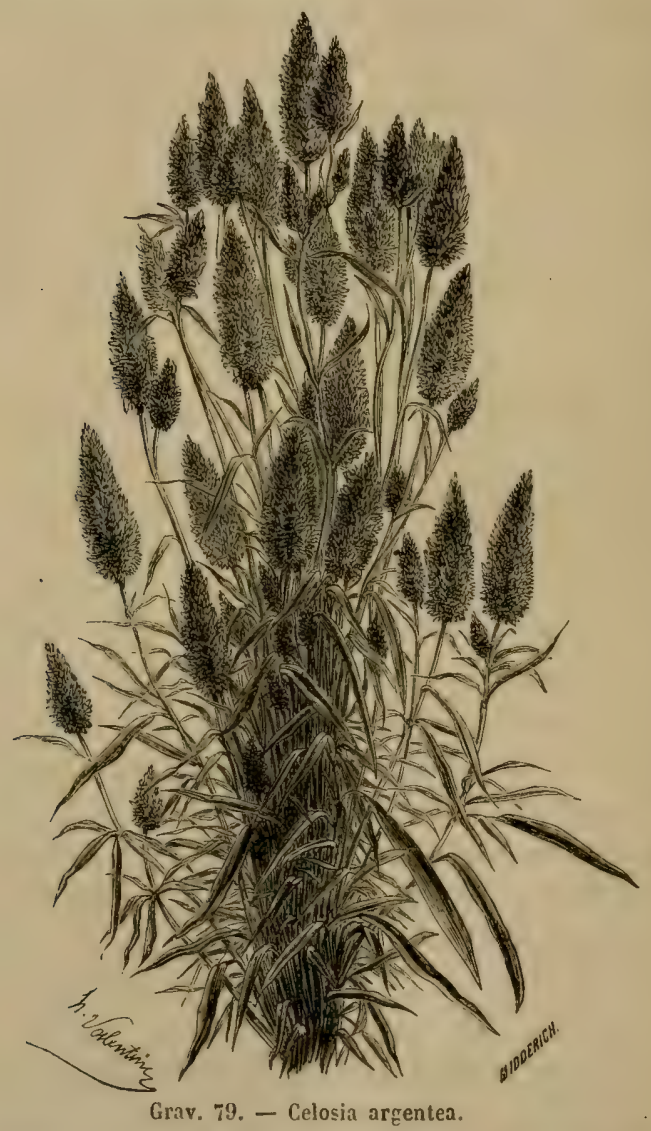

L'argentea," le margaritacea, le trigyna, pourraient peutêtre aussi se croiser et donner, comme le cristata et avec lui, de magnifiques variétés, quand la mode viendra de nouveau ramener ces belles plantes dans nos jardins (grar. 79). 


\section{FAMILLE DES CHÉNOPODÉES.}

Genre Bette. - Bela.

Les plantes qui appartiennent it ce genre ont cing étamines insérées sur un anneau charnu qui entoure l'ovaire, et ce dernier porte deux stigmates ordinairement papillaires et très-bien exposés au pollen des anthères. Il est done essentiel, pour opérer l'hỵbridation, d'enlever les étamines aussitôt que l'épanonissement a lieu, et de poser ensuite, le même jour, le pollen au pinceau. Autant que possible, après avoir préparé le sujet en al:attant la majeure partie de ses branches, il faut opérer sur les deux ou trois lleurs de chaque petit groupe; car, après lit floraison, elles s'accroissent et se soudent à tel point que les graines, au lieu d'etre solitaires, sont toujour's réunies deux on trois sous la mème enveloppe commune, ou du moins sont tellement adhérentes qu'elles ne se séparent pas, même à la maturité, et l'on roit sortir deux à trois plantes de ce qui paraissait une seule graine. Il est rai que l'on peut presque toujours séparer cusuite les jeunes plantes et les repiquer à distance.

Le Beta vulgaris a produit un très-grand nombre de variétés dont les unes, les Poirées, sont cultivées pour leurs levilles, et d'autres, les Betteranes, pour leurs racines. Toutes ces variétis peuvent donner des intermédiaires, soit que l'on cherche is ob. tonir des plantes plus feuillées pour la nourriture des bestiaux, ou des racines plus succulentes, plus sucrées, plus précoces ou plus voluminenses. On peut croiser les Betteraves blanches, jaunes et rougres, obtenir des variétés intermédiaires, et donner a l'agriculture ou au potager quelques races nouvelles. Jignore si des croisements entre le Betu vulyuris et le maritima, le trigyna et même le patula, pourtaient s'opérer et si les résultats en seraient firvorables. Il est lìcheux que l'on s'occupe si pen de l'hybridation des plantes destinées à la grande culture. C'est au hasard seul que l'on doit les variétés que l'on a obte- 
nues, tandis que quelques pieds de ces plantes utiles, transportés dans un jardin et opérés avec habileté et discernement, deviendraient probablement la source de nouvelles richesses agricoles.

Les variétés de Betterares cultivées pour la cuisine sont : la grosse rouge. - La rouge de Castelnaudcry. - La ronde précoce. - De Bassano. - La jaune. - La janme ronde. La jame de Castelnaudary. Ne pourrait-on pas aussi croiser les diverses variétés de nos Betteraves avec celle du Brésil, dont les côtes sont si purement colorées de jaune, d'orange, de rouge et de carmin?

\section{Genre Épinard. - Spünacia.}

L'épinard a quatre étamines et quatre stỵles allongés et amincis qui portent au sommet des stigmates papillaires. Cette piante est diöque, les femelles sont bien séparées des mâles qu'il faut arracher arec le plus grand soin, si l'on reut tenter un croisement, qui, du reste, ne présente d'autre difficulté que l'isolement des femelles.

On voit dans ces dernières les stigmates sortir de bonue heure, et rester très-longtemps propres à receroir le pollen. C'est du reste un des caractères des femelles dioïques de conserver longtemps leur aptitude. Les variétés cultivées et qui peurent toutes se croiser sont: l'ordinaire. - De Hollande. - D'Angleterre. -- De Flandre. - D'Esquermies ou à feuilles de laitue.

\section{FIMILLE DES POLYGONĚES.}

Genre Renouce. - Polygonum:

Le genre très-nombreux des Polygonum ne nous offre que deux sections qui aient de l'intérêt pour l'agriculture ou pour nos jardins. La première est celle des Fayolyyrum, dont la principale esprice est le Sarrasin ou Blé noir. Cette plante, comme 
les autres rspeces de cette section, a huit étamines, dont trois intérieures à antheres qui sourrent 'n dehors, et cing qui alternent avec les divisions du périgone, et par conséquent plus extéricures, et dont les anthères s'ourrent en dedans. Ces dernières répantent leur pollen des le premier jour de l'épanonissement, et les autres le second jour seulement. Alors la fleur se fine. Lovaire triangulaire est terminé par trois stigmates. Ce sont done les cing étumines extérieures qu'il funt s'empre:ser de retrancher dés l'épanouissement des fleurs. Rien n’empêche, il est vaai, de les enlever toutes en mème temps; mais l's trois autres, rapprochées du pistil, altendent le lendemain, comme pour assurer la fécondation, si par hasard les premières ne l'opéraient pas. Le troisième jour la fleur est tlétrie; aussi c'est pendant le premier et le second quil faut imprégner les stigmates au pinceau. J'ignore si les espèces des autres su'ctions pourraient ètre croisées avee les Fagopyrum, mais il est très-probable que les tartaricum, cymosum, emaryinatum, i liruits triangulaires comme le sarrasin, hỵhideraient ce dernier, et l'importance de cette semence nutritive derrait engager quelques expérimentatcurs à croiser ces plantes dans l'espoir d'obtenir de nouvelles variétés de Blé noir.

La seconde section, contenant les Amblygonum, peut interesser, à la fois, l'horticulteur et l'agriculteur. On y remarque diverses varielis du $P$. orientale, à fleurs blanches, à lleurs rouges, etc., pui produisent un fort bel cffet dans nos jardins, et qui peurent domer aussi de la graine en abondance. Ces plantes ont sept étanines, dont les deux inlérieures seulement s'ourrent en dedans; il n'y a que deux stigmates. Lil fécondation artificielle ne mrésenterait pas plus de dilficulté que pour les plantes de l'autre scetion, el, si on parrenait à les croiser avec les especes du groupe des Persicaires, on obliendrail sans doute des plantes trés-remarquables on par leurs fleurs ou par leurs produits en grains farineux. 


\section{FAMILLE DES BÉGONIACÉES.}

\section{Genre Begonia. - Begonia.}

On cultive beaucoup d'espèces appartenant à ce beau genre qui ne prospère bien que dans les serres chaudes, où plusieurs donnent des graines fertiles.

Les fleurs sont monoïques, les máles ont des étamines nombreuses, quelquefois monadelphes; les femelles ont un ovaire infère, surmonté de trois stigmates contournés, très-gros et bipartis. Ces deux sortes de fleurs naissent ensemble dans de vastes panicules qui, ajoutées au feuillage souvent discolor et tonjours élégant de ces belles plantes, en font un des plus riches ornements de nos serres. On peut les féconder avec leur propre pollen, en ayant soin de retrancher une bonne partie de leurs fleurs, et tenter aussi l'hybridation en supprimant toutes les fleurs mâles et conservant cinq à six fleurs lemelles sur chaque pied.

Il est d'autant plus facile d'hybrider les Begonia que les fleurs mâles s'épanouissent généralement avant les fleurs femelles et que l'on peut aussi en conserver le pollen.

Il y a du reste bien peu de plantes sur lesquelles les succès aient été plus nombreux, et l'on ne sait pas où s'arrêteront les nouveaux types que l'on obtient chaque jour. Nous supposons méme que ces créations ne s'arrêteront jamais.

La Flore des serres et des jardins a publié un B. Prestoniensis que l'on suppose issu du $B$. cimnabarina et du $B$. nitida ou peut-être des $B$. rubra et cimabarina, si toutefois, comme le suppose M. Planchon, ce n'est pas simplement une variété de cette dernière.

On cite encore le $B$. Lapeyrousii comme hybride du $B$. hydrocotilefolia fécondé par le $B$. incarnata. "Son port est majestueux, dit M. Van Iloutte, ses tiges robustes, ses feuilles très-grandes, et je ne saurais assez vanter sa beauté. ») 
II. Putzeis a signalé encore dans la Flore des serres et des jardins de nombreux hybrides de Begonia. Le $B$. xanthina a été croisé avec succès par le $B$. rubrovenia; le $B$. fuchsioides par le $B$. nitida; le $B$. samyuiner par le $B$. coccinea, ete.

Un de ces magnifiques hybrides du $B$. xunthinu, croisé par le B. rubrovenia, a resu le nom de B. marmorea, et présente In superbe feuillage préludant à cette belle sírie des $B$. rex que l'on devait découvrir plus tard.

En effet, l'apparition du B. rex a été le signal d'une lutte d'émulation qui dure encore. La postérité du roi a saisi les trônes de tous les jardins. Son alliance arec le B. Reichenheimi a été des plus heureuses. Le B. rex leopardinus n'a pas craint de détrôner son père, ct les serres de Van Iloutte ont vu naitre toute une lignée de princes magnifiquement costumés. a On y trouve, dit MI. Van Hontte, des velours verts incspérés, teints de coloris fantastiques, des pointillages, des marbrures tout i fait inattendues, et un travail de couleurs, de dessins qui simulent de bizarres tissus d'éloffes. Les macules d'argent abondent dans beaucoup de rariétés, ct l'une d'elle porte des feuilles qui paraissent d'argent pur. C'est l'argent rrai sans aucun mélange de leintes de reflets verdatres. ") (Grav. 80 et 81.)

Les $B$. rex et B. xanthina, croisés entre eux et arec leurs hybrides, ont domé des résultats admirables. MIII. Mawet de Liége, qui se sont occupés des semis de ces plantes avec le plus grand succès, meltent tous les ans dans le commerce des plantes extraordinaires. Nous ignorons oì pourront s'arrêter les panachures, les dessins, les zones colorées et l'admirab'e ornementation de ces riches feuillages.

D'innombrables varictés, descendint pour la plupart du B. rex, ont été exposées en 1860 dans les jardins de la Société d' horticulture de Chiswick, ef Angleterre. M. Hoore avait proposé pour ces plantes une classification que nous ne rapportons que pour montrer jusquioù l'hygbridation peut pousser la vatrieti, aussi bien pour le feuillage que pour les fleurs.

I. Noore établit trois sections : 
322 FÉCONDATION NATURELLE ET ARTIFIGILLLE.

$1^{\circ}$ Les Aryentées, dont les feuilles sont entièrement blanches, sans zones ni stries d'aucune autre couleur. Cetle section ne contient qu'une seule plante, le $B$. argentea.

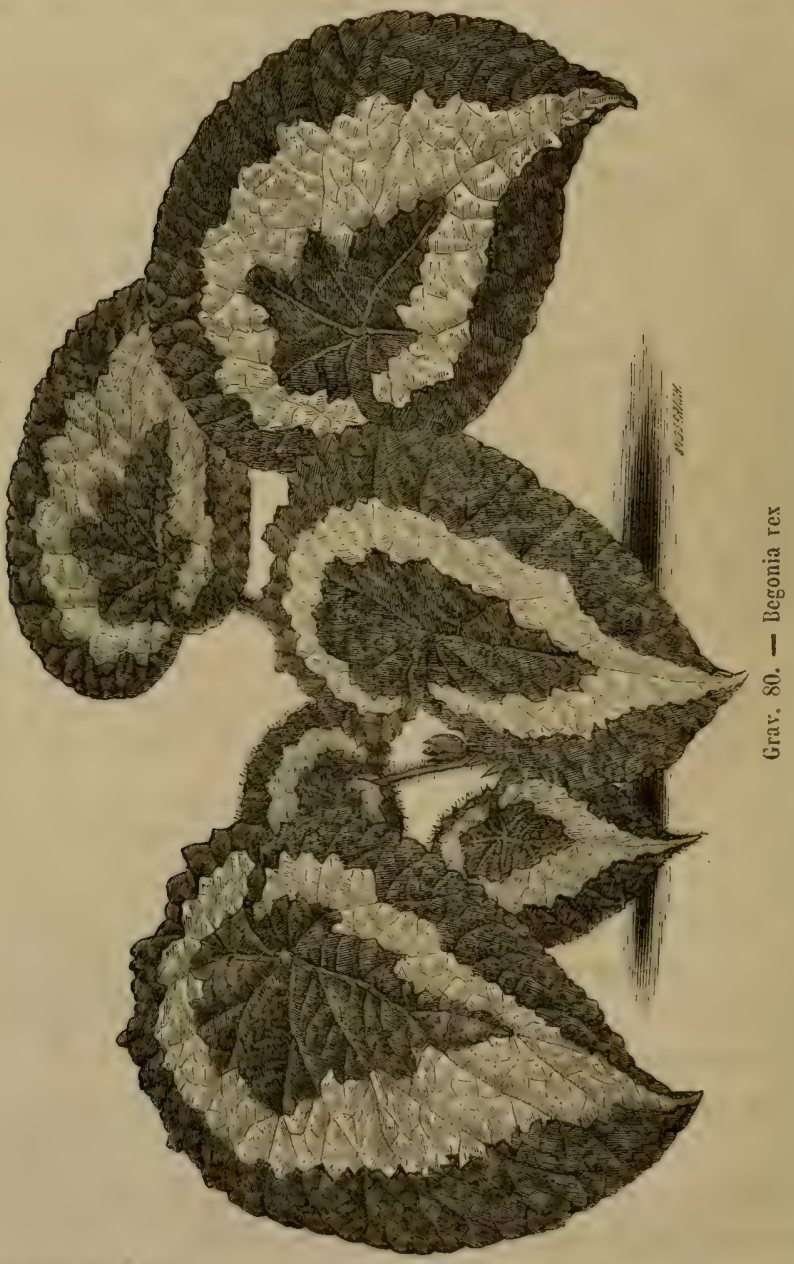

2' Les Zonées, où la face supérieure des fouilles est marquéc d'une zone concentrique dont la couleur est différente de celle du fond. Cette section se sublivise en quatre groupes : A. 
feuilles petites à zones argentée; B. feuilles grandes à zones argentées; $C$. leuilles grandes teintées de rouge, zonées d'argrent et de vert, velues en dessous; 1 ). fenilles entièrement vertes ou rouges.

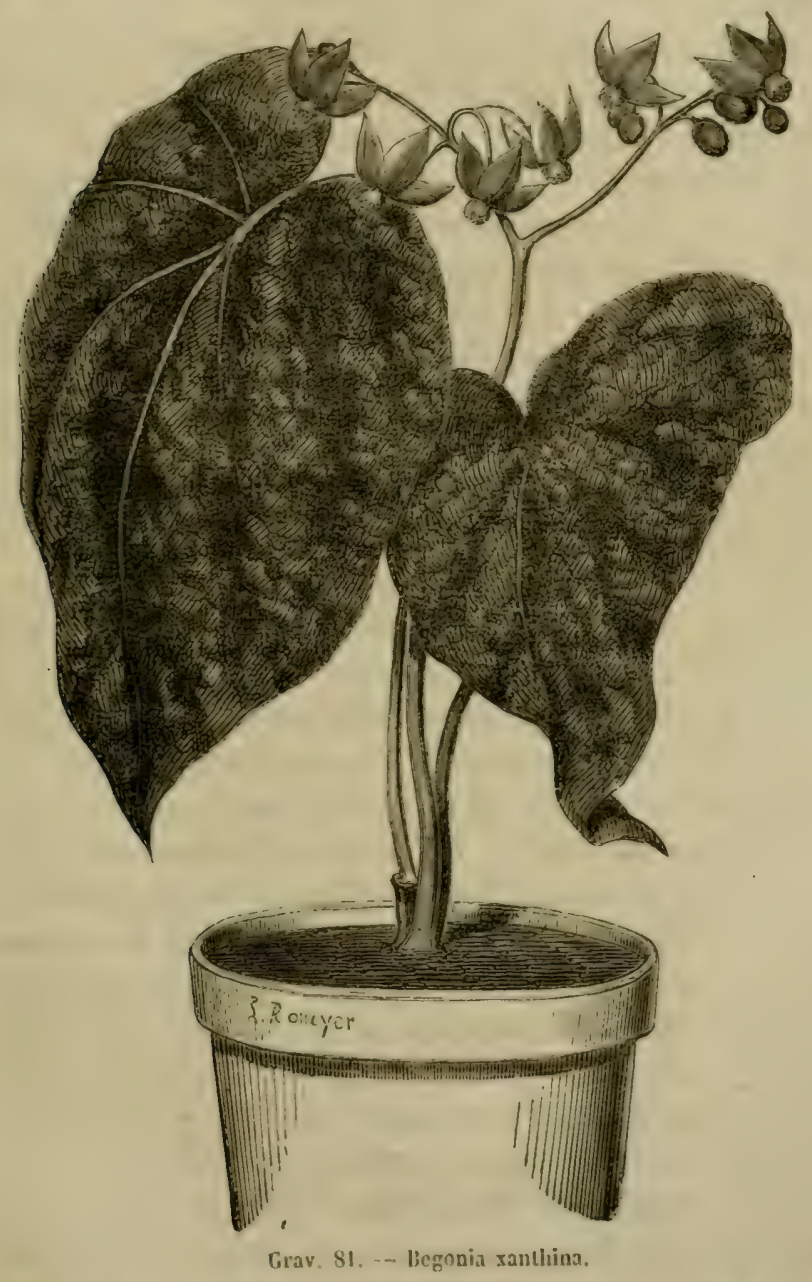

j" Les Bariolées, font les fenilles sont marquées de bandes un de monchelures argenlees on d'une antre munce, mais mon 
zonées, section qui se divise en deux : A. feuilles obliquement ovales; $B$. feuilles palmatilobées.

Les variétés qui peuvent aujourd'hui se ranger sous ces différents titres, sont innombrables et en grande voie de création.

Le croisement dı $B$. rex et du $B$. splendida a donné à MMI. W. Rollisson, le B. grandis, plus grand que l'hybride Leopardina, et le type de nouvelles séries à conquérir.

Des expériences très-intéressantes de M. Regel sur les Begonia sont consignées dans le Gartenflora (janvier et févricr 1858). Elles ont pour objet de reconnaître s'il existe des plantes liylırides qui soient fertiles par le pollen et par le pistil. Il a choisi pour sujet de ses observations les Beyonia (PlatycenIrum Klotzsch) xanthina et rubrovenia, parce que la première de ces plantes avat déjà occipé M. Klotzsch, qui ne voulait voir dans les produits qu'on en arait obtenus que de simples formes et non des hybrides.

La fécondation du B. rubrovenia par le $B$. xanthina a donné naissance à un hybride qui ressemblait absolument, sous tous les rapports, au $B$. xanthina-marmorea, et qui, comme celuici, était fertile par le pollen et par le pistil. Tous les individus issus de celte fécondation se ressemblent parfaitement quant à leurs caractères typiques, et ne diffèrent quelque peu que pour la coloration des feuilles. La grande majorité a des feuilles tachées de blanc, comme celles du $B$. xanthina marmorea; un petit nombre seulement les a colorées en dessus en vert uniforme, et correspondent ainsi au B. xanthina-yandavensis. Il résulte donc de cette expérience qu'il y a des hybrides dont le pollen est bien organisé, et aussi que l'hybride entre detix bonnes espèces présente exactement un même type, et ne peut varier que pour des caractères peu importants.

Un autre résultat a été obtenu dans la fécondation de l'hybride par lui-mêne. Pour celle expérience, M. Regel s'est servi tant du $B$. xanthina-ma'morea que du B. xamthina-gandavensis. Il pensait que les générations obtenues au moven de la féconda- 
tion d'un hybride par lui-mème derraient présenter également un type unique; il en a été autrement : les graines obtenues dans ce eas n'ont donné que quelques formes analogues ia l'hybrite pour leurs caracteres typiques, tandis que les autres, en plus grand nombre, étaient retournées plus ou moins vers l'un on laiutre des parents. It en résultait un étonnant mélange de formes; tantùt les feuilles étaient grandes et larges comme dans le B. xanthinu, tantòt elles étaient plus étroites et plus allongées, comme dans le B. rubrovenia; le plus souvent elles étaient maculées de blane; quelques-unes étaient d'un blane d'argent avec des reines vertes; rarement elles étaient unicolores. Les lleurs rappelaient, plus ou moins, tantôt l'hyybride et tantôt l'un des parents. Quelques pieds constituaient mème des modifications de l'un ou de l'antre de ceux-ci.

"Il résulterait de là que même un hy̧bride fertile ne peut se propager par la fécondation comme type fixe; mais que les générations, provenues de sa fécondation par lui-même, constituent une série de formes qu' on peut conceroir comme rattar'lant l'une à l'autre deux bonnes espèces, et, en outre, que Thribride peut retourner au type paternel ou au type maternel.

"La troisième expérience, dans laquelle l'hỵbride a été fécondé par le pollen de l'un des deux parents, a réussi dans un sens, à saroir : lorsque II. Regel a fécondé l'hybride avec le pollen du B. xanthina. La plupart des plantes qui ont élé ohtenues ainsi étaicnt presque entièrement retournées au $B$. xamthina, et un petit nombre seulement étaient restées intermédiaires aux deux. Le résultat a done ćté à peu près le même que dans la fécondation de l'hybride par lui-mème, puisque linfluence de celui-ci sur les générations consécutives a été souvent presque nulle, et ne s'est montrée que rarement d'une manière appréciable.

"Au point de vue de l'horticulture pratique, ces expériences ont un grand intérèt, puisqu'elles montrent comment on doit procéder pour obtenir beaucoup de formes nourelles. Ainsi, lorsqu'cn a siuss a produire un hybride entre deux bome 
espèces, si celui-ci est fécond, c'est-à-dire s'il possède du pollen bien organisé, il faut le féconder par lui-même; en effet, cette fécondation donne naissance à une bien plus grande variété de formes, que si l'on fécondait ce même hybride par l'un de ses deux parents, ou par une autre espèce roisine.

"Dans la suite de cet article, M. Regel signale et décrit les principales d'entre les formes qui ont pris naissance dans ses expériences de fécondation sur les $B$. rubrovenia et xamthina (Platycentrum rubrovenium Kl.; Pl. xanthinum Kl.). $\Lambda$ la première de ces deux espères, il rapporte les formes qu'il nomme pulcherrima, eximia, marmorata, discolor, splendens, picta et argentea; les trois dernières méritent, dit-il, d'être rangées parmi les plus belles plantes de serre chaude et humide, et clles sont nées à la suite de la fécondation de l'hybride (B. xanthina-marmorea) par lui-même. Les autres sont aussi plus belles de feuillage que le $B$. rubrovenia. Il rattache au $B$ : xanthina trois formes, auxquelles il donne les noms de maculata, argyroneura et discolor, issues toutes les trois de l'hybride B. xanthina-marmorea, fécondé par le B. xanthina.

"Enfin, au Begonia (Platycentrum) rubrovenio-xanthina. M. Regel rapporte les trois formes suivantes : yandavensis (B. xanthina-yandavensis), marmorata (B. xanthina-marmorata), lrevirens, toutes les trois nées à la suite de la fécondation du B. rubrovenia par le $B$. xanthina. »

De nombreux croisements de Begonia ont été faits en 1856 et en 1857 par M. Stange. Nous ne le suivrons pas dans les détails qu'il donne sur les nombreux hybrides qu'il a obtenus, nous constaterons seulement la facilité avec laquelle les espèces de ce genre se marient, et quelques faits particuliers signalès par lui dans cette opération.

Ainsi, il a constaté que des lybrides fécondés par leur propre pollen donnaient des fleurs fertiles et des graines qui reproduisaient en grande partic les formes dont elles provenaient. Le temps donnerait done de lit stabilité à ces hỵbrides, et en ferait des races et des espèces nouvelles. 
II. Stange a atussi observé que certaines formes liybrides perdaient dans leur jeunesse des fleurs mîles en looutons, que peu à peu, aver l'âge, ees lleur's résistaient; plus tard, elles s'épanouissaient arec des etamines sans pollen; plus tard encore, le pollen chait parfait; d'où il concluait avec raison que des hybrides d'abord inféconds peurent devenir féconds par la suite du temps et du déreloppenent. Ce fait vient à l'appui de nos propres observations sur la cause de la sterilité de quelques hybrides, lespucls, trop vigoureux, se multiplient par gemmation au détriment des semences.

M. Stange attache avec raison une grande importance aux conditions de lumière, de temprérature et d'humidité. Il a vu des croisements ne pas réussir dans une serre, et s'effectuer ficilement, entre les mèmes plantes, dans une autre serre dont la température était différente. On sait aussi qu il y a des plantes nocturnes, comme les Tanilles et certains Cactus, quil faut féconder pendant la nuit.

Si l'on arrive à hỵhider les Caladium comme ces beaux $\mathrm{Be}$ yonia, nos scrres seront remplies bientôt de ces éclatants feuillages qui disputent aux fleurs, comme aux oiscaux colorés de la zone torride, les nuances de l'iris ou le feu des pierreries.

\section{FAMILLE DES THYYÉLÉES.}

\section{Genre Daphne. - Daphre.}

Les jolies fleurs tubulées des Duphme ont huit étamines presque dépourves de filets, et fixées sur deux rangs daus le tuhe de la corolle, où elles dominent un stigmate capiti, qui u'est séparé de l'ovaire que par un style trés-court. La fécondatıon n'a lieu qu'après l'épanouissement, et les fleurs restent longtemps ouvertes, car toutes les anthères ne répandent pas leur pollen en mème templs. (In pent done enlever ces organes avec les piners lo premier jour de la for:aison, ef le lendentain imprégner les stignates an pinceau. Iéjà on a oblenu de beaux hỵhrides dans les Muphline, af ave les leelles espriees que l'on 
possède maintenant, on a l'espoir d'augmenter beaucoup les variétés de ce genre. Plusieurs d'entre cux donnent des graines, et si le collina, que l'on regarde comme métis du dauphin et de l'indica, n'en produit pas ordinairement, cela tient peutètre à ce qu'on ne le féconde pas artificiellement. Du reste, le mezereum ou bois-gentil donne partout des fleurs parfaitement conformées, et l'on pourrait utiliser le type à fleurs rouges, la variété à fleurs blanches et aussi la variété à grandes fleurs, récemment obtenue à Effiat, comme porte-graines, que l'on féconderait avec l'indica, le japonica, le lutetiana, le cneorum, le gnidium, etc.; soit en modifiant l'èpoque de floraison de ceux qui ne concordent pas avec celle du mezereum; soit en conservant le pollen par les procédés que nous avons indiqués. Le gnidium, le cneorum et l'alpina grainent aussi très-facilement, comme toutes les espèces indigènes.

Le Daphne Delphini est un hybride obtenu par Fion.

\section{Genre Gnidie. - Gnidia.}

On trouve dans les Gridia, comme dans les Daphne, huit étamines disposées sur deux étages et enfermées dans le tube de la corolle, à la partie supérieure de laquelle les quatre anthères supérieures viennent se montrer. Le style, quoique assez long, ne porte le stigmate qu'au-dessous des étamines supérieures. II faudrait les enlever pour opérer la fécondation artificielle, et l'on obtiendrait sans doute de beaux hybrides si les Gridia fructifiaient facilement en serre. L'aurea, le simplex, le pmifolia se croiseraient probablement. Le sericea, l'aryentea, le laviyata et l'oppositifolia forment une autre série sur laqueile on pourrait aussi tenter l'hybridation.

\section{Genre Pimélée. - Pimelea.}

Le beau genre des Pimélées est caractérisé par deux étamines et un style latéral qui porte un stigmate en tête. Il est toujours facile de féconder artificicllement les différentes espèces de Pimélées. Dans quelques-unes les stigmates ne sont 


$$
\text { 季 }
$$


aptes qu'après la floraison des étamines, comme dans le linifolia; dans d'autres, les organes se développent en même temps; mais, comme les étamines sont saillantes, au nombre de deux seulement, et que la fécondation n'a lieu qu'après l'épanouissement, rien de plus simple que de les enlever. Enfin, il y a aussi des Pimélées où les deux anthères viennent affleurer la partie supérieure du tube de la corolle.

Ces plantes, originaires de l'Australie, se sont probablement déjà croisées sur leur sol natal, et elles ont une grande lendance à s'hyybrider dans nos serres, où l'on en trouve d'admirahles espèces. Le decussatı, qui graine facilement, pourrait être croisé avec d'autres Pimélées, parmi lesquelles nous citerons, comme très-dignes de l'attention des flenristes : les spectabilis, l'affinis, l'Hendersonii, rosea, lanata, linifolia, etc. M. Benoit Morlet, horticulteur à Clermont, a obtenu wine très-belle variété du decussata, qui serait un excellent porte-graine (grav. 82).

\section{FAMILLE DES LAURINEES.}

Genre Laurier. - Laurus.

Les Lauriers ont de six à douze étamines toujours placées sur deux rangs, et un pistil simple presque toujours séparé diö̈quement des étamines. Comme ces plantes sont généralement diö̈ques, on pourrait tenter l'hybridation sans obstacles;

- mais de tels essais ne pourraient avoir lieu que dans les pays chauds. C'est déjà beaucoup si, dans nos serres et au moyen de la fécondation artificielle, nous pouvons obtenir des graines de Lauriers fertiles, en les fécondant avec leur propre pollen.

\section{FAVIILLE DES ARISTOLOCHIEES.}

\section{Genre Aristoloche. - Aristolochia.}

Les Aristoloches forment un des genres les plus curieux dı règne végétal. La corolle, généralement très-grande, souvent 
recourbée comme une véritable pipe, offre toujours à sa base un renflenent ou espèce de chambre presque fermée, dans laquelle six anthères sessiles sont placées sous un stigmate à six

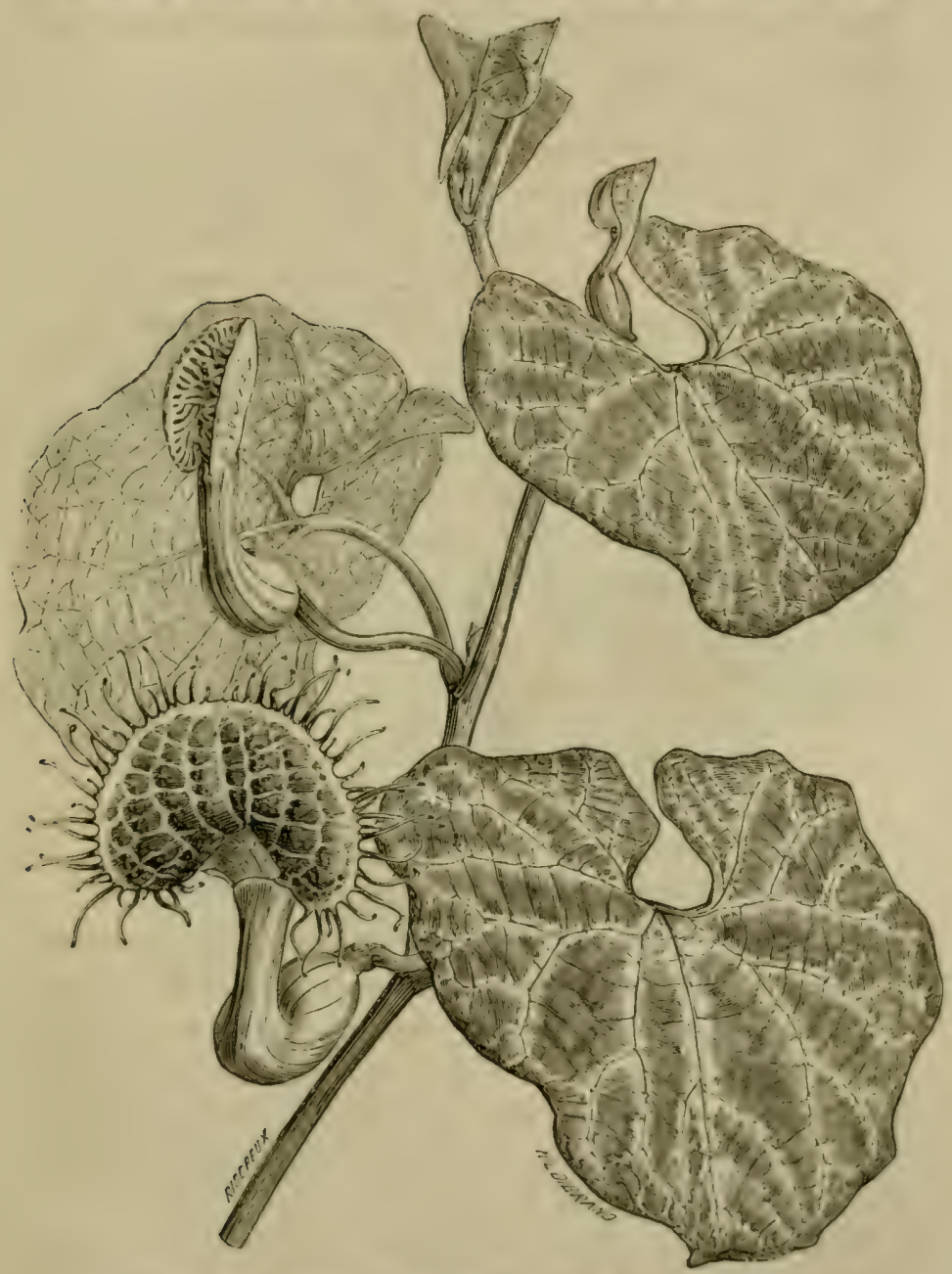

Grav. 85. - Rameau d'Aristoloche fimbriée, de grandeur naturelle.

divisions. Il est trisi-difticile que le pollen des anthères puisso arriver naturellement sur le stigmate, et c'est peut-ètre pour 
celte raison ugue la nature, en enfermant ces organes dans une sorte de chambre close, a ménagé à la partie supérieure une petite onverture quelquefois garnie de poils dirigés en ar. rière, ct qui ne présentant aux insectes aucun obstacle pour entrer, leur en oppose immédiatement pour sortir. C'est peutêtre à leurs ébals dans celte prison végétale que la fécondation est due. Dans tous les cas, on peut la pratiquer artificiellement en ourrant une petite porte avec le canif vers la base de la corolle, et en suppléant arec le pinceau aux ailes et aux brosses dont les insectes sont munis. Je ne crois pas que l'on puisse hybrider les Aristoloches de nos serres ou de nos jardins, mais je pense
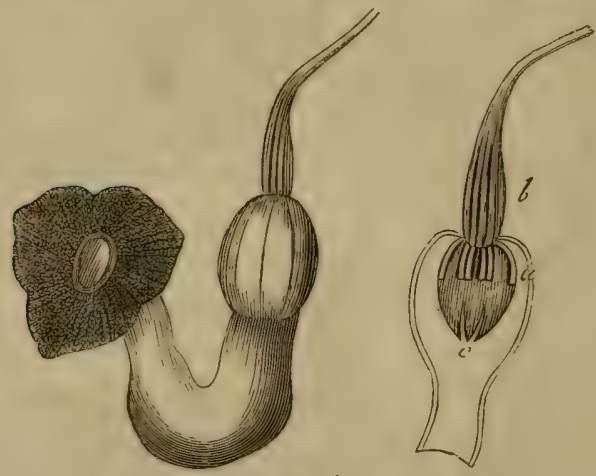

Grav. 84. - Fleur de l'Aristolochia sipho.

$a$, Étamines gynandre $;,-b, 0$ vaire, $-c$, Stigmates.

fu'à moins de mauvaise conformation des stigmates, comme cela est ordinaire dans le sigho, on pourra quelquefois en faire fructifier. Il y a dans ce genre des plantes extrêmement curieuses; nous citerons seulement : l'A. gingls et le labiosa, qui fleurit si bien dans nos serres. MI. Delaire, l'un des premiers horticulteurs qui se soient occupés de la fécondation artificielle, a obtenu un frut de cette dernière.

Nous avons vu souvent des fruits de l'A. sipho, et nous en arons reçu plusieurs fois des graines; mais, malgré le développement normal des péricarpes et des semences, ces dernières étaient toujouŕs stériles (grav. 84). 


\section{FAMILLE DES EUPHORBLACEES.}

Genre Vuphorbe. - Euphorbia.

Limmé avait considéré les fleurs des luphorbes comme ayant donze étamines et un ovaire surmonte de trois styles portant chacun un stigmate simple ou bifide. Les botanistes moderne's, considérant avec raison que les itamines, le plus ordinairement

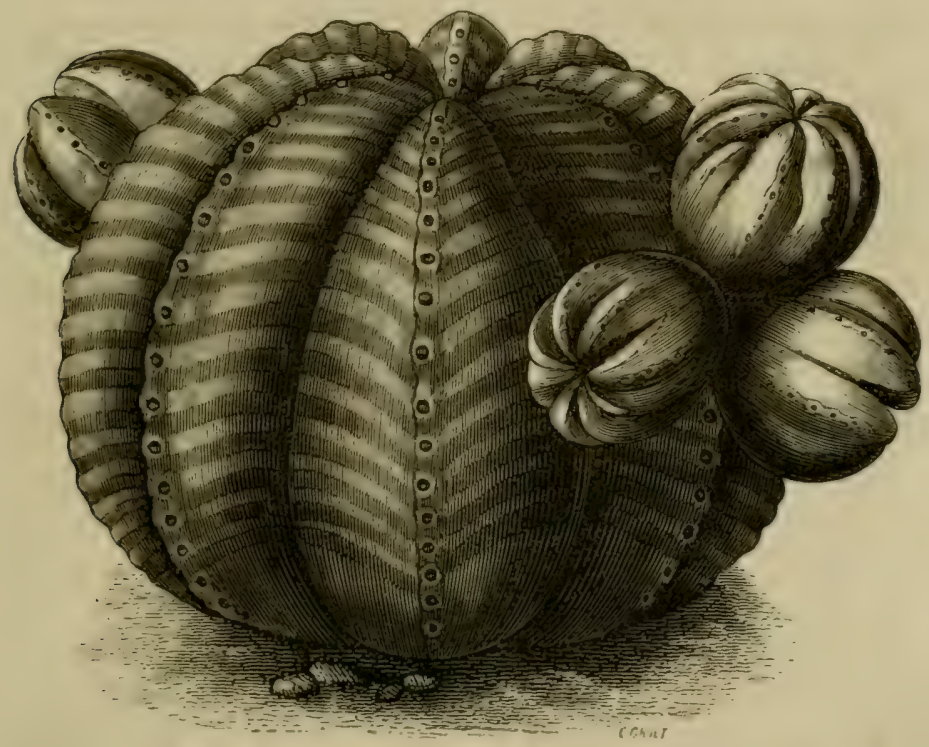

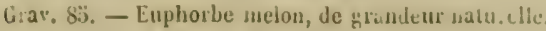

au nombre de douze, sont cependant au nombre varialile de dis à trente-six, ont regardé la fleur de Limné comme un assemblage de fleur's mâles ì une étamine, qui entomrent un seul pistil. Il est difficile, aree dis à trente-six ćtamines qui paraissent successivement, que le pistil mique reste infécond; cest en effet tres-rare quand les plantes croissent naturellement; mais, dans lesplantes cultivées, loraire reste souvent stérile. Cetle stérilité tient, il est vrai, quelquefois a l'inégalité du développement des organes. Le pistil sort asser sourent le premicer, el les stig- 
mates sont flétris quand les fleurs mâles paraissent, ce qui rend la fécondation indirecte, quand elle a lieu. On pourrait done féconder artificiellement les Euphorbes, afin d'en obtenir des graines, et tenter aussi des hybridations sur ce beau genre, 'jui est très-naturel, mais qui présente cependant des types particuliers et assez différents entre eux par le port et le facies.

La fécondation croisée est d'autant plus facile, dans ce genre, que souvent, comme nous venons de le voir, les étamines et le pistil ne se développent pas en même temps.

On pourrait tenter l'hybridation entre les espèces à tiges charnues que l'on cultive comme les plantes grasses, et dont les formes sont aussi curieuses et aussi bizarres. Les unes sont épineuses comme les Cactées, les autres sont privées d'épines. (grav. 85). liais ce sont surtout les espèces à involucre écar. late dont il faudrait tenter de multiplier les variétés. Les E. pulcherrima, cristuta, Breoni, Jacquiniflora, lophogona, splendens, sanguinea, pourraient sans doute s'lrybrider, si ce n'est toutes cusemble, du moins quelques-unes. Déjà le pulcherrima a donné une variété jaunâtre.

\section{FAMILLE DES URTICEES.}

\section{Genre Chanvre. - Cannabis.}

La place importante que le Chanve occupe en agriculture nous oblige à en dire ici quelques mots pour engager les cultivateurs à y chercher des variétés ou à hybrider celles que l'on comnait. Les Chanvres d'Angers et de Piémont, qui sont à peine différents par leurs caractères de l'ordinaire, et qui doment un produit bien plus abondant, pourraient sans doute créer des hybrides. Si l'on observait attentivement les nombreux individus qui composent les champs de Chanvre, on y trouverait aussi quelques variétés qui, peut-être, se maintiendraicnt par les semis et formeraient par la suite des races distinctes propres à l'hybridation. Le Chanrre étant dioïque, la seule difficulté de 
croiscment serait dans l'isolement des pieds femelles dont charque lleur oflre deux stigmates. Les fleurs màles, trèsnombreuses, ont chacune cinc étamines dout les anthères, percées à leur extrémití, répandent daus l'air de gros muages de pollen très-fin. On trouve ausi quelguefois des theurs males dispersies sur les pieds femelles, et plusirurs expériences positives ont démontré que les ovaires peuvent se transformer en graines fertiles sans fécondation.

\section{Genre Houblon. - Humulus.}

Ce que nous venons de dire du Chanrre peut également s'appliquer au Iloublon. On a l'habitude de propager cetle plante par ses nombreux rejets, et je ne salche pas que l'on ait essayé par les semis à obtenir des variétés nouvelles. Les graines avortent mème assez sourent, ce qui n'aurait pas lieu si on imprégnait les ovaires au pinceau. Il y aurait peut-ètre avantage at essayer les croisements cutre les Iloul,lons d'Europe et ceux dAmérique, car cette plante, originaire de ces deux contrées, est également cultivée dans les deux hémisphères. L'isolement serait difficile, et le pollen du Iloublon est peut-être encore plus fin que celui du Chanrre.

\section{Genre Murier. - Morus.}

Les fleurs des Vhûriers naissent en petits chatons qui sont unisexucls. On peut donc retrancher les chatons mâles à mesure qu'ils paraissent, et imprégner les fleurs femelles dont les oraires sont surmontés de deux stigmates. If est essentiel d'opérer lat castration avec beaucoup de soin, et de bien isoler les sujets qui doivent servir de porte-graines, car les quatre étamines des fleurs màles se détendent tout à coup à l'épanouissement comne celles des trties, et répandent en mème temps un pollen très-fin et très-abondant.

Il y a dans les Muricr: deux maces dont on doit chereher it multiplier et anéliorer les variélés: celle du Murier noir, gnci 
l'on considère comme arbre fruitier, et celle du Mùrier blanc, destinée à l'éducation des vers à soie.

Le Mûrier noir a déjà produit une variété à gros fruit qque l'on pourrait essayer de féconder avec d'autres espèces, sans avoir' peut-être beaucoup de chance d'obtenir mieux.

Les Mûriers blancs, beaucoup plus répandus et ayant une grande importance agricole, ont été l'objet d'assez nombreuses recherches; le multicaule, le Morelti à larges feuilles, le rose d'Italie, sont déjà cultivés sur presque tous les points de la France, et permettent d'espérer par les croisements de nombreuses et nouvelles variétés.

Un jardin-appartenant au gouvernement, et spécialement destimé à l'hybridation des plantes utiles à l'agriculture, serait certainement un des établissements qui contribueraient le plus à l'avancement de cetté science.

\section{Genre Figuier: - Ficus.}

Les Figuiers forment un genre très-nombreux qui comprend plus de cent espéces toutes exoliques, à l'exception de l'espèce cultivée et que l'on rencontre sauvage dans le midi de la France. Peut-être existe-t-il déjà des hybrides créés par les insectes dans la longue série d'espèces qui appartiennent à ce genre; mais, comme ils fructifient très-rarement dans nos serres, où l'on n'en cultive, du reste, qu'un très-petit nombre, nous ne nous occuperons que du Figuier ordinaire, considéré comme un des meilleurs fruits qui existent. Nous pensons que l'on pourrait croiser ses différents types et ses nombreuses variétés, et nous recommandons ces essais aux horticulteur's du Midi, les sculs qui puissent espérer quelques succès dans celte opération.

Nous devons dire cependant que le nombre des variétés de Figuiers qui naissent seules dans les régions méridionales est très-considérable. Ce sont peut-être de simples variations ou des hybrides produits par les Cynips, mais on trouve, au milieu de ces produits du hasard, des variétés très-méritantes.

Il est assez difficile de saisir l'époque de la floraison de nos 
Figuier's, car leurs fleur's, très-nombreuses, restent enfermées dans leur réceptacle charnu et tapissent tout l'intérieur de sa eavité. Il faut, quand la figue a acquis une grosseur mojeme, en ouvrir une de temps en temps jusqu'ì ce que l'on s'aperçoive que les fleur's sont développées et que les anthères sont sur le point de répandre leur pollen. On observe alors que les fleurs sont unisexuées; les femelles, ayant chacune un ovaire, un style latéral et deux stigmates, occupent la base et souvent aussi les côtés de la figue; les mâles, avec leurs trois étamines, sont fixées au sommet et renversées de telle manière que leurs anthères pendent sur les fleurs femelles, et les féconderaient nécessaircment, si les étamines avaient toujours du pollen et si les pistils étaient toujours bien conformés.

Il semble nécessaire, pour que la fécondation puisse s'opérer, que le réceptacle soit ouvert à sa partie supérieure. C'est du moins ce qui a lieu naturellement dans les Figuiers saurages.

Risso, qui s'est occupé de la plupart des productions de la Provence, n’a pas oublié le Figuier, dont il a publié l'histoire maturelle. Il partage l'espèce principale en trois sous-types: le premier contient le Caprifiguier proprement dit, le Calymifiguier saurage et celui des bois; le second sous-type est aussi formé de trois sous-espèces, le Figuier du Letant, celui du Midi et celui du Ponent; le troisième comprend le Figuier à fruit cultivé, celui à fruit agréable et l'esculent. Il divise chacune de ces neul' sous-espèces en trois variétés, ce qui fait ringt-sept, et chacune d'elles en plusieurs sous-rariétés. Il parvient ainsi à classer tous les Figuiers qui pullulent dans le Midi.

C'est dans le premier sous-type que se trourent les fruits secs de ces arbors sauvages qui fructifient toujours et naturellement, et dont les étamin's sortent mène quelguefois par l'ouserture yui est all sommet du réceptacle. C'est dans ces mèmes figues que naissent les cynips, petils insectes ailés, qui, par leurs mousenents dans l'intérieur des figues, favorisent lia dissémination du poulen qui ne peut manquer de féconder les pistils.

Le second sous-type offire des fruits qui sont encore un peu 
ouverts au sommet et dont les organes peuvent encore se féconder. Si la fécondation n'a pas toujours lieu naturellement, on l'opère par la caprification, qui consiste à apporter sur ces arbres les cynips que l'on va chercher sur les Caprifiguiers.

Voici comment on procède à cette curieuse opération. Dans les mois de juin et de juillet, quand les rers, qui ont été engendrés dans les figues sauvages, sont prêts à subir leur métamorphose et à se changer en moucherons, les paysans cueillent ces fruits et les portent enfilés dans des brochettes sur les Figuiers domestiques, qui sont alors en floraison. Les moucherons qui sortent des figues sauvages ainsi transportées entrent dans les figues domestiques, y portent la poussière fécondante dont ils se sont chargés en passant à travers les étamines des Caprifiguiers et la font pénétrer jusqu'au centre du fruit, où ils vont déposer leurs œufs.

L'entrée des moucherons produit donc un double effet, celui de porter dans la figue domestique le pollen provenant des figues sauvages, et de causer, dans le premier fruit, par leur. présence et celle des oufs qu'ils déposent, une sorte d'irritation qui y appelle les sucs et occasionne un grossissement en quelque sorte maladif. Les graines sont fertiles.

Les figues du troisième sous-type sont celles que nous cultivons dans nos jardins. Elles ne s'ouvrent pas ou tout au plus a leur maturité; les fleurs mâles sont généralement avortées ou les étamines manquent de pollen; de sorte que leurs graines sont presque toujours infécondes. Ce sont cependant ces derniers Figuiers qu'il laudrait choisir comme pieds mères pour les croisements que l'on voudrait tenter, et il faudrait prendre le pollen sur les figues de la seconde section, plutôt que sur les Caprifiguiers. Quant à la manière de l'appliquer, je suppose que l'on pourrait, sans inconvénient, ouvrir l'œil des figues porte-graines pour appliquer au pinceau la poussière fécondante. J'ignore si des cynips recueillis sur des fruits du second sous-type pourraient agir sur ces figues entièrement fermées et y porter le pollen sur leurs ailes. J'ignore aussi si la floraison de ces diver's Figuiers 
est concordante, et, par conséquent, si l'on pourait opérer avec lacilité. Ce sont autant de questions qu il faudrait étudier en Provence, et je ne doute pas qui un horticulteur instruit, qui voudrait s'occuper sérieusement de l'hybridation du Figuier dans cette partie de la France, n'arrive i multiplier indéfiniment les variétés et à en obtenir de supérieures encore à celles que nous connaissons.

\section{FAMILLE DES PROTÉACÉES.}

\section{Genre Banksie. - Bankisia.}

Lu tres-urand nombre de Banlisia sont maintenant cultivés daus nos serres tempérées, où ils fleurissent assez facilement et où ils grainent mème quelquefois. Leurs quatre étamines peuveni ètre enlevies au moment de l'épanouissement, et le pinceau pent ensuite porter le pollen sur l'extrimité du style renflé en stigmate et imprégné déjì de liqueur miellée sur larjuelle le pollen adhire facilement. On a maintenant assez de Banlisia pour espérer des hybrides, dont plusicurs semblent mène exister; et, si l'on ne pouvait en obtenir, la fécondation artificielle servirait au moins a faire fructifier ces plantes, dont le prix est assez élevé et dont les graines, par conséquent, ont toujours une grande valeur.

\section{FANILLE DES JUGLANDÉES.}

\section{Genre Noyer. - Juglans.}

Les grinds arbres qui composent ce raste et beau genre sont déji assez répandus dans nos jardins, ou plusieurs fructifient; mais il en est une espèce, la seule probablement européenne, que lon rencontre partout el que l'on cultive pour se's fruits. Les fleurs de tous les Nojers sont monoïques; les mâles, en 
longs chatons simples ou rameux, ont de douze à vingt-quatre étamines el répandent en abondance un pollen conleur de soufre. Les femelles, placées à l'extrémité des rameaux, ont deux stigmates sous la forme de petites crètes épaisses et souvent glutineuses, sur lesquelles le pollen reste longtemps adhérent.

Le Juglans regia, ou Noyer ordinaire, a déjà produit plusieurs variétés, dont les unes sont précoces et d'autres trèstardives, avec tous les intermédiaires possibles; d'autres nnt un fruit très-gros. On en trouve à coques dures et à coques tcndres, à fruits ronds, anguleux ou allongés. Il serait facilc d'hybrider ces diverses variétés, d'obtenir des nouveautés et trèsrprobablement des races meilleures. On n'a encore ricn tenté sur le Noyer, dont les fruits sont peu perfectionnés, et on réussirait certainement à croiser les bons Noyers ordinaires avec celui à gros fruit ou avec la variété précoce récemment obtenue. Enfin, on pourrait combiner leur précocité de manière i avoir des races que les gelées printanières ne pourraient surprendre.

On pourrait aussi croiser ensemble les Noyers de l'Amérique du Nord, comme arbres forestiers, ou au moins tenter les croisements entre les espèces de la section des carya, qui contient des arbres superbes et qui ont entre cux de grands rapports. L'isolement des branches sur lesquelles on pratiquerait la fécondation artificielle serait la scule difficulté sérieuse dans cette opération. Il faudrait placer une branche sous cloche, ou l'entourer pendant quelque temps d'un tissu imperméable à l'air.

Le Juglans hickory ou alba, si estimé des Américains pour le charronnage, le cinerea, dont la végétation active laisse bien loin celle de la plupart de nos arbres, le porcina, le nigra, l'amara, qui tous se développent si bien sous notre climat, pourraient peut-êlre aussi se croiser entre eux ou avec le verji ou Noyer ordinaire.

Il existe, dans les pépinières de Versailles, suivant M. Camu- 
sel, un nouveau Noyer, hyjbride du $N$. commun et du $N$. noir, qui se reproduit exactement par ses fruits.

Le $J$. cinerea fructifie à cing à six ans; mais il faut se rappeler que ses noix tombent avaut leur maturilé, pleines d'une malière sclaireuse, et que, selon l'observation de II. Camuset,

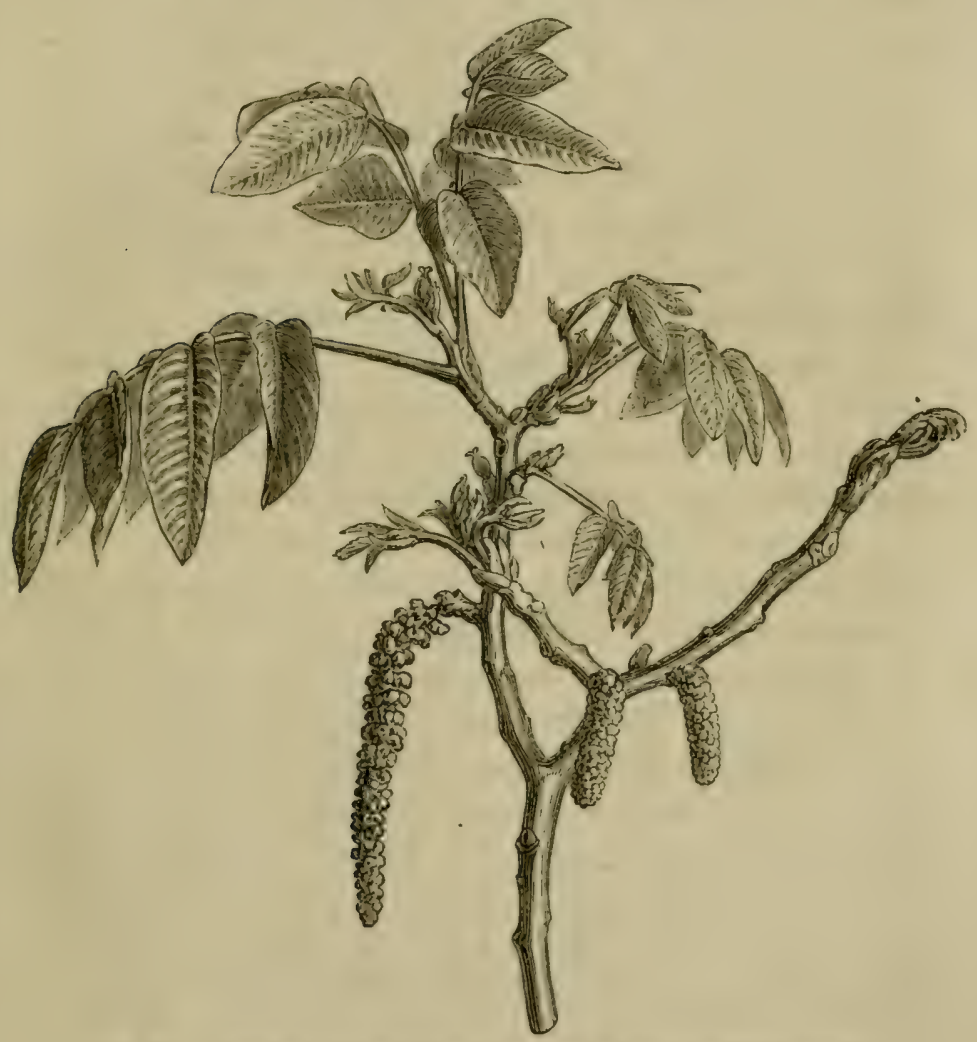

Grav. 86. - Branche de nover offunt des fleurs mäles et des lleurs femelles.

il faut les mettre on las et qu'elles grerment de même que si elles itaient mures. Elles acquierent toute leur maturite sous terre en hiver. Inhanel, cité par Camuset, amnonce la mène chose pour les noix ordinaires (grar. 86). 


\section{FAMILLE DES AMENTACÉES.}

Genre Orme. - Ulmus.

Les Ormes sont hermaphrodites et leurs fleurs ont trois à six étamines et un ovaire aplati, surmonté de deux petits stigmates. On rencontre bien aussi quelques fleurs mâles mêlées aux autres; et, comme les anthères répandent très-facilement leur pollen, la fécondation est toujours assurée, quoique assez souvent indirecte. On pourrait cependant enlever les étamines dès l'épanouissement et féconder le pistil au pinceau, en prenant toutes les précautions nécessaires pour isoler le peu de fleurs qu'il faudrait conserver à chaque bouquet.

Les espèces ou plutôt les variélés indigènes sont nombreuses; les campestris, suberosa, montana, glabra, modiolina, effusa se croiseraient très-certainement, et parmi les espèces exotiques, les fulva, alata, americana, nemoralis, integrifolia et peut-être même les macrophlyylla et pumila de Sibérie pourraient aussi s'hybrider. L'Orme est un bois si utile dans le charronnage et les constructions, que l'on devrait chercher des variétés nouvelles qui pourraient offrir quelque supériorité sur celles qui sont connues. Comme nous avons eu occasion de le faire remarquer plusieurs fois, l'hybridation des arbres forestiers devrait présenter de très-grands avantages; mais, comme les résultats seraient éloignés et qu'il ne résulterait aucun profit direct pour celui qui les obtiendrait, il serait à désirer que le gouvernement établitt, pour ce genre de recherches, un jardin de botanique, dirigé par un homme habile et consciencieux.

\section{Genre Planère. - Planera.}

Très-voisin de l'Orme, ce genre en diffère par ses fleur's polygames et la forme de ses fruits. Aussi n'est-il pas probable que l'on puisse obtenir des croisements entre les deux genres. Et, comme le Planera ne contient que deux espèces, l'aquatica 
et le crenuta, il y a peu de chances d'hybridation entre elles. Yais pent-être en trouvera-t-on d'autres, et, ce que nous venons de dire de la nécessité d'augmenter le nombre des espèces forestières s’applique également au Planeru. La fécondation artificielle serait plus ficile que dans l'Orme; il suffirait de veiller à l'épanouissement et d'enlever complétement les fleurs mâles et celles qui sont hermaphrodites.

\section{Genre Bouleau. - Betulo.}

Les fleurs des Bouleaux sont monoïques; les mâles forment de longs chatons pendant à l'extrémité des rameaux, et les femelles de petits cônes dressés, qui, par la position inclinée des branches du Bouleau, se trouvent ainsi placées au-dessus des mâtes et ne peurent être fécondíes que par celles des rameanx supérieurs. La fécondation artificielle peut done s'opérer comme sur toutes les plantes monoïques, en prenant les précautions convenables d'isolement. On connait maintenant un assez grand nombre de Bouleaux, et ils se ressemblent assez pour qu' on puisse espérer l'hybridation.

L'aspect particulier de ces arbres et le rôle quills jouent dans les pares et les jardins paysagers sont des motils suffisants pour chercher à augmenter leurs variétés.

Outre le Betula alba et les autres espèces européennes, comme le viridis, qui fait le passage aux Aunes, le pubescens, le laciniutu, il y a encore les espèces américaines, telles que le lenta, le nigra, le papyracea, le macrophylla, ste.

\section{Genre Saule. - Salix.}

Les Saules sont de tris-beaux arbres dont on ne tire pas un assez grand parti dans les jardins paysagers. Presque tous sont dioiques, et il semble que cet éloignement des deux sexes ait farorisé le croisement des especes; car, si le genre est distinct et bien siparé des autres Amentacies, les especes passent tellement de lime it l'autre que l'on ne peut guere distinguer que 


\section{FÉCONDATION NATURELLE ET ARTIFICIELLE.}

des groupes bien nets et bien tranchés, et dont les différentes espèces ne sont, pour ainsi dire, que des varićtés.

L'hybridation ne peut offrir aucune difficulté, puisque les sexes sont séparés dans presque tous. Il y a à peine quelques exceptions où l'on trouve des fleurs hermaphrodites ou quelques fleurs mâles mélangées aux chatons femelles. L'isolement se présente parfois tout naturellement, car pendant longtemps. on n'a connu que des individus femelles du Salix babylonicr ou Saule pleureur. Il semblerait que ce Saule est quelquefois hybridé par d'autres, car M. Jacques qui, une fois, a obtenu et scmé des graines, n’a pas cu un seul babỵlonica. M. PoulainIlecquet, pharmacien à Abbeville, a aussi envoyé à la Société royale d'horticulture de Paris un paquet de ces mêmes graines, qu'il suppose aussi liybridées naturellement. Une espèce dédiée à M. Seringe, sous le nom de Seringeana, a été trouvée en Suisse, sur les bords de la Kandel, près du lac de Thoun et aux environs de Vevay. Dans la première localité, on n'a rencontré que des femelles, et dans la seconde, des individus mâles seulement. Ainsi on obtiendrait assez facilement des hybrides; car, dans ces plantes, le pollen n'est pas aussi fin que dans les autres genres dioiques; il est moins pulvérulent; il reste longtemps adhérent aux étamines; et, comme j'ai remarqué très-souvent des graines stériles sur plusieurs chatons femelles, je ne serais pas éloigné de croire que la fécondation n'a lieu que par l'intermédiaire des insectes, toujours très-nombreıx sur ces sortes de fleurs. Une simple gaze, placée sur la fleur femelle, suffirait alors pour éloigner ces messagers ailés.

On cultive déjà de très-belles espèces de Saules. Indépendamment des indigènes, qui sont très-nombreux, on a maintenant des saules exotiques, tels que le babylonica, qui est presque indigène, le japonica, le tetrasperma, le nigra, le paradoxa, le coluteoides, le Humboltiana, etc.

Il y aurait aussi des croisements à tenter sur la section si utile des Osiers ou Viminea, mais cenx qui précisément sont le plus employés pour la vannerie fleurissent très-rarement; ct, 
pour mon comple, je ne connais pas les chatons de l'Osier jaune.

Genre Peuplier. - Populus.

Les Peupliers sont, comme les Saules, des arbres diö̈ques, dont les fleurs mâles ont de huit à trente étamines et dont les femelles ont un ovaire terminé par deux a huit stigmates. Plısieurs de leurs especes sont assez voisines pour qu'on puisse supposer qu'elles se croiseraient; mais il en est qui n'ont jamais donné que des lleur's múles, et d'autres ne fructifient pas sous notre climat, peut-être parce qu'ils ne sont pas assez vieux, ou que, dans la plupart des jardins, ils ne rencontrent pas toutes les circonstances firorables. Ils forment évidemment plusieurs groupes naturels, tels que les Trembles, les Peupliers proprement dits, les Leuce, comme l'alba et le cinerascens. Leur dioëcie rendrait les opérations dı croisement aussi faciles que pour les Saules.

\section{Genre Mètre. - Fagus.}

Ce genre est monoïque; les fleurs mâles sont des capitules arrondis, dont les étamines, au nombre de huit à neuf pour chaque fleur, sont pendantes quand les anthères vont s'ouvrir. Les femelles, placées aux aisselles supérieures, ont deux ovaires terminés chacun par trois styles à trois stigmates. On rencontre dans le Ilêtre commun des pieds dont les graines avortent toujours, mais dans la plupart elles sont fertiles. Ce llètre offre lui-mème plusieurs variétés, comme le pourpre, le cuirré, le lacimié et les panachés, qui s'hybrideraient facilement, mais qui ne lleurissent presque jamais. Il est à regretter aussi que les Hetres que l'on irencontre dans les parties froides des deux Anériques et qui se présentent, comme le nòtre, aree un si beau port, soient à peine cultivés daus nos pares, "t par conséquent presque inconnus en fleurs. 
Genre Chàtaignier. - Castanea.

Les fleurs sont monoïques; les mâles, en longs épis, portent des étamines dont le nombre est indéterminé; les femelles sont munies d'un ovaire qui présente ordinairement six styles qui correspondent à un même nombre de loges. Les stigmates, à peine visibles, terminent les styles.

Le Chàtaignier est à la fois arbre forestier et fruitier. Sous ce dernier rapport, il a dommé un certain nombre de variétés à plus gros fruits, tels que le marron de Lyon, de Lueques, de Lusignan, le gros noir, etc., dont les fruits sont plus gros et plus savoureux, et dont les marrons sont souvent solitaires dans le brou. C'est en partie à l'avortement des loges et des ovules et au développement d'un seul d'entre eux qu'est due la supériorité de ces marrons. Il faut pour cela que sur les six stignates le premier imprégné fasse de suite dérelopper l'ovule qui prend la place de tous les autres, en sorte que si l'on pra- tiquait la fécondation artificielle ou l'hiybridation sur des Chàtaigniers, il serait peut-être convenable de poser le pollen sur un seul stigmate, ou, pour plus de sûreté, de couper les autres. On n'aurait alors, toujours et nécessairement, qu'une seule graine par fruit, et, comme elle ne serail gènée à aucun àge de son accroissement, elle produirait sans doute des sujets plus vigoureux.

Sous le rapport forestier, nous devons nous contenter du Châtaignier ordinaire, qui l'emporte peut-être sur le chêne par les qualités de son bois, que l'on pourrait encore améliorer par des croisements, si on parvenait à introduire et à faire fructifier dans nos jardins les divers Châtaigniers d'Amérique, dont nous connaissons déjà l'americana, mais surtout les belles et nombreuses espèces de l'ile de Java.

\section{Genre Chêne. - Quercus.}

Le chêne est aussi monoïque, comme la plupart des Amentacées. Les fleurs milles, en longs chatons pendants, ont de 
cing à dix étamines, et les femelles offrent un ovaire à trois loges qui renferment ehacune deux ovules. Ici, comme dans le Chàtaignier, il y a avortement dans les ovules après la fécondation, mais plus complétement, car un seul se développe et produit un gland.

Les Chìnes, dont les nombreuses espèces sont répandues dans presque toutes les contrées de l'Europe, de l'Asie et de l'Amérique, sont de tous les arhres ceux qui ont le plus d'importance comme bois de construction et de chauffage. Ils se sont probahlement hỵbridés naturellement, et il serait difficile qu'il en fût autrement, puisque plusieurs d'entre eux semblent formés sur un même type, et sont plutut des rariétés distinctes que des espèces séparées.

Ceux qui nous intéressent le plus habitent nos forèts, où l'on distingue, parmi ceux qui perdent leurs fenilles, le racemosu, le sessiliffora et leurs intermédiaires, les fastigiata, apennima, pubescens, etc. C'est près d'cux que viennent se ranger presque tous ces beaux arbres de l'Amérique du Yord, tels que les rubra, alba, tinctoria, nigra, aquatica, prinos, castanea, bicolor, muerophlylla, etc., dont quelques-uns sont déjì cultivés dans nos pares et nos jardins. C'est la section la plus remarquable du genre, celle dont il conviendrait le plus de cultiver les espéces, pour tâcher ensuite d'hybrider et d'augmenter nos races forestières.

Le cerris, le pseudosuber, l'olivaformis, le to $\approx$ sont aussi de fort belles espèces.

Les Chènes verts sont tous plus méridionaux que les autres. On y remarque de belles espèces indigènes, telles que les ballota, cocciferu, ilex, suber, et une foule d'arbres ćtrangers, parmi lesquels nous citerons seulement les muritima, rigida, virens, hemispharica, cinerea, myrtifolia, etc.

Une école forestière, qui réunirait nos Chènes d'Lurope avec ceux de l'Amérique, des Indes, du Jipon, qui cultiverait au moins cenx qui peusent réussir sous notre climat at fui obtiendrait ainsi des sujets destinés dims leur ìge adulte aux 
348 FÉCONDATION NATURELLE ET ARTIFICIELLE.

essais d'hybridation, serait certainement un établissement utiele $\mathbf{t}$ qui mériterail d'être institué (grav. 87).
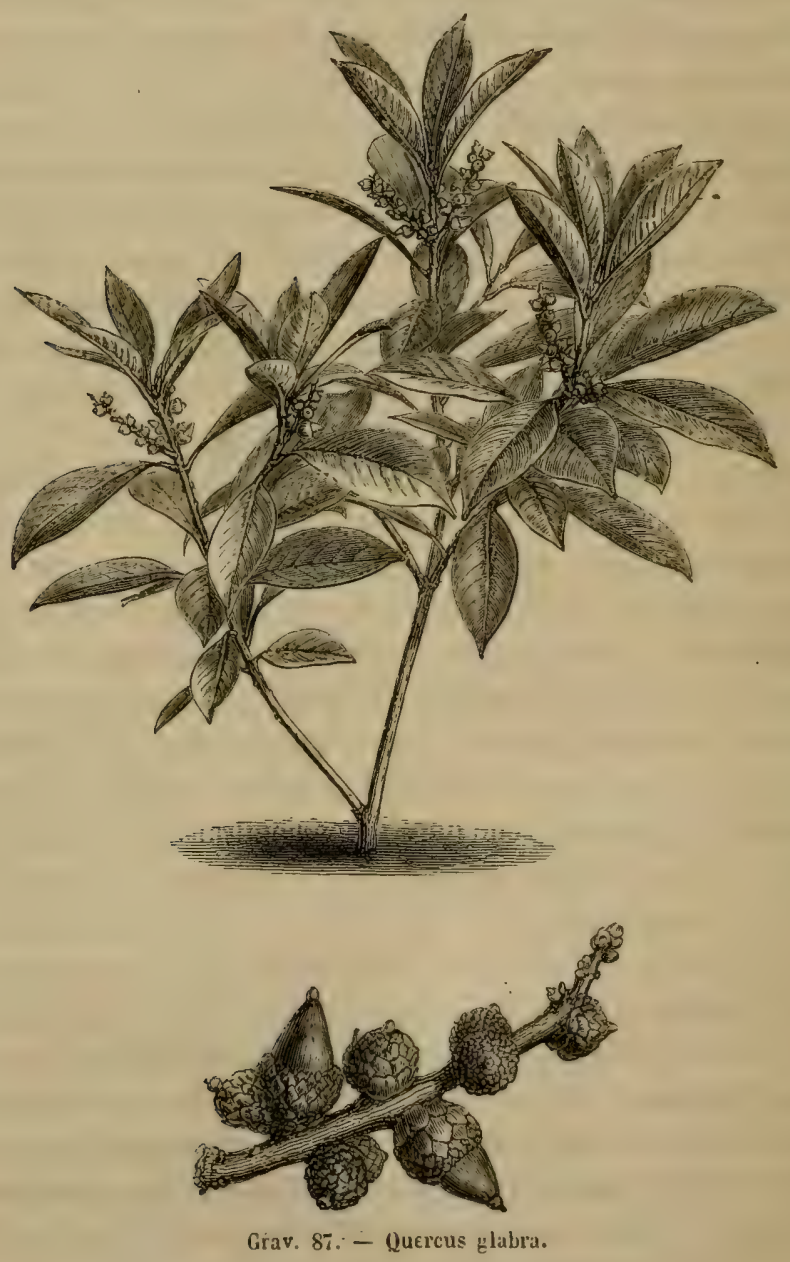

Genre Noisetier. -- Corylus.

Les Noisetiers ou Coudriers forment maintenant sept ì luit espèces distinctek, dont les deux principales, toutes deux undi- 
gènes, les $C$. arellana et tubulosa, sont considérés comme arrbres fruiliers et ont dijà produit un certain nombre de variétes.

On y remarque les arelines rouge et blanche, lit grosse noisetted'Angleterre, les Noisetiers pourpres d'Alyer, à feuilles lacinicées.

Dis le mois de février on aperçoit les fleurs mâles suspendues en longs chatons, qui laissent échapper une grande quantité de pollen de leurs anthères uniloculaires, et les fleurs femelles qui sortent des bourgeons sous forme de petites houppes carminées. Charque ovaire est surmonté de deux de ces petits stigmales qui correspondent à deux ovules dont un avorte le plus ordinairement. Quoique déjai on ait de belles espèces de noisettes, je suis persuade que ce linit peut être encore amélioré par des hybridations d'autant plus faciles, que l'on peut isoler un Noisctier, ct enlever toutes ses fleurs males deux mois avant le développement des fleurs femelles, ce que l'on pourrait igalement faire pour le Nover et pour plusieurs autres Amentacées dont les chatons mâles deviennent visibles très-longtemps avant leur développement.

\section{FAMILLE DES CONIFÈRES.}

\section{Genre If. - Taxus.}

On cultive l'lf commun et sa variété à feuilles pumuchées, celui du Cannada et le purromidal; les autres sont des raretés a peine introduites dans nos cultures. Les fleur's, monoinges ou dioï ues, sont à lunit à dix étamines monadelphes pour les mates, at les lemelles sont très-remaryuables par un stigmate concare qui s'enfonce au sonmet de l'ovaire. It fiut altendre, pour opérer la fiecondation, qu'une petite groutte de liqueur niellée paraisse an sommet de l'ovaire; on pose alors le pollen sur ce liquide. Selon toute apparence, le baccata et le canadensis s'hybrideraient, et il est probable que l'on parriendrait 
aussi à croiser avec les nôtres les $I f s$ de la Chine et du Japon, tels que les macrophylla, mucifera, latifolia, verticillata, etc., si l'on arrive à les faire fleurir dans nos jardins, quand ils auront acquis des dimensions suffisantes:

\section{Genre Genévrier. -- Juniperus.}

Ces arbres sont presque tous dioïques et par conséquent faciles à hybrider. Les fleurs femelles, réunies en petit nombre, sont protégées par leurs écailles et portent un très-petit stigmate sessile sur l'ovaire. Les mâles ont quatre à huil étamines presque sessiles sur leurs écailles. Les graines restent longtemps à mûrir. On cultive quelques Genévriers dans les jardins, tels sont l'excelsa, le virginiana, roisin des Cèdres, le lycia, le prostrata, l'hispanica, le glanca, le phonicea, le sabina, l'oxycedrus, etc., qui peut-ètre pourraient être croisés. Je ne sache pas qu'aucune tentative ait été faite à cet égard.

\section{Gemre Thuya. - Thuya.}

Ces arbres verts sont monoïques; les fleurs femelles, offrant deux ovaires et deux stigmates, sont placées au sommet des rameaux; au-dessous se trouvent les mâles, composées chacune d'une seule écaille et de quatre anthères. La floraison a presque toujours lieu en hiver ou dès le commencement du printemps. Indépendamment de l'orientalis et de l'occidentalis, depuis longtemps cultivés, on trouve encore le pyramidalis, le nepalcnsis, l'articulata, qui fructifie à IIyères, le tartarica, l'australis, le cupressoides et quelques autres que l'on commence à introduire dans nos parcs ou dans nos serres. Les crolsements auraient lieu sans doute sur quelques-uns, en ayant soin d'en isoler complétement les fleurs femelles.

\section{Genre Cypres: - Cupressus.}

L.es Gyprès étant monoïques comme les Thuya, ce que nous venons de dire de ces dernier's s'applique nécessairement 
i ceux-ci. (tn en cullive mantenant dassez lielles espéces; le sempervirens, qui Iructifie si abondamment dans le midi de la France, pourrait servir de pied mère pour tenter des fécondations croisées avec le thuyoirle's ou le torulosa, le sinensis, celui du Népaul, l'horizontul et plusieurs autres très-petits dans nos jardins, et dont la lloraison se fera encore beaucoup attendre.

\section{Genre Pin. - Pimus.}

Genre nombreux qui ne renferme que des arbres à flems monoinques, dont les femelles, rémies en cône, sont placées au sommet des rameanx et formées de deux petits ovaires i stigmaté glanduleux. Les mâles, rassemblées en une fonle de petits chatons, sont situées au-dessous des femelles et offrent chacune une écaille qui porte deux anthères uniloculaires. La Acrnière fleur, c'est-it-dire la plus élevée, est ordinairement femelle et par conséquent la fécondation est indirecte. C'est le pollen de la branche supérieure qui doit féconder la fieur fe. melle de la branche située au-dessous.

Il serait à désirer que l'on tentât sur les Pins, qui sont à la fois arbres d'ornement et arbres forestiers d'un haut intérêt, des essais d'hybridation, dans le but d'augmenter encore leur nombre et d'obtenir de nusvelles qualités de bois. Les pieds mires ne manqueraient pas, ear un grand nombre de Pins sont indigines. I.s syluestris, rubra, maritima, laricio, cembro, et, farmi les exotinues, le strobus ou l'in du lord, le muylio. le cetuariensis pourraient non-seulement être croisés entre cux, mais meore et surtout aree les belles especes dimérigue el d'Asie nouvellement introduites, quand elles seront assez déreloppees pour dommer leurs flemrs dans nos climats.

Cïest un gremre extrèmenent important et qui mériterait, comme le Chene, le sapin, le Chataignier, le Noyer, de former des ícoles particulières destinies à l'utude, à la multiplication et à la création des espèces forestières. 
Genre Sapin. - Abies.

On peut appliquer au Sapin ce que nous venons de dire du Pin, dont le genre ne diffère que par la position solitaire des chatons, par l'isolement des cônes femelles et par les feuilles insérées une à une, et non plusieurs ensemble, dans une gaine, comme dans les Pins.

Les cônes dressés ou inclinés semblent partager les Sapins en deux grandes divisions qui, chacune, sont représentées par une espèce europénne. Le pectinata ou picea a les cònes droits et pourrait être fécondé par le balsamea et plusieurs autres espèces qui se rapprochent de ses caractères. On pourrait tenter sur notre $A$. excelsa, qui a les cônes renversés, des croisements avec l'alba, le nigra, l'orientalis, le canadensis, etc., qui les ont également pendants. Toutefois, je dois dire que je ne connais aucun hybride bien caractérisé dans les arbres verts.

\section{Genre Mélèze. - Larix.}

Les fleur's mâles ont deư anthères comme les Pins, et les fleurs femelles, disposées en cône à écailles rouges, ont deux ovaires et deux stigmates perforés. Les $L$. europxa et americana sont les deux seules espèces qui fleurissent dans nos jardins, et ils se ressemblent tellement qu'il est bien probable que l'hybridation aurait lieu entre eux. Ce serait un essai facile, car les llélèzes fleurissent jeunes, et l'isolement des fleurs femelles n'offrirait aucune difficulté. 


\section{CHAPITRE VIII}

PLANTES MONOCOTYLEDONES.

\section{FAMILLE DES ORCIIIDEES.}

Le nombre des Orchidées s'aceroit tous les jours dans ume si forte proportion, depuis que l'on recherche avec empressement ces admirables végétaux, que l'on ne sait réellement pas i quel chiffre pourront s'élever un jour les membres de cette famille. Toutes les contrées chaudes du globe ont été mises à contribution, et notre zone tempérée nourrit aussi quelques Orchịdé s, moins éclatantes, il est vrai, que celles qui régètent sous le ciel brûlant de la zone torride, mais bien remarquables aussi par leur fraicheur et leur inimitable coloris. A l'exception de ces dernières, e’est-à-dire des espèces indigènes, les Orchidées fructifient rarement dans nos cultures, et jusqu'à présent on s'est peu occupé de faire germer leurs graines; on en a cependant reconnu la possibilité, mais on a remarqué aussi qu'uu grand nombre d'entre elles paraissaient stériles. Il est bien prohabile que cette stérilite des graines que j’ai observée moi-mème sur des Orchidées indigènes ne tient pas aux causes auxquelles on l'altrihue grénéralenent, mais simplenent au manque de fécondation; car M. Scheidweiler a vu dans les serres de Lacken, cn Belgique, un Neotlia picta, et deux autres espèces qui lui sont encore inconmes, en pleine fructification. M. Neumann 
cite le Neottia elata comme croissant partout dans les serres du Muséum, et il dit aussi avoir obtenu des graines fertiles du Calanthe veratrifolia, dont il avait artificiellement fécondé les fleurs. Souvent on voit germer des Orchidées dans la terre qui entoure les plantes que l'on reçoit du Nexique ou du Brésil. Je ne doute pas que l'on n'arrive par la fécondation artificielle à faire fructifier un assez grand nombre d'especes de cette belle famille, et si l'on parvient à les élever de graines, l'hybridation produira, dans ce groupe, plus de merveilles encore qu'elle n'en a opéré dans les Roses, les Pelaryonium, les Dahlia, etc.

Nous regrettons de ne pouvoir consacrer un article spécial à chaque genre de cette belle série végéiale, dont la culture, quoique faisant tous les jours des progrès, n'est pas encore assez avancée pour espérer un grand nombre d'hybrides. Ce ne sont pas des variétés que l'on cultive dans les Orchidées, mais des espèces souvent distinctes, qui peut-ètre se croiseraient aussi facilement que les Cactées et plusieurs autres plantes, mais qui, dans la nature, ont bien peu de chances pour s'hybrider. II arrive même assez souvent aux Orchidées indigrènes d'être infertiles, parce qu'elles ne sont pas fécondées. La disposition toute particulière de leurs organes, la consistance singulière de leur pollen qui presque jamais n'est pulvérulent, sont des causes qui s'opposent à la fécondation naturelle, mais qui faciliteraient au contraire l'hybridation, si l'on parvenait un jour à faire fructifier les belles Orchidées qui ornent nos serres chaudes et à reproduire ces plantes par leurs graines.

Si, pour le moment, on ne doit pas chercher les hybrides, on doit au moins tenter, par tous les moyens possibles, la fécondation artificielle avec le propre pollen de chaque espèce; des essais de ce genre, dus à Ch. Morren, à Neumann et à quelques horticulteurs anglais, ont parfaitement réussi et pronıcttent des succès daus cette opération. G'est pour cela que nous allons donner des indications générales au moyen desquelles pour ra toujours tenter la fécondation sur les Orchidées.

L'ovaire, placé sous le périgone, porte à sa partie supérieure 
et au milien des enveloppes florales une colonne diversement colorée yui est formée du style, du stigmate, du filet et des anthères, dont les positions relatives sont ensuite très-variables. Le style semble sourent de mème nature que le pirigone, et l'on voit tantìt à sa base, tantôt à son' sommet, plus souvent sur un de ses côtés, une espece de fosselte, on une petite pliıpue, plus rarement un tubercule, généralement imprégné d'une humeur visqucuse très-tenace, et qui est le stigmate. Ce dernier est souvent enclavé dans de petits appendices de la colonne qui s'avancent plus ou moins ot isolent, pour ainsi dire, cet organe. On voit anssi la colonne se courber, se renverser, cmportant ainsi le stigmate, qui d’autres fois est caché dans un grand pétale creux nommé labelle ou sabot. C'est au point que, malgré des connaissances botaniques, on ne voit pas toujours distinctement où se troure le stigmate, si dejà on n'a pas l'ha-. bitude de l'organisation des Orchidées exotiques, qui diflèrent aussi entre elles.

Les organes milles sont presque toujours réduits à une seule étamine, dont l'anthère, il est vrai, se partage ordinairement en deux ou quatre parties, ou bien il ! a deux anthères fertiles, el jamais plus. Les filets qui les soutienment sont généralement soudés à la colomne qui porte le stigmate, et les masses polliniques sont placées au sonmet de la colonne ou sur ses còtés, quelquefois rapprochées du stignate, et d'autrefois séparées de lui par des appendices qui rendent la fécondation naturelle presque impossible.

On ne peut supposer, comme dans les autres plantes, que le pollen, dévié par le vent on emporté par les insectes, peut venir, malgre ces olistacles, tomber sur le stigmate, car cet état pulverulent est presque inconnu dans la famille qui nous occupe. Tautit ce pollen est céreux, ressemblant à du véritable cérumen, tantôt il est élastique ou du moins fixé à un corps qui l'est tellement que lit masse pollinique peut ère étirée comme du caoutchouc.

Enfin, il est rare dans ces plantes que le pollen puisse se 
556 FÉCONDATION NATURELLE ET IRTIFICIELLE.

diviser en granules; quand il tombe sur le stigmate, c'est la masse entière ou une partie de cette masse qui est naturellement divisée en plusieurs parties. J'ai vu plusieurs fois, dans

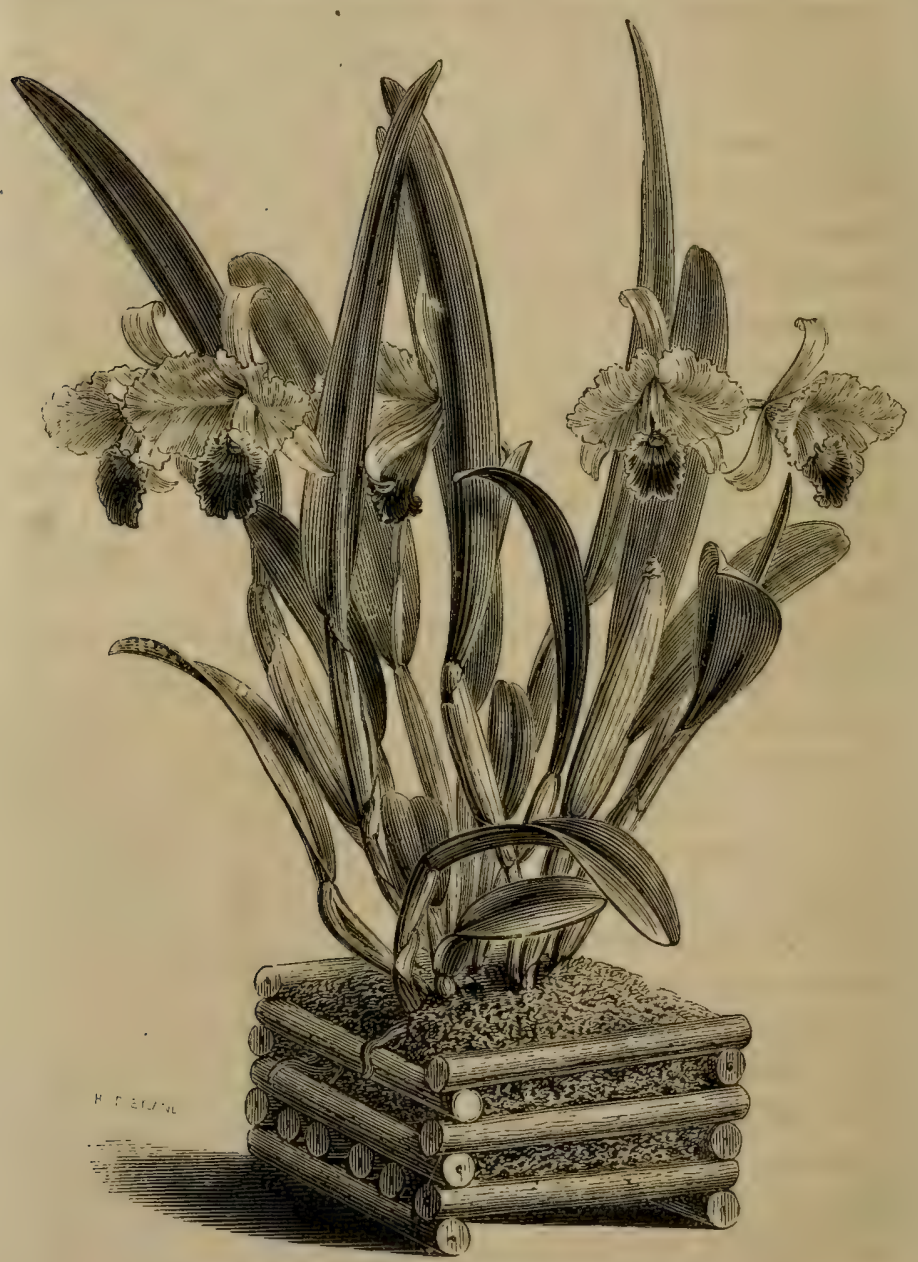

Grav. 88. - Exemple d'Orehidée. - Cattleya de Triana.

les Orchis indigènes, les petites agglomérations de pollen se renverser entièrement et tomber sur le stigmate où elles adhé- 
raient avec force. Jes fleurs se mainlienuent longtemps épanouies, et ce n'est qu'an moment ou elles vont se flétrir que les masses polliniques se détachent el alteignent l'organe femelle, aidées alors par le contournement des parties dupérigone.

Il résulte de ees singulières dispositions que l'on peut toujours facilement détacher les masses polliniques avee une petite pince et les poser tout entières sur le stigmate, ou enlever le pollen céreux et l'étaler au pinceau sur l'organe femelle. Par la mème raison, celui qui est un peu pâteux ou de. mi-pulvérulent pourrait être posé par le même moyen. La seule difficulté sur plusieurs plantes est de trouver le véritable stigmate; mais comme il n'y a jamais sur la colonne qui porte les organes que deux ou trois points que l'on peut supposer stigmatoides, on a toujours asse\% de pollen pour que, dans l'indécision, on puisse en imprégner ces parties douteuses. Je présume que l'on aiderait l'imprégnation de ce pollen gluti-

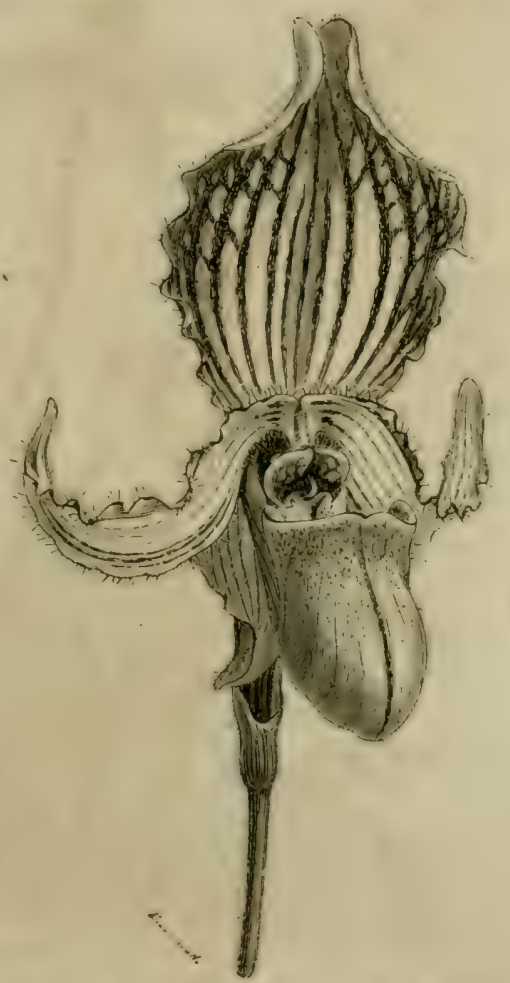

Grav. 8\%. - Fleur du c ypripedium rairiesnum de grandeur naturclle.

neux en mouillant légèrement les stigmates qui ne seraient pas sulfisamment humectés avee un peu d'ean mielles, opération qui m’a réussi daus la fécondation artificielle d'esprees etrangères à cette lamille (grav. 88, 89, 90).

I'ai dit un pen phus haut que les fleurs des Orchideres duraient 
longtemps; c'est en effet ce qui a lieu le plus ordinairement, mais il y a des exceptions à cette règle, et dans ce groupe, comme dans plusieurs autres, on rencontre des fleurs météoriques. On doit, par conséquent, pour ces dernières, pratiquer la fécondation dès le commencement de l'épanouissement.

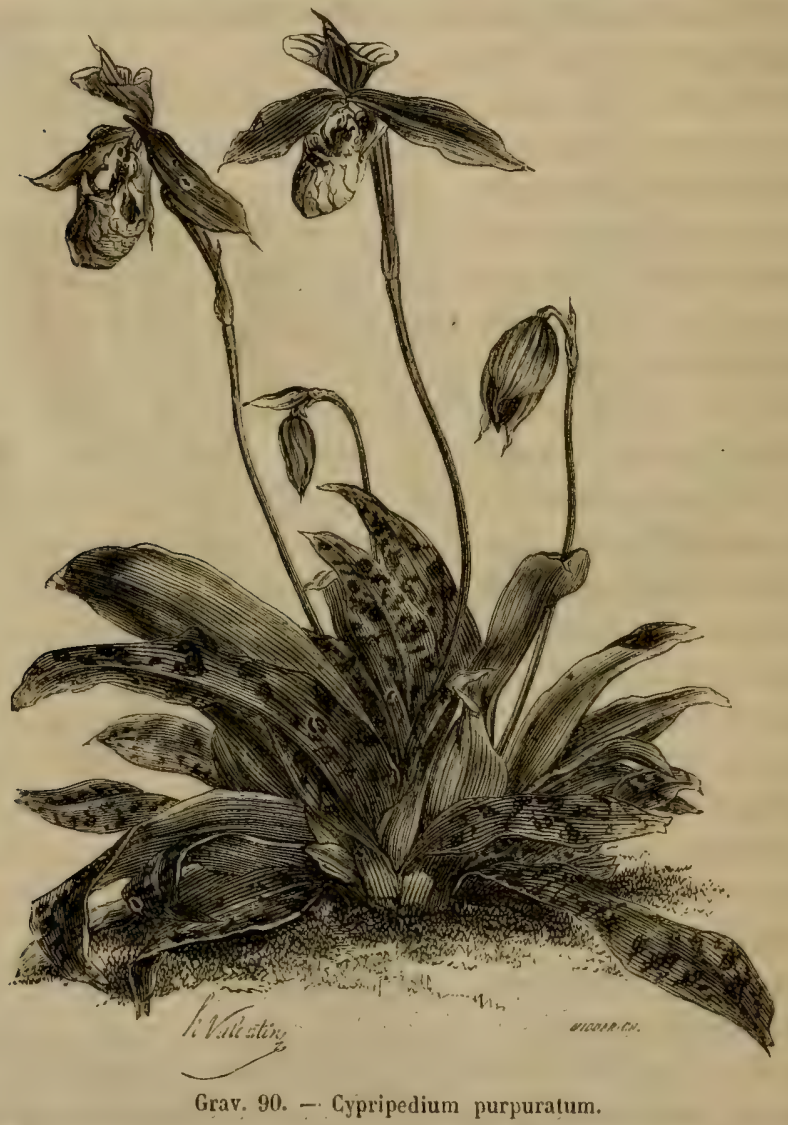

Malgré ce que nous venons de dire de la rareté des hybrides dans les Orchidées, il ne faudrait pas croire que ces hybrides sont inconnus.

On trouve dans le Garlener's Chronicle la citation d'un 
Culanthe que M. Lindley a nonmé C. Dominii, du nom de son producteur, el qui serait intermédiaire entre le $C$. Masuca et $C$. furcuta. M. Dominy aurait aussi obtenu des hybrides de Cattleya. Dans ce mème article, M. Lindley soupçonne l'Aërides maculosum d'être un hybride des $A$. affine et $A$. crispum. Il cite un Saccolobium qui pourrait résulter du croisement du S. gutlatum et du S. Blumei.

Nos Orchis indigènes offrent aussi quelquefois des preuves de ces fécondations croisées tout accidentelles, mais que nous pourrions rendre beancoup plus fréquentes; tels seraient les 0. morio-papilionacea et purpuren-militaris Timbal-Lagrave, 0. simio-militeris Gren. et Godr., 0. simio-purpureu Weddell.

En 1841, M. Weddell avait déjà rencontré des hybrides entre l'Aceras antropophroa et l'Orchis galeata, et vice versî; et, en 1852 , il a revu et analrsé ce phénomène au point de ne laisser plus de doute sur cette existence non prourée, il est rrai, par une opération directe, mais par les analogies et les. passages d'un genre ì un autre genre. Il est vrai de dire que le gemre Acerus et le genre Orchis ne sont différents que dans notre esprit et nos classements de convention, et que, dans la nature, il y a là tout au plus un seul et même type; mais, à moins d'admettre que les espèces ne sont pas fixes et qu'elles passent de l'une à l'uutre, il faut reconnaître dans les cas invoqués une hybridation si probable, qu'elle peut être regardée comme réelle. II. Rœper, en outre, a déjà observé que l'Orchis fusca et l'Orchis militaris peuvent s'hy̧brider entre cux et produire des plantes intermédiaires.

Ces faits sont de nature à engager nos nombreux amateurs d'Orchidées de serre à tenter des hybridations entre espèces d'un genre très-naturel ou entre des genres si voisins quils peuvent être considérés comme n'en faisant qu'un, selon les vues de la nature et non celles de nos conventions arbitraires (C.. Morren, Belgique.horticole, t. III, p. 572).

Des observations toutes récentes, recueillies et discutées avec impartialité dians un mémoire sur le polymorphisme, que 
M. Duchartre vient de publier dans le Bulletin de la Société hotanique (séance du 28 février 1862), viennent à l'appui de la création des hybrides dans les Orchidées. Il arrive tout à coup dans ces plantes des apparitions, sur un mème pied, de fleurs tout à fait différentes par la forme et les coloris. Ce fait n’appartient pas spécialement à la famille des Orchidées, puisque l'on a vu plusieurs fois une branche de Brugnon se développer sur un Pêcher; mais cette observation a plus de valeur peut-être dans cette belle famille, à peine sortie des sites sauvages de ses forêts, que sur un arbre depuis longtemps civilisé et soumis à nos caprices.

Après avoir rappelé que les différents genres d'Orchidées, très-distincts aux yeux des botanistes, tels que les Catasetum, les Myanthus, les Monacanthus, produisaient quelquefois, sur le même pied, des fleurs qui pouvaient appartenir à l'un et à l'autre, comme Lambert l'avait déjà fait remarquer, en 1856 , à la Société linnéenne de Londres, M. Duchartre rapporte l'expérience de M. Bach. "Ce zélé collecteur d'Orchidées, ayant semé, à la Guyane britannique, des graines de Monacamthus viridis sur un tronc en décomposition, en vit naitre plusieurs plantes, dont l'une produisit une hampe chargée de fleurs de. Catusetum tridentatum; M. Schomburgk a vu cette curieuse plante, et il affirme avoir rencontré lui-même des pieds sur lesquels la même hampe portait des fleurs de Monacanthus et de Catasetum, tandis que celui qui fait l'objet principal de sa note réunissait l'organisation florale du Monacanthus à celle du Myanthus. Une conséquence découle nettement de cette instructive observation, et l'auteur n'a pas hésité à la déduire : e'est que les trois genres Catasetum L. C. Richard, Monacanlhus Lindley et Myanthus Lindley n'en forment, en réalité, qu'un seul, dont les espèces peuvent se présenter sous trois formes, le plus souvent distinctes, rarement et accidentellement réunies. Quant à la question de savoir laquelle de ces trois lormes peut être considérée comme fondamentale, M. Schomburgck ne la résout pas définitivement, mais il signale 
un fait qui semble de nature à en faeiliter la solution : cंe.t que plusieurs centaines de pieds, qu'il a olservés croissant spontanément à la Guyane, ne lui ont jamais montré une seule capsule, tandis que, au même lieu, tous les Monacunthus viridis l'étonnaient par leurs fruits gigantesques. On pourrait à la rigueur accepter l'opinion de II. Darwin, qui consiste i regarder ces trois sortes de fleurs comme jount les rôles différents de mâles, de femelles et d'hermaphrodites; mais les nombreus exemples cités par les botanistes et rappelés par M. Duchartre laissent peu de probabilité à cette ingénieuse opinion.

Le principal sujet de la communication de MI. Duchartre est le Vanda Lowii Lindley, originaire des forêts de Sumatra, et qui parait présenter normalement, et non accidentellement, comme les Orchidées précédentes, deux sortes de fleurs qui n'ont entre elles aucune ressemblance.

"La constance qui parait exister dans le dimorphisme des fleurs du Vanda Lowii, dit MI. Duchartre, donne à cette curieuse particularité un intérèt bien supérieur à celui qu' offrent les variations du Catasetum et du Cynoches. En effet, celles-ci étant accidentelles, rares même, rentrent simplement dans la catégorie de ces jeux de la nature qui échappent à toute règle et se refusent à toute explication; au contraire, la production de deux sortes de fleurs par notre Vanda étant un fait constant, parait dépendre de la constitution même de cette plante, ct l'on se sent amené, presque malgré soi, à essarer de l'expliquer. Or, si l'on songe an rôle important que jouent les insectes dans la fécondation des Orchidées spontanées, et an Iransport qu'ils doivent opérer fréquemment des masses polliniques d'une espèce sur le stigmate d'espèces diffërentes; si l'on se rappelle que l'hybridation artificielle parait être facile chez ces plantes, puisque le petit nombre d'essais de ce genre qui ont été faits jusqu'à ce jour ont donné de's résultats heureux; si l'on réfléchit à la difficulté qu'on épronse souvent pour limiter les especes de cette famille, pent-ètre en viemdra-t-on a penser que diverses Orehidées, régardíes conme espèces dis- 
tinctes, pourraient bien n'être que des hybrides, que le Vanda Lowii particulièrement pourrait être issu de l'union des deux espèces différentes, et que ses deux sortes de fleurs, venant sur un même pédoncule, pourraient n'être qu'un nouvel exemple de la remarquable dissociation qu'offre habituellement aussi le Cytisus Adami et qui a été si bien étudiée par M. A. Braun dans son beau mémoire sur le rajeunissement de la nature. ”

Que ces anomalies soient le fait de l'hybridation ou de la simple variation, elles peuvent, dans tous les cas, être considérées comme un des agents les plus utiles pour combattre la spéciomanie qui règne en ce moment d'une manière épidémique chez une certaine classe de botanistes.

Nous renvoyons en outre, au sujet de la fécondation des 0rchidées, à ce que nous avons dit à la page 76 de ce livre sur la fécondation indirecte.

Genre Vanille. - Vanilla.

Ce genre est caractérisé par un ovaire oblong, cylindrique, surmonté d'un style court, terminé par un stigmate concave adhérent au labelle, et par deux anthères ovales insérées sur le style.

Je n'ai pas eu occasion de voir la Vanille en fleur; mais on sait, depuis longtemps, que la fécondation artificielle peut être pratiquée sur ces plantes avec un grand succès, et l'on doit ì Ch. Morren, l'un des hommes dont la Belgique ne doit citer le nom qu'avec orgueil, des observations du plus haut intérèt sur la fructification de la Vanille. Le premier, il a montré des fruits mûrs de cette intéressante Orchidée, et j’ai vu moi-même en 1844, dans sa serre à Liége, IIn pied de Vanille couvert d'un grand nombre de fruits.

Les résultats obtenus par Morren ont déterminé un grand nombre d'horticulteurs à tenter la fécondation sur des plantes de serre qui ne fructifiaient jamais.

C'est sur le Vanilla planifolia que Horren a opéré. Le savant directeur des serres du Muséum, Neumanu, a également fé- 
condé les fleurs du Vunilla aromatica. "Les cinq divisions supérieures du périanthe, dit-il, sont un pecu charnues, ovales, lancéolées, légèrement concaves, et d'un vert très-jaune et comme verni. Le labelle est trilobé, les deux lobes latéraux recourbés en fortme de gouttière, à limbe évasé, un peu échancré, le lobe du milieu rélléchi en dehors, un peu bouclé au centre, à limbe marqué de points proiminents, plus jaunes que le fond, qui est d'un vert blanchatre mat; la colome est blanche et s'élére entre les deux courbures du labelle. Ces fleurs s'ourrent peu, et il n'est pas facile d'opérer la fécondation artificielle, si on n'emploie de petites pinces à cet effet. Le stigmate étant recourbé, on éprouve quelques difficultés pour y appliquer le pollen. A cette occasion jai fait une remarque assez intéressante. Sur les onze fleur's produites par notre Vanille, quatre n'ont pas été fécondées, quatre l'ont été après midi, et trois le matin arant neuf heures. Il n'y a que ces trois dernières qui conserveront leurs fruits ou siliques; on voit, d'après cela, qu'il n'y a pas à espérer que la Vanille se féconde d'elle-même dans nos serres. ")

La durée des fleurs, qui est tout au plus d'un jour, explique la nécessité de procéder de suite à l'imprégnation du stigmate.

La présence de la liqueur miellée indique, selon Morren, la nubilité du stigmate, ct ce savant n’a pas hésité à enlever quelquelois le tablier qui cache le stigmate, sans que pour cela la fécondation en fùt moins assurée. Bien des fois j’ai pratiqué sur d'autres plantes de semblables mutilations sur le périgone, sans que l'acte mystérieux de la fécondation en ait souflert.

\section{Genre Orehis. - Orchis.}

Nous appellerons un instant l'attention des horticulteurs sur le genre nombreux des Orchis. Leur culture n'est pas difficile, et l'abondance de la plupart d'entre eux dans les prairies, sur les pelouses des montagnes ou it l'ombre de nos forèts, est peutitre lis seule canse qui ait empèché d'en faire une culture spé- 
ciale. Mais si les Orchis se maintiennent facilement dans nos jardins, quand l'exposition leur convient, ils ne s'y propagent guère. Et, en effet, des deux tubercules d'un Orchis, l'un se flétrit en donnant naissance à la fleur, l'autre est en réserve pour l'année qui doit suivre. Ce n'èst done que par les graines que ces plantes peuvent se multiplier, et ces graines, dont on ne connait pas encore bien la culture, sont souvent infécondes.

La première condition à remplir serait done de féconder artificiellement les Orchis, en enlevant avec la pointe d'une aiguille les deux petites masses polliniques, enfermées dans les deux poches de l'anthère placée sur le pistil, et en posant ce pollen sur le stigmate visqueux qui est situé au-dessous d'elles. Cette opération pourrait se faire dans les lieux mêmes où naissent les Orchis, dans les prés où ils abondent; il suffirait de marquer les pieds.

Si on parvenait à faire développer ces graines, qui, par ce moyen, sont fertiles, rien n'empècherait de tenter l'hybridation sur toutes ces belles plantes. Plusieurs sont déjà très-sujettes ì varier. J'ai trouvé dans les bois l'O. mascula à fleurs blanches, pourpres, roses, carnées, carminées, etc., le maculata à fleurs blanches, lilacées, violettes, maculées et variées de pourpre en admirables dessins. Le latifolia offre tout autant de variétés; les conopsea, odoratissima, militaris, nigra, pyramidalis, globosa, galeatı, ustulata, etc., varient aussi en couleurs, mais moins que le sambucina à fleurs jaunes ou incarnates. Le bel 0 . fusca varierait sans doute aussi par semis, et je ne doute - pas que l'on obtienne non-seulement des hybrides entre variétés, mais entre espèces, et des planches d'Orchis indigènes variés, élevant leurs grappes magnifiques et de longue duréc, produiraient dans les parterres les plus merveilleux eftets. J'ai souvent cultivé des Orchis dont l'épi avait plus de deux déeimètres de longueur.

Nous avons cité un peu plus haut, en parlant des Orchidées co général, plusicurs exemples d'Orchis hybrides recueillis pay M. Weddell et par N. Timbal-Lagrave. 


\section{RAMLLE DES IRIDÉES.}

\section{Genre Iris. - Iris.}

Les belles lieurs de l'Iris ont une organisation toute particuliver ; elles ont un ovaire infère, surmonté de trois pieces qui remplissent les fonctions de styles, ou qui sont, à proprement parler, des styles ailés. A leur partie supéricure, on remarque une petite duplicature ou une petite lère qui, vue i la loupe, est formée d'un tissu cellulaire tres-apparent et qui s'allonge presque sous forme de papilles. Les étamines, au nombre de trois, ont leurs antheres a la hauteur des stigmates, mais elles s'ourrent en dehors, et leur pollen tombe naturellement sur des poils granduleux qui courrent la partie médiane des trois divisions du périgone qui correspondent aux anthères et aux stigmates. Il est done presque impossible que la fécondation s'opere dircetement; mais souvent, lorsque la fleur se flétrit, les téguments, en se roulant, s'appliquent contre les stigmates et leur transmettent le pollen qu'ils ont resu.

Les insectes peuvent aussi contribuer à l'imprégnation; mais, ce qu'il y a de certain, e'est que très-souvent la fleur des Iris nंest pas fécondéc.

Comme nous l'arons déjà dit ailleurs, plus la fleur a de difficulté à se léconder naturellement, plus l'hybridation devient licile; et c'est, en effet, ce qui a lieu pour les Iris. On retranche, le matin, avant l'épanouissement complet, les anthères que l'on enlive avec: les doigts ou mieux avec de petites pinces, et, dans le courant de la journée, ou le lendemain seulenent, on pose le pullen au pinceau, en ayant soin de l'appliquer non-seulement sur les stigmates, ce qui sulfit cependant, mais encore, et prour plus de certitude, d'en poser sur les poils ou les cellules lubuleuses des trois divisions qui, par leur enroulement, doivent ensuite toucher le stignate. Qunud l'imprégnation a eu lieu sur une ou deus lleurs, il faut supprimer les antres, car beancoup 
d'Iris doment difficilement leurs graines, et le germanica est de ce nombre. Sur plus de cinq cents fleurs de cet Iris, sept à huit seulement ont donné des graines, et celles-là seules avaieut été fécondées.

Les Iris ont la plus grande tendance à varier, même sans lécondation croisée. De nombrouses et très-intéressantes expériences, faites par un horticulteur distinguẻ, M. de Bure, lui ont prouvé que plusieurs espèces bien établies et admises par tous les botanistes n'étaient que de simples variétés. Ainsi, dix-sept plantes provenaut d'un semis d'I. squalens ont tellement varié, qu'aucune n'a représenté exactement son type, et deux de ces plantes étaient des variegata. Un autre semis de la grande variété de ce même squalens a donné vingt-sept plantes fleuries, parmi lesquelles onze s'éloignaient plus ou moins du type, et seize étaient encore des variegata. Des semis de cetle dernière espèce ont souvent donné des versicolor, résultat que j’ai également obtenu. Le sambucina a peu varié entre les mains de II. de Bure, tandis que le variegata lui a donné des sambucina et un pallida, et jamais de versicolor. Le Svertii lui a produit des variétés entièrement nouvelles, et l'Tris de Bure, issu du plicata, n'a pas donné, sur cent quarante-quatre pieds, une seule plante pareille à ses ascendants. Il y avait encore des squalens, un pallida et deux variegata. Que l'on juge, d'après ces faits, des résultats obtenus par l'hybridation, et si l'on en doute, si l'on ne croit pas au succès de cette opération sur les Iris, que l'on aille voir les admirables plantes de M. Lémon.

Il est difficile, en effet, de voir un genre plus modifié que ne l'ont été les Iris par cet habile horticulteur. Les variegata, versicolor, squalens, sambucina, flavescens, plicata, pallida se sont confondus en un scul type excessivement rarié. Le germanica ne lui a pas donné de fruit.

J'ai obtenu de croisements entre les diverses variétés d'l. d'Espagne des graines qui ont admirablement prospéré. Les Iris d'Angleterre se croisent entre eux avec la plus grande ficilité. L'I. pseudoacorus ou lris jaune des marais, qui graine 
FAMILLE IUES IRIDEES.

367

si abondamment, poura tris-probablement itre hybridé par le germanica el plusieurs autres, et donner des plantes incon-

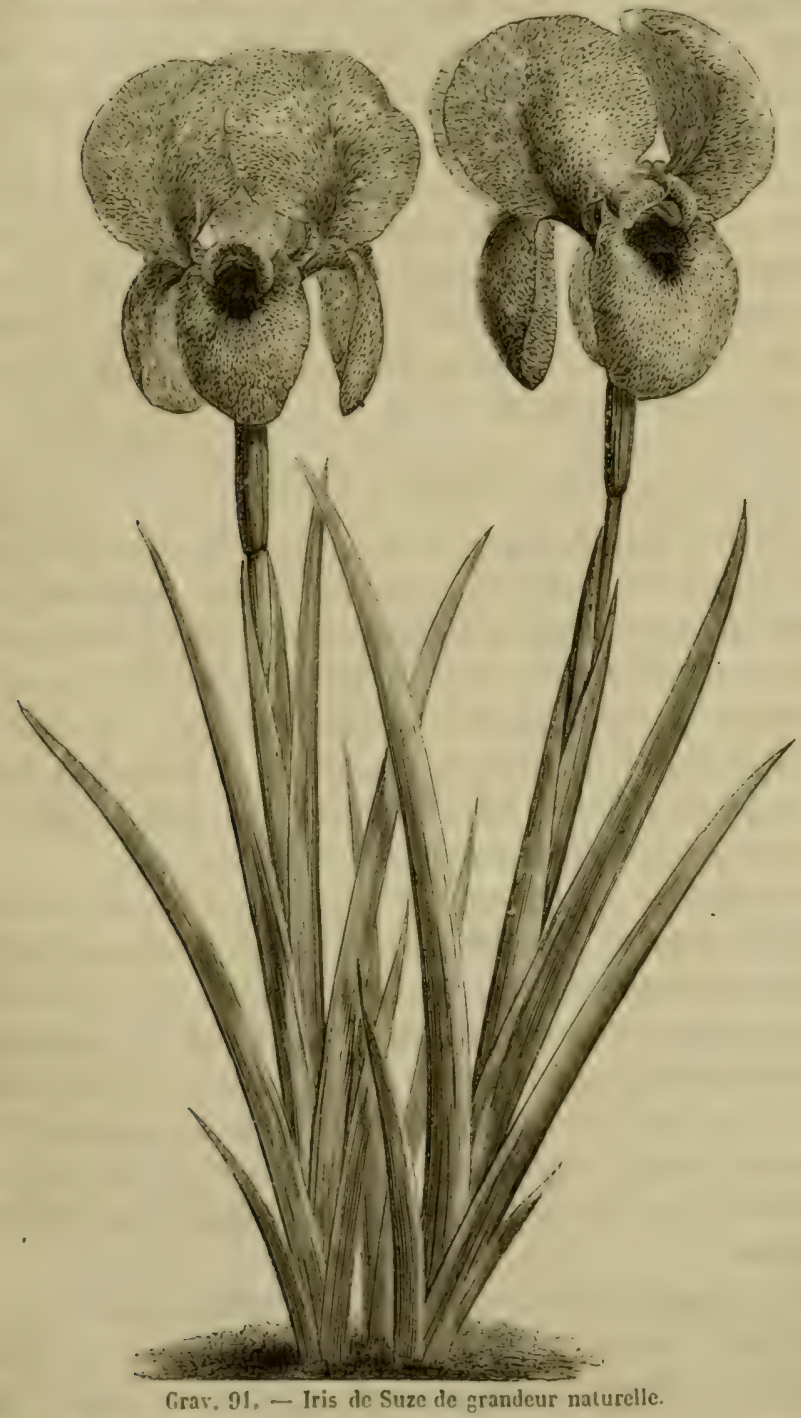

nues. Mais que ne doit-on pas altendre des horticulleurs qui, 
placés sous un climat convenable, pourront hybrider le magntfique Iris de Suze, qui fructifie si bien à Hyères, et que MJ. IIenon a vu donner des graines fertiles à Lyon même et en flein champ, ou de ceux qui, conservant le pollen du gracicux Iris de Perse, parviendront à en imprégner les Iris d'Espagne et ceux d'Angleterre (grav. 91). L'Iris est, comme la Rose et le Dahlia, comme l'Auricule et la Pc ‘sée, un de ces genres inépuisables qui répondent toujours à l'intelligence de leur directeur.

Toutes les belles variétés obtenues de semis par M. Lémon proviennent des $I$. sambucina, squalens, flavescens, plicala, variegata et pallida, et non de l'I. germanica, dont M. Lémon n'a pu obtenir de graines.

\section{Genre Glaieul. - Gladiolus.}

On trouve, dans le Glaieul comme dans l'Tris, trois étamines, dont les anthères sont aussi extrorses. Le style, assez long, se termine par trois stigmates frangés qui s'écartent à l'époque de l'anthèse et qui, très-souvent, ne sont pas fécondés à cause de la position des étamines. Quand la fécondation a lieu naturellement et sans l'aide des insectes, c'est à la défloraison, lorsque le périgone se tord en se desséchant, et applique ainsi le stigmate contre les anthères.

En enlevant, comme pour les Iris, les anthères au moment même où la fleur s'épanouit, on reste maître de choisir le père du porte-graine, et il est bien peu de plantes qui s'hybrident aussi facilement que celles-ci. Déjà on a obtenu de charmantes variétés, mais il faut avoir soin de féconder les deux ou trois premières fleurs de l'épi, c'est-à-dire celles qui s'ouvreni les premières, et de couper le reste, ou, si l'on ne veut pas sacrifier leur belle floraison, les enlever an moins dès qu'elles se flétrissent.

II y a longtemps déjà que M. Souchet a obtenu des lyybrides entre les G. cardinalis, pulcherrimus et blandus, sans l'intermédiaire des $G$. psittacimus et yandawensis, car ce dernicr ćlait encore inconnu. 
Les G. psiltacinus, cardinulis, floribundus et rumosus sont les types de nos admirables collections, et déjà, il y a vingtcimy ans, M. Schneevogt, d'Hlaarlem, asait obtenu de semis et répandu dans le commerce des formes assez nombreuses du G. ramosus, et notamment la variété Gloria mundi, encore remarquable par la grandeur de ses fleurs.

Quelque temps après naquit à Enghien, chez le duc d'Aremberg, ce beau G. yandavensis, proparé par M. Van Houlte en 18 1, heureuse alliance du G. psittucinus et du G. cardinulis.

Le G. yandarensis, qui a produit dès son entrée dans le monde horlicole une si vive émotion, serait une des souches de ces plantes éclatantes encore si récentes dans nos jardins (grav. 92).

D'un autre còté, le G. floribundus, fécondé par le G. ramosus, a donné le G. Leopoldii et d'autres encore qui sont devenus la souche de nouveaux types aujourd'hui multipliés it l'infini.

William Ilerbert, qui s'est beaucoup ocenpé de l'hybridation des Glaïeuls, dit que le G. psittacinus s'est refusé à toute espèce d'union hybride, quoique les tentatives aient été trèsnombreuses et réitérées dans des circonstances très-différentes. Nous ne voyons guère cependant que le G. psittacimus, qui ait pu donner ces tons orangés et écarlates aux variétés nomIreuses du G. yandarensis, et surtout cette couleur jaune pur de l'hybride Gindavensis citrimus obtenu par M. Lemonnier, de Lille.

Après la création du Gladiolus yundarensis, que nous considérons conme issu du psittacinus, cet hybride a été fécondé par les G. floribundus, curdinalis et ramosus. Il a ainsi donné naissance à une immense lignée qui étale aujourd'hui daus nos jardins ses flanmes orangées ou ses étendards ornés des plus vives couleurs.

C'est VI. Souchet, de liontainehleau qui a produit les plus baraux et les plus remarquables hybrides de ces beanx Glaieuts 
déjả hylırides cux-mêmes, et qui les a revètus des nuances les plus pures et les plus variées, des inacules les plus éclatantes et les micux limitées.

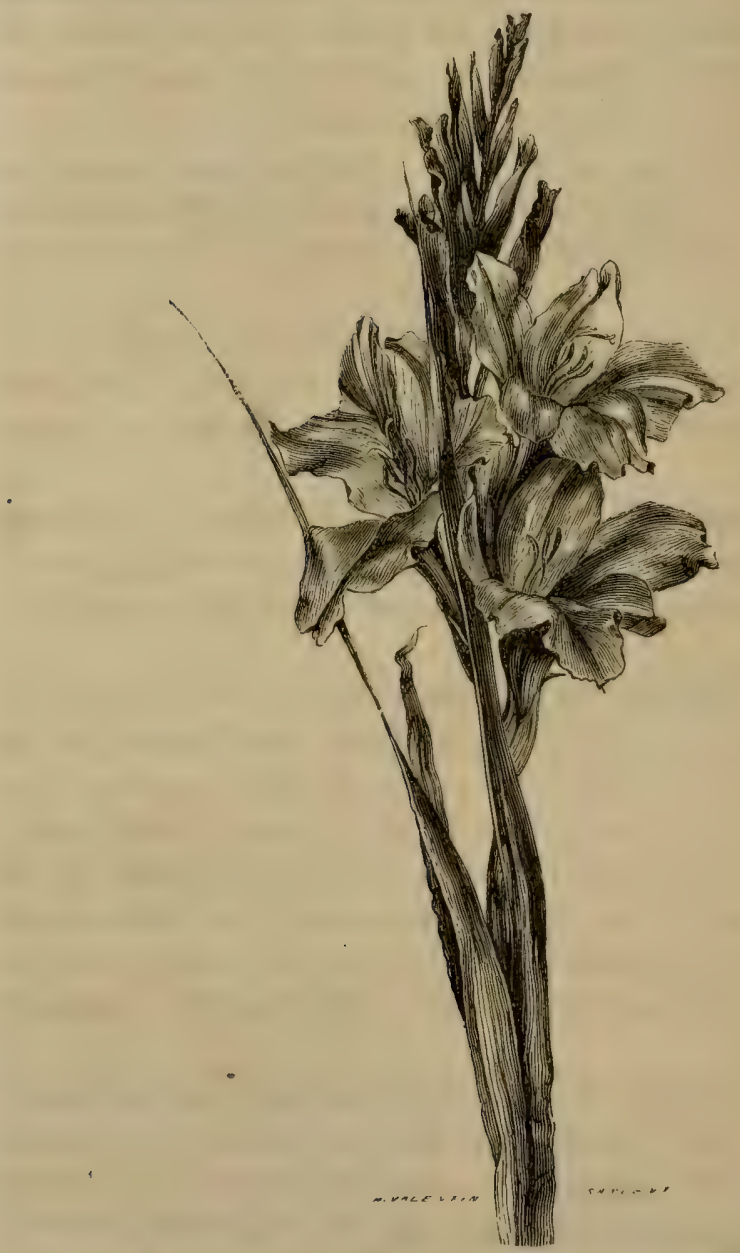

Grav. 92. - Glaïeul hybride de Gandavensis, comtesse de Saint-Iarceau.

Nous pourrions citer comme viriétés les plus remarquables les nouveaux gains de MM. Domage, Mallet, et quelques variétés que nous avous vues en 1860 à l'Exposition de Valognes. 
Il est peou de plantes daus lesquelles les véritables hygbrides, entre espèces tri s-distinctes, se reproduisent avec autant de facilité que dans les Glaïeuls. Les espèces les plus disparates, croisées entre elles, produisent des graines fertiles qui elles-mèmes se reproduisent indéfiniment. A moins de dire que tous les Giaieuls ne constituent qu'une seule espèce, il fiut admettre dans ce genre la fertilité des hybrides.

C'est encore à l'établissement Van Ifoutle que sont dus de noureaux Glaieuls constituant un type très-différent, mais liybride encore de plusieurs espèces distinctes. Ce sont les Glä̈enls nuins, issus du croisement du G. curdinalis avec les G. vemustus, trimaculutus, tristis, etc. Ces plantes s'éloignent des autres Glaieuls par la forme et la différence de coloration de leurs fleurs, ainsi que par l'époque à laquelle elles fleurissent. Dès le mois de juillet, elles ouvrent la série des floraisons. La beauté de leur coloris surpasse même celle du G. yandavensis, et les macules que portent les trois sépales inférieurs sont remarquables par leur couleur généralement claire, qu'entoure toujours une teinte différente, vive et foncée. De plus, les couleurs de ces fleurs et les desseins qu elles forment varient à l'infini.

Voili done encore une nouvelle mine à exploiter, car la facilité avec laquelle les Glaieuls s'hybrident doit nous faire supposer la possibilité du croisement des Glaieuls nains avec les variétés issues du $G$. yandavensis.

du reste, une personne qui réunirait la collection des espices connues de ce beau genre et qui s'occuperait sérieusement de leur union, arriverait, nous n'en doutons pas, à d'admirables résultats.

Pour laire remarquer les progrès de l'horticulture depuis que l'on s'oceupe sérieusement d'hybridations, nous rapporterons ce que disait, en 185̄e, M. Jarques, au sujet du G. psittucinus, qui est un des pàles anceitres de nos éclatants Glaïeuls.

" Je dois cette belle plante à MIII. Lalay et Lémon, qui tous deux la cultivent et l'ont introduite dans le commerce. On lit 
cultive comme toutes les Liliacées du Cap, soit en pots, soit sous chấssis... Elle se multiplie de caïeux et par les graines, qui quelquefois mûrissent, ce qui peut faire espérer d'en avoir des variétés par la suite, et fait ainsi présumer que c'est une espèce franche, les hybrides ne donnant que rarement des graines fertiles. ') (Amnales de Flore et de Pomone, t. II, p. 34.)

Et, en 1858, M. Pépin écrivait dans le mème journal : ( M. Rilkogel, horticulteur distingué, cultive une plante magnifique qu'il vient d'apporter de la Belgique à Paris. C'est le G. ramosus. Je ne connais encore que ce cultivateur qui possède cette plante; il la multiplie et ne tardera pas à la répandre dans le commerce. .)

Genre Ixia. - Ixia.

Presque toutes les Iridées sont des plantes de collection, et les Ixia ne le cèdent, sous ce rapport, à aucun autre genre. $\mathrm{Ce}$ sont des Iris et des Glaïeuls en miniature. Ils exigent un peu - plus de soin, mais fleurissent abondamment dans nos serres et sous nos chàssis. Leurs étamines peuvent être enlevées aussi ficilement que celles des Glaïeuls, et le pollen peut ètre posé au pinceau sur les trois stigmates entiers ou bifides qui occupent le centre de la fleur. Il faut toutefois remarquer que les Ixia sont pour la plupart météoriques, s'ouvrent à des heures fises, et qu'il faut choisir ce moment pour pratiquer la fécondation artificielle. Les diverses variétés du crocata, dont on a fait le grenre Babiana, peuvent se croiser; le flexuosa, le lilacina, le rosea, le tricolor, et surtout le joli et curieux viridiflora, méritent d'être étudiés avec soin sous le point de vue de l'hybridation.

Genre Antholize. - Antholiza.

Nous devons renvojer pour les Antholizes a ce que nous arons dit sur les Glaieuls. Ces deux genres ont beaucoup de 
rapport, mais, en général, les Glaieuls sont plus élégants, plus variés, et peut-être d'une floraison plus certaine que les Antholizes. Du reste, il y a tant d'aualogie cutre ces deux gemres, qu'il y aurait peut-ètre possibilité d'obtenir des hỵbrides entre. eux.

\section{Genre Safran. - Crocus.}

Ice Safrans sont un des plus beaux ornements de la riche famille des Iridées. Ils ont trois étamines à anthères extrorses, ct un style très-long terminé par des stigmates découpés ou frangés. La fécondation s'opère de honne heure, quelquefois mêrie avant la floraison; en sorte que si l'on voulait hybridrer, il ne faudrait pas attendre l'épanouissement, mais écarter les sépales fermés pour enlever les anthères. Le lendemain ou le surlendemain on appliquerait le pollen, en ayant soin de tenir Ioujours les Crocus sous cloche, à cause des nuits froides du printemps et des insectes qui viennent à chaque instant butiner sur les premières fleurs que le soleil fait éclore. Si l'on voit sortir plusieurs fleurs du même tube, c'est-à-dire de la même toufle de feuilles, il ne faut en féconder qu'une et couper les autres à mesure qu'elles paraissent. Une fois la fleur flétrie, on enleve la cloche, l'oviire reste caché sous le sol, et plus tard seulement il s'élève changé en capsule qu'il faut chercher à la surface de la terre et qui reste quelquefois mème un peu enterrée.

Les Crocus, à l'exception du sativus, fleurissent an printemps. Le's espèces cultivées, telles que les vermus, aureus, v'ariégulus, susiamus, ont déjà domé de nombreuses variétés; et, romme elles se ressemblent assez pour supposer qu'elles pourraient se croiser, il sernit i désirer que l'on fasse des essais d'hybridation qui dommeraient certainement des plantes nouvalles. Les Crocus arriveront ì être panachés comme les Tulipes, of le vernus est loin davoir atteint ses limites de variation.

Lianrinulure réclame dans ce genre le Crocus sulivus, qui 
fleurit en automne et dont les longs stigmates offrent une si belle couleur orangée. Il serait peut-ètre possible en Espagne, ou même dans le midi de la France, de croiser cette espèce avec les variétés du vernus, et si l’on n'arrivait pas, par ce moyen, à avoir une plante utile, on aurait certainement, si l'imprégnation pouvait avoir lieu, des variétés ornementales trèsdifférentes de celles que nous connaissons.

\section{Genre Tigridie. - Tigridia.}

Une seule colonne placée au centre de la fleur porte, comme dans les Orchidées, les étamines et le pistil, à l'exception de l'ovaire. Les anthères, au nombre de trois, sont sessiles au sommet de la colonne et répandent leur pollen en dehors. Lce stigmate, placé au milieu des anthères, est à trois lobes bifides, ce qui lui donne six divisions. La fécondation a rarement lieu naturellement, mais on peut l'opérer, soit avec le propre pollen de la plante, soit arec celui d'une autre espèce ou varièté, en choisissant le $T$. pavoria pour porte-graine.

Ces plantes sont météoriques, elles s'ouvrent dans la matinée et en plein soleil. Il faut être attentif à l'heure de l'épanouissement pour enlever les étamines et féconder une heure après.

Le T. conchiflora, apporté en 1805 du Merique, n'est peutêtre qu'une variété du pavonia. On a donné comme hybride un T. aurantiaca. Voilà du moins ce qu'on lit daus un rapport fait par M. Moquin-Tandon à l'Académie de Toulouse : "Cette Tigridie a été obtenue par M. Goudet, archiviste de la mairie. Elle a pour père le $T$. pavonia et pour mère le $T$. conchiflora. Sa fleur n'est pas rouge ponceau comme la première, ni jaune pâle comme la seconde, mais d'une belle couleur orangée. Son oignon, qui est blanc, ressemble à celui du T. conchiflor a. ")

“ La f'oraison de l'hybride s'est montrée un peu plus tardive que celle des deux ascendants, et la fleur, qui dure quelques heures de plus, se trouve un peu plus grande. Les capsules, au lien d'offrir une soixantaine de graines, n'en ont donné que 
deux ou trois, presque toutes infécondes. Cependant, après plusieurs tentatives infructucuses, M. Goudet est parrenu it faire grermer quelques-mes de ces grames qui ont propagé la plante hibride avec tous ses caractires. ) (Annules de Flore et Pomone, 1859-1840, p. 26.)

II. Jarues a gagné, en 1840, deux variétés nouvelles, sous les noms de speciosa el intermedia. Le T. enccinea et l'Herbertii pourraient anssi itre hyluridés. Le calestis, qui est un Huricu, en diffëre darantage, mais il subirait peut-ètre aussi le croisenent et pourrait servir de porte-graine. Je l'ai fécondé avee son propre pollen, et il m'a donné des graines en abondance. Au point où l’on est maintenant, il niest pas douteux que les varietés ne se multiplient à l'infini. Des résultats admirables attendent les amateurs gui voudront s'occiper de res helles Iridées.

\section{FMMILLE DES AMARYLLIDEES.}

\section{Genre Crinum. - Crimum.}

Ces plantes ont plus que de la beauté, c'est de la magnificence dans le port, dans le feuillage et dans la fleur. Six grandes étamines sont placées dans l'atmosphère embaumée de la corolle, et l'on peut les enlever avec les doigts dès que l'épanouissement commence. Il faut aroir soin de supprimer plusieurs fleurs de l'ombelle, ou plutôt, pour jouir de la floraison, on peut leur permettre de s'épanouir en coupant avec l'ongle l'exIrémité du stigmate, et ne fécondant au pinceau que les deux premières fleurs qui s'ouvrent.

Les especes de ce beau genre sont assez nombreuses et ont entre elles assez de rapport pour espérer l'hybridation. Il est probable du moins que l'on aurait chance de sucès en lit tenlant entre les $C$. asiaticum, latifolium, erubescens, americanum, procerum, pedunculatum, aménum, rommelyn, erubescens, cruentum, auyustum, scabrum, amabile, rariabile, 
roseum; ces derniers surtout sont très-heaux et très-rustiques.

Il arrive dans ces sortes de plantes, comme dans plusieurs genres des Liliacées et des Amaryllidées, que les capsules, an lieu de graines, donnent des bulbilles. Il serait curieux de rechercher si ce n'est pas le manque de fécondation qui apporte cette modification au mode de reproduction, et si ce n'est pas là encore une de ces ressources admirables que déploie la nature pour conserver les espèces.

J'ai vu quelquefois, dans plusieurs Liliacées, des graines se reproduire comme à l'ordinaire, mais germer avant d'être mûres et remplir les capsules de bulbilles qui montraient déjà leurs premières feuilles. Ce mode singulier de développenent n'aurait-il pas lieu pour les Crimum, et, dans le cas d'une hybridation, les bulbilles reproduiraient-elles la plante mère senlement ou une variété intermédiaire aux deux ascendants? Ces curieux problemes ne pourront être résolus que par des amateurs favorisés de la fortune qui peuvent sacrifier le termps et l'argent à leurs cultures de prédilection, et qui voudraient s'occuper spécialement d'un des plus beaux genres du règne végétal.

\section{Genre Iremanthe. - IIæmanthus.}

Les Hrmanthus sont, comme les Crinum, des plantes de scrre chaude, remarquables aussi par leurs fleurs éclatantes et leurs fruits en forme de baie. Les fleurs, très-nombreuses, sont rassemblées en tête et chacune d'elles offre six étamines, un style simple et un stigmate un peu trilobé.

Quoique les Hæmanthus aient été partagrés en deux sections, sclon que leurs feuilles sont glabres ou ciliées sur les bords, il est probable que l'hybridation s'opérerait entre leurs diverses espèces, dont plusieurs ne sont peut-être déjà que des hybrides $0 u$ des variétés. Les $H$. coccineus, maculatus, ciliaris, carinaIns, multiflorus, pubescens, tigrinus, puniceus pourraient sans loute se croiser, surtout en prenant pour porte-graine le dernier, qui fructifie assez souvent dams nos serres. Le premirr, 
all contraire, dont les bares arrivent parfors aussi it une bonne maturité, ne donne cependant que des graines stérilis.

Si on ne parvenait pas i hybrider les Hemanthus, on aurait tou-

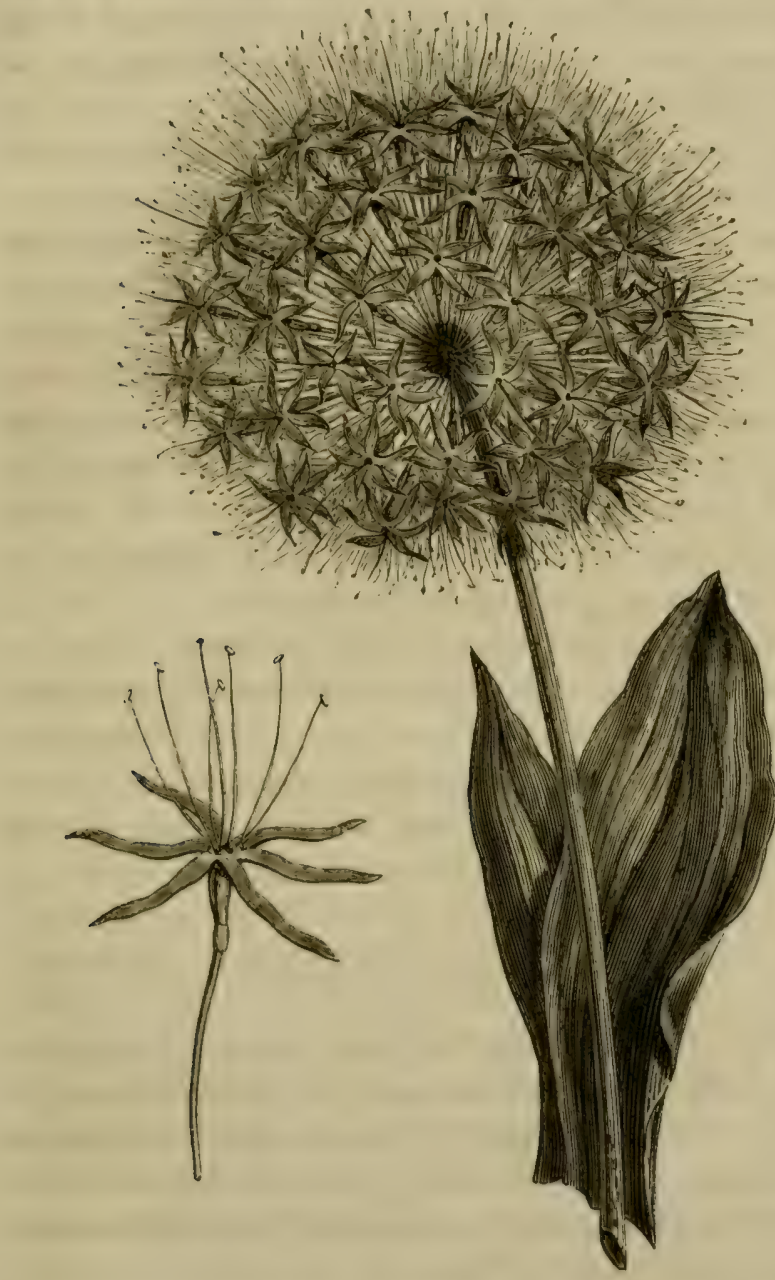

Bัav. 4.7. - Hemanthus toxicarius.

jours la presque certitude de féconder les especes par leur propre pollen, el peut che alors le coccinems lui-meme domerait des 
graines susceptibles de pousser. Il faudrait, dans tous les cas, supprimer la majeure partie des fleurs de l'ombelle, en laisser deux ou trois seulement, enlever les étamines dès l'épanouissement et poser le pollen sur le stigmate en y ajoutant, au besoin, un peu de la liqueur miellée que sécrète la base du périgone (grav. 95).

Nous plaçons à la suite des Hæmanthus l'indication d'un hybride d'himantophyllum, genre voisin appartenant aussi à la famille des Amaryllidées. MI. Duchartre a publié à ce sujet, dans le Journal de la Société impériale et centrale d'horticulture (vol. V, p. 758), un mémoire très-intéressant. Cet liybride a été obtenu par M. Van Houtte, "lequel, dit-il, avait d'abord tenu secrète l'origine de cette remarquable Amaryllidée. ») Cette plante est le résultat du croisement de l'H. Aitoni Stook. père, et de l'H. miniatum Stook. mère, d'où est résulté l'H. Aitoniminiatım, décrit comme espèce par Lindley, qui ignorait son origine, et nommée par lui $H$. cirtanthiflorum.

Les divers individus provenant de ce croisement varient un peu dans la teinte et le plus ou moins de facilité d'épanouissement de leur's fleurs, mais ce sont tous des plantes admirables.

Le pollen et les ovules bien organisés de cet hỵbride le rendent fertile, et il ne pourra manquer d'être croisé de nouveau avec ses parents ou avec des espèces voisines.

\section{Genre Amaryllis. - Amaryllis.}

On trouve dans ces belles fleurs six étamines à longs filets dont les anthères ne s'ouvrent qu'après l'épanouissement. Le style, aussi long que les filets, se termine par trois stigmates qui, d'abord serrés les uns contre les autres, s'écartent à l'époque de la fécondation, tandis que le fond de la corolle s'emplit d'une liqueur miellée.

Il est peu de plantes dans lesquelles l'hybridation soit aussi certaine et aussi facile à opérer. Les anthères sont encore fermées, ainsi que les lobes du sligmate, quand la fleur commence ì s'ou- 
vijir; on peut done les enlever avec les doigts et attendre, pour placer le pollen étranger, que les lobes du stigmate se soient écartés, ce qui n’arrive quelquefois que le second ou le troisième jour de la floraison. Si le slignate est sec, on l'humecte avec une petite quantité d'humeur miellée que l'on puise avec un pinceau dans le fond de la corolle. Indépendamment des belles espèces que l'on importe tous les jours, les Amaryllis ont donné par l'hybridation une multitude de rariétés plus éclatantes encore que leurs ascendants. Tous ceux qui ont pu voir les collections exposées à Gand sont restés étonnés des succès des horticulteurs belges dans ce genre de plantes. Il était impossible de ricn voir de plus beau, de plus éclatant, de plus riche. Ces admirables variétés provenaient, a-t-on dit, de l'hybridation des A. vittata, Johnsonii et pulverulenta: Les hybrides peuvent encore s'hybrider entre eux, mais il vaut mieux y prendre le pollen et le reporter sur les espèces types, qui grainent plus facilement, et ne féconder que deux fleurs et même une seule sur chaque plante; on coupe les autres ou l'on enlive, pour pouvoir jouir de leurs fleurs, la pointe trilobée du stigmate.

LiA. fulyida est encore une belle espèce pour l'hybridation. Le curviflora et le sarniensis peuvent aussi se féconder entre eux. Il est à désirer que l'on tente des croisements sur le Lis de Saint-Jacques ou $A$. formosissima, en portant son pollen sur des plantes qui grainent facilement. On pourrait aussi tenter de féconder entre elles les différentes espèces de la section des Zephiranthes, telles que les candida, roseu, grandiflora, carinuta, ete. Ne pourrait-on pas aussi créer de beaux hỵbrides avec I'A. culyptrata dont les fleurs sont vertes?

Il ne faut pas, toutefois, se dissimuler que le genre Amaryllis, pris dans sa plus grande extension, offe la réunion de plantes assez différentes, destinées à former, sinon des genres distincts, au moins des sections hien tranchées; et si l'on a toujours quelque chance de croiser toules les especes, on en a de hien plus grandes en essavant l'hybridation entre es- 
pèces qui se ressemblent et appartiennent au même groupe.

Dès 1827, le vice-amiral Hamelin a publié un rapport trèscurieux sur la multiplication des Amaryllis et des Crinum, au moyen de fécondations croisées, pratique qui alors était à peu près ignorée en France. C'est à Palerme que ces croisements s'opéraient par les soins du baron Melazzo, et en Angleterre par IV. Herbert.

La plupart des hybrides crééspar M. Aimé Turlure proviennent de la fécondation de l'A. pulverulenta ou tricolor du Brésil avec l'Hyppeastrum viridiflorum. Des variétés très-distinctes de forme et de coloris sont nées de ce mariage de raison. L'avantage de ces hybrides réside surtout dans leur rusticité qui leur permet de montrer en pleine terre leur vigoureuse végétation, et qui n'exigent en hiver que l'abri d'une bâche froide ou de toute autre couverture.

On a pu suivre pendant quelque temps la filiation des hybrides issus de quelques espèces d'Amaryllis. Aujourd'hui les croisements obtenus en France, en Angleterre et en Belgique sont devenus si nombreux, si faciles et si fertiles, que toutes les traces sont perdues, et que l'arbre généalogique de toutes ces plantes magnifiques, qui figurent aujourd'hui aux expositions, ne peut être rétabli.

Linné avait déjà observé que dans l'A. formosissima, ou Lis Saint-Jacques, il sort de l'extrémité du stigmate, pendant les journées chaudes de l'été, une goutte de liqueur limpide et si volumineuse qu'on croit qu'elle est toujours prête à tomber; mais cette liqueur est repompée peu à peu par le pistil, vers les trois ou quatre heures du soir, el ne reparait que le jour suivant sur les dix heures du matin. C'est vers le milieu du jour que la goutte est la plus grosse. Si on secoue les étamines de manière que leur poussière puisse se mêler à cette liqueur, on voit bientôt ce fluide se troubler et devenir jaune. Quelque temps après, lorsque la goutte de liqueur a été entièrement. alsorbée, on trouve la poussière séminale déposée sur le stig- . mate, mais elle est irrégulière et a perlu sa forme primitive. 
Limné en conclut que les grains de pollen ne sont pas alssorliés et ne pénètrent pas à travers le style.

\section{Genre Perce-Neige. - Gialanthus}

On ne cultive dans nos jardins qu une seule espece de P'ereeneige, mais il a produit, depuis très-longtemps, une variété à fleur double, et si lon examinait celle-ci aree attention, on y découvrirait quelquefois des étamines ave lesquelles on pourrait léconder le type à fleur simple. Il est vai que ce dernier est un peu plus précoce que sa varicté, mais il serait lacile, par une exposition différente, d'amener ces deux végétaux à une éporque simultanée de floraison. Je suis convaincu que des semis un peu étendus de cette jolie plante produiraient quelques variétés nouvell's, d'une espèce que l'on remarque à la fois par sa fraicheur et par la saison où elle se développe, pour nous annoncer le retour du printemps.

Le Galanthus plicatus à larges feuilles est plus beau et tout. aussi rustique que le G. nivalis. Ils pourraient certainement ètre croisés et produiraient peut-ètre une race plus robuste et plus florifère que leurs parents.

\section{Genre Pancrace. - Pancralum.}

On trouve dans ces plantes, comme dans les Crimum, six étamines, dont les filets sont soudés par la base en une élégante couronne et dont les anthères oscillantes peurent répandre leur pollen sur un stigmate simple ou papillaire qu'un long slyle amène à la hauteur des anthères.

Tout ce que nous avons dit des Crinum, relativement a la fécondation artificielle, à l'hybridation et aux bulbilles, peut igalement s'appliquer aux Puncratium. Plusieur's d'entre eux, comme le curibum, l'amboine'nse, le sylvestre', ete., sont de serre chande; le muritimum, le rotalum, si sonvent munis de bulbilles, l'illiricum, le nutuns, l'anunicuës, le Linilitii sont de 
serre froide ou tempérée, et quelques-uns mème de pleine terre. Ces belles espèces se cultiveraient facilement dans la même serre que les Crinum (grav. 94).

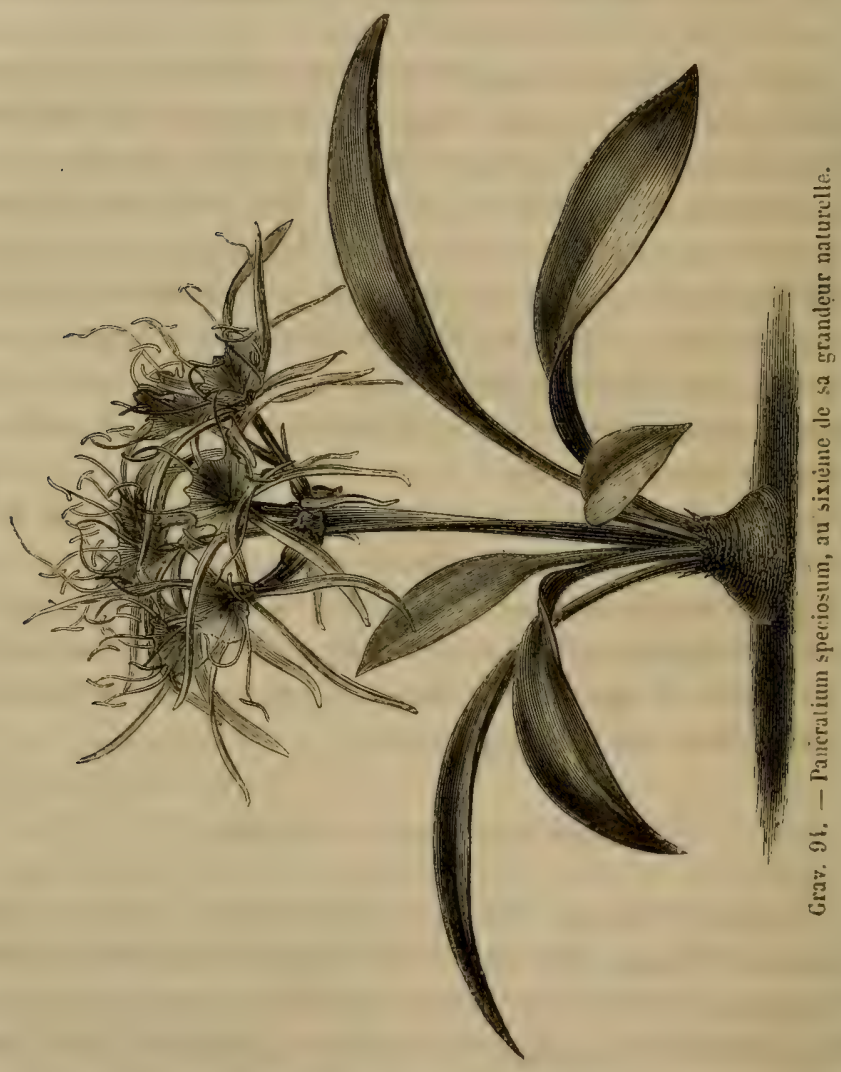

Genre Nareisse. - Narcissul.

Les Narcisses forment un des genres le plus nombreux des Amaryllidées, que l’on désigne aussi sous le nom de Narcissées, à cause de l'importance du genre qui nous occupe. Ces plantes ont six étamincs, souvẹt inégales et enfermées dans le lube du périgone. Les filets sont généralement sondés au tube, et 
amenent trois anthères an sommet de l'ouverture on au fond du godet, et les trois antres un peu au-dessous et totalement incluses. I.e style, assez long, porte un stigmate trifide qui, précisément placé entre les deux séries d'anthères, manque rarement d'ètre fécondé naturellement.

I.es Nircisses sont presque tous de pleine terre et donnent géníralement des graines fertiles, aussi peut-on les hybrider assez facilement, en prenant quelques précautions. Il faut d’abord enlever les anthères, el, comme celles-ci s'ourrent assez souvent avant la corolle, c'est par une petite incision pratiquée au tube du périgone, un peu arant l'épanouissement, qu'il faut les extirper. On attend ensuite le second jour de la floraison pour poser le pollen, el, s'il n'adhérait pas au stigmate, on humecterait celui-ci arec un peu de la liqueur miellée qui se trouve au fond du tube.

Il est peu probable que toutes lis espèces de ce genre puissent se croiser, car elles semblent appartenir à des types assez distincts, on y trouve :

Le $N$. pseudo-narcissus, dont le major et le minor mème ne sont peut-ètre que des variétés. Cette espèce double trèsfacilement, à tel point que, dans les environs de Grasse et dans la majeure partic de la Provence, le type simple est plus rare que la variété double. Celle-ci domnerait sans doute des graines en la fécondant artificiellement, car son stigmate est souvent bien conformé, mais malheureusement l'ovaire avorte presque toujours. Il faudrait alors chercher quclques anthères fertiles, au milieu des pétales multiples de cette rariété, pour fë́conder des fleurs simples. Le N. pseudo-narcissus a été croisé en Ilollande avec le poeticus, et a donné probablement le phanix sulphur, le phonix orange et le sulphur trumpet, qui sont tous stériles.

Ia section des poeticus comprend aussi le biflorus, le poeticus ì fleurs doubles, et plusieurs autres especes ou variétés lu Midi, qui peurent se croiser aree les précédentes et les suivantes. 


\section{8\% FÉCONDATION NATURELLE ET ARTIFICJLLLE.}

Le N. tazetta est celui qui a le plus varié; déjà très-leau dans les prairies des bords de la Méditerranée, où il abonde, il a donné dans les jardins des fleurs bien plus remarquables; c'est à lui, au polyanthes, au stellatus et au chrysanthes, que I'on doit tous ces beaux Narcisses de Hollande, désignés dans les catalogues sous des noms plus ou moins pompeux, et dont les fleurs sont disposées en larges bouquets odorants.

Une division particulière des Narcisses comprend tous ceux dont les feuilles se rapprochent de la forme cylindrique. C'est

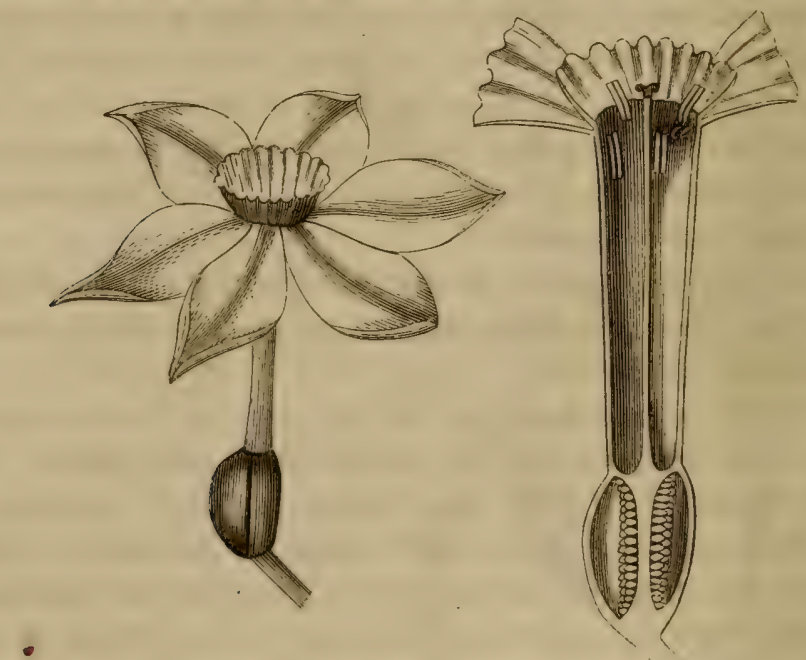

Grav. 93. - Fleui du Narcisse des poëtes - La même, coupéc pour montrer les ćtamines et le pistil.

dans cette section que se trouvent les Jonquilles simples et doubles, et d'autres espèces moins connues et peu cultivées, telles que les bulbocodium, contabricus, aureus, infundibulum, etc. Je présume que l'hybridation aurait lieu entre ces dernières plantes; mais je doute qu'on puisse l'opérer entre celles-ci et celles des sections précédentes. Dans tous les cis, les Narcisses sont loin d'aroir produit toutes leurs variétés, el méritent l'attention des amateurs et des horticulteurs marchands (grav. 95). 


\section{Genre Pérégrine. - Alstrameria.}

On trouve daus les Alstrameria six élamines et un style terminé par un stigmate à trois divisions plissées. En enlevint les anthères lors de l'épanouissement, on féconde eusuite ces plantes artificiellement, en posant an pinceau le pollen sur les lobes du stigmate. Presque tous les genres nombreux en espreces, comme celui-ci, ont, en général, de la tendance à s'hybrider, el comme dejji on cultive bon nombre d'espèces toutes 'assez belles, on peut assurer d'arance beaucoup d'avenir aux Alstrameria.

Les A. ligtu, tricolor, hamantha, antrea, Hoolieri, antanliacu, psiltacina, pelegrina, pulchella, lineatiflora et plusieurs iutres fleurisient dans nos jardins, mais c'est surtout du chiliensis qu'il faut attendre les plus heureux résultats; même sans hybridation, celte plante produit un grand nombre de varictés, et, à plus lorte raison, quand on cherchera à croiser les derniers gains entre eux ou aree leurs ascendants, devrat-on arriver à d'admirables collections.

L'A. Errenbaulti, si remarquable par ses jolies macules, est uncore un charmant hybride, obtenu à Tournay en fëcondant le pistil de l'A. peleyrina avec le pollen de l'A. pulchra. Ses śtamines, qui paraissent bien conformées, ne contiennent pas de pollen.

Ces plantes ont une grande tendance à la variation. Elles domnent naturellement de nombreuses différences dans le coloris; mais, si l'on liybride entre elles aree du pollen mélangé, les A. hamanthu, aureu, pulchella, versicolor, ete., on ne trouvera pas de terme aux divers coloris qui surgissent de cette lacile opération.

\section{FAMLLE ILS BROMELACEES.}

Genre Inanas. - Bromeliu.

Iar cullure de l'Anamas, autrefois réservee a quelques horticulteurs spécians ou conlinée dins lit serre des princes, est 
devenue pour ainsi dire vulgaire et à la portée du simple amateur; aussi a-t-elle fait, dans ces derniers temps, beaucoup de progrès, et les variétés de cet excellent fruit se sont multipliées au point de devenir un jour aussi nombreuses que nos pommes et nos poires.

L'Ananas a six étamines, dont les anthères sont rarement fertiles, mais dans lesquelles on rencontre cependant parfois du pollen. Le pistil n'est presque janais bien conformé; si l'ovaire est intact, le stigmate manque ou est réduil à des appendices dépourvus de papilles et impropres à recevoir le pollen. On trouve cependant des porte-graines parmi les nombreuses variétés d'Anamas aujourd'hui existantes. En les cximinant avec soin, lors de la floraison, on décourre quelques stignates trifides bien couverts de papilles et que l'on peut féconder artificiellement. L' liybridation est un moyen sùr d'obtenir' de nouvelles variétés, et quand on roit quelques graines pendantes, résultat d'une hybridation heureuse, on est presque sûr de variétés nouvelles, qu'il faut attendre quelques années, mais qui dédommagent amplement de la peine que l'on a prise et du temps que l'on a employé. On a déjà des Ananas d'un volume extraordinaire, mais on arrivera par ce moyen à des lruits fabuleux, et aussi différents entre eux pour le volume el la saveur que la groseille à maquereau de nos buissons et les dernières variétés obtenues en Angleterre.

\section{FAMILLE DES ASPARÁGINÉES.}

\section{Genre Asperge. - Asparagus.}

Comme la plupart des Monocotylédones, l’Asperge a encore six étamines; son ovaire simple est surmonté de trois styles à stigmates bifides. L'Asperge officinale ou ordinaire, la seule dont nous ayons à nous occuper est une plante dioïque ou monoïque, et par conséquent facile à hybrider; mais on n'en connait encoroge deux bomes variétés, la verte ordinaire et 
la violette ou Ilollandaise. Elles se croiseraient très-facilement et peut-ètre y aurait-il avantage à essaver cette hybridation. II sultirait d' isoler complétement le pied femelle, en coupant tous les màles, et de vérifier si les porte-graines n'auraient pas aussi quelques fleurs màles au milieu des autres, ce qui arrive quelquefois. Lin supprimant une partie des branches, les graines seraient mieux nourries, plus volumineuses, et l'on aurait plus de chances de succès.

\section{Genre Muguet. - Convallariu.}

Les lleur's ont six étamines à filets courts et à anthères percées de deux pores an sommet, un ovaire et un style simple, un stigmate trifide. Le Nuguet de mai, C. mä̈alis, a déjà produit des variétís à fleurs roses, à fleurs doubles, avec lesquelles on pourrait l'hybrider, mais e'est surtout aree le spicutu et le juponicu qu il faudrait essayer de le croiser; il faudrait, dès la floraison, enlever avec précaution les anthères du maïalis, en tenant les fleur's renversées, c'est-à-dire le stigmate en haut, et lëconder ensuite avec le pollen des autres espèces; laisser sur chaque tige les deux fleur's inférieures seulement et détruire les autres, qui, du reste, avortent souvent naturellement.

\section{FAMILLE DES IJLIACEES.}

\section{Genre Tulipe. - Tulipa.}

L.es Tulipes sont un des plus riches ornements de nos parterres et sont arrivées, par la culture, à un haut degré de perfection qu' elles dépasseront encore. Leurs six étamines à grosses intheres pivotantes sont situées à la hauteur de trois stigmates ippais, glanduleux et sessiles, sur un oraire triangulaire. Le jour où la lleur s'épanouit, les anthères dressées et appliquées te long des filets sont encore intactes et remplies de pollen; on peut les enlever avec les pinces et mème aree les doigts, et deux jours après on peut placer, sur le stigmate, le pollen 
étranger, en prenant avec les pinces ou avec les doigts une étamine avec laquelle on saupoudre une, deux ou les trois divisions du stigmate; car on peut, avec le même porte-graine, 'ssayer trois combinaisons différentes avec du pollen recueilli

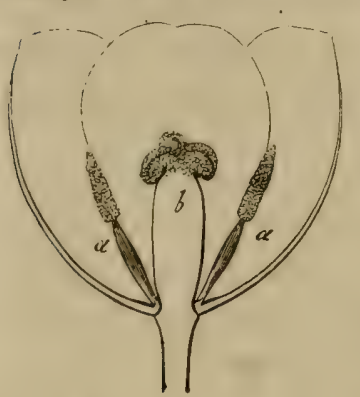

Grav. 96. - Fleur coupée. - u, u, ileux des sic étamines. $-b$, pistil avec stigmate trifide et sessile. sur trois plantes distinctes. La Tulipe destinée à devenir mère doit seulement être entourée d'une gaze liée légèrement sur son pédoncule pour empêchér les insectes de pénétrer dans son calice (grav. 96).

$\mathrm{Si}$, depuis l'époque où l'on cultive les Tulipes, on avait employé ce moyen si simple d'lybridation, au lieu d'abandonner au hasard et aux insectes des fonctions qu'il ćtait si facile de remplir, on aurait des plantes plus méritantes encore
que celles que l'on possède, et dont les plus belles, du reste, sont le résultat de croisements.

La Tulipe des fleuristes, ou T. Gesneriana, est celle dont on s'est principalement occupé. On y distingue deux types, celles à lond blanc ou Flamandes, et celles à fond jaune ou biaurres. "A l'égard de ces dernières, dit le savant rédacteur de l'Horticulteur universel, il est un préjugé aussi absurde que déplorable, qui s'oppose nécessairement à l'amélioration de ce grenre de culture; préjugé que nous avons déjà combattu de toutes nos forces, oralement ou par écrit, et qui consiste à rejeter comme indigne de la collection des Tulipes celles dont la fleur est jaune, et cela quelque grande que soit d'ailleurs leur incontestable beauté. On conçoit quelles privations s'impose le cultivateur qui, cédant à une coutume absurde, jette au fimnier tout le plant qu'il en obtient quand le fond n'est pas blanc. Notez bien que cette blancheur est fort sourent douteuse et n'existe quelquefois qu’à l'extrémité basilaire de l'onglet, et qu'alor's elle est it peine ou même point appréciable. "

Nous partageons tout in fait l'avis de M. Lemaire, ct nous re- 
gardons comne une chance de plus pour l'obtention de nouveaux gains, la possibilité de se servir des fonds jaunes à formes parfaites, ou comme porte-graines, ou comme plantes fécondantes, et nous pensons que les collections y gagneront en beanté et en variété. Il est possible que les fonds hlancs soient plus flatteurs à l'œil, mais qui sait, si l'on avait cherehé à perfectionner aussi les Ionds jaunes en s'en orcupant autant yue des autres, oì l'on serait arrivé à leur égard? et d'ailleurs il en est des Tulipes comme des autres fleurs, comme des Qiillets par exemple, le goût pent être différent. Ainsi, parce que les Giillets llamands sont préférés par plusieurs personnes, est-ce une raison pour rejeter les fantaisies, et se priver de toutes ces lielles plantes et des hybrides qu'elles peuvent former?

Les Tulipes monstrueuses, si curieuses et parfois si éclatantes, ne recerraient-elles pas de notables accroissements si on les fécondait entre elles, et surtout si on les croisait avec les diverses variétés du Gesneriana et du suaveolens.

Cette dernière espèce, plus connue șous le nom de duc de Thol, a produit des plintes moins belles que le Gesneriana, mais bien plus hattives et odorantes. De son croisement avec cette dernière espèce sont nées toutes les jolies Tulipes prícoces qui préludent à la floraison de l'espèce privilégiée des fleuristes. Que de variétés à obtenir encore en croisant ces plantes!

Les T. pracox, oculus solis, Celsiana, sylvestris sont aussi cultivées, mais rarement; il me semble que le Celsiana et le suaveolens produiraient de charmants hybrides. Je crois qu'il y aurait grand avantage à féconder l'oculus solis avec nos helles variétés de Gesneriana, et it introduire dans nos cultures plus de douze espèces de Tulipes qui y sont totalement inconnues, et dont les rapports sont assez grands pour espérer des croisements fructueux, et amener ce beau genre à un degré de perfection qui dépasse de beaucoup le nee plus ultra que l'on croit aroir atteint de nos jours.

Quelques personnes aiment les Tulipes doubles, jusquäi pré- 
sent peu variées. On pourrait augmenter nos richesses en ce genre en recherchant sur ces plantes celles qui conservent des étamines et en employant leur pollen pour hybrider des fleurs simples ou des dragonnes. Quelquefois, mais plus rarement, les fleurs doubles pourraient servir de porte-graine en fécondant leurs pistils, quand par hasard ils sont bien conformés.

II faut attendre longtemps les Tulipes de semis, mais les années s"écoulent si vite, et les amateurs ont quelquefois tant de loisirs à dépenser!

\section{Genre Fritillaire. - Fritillaria.}

Six étamines très-grandes, dont on peut enlever les anthères à la main, et un ovaire surmonté d'un style simple et terniné par un stigmate trifide, caractérisent les Fritillaires. Ces plantes forment deux groupes distincts, les Méléagres et les Couromnes impériales. Les premières ont déjà fourni un grand nombre de jolies variétés, que l'hybridation peut encore augmenter, soit en les fécondant entre elles ou en les croisaṇt avec le pyrenaica; les secondes renferment ces élégantes Couronnes impériales, dont les variétés orangées, rouges ou jaunes, simples ou doubles, peuvent également s'hybrider. N. Delorme a obtenu une variété à fleur jaune, plus belle que l'ancienne, et qui servirait de bon porte-graine pour les croisements de ces belles plantes.

La grande variété nommée Fritillaria maxima est encore une de celles qui donneraient les plus beaux produits. Il suffirait, pour toutes, de placer le pollen sur le stigmate et de supprimer la majeure partie des fleurs, de minire a laisser senlement deux capsules sur un même pied.

On peut placer dans une troisième division le Fritillaria persica, le verticillata, les purpurea, barbata, rhutenica, qui peut-ètre shy̧brideraient entre elles sans grand profit pour l'horticulteur, mais qui donneraient certainement des plantes bien curieuses, si, contre mon opinion, on pouvait les croiser, et surtout la premiere, avec les espèces des denx autres sections. 


\section{Genre Iis. - Lilium.}

Les Lis forment un des plus beaux genres du règne végétal, et ont tous six étamines, un style, un stigmate rifide ou à trois lobes. Les anthères, très-grosses, ne répandent leur pollen qu'après l'épanouissement. On peut donc les enlever le matin, au moment où le calice s'entrourre, et féconder ensuite le stigmate le jour mème ou le lendemain de la floraison.

I; hybridation est, comme on le voit, facile à opérer avec des organes aussi gros et aussi visibles; reste à savoir si elle peut avoir lieu entre toutes les espèces du genre, qui sont trèsnombreuses et présentent d'assez grander différences physiologiques.

On peut partager les Lis en deux sections, cenx à sépales droits et ceux ì sépales recourhés et réfléchis.

Jans la prenière section se troure d'abord le Lis blanc, le Innyiflorum, le peregrinum, le japonicum, le Broussartii, le laucifolium, qui, hybridé avec le précédent, qui n'en est peutètre qu'une variété, a déjà donné de très-belles plantes. Il y a, en Belgique, une grande quantité de jeunes bulbes de ces lis hybridés, dont on attend aree raison des merveilles. M. Senéclauze, dont le mérite est connu de tous les horticulteurs, a obtenu cent graines fertiles en hybridant quatre variétés des $\mathrm{Li}$ lium speciosum et Broussartii.

Près de ceux-là viennent se grouper ces Lis à corolle oransée, tels que le croceum, l'aurantiacum, le bulbiferum, le spectalile, plantes analogues et dont le croisement semble trèspossible.

La seconde section est formée par les Lis à pétales rélléchis, "touticut les Martagons ot toutes leurs variétés, le superbum at le tigrinum, le pyrenaicum, les pomponium, temuifolium, canadense, chalcedonicum, qui ont heancoup de rapports, et entre lesquels, jusqu' à présent, je n’ai pu obtenir l'hỵbridité. Les lancifolium, dans li preniicre section, les Martayons, dans la seconde, me praraissent être de hons porte-grames. 
Les Martagons, dont on connait un très-grand nombre de variétés, obtenues par la culture, se cröisent et fructifient trèsbien. J'ai tenté la fécondation sur eux avec le pollen du Lis

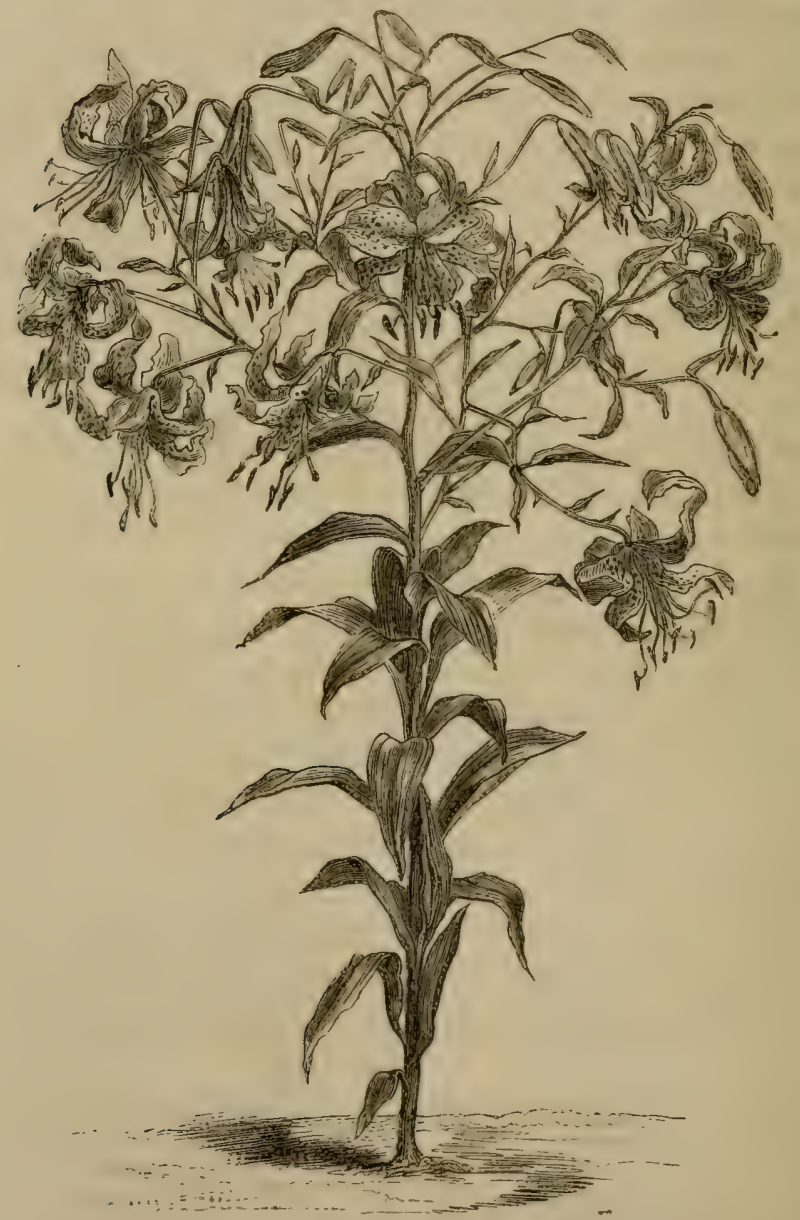

Grav. 97. - Lilium lancifolium var. : corymbiflorum roseum.

blanc et de l'orangé, et j’ai obtemu deux capsules, mais les graines ressemblaient tellement à celles du Martagon, et il y a de si gramdes différences entre ces trois plantes, que je croirais 
plutòt à une fécondation naturelle, qui n'aurait rien d'impossible dans une contrée où les Hartagons sont communs, et où, par conséquent, les insectes auraient pu se jouer de mes précautions (grav. 97).

On sait que plusieurs Lis, tels que le Jis blanc, ne donnent ordinairement des grraines qu'en coupant et suspendant la lige après la féecondation. Peut-être devrait-on agir ainsi, si l'on tenlait sur ces Lis une hybridation quelconque. Lne observation rérente de I. Rivière sur l'Agare potutorum Zucc. indiquerait que ce mode de traitement réussirait aussi sur ce dernier genre. (Bulletin de la Société botanique de France, I. VIII, p. 650.)

Ce fait curieux d'une tige compée et suspendue qui vit longtemps ef qui mùrit ses graines, nest pas particulier au Lis blanc. Il se reproduit chaque fois que l'on a affaire à des planles yui se multiplient facilement et rapidement par leurs racines.

Nous trouvons la preuve de cette opinion dans un mémoire de II. Van den Born, inséré dans la Belgique horticole (février 18621. Voici le procédé qu'il emploie pour faire fructifier le Lis blanc. "Vers l'épogue de la floraison il creuse la terre autour de la plante de manière à mettre le bulbe ì nu, puis il en détache délicatement toutes les écailles el toutes les bulbilles de manière ì dénuder complétement la tige. Cette opération faite, on remet la terre en place et l'on peut s'attrndre, neuf fois sur dix, à roir des capsules bien fournies de graines succéder anx fletrs, et les tiges se maintenir en vie an lieu de se dessécher rapidement comme elles en ont l'haliunde. ")

La loi du balimcenent des organes que nous asons déjä ritée trouve ici une éclatante confirmation. La tige coupée Clait soustraite it la voracté des racines ou plutòt des bulbilles qui épuisaient le déput de nourriture que la végétation y avait annissé. lin sopposint i la multiplication gemmipare, on favorise évidemunent la formation et lat nutrition des graines. Cest

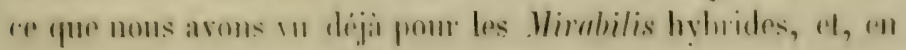


594 FÉCONDATION NATURELLE ET ARTIFICIELLE.

général, pour toutes les plantes hybrides qui ne fructifient pas par suite d'une trop grande vigueur.

Genre Erythuonie. - Erythronium.

Les six étamines des Érythronies, d'abord dressées et appuyées contre un pistil simple, terminé par un stigmate trifide, s'en écartent ensuite et ouvrent leurs anthères. On peut les enlever avant cette époque et féconder artificiellement le stigmate. Les variétés blanches, roses et pourprées de l'E. dens canis existent déjà, aunsi qu'une antre venue de Sibérie. On cultive aussi les $E$. lanceolatum et americamum, qui appartiennent au mème type que l'indigène, en sorte que l'on jeut tenter l'hybridation entre ces différentes plantes.

Genre Yucea. - Yucca.

Les Yucca ont six étamines assez. volumineuses pour quion

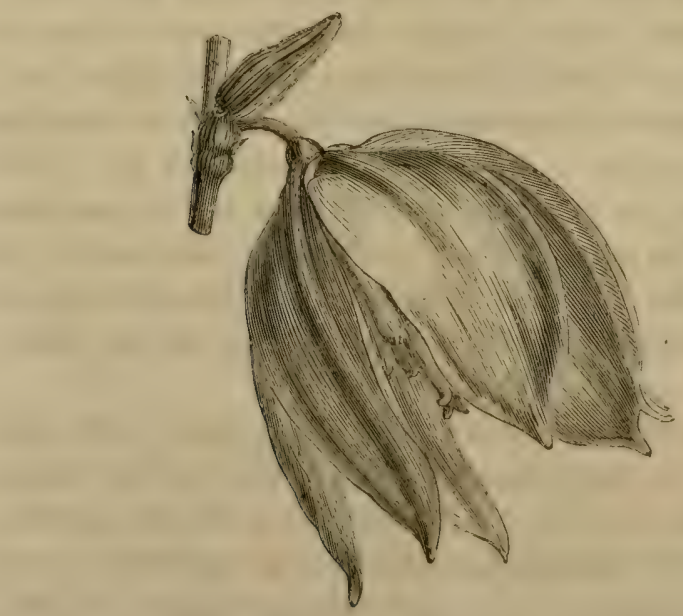

Grav. 98. - Flecir dı Yucea auqustifolia de grancleur naturelle.

puisse les enlever arec les doights, et trois stigmates épais posés 


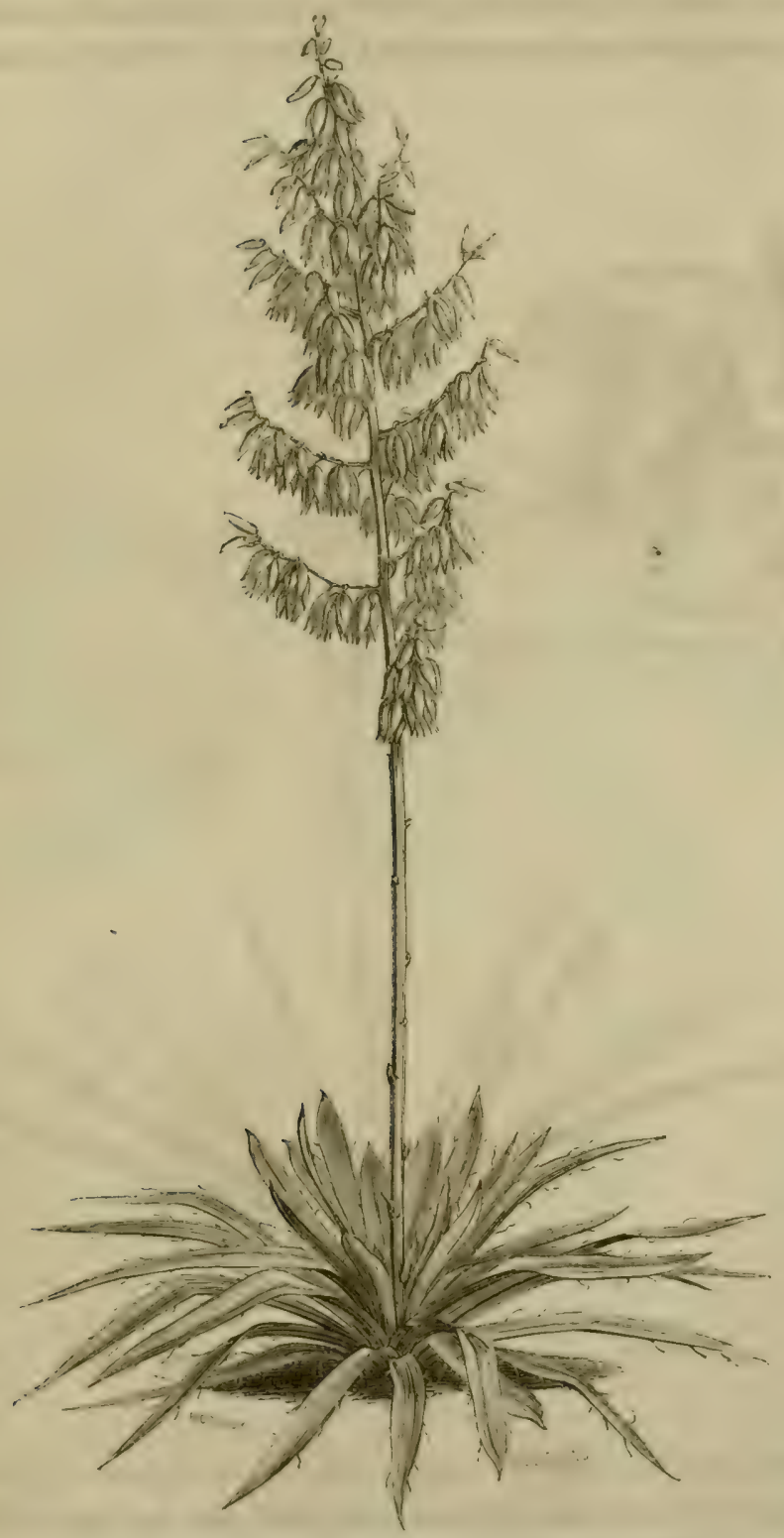

Gras. 99. - Vucen filamentosa media au dixième le grandear. 
596 FÉCONDATION NATURELLE ET ARTIFICIELLE.

sur l'ovaire. L'hybridation pourrait done y être opérée aussi facilement que sur la plupart des Liliacées, et comme les espèces

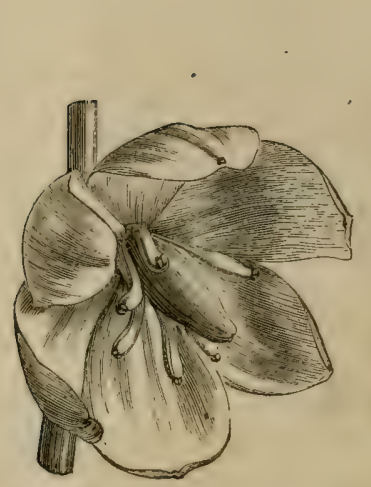

Fleurdı Yucea orchioidesile granileur naturelle.

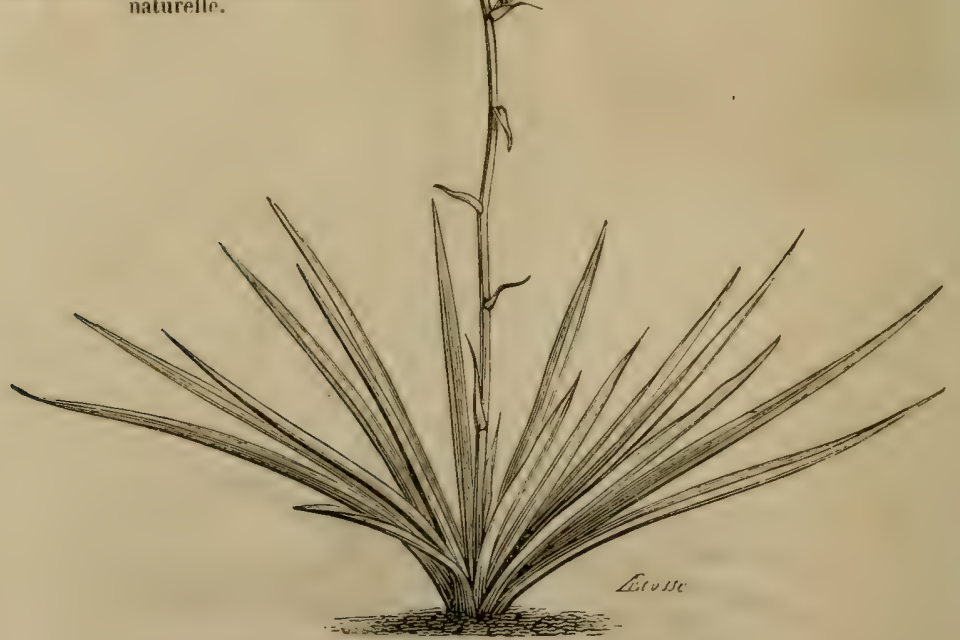

firav. 100. - Yucea nrchioïles au quart de la grandeur naturelle.

sont assez volsines, il serait permis d'en espérer du sucris. Déjà les Y. gloriosu et glaucescens ont donnó des fruitsà Paris. 
II "anrail donc possibilite, en les choisissint pour porte-yraine, de les féconder l'un par l'autre ou par l'aloefolia ou le filcuménInsa; cir je ne pense pas que les autres espèces, peunombreuses, aient encore fleuri daus nos cultures.

Les especes ou les variétés de fucca sont devemes très i lit mode dans ces derniers temps. Nous en reproduisons senlement quelques flem's, et nous arons l'espoir que la plupart de ces formes peuvent se croiser (grav. !), !)! 10), $101,102)$.

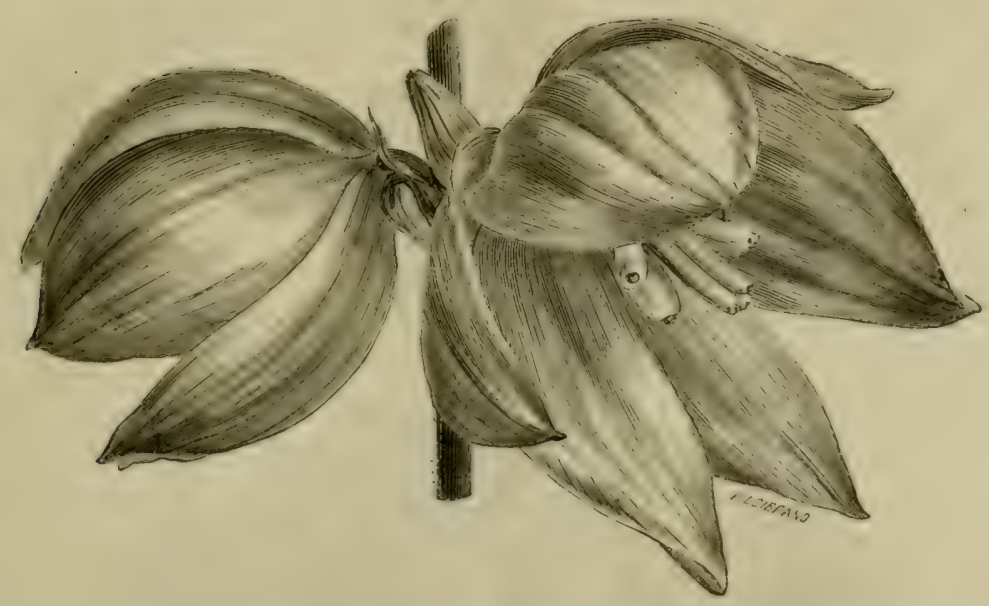

- Grav. 101. - Fleur du Yucca striclu de grandeur naturelle.

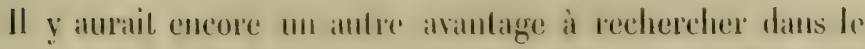
croisenent des diverses especes de Y'ucea : ce serait d'olitenie des varictés plus rustignes ot caprables de supporter en plein aii les froids de nos hivers rigonemx. On pourrail ansi ahandonner des groupes de nos plus heam buece an milieu des gazons, oì res plantes produisent un tres-led affer. 


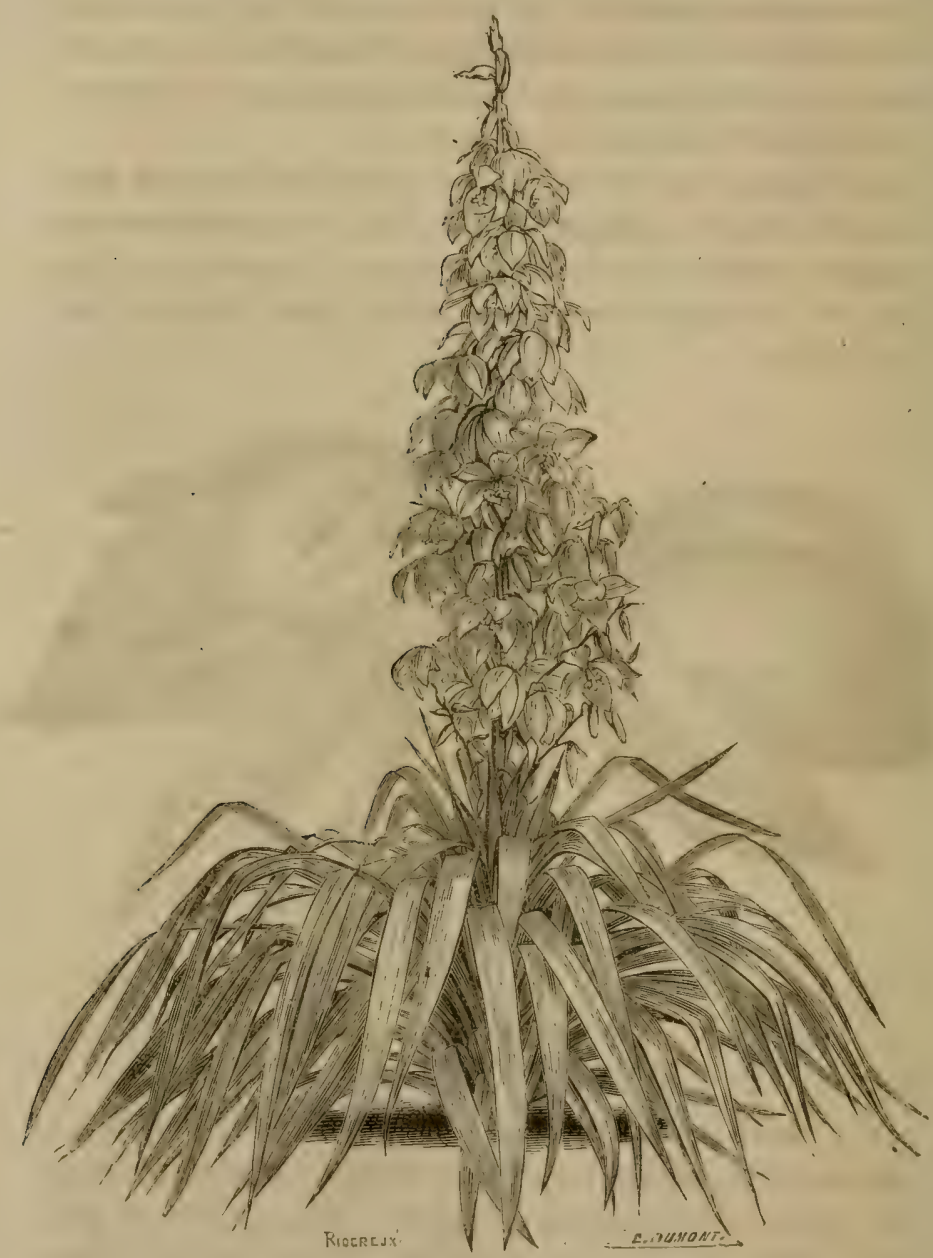

Grav. 102. - Yucca pendula au disième de grandeur naturelle.

Genre Alues. - illoi.

Il existe un très-grand nombre d'espèces d'Aloes, dout plusieurs sont remarquables par la beauté de leurs fleurs et presđue tous par la singularité de leur feuillage. Leurs six étamines 
sont lantòt sirllantes, tantôt incluses ; le stỵle est plus ou moins long, terminé par un stigmate trifide.

Jignore si l'on pourait hybrider les différentes especes l'Dloes, mais si on voulait tenter des croisements sur certaines espèces dont les fleurs sont renflées et les anthères incluses, il laudrait fendre les périgones sur le còté et enlever les citanines avec les pinces.

\section{Genre Scille. - Scilla.}

Lat liecondation s'oprere dans les Sicilles comme dans les autres Liliacies, an moyen de six étamines qui entourent un pistil simple. Liourerture des antheres n'a lieu quiaprès celle de la fleur: "n sorte que, si l'on veul essayer les croisements, on peut tres-ficilement enlever les itamines le premier jour de l'épinouissement.

Plusieurs Scilles sont cultivées comme plantes d'agrément, 'I toutes mériteraient de l'ètre. Elles offrent entre elles assez de diffërence pour linire supposer que l'hybridation entre espèces ne pourrait avoir lien; mais presigue toutes offrent déjà des varietios a lleur's blanche's, a lleur's roses ou lihats, qui permettent d'esperer d'antres feintes par lem mélange. Les Scilla bifoliu, nermianu, liliohyacinthus, companulutu, les untuns, amona, Bertolonii, sibirica présentent ces variélés blanches ou liliatcies, a l'exception peut-ètre de la dernière, qui, ainsi que le bifolia, serait un très-bon porte-graine.

\section{Genre Jacinthe. - Ilyacinthus.}

Iu milieu des six étanines de la Jacinthe se trouse un ovaire trigone, surmonte diun style simple ef d'un stigmate aplati. Le's anlheres sont incluses dans un pirignne c'n forme de grelot, "t le stipmate reste ansisi enfermé aree les organes miles. ()n ne peut dune enlever les élamines, ni pratiquer la fécondation artilicielle sans une petite operation yui consiste i fare une incision en forme de petile porte sur un des coutes de la corolle. 
C'est par cette petite partie soulevée que l'on ôte les anthères, que l'on pose le pollen, et l'on referme ensuite en l'appliquant exactement sur les lignes que l'on a coupées. J'ai fécondé ainsi le belles variétés de Jacinthes simples, dont les fruits ont parlaitement mûri.

On doit noter que dans ces plantes chaque grain de pollen ne produit qu'un seul tube pollinique.

Quoique ce genre contienne un certain nombre d'espèces, ce n'est guère que sur les diverses variétés de l'orientalis que l'on opère l'bybridation; et, quoiqu'il semble, au premier abord, qu’il n'y ait plus rien à espérer, je suis convaincu que, daus les variétés jaunes, dans les bleues et les rouges trèsfoncées, il y a encore de très-boumes plantes à attendre des croisements faits avec soin et discernement. Les Jacinthes doubles, inoins appréciées pour le moment que les simples, ont aussi beaucoup à gagner. En cherchant dans ces variétés celles qui conservent les étamines fertiles pour en féconder des Jacinthes simples à couleur vive, je suis persuadé que l'on parviendra à améliorer encore ces fleurs déjà si belles.

\section{Genie Muscari. - Muscari.}

Ce que nous venons de dire des Jacinthes s'applique igalement aux Muscari que l'on a détachés de ce genre pour en faire un nouveau. On ne pourrait non plus enlever les étamines sans fendre le périgone en grelot de ces fleurs. L'hybridation pourrait, je pense, produire de jolies variétés dans ces végétaux. Le botryoüdes, qui offre déjà des fleurs blanches, joses, bleues, lilas, donnerait encore de nouvelles teintes. Le comosum, que l'on trouve aussi diversement coloré dans les champs, et qui, selon quelques personnes, a fourni l'élégant monstruosum, serait sans doute susceptible de donner encore d'autres plantes, surtoul si on pouvait, en préparant convenablement le monstruosum, c'est-i-dire en retranchant de bonne heure presque toutes se's branches, le foreer de domer quel- 
quies fleurs fertiles ou munies seulement de l'un des deux sexe's.

Lintin, le moschatum et sa variété plus grande, le sessiliftorum et le maritimum, pourraient encore présenter de nouvelles moditications par les croisements.

\section{Gemre Ornithogale. - Ornithogalum.}

Les: Urnithogales sont de belles plantes organisées commue les antres Liliacées, it stigmate simple et obtus, et sur lesquelles I'hifridation peut être tentée aussi aisément que sur les Tulipes el les lis.

Quelyues expèces semblent se grouper en sections nomhrens's "t pourraient peut-être s'hybrider; telles sont d'ahord l'umbellutum, un dame d'onze heures, à fleurs méléoriques, le nutans, l'excupum, le baticum. I' un autre coté, se trouvent les plus helles espèces cultivées : le narbonnense, l'arabicum, le pyramidale, le latifolium, le thrysoides ou aurenm.

Le flarescens et le stachioides, deux variétés du pyrenaicum, forment une autre section qui ne mérite guère d'ètre cultivée. Eufin, les Ornithogales à fleurs jaunes, dont on a fait le genre Gialye'l, en constituent une quatrième, dont les espèces sont assez jolies.

\section{Gemre Ail. - Allium.}

Lees diverse's espèces d'Ail et l'Oignon, qui en fait partie, ont encore des fleurs à six étamines et un ovaire surmonté de trois stiumates plus ou moins longs. Ces fleurs sont nombreuses et rémies en ombelles ou capitules, dont il faut détruire la majeure partie pour rester maître des autres et pouroir leur enlever toutes leurs anthères.

Je ne crois pas, malgré celia, que l'hybridation puisse s'opérer entre les diverse's espèces, dont plusieurs sont de véritables plant's domenent, comme les $A$. üureum, moly, ursinum, roseum, superbum, ete., et encore moins arec le rictoriale, 'jui n'est plas beall el tres-dilférent des antres. 
C'est parnni les espèces ou variétés comestibles que l'on doit tenter l'hybridation en cherchant de nouveaux Poireaux, de nouveaux Oignous, et en essayant de modifier par des croisements bien combinés les saveurs de l'Ail, de l'Échalotte, de la Ciboule; qui font partie de ce grand genre. Ainsi le Poireau A. porrum, qui a maintenant produit des variétés très-grandes, a larges feuilles, pourra sans donte en donner de nouvelles. L'Oignon, Allium c $\left.\alpha^{\prime}\right) a$, a été depuis longtemps modifié, et l'on cultive maintenant le rouge pâle, le paille ou jaune, le blanc hitif, le gros blane, l'Oignon d'Égypte, celui de Nocer'u, l'oiynon patate, etc., variétés qui se croiseraient certainement cusemble et au moyen desquelles on obtiendrait des nouveautés.

Charles Morren rapporte, dans les Aumales de la Société de botanique et d'horticulture de Gand, que le docteur Wiegman fit, dans la famille des Liliacées, un vrai tour de force en produisant des hybrides Oignons-Poireanx. Il sema dans le mème parterre des Oignons et des Poireaux, et, les fleur's onvertes, il lia ensemble les têtes fleuries de ces plantes. Les graines donnèrent des plantes intermédiaires pour la forme et le goût, et les progénitures de ces hybrides lurent elles-mêmes lertiles. On peut dire ainsi que Wiegman créa un légume nouveau. Il est heureux pour ceux qui n’aiment pas les Oignons el qui détestent les Poireaux qu'un tel légume ait disparu des potager's, mais, au point de vue botanique, il eût été bien curieux de s'assurer de sa permanence.

Les A. sativum ou Ail ordinaire, ascalonicum, Echalotte, fislulosum et schonoprasum ou Ciboules, varient aussi par la cullure el pourraient produire encore des variétés nouvelles. P'eutitre l'ampeloprasum, le niyrum et sa variété magicum pourraient-elles servir aussi à croiser les races cultivées et à les améliorer encore. Il y a toutelois un obstacle qui se présente quelquefois pour la lécondation de plusieur's espèces d'Allium, obstacle qui se retrouve dans plusieurs autres Liliacées; c'est li présence des bulbilles au lieu de graines; mais parfois on trouve, au milieu de ces bulbilles, quelques lleurs qui peuvent 
au moins dommer du pollen, si mème elles ne sont pars entierement fertiles.

Comme pouvant former de nouvelles plantes potagères, l'Ail mérite toute l'attention des horticulteurs.

\section{FAMILLE ])ES COLCIICACEES.}

\section{Genre Colehique. - Colchicum.}

C'est à l'automne que les Colchiques fleurissent, et, quoigue leur's capsules ne paraissent qu'au printemps suivant, c’est au moment de la floraison ruiil faut opérer la fécondation. Ils offreut six étamines, qüil e.t lacile d'enlever, et trois styles très-longs, terminés par des stigmates simples recourbés.

C'est le C. antumule, si commun dans nos prés et qui a domné de belles variétés dans les jardins, qu'il faudrait choisir pour porte-graine. Il s'hybriderail avec ses propres variétés, et on pourrait essayer aussi le variegulum, le montanum, l'alpinum et le persicum.

\section{FAMILLE DES MUSACELS.}

\section{Genre Strelitzias. - Strelitzia.}

Les Strelitia ont me organisation tres-curieuse; deux des divisions du périgone, presque tonjours d'un heau bleu, se réunissent ef laissent entre elles un sillon profond où se tromsent placées cinq étamines, dont les anthères sont très-rapprochées, et forment une espece de tube traversi par le style et le stigmate.

Je ne sais pas sil est possible d'hybrider ces plantes, dont deux ou trois espèces seulenent lleurisent dans nos serres, mais on peut an moins employer la fécontation arlificielle avee leur propre pollen pour avoir des graines fertiles. Iteja .I. Ar. Brongniart et M. Delaire avaicut recueilli, par ee moyen, des Eraines fertiles du Strelitzil reginat. En fécondant celte mène: espèce, jai obtenu aussi de magniliques graines parfaitement 
404 FÉCONDATION NATURELLE ET ARTIFICIELLE.

mùres. J'ai opéré sur deux plantes : dans l'une j’ai employé la liqueur nectarifère qui sort en très-grande quantité de la base des fleur's, pour imbiber le stigmate, sur lequel jai ensuite posé le pollen. Dans l'autre, j’ai évité au contraire l'action de cette

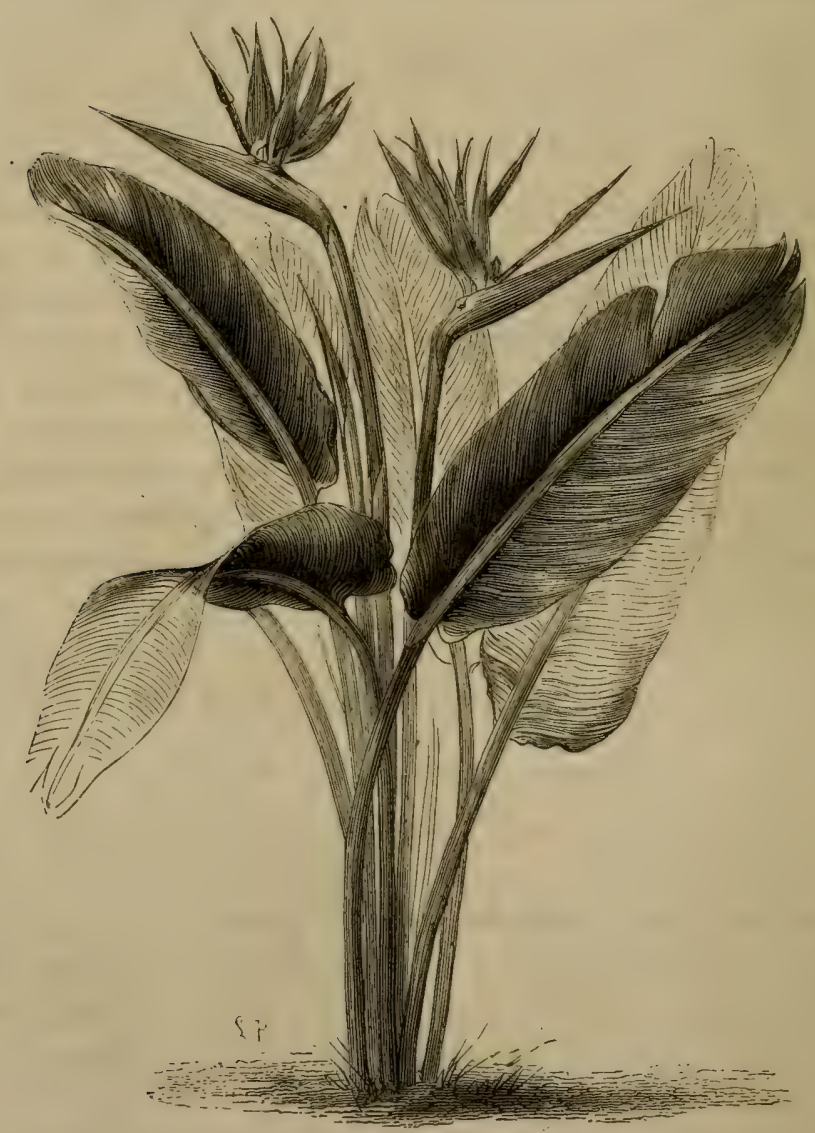

Grav. 105. - Strelitzia reginæ.

liqfueur, que jai lait écouler et que j’ai empêchée d'imprégner le pistil, par de petits tampons de coton que j’ai placés au lond de lafleur; et cette dernière plante, dont le stignate a été inondé de pollen, n’a pas fruetifié (grav. 1(15). 
Voici ce que mon savant et regrettable ami, Charles Morren, écrivait au sujet du Strelitsia. "Lhorticulture belge a produit de nombreuses et remarquables variétés de Strelitziı, rariétés qui ne sont citées par ancun anteur et que nos horticulteurs regrardent comme de rrais hylbrides. Nous citerons les S. rutilans, imperialis, anrora, citrina, vitrea, etc., pour exemples. Il est à noter une le pollen du Strelitzia est singulièrement construit, en ce que, sphírique, sa membrane externe est fort épaisse, finement ponctuée et ne se détache pas de l'interne. Le fait est que nous avons vu obtenir de semis des variétés très-diverses du S. regginx, qu'on nous représentait comme aỵant èté fícondé par le S. angustifolia, le S. parvifolin (juncea) et le $S$. humilis. Nons ajonterons que si les origrines sont exactes, elles prouveraisnt le fait généralement admis sur te continent, mais que M. Ilébert met fortement en doute pour l'Angleterre, à savoir, que les plantes hybrides tiennent leur forme de leur mère et la couleur du père. Tous ces Strelitziu ont en effet l'allure du S. reginx, et ce n'est que pa: la coloration des feuilles, des bractées, du périanthe et de l'anthère, yue ses fleurs diffèrent d'une manière si riche et si étésante. Annules de la Société d'atriculture et de botanique de Gaiml, t. I, p. 41.9.)

\section{FAMILE DES COANELES.}

\section{Genre Balisier. - Camnir.}

Le genre exotique des Balisiers est remarquable par son éta. mine solitaire, dont l'anthère est placée sur le bord du filet, ut par son sț̣le en langunette, terminé par une arête papillaire qui en est le stigmate.

Le C'tmna indica, qui est le plus répandu, donme très-facilemont des graines, ot il en est de même de plusieurs autres espices quand on les cultive en pleine terre, comme les Duhlitu. C"est alors seulement que lemr floraison est ahondante, et l'on 


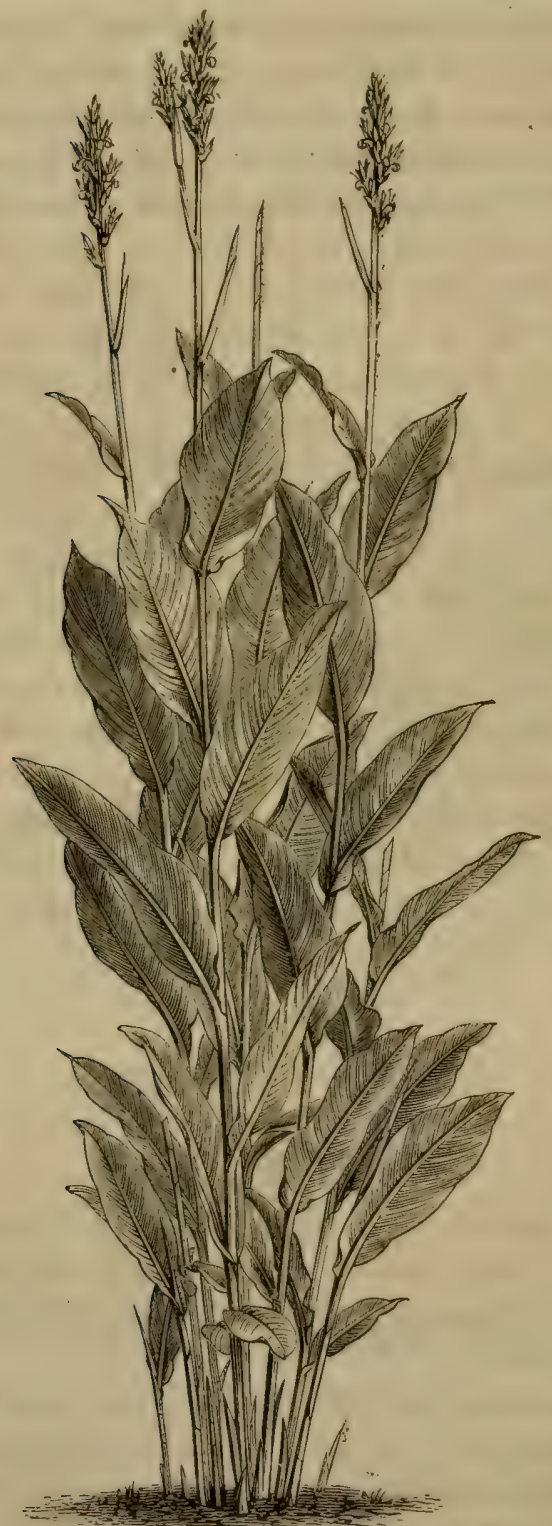

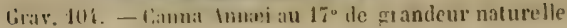


FAMHLE DES CANNEES.

pommait très-certainement les hỵbrider. Les C. iridifora, son

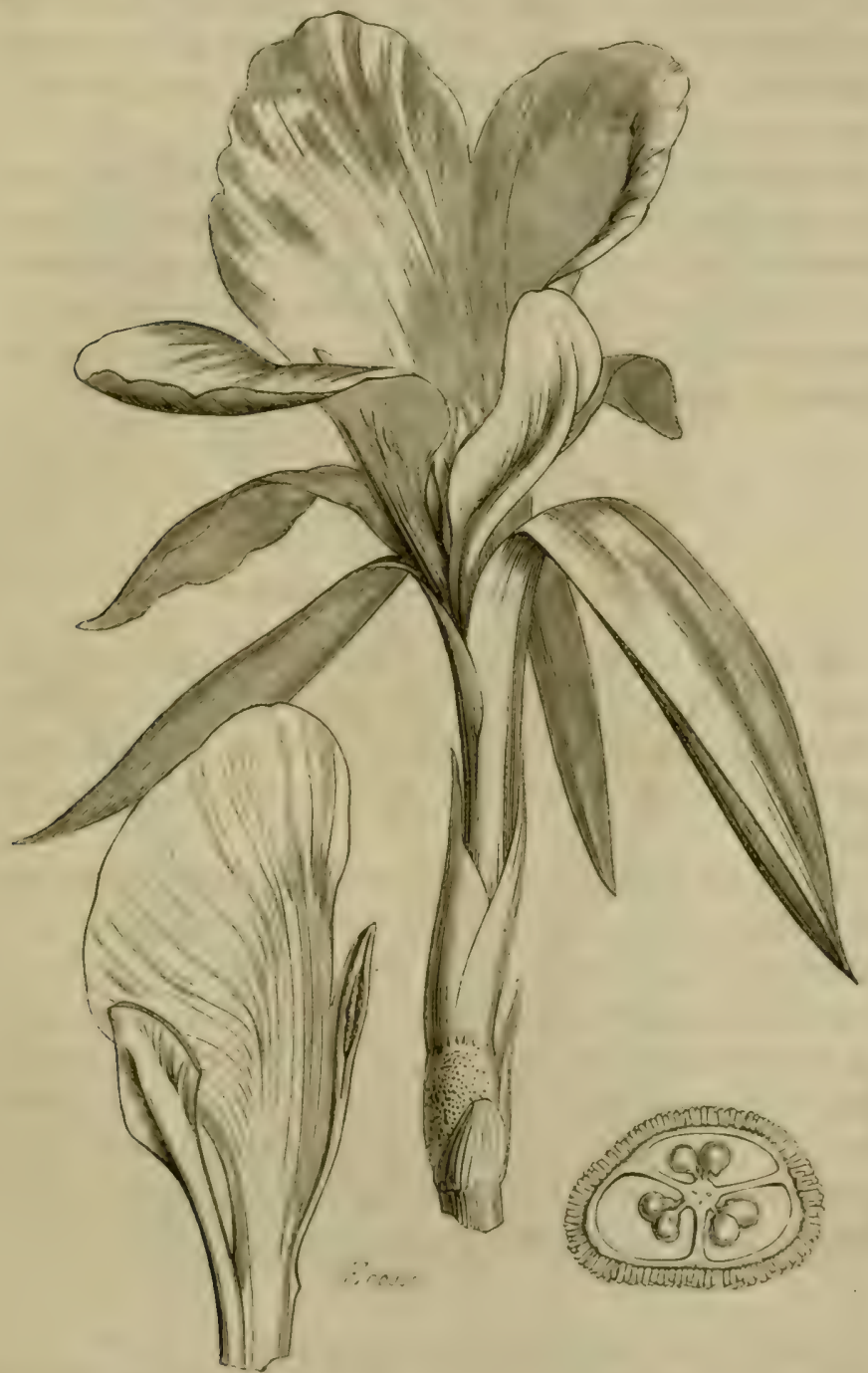

Giav. 10:3. - Fleur du Canna flaccida de grandeur naturelle. - Fragment pétaloîde: asec l'trtamine et le pistil. - Coupe d'unjoraire jeune.

yuimea, speciosu, albiflora, lutea, limbutu, ete., se croiseraient 
très-probablement, et le temps n’est pas éloigné où les Balisiers deviendront de belles plantes de collection comme les Roses trémières, les Asters, les Iris, etc. Celui qui s'occupera sérieusement d'hybrider ce beau genre ne peut manquer d'ohtenir de nombreux succès.

Ce que nous annoncions comme possible en 1845 s'est pleinement réalisé dans ces dernières années. Les Camna, organisés sur le même type, se sont parfaitement croisés et ont donné de bien beaux résultats. Dans quelque temps il deviendra impossible de remonter à l'origine des types comme dans les Fuchsia el dans les Pelaryonium (grav. 10), 105 ).

\section{FAMLLE DES COMMELINEES.}

\section{Genre Éphémère. - Tradescantia.}

On cultive en pleine terre le $T$. virginica, le splendens, le rosea et les variétés blanche et à fleurs roses doubles de la première especce. Un en trouve dans les serres un assez, grand nombre : tels que o discolor; le versicolor, le zebrina, le fuscata.

Toutes ces plantes on! six étamines et un style terminé par un stigmate à trois angles. Les fleurs s'ouvrent le matin et se relerment le jour mème pour ne plus s'ouvrir, en sorte que si l'on voulait tenter l'hybridation, ce serait dès l'épanouissement qu'il faudrait enlever les anthères et opérer de suite la fécondntion. Il n'y aurait guère, du reste, que des variétés du virginicu que l'on pourrait croiser; mais la fécondation artificielle permettrait peut-être de faire fructifier plusieurs espèces qui ne donnent pas de graines dans nos serres.

\section{FAMILLE DES AROÏDÉES.}

Comme uous l'avons fait déjà pour les Orehilées, unus ne pouvons domer que quelques notions générales sur la féconda- 
FAMULIE DES AROIDEEES.

tion de la singruliere famille des . Proidées. Elles sont moins multiplices dans la nature que les Orchidées, et ausi moins cultivies dans nos serres, où quolques especes cependanl tienuent

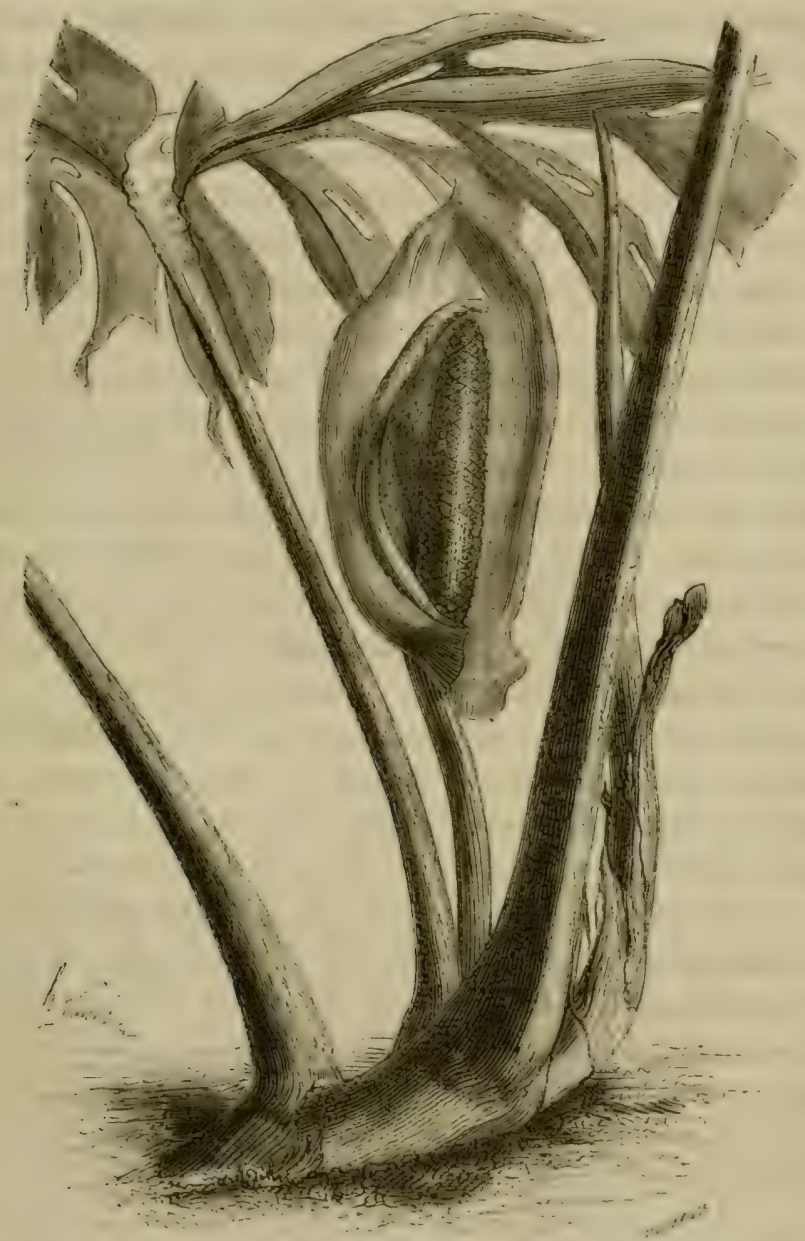

Cirav. Aor, - Intlorescence tlu l'hilodendrum perlusum (famille des .Irö̈lées.) In rang dislingur par leur leaute ef leur leuillage ef la singularite de leurs fleurs. (Ouelques-unes fleurissent, ot peu d'ontre olles fructifient, it l'exerplum des indigenes, comme coptains 
Arum et le Callu. Il est très-probable que, par la fécondation artificielle, on ferait fructifier la plupart des Aroïdées exotiques dont nous pouvons obtenir la fleur. La monoëcie existant ordinairement, ou plutôt les fleurs mâles et femelles étant réunies dans une même spathe, ce que nous avons dit jusqu' à présent des movens employés dans ces diverses circonstances est plus que suffisant pour que l'on opère sans peine l'hybridation on la fécondation artificielle sur ces plantes. Il doit y avoir cependant un moment à choisir pour la pratiquer, car on sait que dans plusieurs de ces végétaux l'anthèse est indiquée par un développement de chaleur assez considérable, et qui se maintient pendant plusieurs heures. N'ayant eu occasion d'étudier aucune Aroïléc de serre chaude, et n'ayant opéré la fécondation que sur le Calla ethiopica, qui a bien fructitie on pleine eau, dans un bassin, je ne puis donner que ces indications générales, et engager ceux qui sont placés dans des circonstances convenables à étudier ce mode de fécondation et à collectionner ces plantes, qui, sans doute, s'accommoderaient parfaitement de la serre à Orchidées (grav. 106).

Les insectes concourent puissamment à la fécondation dr plusieurs Aroïdées. L'odeur infecte que plusieurs espèces rípandent lorsqu'elles fleurissent attire ceux de ces animaux qui ont l'habitude de vivre de viandes corrompues. Les poils rui garnissent souvent l'entrée rétrécic de la spathe dans larquelle les fleurs mâles et les fleurs femelles sont enfermées, tout on permettant par leur fiexibilité l'entrée de ces insectes, s'opposent souvent à leur sortie, et la fécondation s'opère par les angoisses des pauvres prisonniers.

\section{FAMILLE DES GRAMINEEES.}

Cette famille, une des plus nombreuses et. la plus utile du règne végétal, contient, indépendamment de nombreuses espèces d'ornement jour nos jardins, plusicurs gemes qui sont 
l'objet d'une grande culture et qui courrent uns campagnes. Les céréales et une partie des plantes fourragères appartiennent à ce groupe si remarquable.

l.es Graminées, devant servir de nourriture aux hommes et aux animaux, ont été organisées de telle manière que la fécondation e'st tonjours certaine, et que, par conséquent, les graines sont toujours fertiles. Les étamines, dont on trouve six dans le Riz, sont presque partout an nombre de trois seulement, rarement deux, dans les différents genres. Quelquefois les anthères, portées sur de courts filets, restent dans l'intérieur des icailles florales; d'autrefois, et le plus souvent, les filets sont longs, les anthères vacillantes et pendantes comme dans le Seigle, et ce ne sont point les étamines de la fleur qui fécondent son pistil, mais celles de la fleur supérieure et quelquefois relles qui sont les troisièmes ou les quatrièmes dans l'ordre de leur succession en hauteur.

Les pistils sont formés d'un ovaire simple, surmonté de deux ì trois sligmates plumeux, en forme d'aigrette on de pincean, dont les divisions sont droites, obliques, inclinées ou tout à fait pendantes. Le déreloppement de ces organes n'a pas toujours liéu en même temps; mais le plus ordinairement cependant il est simultané. Enfin, on trouve dans cette même famille, et quelquefois sur le même pièl, des fleurs hermaphrodites, màles, femelles et neutres.

La fécondation artificielle, et surtout l'hybridation, n’a, pour ainsi dire, pas été tentée sur la famille des Graminées, mais souvent elle s'est opérée naturellement et sans le secours de I'homme. Il ne pouvait en être autrement pour des plantes que l'on cultive ensenthle, trìs-rapprochies sur d'immenses étendues, et dont le pollen lisse et léger est si ficilement importé par le vent.

Il est à désirer pour l'agriculture que l'on s'occupe de croisements dims plusieurs grenres de cette fimille. On ne rencontremait guire d'autre difficulté que celle d"isoler les port'graines, ce yui pourrait se faire en les cultivant séparément 
dans les jardins. On peut aussi coiffer l'épi pendant quelques jours avec un cornet de papier que l'on serre légèrement sur la tige. La castration exige aussi beaucoup de soin. C'est souvent à quatre heures du matin que les étamines des Graminées sortent des enveloppes de la fleur, et il est essentiel de les enlever de suite, car elles répandent leur pollen immédiatement après. Il faut, en outre, supprimer avec les ciseaux une partic des fleurs de l'épi ou de la panicule, et conserver de prélérence celles de la base ou du milieu.

Enfin, les plus grandes précautions sont nécessaires pour ne pas se laisser voler par les oiseaux les graines que l'on a pris la peine d'liybrider.

Les procédés d'hybridation étant les mêmes, nous ne citerons qu'un petit nombre de genres de celte intéressante famille.

Genue Matis. - Z $Z \ell$.

Le Mais ou Blé de T'urquie a ses fleurs monoïques, les mâles disposées en panicule au sommet des tiges, et les lemelles en épis latéraux, munis de très-longs styles tout couverts de stigmates papillaires. Ces styles pendent le long de la tige et recueillent. sur leurs papilles le pollen qui descend par son propre poids des panicules terminales.

Rien de plus facile que d'hybrider entre elles les nombreuses variétés de cette céréale; il suffit d'abattre le sommet de la tige du porte-graine avant le développement de la panicule, et d'apporter ensuite sur les épis femelles, quand ils sont entièrement léveloppés, des grappes de fleurs mâles que l'on sccour sirr les styles.

J'ai obtenu de cette manière des épis de grains qui offraient plus de sept à huit variétés mélangées à la seconde génération. J'ai hrbride le rostrata avec le jaune et le rouge ordinaire, at ijai détruit son bee; enfin, il n'est aucune variété de celte belle plante qui ure change eneore par l'hybridalion, soit en variant 
la forme de ses épis, soit en parnachant ses prames ou en permutant sia couleur.

\section{Gienre Sorgho. - Sorylum.}

Les Sorylto, cultivés en Afrique comme céréales, orit les lleurs en panicule divisée en une multitude de pédicelles, dont chacun porte d'ux lleurs, une hermaphrodite et une mâle. Les organes femelles sont aptes avant les anthères; 'n sorte que l'on pourait facilement hybrider ees plantes, et notamment les diverses variétés de l'ulepense, qui est la principale espèce. Cés croisements n'auraient d’intérèt que pour les zones méridionales, où cette plante est cultivée en grand.

\section{Genre Panic. - Panicum.}

Les fleur's des l'anie's, dont plusieurs sont cultives sous le nom de: Millet, sont solitaires sur l'épi ou la panicule, et accompangées d'une fleur neutre, plus ou moins complète, quelquelois remplacée par une lleur inâle. On peut leur appliquer ce que nous venons de dire des Sorgho.

\section{Gente Aroine. - Avenu.}

()n ne cultive ginere que quatre especes d'Avoine: ce sont les A. communis, orientalis, strigosa, nuda, dont les trois premières ont les glumes billores et la derniure trillore. Les lleurs sont presque toujours hermaphrodites, quelquefois monö̈gues ou neulres par l'arortement partiel ou complet des orgimes. Les anthères sont à peine saillantes et les stigmates sont inclus. On combait un grand nombre de variétés de ces I voines et surtont de la premiere espece, et il serail facile de les augmenter encore par l'hybridation.

Un pontail, pour l'Aroine, comme poni lit plupart iles Cériales, pratiquer l'hyghidation en grand, en semant dans le mène chanp un certain nombre de variétés mélangées; les 
414 FÉCONOATION NATURELLE ET ARTIFICIELLE.

graines qui en proviendraient seraient semées ensuite dans un autre champ, et, parmi les descendants de ces plintes, on en trouverait très-certainement un bon nombre d'hylrides avec des caractères particuliers, faciles à reconnaitre et que l'on marquerait pour en conserver les graines isolément. On obtiendrait, par ce moyen, à la portée de tous les agriculteurs, des plantes nouvelles qui pourraient l'emporter sur les autres, soit par leur précocité, leur rusticité, soit par leur rendement plus considérable ou d'autres qualités que le cultivateur saurait apprécier à son point de vue.

\section{Genre Riz. - Oriwl.}

Le Riz cultivé a les fleurs disposées une à une; elles ont six étamines et deux stigmates plumeux. On en connait un grand nombre de variétés distinguées principalement par la couleur de leurs graines blanches, rouges, noires ou jaunes. On pourrait, comme pour les autres Graminées, en multiplier les modifications à l'infini, ce qui serait impossible sous notre climat.

\section{Genre Froment. - Triticum.}

Les froments ont ordinairement deux ou trois lleurs dans lat mème glume, et elles sont hermaphrodites. On en connait maintenant plus de quatre cents variétés produites par de vériLables espèces, races ou sous-espèces distinctes, des hybridations et des modifications de climat et même de culture. Nous ne nous y arrêterons pas, et nous renverrons aux travaux de Seringe et de Philippar, qui se sont occupés avec talent de la classification très-dilficile des céréales, et notamment du Blé. Nous croyons que les Froments peuvent ètre hybridés comme les autres Graminées, soit en s'occupant d'un épi isolé, soit en semant à la volée et en mélange les espeices ou les races que l'on se propose de eroiser. Dans le premier cas, il faudra, dies la veille de l'épanouissenıent, entr'ourrir adroitenent les balles de la fleur et enlever légèrement les anthères suns toucher les 
papilles très-1leticales du stigmate. Le lendemain, de bonne heure, on posera le pollen et l'on supprimera an moins la moilié supérienre de l'épi. Il est inconcevahle qu'une opération si simple, et qui peut aroir de si grands résultats, n'ait pass été escilyée sur la plante gui nourrit une partie du genre humain.

Il y a toutefois à remarquer que les Triticum offrent deux races distinctes yni semblent trop diffërentes pour s'hybrider. Ca sont les blés nus, tels que le vulyare, le durum, l'hybernum, ete., et les Blés enveloppés, comme l'Épeautre, T'. speltu, le mono et le diccocum.

Loiseleur thesiongchamps prétenid que les pistils du froment sont liécondes a huis clos, avant l'ouverture des lleurs, par les étamines des fleurs déjà ouvertes.

\section{Gience Esilope. - Eyilops.}

Lat question de lat transformation de l'Eygilops en l'roment, sonlevée par II. Lisprit Fahre, d'Agde, a appelé l'attention sur ce genre de plantes, et l'on a recommu que les digilops pouvaient être parfaitement fécondés par le Blé. Ainsi, l'Égilops ovalu, croisé par un liroment barbu, a donné à plusieurs botanistes une plante désignée sons le non d'E. spelteformis, verrilible hybride fertile, lequel, pendant de nombreuses généritions, reproduit toujours la plante hybride. M. Fabre a cultive frendiant singt ans de suite cet Egilops sans quili se soit modilié.

IIN. Yilmorin el Gr'wuland ont lécondé l'Éyilops ventricosı par le pollen diune variéte barbue du Triticum saticum. Ils n'ont obtenu qu' une seule plante hybride.

"Si nous comparous notre hybride it sa mère, disent-ils, it l'Eyilops ventricora, nous voyous que ce est surtout son épi qui l'éloigne de cette dernière plante, ct qui le rapproche du prire. Iess épillets de l'.Eyilops ventricosa sont fortement renhlés, ventrus vers leur base. " Cette plinte est restée sterile.

II. Cindron a liat de nombreuses expériencess sur la lécondit- 
Lion de l'Éyilops triticoides par le Blé. Il en a obtenu l'E. E. speltaxformis, semblable à celui cullivé par M. Fabre, non-seulement par les organes de la floraison et de la végétation, mais aussi parce que ce prorluit hybride de seconde génération est fertile par lui-mème. II. Planchon (Bulletin de la Société de botanique) voit dans cette observation plus que la conquête d'un fait. "C'est, dit-il, un noureau triomphe du principe de l'induction, auquel les sciences d'observation doivent leurs progrìs. Il faut bien dire, en effet, malgré le désir d'éviter toute polémique irritante, que M. Jordan, en soutenant la cause des hypothèses dans une question d'expérience, s'est préparé d'inévitables mécomptes. D'abord, il a nié que l'ÁEjlops triticoides sortît du même épi que l'Éfilops ovatu; vaincu par l'évidence, il à dû reconnaître son erreur. En second lieu, il a mis en doute l'hylnidité de l'Eyilops triticoides. Nouvelles preuves d'une part, nouvelle délaite de l'autre, et nouvelle pitlinodic. M. Jordan se retranche alors derrière un nouveau rempart d'hypotheses. Il soutient que l'Egilops-blé, de Fabre (Lyilops speltaformis Jord.) est une espèce légitime qui ne dérive en rien de l'Aggilops triticoides. Or, cette prétendue espèce, M. Godron vient de l'obtenir de l'Ágilops triticoides, lécondé par un Froment.

"Depuis lors, M. Groenland a continué ses études sur les hybrides d'AEyilops et des Froments, hybrides qui se perpétuent depuis longtemps par leurs graines fertiles, el ces expériences ont prouvé : l'existence d'une plante hybride devenue fertile sans ètre retournée au tịpe d'un de ses parents. ") (Bullotin de la Société botanique de France, t. VIII, p. 614.)

\section{Gene Seigle. - Secale.}

Les Seigles different des Froments par leurs lleurs réunies deux à deux el par une troisième fleur stérile située au som.* met de l'épillet.

Toutes les variélés cultivées appantiennent à la méme especen, 
le $S$. cerenle, dunt les hybrides ne seraient pas plus difficiles :i obtenir que ceux du liroment.

\section{Gesme Orye. - Ilordeun.}

Le's Orges cultivées ont les fleurs hermaphrodites, it l'exceplinn de quelques fleurs latérales qui sont màles. On en commait quatre espèces, I'hexustichum it six rangs, le vulgare, également a six rangées, le distichum et le zeocriton, gui n'ont tous deux yue deux rangs. Plusieurs de ces espèces ont déjà donné des variétés distinctes, telles que les Orges nues, mais il ne parait pas 'fu'il existe d'hỵbrides bien marqués entre les espèces. Il y aurait des essais à faire pour chercher ces croisements, et lOrge semble, sous le rapport de ses modilications, moins avancée que le Froment, bien que l'on ignore sil patrie ct son origine, connne celle de presque tontes les céréales, qui sont peut-être des modifications créées par l'homme, et dont les types sont devenus méconnaissables.

\section{LAMILLE DES LOUGERLS.}

Voici ce qui on lit dans le Journal d'Agriculture de M. Bixio : "I'armi les moyens les plus puissants de développer le pousuir 小e l'hornue sur les régétaux, l'hybridation étome de plus en flus lohservateur par l'étendue illimitée de ses eflets; en Allemingue, II. liegel croit avoir obtenu des hybrides diuns une fitmille de plantes où la possibilité des croisements semblait dillicile it admeltre, tant ses organes reproducteurs sont imparfaitement connus! Le fait mérite cependiant d'être constaté. II s'igit de la famille des Fouyères.

" On a longtemps dédaiqué d'accorder, dans nos serres, unc filice it ens plantes an feuillage éligant, mais dépourves de fleurs. Anjourd'hui nous niarons pals une serre chame yui niadnetle, rn raison de l'inlinie vantété de leur élégant fernitlinge, les fougeres des contrées intertropicales. On sitt com- 
bicn ces contrées sont riches en Fougères; on trouve daus l'Australic des forêts de Fougrères arborescentes dont les tiges, grosses comme des troncs d'arbres, n'ont pas moins de douze a quinze mètres d'élévation. Les Fougères exoliques sont donc en ce moment en grande faveur. Or, l'observation constate pour le genre Gymnogramma, l'un des plus répandus, que dans les serres où une seule est cultivée, clle se reproduit identique à elle-mème: tandis que si plusieurs espèces sont cultivées côte à côle, il se produit des espèces ou variétés intermédiaires, que II. Regel n'hésite pas à considérer comme des hybrides.

" En Angleterre, M. Ilenderson a observé les nêmes faits, el quoiqu'il ne se prononce pas si ouvertement que M. Regel, quaut à l'hybridation, il est aisé de voir qu'il ne trouve pas d'autre cause probable à assigner à ces phénomènes.

" I'our nous, horticulteurs, dit V. Ysabean, il nous sulfit de constater l'existence du fait et de chercher à en profiter en produisant, sil est possible, de nouvelles espèces et variétés de Fougères, dont les physiologistes auront plus tard à nous expliquer rationnellement la production. ")

Nous n'oserions pas supposer qu'il existe quelque procédé pour hybrider les Fougères, mais l'on croit cependant avoir reconnu quelques hybrides parmi ces plantes.

L'Asplenium Breynii Schwarz a été considéré comme un hybride de l'Asplenium ruta muraria et de l'Asplenium septentrionale.

Bory de Saint-Vincent a publié, sous le nom de Gymmnogramma Martensii, une espèce née dans les serres de Louvain, et qu'il considérait comme un hybride des G. calomelanos et G. chrysophylla. La même Fougère hybride a été retrouvée par le docteur L'Herminier dans les bois de la Guadeloupe. La couleur de celte jolie poussière, répandue sur la face inférieur'e de cette éclatante Fougère, tenait le milieu entre le mat de l'ar'gent et Te brillant de l'or métallique.

Cependant le moyen d'hybridation que recommande Bor'y, et „qui consiste à seconer une fronde fructifiée sur celle d'une autre 
"spece, m peut antener ancun résultat. Les fougrire's sont soumises it lat génération alternante et à de curieuses métanorphoses. Lecurs organes de reproduction ne se montrent que dans le premier àge el avant l'érolution des frondes, dont la liace inférieure ne supporte que des gremmes on des bulbilles, et non de véritables graines.

Nous avons voulu, par celle note, signaler la possibilite de l'existence des hybrides dans les fougeres sans avoir la prélantion d'indiquer un mode pratique de les obtenr.

Nous eroyons qu il faut abandonner an hasard, qui a créé les premières Tulipes, ainsi quiune partie des nombreuses variétés yui décorent maintenant nos jardins, le soin de laire naître itussi les premiers hybrides de Fougères; e’est, je crois, la seule concession que nous lui ayons faite daus tont le cours de motre travail; mais nous reprenons immédiatement nos droits de médiateur, et nous ne pouvons mieux terminer notre tiche qu'en enuruntant les dernières lignes au spirituel al savant rédacteur de la Calronique horticole du Journul d'ugriculfure.

"Nous ne saurions trop engiger los horticulteurs de prolession et les amateurs à essayer toute sorte de croisements hylrides. La joie et l'honneur que procure la conquête d'une hylride nouvelle, nimporte en quel genre, sont au nombre des plaisirs les plus purs que puisse procurer la culture des fleurs.n

J'ai dit tout ce que je savais, tout ce que m'ont démontré lit pratique et de longues observations; jespère apprendre encore, mais les hommes qui cultivent les fleurs ou qui s'adonnent a la recherche des fruits, ceux qui espèrent des nouveaulís dans les légumes et les produits utiles de nos jardins, les forestiors qui voulront marcher dans une voie nouvelle, et les ayriculteurs qui chercheront à itendre leur domination sur des plantes encore inconmues, frouveront peut-ètre dans ce travail quelques documents que je suis heureux de leur offirir. 



\title{
TABLE ALPHABETIQUE
}

\section{DES NOMS DES PLANTES DÉCRITES DANS LE COURS DE L'OUVRAGE}

\author{
L.es noms des familles sont en pelites carrat.es, les noms français sont on \\ caractìres orlinaires et les dénominations latines en italique.
}

Alies. 3i2.

Alricotier, $16 !$, Abutilon, 157.

teacia, 166. Acricin, 166. Aclie, 22\%.

Achiménès, 251. Achimenes, 251. Aconit, 9y. Aconitum, y9. Ailonis, 89. Adonis, 89. Assculus, 138. Ail, 401. Alisier, 191. Allium, 801 . Aloès, 598. Aloes, 398. Alserameria, 585. Allixen 1:y4. Amandier, $16 \%$.

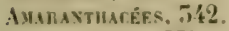
AMAIYI.IDFE, $37 \mathrm{iJ}^{\circ}$ Amaryllis, 57\%. Amaryllis, 57x. AvFrticées, Amygdalus 167. Anayallis 201 Anamas, Tig. Ancolie, 47. Andromeda, 25r.
Androniede, 255.

Anémone, 86 .

Anemone, 86 .

Antholi:a, 572.

Antholize, 57t.

Axтиหmisegs, 280.

Anthyrrinum. 280 .

1 pium, $22 \overline{3}$.

Arocivérs, 267.

Aquilegiu. 97.

Arabis, 115.

Aralia, 225.

Aralie, 295.

InALIACÉEs, 20).

Arhousier, 25!.

Arbutus. 25 '.

Arin. 191.

Aristoloche, 329.

Aristolochia, 529.

Anistoloché, 5\%!?.

Armoriaca, 169).

Anoint:es, 408.

Artichant, "4t.

Arum, 408.

Asclépiade. 267.

Asclepias. 267.

Aspanagistís, 786.

Asparagus, ixti.

Isperge, 786.

Aster, 2500.

Asler, '250.

Astrance, 225.

Astrantia. 29.
Aubépine, 184.

Aubergine, 277

Auricule, 292 .

Aveline, 349.

Arena, $41 \%$.

Avoine, 415.

$A=a l e a, 257$.

Azaliee, 957.

I8

Balisier, 40\%.

Balsamina, 146.

Balsamine, 14ti.

Banksia, 5,9.

Banksie, 5ริ).

Begonia, 520

Begonia, 320.

liègosuch́fs, 780 .

Belle-de-muit. ה100.

Bellis. 25.5.

Benoile, 175.

Jifrnéromifs, Jefr.

Berberis, 106.

Bergamolte, lit.

Belia, 517.

Bette, 317.

Bellerave. 317

Betula. 34.

Bigaraile, 1/7.

Bisnone, 272.

Bifmonia, 272.

liovoviarfirs. y2. 
Blé, 414.

Blé noir, 318.

Blé de Turquie, 12.

Bois gentil, 528 .

Boronia, 152.

Boronie, 152.

Bonragtú́es, 274.

Boule de neige, 226.

Bouleau, 515.

Bouquet parfait, 128.

Brassica, 118.

Bromelia; 585.

Bnoséliacéts, 585 .

Brugmansia, 277.

liruyèce, 250 .

linisson ardent, 184.

Ciactêes, 205.

Calanthe, 359

Cilcéolaire, 285.

Calceolaria, 28.̃.

Calebasse, 19 วั.

Calendula, 240.

Calla, 410.

Calycanthe, 192.

Calycantmées, 192.

Calycanthus, 192.

Camellia, 149.

Camellia. 14\%.

Ciavéliées, 149.

Campanula, 2 15.

Campanule, 245.

Campanulacées, 245.

Canna, 405.

Cannabis, 534.

Caxyées, 405.

Capparis, 121.

Capparidées, 121.

Caprier, 121.

Caprifoliacées, 226.

Capucine, 144.

Cardamine, 115.

Cardon, 245.

Carolte, 221.

CismyophyLéf:s, 128.

Casse, 166.

Cassia, 166.

Castanea, 346.

Catalpa, 272.

Catasetum, 560 .

Catlheia, 5 59.

Cédrat, 147.

Céleri, $22 \overline{5}$.

Celosia, 515.

Célosie, 515.

Centaurea, 240.

Centaurée, 240.

Cerasus. 170.
Cereus, 208.

Cerisier, 170.

Cestre, 277.

Cestrum, 277.

Chanécerisier. 296.

Chanvre, 354.

Châtaignnier, 546.

Chêne, 546 .

Chêne d'Égyple, 237.

Cuéxopodées, 517.

Chevrefeuille, 206 .

Clicorée, 241.

Chicorium, 241:

Chou, 118.

Chou-fleur, 118.

Chou-rave, 118.

Choryะema, 155.

Choryzème, 155.

Chrysanthème, 257.

Chrysanthemum. 2:7.

Ciboule. 401.

Cierge, 208.

Cinćraire, 240.

Cineraria, 240.

Cisle, 126

Gistuxées, 126.

Cistus, 126.

Citronnier; 147.

Citrouille, 198.

Citrus, 147.

Clematis, 82.

Clématite, 82.

Cléome, 122.

Cleome, 122.

Clitoria, 157.

Clitorie, 157.

Coignassier, 191.

Colchicacées, $40 \overline{\text { t. }}$

Colchicum, 405.

Colchique, 405.

Coloquinelle, 199.

Coloquinte, 199.

Colzi, 118.

Comathlnées, 408.

Comrosées, 250.

Concombre, 195.

CoNifìres, 349.

Consoude, 275.

Convallaria; 587.

Convolvolacées. 27i.

Convolvulus, 27.̄.

Coquelicot, 111.

Coreopsis, 257.

Coreopsis, 257.

Cormier, 191.

Corréa, 152.

Correa, 152.

Corydalis, 11:.

Corylus, 548.

Comrye. 198.
Couronne impériale, $\mathbf{7 ( y )}$

Crassula, 216.

Crassule, 216.

Chassulacées, 216.

Cratzyus, 181.

Crinum, 375.

Crinum, 375.

Crocus, 573.

Croix-de-Jérusalem, 152.

Crucifères. 114.

Cucumis, 195.

Gucurbita, 198.

Cucunbitacées, 19 ;.

Cupressus, 500 .

Cyclame, 297.

Cljclamen, 297.

Cydonia, 191.

Cynara, 244.

Cyprès, 550.

Cytise, 155.

Ciftisus, 155.

Vahlia, 254.

Dahlia. 254.

Dapliné, 327.

Daphne, 527.

Datura. 277.

Dancus, 224.

Dauphinelle, 98 .

Delphinium, 98 .

Dentelaire, 298.

Desmanthus, 166.

Dianthus, 128.

Dielytra, 113 .

Digitale, 281.

Digitalis, 281.

Diplacus, 285.

Diosma, 151.

Diosma, 151.

DiPsáćEs, 228.

Dodécathéon, 296.

Dodecatheon, 296.

Uracocéphale, 288.

Dracocephalum, 288.

Echalotte, 401.

Echinocacte, 207.

Echinocactus, 207.

Ellébore, 93.

Endive, 241.

Epacris, 263.

Epacris, 265.

Euhémère, 408.

Epimède, 108.

Epimedium, 108.

Epinard, 518. 
Epine, 18\%.

lipine-vinette, $110 \%$.

Erica, 25is.

lincacérs, 254.

Erysimum, 11\%.

Erythrine, 165.

Erythrina, 163.

Erythronie, 59\%.

Eruthronium. 394.

lischolzic. 115.

Escholzia, 115.

liuphorbe, 255.

Euphorbia, $25 \%$.

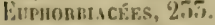

Evon!mms, 15.5.

Faba, 158.

lagus. 515.

Five, 158.

Ficoile, 20.4 .

Ficonnes, 20 \%.

Ficus, 537.

liguier, 357.

Fougieres, 417.

Fragaria. 177.

Fisisier, 177.

liramboisier, 176

Praxinus, 26\%.

lirune, 265.

Fritillaire, 390.

Fritillaria, 390

Froment, \&14.

Fuchsia, 200 .

Fuchsia, 200

V)Manées, 115.

Fisain, 155.

Galanthus, 381.

Genévrier, 550.

liéravié

Geranium, 142.

Gesueria, 267.

liesnerie, 267.

Gesséniées, ¿277.

diesse, 159.

Cineule-de-lion, 2811.

lieum, 175.

limrie, 117.

firrollée, I/k.

firomon, 198.

Giladiolus, 368.

- Ciloxinite, "24!)

(iloxinie, 2 't).

lilaieul, .768.

finidia, 328.

- finidie. $5 \mathrm{~g} x$.
Gimanxíes, 410.

firenadier, $1 ! \mathrm{s}$.

firoseillier, 215.

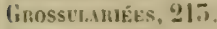

(iuimaure, 17\%.

Gymmo!ramma, 4:8.

Haricot, 161.

Ilélianthème, 128.

IIelianthemum, 128.

Iléliotrope, 27.4.

Heliotropium, 27't.

Ilellébore, 95.

Helleborus, 9:.

Hepalica, 88.

Hépatique, 88.

Hespénidéf, 147.

IIesperis, 117.

Ilêtre, 54:.

llibiscus, 155.

IIbiscus, 155.

IInpocastunérs, 1.78

Ilomsarthe, 576

Homanthus. 376.

Hordeum, 417.

Hortensia, 221.

Houblon, 5isi.

Houx, 155.

Hoved, 155.

llovéc, 155

IIumulus. 550.

Huacinllus, 59!

IIydrangea, 221.

IIjolrangée, 22!.

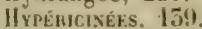

II!)pericum, 159

Ibéride, 117

loeris, 117.

11. 349 .

Ilex, 155.

lpomiea, 275.

InInÉEs. $56 t 5$.

Iris, 765 .

Iris, 505 .

Isopyre, (16.

Ixia, 372.

Ixia. 572

Iacinthe, 549.

Jasuin, 264.

JasuBNÉrs, 20 tit.

Jasminum, stif.

Inularlie, 217.
Jigzannée, 5it!.

Juglans, 700 .

Jujubier, 1:4.

Julienne, 117.

Juniperus, 550.

lialmia, 263.

Kalmia, 2tié

Keunedia, 160.

kemnedie. 160.

\section{I.}

LAdtées, 285

Lactuca, 245.

lagenaria, $19 \%$.

Laitue, 245.

Lantana, 289.

Lantane, 289.

Lavix, 352.

Lathyrus, 159.

Laurier, 329.

Laurier-rose, 271.

LAURIXÉES, 529.

Laurus, 729.

Lavatera, 155.

Lavatère, 155.

LÉGUMIXEUSES, 155.

Lilas, 265.

LuLacées, 587.

Lilium, 391 .

Lin, 153.

Linaire, 280.

linum. 155.

Liriodendrum, 10\%.

Lis, 591 .

Liseron, 275

l.obelia, 246.

Lobélie, 246.

Locheria, 255.

Jonicera, 226.

Lunaire, 115.

Lunaria, 115.

Lupin, 165.

Lupinus, 165.

Luzerne, 156.

Lychnide, 152.

Lyclinis, 15\%.

Magnolia, 105.

M.gxolnacées, $10 \pi$.

Sagnolier, $10 \overline{\text {. }}$

Mais, 412 .

Mahonia, 10 ti.

Mahonie, 106 i. 


\section{4}

Malus, 188.

Malva, 15i.).

Matvacées, 155.

Vamillaire, 20\%.

Mamillaria, 205.

Mandirola, 254.

Jarguerite, 255.

Marronnier, 158.

IIartagon, 591.

Mathiola. 11:.

Ilauve, 155.

Medicago, 156.

Melalenca, 194.

Melalenque, 194.

Melastoma, 195:

MéLastomacées, $19 \overline{7}$

Mélastome, $19 \overline{3}$

Yéléagre, 390 .

Yélèze, 552.

Jélocacte, 207

Melocactus, 207.

Melon, 195.

Melongène, 275.

Mesembryanthemum, 20\%.

Mespilus, 185.

Métrosidéros, 194.

Netrosideros, 19\%.

Mignardise, 128.

Mille-pertuis, 159.

Mimosa, 166.

llimose, 166.

Mimule, 282.

Himulus, 282.

Nirabilis, 500.

Monitcanthus, 3600.

Monarda, 286.

Honarde, 286.

Morelle, 275.

Morus, 53 ذ.

Mouron, 291.

Yuflier. 280.

Juguet, 587.

Mûrier, 350 .

MusacÉEs, 405.

Muscari, 400:

Muscari, 400.

Myanthus. 560

Myntaḉes, 195.

Myrthe, 195

Mịrllins, 195.

Narcisse, 585

Narcissus, 38.3.

Navet, 119.

Navelte, 119.

Nétlier, 185.

Nelombo, 108

Nelumbirm, tos.
TIBLE ALPII IBETIOUE

I Nénufar, 109.

Neottin, 554 .

Nerium, 271.

Nicotiana, 278.

Nigella, 96 .

Nigelle, 96.

Noisetier, 548.

Noyer, 559.

NyctaginéEs, 500 .

Nymphra, 109.

Nท̈rPH FacÉEs, 108

Eillet. 128 ,

OEnothera, 202.

E.vothéRÉks, 200.

Uirnon, 401.

Oléandre, 271.

Olivier, 264.

Olxu, 264.

OMeellifìres, $22 \bar{i}$.

Unagraire, 202.

Onobrychis, 158.

Opuntia, 215.

Uranger, 147.

ORCHIDES, 555.

0rchis, 565.

Orchis, 565 .

Oreille-d'ours, 292.

Orge, 417.

Oryza, 414.

Urine, 542.

Ornithogale, 401.

Ornithogalum. 401.

Orobe, 160.

Orobis, 160.

Orpin, 217.

Oxalide, 147.

Oxalis, 147.

Pronia, 101.

Pancrace, 581.

Pancratium, 581 .

Panic, 415.

Panicum, $41 \overline{\text { s. }}$

Papaver, 111.

Papavéracées, 111.

Pâquerelte, 235.

Passe-rose, 13i.

Passiflora, 122.

Passillore. 12.2.

Passiflonées, 122.

Pastèque, 195.

P.istisson, 198.

Patate, 275.

Pivie, 168.

Pavia, 158.
Pavia, 158.

Pavot, 111.

Pêcher, 168.

Pelargonium, 142.

Pelargonium, 142.

Pensée, 124.

Penstemon, 281.

Penstemon, 281.

Pepo, 198.

Perce-neige, 585.

Perégrine, 585.

Persica, 168.

Persicaire, 518.

Persil, 225.

Pervenche, 271.

Pe-tsaie, 118.

Petunia, 280.

Pétunie, 280.

Peuplier, 545.

Pharbitis, 275.

Phasęolus, 161.

Philadelphus, 194.

Phlomis, 288.

Phlomis. 28s.

Phlox, 272.

Phlox, 272.

Phylica, 154.

Phylique, 154.

Pied-d'alouelle, 98.

Pigamon, 85.

Pimelea, 528.

Pimelée, 528.

P'in, 53 I.

Pinus, 551.

Pisum, 158.

Pivoine, 101.

Planère, 3 :2.

Planera, 342.

Plectopoma, 25i.

P'enabiginées, gegs.

Plumbrgo, 298.

l'oirée, 517.

Poirier, 18:.

Pois, 158.

Pois de senteur, 15!?.

Polémoniacées, 272.

Polygala, 125.

Polygala, 125.

Polygalées, 125.

Polygonées, 518.

Polygonum, 518.

l'omme de terre, 27i.

J'ommier, 188.

Populus, 545.

Portulaca, 20\%.

Pontulackes, 205.

Potentilla. 17x.

Potentille, 178.

Potiromon, ins.

Potiron, 198. 
Pourpier, 20\%. Primevère, 29:. Primula, 292.

P'Ruveacếes, ¿gl Prurkickes, Jîy. J'runier, 170.

Prumus, 170.

Pseudi-acacia. $15 \overline{\text { T. }}$

Puluonaire, 275.

Pulmonaria, 275.

P'mica, 195.

l'yrus, 18ì.

Huaralaim. 114. (luercus, 546.

limlis, I"21.

lianunculus, y1. liaphanus, 121.

Kave, 119 .

Haquelte, 215.

Reine-Jlarguerile. 250 .

lienoxculacees, 8\%.

lienoncule, 91.

lienonée. 518.

liésélar. 126.

lieseda, 120.

Résedunés, 1ㅇ․

liausiées, 155.

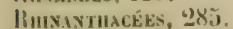

Rhododenirum. $25 !$.

Ribes, "215.

Kiz, \$14.

liobinia, 157.

llobinier, 157.

llochear, 216.

Rochea, 216.

Romine, 2f5.

Honce. 176.

Rosia, 181 .

Kosiches. 167.

liosige, 259.

lio-ier, 181.

liubus, 176.

Rulabiga, 11 \%.

Ituracées, 1 il.

Siffran, 575.

Similoin, 1 ¿8.

Salix, 5 is.

Silsilis, 242.

Sulvia, 285 .
Sapin, 532.

SAnifisacé, 159.

Sarrasiu, 518.

Sauge, 28;

Sanguinaire, 11:.

Sanfuinaria, 112.

Saule, 5/5.

Saxifraga, 22)1.

Saxifiuge, 201.

Sixifnigées, 221.

Scabieuse, 228

Scrabiosa, 288.

Scilla .799.

Scille, 599

Scutellaire, 286 .

Scutellaria, geti.

Secule, 416.

Sedum, 217.

Seigle, $\$ 16$.

Sempervivum, ⒘

Seringat. 19\%.

Sida. 137.

Sirla. 157.

Silene, 151.

Silerie, 131.

Sinupis, 121.

Sisymbrium. 115.

Solaxées, 270.

Solanum, 275.

Sorbier, 191.

Sorbus, 191.

Sorgho. 415.

Sorglum, $41 \overline{5}$

Sonci. 240.

Spinacia, ils.

Spiraa, 171.

Spirée, 171.

Stapelia, 262.

Statice, 298.

Statice, 298.

Stramoine, 277.

Sitrelitzia, 405

Strelit:ia, 405.

Symphytum, 27j.

Srnanthérées, 250.

Syplocampilos, 277.

Syphocampilos, 267.

Syringa, 205.

\section{'}

Talac, 278.

Ta.ens. 5.19.

'Thalictrum, 8.

Thuya, "250.

Thuiya, 250.

Turmilés, 507.

Tiyrilla, э74.
Tiuridic, 574 .

Tilia. 157.

Tuncées, 157.

Tilleul, 157.

Tomale, 275.

Tradescantia, 408.

Tragopoyou, 21․

Truble. 157.

Trevirania, 251

Trifolium. 157.

Trificum, 411.

Trolle, 92.

Trollius, !ly.

Tropocolum, 1/4.

Tulipa, 587.

Tulipe, 587.

Tulipier, 10 '.

Turritis. $11 \mathrm{~b}$.

Tydea, 251.

Ulmus, 5는

UnTicées, Jüt.

landa, Jiti.

Yanilla, 56'.

Vanille, j6it.

Verbena, 289.

Vendiracéts. '289

leronica, 285.

Véronique, 28i.

Verreine, 289.

Viburnum, 2206.

Jictoria, 111.

Vigne, 159.

Vinca, 271.

riola, 12k.

Viomatés, 1ㄴ.

Violelle, 124.

Viorme, 2206.

I'itis, 159.

Ysopirum, 56.

Finces, 59?.

liucra, 59k.

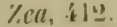

Yisuial, 256.

Vimnia, 256.

Ziz!llules, isi. 



\title{
TRAITÉ DES PLANTES FOUR
}

\author{
DEUXIÈME ÉDITION
}

4

1 vol, in 8 de Бria pages el 40

La Flore des prairies. naturelles et artificielles de France ef de l'Furope centrate.

Description, usage, qualités et cullure de toules les planes qui peuvent servir å la nourrilure des animaux, à la création et à l'entı elien des prairies. gravires. - Pri: Composilion des prai lites du sol. - ch Semis et entrelien animaux et plinte grais et amendem

\section{i'TUES}

SUR LA

GÉOGRAPHIE BOTANIOUE DE L

9 gros vol. avec planches. - Prix * 00 ।

i.

BOTANIQUE POPULAIF

ÉLÉMENTS DE BOTANYUE

:

DIRETES A L'AGRICULTURE ET

I vol. In-18 de 360 pages avec gravures, - Pris

LA VIE DES FLELRS

OU LES MEURS DES - YEEGETA

1 vol. In-18.- Prix : 9 fr. 50

\section{DICTIONNIIRE RAISONYÉ DES TERIIES}

ET DES FAMILIES NATURELI

PAR LECOQ ET JU!LLET

1 rort volume in-8. - Prix $z$ o fr.

\section{EXTRAIT DU CATALOGUE DE LA LIBRAIRIE A}

cercurxume (Cours d'), nar DE Gaspanis, 6 vol, in-8 et $25 \overline{5}$ gravn

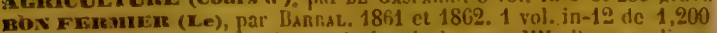

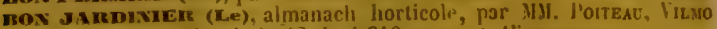
Nebvans, P'érix, 1 vol, in-12 de 1,616 page's et 13 gravures. . . .

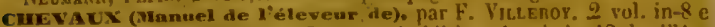

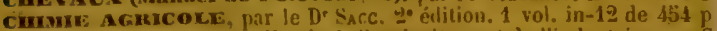
cmome usuexex, appliquée ì l'agriculture et à l'industrie, par $\mathrm{S}$ par Brostreix surla $4^{\circ}$ édition. 1 vol. in-18 de 521 pages et 225 gravt

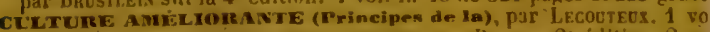

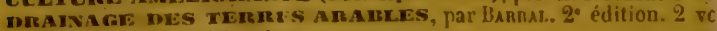

$\$ 45$ gravures el 9 planche's. . . . . . . . . . . . .

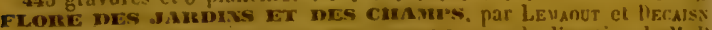

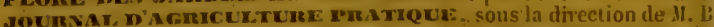

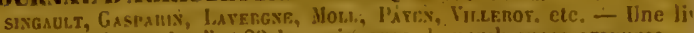
in 4 , paraissant les 5 el 20 du mois. avec te nombreses gravures. -

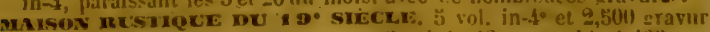

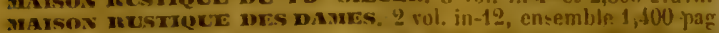

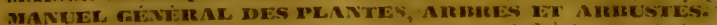
de 20,000 plantes inligènes ou de serre, 4 vol. a 2 iolonnes, . : . . reoularer.i.re (i.e), par Cr. JACQUE. 1 vol. in-12 et 120 gravares. . .

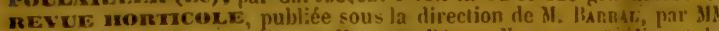

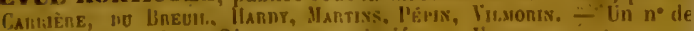
ler pt 16 du mois, et 21 gravures coloriées. - Un an.

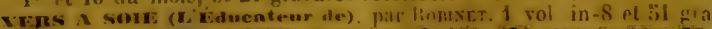

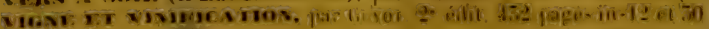




$$
\text { . }
$$





